
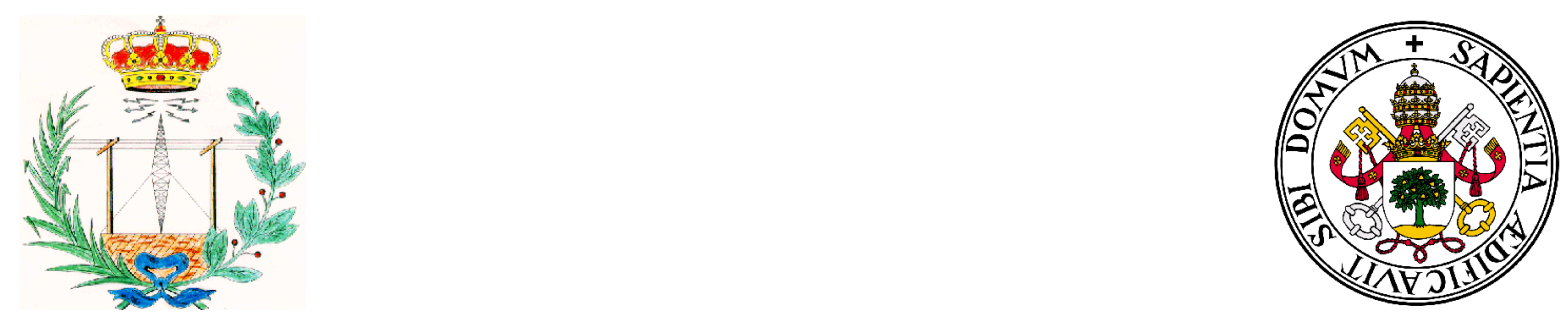

UNIVERSIDAD DE VALLADOLID

Dpto. de Teoría de la Señal, y Comunicaciones e Ing. Telemática

Escuela Técnica Superior de Ingenieros de Telecomunicación

Tesis Doctoral

\title{
Supporting orchestration of blended CSCL scenarios in Distributed Learning Environments
}

\author{
AUTOR \\ Luis Pablo Prieto Santos \\ Ingeniero de Telecomunicación
}

DIRECTORES

Ioannis Dimitriadis Damoulis

Dr. Ingeniero de Telecomunicación

Juan Ignacio Asensio Pérez

Dr. Ingeniero de Telecomunicación

Julio de 2012 



\section{Abstract}

The progressive introduction of Information and Communication Technologies (ICT) in education at all levels has transformed the classroom, adding a technological layer to the already complex environment where learning and teaching take place. Teachers and other actors are expected to incorporate new research-driven technologies and approaches, re-contextualizing them to their own settings and using them effectively towards a number of learning goals. This technological (but also pedagogical) complexity has been recently acknowledged by the TechnologyEnhanced Learning (TEL) research field as one of the main challenges for the upcoming years, under the label "orchestration". This dissertation revolves around this emergent notion of orchestration, identifying three challenges for researchers and teachers, and providing technological and conceptual tools to overcome those challenges. More specifically, the increasingly common educational context of blended Computer-Supported Collaborative Learning (CSCL) practice that uses Distributed Learning Environments (DLEs) has been chosen to analyze and evaluate these tools, as an example of formal educational setting where this orchestration is especially difficult and critical.

The first challenge that TEL researchers encounter when approaching orchestration, is the lack of clarity of such concept, as it is employed by that multi-disciplinar research community with a variety of meanings. Emerging from a deep literature review of orchestration in TEL, we propose "5+3 Aspects", a descriptive conceptual framework to help researchers frame and characterize TEL orchestration, which can be used as an analytical lens to understand how orchestration happens in a concrete educational setting, and as a basis for the development of further research instruments that aim at making TEL innovations more easily applicable in authentic (physical or virtual) classrooms.

The second orchestration challenge we have identified, in this case mainly for TEL teachers and practitioners, is the scarcity of concrete advice that they face (especially non-expert ones), in performing such orchestration in their classrooms. In this dissertation we propose "atomic patterns" as a conceptual tool to help teachers in bridging the gap between researchers' often de-contextualized advice and the contextualized performance that they have to deliver in their concrete classrooms. These atomic patterns are based on the concept of design patterns as a tool to capture expert advice about complex practices, but in this case customized for the peculiarities of orchestration. Also, a multi-level pattern approach for the elicitation and use of atomic patterns, e.g., in teacher professional development, is proposed in this dissertation.

The third orchestration challenge, which teachers face when orchestrating TEL, and especially when orchestrating blended CSCL in DLEs, is the lack of adequate technological support for such orchestration, especially regarding the deployment of learning designs (e.g. done with 
computerized authoring tools) across the de-centralized infrastructure of a DLE (which includes not only a Virtual Learning Environment, but also external tools such as "Web 2.0" tools), and their flexibility in run-time. In this dissertation we propose the Group Learning Unified Environment - Pedagogical Scripting (GLUE!-PS), a service architecture and an underlying data model to deploy and manage in enactment-time the activities of a learning design, which supports multiple authoring tools and multiple combinations of DLEs.

The research methodology used to frame this dissertation is the engineering method, which has been followed iteratively for each of the proposed tools, in incremental phases of information, proposal, analysis and evaluation. The evaluation of our proposals, guided by the CSCL Evaluand-oriented Responsive Evaluation Method (EREM), was characterized by orchestration's focus on authentic settings and the multi-disciplinarity of the TEL and CSCL fields. These factors led us to evaluate through expert panels, teacher professional development actions, or authentic classroom experiments, using a variety of mixed methods techniques for data gathering and analysis.

The evaluation results suggest that the proposed tools are useful for researchers and teachers to orchestrate learning in authentic settings, and that such proposals have a high likeliness of being used in real practice in the immediate future. However, the evaluation also found shortcomings, which hint at paths for future research, such as developing more normative frameworks for orchestration, investigating the possibilities of co-orchestration (with students or technological systems as more prominent actors), or finding ways to scale up the innovations developed during the dissertation. 


\section{Resumen}

La progresiva introducción de las Tecnologías de la Información y las Comunicaciones (TICs) en la educación a todos los niveles ha transformado el aula, añadiendo una capa tecnológica al ya complejo entorno donde tienen lugar el aprendizaje y la docencia. Se espera de los profesores y otros actores que incorporen nuevas tecnologías y aproximaciones promovidas por la investigación, re-contextualizándolas para usarlas en sus contextos concretos, y usándolas de manera efectiva para conseguir una serie de objetivos de aprendizaje. Esta complejidad tecnológica (y pedagógica) ha sido descrita recientemente en el campo de investigación del Aprendizaje Mejorado por Tecnología (TEL en sus siglas inglesas) como uno de los principales desafíos de los próximos años, denominándola "orquestación". Esta tesis gira alrededor de esta noción emergente de orquestación, identificando tres problemas concretos para los investigadores y para los profesores, y proporcionando herramientas conceptuales y tecnológicas para superar dichos desafíos. Más concretamente, hemos elegido analizar y evaluar estas propuestas en el contexto cada vez más común del Aprendizaje Colaborativo Soportado por Ordenador mixto (blended CSCL, en sus siglas en inglés), que usa Entornos de Aprendizaje Distribuidos (DLEs en su acrónimo inglés), como ejemplo de contexto educativo donde esta orquestación es especialmente crítica y difícil.

El primer desafío que los investigadores en TEL se encuentran cuando se aproximan al problema de la orquestación, es la falta de claridad del concepto, ya que en dicha comunidad científica multi-disciplinar el término se usa con variedad de significados. Proponemos "5+3 Aspectos", un marco conceptual descriptivo emergente de una revisión de literatura sobre orquestación en TEL, para ayudar a los investigadores a enmarcar y caracterizar la orquestación en TEL. Este marco puede usarse como "lente analítica" para entender cómo ocurre la orquestación en un entorno educativo concreto, y como base para el desarrollo de instrumentos de investigación adicionales que tengan por objetivo el hacer que las innovaciones de TEL sean más fácilmente aplicables a entornos (físicos o virtuales) auténticos.

El segundo reto de orquestación que hemos identificado, en este caso especialmente para profesores y otros practicantes de TEL, es la escasez de guías concretas que sufren (especialmente los no-expertos), a la hora de realizar dicha orquestación en sus clases. En esta tesis proponemos los "patrones atómicos", como herramienta conceptual para ayudar a los docentes a cubrir el hueco entre las guías, a menudo descontextualizadas, de los investigadores, y las actuaciones contextualizadas que los docentes deben realizar en sus clases concretas. Estos patrones atómicos están basados en el concepto de patrones de diseño como herramientas para capturar guías de expertos sobre prácticas complejas, pero en este caso modificadas para hacer frente a las particularidades de la orquestación. También se propone una aproximación basada en patrones 
multi-nivel para la extracción y uso de los patrones atómicos, p.ej., en desarrollo profesional docente.

El tercer problema de orquestación, que los profesores encaran cuando hacen orquestación de TEL, y especialmente de CSCL mixto en DLEs, es la falta de un apoyo tecnológico adecuado para dicha orquestación, especialmente en cuanto al despliegue de diseños de aprendizaje (p.ej. realizados con herramientas de autoría computerizadas) a lo largo de la infraestructura descentralizada del DLE (que incluye no sólo un Entorno Virtual de Aprendizaje, sino también herramientas externas tales como las herramientas "Web 2.0"), así como su modificación flexible en tiempo de ejecución. En esta tesis proponemos el Entorno Unificado de Aprendizaje en Grupo - Guiado Pedagógico (GLUE!-PS, por sus siglas en inglés). GLUE!-PS es una arquitectura de servicios y un modelo de datos subyacente para desplegar y gestionar en tiempo de ejecución las actividades de un diseño de aprendizaje, que soporta múltiples herramientas de autoría y múltiples combinaciones de DLEs.

La metodología de investigación usada para enmarcar esta tesis es el método de ingeniería, el cual se ha seguido iterativamente por cada una de las herramientas propuestas, en fases incrementales de información, proposición, análisis y evaluación. La evaluación de nuestras propuestas ha sido guiada por el Método de Evaluación Receptivo centrado en el Evaluando CSCL (en inglés, CSCL-EREM), dado el foco de la orquestación en los contextos educativos auténticos y la multi-disciplinariedad del TEL y el CSCL. Estos factores nos han llevado a evaluar las contribuciones a través de paneles de expertos, acciones de desarrollo profesional docente o experiencias de aula auténticas, usando una variedad de técnicas de método mixto para la recogida de datos y su análisis.

Los resultados de la evaluación sugieren que las herramientas propuestas son útiles para los investigadores, y para los docentes en la orquestación del aprendizaje en entornos auténticos, y que dichas propuestas tiene grandes probabilidades de ser usadas en la práctica real en el futuro inmediato. Sin embargo, la evaluación también ha encontrado defectos, los cuales apuntan hacia caminos de investigación futuros, tales como el desarrollo de marcos más normativos para la orquestación, la investigación de las posibilidades de la co-orquestación (con los propios estudiantes o sistemas tecnológicos como actores más prominentes), o el encontrar maneras de escalar las innovaciones desarrolladas durante la tesis. 


\section{Contents}

\begin{tabular}{|l|l}
\hline Abstract & I \\
\hline
\end{tabular}

\begin{tabular}{ll|l} 
Resumen & III
\end{tabular}

\begin{tabular}{lll}
\hline & Introduction & 1
\end{tabular}

1.1 Dissertation goals and contributions . . . . . . . . . . . . . . . . 4

1.2 Research methodology . . . . . . . . . . . . . . . . . . . . . . . . . . . . . . . .

1.3 Document structure . . . . . . . . . . . . . . . . . . . . . . 15

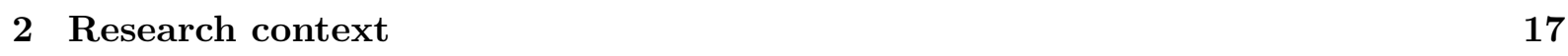

2.1 Introduction . . . . . . . . . . . . . . . . . . 17

2.2 From TEL to blended Computer-Supported Collaborative Learning . . . . . . . . 19

$2.2 .1 \quad$ Computer-Supported Collaborative Learning (CSCL) . . . . . . . . . . . 19

$2.2 .2 \quad$ Learning Design, CSCL scripts and the CSCL life-cycle . . . . . . . . . . 20

$2.2 .3 \quad$ Blended learning and blended CSCL . . . . . . . . . . . . . . . . . . 22

2.3 Orchestration in TEL and CSCL $\ldots \ldots \ldots \ldots \ldots \ldots \ldots \ldots$

$2.3 .1 \quad$ A literature review on orchestration . . . . . . . . . . . . . . . 24

2.3.2 Examples of research under the orchestration "umbrella" . . . . . . . . 26

$2.3 .3 \quad$ First synthesis attempt on orchestration . . . . . . . . . . . . 28

2.4 VLEs, PLEs and DLEs $\ldots \ldots \ldots \ldots \ldots \ldots \ldots \ldots \ldots$

$2.4 .1 \quad$ Virtual Learning Environments (VLEs) . . . . . . . . . . . . . . . . 31

2.4.2 Personal Learning Environments (PLEs) and other platforms used for

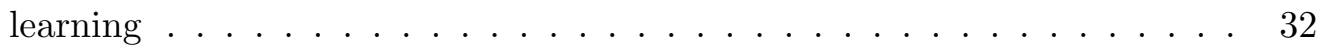

2.4.3 Distributed Learning Environments (DLEs): Beyond the VLE/PLE . . 34

2.5 Orchestrating Blended CSCL in DLES . . . . . . . . . . . . . . . . 37

2.5 .1 Towards a synthesized view of of orchestration in TEL and CSCL . . . . 37

2.5.2 Teacher practice in the orchestration of DLEs: the need of conceptual advice 39

2.5.3 Learning Design and the "deployment gap" . . . . . . . . . . . . 40

2.5.4 Flexibility: From improvisation to the problem of real-time management and adaptation of the learning activities in DLEs . . . . . . . . . . . . 43

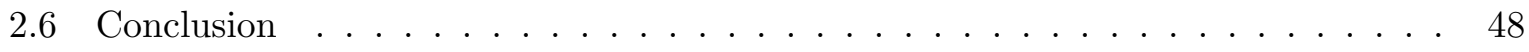

\begin{tabular}{|lll|}
\hline 3 & '5+3 Aspects' & 51 \\
\hline
\end{tabular}

3.1 Introduction . . . . . . . . . . . . . . . . . . . . . . 51

3.1 .1 A note on methodology . . . . . . . . . . . . . . . . 54

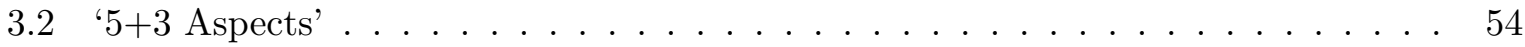

3.2 .1 Aspects of orchestration $\ldots \ldots \ldots \ldots \ldots$ 
3.2 .2 What is orchestration? A definition . . . . . . . . . . . . . . . 61

3.3 Evaluation of $5+3$ Aspects $7 \ldots \ldots \ldots \ldots \ldots \ldots$

3.3 .1 Context and method of the evaluation . . . . . . . . . . . . . 62

3.3.2 A panel of related TEL/CSCL researchers (RP1) . . . . . . . . . . . 66

3.3.3 A wider panel of internationally-recognized TEL/CSCL orchestration re-

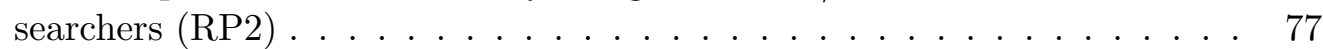

3.3 .4 Partial conclusions of the study . . . . . . . . . . . . . . . 93

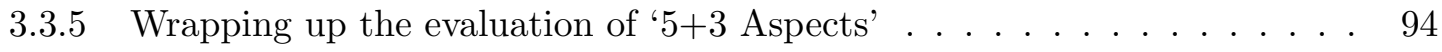

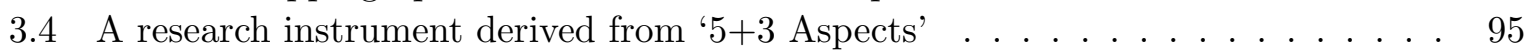

$3.4 .1 \quad$ Structure of the guide $\ldots \ldots \ldots \ldots \ldots \ldots$. . . . . . . . . . . . . . . . . . . . . . . . 97

3.4 .2 Preliminary evidence of usefulness . . . . . . . . . . . . . . . . . 98

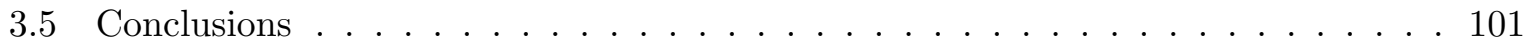

\begin{tabular}{|lll}
4 & Atomic patterns as conceptual tools for orchestration & 105
\end{tabular}

4.1 Introduction . . . . . . . . . . . . . . . . . . . 105

4.1 .1 A note on methodology . . . . . . . . . . . . . . . . 108

4.2 Atomic patterns for orchestration $\ldots \ldots \ldots \ldots \ldots \ldots$

4.2 .1 The origin of atomic patterns . . . . . . . . . . . . . . . . . . . . . . . . . . . .

4.2 .2 What is an "atomic pattern"? . . . . . . . . . . . . . . . 116

$4.2 .3 \quad$ Atomic patterns vs. other pattern approaches . . . . . . . . . . . . . 120

$4.2 .4 \quad$ Analyzing atomic patterns from an activity theory perspective . . . . . . 123

4.3 Multi-level pattern approach to orchestration . . . . . . . . . . . . 125

4.3 .1 Overview of the approach . . . . . . . . . . . . . 126

4.3 .2 The approach in use . . . . . . . . . . . . . . . . . . . 127

$4.3 .3 \quad$ Advantages, disadvantages and challenges of the approach . . . . . . . . . 132

4.4 Other uses of atomic patterns . . . . . . . . . . . . . . . 133

4.4 .1 Representing orchestration through atomic patterns . . . . . . . . . . . 133

4.4 .2 Using atomic patterns to develop technology . . . . . . . . . . . . . . 137

4.5 Evaluating atomic patterns $\ldots \ldots \ldots \ldots \ldots$

4.5 .1 Context and method of the evaluation . . . . . . . . . . . . . . 140

4.5.2 Iteration \#1: First attempt at an atomic pattern teacher workshop in primary education $(\mathrm{TW} 1) \ldots \ldots \ldots \ldots \ldots \ldots \ldots$

4.5.3 Iteration \#2: A second atomic pattern teacher workshop in primary education (TW2) . . . . . . . . . . . . . . . . . 154

4.5.4 Iteration \#3: Generation of a new atomic pattern catalogue, and first teacher workshop in higher education (TW3) . . . . . . . . . . . . . 160

4.5.5 Iteration \#4: A second higher education teacher workshop (TW4) . . . . 175

4.5 .6 Wrapping up the evaluation of atomic patterns . . . . . . . . . . . . 192

4.6 Conclusions, relevance and future work on atomic patterns. . . . . . . . . . . . 197

$\begin{array}{lll}5 & \text { GLUE!-PS } & 201\end{array}$

5.1 Introduction . . . . . . . . . . . . . . . . . . . . 201

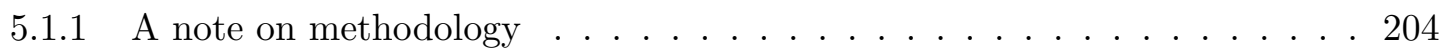

5.2 GLUE! Pedagogical Scripting (GLUE!-PS) . . . . . . . . . . . . . . . . . . . 206

5.2 .1 Requirements and guiding principles of GLUE!-PS . . . . . . . . . . . 207

$5.2 .2 \quad$ GLUE!-PS architecture $\ldots \ldots \ldots \ldots$. . . . . . . . . . . . . . . . 212 
5.2.3 GLUE!-PS Lingua Franca (GLUE!-PS LF): A data model to deploy and manage learning designs in DLEs . . . . . . . . . . . . . . 220

5.2 .4 GLUE!-PS functionality: Main use cases . . . . . . . . . . . . . . . . . . . 223

5.3 Analyzing GLUE!-PS as a tool for orchestration . . . . . . . . . . . . . . 236

5.3 .1 GLUE!-PS within the '5+3 Aspects' conceptual framework for orchestration 236

5.3.2 Analyzing GLUE!-PS support for orchestration through atomic patterns . 238

5.4 GLUE!-PS reference implementation (GLUE!-PS RI) . . . . . . . . . . . . . . . 244

5.4 .1 Technologies involved . . . . . . . . . . . . . . . . . 245

5.4 .2 Evolution of the GLUE!-PS RT . . . . . . . . . . . . . . . . . . . . 246

5.4 .3 Current state of the GLUE!-PS RI . . . . . . . . . . . . . . . . . 246

5.5 Evaluating GLUE!-PS . . . . . . . . . . . . . . . . . . . . . . 255

5.5 .1 Context and method of the evaluation . . . . . . . . . . . . . 255

5.5 .2 Iteration \#1: A first analytic evaluation of GLUE!-PS $\ldots . . \ldots .260$

5.5.3 Iteration \#2: Evaluating the first GLUE!-PS prototype in workshops . . . 266

5.5.4 Iteration \#3: Extending VLE support in GLUE!-PS - a wiki-based exper-

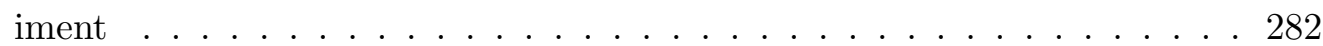

5.5.5 Iteration \#4: Adding run-time changes support and evaluation in workshops and wiki-based experiments . . . . . . . . . . . . 293

$5.5 .6 \quad$ Wrapping up the evaluation: Global evaluation conclusions . . . . . . . . 319

5.6 Conclusions, relevance and future work around GLUE!-PS . . . . . . . . . . . . . 321

\begin{tabular}{|lll}
6 & Conclusions and future work & $\mathbf{3 2 7}$
\end{tabular}

6.1 Conclusions of the dissertation . . . . . . . . . . . . . . . . . 327

6.2 Future lines of work $\ldots \ldots \ldots \ldots$. . . . . . . . . . . . . . . . . . . . . . . . . . . . .

\begin{tabular}{ll}
\hline References & 335
\end{tabular}

\begin{tabular}{|l|l|}
\hline A The Orchestration Interview Guide (OIG) & 361
\end{tabular}

A.1 General guidelines . . . . . . . . . . . . . . . . . . . . . . 362

A.2 Questioning guide . . . . . . . . . . . . . . . . . . . . . . 362

A.2.1 Design/Planning . . . . . . . . . . . . . . . . . . . . . . . . . . . . . . . . . . . . . . . . . . . . .

A.2.2 Regulation/Management . . . . . . . . . . . . . . . . . . 363

A.2.3 Adaptation/Flexibility/Intervention . . . . . . . . . . . . . . . . . . . . . . . . . . . . . . . . . . . . .

A.2.4 Awareness /Assessment . . . . . . . . . . . . . . . . . . . . . 364

A.2.5 Roles of the teacher and other actors . . . . . . . . . . . . . . . . . . . . . . . . . . . . . . . . .

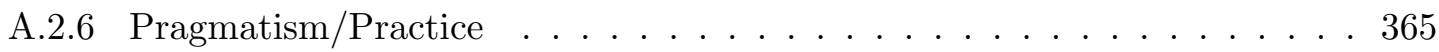

A.2.7 Alignment/Synergy . . . . . . . . . . . . . . . . . . . 365

A.2.8 Models/Theories . . . . . . . . . . . . . . . . . . . . . . . . . . . 365

\begin{tabular}{|ll}
\hline B Orchestration Atomic Patterns Catalogue (OAPC) & 367
\end{tabular}

\begin{tabular}{|lll}
\hline C An analysis of existing Learning Design languages and learning platforms & 371
\end{tabular}

C.1 Analysis of Learning Design languages . . . . . . . . . . . . . . . . . . . . . . . . . . . . . . . . . . . . . .

C.1.1 IMS Learning Design. . . . . . . . . . . . . . . . . . . . . . . . . . . . . . . . . . . . . . . . . . . .

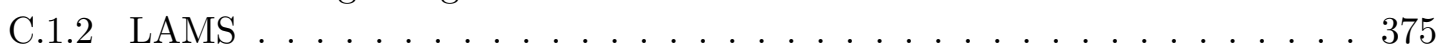

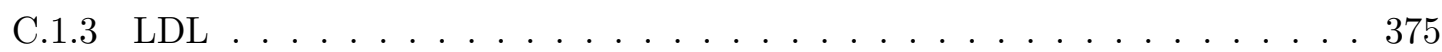

C.1.4 LAS (SCY-SE) . . . . . . . . . . . . . . . . . 376 
C.1.5 CompendiumLD . . . . . . . . . . . . . . . . . 378

C.1.6 Ad-hoc, non-computational languages . . . . . . . . . . . . . . . 378

C.2 Discussion . . . . . . . . . . . . . . . . . . . . . 381

C.3 Learning environments and Learning Design scripting . . . . . . . . . . . . . . . 382

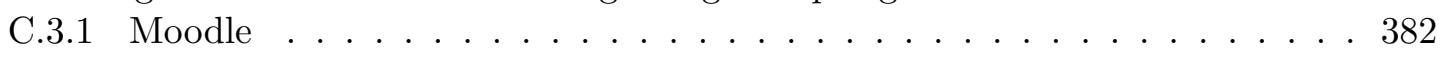

C.3.2 LAMS (Learning Activity Management System) . . . . . . . . . . . . . . 384

C.3.3 Dokeos. . . . . . . . . . . . . . . . . . . . 386

C.3.4 Blackboard . . . . . . . . . . . . . . . . . . 387

C.3.5 MediaWiki . . . . . . . . . . . . . . . . . . . . . 387

C.3.6 Elgg . . . . . . . . . . . . . . . . . . . . . . . . . . 389

C.4 Using standards to deploy learning designs . . . . . . . . . . . . . . . . . 390

C.5 Summing up . . . . . . . . . . . . . . . . . . . . . . 391

D GLUE!-PS Lingua Franca (GLUE!-PS LF) 395

D.1 Overview of the data model . . . . . . . . . . . . . . . . . . 395

D.2 Detailed description of the data model . . . . . . . . . . . . . . . . . . 397 


\section{List of Figures}

1.1 Schematic view of the context, goals, contributions and evaluation of the thesis

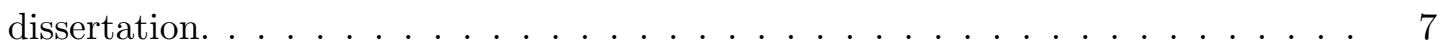

1.2 Simplified view of the engineering methodology followed during the dissertation . 9

1.3 Detailed view of the engineering methodology followed during the dissertation, including the iterations followed $\ldots \ldots \ldots \ldots \ldots 10 \ldots \ldots$

1.4 Graphical template for the design of an evaluation using the CSCL-EREM model 12

1.5 Graphical representation of the general flow of data gathering and analysis techniques employed in each of the evaluation happenings during the dissertation . . 13

2.1 Diagram representing the research context of the dissertation, reviewed in this

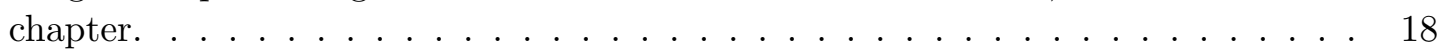

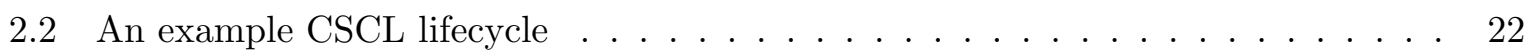

2.3 Diagram with common orchestration concepts . . . . . . . . . . . . . . . 28

2.4 Concept diagram of orchestration, with the elements grouped in categories. . . . 30

2.5 Authentic example of a wiki being used as a learning platform . . . . . . . . . 33

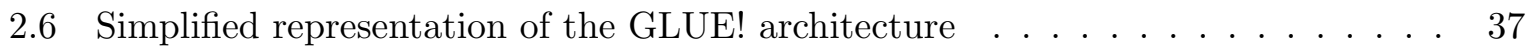

2.7 Graphical representation of the "kernel and rings" model for classroom orchestration 45

2.8 Summary of the technological support for orchestration provided by different

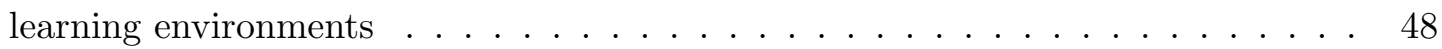

3.1 Relative probability of a paper having the word 'orchestration', given that it had

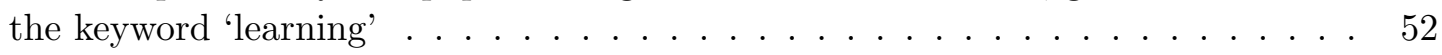

3.2 Parts of the thesis diagram concerned by chapter 3 . . . . . . . . . . . . . 53

3.3 Detailed view of the engineering method followed during the research work around

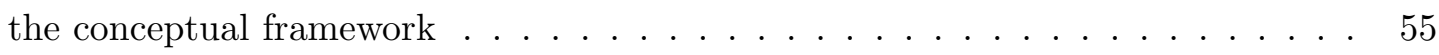

$3.4 \quad$ Graphical representation of the ' $5+3$ ' orchestration conceptual framework . . . . 59

$3.5 \quad$ Alternative graphical representation of the ' $5+3$ ' orchestration conceptual frame-

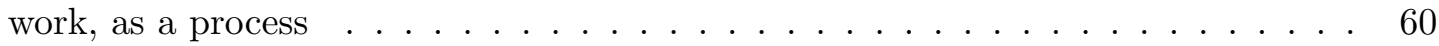

3.6 Graphical representation of the research questions, issues, topics and informative questions used during the evaluation of ' $5+3$ Aspects' $\ldots . . . . . . . .663$

3.7 Evaluation design for the "5+3 Aspects" conceptual framework . . . . . . . . . . 65

3.8 Graphic representation of the data gathering and analysis techniques flow during the evaluation of $5+3$ Aspects $] \ldots \ldots \ldots \ldots \ldots 6$

3.9 Graphical representation of the framework . . . . . . . . . . . . . . . . . 68

3.10 Graphic representation of the participants' research expertise and prior orchestration knowledge in the RP1 study . . . . . . . . . . . . . . . . . 70 
3.11 Graphic representation of the participants' quantitative evaluation of the completeness of ' $5+3$ ' framework in the RP1 study . . . . . . . . . . . . . . . . . 74

3.12 Graphic representation of the participants' quantitative evaluation of the usefulness of the ' $5+3$ ' framework in the RP1 study . . . . . . . . . . . . . . 75

3.13 Graphical representation of the responses to the main profiling questions in the

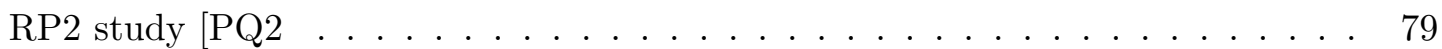

3.14 Word cloud representing the relative importance of keywords in RP2 participants' prior definitions of orchestration $\ldots \ldots \ldots \ldots$. . . . . . . . 80

3.15 Graphic representation of the participants' quantitative evaluation of the completeness of the $5+3$ ' framework in the RP2 study . . . . . . . . . . . . 86

3.16 Graphic representation of the participants' quantitative evaluation of the usefulness of the ' $5+3$ ' framework in the RP2 study . . . . . . . . . . . . . . . . 91

3.17 Graphic representation of the partial conclusions of the evaluations on the ' $5+3$ Aspects' conceptual framework . . . . . . . . . . . . . . . . 94

3.18 Graphic representation of the global conclusions of the evaluations on the $5+3$ Aspects' conceptual framework . . . . . . . . . . . . . . 96

3.19 Proposal for the graphical representation of a new framework proposal that integrates some of the evaluation feedback . . . . . . . . . . . . . . . . 104

4.1 Parts of the thesis diagram concerned by chapter 4 . . . . . . . . . . . . 107

4.2 Detailed view of the engineering method followed during the research work around

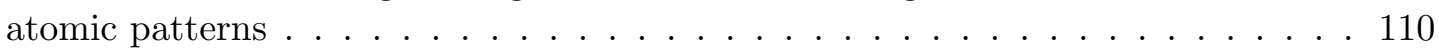

4.3 Screenshot of a Group Scribbles activity, taken from the observations at the pri-

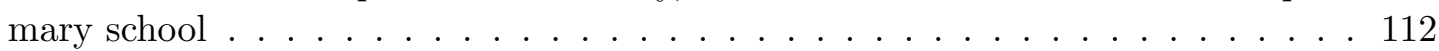

4.4 Graphical representation of the enactment analysis of an observed Group Scribbles

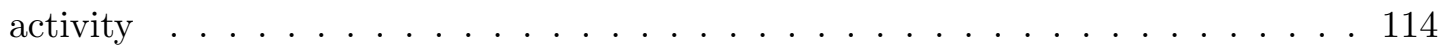

4.5 Graphical representation of the tensions found in the atomic pattern analysis from an activity theory perspective . . . . . . . . . . . . . . 124

4.6 The four main phases of the multi-level pattern approach to support orchestration 127

4.7 Example design analysis of a teacher's CSCL learning design $\ldots \ldots$. . . . . . . . 128

$4.8 \quad$ Example enactment analysis of a teacher's CSCL learning design . . . . . . . . . 129

4.9 Example representation of a learning design. Basic template depicting roles, social levels, tasks and tools. . . . . . . . . . . . . . . . 135

4.10 Example representation of a learning design. Completed template with the atomic patterns present in each phase . . . . . . . . . . . . . . 137

4.11 Example teacher-generated representation of a learning design enriched with atomic patterns, taken from one of the teacher workshops . . . . . . . . . 138

4.12 Graphic representation of the evaluation design of atomic patterns as a conceptual tool for orchestration . . . . . . . . . . . . . . . . . . 142

4.13 Graphical representation of the research questions, issues, topics and informative questions used during the evaluation of atomic patterns, as the research effort

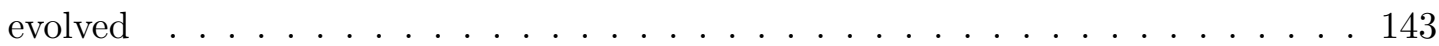

4.14 Graphic representation of the data gathering and analysis techniques flow during the evaluation of atomic patterns $\ldots \ldots \ldots \ldots \ldots \ldots$. . . . . . . . . . . . . . . . .

4.15 Example atomic pattern cards used in the workshop, both for design-time and

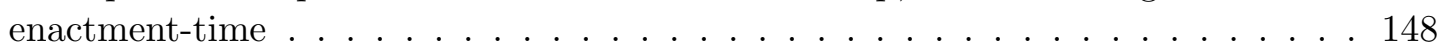


4.16 Example role card handed out to teachers during the role-playing in the workshop 149

4.17 Original design draft done by teachers during the workshop (left). Design enriched with design-time atomic patterns (right) $\ldots \ldots \ldots \ldots \ldots$. . . . . . . . . . . . . 150

4.18 Graphical representation of the partial conclusions to Iteration \#1 . . . . . . . . 154

4.19 Graphical representation of the partial conclusions to Iteration \#2 . . . . . . . . 160

4.20 Graphical representation of selected quantitative evidence from the questionnaires in the evaluation of the third iteration (TW3) $\ldots \ldots \ldots \ldots$. . . . . . 165

4.21 Graphical representation of the partial conclusions to Iteration \#3 . . . . . . . . 176

4.22 Photograph taken during the TW4 workshop . . . . . . . . . . . . . . . . . 184

4.23 Graphical representation of the partial conclusions to Iteration \#4 . . . . . . . . 193

4.24 Graphical representation of the partial conclusions leading to the global evaluation conclusions on atomic patterns as a conceptual tool for orchestration . . . . . . 196

5.1 Parts of the thesis diagram concerned by chapter 5 . . . . . . . . . . . . . 202

5.2 Detailed view of the engineering method followed during the research work around

5.3 Cartoon representation of the "deployment gap" between computer-interpretable LD languages and the variety of widespread learning platforms . . . . . . . . . 208

5.4 GLUE!-PS simplified architecture . . . . . . . . . . . . . . . . . . . 213

5.5 GLUE!-PS simplified architecture, including the use of the GLUE! architecture

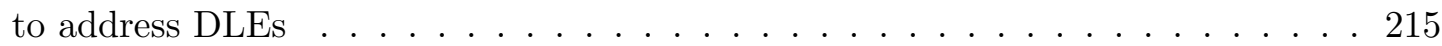

5.6 Summary of the main scripting characteristics supported by learning design languages and learning environments . . . . . . . . . . . . . . . . 221

5.7 GLUE!-PS data model . . . . . . . . . . . . . . . . . . . . . . . . . . . . 223

5.8 GLUE!-PS block interactions diagram for Use Case I . . . . . . . . . . . . . . . . 225

5.9 GLUE!-PS sequence diagram for Use Case 1 . . . . . . . . . . . . . . . . . . . . 227

5.10 GLUE!-PS block interactions diagram for Use Case Ib . . . . . . . . . . . . . . . . 229

5.11 GLUE!-PS sequence diagram for Use Case Ib . . . . . . . . . . . . . . . . . . . . 230

5.12 GLUE!-PS block interactions diagram for Use Case II . . . . . . . . . . . . . . . 231

5.13 GLUE!-PS sequence diagram for Use Case II . . . . . . . . . . . . . . . . . . . . 233

5.14 GLUE!-PS block interactions diagram for Use Case IIb. . . . . . . . . . . . . . . 234

5.15 GLUE!-PS sequence diagram for Use Case IIb . . . . . . . . . . . . . . . . . . . 235

5.16 Screenshot of the WebCollage LD authoring tool, showing a learning design based on the Jigsaw pattern and an initial group particularization . . . . . . . . . . . 248

5.17 Screenshot of the Pedagogical Pattern Collector LD authoring tool, showing a simple role-playing collaborative scenario f . . . . . . . . . . . . . . . 249

5.18 Screenshot of a Moodle VLE where a Jigsaw-based learning design has been deployed using GLUE!-PS . . . . . . . . . . . . . . . . . . 250

5.19 Screenshot of a MediaWiki installation where a collaborative learning design has been deployed using GLUE!-PS . . . . . . . . . . . . . . . . . 252

5.20 Screenshot of the GLUE!-PS RI user interface, showing the initial page to upload new designs and the page to create a new particularization of a design . . . . . . 253

5.21 Screenshot of the GLUE!-PS RI user interface, showing the main interface for the

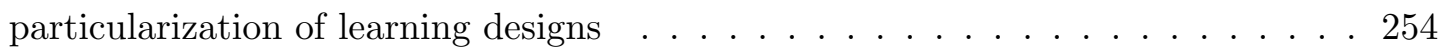

5.22 Graphical representation of the research questions, issues, topics and informative questions used during the evaluation of GLUE!-PS . . . . . . . . . . . . . . 257 
5.23 Graphic representation of the evaluation design of GLUE!-PS as a technological tool for orchestration . . . . . . . . . . . . . . . . . . . . . . 259

5.24 Graphic representation of the data gathering and analysis techniques flow during the evaluation of GLUE!-PS . . . . . . . . . . . . . . . . . . . 261

5.25 Converting roles and groups in GLUE!-PS: from IMS-LD to Moodle through the GLUE!-PS data model . . . . . . . . . . . . . . . . . . . . . . . 263

5.26 Summary of the intrinsic and extrinsic characteristics of the Planet Game script, and how they are preserved throughout the translations . . . . . . . . . . 264

5.27 Graphical representation of the research questions, issues and partial conclusions of Iteration \#1 in the GLUE!-PS evaluation . . . . . . . . . . . . . . . . 265

5.28 Example mockup screens used in the first usability tests of the GLUE!-PS GUI

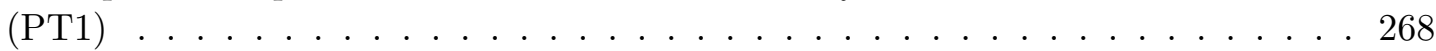

5.29 Photos taken during the TW3b evaluation happening $\ldots \ldots \ldots$. . . . . . 272

5.30 Photo taken during the TW5 evaluation happening . . . . . . . . . . . . . . . 276

5.31 Graphical representation of the issues, topics and partial conclusions of Iteration

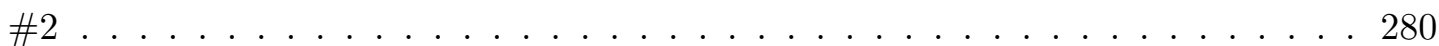

5.32 Graphical representation of the design done by the teacher in happening AE1 . . 284

5.33 Screen capture of the (outdated) first version of the GLUE!-PS GUI . . . . . . . 286

5.34 Screen capture of the Jigsaw learning design used in happening AE1, once it was deployed in a wiki by GLUE!-PS . . . . . . . . . . . . . . . . . . 288

5.35 Graphical representation of the issues, topics and partial conclusions of the Iter-

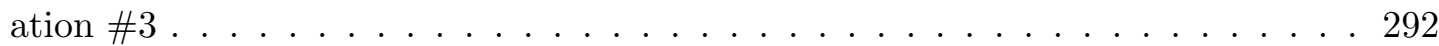

5.36 Examples of the paper prototypes of the GLUE!-GUI used during Iteration \#4 . 294

5.37 Screen capture of the Pyramid-based learning design used in happening AE2, as represented by the new version of the GLUE!-PS GUI. Includes basic explanation

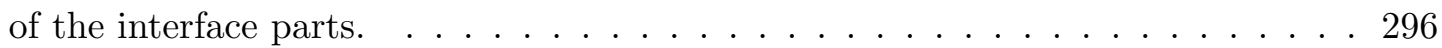

5.38 Graphical representation of the design done by the teacher in happening AE3 . . 301

5.39 Screen captures of the Jigsaw learning design used in happening AE3, once it was deployed in a wiki by GLUE!-PS . . . . . . . . . . . . . . . . . . . 301

5.40 Photo of the enactment of one of the face-to-face sessions in evaluation happening

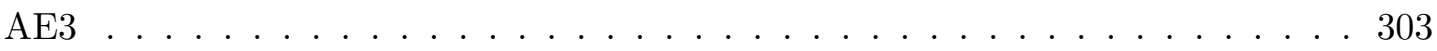

5.41 Student list used to conceptualize the run-time regrouping of students for the Jigsaw design in AE3 . . . . . . . . . . . . . . . . . . . 304

5.42 Graphical representation of the example learning design that participants in workshop TW6 had to implement using WebCollage and GLUE!-PS . . . . . . . . . . 310

5.43 Photos taken during the face-to-face session of evaluation happening TW6 . . . . 311

5.44 Graphical representation of the issues, topics and partial conclusions of the Iter-

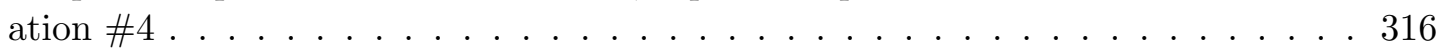

5.45 Graphical representation of the partial conclusions leading to the global evaluation conclusions on GLUE!-PS as a technological tool for orchestration . . . . . . . . 322

C.1 IMS-LD metamodel . . . . . . . . . . . . . . . . . . . . . . 373

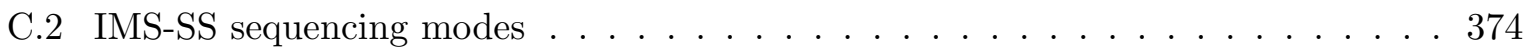

C.3 LDL simplified meta-model . . . . . . . . . . . . . . . . . . . . . . 376

C.4 "Design" LAS example . . . . . . . . . . . . . . . . . . . . . . . . . . . 377

C.5 Example activity in CompendiumLD . . . . . . . . . . . . . . . . . . . . 379 
C.6 Example of MUITIC integrated working plan . . . . . . . . . . . . . . . . . . 380

D.1 Summary of the main scripting characteristics supported by learning design languages and learning environments . . . . . . . . . . . . . . 396

D.2 GLUE!-PS data model . . . . . . . . . . . . . . . . . . . . . . . . . . . . . 396 



\section{List of Tables}

3.1 Main data sources used during the RP1 researcher panel . . . . . . . . . . . . . . 67

$3.5 \quad$ Main data sources used during the RP2 researcher panel . . . . . . . . . . . . . 78

4.1 Catalogue of design atomic patterns encountered in activities of the Cigales School 113

4.2 (Partial) Catalogue of enactment routines, classified by type of task $\ldots$. . . . 118

$4.3 \quad$ Example atomic pattern expressed using the Adaptation Pattern template . . . . 122

4.4 Learning design of one of the workshop sessions in a teacher workshop, using a Pyramid CLFP as the general structure, and indicating the atomic patterns that were used to enrich it . . . . . . . . . . . . . . . . . . 131

4.5 Main data sources used during the TW1 workshop . . . . . . . . . . . . . . . . 149

4.6 Selected findings and evidence from the TW1 workshop, on the issue of atomic patterns' usefulness (T1a) . . . . . . . . . . . . . . . . . . 151

4.7 Selected findings and evidence from the TW1 workshop, on the influence of the workshop's own orchestration in the atomic patterns' usefulness (T2) . . . . . . . 152

4.8 Selected findings and evidence from the TW1 workshop, on the issue of atomic patterns' mirroring qualities (T3) . . . . . . . . . . . . . . . . 152

4.9 Main data sources used during the TW2 workshop . . . . . . . . . . . . . . . . 156

4.10 Topics, findings and selected supporting evidence from the TW2 workshop. . . . 158

4.11 Main data sources used during the TW3 workshop . . . . . . . . . . . . . . 163

4.13 Selected findings and evidence from the TW3 workshop, on the influence of the workshop's own orchestration in atomic patterns' perceived usefulness (T2) . . . 168

4.14 Selected findings and evidence from the TW3 workshop, on the influence of participants' experience in the perceived usefulness of atomic patterns (T4) . . . . . 170

4.15 Selected findings and evidence from the TW3 workshop, on the influence of participant beliefs in atomic patterns' perceived usefulness (T5) . . . . . . . . . . 172

4.16 Selected findings and evidence from the TW3 workshop, on the usefulness of embedding atomic patterns in educational technologies (T6) $\ldots$. . . . . . . . . 173

4.17 Main data sources used during the TW4 workshop . . . . . . . . . . . . . . 179

4.19 Selected findings and evidence from the TW4 workshop, on the influence of the workshop's own orchestration in atomic patterns' perceived usefulness (T2) . . . 186

4.20 Selected findings and evidence from the TW4 workshop, on the influence of participants' experience in the perceived usefulness of atomic patterns (T4), including the teaching experience of the participants providing the evidence. . . . . . . . 187

4.21 Selected findings and evidence from the TW4 workshop, on the influence of participant beliefs in atomic patterns' perceived usefulness (T5)] . . . . . . . . . . 189

4.22 Selected findings and evidence from the TW4 workshop, on the usefulness of embedding atomic patterns in educational technologies (T6) . . . . . . . . . . 190 
5.1 Behavior of the GLUE!-PS Manager service, represented as REST resources . . . 220

5.2 Analysis of the orchestration support provided by GLUE!-PS, according to the different aspects in the " $5+3$ " framework . . . . . . . . . . . . . . 237

5.3 Atomic pattern support analysis for GLUE!-PS (excerpt). First stage: implemen-

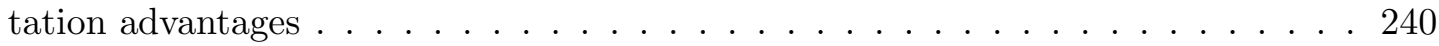

5.4 Atomic pattern support analysis for GLUE!-PS (excerpt). Second stage: technological and user-perspective issues $\ldots \ldots \ldots \ldots \ldots 242$

5.5 Atomic pattern-oriented roadmap for GLUE!-PS RI . . . . . . . . . . . . . . . . 244

5.6 Summary of the roadmap followed in the implementation of the GLUE!-PS RI . 246

5.7 Main data sources used during the TW3b workshop . . . . . . . . . . . . . 271

5.9 Main data sources used during the TW5 workshop . . . . . . . . . . . . . . . . 277

5.10 Topics, findings and selected supported evidence from evaluation happening TW5 279

5.11 Main data sources used during the AE1 evaluation happening . . . . . . . . . . . 285

5.12 Topics, findings and selected supported evidence from evaluation happening AE1, around topics T1 (deployment ability), T2 (time-efficiency) and T4 (run-time

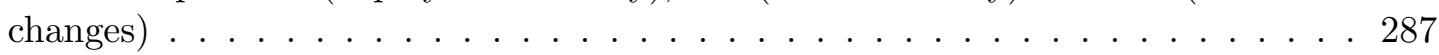

5.14 Main data sources used during the AE2 evaluation happening . . . . . . . . . . . 296

5.16 Main data sources used during the AE3 evaluation happening . . . . . . . . . . . 302

5.19 Main data sources used during the TW6 evaluation happening . . . . . . . . . . 311

5.20 Findings and selected supporting evidence from evaluation happening TW6, around topics T1 (deployment ability) and T2 (time-efficiency) . . . . . . . . . 312

5.22 Findings and selected supporting evidence from evaluation happening TW6,

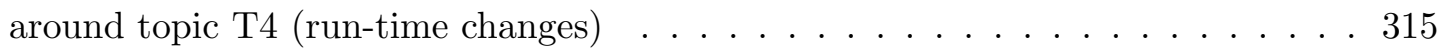

B.1 Master catalogue of uncovered atomic patterns . . . . . . . . . . . . . 367 


\section{Chapter 1}

\section{Introduction}

Summary: This chapter describes the general research context of the dissertation, its goals and the methodology followed to attain such goals. The dissertation deals with the increasingly popular concept of "orchestration" in Technology-Enhanced Learning (TEL), which is the coordination of learning activities occurring at different contexts, social levels and using multiple (digital or nondigital) tools. Particularly, we intend to provide technological and conceptual tools to support this kind of coordination in blended Computer Supported Collaborative Learning (CSCL), in the technological context of Distributed Learning Environments (DLEs) that include a Virtual Learning Environment and external tools. By following the engineering method of informing, proposing new solutions, analyzing and evaluating them, in an iterative way, we propose three contributions targeted mainly at researchers and teachers, to solve three outstanding orchestration problems of this kind of scenarios. For the evaluation of our contributions, we propose to use a mixed methods approach framed on a responsive, evaluand-oriented model for evaluation of CSCL (CSCL-EREM). Finally, this chapter also outlines the general structure of the rest of the dissertation.

The increasing use of Information and Communication Technologies (ICT) in the realm of education at all levels has opened a host of new opportunities, enabling new approaches to teaching and learning, as well as modifying existing ones. The field of Technology-Enhanced Learning (TEL, [Bal09]) studies these new opportunities and approaches. However, with each new technology not only come new opportunities, but also new challenges regarding its use, as well as whether and how such use actually enhances learning.

In current research and practice of TEL, be it either face-to-face, distance, or the combination of both (known as blended learning Gra05), we can observe several trends. From a purely technological perspective, apart from the usage of Virtual Learning Environments (VLEs 1 . Dil02b]), other ICT tools such as "Software-as-a-Service" (SaaS) and "Web 2.0" tools O'R05 are also being utilized more and more often [Con10b. The combination of these tools with other digital and non-digital ones (digital whiteboards, cameras, laptops, pen and paper, etc.) have prompted researchers to talk about the current and future learning environments as complex technological ecosystems Luc08. On the other hand, from a pedagogical perspective, "traditional" lectures and purely content-based online courses are being substituted or complemented with multiple other approaches, such as (Computer-Supported) Collaborative Learning (CSCL, Sta06a]), Inquiry-Based Learning (IBL, Gre96) or Project-Based Learning (PBL, Blu91).

\footnotetext{
${ }^{1}$ Also known as Learning Management Systems, or LMSs. Throughout the dissertation, we will use the term "VLE" when referring to these two mostly interchangeable concepts.
} 
The coordination of this increasing number and variety of elements, normally performed by teachers or other practitioners (tutors, instructional designers, etc.), is being referred to by the TEL research community as "orchestrating learning" [Bal10]. Although this coordination is crucial in most forms of TEL, this coordination is especially critical in areas such as ComputerSupported Collaborative Learning (CSCL Dil09a]), where orchestration implies coordinating "supportive interventions across multiple learning activities occurring at multiple social levels" Fis06. This idea of orchestration not only addresses interesting challenges in TEL research, but also has implications on how TEL research is done, with an emphasis on the usefulness of research outcomes for average practitioners and its applicability in authentic, "messy" environments (as opposed to e.g., expert teachers or controlled laboratory experiments [Dil09b]).

Although practitioners have been "orchestrating" TEL ever since the field was born (and before, as it is a hallmark of all teaching), TEL research and practice have mostly concentrated on the use of only one or a few technologies or approaches, until very recently. However, nowadays it is not uncommon to see in a classroom multiple digital and legacy technologies being used, as well as different teaching and learning approaches being put in practice, even by the same teachers. This fact has rightfully won classrooms the title of "messy" for research [Col04]. Thus, currently there exist few tools (either technological or conceptual) specifically conceived to support teachers in the coordination of these multiple elements, often preventing teachers from using more than a few of these tools and concepts, nor combining them in optimal ways to obtain what some authors have called "synergistic scaffolding" Tab04.

Let us consider the (comparatively simple) example of a teacher managing a blended learning course through a simple VLE like Moodle ${ }^{2}$ for a class of 16 students. Let us imagine that she intends to enact a technology-enhanced collaborative learning series of activities, following the Jigsaw strategy [Aro92]. Our imaginary teacher has a number of tools at her disposal (the VLE and its internal tools like chats and fora, the Jigsaw strategy itself, a digital whiteboard, etc.) which, separately, should allow her to accomplish the different parts of her pedagogic ideas (i.e. her learning design). However, the conceptualization and preparation of the required infrastructure (e.g. forming the groups in Moodle so that they cannot see each other's work, assigning people to different groups, creating the different chat activities, etc.) would take a long time and would be highly error-prone, even if the teacher is knowledgeable about ICT (and most teachers are not). Even if she could take a ready-made design from other teachers, or if she could use a specialized learning design authoring tool like Collage [HL06a], this creation of the needed ICT infrastructure according to the learning design ideas (which we will call "deployment") normally has to be done manually. Moreover, in the case of unexpected occurrences during the enactment of the activities (e.g. some students not showing up, the teacher wanting to add additional supporting resources or activities on-the-fly), the modifications to the ICT infrastructure would also be tedious and error-prone.

This kind of VLE-centric scenario is nowadays very common in e.g., higher education institutions all over the world. However, in the last few years there is an increasing trend of TEL practitioners trying to go beyond the walls of the institutional VLE, and using tools available from the web at large, especially the so-called "Web 2.0" tools (wikis, blogs, social media, shared apps, etc.) O'R05, to enhance education Con10b Ben12. A similar trend of expansion and customization of the (technological) learning environment, in more student-centric pedagogical

\footnotetext{
2http://moodle.org (Last visit: 08 Apr 2012)
} 
approaches, is the appearance and proliferation of the so-called Personal Learning Environments (PLEs, see [Wil06]).

We can imagine that, if a teacher finds obstacles in the case of a simple, centralized learning environment like a VLE or PLE, a multi-ICT tool environment in which both VLEs/PLEs and "Web 2.0" tools are used, would be even harder to orchestrate: instances of external tools (e.g. separate documents in a shared text editing application) have to be created, they have to be linked somehow from a central point (e.g. the Moodle VLE), etc. This kind of technological context, which some researchers have called Distributed Learning Environments (DLEs, Mac10]), provide a good example of the "messier environments" that TEL practitioners might be heading into in the next few years.

Along with these problems emanating directly from the technological context of DLEs, there is another, more subtle kind of problems, which is that of the conceptual tools that practitioners have today in order to achieve the aforementioned "synergistic scaffolding" with the multitude of ICT tools at their disposal. In other words, it is not enough to have technologies that let us handle the host of learning affordances that VLEs and "Web 2.0" tools offer; we also need to know how to use those tools, in the concrete context of our classrooms, in a way that is aligned with our pedagogical goals. Currently, much of the more innovative TEL practice in the classroom is being done, not by average teachers, but rather by the ones that are more knowledgeable about ICT and pedagogy. In an effort to provide an answer to this issue, educational institutions invest in professional development programs to develop their teachers' technological capabilities. However, even after attending these courses, the average teacher is still reluctant to use available ICTs for anything but the most basic tasks (let alone complex pedagogical techniques such as CSCL).

All the aforementioned problems exemplify some of the reasons why the results of much TEL and CSCL research may not have been widely adopted in the "messy classrooms" of our schools and universities Hop93 [Cub01] [Wat06] [Her08], and why the study and support of the problem of orchestrating learning is relevant for current and future education, if ICT is to have any role in it.

In this context, the general problem that is addressed in this dissertation is the provision of technological and conceptual tools to support the orchestration of blended Technology-Enhanced Learning, focusing especially in activities with a collaborative component (where orchestration is even more critical), in the technological context of Distributed Learning Environments (DLEs - which include multiple ICT tools from different domains, see [Mac10]). Since this problem is novel and still ill-defined in the field of TEL, also a clarification of the concept of orchestration in TEL and CSCL research will be needed. This clarification can prove to be a useful tool not only for the author during this dissertation, but also for other researchers interested in the increasingly ubiquitous problem of orchestrating technology-supported learning.

However, providing tools to support all aspects of orchestration is beyond the scope of a single $\mathrm{PhD}$ thesis (especially taking into account that orchestration has not been clearly defined and delimited as a research problem yet). Thus, in the work presented here we have concentrated on a number of specific, outstanding (conceptual and technological) orchestration problems:

- Orchestration deals with the design and real-time management of a learning situation, in order to achieve synergistically a number of desired outcomes [Fis06] Bal10]. The design 
of learning scenarios has been extensively studied by the discipline of Learning Design Kop06, which has produced a plethora of learning design languages and tools. However, few learning designs can be deployed in the execution environments currently in use (e.g. mainstream VLEs like Moodle), forcing teachers to do this deployment manually, a process that is tedious and error-prone. This problem is even more acute in the case of Distributed Learning Environments (DLEs) due to their de-centralized nature. Moreover, after deployment, the learning activities have to be managed in enactment-time by practitioners. The ability to make changes to the deployed technological infrastructure during run-time, to better align its elements in the face of unexpected occurrences and opportunities in the classroom, is one of the main tenets of "orchestrating learning". However, making this kind of run-time changes to the de-centralized infrastructure of a DLE is difficult, even for ICT-expert practitioners, since tools from multiple domains are involved and, as it happens when deploying, translating from pedagogical concepts like activities or groups to tool-specific terms is not straightforward. Thus, we see that the deployment and real-time management of blended CSCL activities in DLEs is severely undersupported. [Technological problem for teachers]

- Taking into account that most teachers are not experts either in TEL, nor in ICT, finding good pedagogical uses of ICT when orchestrating blended TEL scenarios (i.e. when designing and enacting those scenarios) is as problematic and crucial [Law08] as the technological challenges mentioned above. Due to the myriad of elements involved, the orchestration of ICT in learning situations is a complex practice that is difficult to express, teach and enact. Design patterns [Ale77 represent practices of value, which provide conceptual tools for the praxis of design, which have been applied to a number of disciplines, from architecture to software engineering. Having a similar kind of tool for the practice of orchestration could prove very useful for many non-expert practitioners. [Conceptual problem for teachers]

- Furthermore, "orchestrating learning" is an emerging metaphor with rather nebulous definitions and frontiers. Thus, it is difficult for researchers to clearly communicate and accumulate knowledge about orchestration of learning until a clearer definition of the process and its main aspects is found. [Conceptual problem for researchers]

In order to present our approach to tackle these problems, the rest of this chapter is structured as follows: the next section details the main goal of the dissertation, as well as the partial objectives that have been set towards such goal; Section 1.2 describes the research methodology that has been used throughout the dissertation; and Section 1.3 summarizes the structure and contents of the rest of the dissertation document.

\subsection{Dissertation goals and contributions}

Given the research context mentioned above, we can formulate the main objective of the thesis as: "To design, implement and evaluate technological and conceptual tools to support the orchestration of blended Computer-Supported Collaborative Learning (CSCL) activities involving Distributed Learning Environments". Given the breadth of pedagogical practices that Technology-Enhanced Learning (TEL) includes, we have chosen to study more specifically the 
case of CSCL, as it is one of pedagogies where orchestration is most complex and critical (often including multiple technology-supported tasks at different, changing social levels and group sizes). Also, we have chosen Distributed Learning Environments as our main technological context, to anticipate the complex challenges that these increasingly common, de-centralized environments might pose for teachers in the immediate future. In order to achieve the aforementioned main objective, we propose to tackle the orchestration problems hinted at in the previous section, which in turn translate to the three partial objectives of the dissertation, summarized below and in Figure 1.1:

1. To clarify the concept of orchestration in TEL/CSCL research.

The metaphor of orchestration is relatively recent in the field of TEL. Since orchestrated teaching and learning is the central activity that we want to support, it is very important to understand it, the elements and processes that it comprises and what challenges and obstacles exist to a successful orchestration. Current research on the problem of orchestration is highly heterogeneous and the lack of a common theoretical or conceptual framework may prevent accumulation of knowledge on the subject. Thus, one of the main contributions of this dissertation is to propose and evaluate a definition and conceptual framework of the process of orchestration, to help the research community (and other stakeholders) in devising solutions to support such orchestration. In fact, research instruments based on this conceptual framework can also be devised, to help characterize orchestration in concrete educational settings. This framework and associated instruments can be used to analyze the context of blended CSCL activities in DLEs, to extract main orchestration challenges, and to guide our proposal of tools to support orchestration in such settings (see the following objectives).

2. To provide non-expert teachers with conceptual support on the orchestration of blended CSCL activities in Distributed Learning Environments.

According to socio-cultural activity theory [Eng99, human activities are composed of actions and operations of different granularity levels. In order for the learning experience to be orchestrated, not only the designed activities have to be devised towards certain pedagogical goals, but also the actions and operations taken during the enactment of the activities have to be aligned with those goals, so that they really are "supportive interventions" Dil09a Pri11f]. In this sense, best practices formalized as patterns are a common way of eliciting, communicating and reflecting about complex practices at different granularity levels [Ale77]. Another contribution of this dissertation is the proposal of atomic, contextualized patterns as conceptual tools to support the orchestration of blended learning activities involving multiple ICTs. Albeit the nature of these best practices is largely context-dependent, these patterns can be used as mediating tools by teachers (especially non-expert ones) to better align their orchestration actions with the desired outcomes of the learning activities. Moreover, an approach for the elicitation, development and evaluation of this kind of best practices is another contribution of this thesis, so that researchers and other stakeholders can help teachers in orchestrating TEL/CSCL, e.g., through professional development actions. This set of practices and approach has been informed by the aforementioned conceptual framework developed during the dissertation. In turn, these patterns can inform the development of technological tools to support orchestration (see below), as an expression of the kinds of actions that teachers 
normally take when orchestrating CSCL activities in Distributed Learning Environments.

3. To provide technological support for the orchestration of CSCL activities in Distributed Learning Environments.

The current gap between learning designs produced using a variety of authoring tools, and their deployment across a DLE (including one of many possible VLEs/PLEs, and a variety of learning tools) can be bridged by a technological infrastructure that translates between learning designs and their implementation in this heterogeneous environment. Thus, this dissertation proposes to design, develop and evaluate a technological infrastructure to support the orchestration of blended CSCL activities in DLEs, without tying users to a particular VLE/PLE or Learning Design authoring tool. One contribution of the dissertation is the proposal of a data model that serves as a lingua franca to translate between the concepts of the different Learning Design languages and different computerized learning environments. Another important contribution of the dissertation is the proposal and evaluation of the architecture and reference implementation of a technological infrastructure to support the deployment of blended learning designs in Distributed Learning Environments, based on this intermediate data model. In a similar way, the real-time management of blended learning activities in Distributed Learning Environments is challenging even for ICT-expert practitioners, as it involves the management of learning processes at different social levels, involving multiple dislocated tools. Thus, providing an explicit way to view and manipulate learning activities and other crucial activity elements such as participants or tools, during the execution of activities, could be very valuable to increase the feasibility of the orchestration of activities in these heterogeneous learning environments. Another contribution of this dissertation is to extend the aforementioned technological infrastructure to enable teachers to flexibly manage different aspects of the blended learning activities during runtime (e.g., changes in participant, group and tool configuration within the activity flow). The technological support provided should be informed both by the conceptual framework (to understand more clearly the impact of our proposals on the different orchestration aspects), and the aforementioned set of best practices developed during this dissertation (to understand the kinds of action that end users - i.e., teachers - take during such orchestration).

These three partial objectives tackle three outstanding orchestration challenges emerging from the literature and our own observations (see Chapter 2). Even if each of the proposed contributions addresses one of these problems separately, and can be used separately, they are not independent of each other. Rather, these contributions have informed each other during the research process, as hinted above. Nevertheless, not only these contributions are related through their respective development processes, they can also be combined in their use. In this way, they are completely compatible with each other, and they can be (and have been) applied in a combined manner, by the different actors (i.e., teachers and researchers), to concrete authentic CSCL scenarios. For instance, the set of best practices (classified using the conceptual framework) was used to structure a series of professional development actions (workshops) with university teachers, in which they learned how to orchestrate CSCL activities using DLEs. Later in these workshops, the same teachers used the technological support developed in this dissertation to deploy and manage in real-time the learning activities that they had devised. 


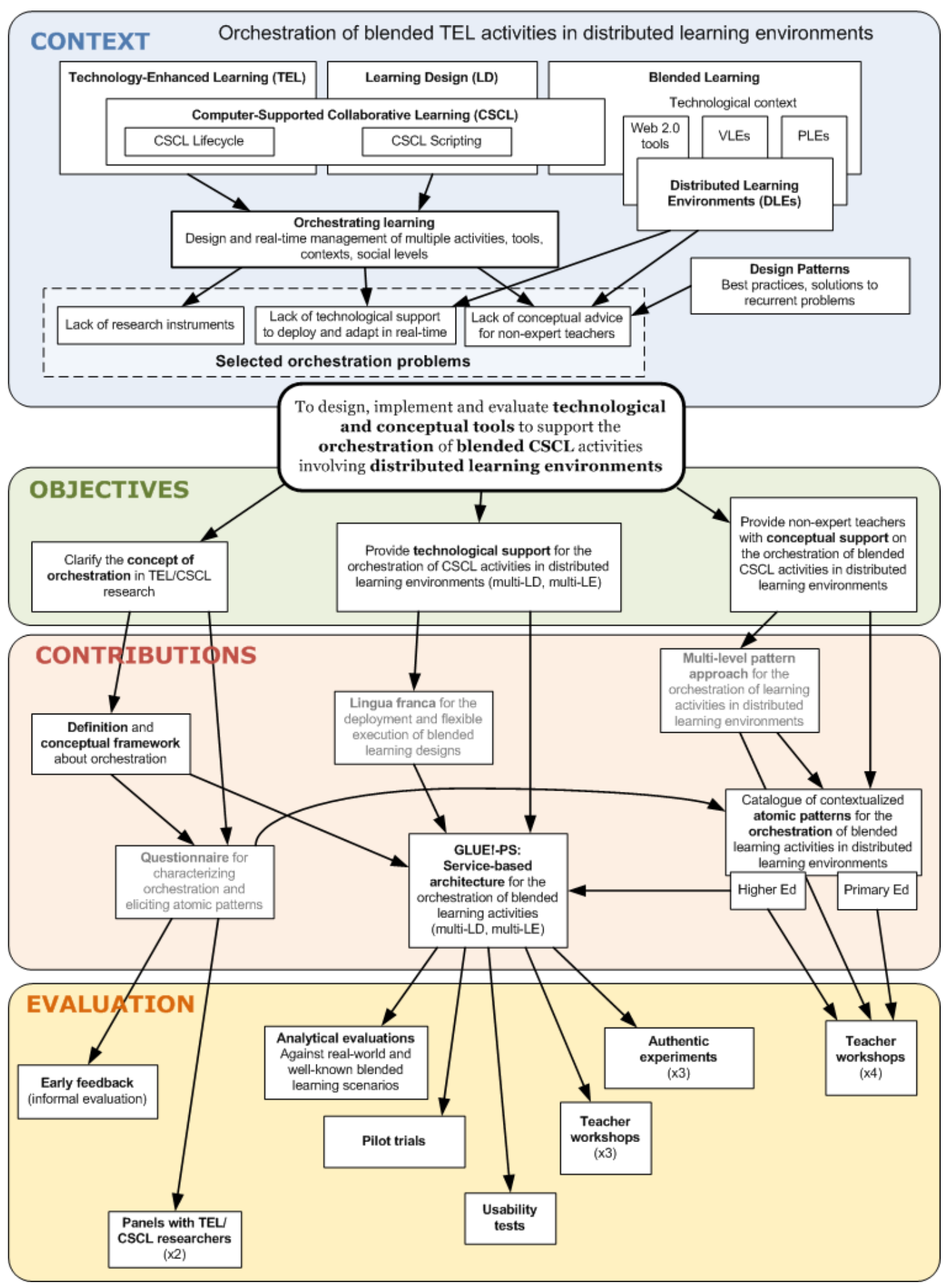

Figure 1.1: Schematic view of the context, goals, contributions and evaluation of the thesis dissertation. Contributions in gray are those of secondary importance. Number of evaluation happenings expressed in brackets, e.g., 'Teacher workshops (x2)'. 


\subsection{Research methodology}

Engineering method The problem of orchestration in Distributed Learning Environments is framed in the multidisciplinary problem domain of TEL, and in our case with a special focus on CSCL. In accordance with the observations and recommendations of many authors in the CSCL field [Sut06] [Str07, we have selected a hybrid, iterative methodology such as the engineering method [Adr93 [Gla95], to be the general methodological framework of the thesis, following four general phases in an iterative way: informational, propositional, analytical and evaluative. This multidisciplinary engineering-oriented research methodology highlights the importance of the evaluation phase to demonstrate the trustworthiness of the proposals. In this sense, although the results of the evaluation phase will be presented at the end of each contribution chapter, the reader should keep in mind that the partial results of each evaluation feeds the other phases in later iterations, as well as the ongoing iterative processes of the other contributions.

In order to validate our contributions, we have chosen to follow an observational method (as opposed to historical or controlled methods, see [Zel98]), collecting relevant data as an experience develops. Despite the weaknesses that are typically associated with this type of validation (e.g. it is not always possible to generalize its results), this method is the most adequate to address the posed research questions around orchestration, which is an emergent area of TEL (thus forsaking historical methods) that emphasizes the role of human actors and the importance of authentic educational settings Dil09b (thus forsaking controlled methods). Moreover, as researchers we are more interested in ascertaining the appropriateness of the proposed tools for orchestration in authentic settings and in evaluating the affordances that they provide, rather than in measuring their effects.

Within this observational approach, and given the constraints for research in orchestration mentioned by [Dil09b], we have to consider carefully the social context, combining theory and praxis to focus on real practitioners' needs. Thus, for each of the three partial objectives presented in the previous section, the four phases of information, proposition, analysis and evaluation have been followed iteratively until a 'satisficing ${ }^{3}$ solution is reached. In this kind of "nested engineering method" structure, each cycle has informed subsequent cycles along the same objective, but also provided valuable information to the other lines of work. Discarding the cyclical nature of the method to abstract the main activities towards the different objectives, we can represent the followed methodology graphically in a simplified way (Figure 1.2).

However, such a linear view of the methodology might be misleading to understand the process followed during the dissertation work (and the nature of the engineering method itself), as its main defining quality is its iterative nature. In fact, for each of the three mentioned contributions several cycles were followed, proposing and developing/analyzing the proposals, and eventually evaluating them. A more accurate (albeit more complex) view of the process followed during the dissertation is shown in Figure 1.3, where the informational, propositional, analytical and evaluative phases of each iteration are clearly marked. A more detailed and concrete view of the methodology and tasks followed to approach each contribution can be found in the introduction to their respective chapters (Chapters 3, 4, 5).

\footnotetext{
${ }^{3}$ Term coined by Herbert Simon which identifies the decision making process whereby one chooses an option that is, while perhaps not the best, good enough Sim82.
} 


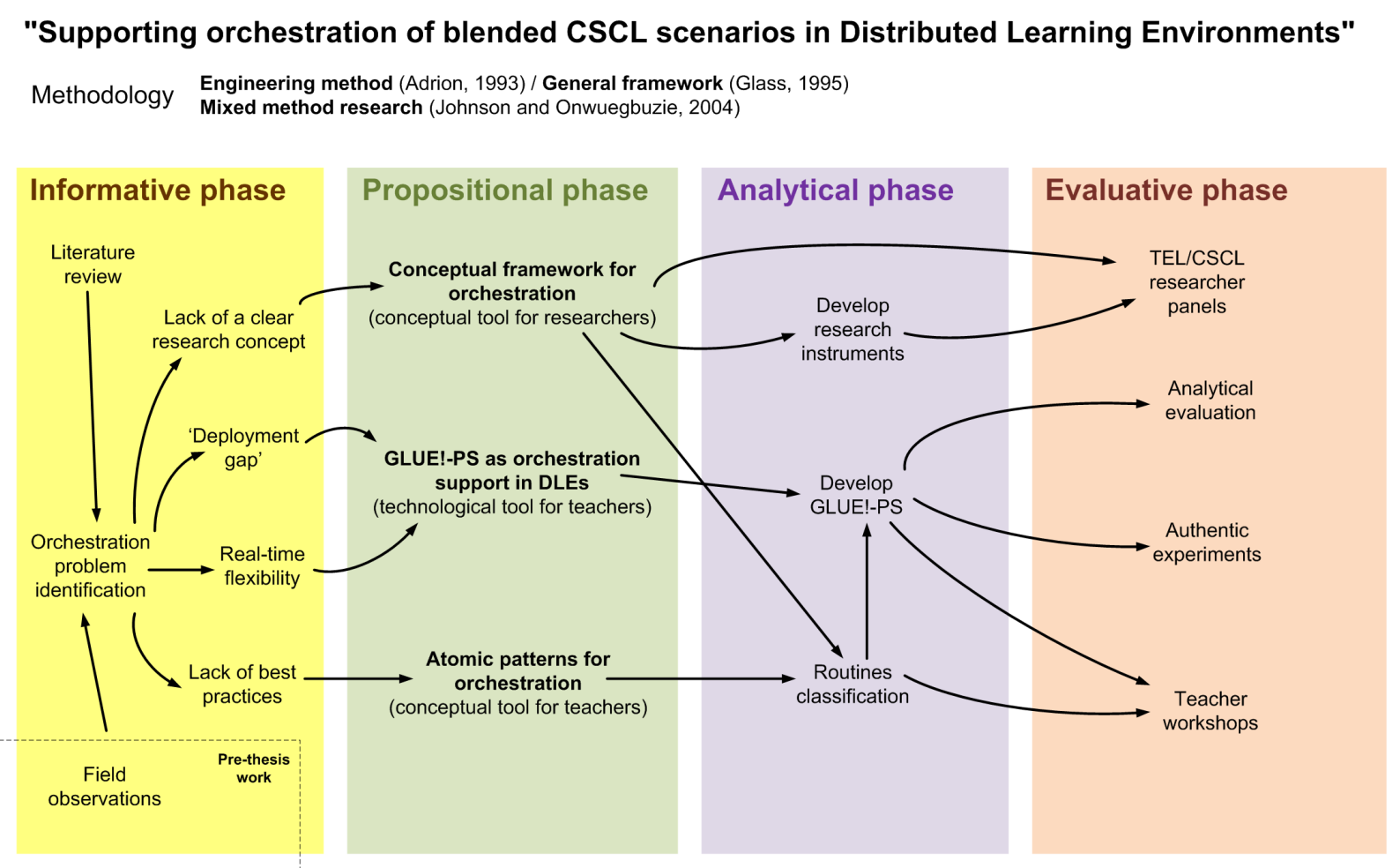

Figure 1.2: Simplified view of the engineering methodology followed during the dissertation

Evaluation model: CSCL-EREM The evaluative phases of the engineering methodology that we have followed demand further discussion. The evaluation of CSCL systems and innovations has shown to be a new and challenging field. Such a challenge originates from the combination of the innate difficulty of evaluation and the emergence of CSCL. In this sense, Treleaven argues that "evaluation in these contexts challenges traditional approaches to evaluation and require new theoretical frameworks to guide analysis and interpretation" Tre03. Given the focus of TEL (and especially CSCL research) in the social context, as well as the importance of contextual factors in the idea of orchestration itself, it follows that our evaluation experiences should be situated, whenever possible, in authentic educational settings. This fact points in the direction of naturalistic approaches to the evaluation of our proposals.

Along these lines, Jorrín-Abellán and Stake propose a "responsive evaluation" framework to be used by CSCL practitioners across disciplines: the CSCL Evaluand-Oriented Responsive Evaluation Model (CSCL-EREM, [JA09b]). This framework is intended to guide the CSCL researcher in evaluating educative innovations, learning resources, teaching strategies or technological support, having in mind that the effects of CSCL can not be reassumed along a single variable, but rather as a chain reaction in which each event gives meaning to the next [Sal92]. One of the main features of this evaluation model is that it is not stakeholder-oriented (e.g. directed at researchers vs. at teachers), but rather "evaluand-oriented" (i.e. focusing on what we want to evaluate - the evaluand - rather than in the differences of the evaluators). Other is the inclusion of multiple data gathering techniques, and member checking with the aim of 
CAP. 1

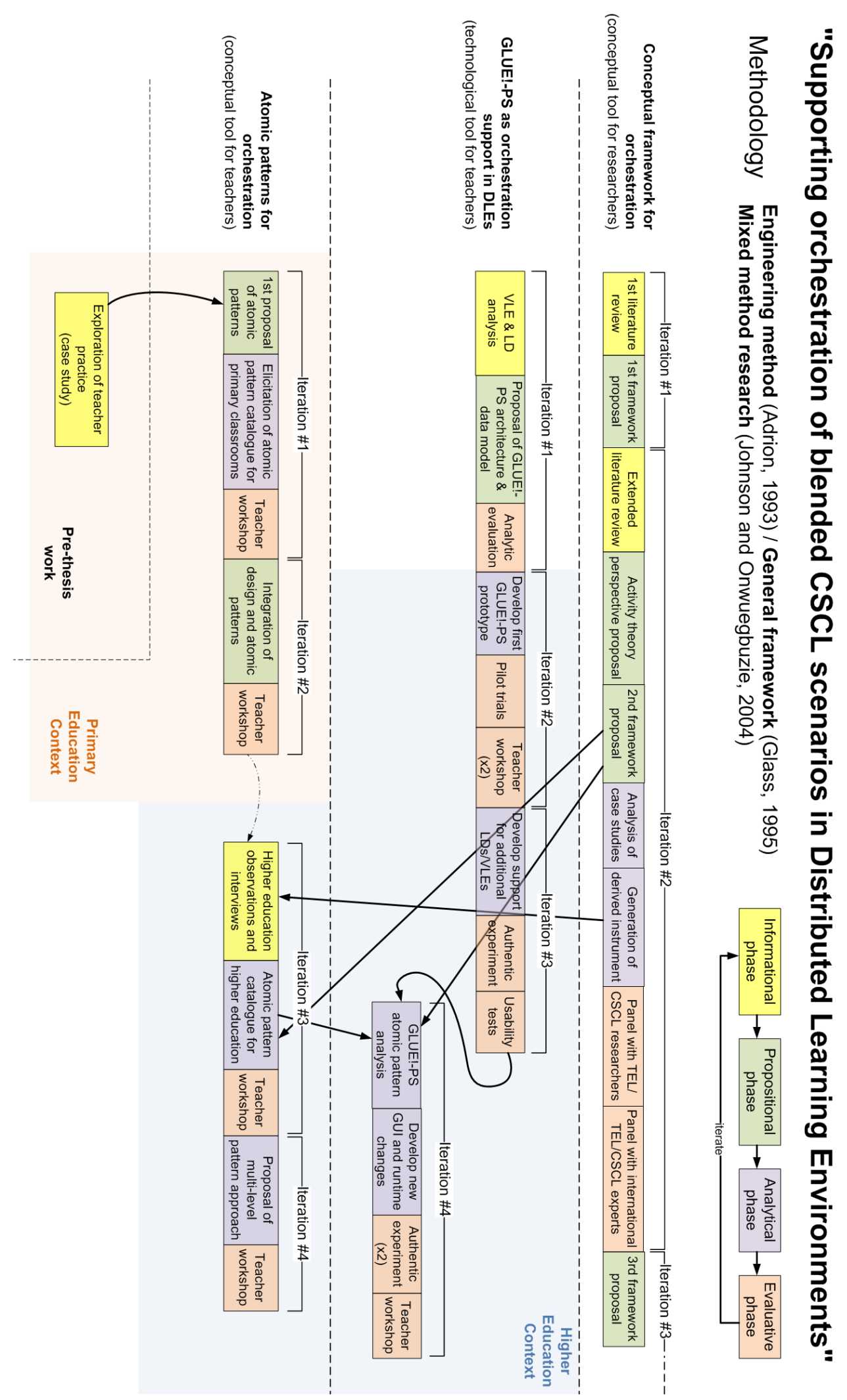

Figure 1.3: Detailed view of the engineering methodology followed during the dissertation, including the iterations followed 
generating rich enough evaluation outcomes [JA09b].

This model is part of the "fourth generation" of evaluation, which aims at responding to participants instead of measuring them (first generation), describing them (second generation), or judging them (third generation). The model is oriented more to the activity, the uniqueness and the plurality of the evaluand. Its design is slowly developed, with continuing adaptation of evaluation goal-setting and data gathering while the people responsible for the evaluation become acquainted with the evaluand and its context JA09a. In this approach, Issues are suggested as "conceptual organizers" for the evaluation study, rather than hypotheses or objectives. An Issue is a matter of contention which is of special concern or importance. Issues are ideas about the evaluated that have tension or potential tension, that there is a trade-off between two ways of treating the evaluand. Stories and/or data included in the evaluation report address the issues defined for the evaluation, providing pathways to reach a deep understanding of the studied reality. The statement of an issue can take the form: "For this case, with certain purposes in mind, certain actions have been taken, with implication for certain outcomes and well-being, with lack of agreement as to the desirability of the outcomes and appropriateness of the original actions".

Moreover, this responsive CSCL evaluation approach acts as a boundary object [Sta89] Sut06], determining a model both plastic enough to adapt to local needs and constraints of the several stakeholders employing them, and robust enough to maintain a common identity across different CSCL communities and possible CSCL systems and innovations to be evaluated (such as the different contributions proposed in this dissertation). This "evolving model" needs to be completed by CSCL practitioners.

Thus, the CSCL-EREM aims to provide clear, understandable and action-oriented guidance to CSCL practitioners involved in the evaluation of CSCL systems. The model's core parts are three facets (perspective, scope and method) that summarize the main characteristics that could be taken into account while conducting an evaluation of a CSCL system. The model also defines four question-oriented practical courses (or pathways) according to the possible evaluands that can be evaluated (CSCL programs/innovations/courses, CSCL tools, CSCL teaching strategies/learning resources and CSCL projects). Finally, it also provides a representation diagram with the aim of helping evaluators to plan an evaluation (see Figure 1.4), which we have used throughout our evaluations to describe the main features of each evaluation effort. Finally, the model describes a set of recommendations to write the report of an evaluation using the current model. The model provides clear and practical guidance to those CSCL practitioners that are novice in evaluation (such as the author), by proposing a particular organization of the complexity of the field. This way, the model can be interpreted as an effort to minimize the evaluation uncertainty.

For each of the three evaluations described in the contribution chapters (Chapters 3,4 and 5), we will describe the evaluation using the three facets of the CSCL-EREM model: the Perspective (the point of view based on which an evaluation process can be designed and carried out, including the main goals of evaluators), the Ground of the evaluation (the state of the environment in which a CSCL system exists, including the characteristics of the evaluation, the participants and the settings in which we evaluate) and the Method of the evaluation (the sequence of steps that lead the evaluation process, involving reasoning, observations, data collection, data processing, analysis and interpretation). In this regard, the model encourages the 


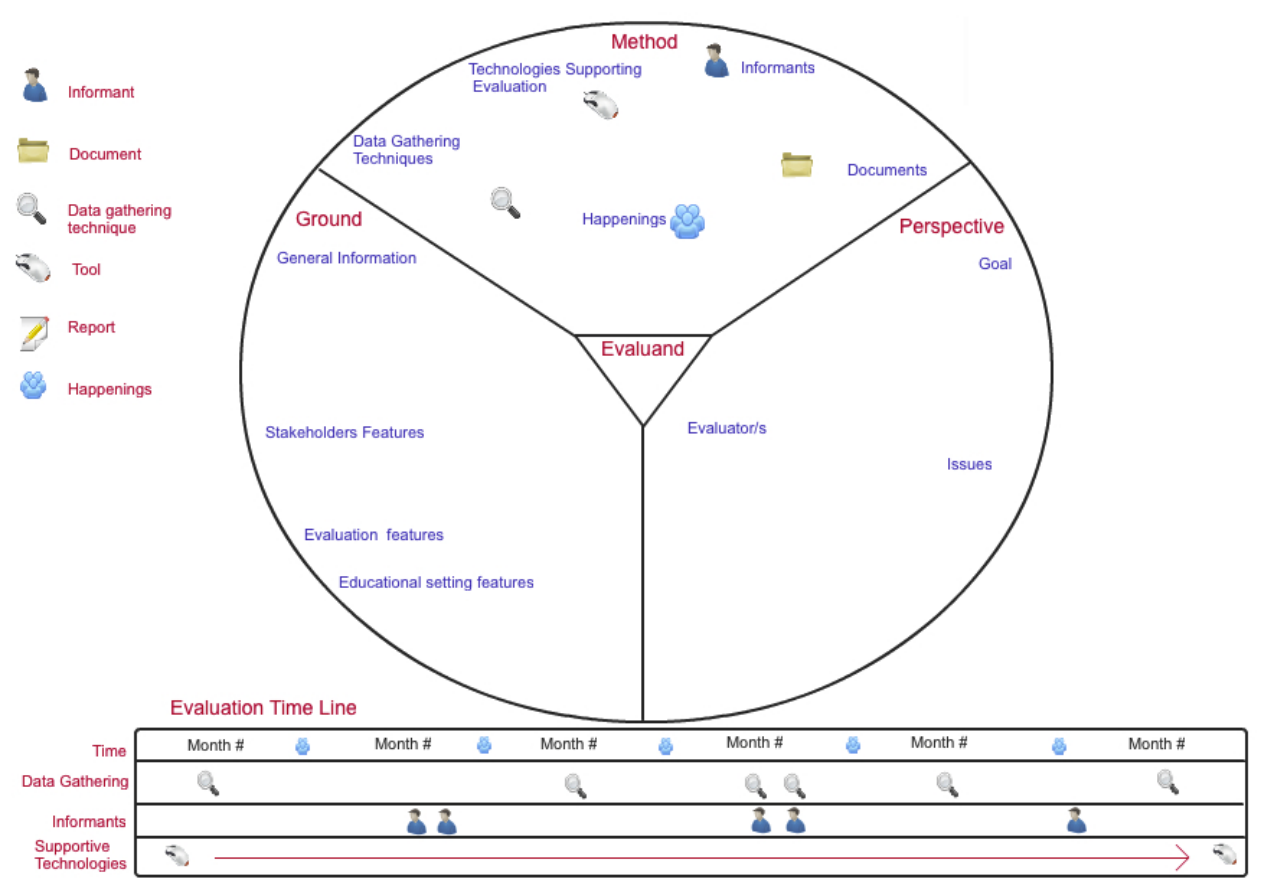

Figure 1.4: Graphical template for the design of an evaluation using the CSCL-EREM model JA09b

use of mixed data gathering techniques as well as a variety of informants, in order to provide multiple perspectives to enrich the evaluation process. The Method also includes the description of the Happenings or data gathering events that focus much of the evaluation work.

In this regard, throughout this dissertation we have defined a number of evaluation happenings for each of our contributions:

- The proposed conceptual framework on orchestration will be evaluated to ascertain its completeness and usefulness for TEL and CSCL researchers. Two panels of researchers (one composed by younger researchers, the other composed by international experts in the subject of orchestration) have been conducted, in order to assess the framework's completeness and usefulness for these research communities, at different levels.

- The approach to the elicitation and application of orchestration best practices, and the set of resulting best practices (in the form of 'atomic patterns'), have been evaluated in an iterative manner in two different educational contexts. First, two teacher professional development workshops were used to evaluate the approach and patterns in primary education. Afterwards, the same approach was refined iteratively to adapt it to higher education teachers and blended CSCL practice using DLEs, and it was evaluated through another two professional development workshops with university teachers from multiple disciplines.

- Regarding the technological infrastructure for orchestration, its development and evaluation have also been iterative and incremental in terms of the authenticity of the 
data gathered. First implementations were evaluated analytically, and by doing limited deployment experiments in real educational scenarios and in two teacher workshops. As the development of the infrastructure went on, up to three authentic educational scenarios were deployed and enacted with this infrastructure, and it was also evaluated through an additional teacher workshop.

Mixed methods approach As we have mentioned, the CSCL-EREM framework proposes the use of profuse data gatherings based in the mixed methods approach Gre01] Cre03], which is generally considered an adequate way of exploring the different perspectives and multiple factors that affect TEL and CSCL situations [Sut06] STtr07]. In this dissertation we have employed and adaptation of the mixed evaluation method described in Mar06b], which combines quantitative (for showing trends) and qualitative (to confirm or reject those trends as well as to understand them and identify emergent features) data gathering techniques. In this dissertation, due to the exploratory and emergent nature of orchestration of CSCL, our analysis has prioritized the qualitative perspective, using quantitative data gathering and analysis to increase the trustworthiness of our findings and conclusions, "triangulating" 4 the available evidence. This combination of data sources and analysis techniques frames our method within the mixed-evaluation-method approach [Joh04b], which advocates for the opportunistic selection of qualitative and quantitative data collecting and analysis techniques in order to achieve the desired evaluation goals.

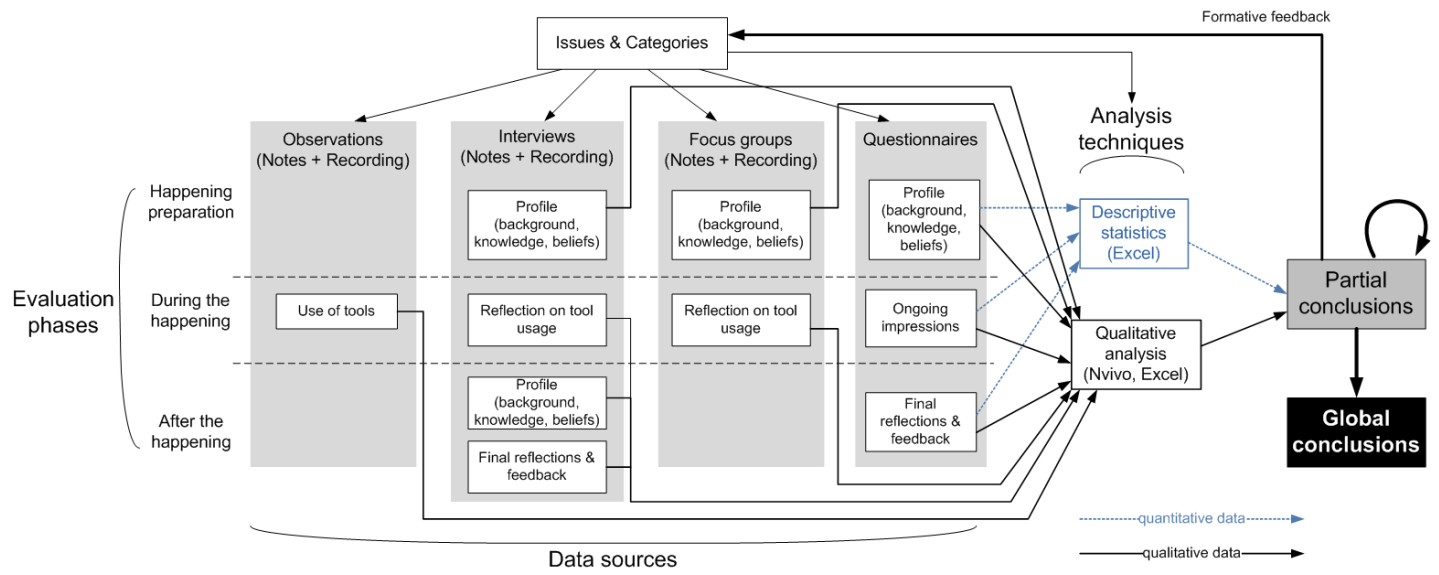

Figure 1.5: Graphical representation of the general flow of data gathering and analysis techniques employed in each of the evaluation happenings during the dissertation, adapted from Mar06b

In our mixed-evaluation method (see Figure 1.5), the study starts with the definition of a series of issues (to focus the evaluator's attention) and a scheme of categories for analysis. This can be done empirically, based on the results of past experiences, or theoretically, according to the evaluation objectives. This scheme is refined during the study by the specialization of existing issues and categories or the addition of new ones that emerge from the analysis. The evaluation is a longitudinal process that evolves cyclically throughout the research effort.

\footnotetext{
${ }^{4}$ I.e. comparatively analyzing and critically reviewing evidence proceeding from different sources Gub81.
} 
Qualitative and quantitative data is gathered to obtain information about the participants' profile and background, the use of the proposed contributions, and reflections on such use after the events. We use qualitative sources such as open questionnaires (useful for obtaining quotations), observations, semi-structured interviews and focus groups, and quantitative sources such as closed questions (to obtain measurement data). The quantitative data is aggregated and preprocessed using descriptive statistical analysis while the qualitative information is accumulated and structured into categories of analysis facilitating the interpretation of the arguments. The concrete data sources used and the moments of data gathering during each of the evaluations differ due to the circumstances, including time or accessibility constraints.

In the first stages each type of analysis is performed independently, providing partial conclusions that can be confirmed or rejected by triangulation, or that can produce a new cycle of the evaluation process in order to gain insights about an emergent aspect. The main products expected from this process are the refinement of the initial scheme of issues and categories, and the conclusions that provide formative feedback on different aspects of the learning situation, and which finally crystallize in a series of global conclusions of the evaluation.

It is also important to note that, although this multi-method study has been completed mainly by a single researcher (the author of this document), other researchers (components of the GSIC/EMIC research group) have helped in reviewing the design of the study, the careful preparation of the materials and the orchestration of the happenings, as well as the thoroughness of interpretation. This "member checking" [Sta05 is a crucial technique that contributes to the revision and improved formulation of the conclusions. However, given the complexity of the research effort proposed in this dissertation (to be carried out mainly by only one evaluator), and following some of the CSCL-EREM practical recommendations, we have selected as few different data gathering/analysis techniques as possible (to avoid data saturation). Also, following the parsimony principle, we have tried to use similar evaluation rationales and methods across each contribution as much as possible.

Presentation of evidence Several remarks need to be made about the way we have chosen to present our findings and supporting evidences throughout this dissertation. Rather than providing a more narrative, thick description style of evidence portrayal (which is typical of many qualitative methodologies [Sta10]), or a purely analytical, table-based one, we have chosen to provide our findings and supporting evidence through a hybrid approach between those two forms. In the evaluation sections throughout this dissertation we provide tables showing the issues under study, the findings uncovered and a selection of supporting (qualitative and quantitative) evidence, and we accompany them with short narratives summarizing those findings on each of the issues. We believe this hybrid form supports the reading styles of different readers, which might come from different disciplines and perspectives (e.g. those wanting to go deep into the raw evidence vs. those rapidly scanning for the main results), while maintaining the length of the document under control. Moreover, additional pieces of evidence (e.g. photos, document information, video snapshots) are included, to illustrate the multiple data gathering formats used during the evaluations. 


\subsection{Document structure}

In Chapter 2 we can find a literature review of the use of the term "orchestration" in TEL/CSCL literature. Also, the main technological and pedagogical contexts of the thesis are described in this chapter: the blended learning approach (especially its ICT-supported form) is reviewed; current TEL research on Virtual Learning Environments and "Web 2.0" for education are also summarized, as well as how those trends gave birth to the notion of Distributed Learning Environments (DLEs). Finally, we take a look into how orchestration of TEL/CSCL in DLEs is approached currently in authentic educational settings.

Chapter 3 includes the first conceptual contribution of the thesis: the ' $5+3$ Aspects' conceptual framework for orchestration in TEL, and the definition of orchestration derived from it. The evaluation of the framework by two panels of TEL/CSCL researchers (one of international experts on orchestration, another with younger TEL/CSCL researchers) is described. Moreover, the first research instrument derived from the framework (an interview and reflection guide on orchestration) is presented as a first practical application of the framework.

The other conceptual contribution of the dissertation, the notion of "atomic patterns for orchestration", is described in Chapter 4 . We begin by reviewing briefly the concept of design patterns, and then describe a two-year case study in which a pattern approach was applied to the professional development of primary school teachers that needed to orchestrate highlytechnologized classrooms. This chapter also describes how that approach was translated to a higher education context, and further developed to propose a multi-pattern approach to help non-ICT-expert teachers to orchestrate TEL and CSCL activities. We also describe how this approach and the notion of atomic patterns was evaluated iteratively through several professional development workshops at the University of Valladolid.

Chapter 5 is devoted to the main technological contribution of the dissertation: the Group Unified Learning Environment - Pedagogical Scripting (GLUE!-PS) architecture and data model. In this chapter we highlight the problems of deploying learning designs into DLEs, and we show the GLUE!-PS as a cost-efficient way of solving such "deployment gap" for a teacher who uses a Learning Design authoring tool and a mainstream institutional VLE/PLE (plus external tools). Also, the problem of flexible execution of the activities is discussed, and how GLUE!-PS provides means towards this flexibility in DLEs (drawing also from the best practices extracted as patterns in Chapter 4). Finally, we describe the development of a reference implementation of the GLUE!-PS architecture, and how it was evaluated iteratively by teachers using such implementation, both in professional development actions and authentic CSCL situations.

Finally, the dissertation's conclusions are drawn out in Chapter 6, highlighting the relevance of our findings for the CSCL and TEL research communities, as well as their implications for other educational contexts and related research areas. Furthermore, limitations of the presented work and directions for future research are outlined.

The dissertation's appendices include supplementary material such as the interview guide derived from the ' $5+3$ ' framework (Appendix A), the complete catalogue of atomic patterns extracted during the thesis's work (Appendix $B$ ), the analysis of LD languages and learning platforms that led to the proposal of the GLUE!-PS data model (Appendix C), or the detailed definition of the GLUE!-PS data model itself (Appendix D). 



\title{
Chapter 2
}

\section{Research context: Orchestration in TEL and Distributed Learning Environments to support Blended CSCL}

\begin{abstract}
Summary: This chapter reviews the central concept of this thesis (that of "orchestration"), as it is being currently used in the TEL and CSCL research communities. Also, the main educational and technological contexts of the dissertation are discussed: first, we review the notion of ComputerSupported Collaborative Learning (CSCL), and how it can be promoted using the blended learning format, which combines face-to-face and online activities. From the general research fields of TEL and CSCL, we summarize in depth the emergence of the notion of "orchestration" as a concept that emphasizes the complexity of teachers' work in authentic TEL and CSCL settings. This leads us to the first contribution of this thesis, a first synthesis of the TEL/CSCL literature dealing with orchestration. Afterwards, we review the notions of Virtual and Personal Learning Environments (VLEs and PLEs), that are often used to support blended learning. We also highlight the increasing need to extend or complement the VLE/PLE-provided tools with other external ICT tools, especially "Web 2.0" tools (wikis, blogs and other social media). Distributed Learning Environments (DLEs), which combine a VLE/PLE and external tools, are presented as one of the main outcomes of this need. Orchestrating learning in this technological and pedagogical context entails many difficulties, some of which are also described in this chapter. These selected problems (the lack of a clear "orchestration" concept, the lack of best practices for orchestration, and the difficulty of deploying learning designs and adapting them in run-time) will be explored throughout the rest of this dissertation.
\end{abstract}

\subsection{Introduction}

Information and Communication Technologies (ICTs) are being used more and more often in the field of education at all levels. In the last few years, the increasing technological and pedagogical complexity of authentic learning situations (as opposed to laboratory experiments where most of the variables and uncertainties are controlled) has prompted researchers to talk about the "classroom as an ecosystem" [Luc08]. In parallel with this systemic view of the educational setting, the complex task of teachers (and other actors) in coordinating the learning processes of students, across the different (digital and non-digital) tools, tasks and social levels has been 


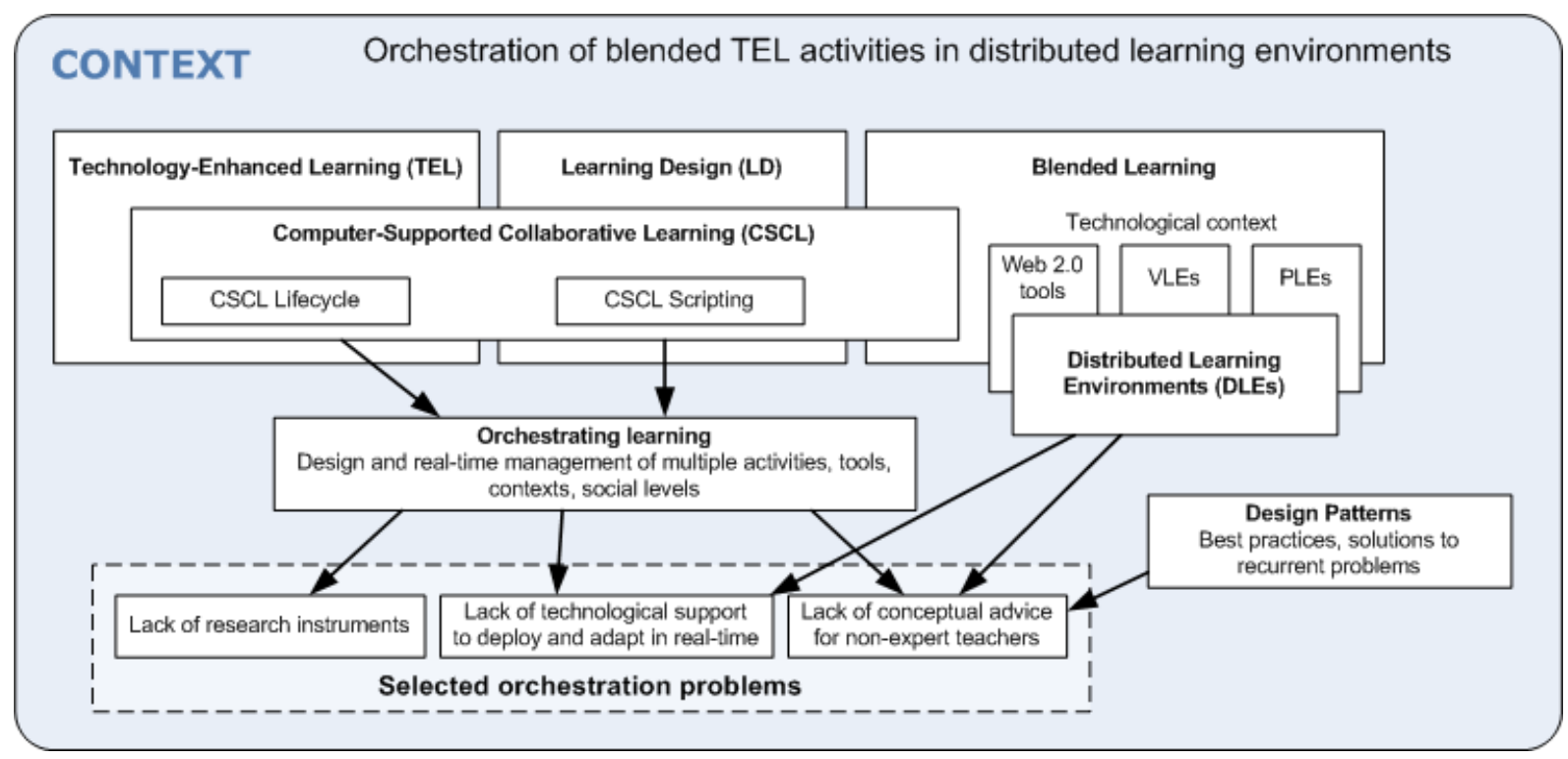

Figure 2.1: Diagram representing the research context of the dissertation, reviewed in this chapter. See also Figure 1.1

termed "orchestration" Fis06]. Orchestration, under this new meaning, has been posed as one of the most important research challenges in the field of Technology-Enhanced Learning [Sut09], and it is especially critical in the case of blended Computer-Supported Collaborative Learning (due to the complexity of the social structures and activities that have to be orchestrated in this kind of pedagogy). This dissertation revolves around this recent notion of orchestration of technology-supported learning, and tries to provide technological and conceptual tools to support different actors (mostly teachers, but also researchers) in such complex coordination.

In this chapter we draw from the TEL and CSCL literature to frame the research area of orchestration (also represented in Figure 2.1). After describing the broad area of TechnologyEnhanced Learning, we center on the multidisciplinary field of CSCL, and especially on the concept of scripting, as one common way of managing and coordinating (thus, orchestrating) CSCL activities. Then, we discuss the notion of orchestrating learning, used first in the field of education in general and, more recently, as critical for the practice of teaching and learning supported by ICT. We also review an increasingly common technological setting in education, which is the use of Virtual Learning Environments and other web-based platforms to support blended learning, and their evolution into more distributed learning environments that go beyond the walls of the institutional VLE. Finally, we motivate and frame within the TEL/CSCL literature three selected orchestration problems, both for researchers (the lack of clear definitions and research instruments for orchestration) and for teachers (the lack of best practices for teacher orchestration of CSCL in DLEs, and the lack of technological support for the deployment and real-time management of CSCL activities in DLEs).

It is worth noting that parts of the literature review in the present chapter have already been published by the author in different fora. For example, [Pri10b] and [Pri11e] contain much of the work on the notion of orchestration, Pri11g and $[$ Pri11d $]$ on the role of patterns and best practices in orchestration, and Pri09] on the improvisatory nature of teaching and the need for 
real-time flexibility in CSCL.

\subsection{From TEL to blended Computer-Supported Collaborative Learning}

As it has been mentioned already, this dissertation is framed within the wide research area of Technology-Enhanced Learning (TEL). TEL is a field of study that aims at facilitating and improving teaching and learning through technology [Joh04a] [Bal09]. In its infancy, the technology included audio- and video tapes, but the advent of computers and computer networks is the one that has most heavily influenced TEL Kos96. Albeit orchestration has been denominated a "Grand Challenge" of TEL in general [Sut09], in this dissertation we will mainly explore a concrete subset of the myriad of situations and settings that TEL encompasses (since it covers both face-to-face and distance, formal and informal learning). More concretely, we will concentrate on those forms of TEL that try to promote learning through collaboration (commonly denominated Computer-Supported Collaborative Learning, [Sta06a], integrating both face-to-face and online learning activities (i.e. blended learning, Gra05]). In this section we will review the most relevant concepts and literary sources for CSCL, making special emphasis on two concepts which are closely related to orchestration, that of scripting and the "life-cycle" of CSCL activities. We will also review the concept of blended learning, and the peculiarities and challenges that blended CSCL activities pose.

\subsubsection{Computer-Supported Collaborative Learning (CSCL)}

The field of Computer-Supported Collaborative Learning (CSCL) emerged in the 1990's, as a sibling research community to Computer-Supported Collaborative Work (CSCW) Ell91, which in turn studies collaboration processes and its ICT support in the workplace. In one of CSCL's seminal papers, Koschmann Kos96 presents CSCL as emerging from Instructional Technology (i.e. using technology for instructional purposes). CSCL is a "multidisciplinary research field inspired by the power of collaborative learning and by the promise of computer technologies to support collaborative learning" [Sta11]. Further description and historical accounts of the field can be found in Sta06a [Dil09a. Common gathering fora for this international research community, besides the ones promoted by the general TEL community, include the International Journal of CSCL (ijCSCL ${ }^{17}$ or the International Conference on CSCL ${ }^{2}$, which is held biennially.

As many other sub-communities in TEL, CSCL is a multi-disciplinar field that "builds on conceptual frameworks and analytic approaches of many academic fields, including education, psychology, communication, computer science and social science" Sta11. However, the main distinctive feature of CSCL is that it specifically approaches the support of social interactions among the students themselves, with a teacher playing a mediator or facilitator role Sta06a. In CSCL, a variety of quantitative and qualitative research methods are used, often combining them to develop richer understandings of complex phenomena. Likewise, research in CSCL may involve both laboratory and classroom studies, formal and informal learning settings, different

${ }_{1}^{1}$ http://ijcscl.org/ (Last visit: 09 May 2012)

${ }^{2}$ See, e.g., http://www.isls.org/csc12011/ (Last visit: 09 May 2012) 
temporal scales and the study of a wide range of influential factors. This multi-disciplinarity and the reliance on mixed research methods Cre03 should not be understated, and should be taken into account in any research effort framed inside the CSCL research field. In fact, those two aspects have heavily influenced the approach and methodology of this dissertation, within the general framework of the engineering method [Adr93].

\subsubsection{Learning Design, CSCL scripts and the CSCL life-cycle}

As we have seen, CSCL is a multidisciplinary research field that seeks to enhance learning by providing (networked) computer or ICT support for collaborative learning (CL), "in which particular forms of interaction among people are expected to occur, which would trigger learning mechanisms, but there is not guarantee that the expected interactions will actually occur" Di199. This "expectation" leads us into one common approach for supporting CL (and CSCL): scripting. Collaboration scripts [O'D92] [Fis07] focus on the specific activities that learners are expected to engage in, especially activities that research relates strongly with learning (e.g. elaboration, explanation, argumentation and question asking).Scripts are aimed at making the collaboration processes more productive [Dil07c], as they are assumed to lead to higher level cognitive processing and therefore to better learning outcomes. Thus, we could say that CL scripts are designs for assisting practitioners in organizing and structuring (i.e. orchestrating) collaboration that serves to guide learners through complex workflow or learning processes in order to improve educational benefits [Fis07]. Within the general idea of CL scripts, CSCL scripts often imply that the script has some kind of computational representation [Mia05].

Scripting has been studied from different perspectives, from the cognitive to the educational or the computational Fis07. Nevertheless, we can broadly classify scripts in two categories: micro- and macro-scripts. Micro-scripts are typically designed to support the development of internal representations for particular courses of action in particular situations (e.g., learning how to argue), often at the level of particular utterances [Wei09]. Macro-scripts are more focused on the coordination of didactic methods that facilitate the generation of educationally productive interactions among learners. These scripts describe activities on a coarser grain, involving organizational issues such as who collaborates with whom, in which roles participants act, and what the task distribution among groups is. Macro-scripts often structure collaboration by managing resources and deliverables and by defining roles and phases in order to produce specific interactions that lead to situations of effective learning [Dil07d]. In the rest of this document we will refer most often to macro-scripts, and thus we will label them just "scripts" from now on, for simplicity.

CSCL research has produced several conceptual frameworks for the design and description of scripts [Dil02a] [Dil06] [Kol06] [Kob07] [Haa07] [Wei09]. For instance, Dillenbourg [Dil02a] proposed a conceptual framework for the comparison and design of scripts. In his work, scripts are described as a sequence of phases, characterized by: the type of task to be accomplished, group formation, distribution of task within and among groups (including activity and role assignment, physical or virtual resources, etc.), the type and mode of the interaction (co-located, synchronous, text-based, etc.), and the timing of the phase. Later, Dillenbourg and Jermann Dil06 expanded this description to include individual and whole-class activities, forming a $d i$ dactic envelope around the core collaborative script. Kollar, Fischer and Hesse [Kol06] developed another conceptual framework by comparing CSCL scripts and instructional psychology scripts. 
From this analysis a number of concepts emerged, in order to classify scripts: objectives, activities, sequencing, roles and types of representation. Kobbe et al. [Kob07] proposed another conceptual framework for specifying scripts, based on the works by Dillenbourg, Kollar, and colleagues. Their goal is specifying scripts in a way that is independent of the design decisions like tool choice or concrete group composition (thus, as abstract and context-independent as possible). The authors distinguish between components and mechanisms in a script. Components include the participants, the activities, the roles, groups and the resources, while script mechanisms include task distribution, group formation and sequencing.

The scripting approach can be said to be part of the more general field of Learning Design Kop05. The design of learning situations (understood widely as the preparation of instructional materials, activities, information resources and/or evaluation), not only is a crucial step in the orchestration of such learning situations, but also has been present in education, in one form or another, for ages. In modern times, educational science has proposed slightly different flavors and methodologies of design (e.g. instructional design [Mer94], learning design [Lau02] ...), with varying degrees of acceptance by the practitioners. With the advent of ICT, distance learning and e-learning, the dream of being able to model computationally, automatically execute and reuse learning situations has been driving many research and standardization efforts. This aim gave rise to Educational Modeling Languages (EMLs): frameworks that contain important concepts, processes and relations for the modeling of educational systems or sub-systems, Kop04 [Bot07, such as the IMS-LD specification IMS03a. These computational models can be used to express collaborative scripts, and eventually to offload part of their management (and thus, part of their orchestration) to computers.

Looking at the practice of Learning Design in general (and of CSCL scripting in particular) from a temporal perspective, we can establish a "life-cycle" for the learning activities that appear in a script (or, more generally, in a learning design). Weinberger et al. [Wei09] define this life-cycle as having four phases: specification, formalization, simulation and deployment. Other authors have attempted different classifications, such as design, instantiation and enactment HL06b] VF09b. Alternatively, others have used the term "operationalization" instead of instantiation (i.e. design, operationalization and execution) Vig08a. Other researchers go further beyond the life-cycle of the script itself, and define a more general "CSCL life-cycle" composed of design, instantiation, enactment and evaluation of the learning activities [GS09 (see Figure 2.2. These different terminologies often seem to derive from the kind of educational context that the researchers have in mind when proposing the framework, and more specifically, the actors involved (e.g. is the design done by a common school teacher, or by an specialized learning designer in a distance university?).

Despite the differences in terminology, all approaches share the idea that CSCL scripts traverse a process, from the CSCL script's conception in the mind of its author, up to its enactment in a concrete computer-supported scenario, and its eventual refinement in further cycles. This is a complex process in which multiple human or computer agents can intervene $\mathrm{MC} 12 \mathrm{~b}$. In fact, some authors point out that this process does not need to be linear, with perfectly differentiated phases Vig08a], a fact that is confirmed by other authors that studied the practice of learning design in VLEs [Ber05], which is the kind of technological environment where many of the CSCL scripts worldwide are enacted (or executed, depending on the terminology used) today. Given the importance of Learning Design and scripting in supporting orchestration 


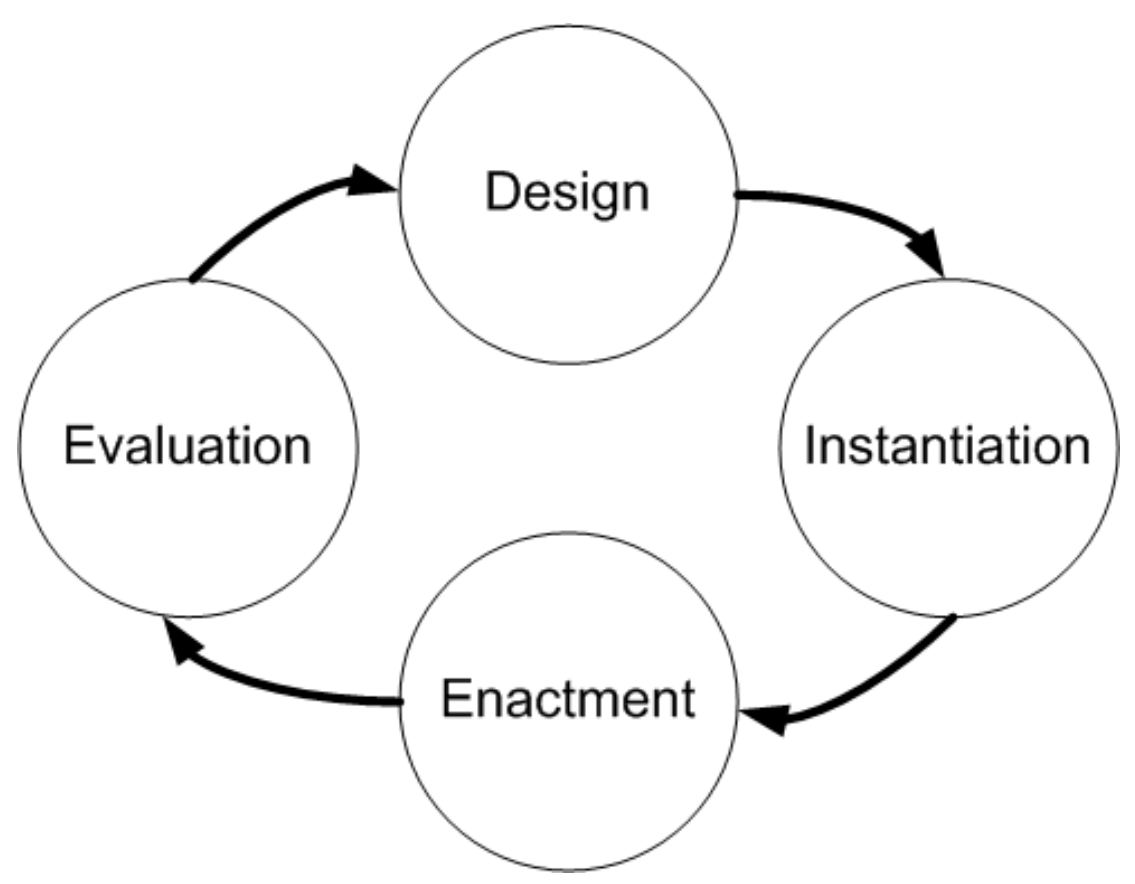

Figure 2.2: An example CSCL life-cycle, with the phase terminology from GS09

of CSCL (and TEL in general), any efforts to support orchestration of CSCL should take into account this cyclical structure (as we will see in Section 2.5).

\subsubsection{Blended learning and blended CSCL}

Contrary to some beliefs, the field of TEL does not equate to online learning or distance education. In fact, TEL covers both online learning, face-to-face learning where technology intervenes, and combinations of both (and conversely, so does CSCL for collaborative learning). This last flavor of education that combines both face-to-face classroom activities and other kinds of online learning, is often referred to as blended learning Osg03 Gra05. So and Brush define blended learning as "any combination of learning delivery methods, including moreover face-to-face (f2f) instruction with asynchronous and/or synchronous computer technologies" So08. Other authors use the term 'blended' from a technology perspective, referring both to the combination of $\mathrm{f} 2 \mathrm{f}$ with technology-supported activities, as well as pure online learning that enriches educational experiences by mixing virtual and real simultaneously [Kop05]. Given these ambiguous definitions of blended learning, Pérez-Sanagustín, after a literature review, proposes a wider different definition, that included "blend of spaces, blend of activity types (formal and nonformal) and blend of technologies to integrate the activities", thus proposing blended learning as "learning through combinations of formal and informal activities occurring in different spatial locations which are mixed and integrated into the same learning setting using technology" [PS11]. In this dissertation, however, we will look mostly at blended learning occurring in formal settings (e.g. schools, universities), which still account for most of the collaborative learning situations that are orchestrated every day. Moreover, blended learning is closely related to other areas within TEL such as "ubiquitous learning" Bru08]: learning which is connected across all the 
stages and places on which we play out our lives (also associated with fields like ubiquitous computing [Wei91]).

As part of the TEL area, (computer-supported) collaborative learning activities can be promoted also using a blended learning format. In fact, the usage of technology to facilitate blended learning approaches (i.e. to ease the transition between classroom activities and learning activities in other contexts) is a common feature of modern education, especially in higher education, e.g. in the framework of policies such as the European Space of Higher Education [Dec99]. These policies also encourage the use of active pedagogies such as collaborative learning, thus making blended CSCL scenarios very relevant in the near future, especially for higher education.

The process of designing and enacting (i.e. orchestrating) this kind of blended CSCL scenarios, in order to achieve effective collaborative learning is highly challenging. Both the learning flow and the technological support for the enactment have to be designed for promoting and enhancing interactions among learners. In the CSCL literature we find several challenges for CSCL than can also be applied to blended CSCL situations [PS11]: First, collaboration has to be guided, by choosing an appropriate sequence of activities and a particular role distribution to elicit the appropriate interactions for generating understanding [Dil07a]; Second, the use of technology has to be always driven by the educational considerations defined by the collaborative guide proposed, taking special note of the way in which their functionalities effectively connect activities and spaces to support and enhance the learning purposes Ros02]; Third, the activities and actions of the learners occurring at different spaces should be integrated into the same learning setting, often through the data flow used and produced in each activity Dil07b.

Interestingly enough, the most common way in which blended learning activities are currently "connected", i.e. the "data flow" mechanisms used, are based around the World Wide Web. More concretely, higher education institutions very often employ software platforms known as Learning Management Systems (LMSs) or Virtual Learning Environments (VLEs). Other institutions and researchers, seeing limitations in the VLE approach, have proposed equivalent but different technological platforms, dubbed Personal Learning Environments (PLEs), or just advocate the use of so-called "Web 2.0" tools to enhance blended learning. In Section 2.4 we review those concepts as the main technological background of the dissertation, and the challenges they pose for successful, blended CSCL orchestration.

\subsection{Orchestration in TEL and CSCL}

Common English dictionaries give us definitions of "orchestration" such as "arranging things to achieve a desired effect" [Hou00] or "to arrange or combine so as to achieve a desired or maximum effect" [Mer10]. The concept of teaching practice as an activity similar to that of an orchestra conductor is not a new one [Kov01] [For02]. This metaphor of orchestration, however, has experienced a revival in the past few years, as researchers in the fields of TechnologyEnhanced Learning (TEL) and Computer-Supported Collaborative Learning (CSCL) realize the difficulties that many teachers encounter as they try to incorporate into their practice the results of those fields' advances. In order to reap the benefits of technology-enhanced and collaborative learning situations, teachers have to cope with coordinating different activities, to be done using 
different tools (technological or not) by students grouped in a variety of manners [Fis06] [Dil09a], sometimes even across different contexts (e.g., in blended learning that combines face-to-face and online activities). The criticality of this coordination in the enactment of CSCL practice (where collaboration and learning can be made ineffective by poor management of those elements) has shifted the focus of some researchers to the support of teachers and learners as they wade through this myriad of factors in complex CSCL scenarios [MS10].

In this section we will deepen on the multiplicity of references to "orchestration" in the TEL and CSCL literature, in order to reach a first synthesis of its operative definition and main components. In order to reach this definition, not only we have reviewed the literature that mentions this concept of orchestration, but we have also analyzed different examples of research efforts that try to support this process using technology, so that we can extract the (often implicit) definition of orchestration that underlies those efforts.

\subsubsection{A literature review on orchestration}

The orchestration metaphor has existed in educational literature for a long time. It is one of the many metaphors that draws similarities between teaching and other forms of human creative behavior (specially, artistic expression): theater (see, for example, the IMS Learning Design specification [IMS03a]), improvisation [Saw04] or, as it is our case, musical orchestration and orchestra direction. The parallels between orchestration and teaching are obvious if we consider that teachers have certain (pedagogical) goals, decide what will be the flow of the class, and also decide when the lesson is over [Kov01] [For02]. Thus the assertion of teachers "orchestrating classroom discourse" [Jur05], or that orchestration is "the process of managing a whole learning group in such a way as to maintain progress towards the learning outcomes and improvement of practice for all" Moo01]. Chamberlain and colleagues define orchestrating learning as a process that leads to knowledge construction in a student-centered approach under teacher guidance Cha01, while Watts refers to orchestration as a way to know and understand students who have diverse preferences, and appreciate and create variety of approaches to support their learning Wat03.

In the field of CSCL, the most prominent proponents of the orchestration metaphor in the past few years have been Frank Fischer and Pierre Dillenbourg who, in 2006, posed orchestration as "the process of productively coordinating supportive interventions across multiple learning activities occurring at multiple social levels" Fis06. This first definition was later on expanded by the same authors, who cited some of the main dimensions of this coordination $($ Dil07c $]$ :

- Management of the cognitive dimension of the process, coordinating the learning processes taking place at different social levels (e.g. individual work, group work, whole-class activities).

- Coordination of the pedagogic dimension of the process, by adapting the designed activities to the real occurrences of the classroom.

- A technological dimension of orchestration should also be taken into account in CSCL scenarios, coordinating the transactions among the different software components of the scenario. 
In a similar way, Dil08a refers to teachers that orchestrate activities in different dimensions (social, pedagogic and technological) in order to achieve specific goals. More recently, some of the same authors expanded this definition of orchestration, towards the goal of successfully integrating CSCL in a broader learning environment that combines different activities, occurring at different social levels, contexts and media Dil09a. They note that several kinds of coordination should take place:

- Coordination of the workflow of activities that occur at the different social levels (e.g. by using macro-scripts).

- Coordination of the different scaffoldings used (the teacher, the peers, materials, software) in order to obtain synergies among them.

- Coordination of regulation mechanisms in the activity, be them either auto-regulation or external regulation.

- Coordination of individual motivation and social processes.

- Adaptation of the activities to classroom occurrences, and fading of any external scripts as they are being internalized by the participants.

- Providing teachers with monitoring tools that enable them to monitor the collaboration and adapt the environment to this information flexibly.

The first of these forms of coordination (coordinating the flow of activities) is the one that has gathered more attention and effort so far in CSCL, even before the rise of the orchestration metaphor itself, in the already mentioned concept of scripting. The use of scripts in education was originally derived from the "teaching as performance" metaphor [Gag78] [Del95], which viewed teachers as performers, actors that played a pre-defined script, represented in many cases by the textbook. Please refer to Section 2.2.2 for a more complete review on the concept of scripting. From an orchestration perspective, let us just say that in CSCL, scripts can be used to guide and structure complex collaborative scenarios, where a computational representation of the script (e.g. using the IMS-LD specification [IMS03a]) can serve to automate the workflow (e.g. by using Virtual Learning Environments with script playing abilities). However, this kind of script automation has been criticized as being potentially inflexible [Dil02a [Dil07d], and it is still not widely used in authentic teaching practice.

As we can see, a number of concepts and dimensions have been presented so far, even by the same authors, in a rather disorganized way. The STELLAR Network of Excellence [STE10b] acknowledged orchestration as an important emergent topic in TEL, by naming it one of TEL's "Grand Challenges". Interestingly, in its initial exploration of the concept [STE10a], four main research questions around the topic are posed, dealing with the role of teachers in orchestrating learning, the role of assessment and technology in learning, the relationship between high-order skills, knowledge domain and technology, and finally, about the learning trajectories of a person. These divergent questions, which had for the most part little to do with the definitions provided above, exemplify perfectly the increasing range of uses (some would say, the current state of confusion) of the concept of orchestration in TEL. In fact, the STELLAR NoE itself has also made attempts to clarify this situation by providing documents where different TEL experts express their opinions on the subject Dil11a. 
It is worth noting that, even if the concept of orchestration can be stretched to be applied to almost any learning situation, the word "orchestration" is almost invariably used in the context of formal learning, tied very often with the concept of a "classroom" where there is a teacher or "more knowledgeable other". This includes a main body of work in the context of faceto-face lessons in a (physical) classroom [Bea10] [Dil10], but also distance learning (especially the support for the management of learning) and the growing research area of blended learning and integrated learning [Dil07d] [Car09] [dlFV10]. The workplace is another possible context for orchestrated learning experiences [Sut09].

It may also be interesting to look briefly at how the metaphor of orchestration has been used in other research areas, even if an exhaustive and deep literature analysis of every field that mentions orchestration exceeds by far the scope of this dissertation:

- In the area of service-oriented architectures, orchestration has been used to denote the (often automated) combination of services to create higher level services and processes [Pel03]. This concept is also very related to that of choreography (another arts-related metaphor), which in this case refers to the concrete communication protocol (i.e. the messages exchanged) between the different services that are to be orchestrated [Dom06. It is interesting to note that in this context orchestration "always represents control from one party's perspective" Pel03. This is often the case with orchestration in TEL and CSCL, which normally depicts the teacher's perspective of the complex teaching/learning process. The choreography metaphor, on the other hand, is more collaborative, and allows involved parties to describe their part in the interactions.

- In the area of human-computer interaction (HCI) and in computer-supported collaborative work (CSCW), references to orchestration are not unheard of, either. [Dav09] analyze and model orchestration of interactive systems, and conclude that adaptation and plasticity of HCI systems can provide partial answers to it. Some of the same authors also proposed formalisms to model individual and collective activities (in a sort of musical staff notation) [Dav06.

- In the area of cognitive psychology, several other authors [Gra00 Mcc10 have used the term orchestration to emphasize that writing processes must be activated and coordinated by a control structure, such as the monitor in the Hayes and Flower model [Hay80].

\subsubsection{Examples of research under the orchestration "umbrella"}

Now that we have a first idea of what orchestration means in the fields of TEL and CSCL, we will take a look at four different examples of concrete research initiatives to support the process of orchestration. We will then attempt to map those efforts to the concepts of orchestration depicted above, to see what particular definitions of orchestration emerge from them, and use those definitions to confirm or expand our conception of the metaphor.

- One concrete effort to improve the process of orchestration, co-authored by Dillenbourg himself, can be observed in Ala09. In this work, Alavi and colleagues take a very concrete kind of learning scenario (recitation sections, where students work individually or in small 
groups solving problems, with teacher assistants helping on demand), and try to improve the orchestration process. Since this kind of scenario cannot have a clear, fixed script, the authors opted for improving the awareness of the progress of each group, through the use of multi-colored lamps. This helps teachers in planning their support and in optimizing their itineraries through the classroom. This work, obviously, uses Fischer and Dillenbourg's conception of orchestration ("managing the flow of activities across different social planes") as the starting point, but it concentrates on enhancing the awareness processes of the teacher.

- Another effort that also uses the same concept of orchestration as the starting point is [PS09c (with additional partial results detailed in [PS09b and [PS09a]). However, the authors in this case concentrate on very different educational settings: scripted blended learning scenarios (see Section 2.2), using computationally interpretable scripts for automation of the flow of activities. The authors propose a conceptual framework of factors that affect group management of CSCL scripts, aiming to implement flexible solutions for supporting group management in the design and enactment of such scripts. This work does not give a formal definition of orchestration, but its underlying assumption is that group management plays an important role in it.

- Niramitranon and colleagues [Nir07 propose what they call a Classroom Orchestration Management Language (COML), a computationally interpretable language to communicate the two main parts of their system for the design and facilitation of one-to-one technology classrooms (i.e. where each student has one computer device). They propose a prototype for designing and later automating to a certain extent several aspects of classroom management: a simple taxonomy of tasks, resources from a limited set of resource types, the presentation means of each task, and the actors of the tasks. The prototype for the enactment tool is based around a simple shared whiteboard tool, where information is interchanged in the form of "virtual stickers" [DiG06] [SRI08a]. Their work does not include any definitions of what they understand by orchestration, but the underlying idea seems to be that orchestration entails designing/scripting the aforementioned aspects of classroom management (basically, dictating what boards each of the participants sees according to the lesson design).

- Finally, Carell and Schaller Car09 try to ascertain how "Web 2.0" applications O'R05 can be orchestrated to support face-to-face TEL situations. They develop a case study in a face-to-face CSCL setting in higher education, where tools such as blogs or wikis were used to support collaborative learning. In their work, the authors do not provide a definition of what they understand as orchestration, and in the conclusions the term "orchestration" is lost, substituted by something more akin to the usage of multiple tools (in this case, the "Web 2.0" tools) to perform a set of activities.

As we can see, even if these works share the keyword orchestration, and all pertain to the field of CSCL, very different operative definitions of orchestration emanate from them. We can also try to map these works' conceptions of orchestration to the general notions of orchestration presented above (see figure 2.3). As we can see, all of them can be mapped to some of the factors derived from our literature review, and all of them can be seen as partial solutions to the complex challenges that orchestration poses for teachers. 


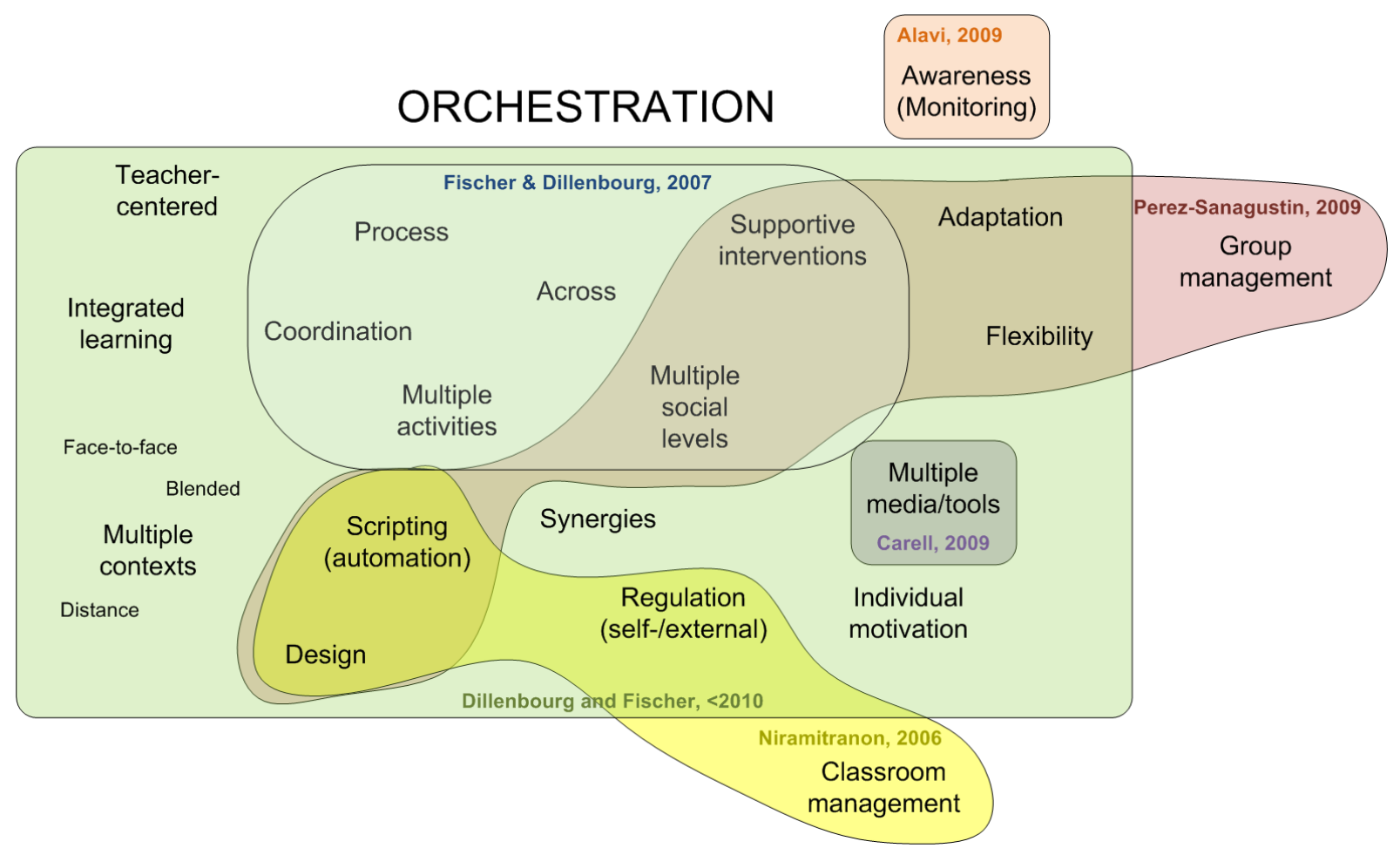

Figure 2.3: Diagram with common orchestration concepts, derived from the works of Fischer and Dillenbourg, and mapping of the four research examples analyzed onto those concepts

\subsubsection{First synthesis attempt on orchestration}

With all the information from the literature review described above, we can try to distill one possible operative definition of what is the orchestration of TEL and CSCL activities. By clustering and categorizing the concepts and factors that have appeared so far in this chapter, we can reach a more organized view of the metaphor, which can be seen in Figure 2.4. This diagram includes not only explicitly mentioned aspects of orchestration, but also others which are implicit in the reviewed work, or emanate from observed examples of real teaching practice, or have a clear relationship with our understanding of orchestration, such as:

- One important missing (or incomplete) element of the definitions of orchestration is the temporal element, represented by the question "when does orchestration happen?". This question has clear ties with the concept of "CSCL life-cycle" mentioned in Section 2.2.2. Many works assume that orchestration is done during the enactment of the activity, and most of them also assume that the enacted activity has been designed and thus, this design helps in the orchestration of the activity, by providing a structure for the elements that have to be orchestrated. Some works on the evaluation of CSCL activities [JA09a, also hint at the possibility that evaluation has to be integrated in the design and enactment and thus, evaluation may become another element to be orchestrated by the teacher.

- A more fine-grained temporal element can be distilled from the word "across" that appears in Fischer and Dillenbourg's original definition. Orchestration across different activities 
and contexts seems to imply that orchestration is more critical in the transitions between activities, contexts or social planes, but also when the activity occurs in several of these planes concurrently. That is not to say that teachers do not orchestrate when they (or their students) are immersed in a single task, but we could assume that in those moments orchestration falls to a more background position. Also, the frequency of these "orchestrationally critical episodes" is an important factor, marking the tempo of the orchestration (continuing with the musical analogies).

- Another important gap in the literature about orchestration is exemplified by the question "How is orchestration done?". In fact, this is the question that many a work on orchestration aims to answer. Even if no single formula can be given, research efforts (and real practice) in orchestration seems to polarize around two ways of orchestrating: the automated, technologically-mediated orchestration provided by computationally represented scripts (e.g. using IMS-LD specification and players), and the manual, socially-mediated way of orchestrating, where teacher uses spoken word and gestures to manage the flow of the activity. However, this is not a binary distinction, but rather a continuum, a design tension [Tat07], where each researcher or teacher chooses to position him/herself.

- Finally, the processes that orchestration is composed of ("What processes does it entail", in the figure), extracted from the literature sources, have been completed with others that have been made apparent by obvious relationships with existing concepts: assessment of the progress of the class (tied to the awareness mechanisms and leading to the "supportive interventions" mentioned by Dillenbourg and Fischer); re-design of the activities, tied to these interventions and to the concepts of "adaptation" and "flexibility"; and management of tools, timing and the workflows of the activity, which are not explicitly mentioned as composing processes of orchestration, but are readily apparent when we observe orchestration in practice.

Thus, with all these considerations in mind, and taking Fischer and Dillenbourg's definition as the center of our own definition and categorization, we could provide a first definition of orchestration:

Orchestration is the complex process of coordinating a teaching/learning situation, from the point of view of the teacher. Orchestration aims to manage (or subtly guide) the different activities occurring at different educational contexts and social levels, using different resources and tools in a synergical way. Orchestration is specially critical in the transitions and concurrencies between those elements, and it is often guided by a design (in the form of a script or not), that may be flexibly modified during the enactment (automated or not) of the activity, in response to emergent occurrences.

This new definition of orchestration can be used to guide our efforts in the development of tools that support non-expert teachers in the orchestration of CSCL activities (the aim of this $\mathrm{PhD}$ thesis). Since supporting all aspects of orchestration in every educational setting would greatly exceed the scope of the dissertation, we will focus on the orchestration of blended CSCL activities occurring in formal education, which are often supported using a specific kind of technological infrastructure. The following section provides an overview of the main technological 


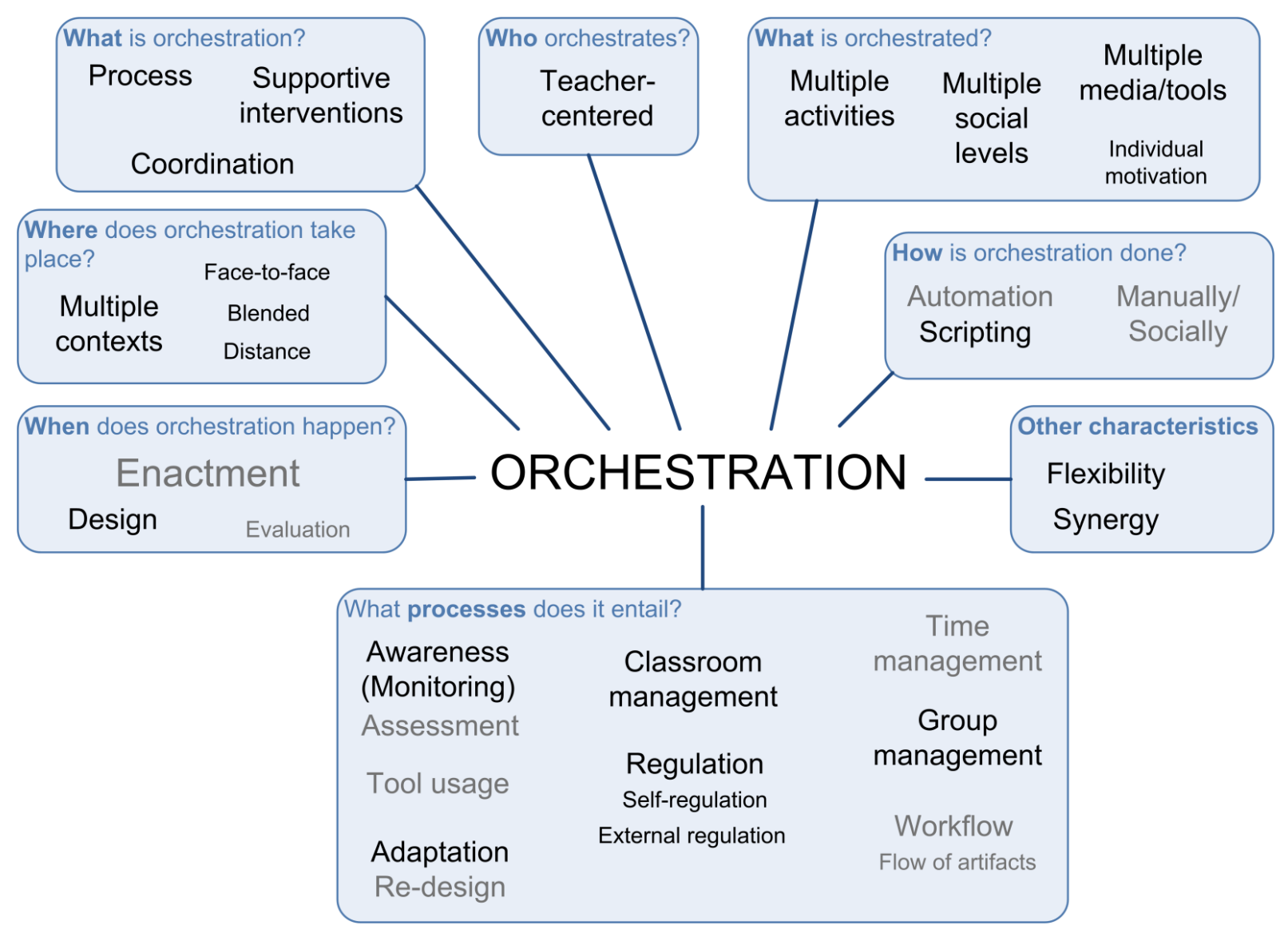

Figure 2.4: Concept diagram of orchestration, with the elements grouped in categories. Grey concepts are inferences, not appearing explicitly in the literature. 
background of the orchestration in these settings: the different learning platforms where most of the blended CSCL activities take place today.

\subsection{Virtual, Personal and Distributed Learning Environments (VLEs, PLEs and DLEs)}

\subsubsection{Virtual Learning Environments (VLEs)}

Virtual Learning Environments (VLEs) are software platforms for learning, which have been defined as "the components in which learners and tutors participate in online interactions of various kinds, including online learning" JIS12. Many authors, however, consider that the 'online interactions' element is not mandatory [Sti00 [Dil02a. Another term commonly used to refer to such platforms is Learning Management Systems (LMSs), which themselves are an evolution of Content Management Systems (CMSs), thus marking an evolution from contentoriented approaches to learning to more constructivist learning environments [Jon99] that are still mainly teacher-oriented. In the context of this dissertation "VLE" and "LMS" will be used interchangeably with preference of VLE over LMS.

Despite the ambiguities in definitions and terminology, and the variety of implementations of VLEs, normally these systems show several common features Dil02a): a) VLEs are designed information spaces in which multiple authors can produce both structured and unstructured information; b) VLEs are social spaces that promote interactions and discussions both synchronously or asynchronously; c) VLEs are explicitly represented ranging from textbased interfaces to complex 3D graphical systems; d) VLE students are also actors, producing (rather than consuming) contents; e) VLEs are not restricted to distance education, thus supporting also face-to-face and blended learning; f) VLEs integrate heterogeneous technologies (often including a variety of tools supporting different kinds of tasks), and multiple pedagogical approaches; g) Most VLEs overlap with physical environments at some point, since learning activities may involve the use of non-computerized resources or interactions among participants.

Examples of VLEs that are widely used ${ }^{3}$ by teachers and institutions on a worldwide scale include: Moodle 4 LAMS Saka: 4 , Blackboard 7 , or .LRN ${ }^{8}$. These widespread examples are all based on web technologies, following a three-tiered client-server architectures [Eck95]. Among these platforms we can encounter both open source projects backed up by large user communities (e.g. Moodle), as well as proprietary platforms owned by enterprises (e.g. Blackboard), implemented in a variety of programming languages.

VLEs have been reported to be beneficial for educators and students in many studies (see, e.g. Kat10]). Moreover, since most of them allow the definition of activities and social structures of users (e.g. groups), and the use of a variety of tools (e.g. chats, fora, shared text editing), VLEs can be considered important examples of CSCL systems used today in authentic learning

\footnotetext{
${ }^{3}$ Please refer to Appendix C for some statistics of VLE usage.

${ }^{4}$ http://moodle.org (Last visit: 11 May 2012)

5 http://www.lamsinternational.com/ (Last visit: 11 May 2012)

6 http://www.sakaiproject.org/ (Last visit: 11 May 2012)

7 http://www.blackboard.com (Last visit: 11 May 2012)

http://dotlrn.org (Last visit: 11 May 2012)
} 
contexts [Jon05]. Given the first definitions of orchestration given in Section 2.3.3 ("... to manage (or subtly guide) the different activities occurring at different educational contexts and social levels, using different resources and tools..."), we can also assert that VLEs can be a central piece in the orchestration of blended CSCL scenarios that utilize such platforms.

\subsubsection{Personal Learning Environments (PLEs) and other platforms used for learning}

Even if they are the most successful due to the wide institutional support they have garnered, VLEs are not the only widespread learning platforms. Personal Learning Environments vH06 are software systems that "help students take control of and manage their own learning". PLEs directly involve learners in the access, aggregation, configuration and manipulation of lightweight tools and resources (unlike VLEs in which educators or other staff select and manage the resources and tools that students should use) [Sev08]. Examples of PLEs include the one developed in the PLEX project Wil06, or the Southampton Learning Environment (SLE) Whi11. In general, PLEs take a non-hierarchical approach that promotes students' selfarrangement during their learning process. However, due to the promotion of collaboration and groupwork, PLEs may also be very useful for the design and enactment of certain collaborative learning situations.

Although PLEs are considered a very promising field in TEL, currently PLE use is much less widespread than VLE use, although some institutions have started to endorse them officially (see, e.g. the Southampton Learning Environment, or SLE, at the University of Southampton [Dav10]). PLEs share many of the technical characteristics of VLEs (web technology, threetiered client-server architectures, etc), although their approach is quite different, centering more in features delivered to (or produced by) each individual, and not so much to the management of a whole class or course. Nevertheless, the differences among them are starting to blur, as traditional VLEs support more customization features for students.

There exist other software platforms that have been used in the last few years to support (technology-enhanced) learning. Wikis, for instance, are software systems that enable the creation and management of content structured as interlinked web pages Leu01]. Probably the most prominent example is the Wikipedia 9 , an online encyclopedia built by an open, large community of contributors, and which now rivals established printed equivalents such as the Britannica. In wikis, the contents of a web page (e.g. one concept in the online encyclopedia) can be edited by multiple users, working in collaboration, and can include hyperlinks to other wiki pages or to external resources. Wikis are often also three-tiered web applications, developed in a number of programming languages, although probably the most widely used wiki engine is MediaWiki $\sqrt{10}$, a PHP wiki engine. Even if wikis were not developed with learning or teaching in mind (e.g. they do not include concepts such as course, or advanced grouping functionality), they are known to be used in the delivery of courses in some institutions, using active pedagogical approaches such as collaborative learning (see e.g., [For07] [JA07] [MM08] [Doe09], and Figure 2.5].

Similarly, Social Networks are software systems that allow users to create personal profiles and build (virtual) social networks, that connect them with other users [Len07]. Most prominent

\footnotetext{
9 http://wikipedia.org (Last visit 11 May 2012)

10 http://www.mediawiki.org (Last visit 11 May 2012)
} 


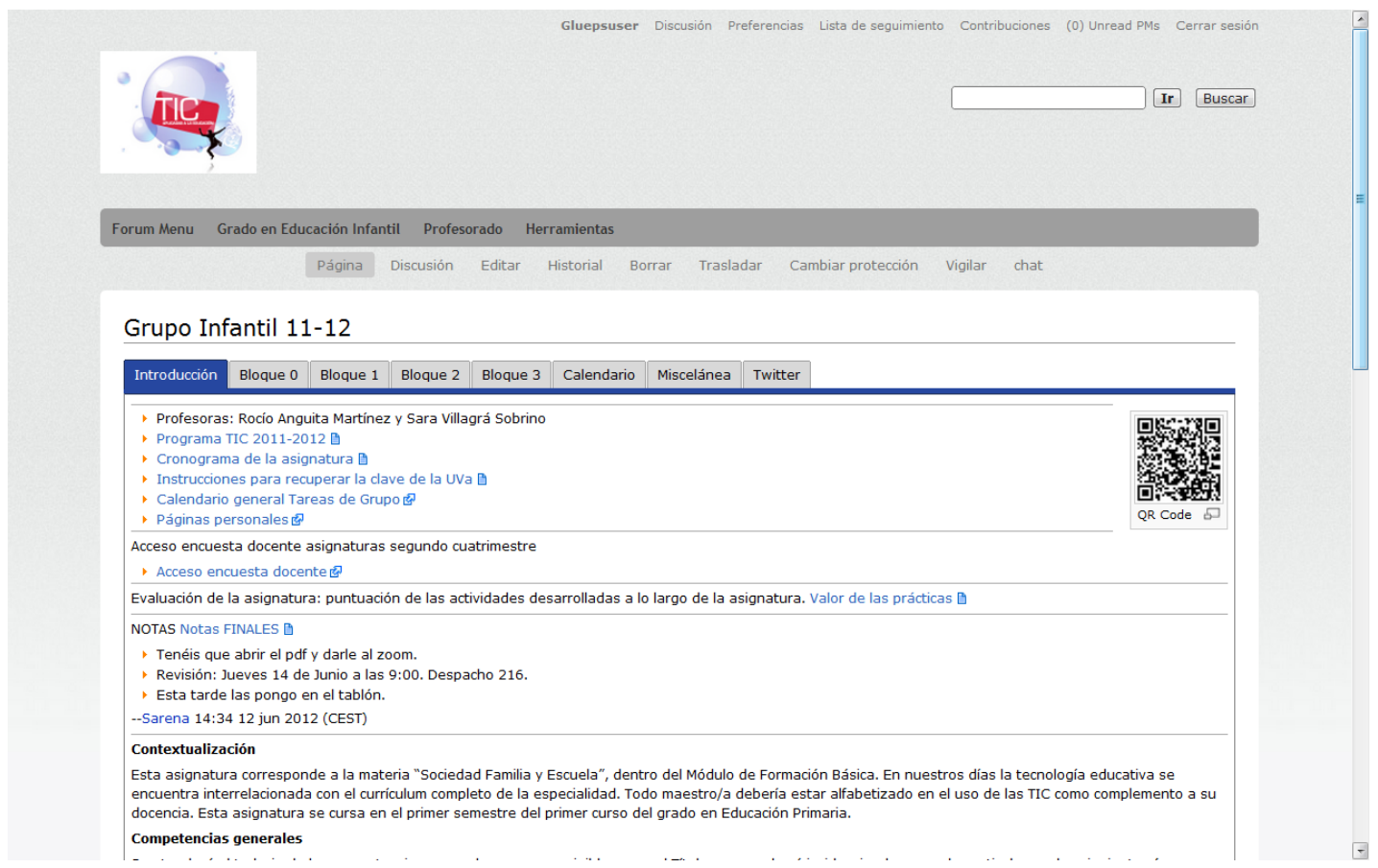

Figure 2.5: Authentic example of a wiki being used as a learning platform

examples of social networking sites include Facebook 11 LinkedIn ${ }^{12}$ or MySpace ${ }^{13}$, which have millions of users worldwide. The quick adoption of this kind of sites, especially among the young population, has prompted some researchers to propose them as suitable platforms for promoting learning, especially learning with a social component such as collaborative learning Mag09 SF11]. As in the case of wikis, these social software systems generally were not conceived with teaching and learning in mind.

As we can see in the example of wikis and social networks, in the last few years, teachers and researchers have started exploring new technological tools available in the World Wide Web, to support teaching and learning. From the point of view of their software design and architecture, all these platforms are very similar, and mainly they serve as "integrators", common access points towards other services offered by the platforms themselves or by third parties. In this sense, we will refer to all of them generically as "learning platforms".

In fact, both wikis and social networking sites are examples of what has been termed "Web 2.0" tools. As Tim O'Reilly notes, "Web 2.0" does not have a hard definition or boundaries, although it is said to have a number of core principles: service orientation, users as co-developers or content generators, software above the level of a single device, lightweight interfaces, development and business models, etc. [O'R05. Examples of "Web 2.0" tools include the aforementioned wikis and social networking sites, as well as other websites with an important component of usergenerated content (blogs, micro-blogs, photo sharing sites, etc). This kind of tools are being used increasingly in education [Con10c] Ben12], in an effort to leverage their affordances for knowl-

\footnotetext{
${ }^{11}$ http://www.facebook.com (Last visit 11 May 2012)

12 http://www.linkedin.com/ (Last visit 11 May 2012)

13 http://www.myspace.com (Last visit 11 May 2012)
} 
edge construction and collaboration, but also because students very often are already familiar with them. Also, other teachers and researchers see these tools as ways to escape the limited amount of built-in tools that a typical VLE offers Bow11.

\subsubsection{Distributed Learning Environments (DLEs): Beyond the VLE/PLE}

There are studies that report educators' concerns regarding the use of VLEs, such as the burden that setting up the infrastructure that the learning situations entail (compared to traditional face-to-face classes) Bow11, or the reduced set of built-in tools that the VLE provides Con10a Bow11. This last concern is particularly important, since this lack of built-in tools limits the amount and nature of learning situations that a teacher can propose when working with a VLE. This might be one of the reasons why many VLEs are simply used as document repositories and for administrative tasks (course announcements, submissions of homework) Kat10.

In this context, several research initiatives have emerged in the recent years, to expand learning environments beyond the traditional definitions of VLE and PLE, making VLEs more customizable, making PLEs more integrated into the institutional community, while opening them and leveraging the affordances of publicly available web applications (especially so-called "Web 2.0" tools) Mac10]. Alario-Hoyos and Wilson AH10 describe and compare several approaches to the integration of external tools in VLEs:

- The IMS Learning Tools Interoperability specification (IMS-LTI [IMS12]) is a standards-based approach that defines a complex contract, aimed to a tight integration of a wide diversity of tools, ranging from simple web applications to domain-specific learning environments and assessment tools. This tight integration allows VLEs to provide and extract information from the learning tools, which can be useful for learning situations (e.g. monitoring information about the progress of a task). However, it imposes severe requirements on both VLEs and prospective learning tools, which might explain why main VLE providers have been reluctant to implement the standard (and it is unlikely that general-purpose tool providers like Google will also comply with such restrictions).

- The Basic LTI IMS10 is a simplified subset of the IMS-LTI specification, offering a more lightweight contract focusing on the loosely-coupled integration of web applications. Basic LTI only exposes a single URL destination and a single POST method to provide access to the functionality of external tools, effectively limiting (and simplifying) the interactions between the platforms and the tools. This standard has garnered more positive reactions from VLE vendors and content publishers, who have begun its implementation. This approach has a disadvantage, however: in Basic LTI it is the teacher who has to manage the external resources being integrated (e.g. in the case of using Google Documents, creating and configuring the document, and linking it from the Basic LTI tool provider).

- Apache Wookie $e^{14}$ follows a different approach to tool integration. The Wookie server is an implementation of the widget server architecture, a standalone server application that can manage $\mathrm{W} 3 \mathrm{C}$ widgets $\mathrm{W} 3 \mathrm{C} 09$. This architecture, which was not designed with

\footnotetext{
${ }^{14}$ http://incubator.apache.org/wookie (Last visit 11 May 2012)
} 
learning tools in mind, allows for the instantiation and the subsequent management of widget instances, the configuration of these instances, the installation and deployment of new widgets, and the storage of persistent information that can be shared between them.

On the other hand, MacNeill and Kraan Mac10 take a wider approach, comparing not only the new approaches to learning platforms that used the VLE as a starting point, but also other approaches originating from the PLE and other fields, coining the term "Distributed Learning Environment" (DLE), and abstracting their main architectural features into five "models":

1. One system in the cloud, many outlets. In this model, a collection of services is gathered in one place, and from there they are broadcasted to a range of platforms. The platforms range from an existing VLE to a smartphone application, and the aforementioned Apache Wookie is mentioned as an example of this approach.

2. Plug-ins to existing VLEs. This model is premised on the possibility to extend the functionality of an existing VLE with a plug-in (a dedicated piece of software that exists solely to provide additional features to a host system). Since this plug-in is tightly bound to the host system, it is possible for a VLE to launch the plug-in and provide rich user identity, course, role and other session information (and similar kinds of information can be expected in return). The aforementioned Full LTI specification or the Icodeon SCORM player ${ }^{15}$ are examples of this model.

3. Many widgets from the web into one widget container. This model represents the typical mash-up of a variety of sources using nothing but Software as a Service applications (e.g. Netvibes ${ }^{16}$ ). This is also one of the archetypal implementations of a Personal Learning Environment. As such, each user has to assembly and/or customize the learning environment, and generally it lacks interactive, collaborative features such as discussion forums or other tools now considered typical in CSCL scenarios.

4. Many providers and many clients. As it happens with email, it is possible to federate an infrastructure out of many similar clients and servers. Google Wave (a recently-shut Google service) was one example of this type of infrastructure. In this service, a message or 'Wave' could be started by one person in one client, and replicated via public and private servers to the users of other clients. In this way, it was possible to create synchronous, highly interactive collaborative workspaces (waves). These technologies also allowed other widgets and services to be integrated into their messages (waves), and those waves could be embedded in other web platforms.

5. Both a provider and a client. In this model, the platform does the provision and consumption of tools/content directly, and to equal degrees, following the ideal of Service Oriented Architectures (SOA) Pap03. As an example, the future version 3 of the Sakai VLE is said to expose some of its content via W3C widgets to a blog and a smartphone app, and some of its functions to a social networking sites.

15 http://www.icodeon.com/product.html (Last visit 11 May 2012)

${ }^{16}$ http://www.netvibes.com (Last visit 11 May 2012) 
As an important sidenote, we should highlight that the term "Distributed Learning Environments has also been used in the field of educational technologies with a different meaning. On the wake of the Internet-era, in the late-1990's and first-2000's, several publications used that term to refer to environments "that give students access to a wide range of resources teachers, peers, and content such as readings and exercises - independently of place and time" Ala04. What this means, basically, is that they were Learning Management Systems that could be accessed through the Web, and that allowed distributed (i.e. distance) learning. In this sense, those distributed learning environments can be said to be ancestors of current LMSs/VLEs (in fact, the aforementioned Blackboard VLE is mentioned as one of such systems). In the rest of this dissertation, however, we will use the term "Distributed Learning Environment" with the meaning used by [Mac10], to denote learning environments composed of a VLE, PLE or similar learning platform, used together with other external tools (especially, "Web 2.0" ones), to support a learning scenario.

As we can see, there are many different approaches to DLEs, using very different architectural models. One such approach to the integration of external tools into VLEs (and hence, an approach to DLEs) is the GLUE! architecture AH10. The Group Learning Unified Environment (GLUE!) is a service-oriented architecture that aims at decreasing the average development effort that should be made to support the basic integration of several external tools, developed with multiple technologies, in different VLEs. This architecture defines a REST-based contract Fie02, follows a simple, loosely-coupled integration model, and uses the extension interfaces provided by external tools and VLEs.

The GLUE! architecture is composed by three main elements (see Figure 2.6). A set of VLE Adapters that deal with the specificity of each of the supported VLE's contracts (generally, developed as a plug-in for each VLE), provide an interface for the interaction of educators and students with GLUE!. Its main functionality is to show the list of available tools, and afterwards to request the creation and configuration of tools instances depending on the selected tool, but also on the number of users and groups that have been defined for that activity in the VLE. The central service in the architecture, the GLUElet Manager service, manages the process of creating and configuring instances. Finally, the Tool Adapters deal with the specific tool contracts in order to create the needed tool instances (since each tool adapter knows about the specifics of the tool's contracts). The Tool Adapters also contain specific or generic configuration templates for the tool they abstract. The GLUE! architecture offers a light integration of the basic features of external tools, maintaining the main functionality of VLEs, regarding the definition of groups, roles and learning designs. This architecture is extensible by developing new VLE and Tool Adapters (potentially by third parties), in order to increase the number of tools that can be integrated in different VLEs, and the range of VLEs supported.

In the scope of this dissertation, our technological proposals and research work will focus mainly on DLEs based on the GLUE! architecture. This decision was prompted by three main reasons: a) Given its ability to manage external tool instances, GLUE! can be a very useful tool for orchestrating the external tools that are part of a DLE, offloading part of the orchestration load from the teachers or the systems that we propose; b) GLUE! was specifically designed for learning environments, while remaining VLE-agnostic, a characteristic that we also desire for our proposals, given the fragmented and confusing learning platform panorama presented above; and c) Pragmatically speaking, GLUE! supported the two learning platforms in which our proposals would be first evaluated (namely, Moodle and the MediaWiki engine). It should be 


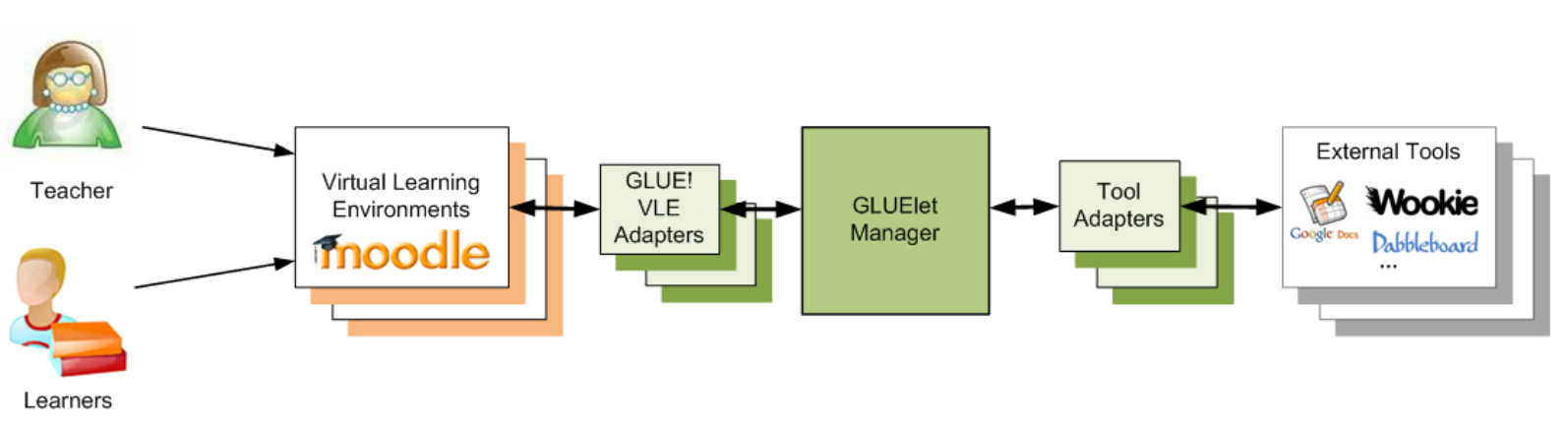

Figure 2.6: Simplified representation of the GLUE! architecture

noted, however, that neither GLUE! nor our technological proposal (see Chapter 5) are restricted to these two learning platforms.

\subsection{Orchestrating Blended CSCL in DLEs: Current practice and orchestration problems}

After reviewing the main literature sources relevant to orchestration in TEL and CSCL, blended learning and the technological context of Distributed Learning Environments, we can readily see how such orchestration can prove challenging. In this section we highlight only a few of the involved challenges, indicating how they are currently dealt with in literature or in practice. This analysis will serve as an introduction to the dissertation contributions that address each of those problems, and which will be presented in the following chapters.

\subsubsection{Towards a synthesized view of of orchestration in TEL and CSCL}

As we saw in Section 2.3, the usage of the word "orchestration" in the TEL and CSCL research communities is not exemplar in its coherence, with multiple aspects being highlighted by different authors. In Section 2.3.3 we attempted to synthesize a first definition of orchestration, but still such definition is not very adequate in order to operationalize a research effort from it.

In fact, this attempt by the author to clarify and synthesize the notion of orchestration in TEL/CSCL is not unique. There is a growing community of researchers that has been discussing this issue for the past few years. A first declaration of intentions was made by the STELLAR NoE in [Sut09]. In fact, the aforementioned synthesis and definition of orchestration by the author were presented in a workshop devoted to orchestration, at the International Conference of CSCL in 2011 Pri11d. More recently, also in the framework of the STELLAR NoE, a collective publication was made by Dillenbourg and many of the researchers in this small community Dil11a. The author and colleagues also were part of this deliverable Dim11a. Moreover, an evolved version of this "collective paper" is now under review at a well-known journal in the field of educational technology.

Albeit different concepts and issues are raised by each member of this community, there are two ideas that are slowly emerging from the discussions: a certain vision of an integrated 
learning based on scenarios that combine multiple social planes of activities and multiple tools; and empowering the teachers to manage this kind of learning in real time Dil11a. Additionally, Dillenbourg also mentions a series of requisites of any research effort towards supporting orchestration Dil09b]:

1. The proposals have to be teacher-centered, since orchestration is, by definition, a teacher process.

2. The solutions and research efforts have to be relevant to the curriculum, since teachers have little time to spare with experiments that do not advance their curricular goals.

3. The solutions have to take into account events in the different social planes, e.g. by providing workflows and scripts to help teachers in the orchestration.

4. The proposals have to take into account the co-existence with legacy tools in the classroom, using technology only for its added value.

5. The proposals have to take into account time management issues, as teachers need to be able to shorten, interrupt, resume or reschedule activities.

6. The solutions have to be flexible, not only in the time axis, but also in other aspects such as group formation, tool choice, etc.

7. The research efforts have to be sustainable, and they have to be designed, not for the motivated or gifted teacher, but rather with the average teacher and the common contexts in mind.

Even though these emergent trends are important, and should be taken into account in our endeavor to provide tools to support orchestration in TEL and CSCL settings. Yet, these trends are not enough to structure a whole research endeavor around them, since they do not help researchers in communicating about the differences and similarities among their orchestrationrelated research, and they do not provide a holistic view, an "analytical lens" upon which to analyze and eventually evaluate research proposals. Thus, the first problem to be solved in the field of TEL orchestration is a researcher problem: What exactly is orchestration, and how can we say whether we are improving it (or even affecting it)?. We hypothesize that having a clearer conceptual framework of orchestration will not only help us in researching new tools to support particular aspects of orchestration, but also will help in developing more general TEL solutions that support all these aspects by integrating particular methods and tools in a synergistic way. Moreover, from this kind of conceptual framework we can derive practical tools and instruments for other researchers to use, that can guide and help them in implementing innovations that are "orchestrable". One such framework is presented and evaluated in Chapter 3, representing one of the main dissertation contributions. Furthermore, this framework (along with the aforementioned slowly-forming consensus) has been used as base material for the design and evaluation of the other two main contributions of the dissertation: atomic patterns (see Chapter 4) and GLUE!-PS (Chapter 5). 


\subsubsection{Teacher practice in the orchestration of DLEs: the need of conceptual advice}

As digital technologies become more and more pervasive in education at all levels, ICT tools are becoming a commodity that coexists with other legacy tools (e.g. pen and paper, books, or traditional blackboards). Thus, classrooms are becoming a complex ecosystem of technologies and tools that can be used and combined in many ways to support learning processes of students Zha03. On a parallel trend, pedagogical research has long been advocating for methods and practices that are more complex than the unidirectional flow of information typical in traditional lectures (see, for example, [Bru08] and [JA09a]). However, the introduction of a new technology alone does not guarantee improved learning experiences, or greater learning outcomes. Over and over again, we have seen how ICT, in their different incarnations, are applied to education in a way that only leads to technology underusage and mimicry of the uses of older technologies (see [Hop93 [Cub01] Wat06] [Her08]). Even though research projects that consider both pedagogy and the development of technical skills in tandem do exist Mis06] She09] Bey10, most current examples of professional development programs for teachers concentrate mainly on the technical capabilities of these new tools, divorced from actual teaching practice Jun05.

As we saw in Section 2.3, teachers trying to enact learning activities in one of these technology-enhanced environments (be it in a face-to-face classroom, a VLE-centered distance course or a blended combination of both), will have to coordinate the different tasks that are to be performed using the variety of available technologies, ICT-based (networked computers, digital whiteboards, etc) or not (pen and paper, blackboards, books, and the like). This coordination is made even more complex in the case of teachers trying to apply collaborative learning techniques to their classrooms (i.e. orchestrating CSCL), since the activities will be spread over several social levels (individual work, small-group work or whole-class activities).

The enactment of activities (and especially collaborative learning activities) in a technologically-enriched classroom is a very complex process that has to take into account a multitude of contextual, technical and pedagogical factors (thus the metaphor of orchestration). One way of dealing with complex activities (especially for non-expert practitioners) is through the usage of patterns. Design patterns were proposed by [Ale77] in the field of architecture, as a way of representing successful solutions to recurrent problems in a field of practice (in Alexander's case, architectural design). The basic idea of design patterns is to present a recurrent problem that appears in a field of practice, and to describe the core of a successful solution to that problem, in a way that allows it to be reused throughout different contexts.

This approach provides several advantages for practitioners (especially non-expert ones): first, its problem-orientedness allows for easy location of solutions when the practitioner faces a problem; second, it allows practitioners to be more productive, as they are free to concentrate in the creative solution of unsolved problems (to which no patterns already exist). Design patterns also have the goal of making expert knowledge available to non-experts, as well as to serve as a tool for communication among practitioners in a field. Patterns have been successfully applied to fields as disparate as software development Gam95] or the design of CSCL activities [HL09].

A variant of the design patterns approach could be employed to try to make a complex activity (the orchestration of technology-enhanced, collaborative learning activities) available and understandable for non-expert practitioners. Taking into account the known properties of 
design patterns, we hypothesize that teachers would be able to easily find solutions to recurrent problems in the orchestration of activities. Also, that a pattern approach would allow teachers to apply and combine these core solutions to lower-level problems when enacting activities with ICT, allowing them to concentrate on higher-order issues, such as creating an atmosphere of productive discussion and a sense of agency in the process of learning [Mer07]. These social and motivational aspects of the activities are emergent and largely contextual, and normally cannot be predicted in the design phase of an activity. Indeed, these latter aspects can very often be neglected as a result of teachers having to solve the myriad of minute technical and management problems that appear when ICT is present in learning activities.

To the best of our knowledge, patterns have only recently been applied to the area of teacher enactment with ICT tools [DeB11. However, the concept of patterns is not completely unknown in performance-like practices (and we consider teachers' activity enactment as such, see [Wha02] and [Hum02]). [Bor89 and [Sud93] establish patterns as a basis for improvisational conduct in teaching and, more recently, studies by [Bea10] examine how musical metaphors such as orchestration and jazz improvisation can illuminate our understanding of the patterns inherent in classroom behaviors. Also, in a related but different area, the design patterns approach was taken by [HL09] to support teachers in the design of CSCL scripted activities (especially practitioners not expert in CSCL design).

Although the metaphor of orchestration has a long history in educational literature, there is a dearth of studies on how the orchestration of multiple ICT tools is performed by practitioners, since most studies concentrate on the use of only one tool (see, for example, Bea10). In the concrete case of Distributed Learning Environments, due to their recent emergence, we have not found in the literature any detailed account of how teachers approach their practice in such environments. Given the lack of useful conceptual models or frameworks on how this orchestration is done by teachers, we could employ inductive (bottom-up) research methods [Gla67] Bar04 in order to propose conceptual tools directed at teachers, e.g. in the form of patterns and best practices. Thus, this dissertation not only tries to understand what researchers mean by "orchestration": we also aim at providing teachers with tools that help them in attaining the needed skills for better orchestration.

\subsubsection{Learning Design and the "deployment gap"}

Although the concept of planning and preparing the learning activities to be undertaken by learners is as old as teaching itself, Learning Design as a research discipline (mainly related to e-learning systems and other technologies for education) is relatively recent. Koper [Kop06 defines Learning Design as "the description of the teaching-learning process that takes place in a unit of learning (e.g. a course, a lesson or any other designed learning event)". Multiple languages for the computer-interpretable specification of learning designs have been proposed, e.g. LDL [Fer08], PoEML [Cae08] and, especially, the IMS-LD specification [IMS03a, which became the most widely cited standard for this purpose. Along with these languages, a number of authoring tools were also created to help in describing those designs (e.g. Reload ${ }^{17}$ or Collage ${ }^{18}$, as well as tools for their execution (such as CopperCor ${ }^{19}$ for IMS-LD or LDI [Fer08] for LDL). With

\footnotetext{
${ }^{17}$ http://www.reload.ac.uk/ldesign.html

18 http://www.gsic.uva.es/collage

19 http://coppercore.sourceforge.net/
} 
these languages and tools, learning designers hoped to be able to describe, execute and reuse their designs on a large scale.

However, IMS-LD and other computer-interpretable learning design languages and tools have failed in gaining widespread acceptance. One of the possible reasons for this lack of adoption might be the scarcity of authoring tools that are usable by teachers [Neu10]. However, even if there exist LD authoring tools that try to overcome this limitation (such as Collage HL06a for CSCL designs), an even bigger obstacle lies in the (automatic) setup and execution of learning designs. Currently, few alternatives exist for the automatic deployment of learning designs, and these alternatives tie the practitioner to one specific implementation of execution environment. The standards-based approach of IMS-LD assumed that, once the specification was in place, developers of learning environments (such as VLEs) would implement or integrate IMS-LD players in their software. Still, with the exception of .LRN and its extension to execute IMS-LD (not part of the default .LRN distribution, see [Esc07]), none of the major learning environment developers has adhered to the specification, whose development has now stalled, and is being criticized for its complexity and the difficulty in implementing compliant execution environments [Der10] [Neu10], even if there are studies highlighting that IMS-LD's conceptual structure is not the main barrier for adoption Der11. Indeed, we can find in the literature several other efforts to go from the design of the activities to their execution (see, e.g. Fer08 [Nod08]), but they all require deep knowledge of the respective specification languages, and considerable efforts in deploying them into customized or specialized execution environments.

Thus, despite the progress of the Learning Design field (in the form of design methods and tools) and the existence of standards to help in the definition and reuse of learning designs (such as the IMS-LD specification), these computationally-represented scripts are not widely used. For many practitioners the design of learning situations is restricted to aide memoires scribbled in a notebook, or high-level lesson plans required by educative administrations, while in other cases it is not even in written form, and it lives on the teacher's head. An exception to this are large institutions, often specialized in distance learning, which may have invested in specialized "learning designers" to aid in the task of formalizing the scripts. In most other institutions, with more modest budgets (or less interested in automating course delivery), Educational Modeling Languages (EMLs Kop04) such as IMS-LD normally are not used by teachers in everyday practice Neu10.

Against this background, nowadays a teacher working with a VLE (often provided/mandated by her institution) has very slim chances of being supported in deploying a learning design she has crafted. Indeed, often her only option is to create the infrastructure that reifies the design in the terms and concepts of the available VLE by hand. This process is tedious, often taking long hours, and increasingly error-prone as the design complexity rises. The problem is even more acute in the case of (scripted) collaborative learning since, as we saw in Section 2.2.2, it often requires the management of changing student groups accessing different resources, throughout the different activities in the scenario.

To unblock this situation, making learning designs and their execution more widely available to teachers, other non-standard approaches have been tried. The LAMS platform Dal03, for example, provides teachers with an environment including intuitive graphical authoring tools (inspired by, but not compliant with IMS-LD), as well as execution and monitoring features. More recently, the SCY project Lej09b has also developed a learning design tool and execution 
environment, intended for science teaching and learning. However, despite their value as integrated environments that allow for design and enactment of learning situations, these approaches have a common problem: they are "walled gardens" in the sense that, in order to enjoy their advantages, users (e.g. teachers) have to embrace the whole approach, adopting both the design tools and the execution environments provided. Although seemingly unimportant, this requirement poses an important practical obstacle for the adoption by teachers and other practitioners, who normally have to use a VLE chosen by their institution. Moreover, these integrated systems present the same problems, regarding the limited number and variety of built-in learning tools, that VLEs suffer Bow11.

This task of deploying a learning design is even less supported in the case of the DLEs depicted above (Section 2.4), which combine VLEs and other ICT tools (e.g. "Web 2.0" tools). Very few solutions exist to the problem of supporting practitioners in the deployment of learning designs in such de-centralized environments. In this increasingly common technological context, a teacher whose institution has not chosen one of the aforementioned LD-enabled learning environments (and let us remember, most of the major VLEs are not of this kind) is "out of luck". This teacher will have to design the activities as she sees fit, and then transform that design into VLE-specific concepts (such as groups, activities, etc.) manually, across the different DLE domains (e.g. in her Moodle VLE, in Google Docs, in her wiki server, etc.). This process is even more time-consuming and error-prone than it was in the case of a single VLE. Furthermore, the reusability of these designs will be even more limited, since the whole manual deployment process has to be undertaken again throughout the different domains, in case she wants to repeat the same (or a slightly modified) design in a different context. Looking at the different kinds of DLEs that were presented in Section 2.4, we can see how such deployment would take place:

VLE + external tools (Basic LTI) The Basic Learning Tool Interoperability (BLTI) specification [IMS10] is one of the main ways of integrating external Web 2.0 tools into VLEs, which is gaining acceptance among VLE vendors due to the simplicity of its implementation 20 . As we saw in Section 2.4.3, the specification focuses on loosely coupled integration during execution of the learning activities. Due to the inclusion of BLTI in the IMS Common Cartridge specification (IMS-CC [IMS11]), and even if this specification is more intended towards content distribution, a learning design could be expressed in that format (including external tools and resources) and deployed to a VLE. However, IMS-CC lacks the expressivity necessary to deal with most collaborative learning designs. Moreover, manual administrative steps are needed to deploy the parts of the design outside the VLE (e.g. the creation of instances of the external tools/services, such as e.g. each Google Documents document needed by groups and students), and to link the activities in the VLE to such resources.

VLE + external tools (GLUE!) The GLUE! architecture AH10 is another loosely coupled approach for the integration of VLEs and external tools. Its main advantage is that it manages the whole life-cycle of the tools external to the VLE (e.g. external tool instance creation and configuration). However, the GLUE! architecture currently does not support the deployment of learning designs, and so teachers using this architecture have to implement their designs by hand. That is, the teacher would have to translate the activities,

\footnotetext{
${ }^{20}$ The BLTI website lists 22 learning environments that have been certified as compliant with the specification, including some of the main players like Moodle or Blackboard.
} 
groups, etc. to VLE concepts in her mind, and then deploy the internal and external tools/services manually, through the VLE's graphical interface.

One system in the cloud, many outlets (Widgets) Widget engines such as Apache Wookie are another way of integrating external tools in VLEs and other platforms, which already has been implemented in several VLEs, social networks and portals. In this case, the widget engine also manages the life-cycle of the external tool. The support for the deployment of learning designs in this case depends on that of the host platform (i.e. VLE, social network etc.). Since most platforms do not support this kind of deployment, and there is no standard way of referencing these widgets from a learning design, the teacher would have to deploy the design in the platform and reference the widgets manually ${ }^{21}$.

Widget container as Personal Learning Environment One of the archetypical implementations of a PLE is to gather a mashup of different widgets (i.e. Software as a Service applications). As noted by [Mac10], this kind of environments requires manual assembly, and currently they lack interactive, collaborative features such as forums or chats. Even if the concepts of PLE and learning design seem somewhat opposite, they are not necessarily incompatible. Nevertheless, since there exists no PLE that supports Learning Design specifications, teachers again lack support for deploying learning designs in this kind of environments.

Others Other models of DLE are also possible, such as implementing learning scenarios based on (now extinct) Google Wave ${ }^{22}$ Kra09, or the upcoming Sakai 3 VLE (which acts both as consumer and provider of information for Web 2.0 applications). However, these technologies are still in an experimental stage, and their educational uses (especially regarding Learning Design) are unclear.

As we can see, not only deploying learning designs (expressed in whichever Learning Design language we have chosen) in our choice of learning environment (e.g. a VLE) is undersupported and requires multiple manual steps in most cases. It is even more critical in the case of decentralized learning environments such as DLEs, since we have to ensure that the design is deployed coherently across different domains (which currently requires additional manual operations).

\subsubsection{Flexibility: From improvisation to the problem of real-time manage- ment and adaptation of the learning activities in DLEs}

In the previous section, we have explored the challenges and difficulties that teachers encounter as they design CSCL situations, and try to set them up for students to perform them, in Distributed Learning Environments. However, as we saw in Section 2.3, orchestration not only refers to the design and preparation of the learning activities, but it especially entails the real-time management of the learning processes, especially regarding the adaptations of those

\footnotetext{
${ }^{21}$ As an exception to this, the TENCompetence project (http://www.tencompetence.org) succeeded in integrating widgets into an IMS-LD player. However, it is still unclear how such a player would be integrated in other environments such as VLEs (and at what cost).

${ }^{22}$ http://support.google.com/wave/bin/answer.py?hl=en\&answer=1083134 (Last visit: 25 Jun 2012)
} 
activities to unexpected events. This is a well-known problem of the CSCL scripting approach (in which the plans and expectations are explicited and somehow reified into the technological support): the flexibility of learning designs and learning environments. In this section we will review the most relevant literature on the subject, and we will see how this flexibility is still challenging, especially in DLEs.

When enacting CSCL activities, one of the main tensions that emerges during the enactment is that of scripting (following a planned sequence of tasks) vs. improvisation (deviating from those plans, to address emergent and/or unexpected events) [Dim07] Tat07] [Dil02a]. In teacher enactment, the concrete form that classes take is not predefined to the last word in advance, but rather it is largely improvisational [Saw01]. Despite some attempts to "teacherproof" education by scripting the lessons down to a very low level (almost word-for-word [Sla01] Eng80]), the plans for the most exhaustively prepared lesson usually remain at a higher level of abstraction than the final enactment. Thus, the element of improvisation has always been present, in one way or another, in the art of teaching [Saw04].

Improvisation as a metaphor for teacher discourse in class has been around for a long time now, for example in the writings of Erickson [Eri82] and Yinger [Yin87]. These writings use the concept of improvisation to study and develop discourse strategies and classroom discourse patterns, extracted from the practice of expert teachers, that can be useful in the education of novel teachers. In fact, much of this interest in improvisation comes from the fact that expert and modellic teachers are known to improvise more (and more effectively) than novel teachers [Bor89] [Yin87], producing more open-ended plans for their lessons than their novel counterparts. As Brown and Edelson put it, teaching is disciplined improvisation, "a dynamic process involving a combination of planning and improvisation" Bro01. It is disciplined because it occurs within some level of structure and framework. In fact, expert teachers are known to use more routines and activity structures, i.e. patterns, but combined in a more creative way. This concept of patterns [Ale77] as a basis for improvisational conduct is well established in literature [Bor89] Sud93.

Thus, we should be aware that, while a TEL or CSCL situation is normally prepared (especially if a formal learning design or script is in place), teachers will naturally expect changes to be made in the plans, if an opportunity or an extraneous event appears. Thus, we could think of any lesson as being at some point in a continuum between structure/script and flexibility/improvisation [Dim07. That is the main reason why the notion of "adaptation" also appears in much of the research work on orchestration.

Up to now, we have seen how scripting is a common mechanism to structure face-to-face (and computer-mediated) collaborative learning. However, even if CSCL scripts have obtained positive results in fostering effective learning in certain situations, they have also been criticized for offering a too rigid support [Dil02a] Dil07d, which could render them ineffective in the face of unexpected events that often occur in the classroom.

One possible approach to implementing this kind of flexibility in macro CSCL scripts is to analyze the scripts and separate the intrinsic constraints of the script (i.e. the characteristics that are crucial for the useful interactions to occur) from the extrinsic constraints (the arbitrary implementation decisions that make up the rest of the script, and which could be changed without the script losing its sense) Dil07d. Ideally, the runtime of the script should allow 


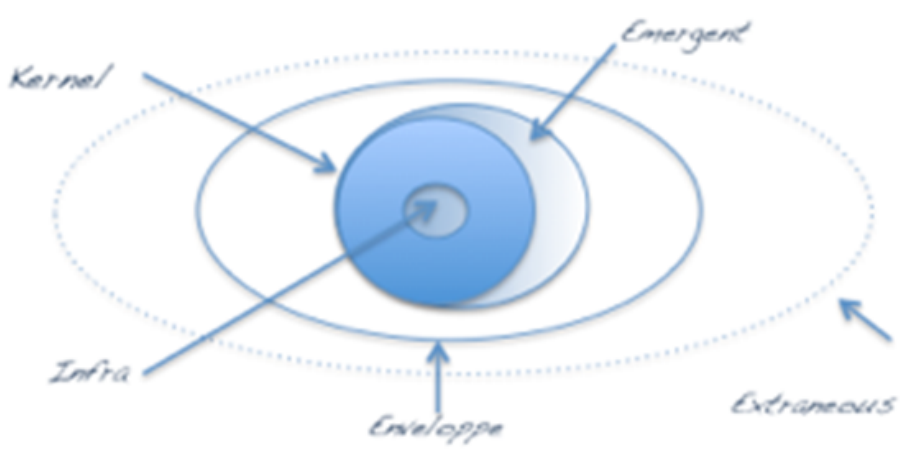

Figure 2.7: Graphical representation of the "kernel and rings" model for classroom orchestration, taken from Dil11a

the modification of these extrinsic constraints, while leaving the intrinsic part of the script unchanged.

More recently, Dillenbourg proposed a different conceptual framework to look at the events and constraints that prompt this need for flexibility [Dil11a], when approaching the orchestration of a classroom. According to this model (which is represented graphically in Figure 2.7), there are a number of events and constraints that are present in the learning designs (the activities, contents, etc. that are expected to trigger the learning process, including monitoring and individualization, if needed), which he calls the kernel. This kernel is the main object of study of Learning Design and Instructional Design. The model, however, suggests that in an authentic classroom situation there is plethora of other events and restrictions that can modify the orchestration of the situation: emergent activities (designed but contingent activities, such as debriefing about student-generated content), envelope activities (not designed but necessary activities that consolidate the kernel, such as making students copy into their notebooks the contents of the blackboard), extraneous events (unavoidable and unexpected, such as dropouts, latecomers, or kids copying the solution from their peers) and infra activities (not designed but necessary logistic issues, such as setting up the computers for the class or opening books on the right page). All these events, which researchers often control carefully when conducting experiments, can modify greatly the course of a lesson and should be taken into account when supporting orchestration (e.g. through technological systems). Thus, Dillenbourg hints at the need of flexible tools that support orchestration.

In CSCL literature, several approaches and tools have been proposed in order to add flexibility to collaborative scripting systems:

- Adaptive Collaboration Scripting (ACS) Adaptive Collaboration Scripting (ACS) is a framework for adding certain flexibility features to a scripting engine, proposed by Demetriadis and Karakostas [Dem08]. The framework follows the aforementioned concepts about the separation of intrinsic and extrinsic constraints Dil07d. ACS tries to adapt the execution of a script automatically (during its runtime, but also in its design, instantiation and setup), in order to account for a number of different events, such as user characteristics and (extrinsic) script characteristics. Thus, for example, this framework would allow for on-the-fly changes in group formation (e.g. if students fail to show up), in 
the deadlines of activities, or by providing additional support to novice learners that need extra scaffolding. In this work, flexible design, setup and enactment tools are necessary in order to adapt (i.e. to optimize) the activity to the circumstances of the classroom, most of which are known in advance, but which may also be emergent. However, the implementations of this framework so far are fragmentary, covering only one script at a time. As a complement to the framework, a prototype method (DeACS) has also been proposed, in order to uncover adaptation patterns (specific, recognizable ways in which a script can be changed, when triggered by certain events, see also Section 4.2.3) that may be used in future implementations of the framework Kar09.

- Anticipating flexibility through assessment A different perspective in the addition of flexibility to scripted environments is the one proposed in VF09a: The main idea is to embed assessment activities into the script, which can act as triggers for conditional enactment of parts of the script. For example, if the assessment activity indicates that insufficient knowledge has been gained by a group, an additional task (which was included in the design, but had been hidden up to that point) would be made available to that group. This proposal exploits a concept related to that of flexibility, which some authors have called contingency SRI08b]. In a contingent class, the teacher embeds conditional avenues for the activities, specially if the outcome of a phase is uncertain. Contingency (as well as other flexibility features in scripting) is supported by formalized Learning Design languages, such as IMS-LD IMS03a]. However, the complexity of implementing it has derived into partial, non-standard implementations in the different IMS-LD compliant script environments. The prototype implementation of this system involves the combination of the Collage authoring tool HL06a for designing the script, and the Grail IMS-LD player dC07 and a Wiki for its enactment. Currently, the execution is not fully automatic and, in fact, requires deep knowledge of the tools and the IMS-LD specification for the flexibility mechanisms be used.

- WikiPlus Another attempt to provide a more flexible CSCL system, but from a very different perspective, is the one proposed by Doebeli and Notari Doe09], using a modified version of a wiki to regulate learners' activities. They called this kind of system a WikiPlus. This system allows teachers to adapt the script whenever non-predicted learner activities come to happen. A prototype of this WikiPlus concept has been implemented, based on Twiki [Tho09]. Basically, its aim is to extend the basic functionality of a wiki (basically, to let non-technicians generate static web content) towards something more dynamic (to allow non-technicians to define processes, in this case, learning processes). In this kind of systems, technical knowledge is still required to generate wiki templates that represent a macro script (see chapter 2.2.2). Later on, the teacher instantiates the script by filling in the template, and finally students and teachers enact the script. The distinguishing features of this WikiPlus system, according to its authors, include the fact that it can be used by teachers with relative ease (as opposed to many scripting environments, which require specialized technical and pedagogical knowledge), its ability to accommodate rapidly any kind of script (many scripting environments only support a limited variety of scripts) and, especially, its ability to be modified on runtime (i.e. flexibility) without specialized help.

As we can see, all these efforts propose ad-hoc, self-contained environments that are not compatible with systems that are widely used currently by teachers and institutions. In fact, 
none of them uses any tools external to the VLE (or equivalent learning environment), or hints at any way in which these approaches could be used in the wider context of Distributed Learning Environments. However, given that the need for flexibility seems to be an inherent quality of any authentic learning scenario, it would be interesting to know how teachers are currently facing flexibility in VLEs and DLEs, using three examples among the multiple systems described in this chapter (Section 2.4). Here, the concept of CSCL life-cycle described in Section 2.2.2 will be useful to structure our discussion:

- Moodle VLE. A teacher using this VLE, as we saw in Section 2.5.3, does not have any special support in designing or deploying her learning designs, and thus the designs are reified manually by the teacher using Moodle's graphical interface. Albeit the deployment process is cumbersome and error-prone, this kind of approach (which some authors label 'bricolage' [Ber05]) in which the teacher manipulates the execution environment in a trialand-error way, proves to be quite flexible. Extraneous events such as dropouts or latecomers can be addressed directly using the VLE's user interface (although complex changes in groups may prove tedious), and new resources and tools can be added with relative ease. The lack of an enforced activity sequence, which some CSCL researchers might see as a weakness, proves in this case to be one of Moodle's strengths.

- LAMS VLE. As we have already mentioned, LAMS is a VLE that allows for the design and enactment of learning activities. In LAMS, the instantiation process (i.e. transforming an "abstract" activity in a learning design into concrete activities and resources for each of the groups/individuals that have to perform it) can be done, transparently for the end users, just before starting each activity. Thus, activities in a learning sequence can be modified in a flexible way, as long as that activity has not started yet. This allows teachers to cope with many extraneous events (e.g. dropouts or latecomers to an activity), since they will be included in the activity as - and if - they come. The problem with LAMS, however, is that it is a VLE, and as such we are restricted to the built-in tools available in the system for the enactment of our learning situations. It remains, however, a solution that provides a considerable flexibility, which may be the reason why it is one of the most widespread LD-oriented systems today.

- Ad-hoc, wiki-based DLE. As it has been mentioned, a number of practitioners and researchers have used wikis as learning platforms. Not only that, but this kind of wikibased environments can also be used as the central access point of a DLE, integrating a number of external tools with ad-hoc plugins or simple hyperlinks [Ang10. The design of this kind of DLE-supported courses (which can be complex and highly collaborative) is currently instantiated and deployed by teachers manually, by editing multiple wiki pages somehow representing the activities and tools involved. As in the case of the Moodle VLE, this tedious and error-prone process is highly flexible, since any wiki page can be edited at any time, adding or deleting resources, or changing groups by modifying the text where the group components are listed (although this, still, can be challenging for complex collaborative techniques like the Jigsaw Aro92). Albeit this approach to DLEs has its deficiencies (lots of manual steps and editing, lack of an enforced sequence of activities, lack of a strong separation of contents - i.e. copying is easy), it is very flexible, which is the reason why there are teachers that have opted for this unstructured platform for supporting their courses. 


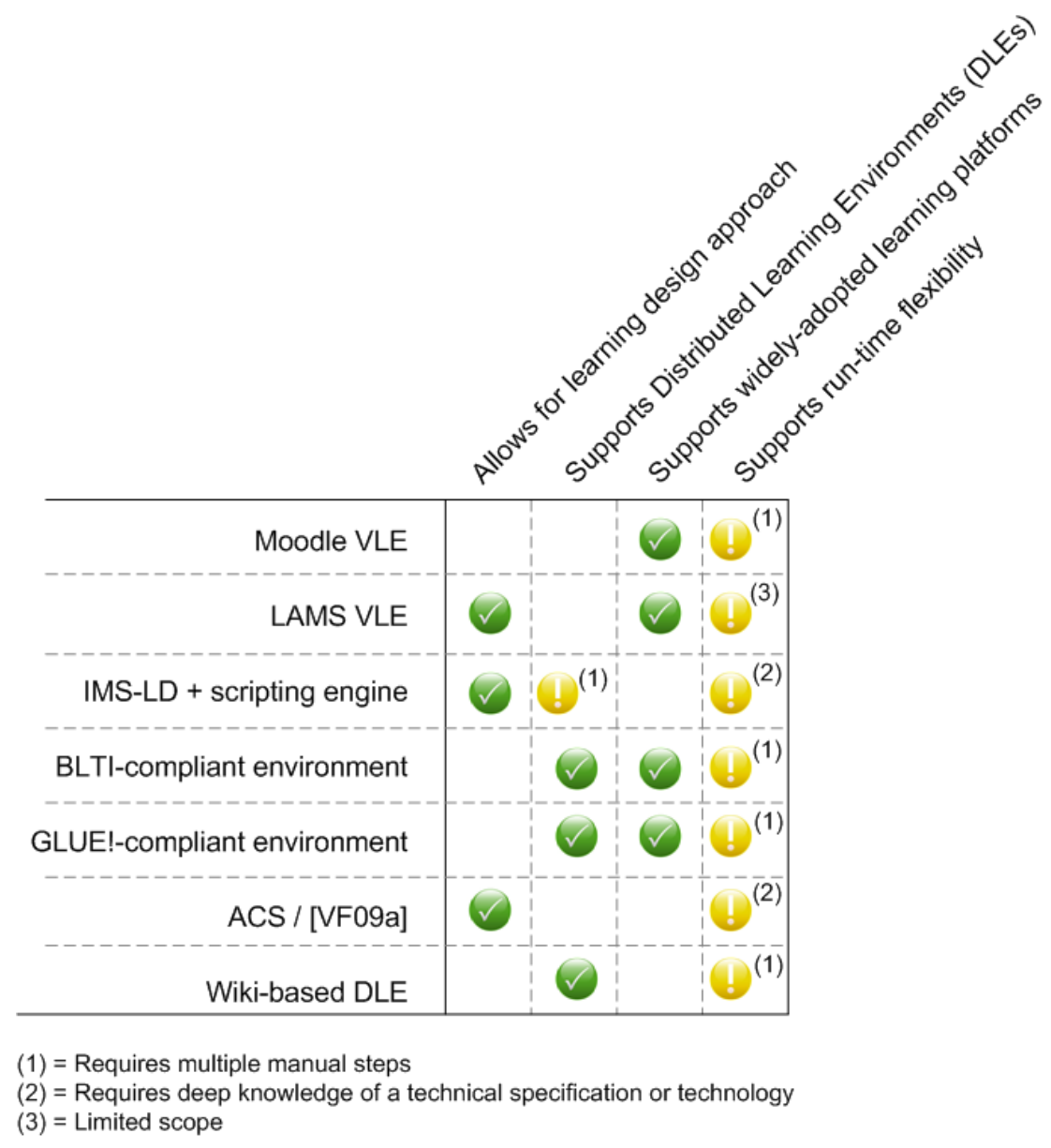

Figure 2.8: Summary of the technological support for orchestration provided by different learning environments. As it can be seen, none of the presented learning environments comply with the four conditions that a practitioner in an authentic setting requires.

From this literature review and examples we can thus conclude that, not only deploying learning designs (especially CSCL ones) is severely undersupported in DLEs based on currently widespread learning platforms. Moreover, providing flexible technological support for the orchestration of CSCL activities in this kind of learning environments is still a challenge that is largely unsolved (see the summarizing table in Figure 2.8). Thus, in this dissertation we will propose a technological infrastructure that allows for this deployment of learning designs across DLEs within the currently fragmented Learning Design and learning platform panorama. Moreover, this infrastructure should be able to support the flexible adaptation of such deployments in run-time.

\subsection{Conclusion}

ICTs are slowly permeating all aspects of our society, and education is no exception. We are expecting teachers and students to tap into the potential of computers and the Web, and 
to reproduce in their classrooms the complex pedagogical and technological tools that research fields like TEL and CSCL constantly produce. However, there is an increasing concern about how those different approaches and technologies, which are often tested in an isolated and controlled manner, can be applied in authentic settings in a synergistic way. The emergent notion of 'orchestration' tries to address this concern.

Yet, a literature review on the usage of the word orchestration reveals that TEL and CSCL researchers use the word with slightly different meanings and flavors, leading to an overuse of the word, to the point that it may become a "buzzword". This state of confusion about what we mean by orchestration and which processes it entails, do not favor the accumulation of knowledge and the production of TEL that supports teachers in the complex endeavor of enacting such innovations in authentic educational settings. Clearly, more accurate definitions and models of orchestration are needed, which can help researchers develop innovations and new research instruments to understand and measure their effects in authentic settings.

With the advent of ICTs to education, the life-cycle of teaching practice continues to be essentially the same, if looked at a very high level: preparing their lessons, enacting them and evaluating the result. However, as TEL researchers we are providing them with new tools, new concepts and pedagogies which they are expected to appropriate and apply effectively, shaping their practice in new ways. We have looked into one concrete pedagogical context (blended Computer-Supported Collaborative Learning), and into an increasingly common technological context in which it is being applied (Distributed Learning Environments that include learning platforms and external tools), to study in depth the orchestration challenges that a teacher faces at the intersection of those contexts. The first challenge that we came across is the lack of concrete orchestration advice about how all the different elements in the classroom (or outside it, since we are considering blended learning) can be productively combined, from technological artifacts to pedagogical actions, in the concrete context of their own classrooms. The wide array of such tools available in Distributed Learning Environments only make such decisions more painful.

As we have seen, Learning Design and, especially, CSCL scripting is a common way of structuring collaborative activities enhanced with technology. However, technological support for orchestration of learning designs in DLEs is clearly lacking, if we consider a teacher in an authentic setting, who normally only has access to one institutional Virtual Learning Environment. The first problem is that, despite the availability of a number of LD authoring tools and specifications, there is currently no easy way of automatically deploying learning designs into DLEs that include widespread learning environments. Thus, for most teachers this deployment requires a considerable effort of creating, organizing and linking resources directly using the learning environment's interface. Moreover, even if a teacher manages to deploy her ideas into this sparse learning environment, it is a well-known fact of life (and especially, life in the classroom), that unexpected events may happen, and that those events may prompt changes in the technological support to be addressed. The technological support for real-time adaptations of the design deployed across the DLE is another clear open challenge.

These four problems that we have emphasized here, one from the point of view of researchers, and the other three mainly from the teacher perspective, will be addressed in turn in the following three chapters. 



\title{
Chapter 3
}

\section{'5+3 Aspects': A conceptual framework for orchestration in TEL}

\begin{abstract}
Summary: The term "orchestration" is being used in the TEL and CSCL research fields increasingly often, albeit it is still a rather diffuse concept, used with different meanings and connotations. In order to provide the TEL researcher with a first (conceptual) tool to do research about orchestration, an extensive literature review was conducted (see previous chapter), from which a conceptual framework has emerged. This chapter presents the ' $5+3$ Aspects' conceptual framework and a working definition of orchestration in TEL, which can be useful for researchers as an analytical lens to characterize orchestration in concrete educational settings, as well as for framing and communicating about orchestration-related research. Also, the framework evaluation results from two researcher panels (one with international experts, another with younger researchers) are presented in this chapter. The evaluation data shows that the framework's is portrayed as complete and useful by the participant researchers (although these opinions were not unanimous), and that the framework has a certain didactic potential for younger researchers. Finally, a first research instrument derived from the framework (a questionnaire and reflection guide) is presented, providing preliminary evidences of its usefulness.
\end{abstract}

\subsection{Introduction: The relevance of orchestration in TEL}

One of the first questions that arise when the word "orchestration" is brought up in conversation among TEL researchers is: "What do you mean by orchestration?". In our literature review on the concept of orchestration (Section 2.3), we have found several definitions of the term, and several research efforts working around "orchestration" that had, surprisingly, very little in common. Nonetheless, the word is being used increasingly often, as we can see by doing simple prospective searches such as the one in Figure 3.1, taken from the Google Scholar Trend Miner 1 .

In fact, this increase in the interest in "orchestrating learning" has reached a point where the metaphor (if we can consider it a metaphor) has almost become a "buzzword" in the opinion of some researchers (as we will see in Section 3.3). As an example, the STELLAR Network of Excellence [STE10b] has posed "orchestrating learning" as one of the main challenges of the TEL

\footnotetext{
${ }^{1}$ http://ats.cs.ut.ee/u/kt/stuff/scholartrend/(Last visit: 15 May 2012)
} 


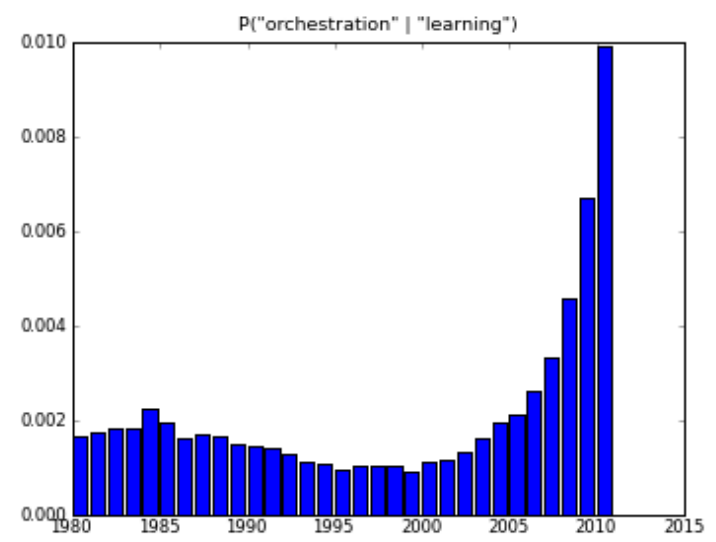

Figure 3.1: Relative probability of a paper having the word 'orchestration', given that it had the keyword 'learning'

area. However, the text of this challenge in the STELLAR website ${ }^{2}$ states that "orchestration takes up the challenge of the actual implementation of all the interactions needed for a successful scenario", practically equating orchestration with anything that happens in the enactment of a learning situation, other than the learning itself.

As we can see in Figure 3.2 , this dissertation proposed generally to provide "technological and conceptual tools to support orchestration". In this chapter we describe the first of these tools: a conceptual framework for orchestration in TEL, and a definition of orchestration derived from it. We hypothesize that clearer concepts of what orchestration is in the field of TEL, and what it entails, can help researchers in structuring and analyzing their innovations in authentic TEL settings. Moreover, this kind of conceptual framework can also help the TEL research community to communicate with each other (by providing clear aspects and definitions that can be referred to), accumulate knowledge on the subject, and it may even have a didactic value for novel researchers arriving to the area of orchestration, by giving them a starting point for their efforts. Even if this kind of framework could also be useful for teachers-researchers or even teachers in general, to reflect about their practice, in this dissertation we mostly explore its usefulness for researchers (with only indirect evidence of its usefulness for teachers).

Not only did we provide this first conceptual tool for researchers. Rather, we also started developing other research instruments based on this framework, which we have used during the dissertation. A first example of these derived instruments is a questionnaire or reflection guide to delve in orchestration aspects of a concrete TEL/CSCL setting. This (admittedly minor) contribution is also presented as part of the dissertation, in this chapter.

During this chapter we will present this conceptual framework, called ' $5+3$ Aspects', and we will evaluate it with regard to the following research question: "Can we provide conceptual tools for researchers to clarify and support orchestration-related research?" (RQ1.1).

This chapter is structured as follows: after a brief description of how we have applied the engineering method to our research about the proposed conceptual framework, the conceptual

2 http://www.stellarnet.eu/d/1/1/Orchestrating_learning (Last visit: 29 May 2012) 


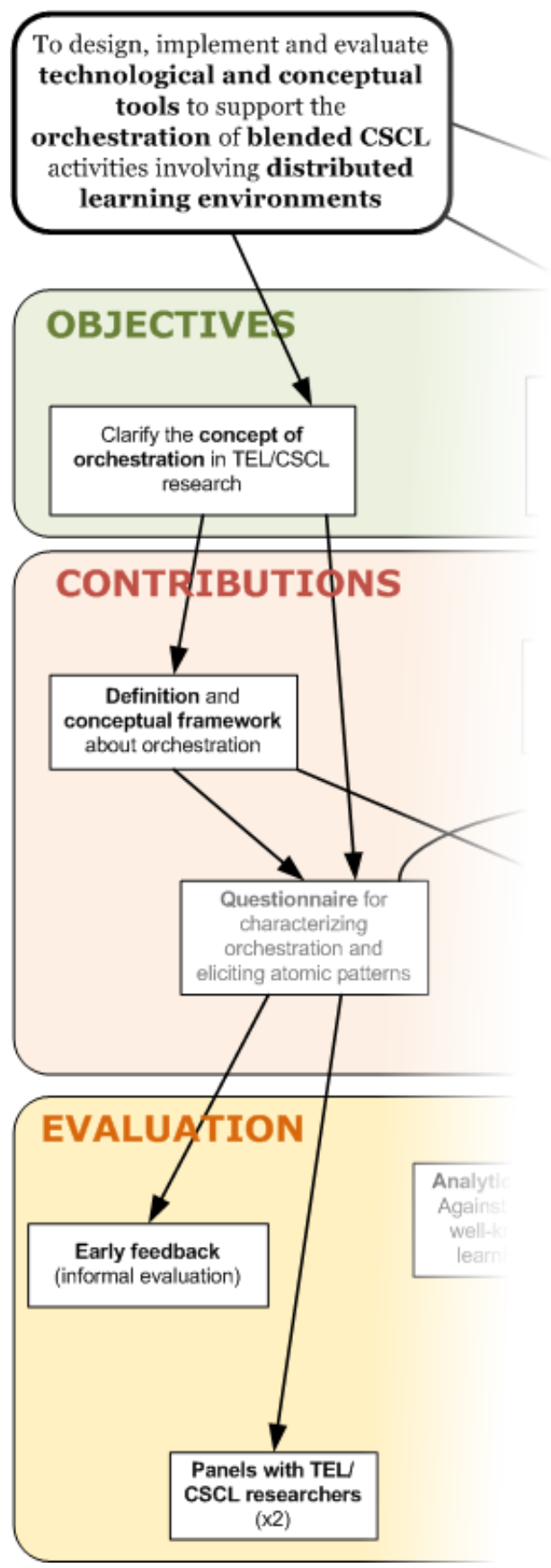

Figure 3.2: Parts of the thesis diagram concerned by chapter 3

framework itself is described, leading to a new, more concrete definition of orchestration in TEL (Section 3.2); afterwards, we detail the evaluation performed on the framework, especially in two TEL/CSCL researcher panels (Section 3.3); finally, we present a first research instrument derived from the framework (Section 3.4), and close the chapter with a few concluding remarks about the relevance of these contributions (Section 3.5). 
It is worth noting that parts of this chapter have already been published by the author and colleagues in international conferences and peer-reviewed TEL journals. More concretely, in Pri11d we can find the first synthesis of orchestration (see also Figure 2.4, and in Pri11e we can find a first proposal of the ' $5+3$ Aspects' framework for orchestration (see Section 3.2 ).

\subsubsection{A note on methodology}

As described in the introductory chapter, in this dissertation the engineering method Gla95 has been used to provide the general methodological structure of the dissertation. This method proposes cyclical iterations of four phases of information, proposition, analysis and evaluation [Adr93]. This iterative process has been also followed over the ' $5+3$ Aspects' conceptual framework presented here. As we can see in Figure 3.3, during this dissertation we have completed two iterations, and at the end of the dissertation we are ready to make a proposal for the third iteration. These iterations include:

1. A first literature review of orchestration (see Section 2.3), which lead to a first effort to synthesize a definition of orchestration (already presented in Section 2.3.3). This first synthesis was presented at a workshop during the International Conference of CSCL 2011, gathering positive feedback (which can be seen as a first, prospective evaluation of its value for the TEL/CSCL research community).

2. The literature review was expanded with the help of other young researchers, and the concepts found were clustered in eight aspects, which were presented as a conceptual framework and augmented with the analysis of three case studies using the framework as an analytical lens. These three products were published at the International Journal of TEL Pri11d. This framework has been used to analyze the orchestration support provided by the other dissertation contributions (such as the "atomic pattern catalogue", see Appendix B, or the GLUE!-PS system, see Section 5.3.1). Moreover, a research instrument was derived from the framework in the form of a questionnaire (Section 3.4), that has been used to elicit orchestration practice in blended CSCL scenarios during this dissertation (Chapter 4). Finally, two panels with TEL/CSCL researchers (one with a mix of researchers from nearby research groups, the other one with international experts on the subject) were conducted to evaluate the completeness and usefulness of the framework (Section 3.3).

3. The feedback from the aforementioned panels has been used to further refine the framework (see Section 3.5), on which we will continue working iteratively (e.g., by deriving new research instruments).

\section{2. '5+3 Aspects': A definition and conceptual framework for orchestration of Technology-Enhanced Learning}

There have been few attempts to provide conceptual frameworks that account for the different aspects and characteristics of orchestration of learning activities, especially those involving new technologies. Those sources that do provide conceptual frameworks either lack 


\section{"Supporting orchestration of blended CSCL scenarios in Distributed Learning Environments" Contribution \#1 - Conceptual framework for orchestration}

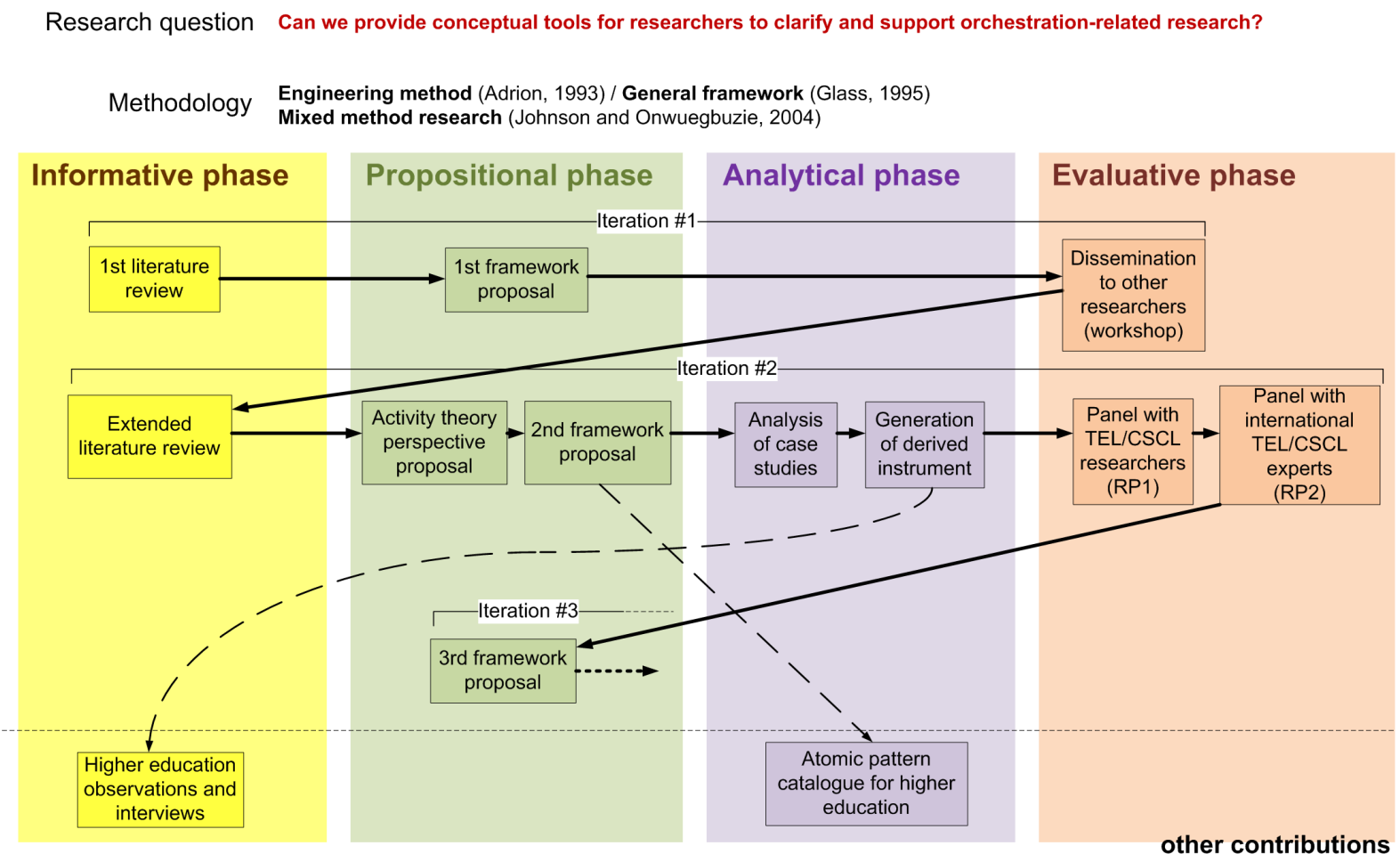

Figure 3.3: Detailed view of the engineering method followed during the research work around the conceptual framework

extensive literature reviews to back them up (being more based on their authors' own research and personal experience, see [Dil07d] Dil10, for example), or provide mere lists of issues and research challenges Bal10. Moreover, there are several TEL research works that mention orchestration and delve into some particular aspect of it, but do not give explicit definitions or conceptual frameworks for it. A notable and recent exception to this lack is [Ham11], in which the notion of orchestration of collaborative learning and creativity is reviewed from a purely educational perspective, describing the available literature from the point of view of its pedagogical bases, teachers (pre- and real-time) activities and the opportunities and challenges for teacher activities.

We have tried to address these deficiencies in this dissertation, by reviewing the TEL literature related to the concept of 'orchestrating learning', and trying to provide a cohesive framework that includes the aspects most often mentioned by TEL researchers in relation to this concept. We believe that this kind of framework can be useful as an analytical lens when researching TEL settings (especially authentic, complex classroom settings, as mentioned above) as a way to structure the information available to the researcher and to detect challenges and eventual solutions to aid orchestrating learning in such settings. An account of the reviewed literature can be found in Section 2.3, rather than presenting a list of all the reviewed literature here, and for brevity's sake, we have chosen to cluster the main aspects mentioned in the available 
literature, presenting them in thematic groups, along with a selection of the literature sources that mention these themes.

\subsubsection{Aspects of orchestration}

When going through the orchestrating literature in the fields of TEL and CSCL, there are eight main themes that are commonly addressed:

Design/Planning As it can be seen from the definitions of orchestration provided in Section 2.3, an important component of orchestration is planning the learning activities that will be coordinated. This planning ahead, often referred to as learning design [Kop05] or simply, design, can be related to the areas of instructional planning and instructional design, which have a long tradition in education. This aspect also relates to the idea of orchestration coming from music (i.e. which musical instruments will play which parts of a musical composition). In education, it has been argued [Mor06 that orchestrating can be better achieved via designing collaborative learning lesson plans that are rich enough to accommodate the nine elements of Gardner's model of intelligence Gar85. There is an even greater need in TEL for adequate planning of the activities and technological tools that will be used to enact them, so that the objectives of a learning activity can be achieved. In this sense, we can see that the design and enactment of flow control in elearning environments (also known as "scripting", see Section 2.2.2 ) is very closely related to orchestration, including the work in the IMS Learning Design specification [IMS03a) and other scripting frameworks (e.g. Kob07]). Consequently, the importance of design and scripting is mentioned in several orchestration frameworks [Dil09a] [Dil10]. However, it is important to note that the preparation and enactment of orchestrated activities do not necessarily follow a linear process; for example, Ber05 depict a "bricoleur" teacher that designs activities and re-designs them as the course progresses. This adaptation of designs is one of the hallmarks of orchestrated TEL activities, as we will see below.

Regulation/Management The idea of orchestrating learning trades upon the virtues of managing the processes of learning and teaching in order to maximize outcomes on a variety of fronts Wat03. According to Watts, well-orchestrated learning takes place when "it all comes together", when a teacher stages personally satisfying sessions that 'chime' with learners' favored modes of learning which, at the same time, are modulated by the demands and characteristics of the subject under consideration. Thus, an important aspect of many works on orchestration is the regulation of learning activities (either external regulation or self-regulation, as mentioned by Dil09a]). Issues related to class, time, workflow and group management, which appear in numerous orchestration works (such as Dil07d [Dil08b] Nir10] Dil10]), can also be gathered under this theme. This regulation and management of learning processes and their constraints can be done either "manually" through social interactions [Dim07] Ala09] JJer09] or mediated and/or automated by technological means. In the latter case, this is normally done by specifying the learning activities through computer-interpretable scripts [Wei09 [Gru10] diFV10]. Also related to this theme are the works of [Wec07] on the "fading" of scripts as a way in which this regulation/management may be modified over time as learners progress. 
Adaptation/Flexibility/Intervention Another aspect that can be derived from the definitions of orchestration provided above, as well as from many other sources dealing with orchestration Dil07d [Dil09a] Dil10], is that of orchestration as intervention; that is, the act of changing and adapting the design/plan to both the local context of the classroom and the emergent occurrences during the enactment of learning activities. This often requires the management of learning activities, either through social mechanisms which are naturally flexible, or through technological systems that are flexible enough to handle those adaptations Dil07d Kar09.

Awareness/Assessment Since the concept of orchestration revolves around making interventions in response to classroom context and emergent occurrences, awareness of what is happening in the classroom and within the learners' minds is crucially important in any well-orchestrated learning scenario Dil09a [Bal10]. Ala09] demonstrates how simple awareness mechanisms can enhance the orchestration of a certain type of classroom, and the concept is further developed by [Dil10]. Assessment (either formative or summative) can provide insight into the progress towards the intended learning outcomes being gradually achieved by the learners, thus allowing for adequate adaptations of the learning design (see above). For example, the formative e-assessment case studies analyzed in Pac10 show that defining the roles of the key players (teacher, learner as an individual, and peers) is crucial in order to orchestrate formative assessment in the teaching process. Although the use of technology in the formative assessment process has a socio-technical impact in orchestration, its high current and future potential is shown by [Pel10]. In fact, the awareness provided by formative assessment is as beneficial for learners (to improve their learning), as it is for teachers (see, e.g., Wat03, where the orchestration process is defined as adapting the teacher's teaching style to the learner's learning style in a concrete context).

Roles of the teacher and other actors The body of research work around orchestration focuses primarily on the perspective of the teacher (as expressed explicitly by [Dil10] and [Bal10]), where teacher presence is essential to achieve orchestration [Ken07]. This has prompted many advocates of learner-centered approaches to dismiss the concept of orchestration altogether. However, nothing precludes the concepts presented here from being used by learners themselves, shifting the load of orchestrating the activities from the shoulders of teachers. In fact, some authors argue that teachers in orchestrated learning are "guiders, not a knowledge source" [Cha01] [Nat07], and that there is a correlation between concepts of orchestrating learning and principles of constructivist pedagogy Cha01 Ric03 as a means of facilitating authentic learning [Ric03 [Nat07. There is a range of possibilities for more learner-driven orchestration, from approaches where learners directly affect the awareness mechanisms [Ala09], to scripted environments where scripts are progressively faded [Wec07], or scenarios where only the widest goals and activities are set by the teacher, and the learning tasks and their coordination are handled by the students. Thus, even if TEL orchestration literature tends to be teacher-centric, more learner-centric approaches could also be accommodated into the "orchestration umbrella".

So far, we have identified five main aspects of the literature regarding what orchestration entails in TEL. However, there is an dearth of advice about how to design and support wellorchestrated learning experiences. The main exceptions to this are the works of Dillenbourg, 
which take a more normative approach to the orchestration of face-to-face classrooms. In this and other related works we can find three aspects that are not concerned so much with what orchestration is, but rather how it should be done:

Pragmatism/Practice Dillenbourg and others have noted that many of the ideas behind the concept of orchestration deal with making TEL research results available to average (as opposed to TEL-expert or enthusiastic) teachers, and catering for the constraints of authentic classroom settings especially [Dil09b [Dil10] Ham11]. This emphasis on pragmatic research efforts that are scalable and sustainable, and on changing everyday teaching practice in authentic settings (as opposed to controlled experiments) seems to be a common concern of current TEL research (hinted at by the fact that the latest international CSCL and EC-TEL conferences have had "practice" as one of their main themes).

Alignment/Synergy Probably the single most often cited characteristic of a well-orchestrated learning experience is the coordination of the elements to be orchestrated (learning activities at various social levels, tools and scaffoldings used, including teacher and peer actions) so that they provide what Tabak [Tab04] labeled 'synergistic scaffolding' [Dil09a] Dil10] Pri11f]. The alignment of as many of these elements as possible, through the changing conditions of a learning situation, in order to attain the learning goals that are desired at different levels, is one of the few clues given to the TEL practitioner who intends to better orchestrate a learning scenario. It has already been pointed out that to align the intended learning outcomes of a course with teaching and learning is a big challenge Big99. Wat03 introduces new factors in this equation, such as the engagement of students to meet the intended outcomes and the learners' method of learning, which is influenced by innate issues like gender or culture.

Models/Theories The increasing complexity of current and future learning scenarios urges the development of more robust theories and models of orchestrating learning [Nat07]. A number of models of orchestration are proposed in the literature, but they are strongly heterogeneous and very context specific. Weinberger's model of orchestration for CSCL environments [Wei07] is implemented via guiding scripts. Cha01 implemented the orchestration of an engineering course via a cyclic learning pedagogical model. Orchestrating the process of listening in language learning courses is also implemented via cyclic pedagogical models and activating meta-cognition and cognitive skills Van03. [Sha09] describe a ' 2 x3' model to orchestrate assessment in collaborative work, emerging from the interaction of three elements (knowledge cognition, collaboration, and regulation) on two levels (individual and group). Another model of five practices is proposed to orchestrate mathematics learning [Ste08]. Less explicitly, other researchers propose what can be considered, to a large extent, orchestrating learning models. For instance, $\mathrm{Abd} 08$ view the process of orchestrating project-based learning from a Cybernetics perspective. Abd09 describe a pedagogical model for orchestrating laboratory education based on Kolb's experiential learning and by utilizing virtual and remote experimental technologies. [Pri11f] hint at using Activity Theory Eng99 as a theoretical lens to analyze the orchestration of learning experiences and the alignment of the mediational means used towards the learning objectives at different granularities. Nevertheless, a deeper theoretical analysis backed up with empirical data is needed to alleviate the lack of general theories and models that can inform researchers and practitioners in orchestrating learning. 
This conceptual framework is represented graphically in Figure 3.4. As we have mentioned, these two sets of concepts are related to each other in different ways. Although a closed model of relationships among these elements is not provided (since the concrete relationships and forms that each aspect takes will vary from context to context), we can readily see how the ' 5 ' and the ' 3 ' aspects are somehow orthogonal, with the latter three being transversal to the others (e.g. the application of Pragmatism due to the authentic context's constraints can affect all five elements, from the Role of the different actors to the way the Design is done).

The reviewed literature on orchestration has also spotted, although not studied in detail, the influence of other aspects such as the motivational/emotional ones, or the role of evaluation and other after-the-experience processes common in learning scenarios. Other authors point at wider-scope issues, and argue that educational institutions should be restructured to enable orchestrated learning that meets the needs of future education [Nat07]. We have, however, chosen to leave those out of our framework, in order to make it simpler and easier to remember. Indeed, further research is needed in these aspects in order to provide a more complete view of the orchestration of TEL scenarios.

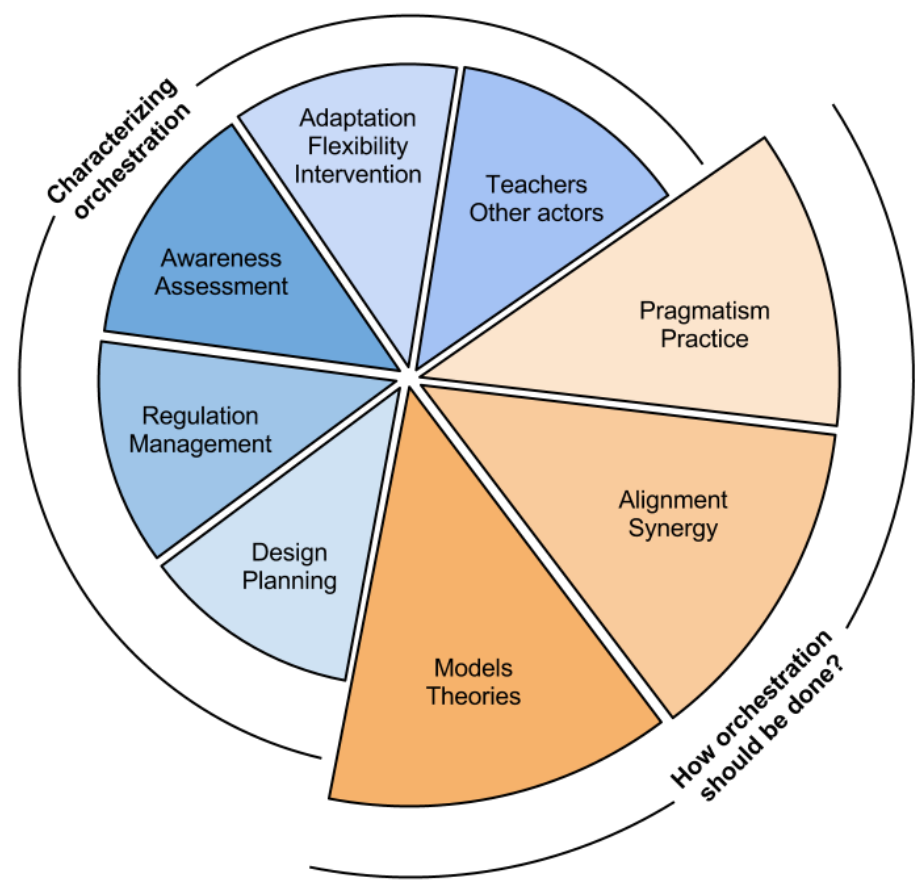

Figure 3.4: Graphical representation of the ' $5+3$ ' orchestration conceptual framework

We can also choose to see these aspects in an alternative, process-like view of the framework 3 (see Figure 3.5), depicting a simple example situation set in a primary school classroom. Thus, we can see how theories and models (sometimes explicit, but often implicit) inform the teacher (the main actor in the example, given the children's low self-regulation ability) in the

\footnotetext{
${ }^{3}$ This and other views, along with a short presentation of these concepts, can be found in a visual presentation of the framework, available at http://prezi.com/aa2vighak7hh/orchestration-in-tel-cscl-as-easy-as-53/ (Last visit: 16 May 2012).
} 
design/planning of the learning experience (guided by her pedagogical models that promote the use of collaboration, and constrained by the time constraints of the school lessons). This design is then put into practice (i.e. deployed and enacted) pragmatically in a specific context (again, constrained by the available resources such as hardware, trying to align them towards the goals about content and collaboration competences). During the enactment of the learning experience, the orchestration consists of processes of regulation, assessment, and adaptation (which, again, the teacher tries to align, guided by her inner models and constrained by the classroom context). During the whole process, the teacher tries to align these elements so that they contribute synergistically to achieve the desired learning outcomes.

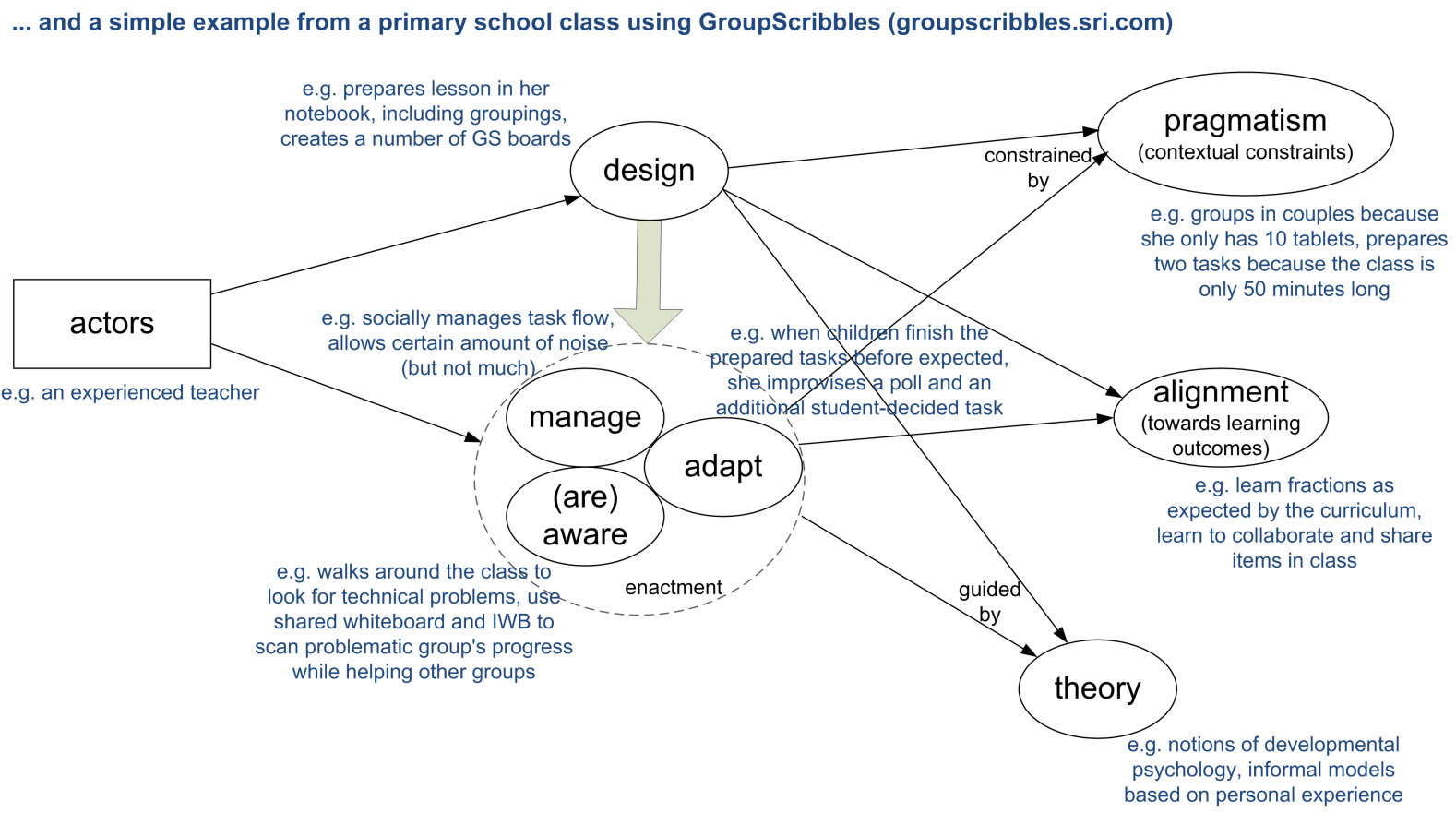

Figure 3.5: Alternative graphical representation of the ' $5+3$ ' orchestration conceptual framework, as a process

The ' $5+3$ Aspects' framework presented so far can be considered as our contribution to a more general and cohesive model of orchestrating learning, especially aimed at TechnologyEnhanced Learning. However, in Pri11e, the authors reckoned that the usefulness of this framework for TEL researchers has to be further grounded in empirical data. As we have mentioned throughout Chapter 2, "orchestration" is a problem that affects the whole of TEL as a research field, and thus this classification of aspects can be applied to any TEL setting (although it is especially indicated for more formal, teacher-centric learning). However, within the multiple existing TEL approaches, CSCL is one of the most complex to orchestrate, due to the different social levels, tools and activities often involved. Thus, the framework might be especially relevant for CSCL research, and in the rest of this dissertation (sp. Chapters 4 and 5 the reader will find this conceptual framework applied to concrete CSCL settings, as a first example of its use in an actual research effort. 


\subsubsection{What is orchestration? A definition}

Thus, taking these eight aspects into account, we could propose a different, synthetic definition of orchestration that encompasses the reviewed literature:

The process by which teachers and other actors design, manage, adapt and assess learning activities, aligning the resources at their disposal to achieve the maximum learning effect, informed by theory while complying pragmatically with the contextual constraints of the setting.

This definition is slightly shorter than the one we gave in our first synthesis of the literature (Section 2.3.3), which was extended from the ones given by Dillenbourg and colleagues. In this second attempt we have left out certain elements, such as "social levels" (which is a typical CSCL element, which may or may not be as relevant to other TEL approaches) or the references to "transitions and concurrencies" (which was mostly a conjecture not directly based on the literature), and we have reformulated others to make the definition briefer. This definition introduces other elements ("assess" and "guided by theory"), which emerged from the expanded literature review and were not originally present in Dillenbourg et al.'s definitions.

In fact, this definition has been distilled to include the eight aspects of the framework in a sentence as short as possible. Even with that aim in mind, the definition is quite complex but broad enough to accommodate most of the orchestration-related research that we have reviewed throughout the field of TEL. In the following section, we will show how this definition and the conceptual framework have been tested by two different sets of TEL researchers, to ascertain their usefulness and completeness.

\subsection{Evaluation of ' $5+3$ Aspects': Two researcher panels}

As it has been described in the introductory chapter of this dissertation, and at the beginning of this chapter (Section 3.1), we have followed the engineering method Adr93 to structure our research towards providing a conceptual tool to support researchers in understanding the concept of orchestration in TEL, and conducting orchestration-related research. Throughout this chapter we have proposed ' $5+3$ Aspects' as such conceptual tool to support researchers. However, it is in the evaluation phase of each iteration where the trustworthiness of this proposal should be demonstrated.

The first iteration of this line of work (see Figure 3.3 included an "informal evaluation" by the CSCL community interested in the topic. The form that this evaluation took was to present our first synthesis of the concept (see Figure 2.4 and Section 2.3.3) in an international workshop titled "How to integrate CSCL in classroom life: Orchestration", held at the International Conference on CSCL, in September 2011 Nus11. Although the workshop was not recorded, the synthesis was received favorably, and in fact, Miguel Nussbaum, chair of the workshop, used Figure 2.4 in his wrap-up presentation at the end of the workshop. Thus, we can gather that, even if there are very different views on orchestration among CSCL researchers (as the discussions in the workshop showed), at least our synthesis seems reasonable enough to be presented 
as a summary of common understandings among the sub-community of international experts interested in the subject of orchestration in CSCL.

Later that same year, the author and colleagues proposed the " $5+3$ framework" as a step forward from this synthesis Pri11e. This framework has been used to structure and shape several parts of this dissertation, such as classifying the atomic pattern catalogue presented in Chapter 4, analyzing the technological support for orchestration that the GLUE!-PS system provided (see Section 5.3.1), or deriving questionnaires from it (see Section 3.4) in order to elicit orchestration atomic patterns. Overall, the author can assert that " $5+3$ " helped him conceptualize his proposals for this dissertation, and the reader can appreciate throughout the rest of this dissertation how the framework can be used within a concrete research effort.

However, apart from this rather subjective evaluation of the framework presented above, it is clear that the usefulness of the ' $5+3$ ' framework for researchers beyond its own authors needed to be assessed. One possible way to validate the framework would be to convince external researchers to use it in their research and ask them to assess how much the framework helped. This option, however, was unlikely until examples of framework use (such as this dissertation) were available. Given the timeframes that TEL research normally involves (compared with the timeframe of this dissertation), and the difficulties of convincing a sizeable number of researchers of using a new framework (especially if it has not been validated before), such an option was unfeasible, and thus, alternative ways of evaluating the framework were needed.

Given the lack of established methodologies in education in order to validate conceptual frameworks, we have borrowed an approach from other fields where this sort of validation is more common (e.g. nursing, see [Faw89] Whal05]), taking a consensus-based approach (see [Moo05]) among the framework's main potential users (TEL and CSCL researchers). This method reflects the "collective wisdom" of the field, and generally has the advantage of addressing cases of a lack of standardization and terminology, and the potential to prompt practitioner acceptance Moo05] (both goals of our framework, whereby "practitioner" means "research practitioner", i.e. researchers). This method, however, may not necessarily produce the best result, since the incorporation of so many people's ideas can lead to a loss of conceptual integrity. Nevertheless, the method followed, in a way similar to the one depicted by [Lég11], was considered more appropriate than other alternative forms of expert validation which have a stronger emphasis on forecasting trends, such as the Delphi method [Lin75], since our interest was rather directed towards the evaluation of usefulness in a certain practice.

In the rest of this section we describe the evaluation of the second iteration of research in two short panel studies aimed at evaluating the value of our ' $5+3$ Aspects' proposal as a conceptual tool to support TEL researchers interested in orchestration. These two panels, performed online with a selection of TEL and CSCL researchers, were conducted in the Spring of 2012, try to answer the following research question (already mentioned in Section 3.1): "Can we provide conceptual tools for researchers to clarify and support orchestration-related research?" (RQ1.1).

\subsubsection{Context and method of the evaluation}

As outlined in Section 1.2, we have used the CSCL-EREM framework JA09b to design the evaluation phase of this second iteration in the research on ' $5+3$ Aspects'. Thus, our evaluations 
try to explore an evaluative tension (or Issue, in CSCL-EREM terminology), derived from our main research question (RQ1.1, above): "Does ' $5+3$ Aspects' clarify the notion of orchestration, supporting orchestration-related research?" (I1), through each evaluation iteration (both for the present second iteration, and for future iterations). This issue can be explored through informative questions grouped into several topics (see Figure 3.6), both defined by us at the start of the evaluation, but also emergent while gathering and analyzing data, in a "progressive in-focus" Sta10]. These topics can be exemplified by the following questions:

T1 (participants' profile) What are the participants' background and prior knowledge on orchestration, and how do they affect their perception of ' $5+3$ Aspects'?

T2 (framework's completeness) Is ' $5+3$ Aspects' a coherent and complete view of orchestration in TEL/CSCL?

T3 (framework's usefulness) Would researchers use '5+3 Aspects' in their research practice? with what purpose?

Each of those topics is in turn informed by several informative questions that try to probe for information. This conceptual organization of the data from the evaluation is adapted from Huberman \& Miles's anticipated data reduction procedure (typical in qualitative data analysis [Hub94]).

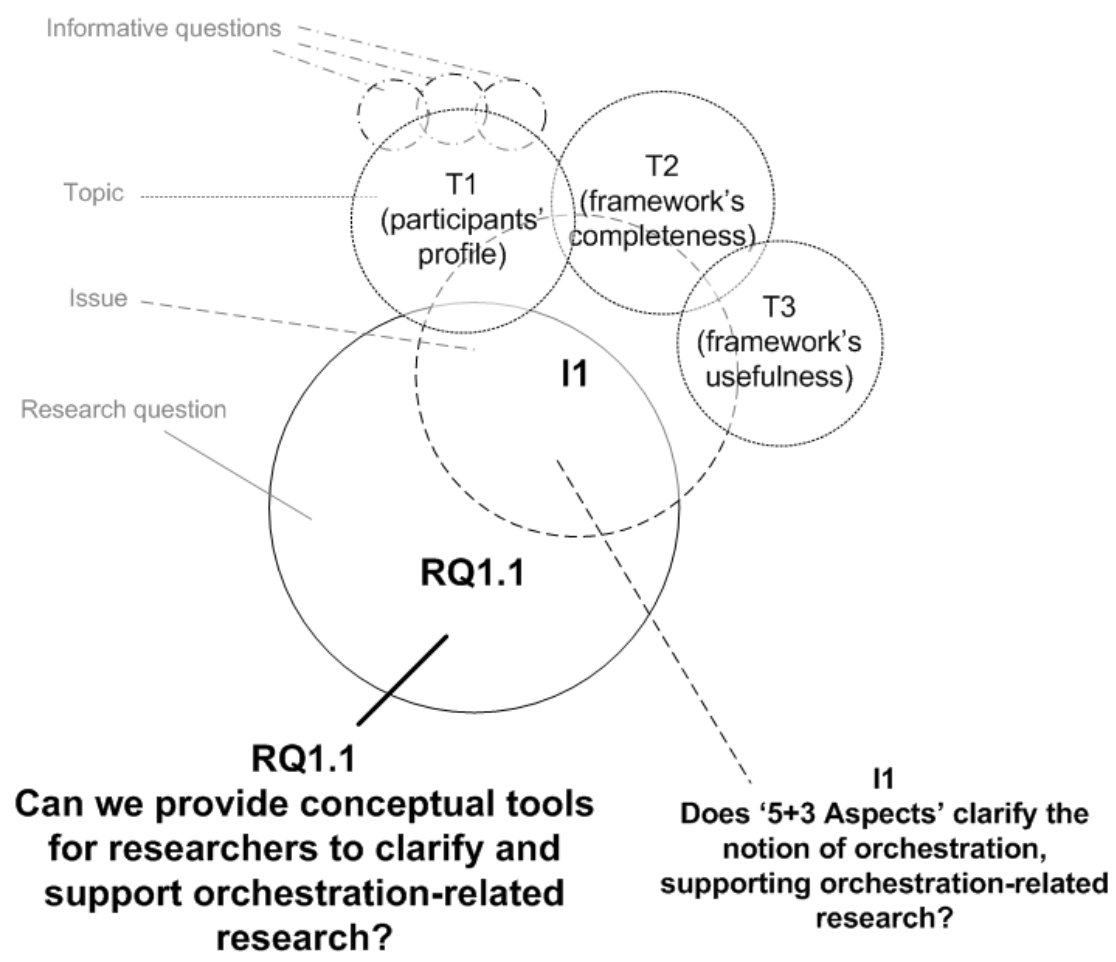

Figure 3.6: Graphical representation of the research questions, issues, topics and informative questions used during the evaluation of ' $5+3$ Aspects' 
With all of the above in mind, we can represent our evaluation design for the ' $5+3$ Aspects' contribution of the thesis through the diagrammatic view provided by the CSCL-EREM framework(Figure 3.7). As we can see, our Evaluand in this case is the ' $5+3$ Aspects' conceptual framework, as a tool to support researchers in understanding and conducting orchestrationrelated research. Our Perspective as evaluators can be described through our Goal, which is to support researchers in their research practice related to orchestration in TEL/CSCL. The main Evaluator was the author of this dissertation, although at different points of the evaluation, he was supported by 2 more expert researchers from the GSIC-EMIC group, who had previous experience in the evaluation in this kind of technological and educational contexts. The central Issue in this evaluation, that we have mentioned already, was the tension represented by the I1 question in Figure 3.6 ("Does ' $5+3$ Aspects' clarify the notion of orchestration, supporting orchestration-related research?"). However, this tension was resolved through the exploration of the aforementioned four topics that explore different orchestration aspects (T1 through T3).

The Ground of our evaluation is represented centrally by the GLUE!-PS architecture and data model as they have been presented throughout this chapter. The main Stakeholders of the evaluation have been the 46 researchers participating in the researcher panels. This Evaluation took place during the Spring of 2012, involving three members of a trans-disciplinar research group (including the author).

Finally, the Method we have chosen in the evaluation included one main Data gathering technique (due to the limitations of data gathering over such a distributed array of participants, many of which had important availability constraints): online questionnaires. The Supporting technologies used during the evaluation included MS Exce ${ }^{4}$ for quantitative and qualitative analysis, or GoogleForms 5 for web-based questionnaires. Our Informants were the aforementioned 46 researchers (including 22 younger researchers from nearby research groups and 24 international experts on the subject of orchestration), and the Documents that were available for analysis included researcher-generated reflection guides (see Section 3.4) based on the framework. The evaluation was composed of two very similar studies (Happenings in CSCL-EREM terminology), which would be conducted in series: 1) a "pilot" researcher panel (RP1) with TEL/CSCL researchers from four research groups at three Spanish universities, which would serve mainly to gather evidence from younger researchers, and to polish any glaring defects in the study method; 2) an international experts panel (RP2) with internationally recognized TEL/CSCL experts in the topic of orchestration, which would gather more extensive data about the perceived usefulness and completeness of the framework. Both phases were to be conducted via online interaction (e.g. online resources and questionnaires), due to the difficulty of physically gathering such an array of people synchronously in one place.

It is also worth mentioning the way we have applied the mixed methods approach to the data gathering and analysis during these evaluations. As outlined in Section 1.2 , we have applied a variation of the mixed methods approach to studying CSCL, first proposed in Mar06b. In the concrete case of the evaluation of ' $5+3$ Aspects', we have followed a simplified version of the flow depicted in Figure 1.5, to account for the contextual differences in this study (i.e. the fact that we mainly used online questionnaires as data sources). A particularized data gathering and analysis diagram can be seen in Figure 3.8. As we can see there, several quantitative/qualitative

${ }^{4}$ http://office.microsoft.com/en-us/excel/ (Last visit: 6 Jun 2012).

5 http://www.google.com/google-d-s/forms/(Last visit: 6 Jun 2012). 


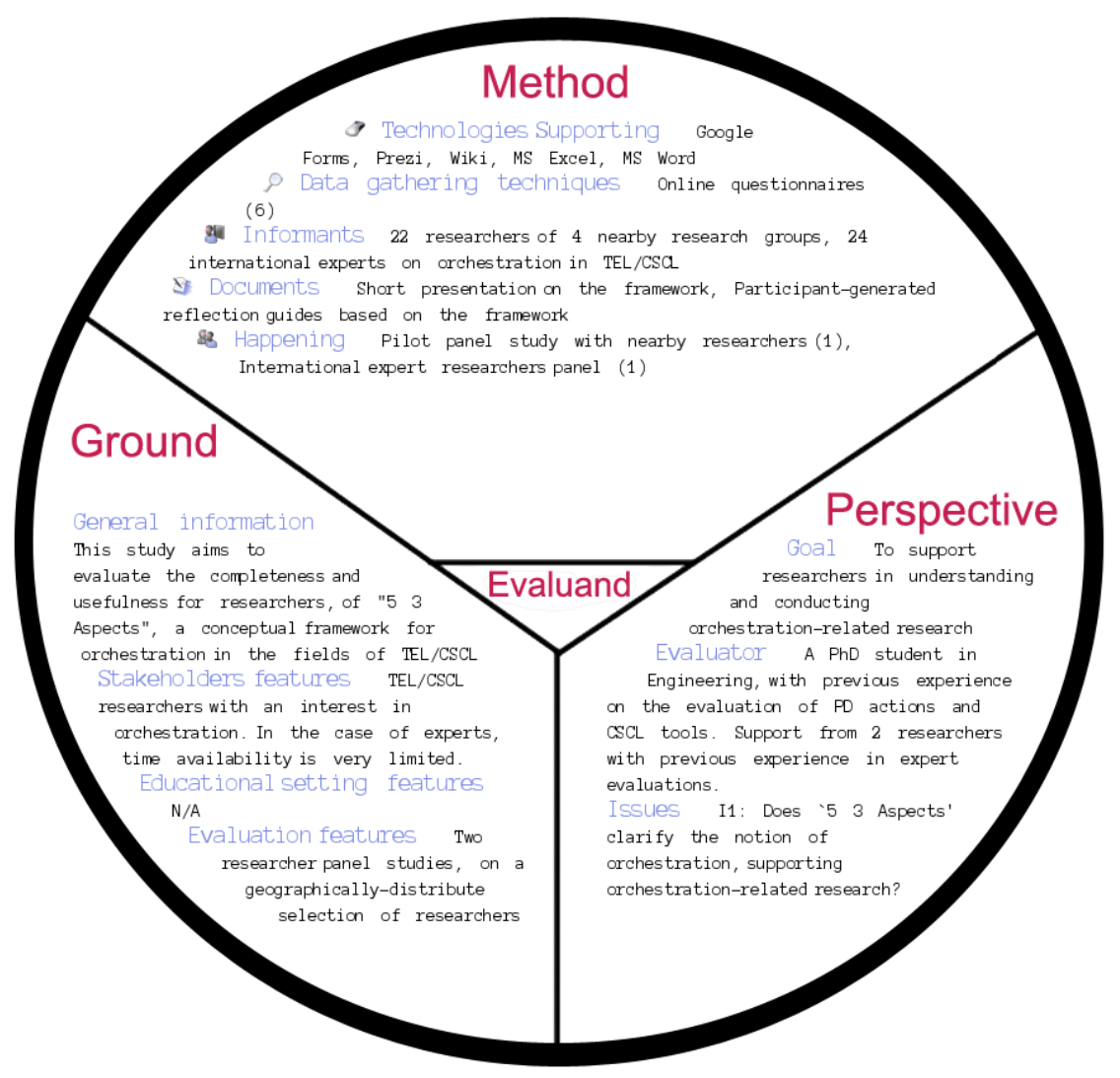

\begin{tabular}{|l|l|l|}
\hline Time & $13-27$ March 2012 & 20 Apr - 16 May 2012 \\
\cline { 2 - 3 } Data Gathering & Online questionnaires (3) & Online questionnaires (3) \\
\cline { 2 - 3 } 214 Informants & $\begin{array}{l}\text { 36 researchers from nearby research } \\
\text { groups }\end{array}$ & $\begin{array}{l}25 \text { international expert researchers } \\
\text { on orchestration }\end{array}$ \\
\cline { 2 - 3 } Supportive Technologies & Wiki, Prezi, Googleforms & Wiki, Prezi, Googleforms \\
\hline
\end{tabular}

Figure 3.7: Evaluation design for the " $5+3$ Aspects" conceptual framework

questions within the different questionnaires have been used to obtain triangulated evidence about the participant researchers' profiles, feedback and reflections about the usage of ' $5+3$ Aspects'. All this (mostly qualitative, but also quantitative) data was then analyzed, using qualitative and quantitative (descriptive statistical) analysis techniques, triangulating evidence where possible. This analysis led us to partial conclusions which were used to decide the changes to focus to be made in the following evaluations, as well as to generate our (provisional) global conclusions about ' $5+3$ Aspects' as a conceptual tool for researchers.

Finally, we should note that, as highlighted in Section 1.2 , in this section we have chosen the "findings/evidence tables + summarizing text" formula to present the evaluation findings and evidence, in order to keep the evaluation's length under control, while allowing readers to 


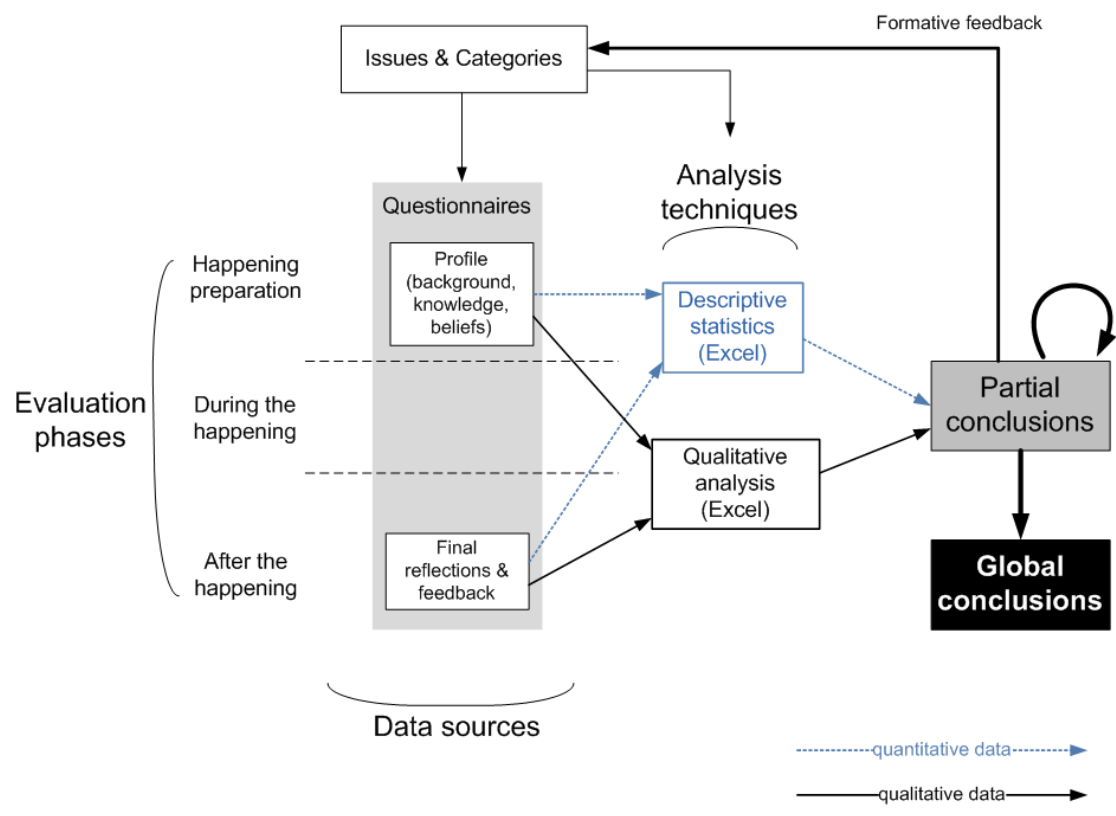

Figure 3.8: Graphic representation of the data gathering and analysis techniques flow during the evaluation of ' $5+3$ Aspects', adapted from [Mar06b.

define their reading patterns more flexibly.

\subsubsection{A panel of related TEL/CSCL researchers (RP1)}

\section{Context and methodology of the study}

The first research panel was designed to follow a process in several phases, some of them mandatory (in normal print), and some of them optional (in italics). The optional part was aimed towards the evaluation of the first research instrument derived from the conceptual framework (see Section 3.4), and thus was deemed less important. The study phases were:

1. Subjects were presented with a short profiling questionnaire [RP1-Q1] about their background and expertise (including a self-assessment on orchestration knowledge)

2. Subjects were presented with a short reading material $]^{6}$ (see Figure 3.5) (in this case, a Prezi $1^{7}$ visual presentation, see Figure 3.9 depicting the definition and conceptual framework on orchestration, and graphical representations of it (and links to the full framework article [Pri11e], in case the participant was interested)

3. Subjects were asked to fill in a questionnaire appraising the framework [RP1-Q2]. This questionnaire included, among others, the following items, both in quantitative and qualitative terms, in order to obtain triangulated data about our topics of interest (in brackets):

\footnotetext{
${ }^{6}$ Available online at http://prezi.com/aa2vighak7hh/orchestration-in-tel-cscl-as-easy-as-53/ (Last visit: 16 May 2012).

http://www.prezi.com (Last visit: 09 Apr 2012)
} 
- Self-assess again your orchestration knowledge (to gather perceived gains in knowledge about orchestration) (T1, participants' profile)

- Is the framework logical? (T2, framework's completeness)

- Is it relevant to TEL/CSCL? (T2, framework's completeness)

- Are the framework's concepts clear? (T2, framework's completeness)

- Are the graphical representations clear? (T2, framework's completeness)

- Do you think the framework is complete? Which parts may be missing? (T2, framework's completeness)

- Propose any other enhancements to the framework that come to mind (T2, framework's completeness)

- Did the framework provide insights on orchestrating TEL/CSCL? (T3, framework's usefulness)

- Did the questionnaire provide insights on orchestration of concrete settings? (T3, framework's usefulness)

- Would you use the framework in your research practice? (T3, framework's usefulness)

4. Participants were asked to think about a real, concrete TEL/CSCL context in which they were developing an orchestration-related research effort (or any concrete TEL/CSCL context that they knew in depth). Then, they would read the reflection guide (see Appendix A). If inclined to do so, participants were encouraged to write down (in a paper or in the document itself) any problems, solutions and other ideas that emerged as they answered the questions. The document could be then sent to the author. (Optional)

5. Participants were to answer an instrument evaluation questionnaire [RP1-Q3] to assess the value of the reflection guide. (Optional)

As the reader may have noticed, several questionnaires and participant-generated documents have been used to gather data about the framework's evaluation. Table 3.1 lists them along with the codes used in the finding/evidence tables.

Table 3.1: Main data sources used during the RP1 researcher panel

\begin{tabular}{|lll|}
\hline Source & Kind of evidence & Codes \\
\hline \hline First profiling questionnaire & $\begin{array}{l}\text { Qualitative \& Quantita- } \\
\text { tive }\end{array}$ & RP1-Q1 \\
\hline $\begin{array}{l}\text { Second questionnaire, about the conceptual } \\
\text { framework's support for researchers }\end{array}$ & $\begin{array}{l}\text { Quantitative \& Qualita- } \\
\text { tive }\end{array}$ & RP1-Q2 \\
\hline $\begin{array}{l}\text { Third questionnaire, about the derived instru- } \\
\text { ment's usefulness }\end{array}$ & $\begin{array}{l}\text { Quantitative \& Qualita- } \\
\text { tive }\end{array}$ & RP1-Q3 \\
\hline $\begin{array}{l}\text { Researcher-generated reflection guides, using the } \\
\text { derived instrument }\end{array}$ & Qualitative & RP1-D \\
\hline
\end{tabular}

Regarding the panel's participants, for the first panel study, researchers from four research groups in three Spanish universities were contacted. The research groups chosen were: 


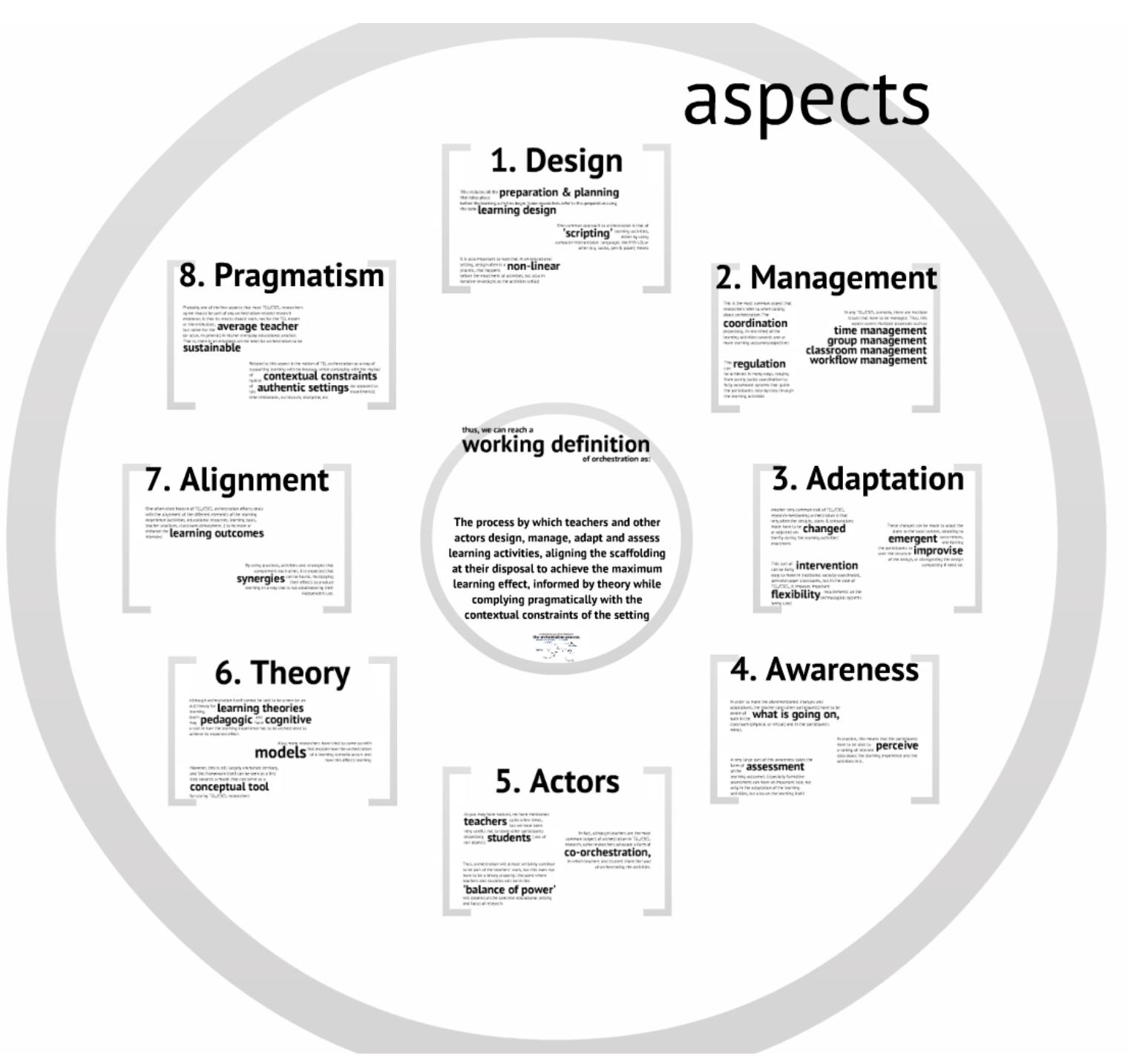

Figure 3.9: Graphical representation of the framework, taken from the multimedia presentation that experts had to go through during the study

- The GSIC-EMIC group 8 at the University of Valladolid, a large (20+ researchers) multidisciplinary group dedicated mostly to the study of CSCL (the author is a member of this group)

- The CETIE group ${ }^{9}$ at the University of Valladolid, a small (4 researchers) educational research institute with close ties to the GSIC-EMIC group

- The GTI groun ${ }^{10}$ at the Universitat Pompeu Fabra in Barcelona, a medium (13 researchers) research group dedicated to Human-Computer Interaction, 3D graphics and TechnologyEnhanced Learning

8 http://gsic.uva.es (Last visit: 9 Apr 2012)

9 http://www.cetie.uva.es (Last visit: 9 Apr 2012)

10 http://gti.upf.edu/ (Last visit: 9 Apr 2012) 
- The GAST groun ${ }^{11}$ at the Universidad Carlos III in Madrid, a large (50 researchers) research group dedicated to various areas of Telematics Engineering, including TechnologyEnhanced Learning

These four groups were chosen mainly for pragmatic reasons, in order to ensure a minimum number of participants (which would be otherwise unlikely for the evaluation of such an unknown new framework). These four research groups are close to the author in the sense that they have a history of joint work, both at the personal and at the group level. Moreover, the fact that three of those groups are part of a joint Spanish research project ${ }^{12}$ whose main theme is the orchestration of ubiquitous learning activities across web, 3D and augmented reality (AR) spaces, ensured that a certain number of the contacted researchers would have an interest in the concept of orchestration. This selection of groups obviously presents disadvantages for the representativeness of the study (i.e. results will not be generalizable to the whole population of TEL/CSCL researchers), and presents a number of biases (e.g. a majority of the selected groups' participants have a technological background). Nevertheless, the necessity of gathering data from a sizeable number of participants (due to the limitations in terms of data source variety, since only questionnaires were being used) outweighed these problems - even if they should be taken into account when drawing conclusions from the data.

From these four groups, a total of 36 researchers were contacted (18 from GSIC-EMIC, 3 from CETIE, 2 from GTI and 13 from GAST). Of this population of 36 researchers, 22 (61.1\%) answered the questionnaires evaluating the framework (14 from GSIC-EMIC, 2 from CETIE, 2 from GTI and 4 from GAST). The main results of both questionnaires are given below.

\section{Findings and evidence}

The responses of the participants revealed a number of findings around our three main topics of interest:

Participants' profile (T1) From the profiles of the 22 participants answered the questionnaires, we can draw the following picture (see Table 3.2, and also Figure 3.10 below): they were fairly novel researchers (many of of them were actually PhD students, and none of them had 20 or more years of research experience), although most of them declared having some degree of experience in TEL, either as a researcher or as a practitioner. Degrees of experience were comparable for the field of CSCL (both as researchers and as teachers/practitioners). When asked about their knowledge on the area of orchestration in TEL/CSCL quantitatively, they self-assessed their knowledge in the mid-lower part of the scale (see Table 3.2, and Figure 3.10). Regarding the background of the participants, most of them were technology-related (e.g. up to 15 came from technological backgrounds), but there was also a noticeable amount of participants from an Educational background (6 participants), or stating a multi-disciplinar background (1 participant).

\footnotetext{
${ }^{11}$ http://www.gast.it.uc3m.es/ (Last visit: 9 Apr 2012)

${ }^{12}$ EEE-Web, TIN2011-28308-C03-02.
} 


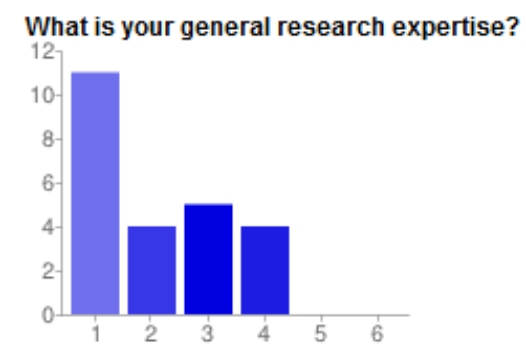

PhD student/Recent doctorateWorld-recognized research expert/ More than 20 years of research experience

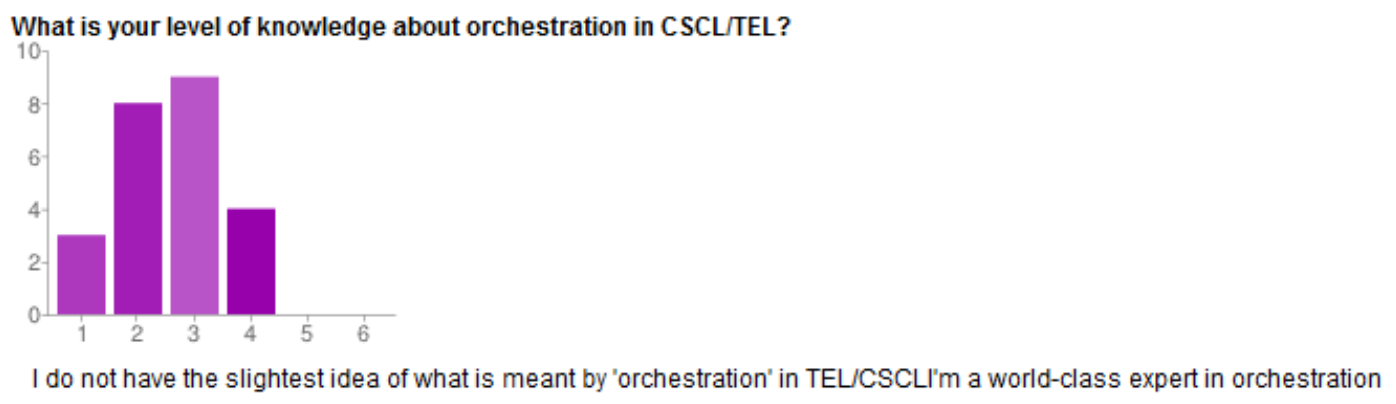

Figure 3.10: Graphic representation of the participants' research expertise and prior orchestration knowledge in the RP1 study

Regarding their initial conceptualizations and attitudes about orchestration, participants showed a variety of attitudes towards it, from interest to lack of knowledge. Other qualitative answers referred concerns about the notion of orchestration (particularly, its current use as a "buzzword", see Table 3.2). But the most interesting were the informal definitions of orchestration that were derived from the participants' qualitative answers, which mention (directly or implicitly) many of the orchestration aspects portrayed in our literature review: coordination, design, management, scripting, adaptation, groups, tools, resources... Table 3.2 provides some significant examples.

Table 3.2: Selected findings and evidence from the RP1 panel, concerning the topic of the participants' profile (T1)

\begin{tabular}{ll}
\hline Findings & Selected supporting evidence \\
\hline Certain experience with CSCL & Most of the participants declared having some degree of experience in CSCL, \\
& either as a researcher (18 out of $22,81.8 \%$ ) or as a practitioner $(17,77.3 \%)$ \\
& [RP1-Q1] \\
& Participants assessed their CSCL expertise in the mid-lower range of the scale \\
& (avg=2.45, std=1.12 as researchers, avg $=2.5$ std=0.93 as practitioners, on a \\
& $1-6$ Likert scale) [RP1-Q1] \\
& Most of the participants declared having some degree of experience in TEL, \\
& either as a researcher (16 out of $22,72.7 \%$ ) or as a practitioner $(21,95.4 \%)$ \\
& [RP1-Q1] \\
Certain experience with TEL & Participants assessed their TEL expertise in the mid-lower range of the scale \\
& (avg=2.41, std=1.21 as researchers, avg=2.68 std=0.89 as practitioners, on a \\
& 1-6 Likert scale) [RP1-Q1] \\
\hline
\end{tabular}


Table 3.2 (continued from previous page)

\begin{tabular}{l}
\hline Findings \\
Dominance of GSIC-EMIC perspec- \\
tives \\
Fairly young participantship \\
Multi-disciplinar background, with \\
technical bias
\end{tabular}
Selected supporting evidence

22 participants answered the questionnaire evaluating the framework (14 from GSIC-EMIC, 2 from CETIE, 2 from GTI and 4 from GAST) [RP1-Q2]

$45.5 \%$ of them were PhD students, none of them had 20 or more years of research experience [RP1-Q1]

Participants assessed their own research experience in the lower end of the scale $(\operatorname{avg}=2.18, \mathrm{std}=1.39$ in a $1-6$ Likert scale) [RP1-Q1]

Orchestration as a buzzword [to the question: What is your general opinion on orchestrating CSCL/TEL?]

[...] I believe orchestration is a highly overused buzzword [RP1-Q1]

[to the question: What is your general opinion on orchestrating CSCL/TEL?]

[...] IMHO [In My Humble Opinion] it is unnecessary and too vague for researchers [RP1-Q1]

Orchestration as requiring teacher effort

[to the question: What is your general opinion on orchestrating CSCL/TEL?] [...] sometimes, I think that the improvement of including orchestration in courses is not worth, because it requires a lot of effort for teachers, for only a slight improvement. I think that sometimes [it] is not efficient. [RP1-Q1]

Prior definitions of orchestration: coor- $\quad$ [to the question: What is your general opinion on orchestrating CSCL/TEL?] dination

I think orchestration is a specific application of "coordination" to educational settings [...] It concerns the teacher in most educational situations, but not necessarily all of them [RP1-Q1]

Prior definitions of orchestration: coordination [to the question: What is your general opinion on orchestrating CSCL/TEL?] I understand that orchestration deals with the coordination of technologysupported processes that may take place at different levels (e.g. individually, small groups, big groups) of a collaborative learning situation [RP1-Q1]

Prior definitions of orchestration: design [to the question: What is your general opinion on orchestrating CSCL/TEL?] $[\ldots]$ my understanding of orchestration is very close to that of learning design [RP1-Q1]

Prior definitions of orchestration: design, content, technology, context

[to the question: What is your general opinion on orchestrating CSCL/TEL?] I think that orchestration consists in the design of a learning experience taking into account the different elements that intervene in it, clarifying both the mechanics and dynamics of such system [...] I think that orchestration should include aspects related to the content, as well as the technologies used, the involved people, their roles and the space-time in which the activities develop [...] It is necessary to place the orchestrated experience in a wider frame, to acknowledge not only the experience, but also the context it is placed in [RP1-Q1]

Prior definitions of orchestration: integration, tools, groups, management

[to the question: What is your general opinion on orchestrating CSCL/TEL?] For me orchestration relates to how teachers handle the great diversity of factors in TEL and, specially, CSCL-based experiences. The teacher needs to configure many tools and groups, coordinate the flow of information and artifacts between groups, provide instructions to the students and control how they are developing in their group-work. [RP1-Q1]

Prior definitions of orchestration: management, resources, groups, design, enactment

[to the question: What is your general opinion on orchestrating CSCL/TEL?] "Orchestrating" evokes the tasks that the teacher (or an LMS) would do to manage CSCL activities: give instructions, provide resources, form groups, etc. [...] From what I hear at the GSIC lab, orchestration includes design, preparation and enactment [...] [RP1-Q1]

Prior definitions of orchestration: [to the question: What is your general opinion on orchestrating CSCL/TEL?] I scripting take orchestration as a nice synonym for scripting. It justs shifts the metaphor [...] [RP1-Q1]

Prior definitions of orchestration: [to the question: What is your general opinion on orchestrating CSCL/TEL?] scripting, improvisation, design, adaptation

[...] evolves from conventional guided collaborative learning situations in the field of CSCL, and promotes in the practitioner the ability to improvise and implement well-known routines at the proper time in specific scenarios. It can start with a design that describes the coarse grained case and then, on-the-fly be free to introduce such modifications in the plan [...] [RP1-Q1] 
Completeness and coherence of the framework (T2) By analyzing the responses to the framework assessment questionnaire [RP1-Q2], both quantitatively and qualitatively, we can extract the following findings regarding the completeness and coherence of ' $5+3$ Aspects' (see also Figure 3.11): the framework was deemed logical by participants, especially in the quantitative questions. However, if we look at the qualitative responses (see Table 3.3), we can find some participants who raise doubts about the framework's logical structure (e.g. some elements being transversal or orthogonal to others), or the relative relationships or hierarchies between certain framework aspects (especially Design, Management and Adaptation).

Regarding the framework's clarity, again participants provided rather positive feedback on the quantitative side (see Figure 3.11 and Table 3.3), but also expressed doubts about the clarity and/or terminology of certain aspects, such as Pragmatism, Awareness or, especially, Theory.

Table 3.3: Selected findings and evidence from the RP1 panel, concerning the topic of the framework's completeness (T2)

\begin{tabular}{|c|c|}
\hline Findings & Selected supporting evidence \\
\hline $\begin{array}{l}\text { Participants thought the framework } \\
\text { logical }\end{array}$ & $\begin{array}{l}\text { The framework was deemed "logical" by participants }(\operatorname{avg}=5.09, \mathrm{std}=0.53 \mathrm{in} \\
\text { a } 1-6 \text { Likert scale) }[\mathrm{RP} 1-\mathrm{Q} 2] \\
\text { Only } 1 \text { out of } 22 \text { participants valued the framework in the negative part of } \\
\text { the "logical" question [RP1-Q2] }\end{array}$ \\
\hline $\begin{array}{l}\text { Doubts about the logical organization } \\
\text { of the framework }\end{array}$ & $\begin{array}{l}\text { [to the question: explain why you do not think the framework's concepts are } \\
\text { clear] I don't see very clear if "actors" are aspects in the same way "design", } \\
\text { "management", "adaptation" and "awareness" are "aspects". I mean, actors } \\
\text { are people, while the rest are activities. [RP1-Q2] } \\
\text { [to the question: explain why you do not think the framework's concepts are } \\
\text { clear] I would also see that in a framework for successful CSCL, Awareness } \\
\text { stood up in the first level, but I don't see it meets the metaphor of orchestra- } \\
\text { tion, [...] Actors can be a relevant object of study, of course, but again at a } \\
\text { complete different level [RP1-Q2] } \\
\text { [to the question: explain why you do not think the framework's concepts are } \\
\text { clear] [...] there is in my opinion an issue regarding the granularity and the } \\
\text { essence on some of the components. Actors, theory and in some way pragma- } \\
\text { tism could be seen as transversal/orthogonal components of the model. In a } \\
\text { way they conform ground aspects to be taken into account when orchestrating } \\
\text { [RP1-Q2] }\end{array}$ \\
\hline $\begin{array}{l}\text { Logical organization: design, } \\
\text { adaptation and management }\end{array}$ & $\begin{array}{l}\text { [to the question: explain why you (do not) think the framework is logical] } \\
\text { Adaptation to me is an issue that concerns management. On a logical level, } \\
\text { it is "part of" management [RP1-Q2] } \\
\text { [to the question: explain why you (do not) think the framework is logical] I } \\
\text { would consider to split the management aspect into several ones. [RP1-Q2] } \\
\text { [to the question: explain why you (do not) think the framework is logical] } \\
\text { From my point of view, "learning designs", "workflow" and "adaptation" are } \\
\text { concepts with a lot of in common, but they are in three different aspects (1, } 2 \text {, } \\
\text { and 3). I think they should be in the same aspect, and the framework should } \\
\text { give a clear relationship among these three concepts [RP1-Q2] }\end{array}$ \\
\hline $\begin{array}{l}\text { Participants thought the framework } \\
\text { clear }\end{array}$ & $\begin{array}{l}\text { The framework was deemed "clear" by participants }(\operatorname{avg}=4.95, \text { std }=0.40 \text { in a } \\
1-6 \text { Likert scale) }[\mathrm{RP} 1-\mathrm{Q} 2] \\
\text { No participant valued the framework in the negative part of the "clear" ques- } \\
\text { tion [RP1-Q2] }\end{array}$ \\
\hline Unclear concepts: Pragmatism & $\begin{array}{l}\text { [to the question: explain why you do not think the framework's concepts are } \\
\text { clear] Pragmatism is a little bit unclear for me. I do not know if that is the } \\
\text { right word [RP1-Q2] }\end{array}$ \\
\hline Unclear concepts: Awareness & $\begin{array}{l}\text { [to the question: explain why you do not think the framework's concepts are } \\
\text { clear] I have understood "awareness" as group awareness (i.e. know what } \\
\text { others are doing) but it may be intended to mean "know how well I/we are } \\
\text { doing". In this case I would look for another term. [RP1-Q2] }\end{array}$ \\
\hline
\end{tabular}


Table 3.3 (continued from previous page)

\begin{tabular}{|c|c|}
\hline Findings & Selected supporting evidence \\
\hline Unclear concepts: Theory & $\begin{array}{l}\text { [to the question: explain why you do not think the framework's concepts are } \\
\text { clear] I think the "guided by theory" part needs a revision to integrate it } \\
\text { with the rest of the model [...] it should be oriented towards the pedagogic } \\
\text { and cognitive aspects of learning [RP1-Q2] } \\
4 \text { participants mentioned the aspect "Theory" in the qualitative answers } \\
\text { about the framework concepts' clarity }\end{array}$ \\
\hline $\begin{array}{l}\text { Participants thought the framework } \\
\text { complete }\end{array}$ & $\begin{array}{l}\text { The framework was deemed "complete" by participants (avg }=5.27, \text { std }=0.83 \\
\text { in a } 1-6 \text { Likert scale) [RP1-Q2] } \\
\text { Only } 1 \text { out of } 22 \text { participants valued the framework in the negative part of } \\
\text { the "complete" question [RP1-Q2] }\end{array}$ \\
\hline Missing aspects: cognition/pedagogy & $\begin{array}{l}\text { [to the question: state any missing concepts from the model] The cognitive } \\
\text { and pedagogic aspects I do not think are described clearly enough in the } \\
\text { model [...] [RP1-Q2] }\end{array}$ \\
\hline $\begin{array}{l}\text { Missing aspect: technology and } \\
\text { resources }\end{array}$ & $\begin{array}{l}\text { [to the question: state any missing aspects from the framework] If we make the } \\
\text { analogy with language, I'd say that the model captures the subject (actors), } \\
\text { the verbs (what), the adverbs (how), but leaves out the direct and indirect } \\
\text { objects (the elements to which the verbs are applied [RP1-Q2] } \\
\text { [to the question: state any missing aspects from the framework] [...] as teachers } \\
\text { use resources to achieve their learning outcomes, I would make it more [salient] } \\
\text { [RP1-Q2] } \\
\text { [to the question: state any missing aspects from the framework] Where is the } \\
\text { role of "technology"? [RP1-Q2] }\end{array}$ \\
\hline $\begin{array}{l}\text { Participants thought the representa- } \\
\text { tions were fairly helpful }\end{array}$ & $\begin{array}{l}\text { The graphical representations were considered fairly "helpful" by participants } \\
\text { (avg=4.86, std }=0.94 \text { in a } 1-6 \text { Likert scale) }[\mathrm{RP} 1-\mathrm{Q} 2]\end{array}$ \\
\hline $\begin{array}{l}\text { Participants thought the definition of } \\
\text { orchestration was fairly accurate }\end{array}$ & $\begin{array}{l}\text { The provided definition of orchestration was considered "accurate" by partic- } \\
\text { ipants (avg }=4.86, \mathrm{std}=0.66 \text { in a } 1-6 \text { Likert scale) }[\mathrm{RP} 1-\mathrm{Q} 2]\end{array}$ \\
\hline
\end{tabular}

In the end, most participants explicitly valued the the framework as complete (see Figure 3.11 and Table 3.3). However, it is interesting to mention that a small part of the participants (3) mentioned the resources and technologies (i.e. the "things that are orchestrated") as an important missing part of orchestration in the framework.

As we can see in the quantitative graphics (see Figure 3.11), the definition of orchestration and the graphical representations provided alongside the framework were also highly rated, albeit in a lesser degree than the previous parameters (see also Table 3.3).

Usefulness of the framework (T3) In this regard, the mixed methods analysis over the [RP1-Q2] questionnaire indicates that participants saw the framework as moderately usable for their research practice in the future (see Figure 3.12 and Table 3.4 for more details). In general, the framework was seen as useful because of the holistic view it provided about the practice of CSCL in authentic settings. Some of the participants even mention its usefulness as a sort of "checklist" of aspects to take into account when approaching a CSCL situation. Others found it useful in order to frame their own research work within the orchestration of TEL/CSCL, thus helping them motivate their research problems.

Some of the aspects that may have made it less attractive for some participants include the fact that there is a need of further examples and best practices in order to orchestrate better (an aim that, in this dissertation, is rather targeted by the "atomic patterns" contribution, see Chapter (4). Indeed, participants scored it only above average with regard to its ability to provide new insights (see Table 3.4), probably indicating that none of the concepts in the framework is entirely novel. 


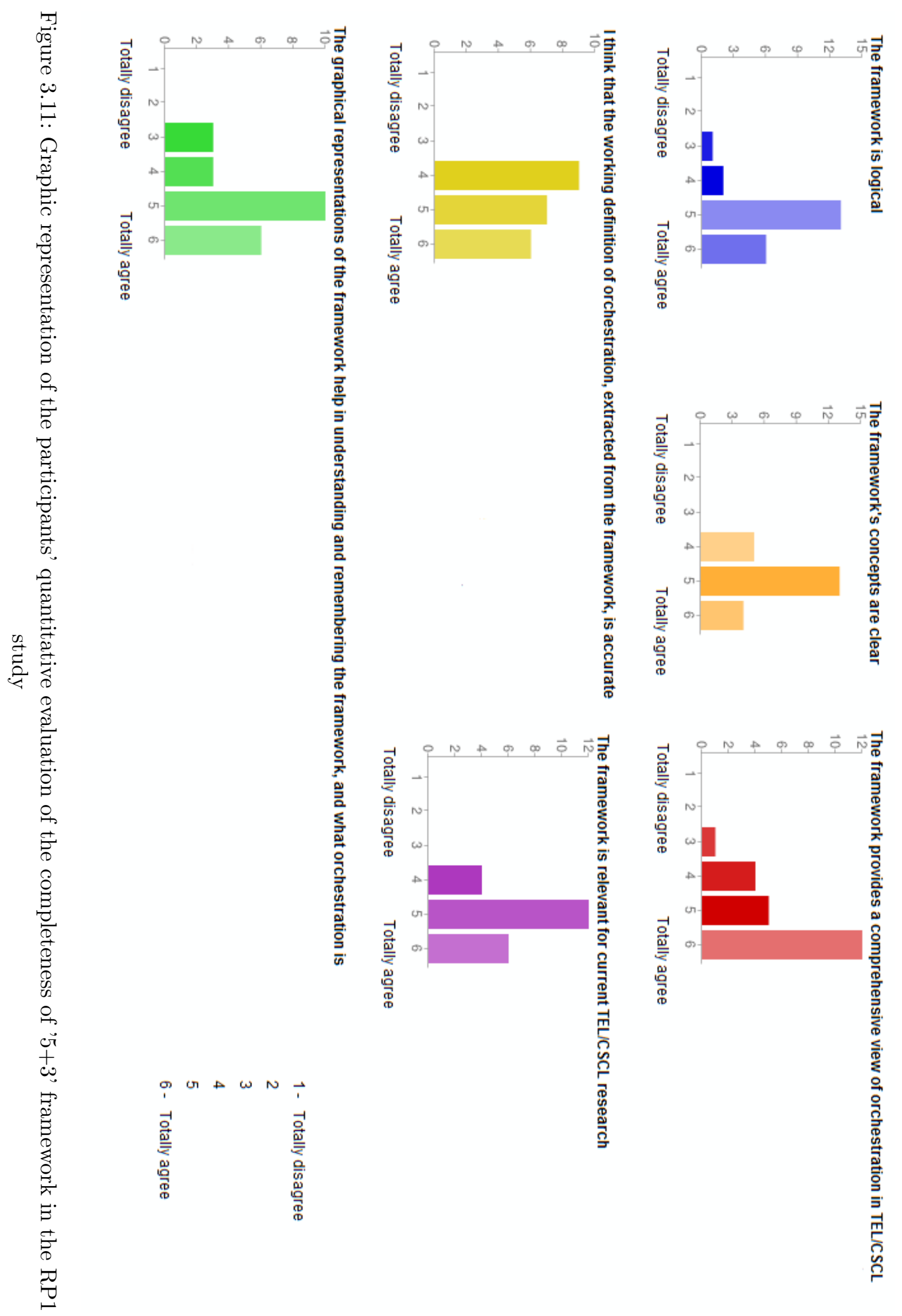



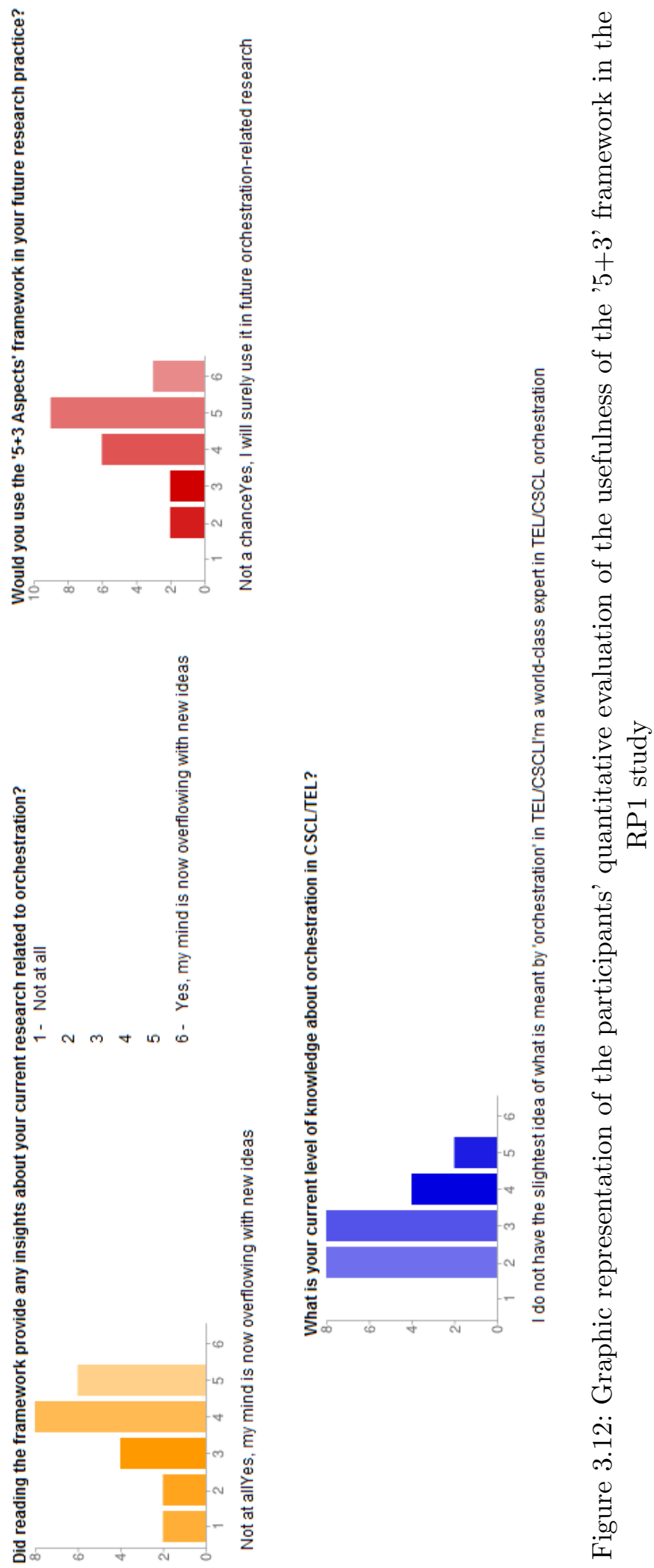
Table 3.4: Selected findings and evidence from the RP1 panel, concerning the topic of the framework's usefulness (T3)

\begin{tabular}{|c|c|}
\hline Findings & Selected supporting evidence \\
\hline $\begin{array}{l}\text { The framework is moderately useful } \\
\text { for research practice }\end{array}$ & $\begin{array}{l}3 \text { out of } 22 \text { participants stated that they would surely use the framework in } \\
\text { their practice ( } 6 \text { in the } 1-6 \text { Likert scale) [RP1-Q2] } \\
\text { Participants stated that they were mildly likely to use the framework in their } \\
\text { research practice (avg }=4.41, \text { std }=1.24 \text { in a } 1-6 \text { Likert scale) }[\mathrm{RP} 1-\mathrm{Q} 2]\end{array}$ \\
\hline $\begin{array}{l}\text { The framework does not portray novel } \\
\text { concepts }\end{array}$ & $\begin{array}{l}\text { Participants mildly agreed with the assertion of the framework providing new } \\
\text { insights (avg }=3.64, \text { std }=1.5 \text { in a } 1-6 \text { Likert scale) }[\mathrm{RP} 1-\mathrm{Q} 2]\end{array}$ \\
\hline Usefulness as checklist & $\begin{array}{l}\text { [to the question: explain why you would (not) use the framework for your } \\
\text { research practice] I find it useful as a check-list for the design process [...] } \\
\text { Also, it could be useful to design tools aimed at supporting specific parts of } \\
\text { the orchestration process [RP1-Q2] }\end{array}$ \\
\hline $\begin{array}{l}\text { Usefulness as holistic view of } \\
\text { practice-oriented CSCL }\end{array}$ & $\begin{array}{l}\text { [to the question: state any insights elicited by the reading of the framework] I } \\
\text { think it collects simply and concisely the main issues for successfully putting } \\
\text { CSCL into practice [RP1-Q2] } \\
\text { [to the question: state any insights elicited by the reading of the framework] } \\
\text { It combines not only the elements that should be present but also the criteria } \\
\text { on how they should be combined [RP1-Q2] } \\
\text { [to the question: state any insights elicited by the reading of the framework] } \\
\text { The enumeration of all these aspects offers a more holistic view than what I } \\
\text { previously had [RP1-Q2] }\end{array}$ \\
\hline Usefulness to frame CSCL research & $\begin{array}{l}\text { [to the question: state any insights elicited by the reading of the framework] } \\
\text { This framework could help me to contextualize the interest and purpose of } \\
\text { my research work [RP1-Q2] }\end{array}$ \\
\hline $\begin{array}{l}\text { Need for further examples and best } \\
\text { practices }\end{array}$ & $\begin{array}{l}\text { [to the question: explain why you would (not) use the framework for your re- } \\
\text { search practice] It would be very interesting to make a companion guide to the } \\
\text { framework where all the aspects are reviewed, indicating with examples [...] } \\
\text { what happens in the process of orchestration and the implications of touch- } \\
\text { ing, eliminating or modifying these aspects [...] caveats and best practices } \\
\text { [RP1-Q2] }\end{array}$ \\
\hline $\begin{array}{l}\text { Potential as pedagogical tool for re- } \\
\text { searchers }\end{array}$ & $\begin{array}{l}\text { Participants' average self-perceived knowledge about orchestration was risen } \\
(\operatorname{avg}=2.63, \text { std }=0.9 \text { in }[\mathrm{RP} 1-\mathrm{Q} 1] \text { vs. } \text { avg }=3, \mathrm{std}=0.91 \text { in }[\mathrm{RP} 1-\mathrm{Q} 2])\end{array}$ \\
\hline
\end{tabular}

Finally, there were also certain hints of the potential of the ' $5+3$ Aspects' framework as a pedagogical tool for novel researchers approaching orchestration for the first time. For example, the participants rated their knowledge about orchestration in [RP1-Q2] as higher than the one in the first questionnaire [RP1-Q1] (this was a purposeful question to capture significant knowledge variations after completing the study). Albeit this difference is not statistically significant, and there is no appreciable correlation between this difference and the researcher experience, we can probably infer a certain usefulness of the framework and the reading material as a tool to communicate or teach about the subject of orchestration in TEL/CSCL.

\section{Conclusions and modification towards the following panel}

Overall, the RP1 study can be considered a success, since a considerable number of participants answered the questionnaires, and the quantitative and qualitative feedback obtained about the conceptual framework was rather positive. We can summarize our partial conclusions from this panel in the following manner, referring to the different topics of interest:

T1 (participants' profile) It was found that participants' prior conceptions of orchestration were coherent with the literature review conducted so far, highlighting aspects such as 
coordination, design/scripting, adaptation, or management [RP1-T1-PC1]. However, it was interesting to find that some of these researchers also shared our observation of the orchestration concept becoming something of a "buzzword" in TEL/CSCL research. [RP1T1-PC2]

T2 (framework's completeness) The framework was generally considered logical, clear, and complete by the participants [RP1-T2-PC1]. Moreover, the definitions and representations were also considered accurate and useful, albeit to a lesser extent, probably indicating that further refinements are needed in them [RP1-T2-PC2]. There were some concerns, however, about the structure and terminology of the eight aspects, which could be improved [RP1-T2-PC3].

T3 (framework's usefulness) The framework was considered by the participants (who were not necessarily involved directly in orchestration-related research) moderately useful for research practice [RP1-T3-PC1]. The main usefulness participants found in the framework was as a holistic view of TEL/CSCL practice in authentic settings, as a list of issues to consider [RP1-T3-PC2]. Even if the framework was not considered too novel or revolutionary, there were certain hints that it may have a certain pedagogical value for younger researchers [RP1-T3-PC3].

However, we still could not assert the completeness and usefulness of the framework, especially considering the contextual caveats of the study, such as the fact that most researchers were from the nearest circle to the author, and that few of them had a large TEL/CSCL research experience.

Despite this study's primary goal as a "pilot" for the experts' study (RP2, see next section), some responses by participants provided very interesting matter for reflection and refinement of the framework. Especially interesting were remarks regarding the framework's logical structure and terminology, the role of technology in the framework, or the separation of some elements as transversal to the others. However, given the aforementioned contextual caveats, we chose to hold up any major changes in the framework until further confirming feedback was provided in the RP2 study by the international experts. There were, indeed, a number of minor modifications that were suggested (directly or indirectly) by participants, which were applied to the form and structure of the study, towards the enactment of RP2 (we will not list them here for brevity's sake).

\subsubsection{A wider panel of internationally-recognized TEL/CSCL orchestration researchers (RP2)}

\section{Context and methodology of the study}

For this second panel of researchers, the method remained largely the same as the one in the RP1 study (described at the beginning of Section 3.3.2), including the mandatory part about the conceptual framework (profiling questionnaire, multimedia presentation and assessment questionnaire), and the optional part about the derived instrument (following of the reflection guide and final questionnaire). Table 3.5 summarizes the data sources used in the evaluation. 
Table 3.5: Main data sources used during the RP2 researcher panel

\begin{tabular}{|lll|}
\hline Source & Kind of evidence & Codes \\
\hline \hline First profiling questionnaire & $\begin{array}{l}\text { Qualitative \& Quantita- } \\
\text { tive }\end{array}$ & RP2-Q1 \\
\hline $\begin{array}{l}\text { Second questionnaire, about the conceptual } \\
\text { framework's support for researchers }\end{array}$ & $\begin{array}{l}\text { Quantitative \& Qualita- } \\
\text { tive }\end{array}$ & RP2-Q2 \\
\hline $\begin{array}{l}\text { Third questionnaire, about the derived instru- } \\
\text { ment's usefulness }\end{array}$ & $\begin{array}{l}\text { Quantitative \& Qualita- } \\
\text { tive }\end{array}$ & RP2-Q3 \\
\hline $\begin{array}{l}\text { Researcher-generated reflection guides, using the } \\
\text { derived instrument }\end{array}$ & Qualitative & RP2-D \\
\hline
\end{tabular}

Regarding the participants on this second study, a list of internationally-recognized experts in the area of orchestration within the TEL and CSCL research fields was elaborated. This list was produced by choosing the first authors from the main publications of the literature review in Section 2.3, as well as from the list of authors in the aforementioned orchestration workshop at the CSCL 2011 international conference [Nus11] and the "collective paper/issue" on orchestration that derived from the STELLAR document on orchestration [Dil11a] (many of which had been hand-picked, e.g. by Pierre Dillenbourg when writing his report on the subject). All in all, 31 experts from across the world were contacted, of which 25 agreed to participate. Out of this population of 25, 24 experts finally completed the mandatory part of the study.

\section{Findings and evidence}

By analyzing the quantitative and qualitative evidence gathered, we can describe a number of findings regarding our three main topics of interest, plus a number of emergent general ideas about this study and orchestration as a whole, which we will address under a fourth emergent topic (T4).

Participants' profile (T1) From the answers of the 24 participants to the profiling questionnaire [RP2-Q1], we can conclude that the participants in this study were much more experienced researchers, both in general research expertise (e.g. with an average expertise over 17 years, see Figure 3.13 and Table 3.6), as well as in the fields of TEL and CSCL (with averages over 15 and 12 years of experience, respectively). Moreover, participants were also seasoned CSCL and TEL practitioners. When asked to assess their knowledge on the area of orchestration in TEL/CSCL, they self-assessed their knowledge in the mid-higher part of the scale (see Figure 3.13 and Table $3.6 \sqrt{13}$. Regarding the background of the participants, they were a heterogeneous mix of mostly Education backgrounds (45.8\%), Computer Science and Engineering (25\%), and multidisciplinar backgrounds $(29.2 \%)$. It is interesting that in this case, even if it is definitely a hard to pinpoint mixture of expertises and background, it is more inclined towards the educational side, while RP1 participants had a more technology-oriented background in average.

Apart from gathering data about the participants' experience and background, this questionnaire prompted the international experts to provide their own definitions of what orchestration is. The qualitative responses to these questions themselves are highly interesting, and

\footnotetext{
${ }^{13}$ This fact itself is interesting. Experts were either very modest, or not entirely sure of what counted as orchestration in TEL. This might be another hint that the concept of orchestration in TEL/CSCL is currently ambiguous.
} 


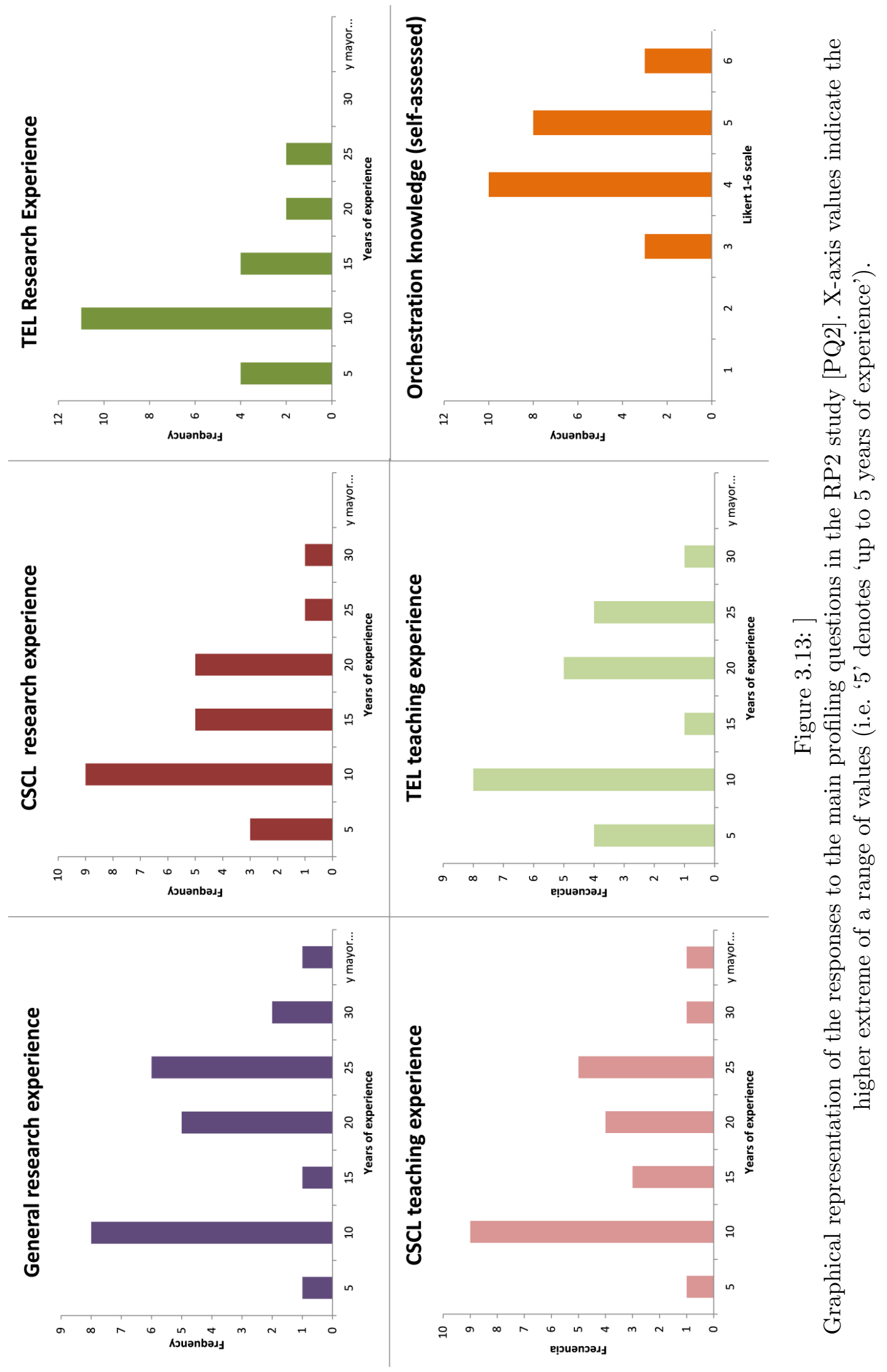


CAP. 3

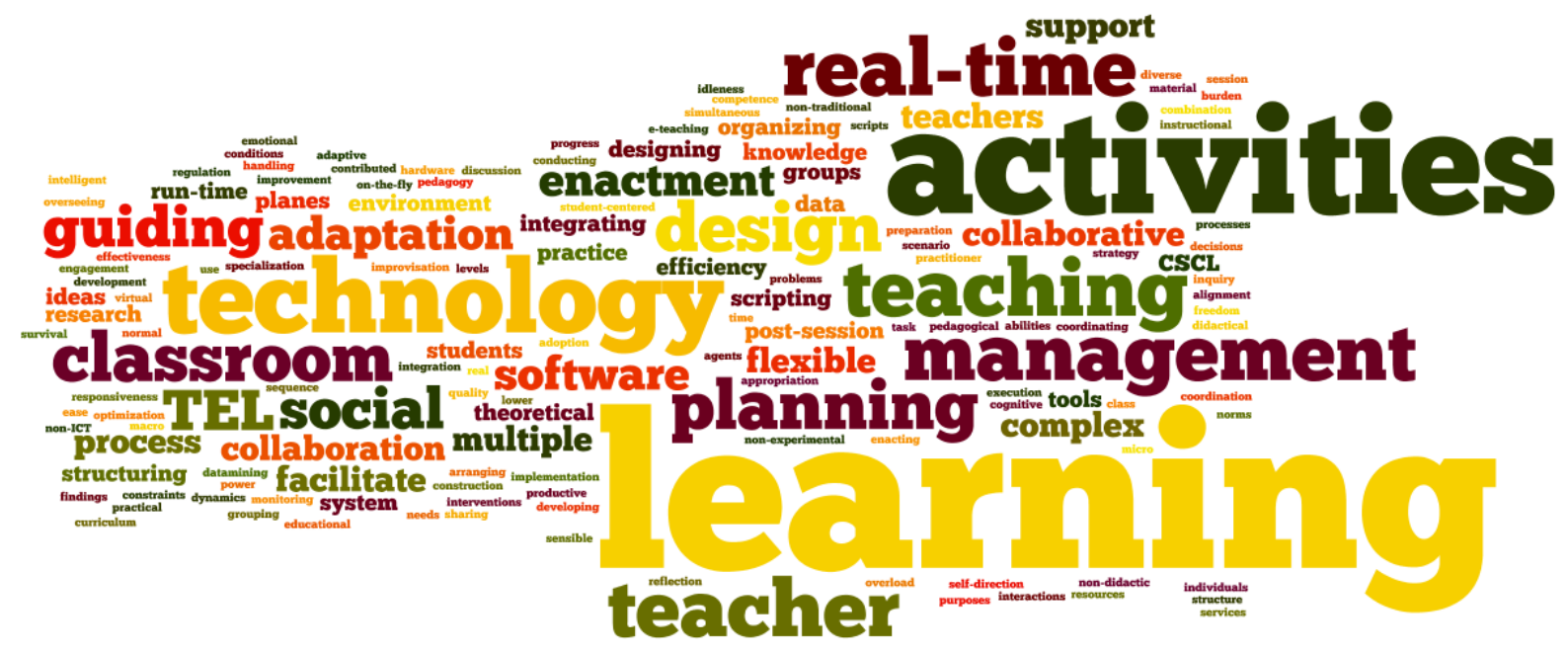

Figure 3.14: Word cloud representing the relative importance of keywords in RP2 participants' prior definitions of orchestration

analyzing them could provide us with another valuable synthesis (apart from the one presented in Section 2.3.3 of what TEL and CSCL researchers think orchestration is. Figure 3.14 represents graphically the main keywords that appeared in those definitions ${ }^{14}$.

As we can see, the picture that these definitions paint is not very different from the ones provided in this dissertation, in Figure 2.4, or the ones appearing in the " $5+3$ Aspects" framework. We do have, however, first indicators of their relative importance. Apart from obvious keywords in TEL, like "technology" or "learning", we can notice that, for the experts, orchestration seemed to be about the learning activities, and how teachers manage (or guide) them in real-time, often in the classroom. However, we can also see that designing and planning have an important role in these definitions. Other concepts that appear, on a slightly lesser degree are flexible adaptation, complexity, integration, or multiplicity (of activities, tools, etc.). Additional complete examples of these definitions can be found in Table 3.6. In these definitions we can see that the most repeated notions remain the same as in our literature review or the previous panel, here the breadth of concepts is larger, probably accounting for the variety of backgrounds and perspectives represented in the study.

Table 3.6: Selected findings and evidence from the RP2 panel, concerning the topic of the participants' profile (T1)

\begin{tabular}{ll}
\hline Findings & Selected supporting evidence \\
\hline Participants are experienced & Participants stated many years of research experience $($ avg $=17.8$ yrs, std=8.32 \\
TEL/CSCL researchers & yrs) [RP2-Q1] \\
& Participants stated many years of TEL research experience (avg=15.8 yrs, \\
std=7.86 yrs) [RP2-Q1]
\end{tabular}

\footnotetext{
${ }^{14}$ These word clouds have been produced by extracting keywords from the definitions and using Wordle, see http://www. wordle.net/ (Last visit: 17 May 2012).
} 
Table 3.6 (continued from previous page)

\begin{tabular}{|c|}
\hline Findings \\
\hline $\begin{array}{l}\text { Participants are experienced } \\
\text { TEL/CSCL practitioners }\end{array}$ \\
\hline $\begin{array}{l}\text { Practitioners considered themselves } \\
\text { fairly knowledgeable about orchestra- } \\
\text { tion }\end{array}$ \\
\hline $\begin{array}{l}\text { Multi-disciplinar background with an } \\
\text { educational bias }\end{array}$ \\
\hline
\end{tabular}
Selected supporting evidence

Participants stated many years of CSCL research experience (avg $=12.08$ yrs, std $=6.53 \mathrm{yrs})[\mathrm{RP} 2-\mathrm{Q} 1]$

Participants stated many years of TEL teaching practice experience (avg $=12.82$ yrs, std=7.99 yrs) [RP2-Q1]

Participants stated many years of CSCL teaching practice experience (avg $=10.39$ yrs, std=6.37 yrs) [RP2-Q1]

Participants self-assessed their knowledge about orchestration as mid-high (avg $=4.46$, std $=0.88$, in a 1-6 Likert scale) [RP2-Q1]

Multi-disciplinar background with an Prior definitions of orchestration: inte-

Participants stated Education backgrounds (11 participants, 45.8\%), Computer Science and Engineering backgrounds (6 participants, 25\%), and multidisciplinar backgrounds (7 participants, 29.2\%) [RP2-Q1]

gration, teachers, software

Prior definitions of orchestration: activities, scripting, management, preparation

\begin{tabular}{l} 
Prior definitions of orchestration: man- \\
agement, teacher, activities, social \\
planes, constraints \\
\hline Prior definitions of orchestration: de- \\
sign, adaptation, enactment
\end{tabular}

Prior definitions of orchestration: ease, efficiency, flexibility, technology, teaching, learning

Prior definitions of orchestration: coordination, teaching, technology, student-centric, real classroom, enactment

Prior definitions of orchestration:
teachers, multiplicity, complexity

[to the question: what does orchestration of TEL/CSCL mean for you?] Integrating/articulating run-time teachers' activities and educational software [RP2-Q1]

[to the question: what does orchestration of TEL/CSCL mean for you?] It is about both the learning activities [...] we want students to perform (so, it includes a.o. scripting approaches etc.) and the way we manage to do this in learning environments (so it involves management). It is not solely about classroom management, but it could also be virtual classroom management. It is not solely about the ad hoc activities on the spot, but also on the preparation [...] [RP2-Q1]

[to the question: what does orchestration of TEL/CSCL mean for you?] The real time management by the teacher of multiple activities, across several social planes, and of multiple constraints [RP2-Q1]

[to the question: what does orchestration of TEL/CSCL mean for you?] Actually three parts: the initial design of a TEL scenario [...], adaptation of the TEL scenario by a practitioner for her purposes [...], on-the-fly enactment, incl. on-the-fly decisions based on unforeseen issues during enactment [...] From my perspective, orchestration should be understood as encompassing all three stages [RP2-Q1]

[to the question: what does orchestration of TEL/CSCL mean for you?] Making it EASY and EFFICIENT for practitioners to FLEXIBLY and ADAPTIVELY use technology in teaching and learning [RP2-Q1]

[to the question: what does orchestration of TEL/CSCL mean for you?] What is it: organizing and coordinating teaching practices when implementing technology-enhanced student-centered learning activities in a real classroom. What is not (the boundary): 1) not traditional teaching practices which do not involve technology, 2) not in an "experimental" design context in which the class deviates from normal class, 3) not about lesson planing but about lesson enactment, 4) not about didactic teaching but student-centered learning activities [RP2-Q1]

[to the question: what does orchestration of TEL/CSCL mean for you?] [...] I look at orchestration especially in terms of what can be done to support teachers in the complex process of creating the multifaceted conditions that are conducive to learning. Those conditions are of many types, including: social $[\ldots]$, emotional $[\ldots]$, temporal $[\ldots]$, structure $[\ldots]$; cognitive $[\ldots]$, material [...] [RP2-Q1]

\begin{tabular}{|c|c|}
\hline Doubts about use in academia & $\begin{array}{l}\text { [to the question: what is you opinion about orchestration in CSCL/TEL?] [...] } \\
\text { I wonder if it's a good idea for academics to deal with this. It seems more like } \\
\text { a product oriented question; probably people who offer real products will be } \\
\text { better at it [RP2-Q1] }\end{array}$ \\
\hline Orchestration as buzzword & $\begin{array}{l}\text { [to the question: what is you opinion about orchestration in CSCL/TEL?] } \\
\text { Buzz word, to a large extent. Re-discovering known basic issues and ideas. } \\
\text { Some real research questions, however [RP2-Q1] }\end{array}$ \\
\hline Orchestration as fuzzy & $\begin{array}{l}\text { [to the question: what is you opinion about orchestration in CSCL/TEL?] [...] } \\
\text { the concept is still too fuzzy, and everyone concerned with it seems to have a } \\
\text { different understanding of what the term is about. Usefulness of the metaphor } \\
\text { will only be observable once the term is clearly established and new research } \\
\text { questions [..] come along that are based on the term [RP2-Q1] }\end{array}$ \\
\hline
\end{tabular}


Table 3.6 (continued from previous page)

\begin{tabular}{|c|c|}
\hline Findings & Selected supporting evidence \\
\hline & $\begin{array}{l}\text { [to the question: what is you opinion about orchestration in CSCL/TEL?] } \\
\text { good [...] the problem is that it is used in several ways and everybody has his } \\
\text { own idea (not necessarily a concrete definition) of what orchestration really } \\
\text { means [...] I do not think it is overused, but it is definitely kind a fuzzy } \\
\text { concept. [RP2-Q1] } \\
\text { [to the question: what is you opinion about orchestration in CSCL/TEL?] } \\
\text { perfectly, wonderfully fuzzy. Lets leave it that way. (easier, down in the weeds) } \\
\text { [RP2-Q1] }\end{array}$ \\
\hline $\begin{array}{l}\text { Orchestration as important, unsolved } \\
\text { problem }\end{array}$ & $\begin{array}{l}\text { [to the question: what is you opinion about orchestration in CSCL/TEL?] [...] } \\
\text { in practice it has always been an issue, since without orchestration of learners } \\
\text { and resources there's no way to make collaborative learning happen. There is } \\
\text { still a lack of computer support for orchestration in real-world environments } \\
\text { [...] I guess there's still a lot to do if we want to consider orchestration also from } \\
\text { a formal educational planning perspective (which I am not really convinced } \\
\text { of) [RP2-Q1] } \\
\text { [to the question: what is you opinion about orchestration in CSCL/TEL?] A } \\
\text { valuable concept that needs further research and clarification. Also needs to } \\
\text { be related to/distinguished from coordinate concepts, such as learning design, } \\
\text { scripting, authoring, lesson planning [RP2-Q1] } \\
\text { [to the question: what is you opinion about orchestration in CSCL/TEL?] } \\
\text { Good and important [RP2-Q1] }\end{array}$ \\
\hline $\begin{array}{l}\text { Orchestration as novel awareness of } \\
\text { teacher work }\end{array}$ & $\begin{array}{l}\text { [to the question: what is you opinion about orchestration in CSCL/TEL?] It } \\
\text { denotes a key evolution of the field, some novel awareness of the reality of } \\
\text { teachers' work [RP2-Q1] }\end{array}$ \\
\hline $\begin{array}{l}\text { Orchestration as umbrella term hiding } \\
\text { complexity }\end{array}$ & $\begin{array}{l}\text { [to the question: what is you opinion about orchestration in CSCL/TEL?] } \\
\text { [...] Many current TEL solutions seem to "orchestrate" one issue or another, } \\
\text { involving design aspects, instantiation, enactment, integration, group forma- } \\
\text { tion, [...] sometimes the use of the term contributes to somehow "hiding" the } \\
\text { actual type of research proposal being reported! [RP2-Q1] } \\
\text { [to the question: what is you opinion about orchestration in CSCL/TEL?] Or- } \\
\text { chestration "hides" a really complex life-cycle that, as you know, we consider } \\
\text { as particularly "flexible" with entwined phases [RP2-Q1] }\end{array}$ \\
\hline
\end{tabular}

The profiling questionnaire also had a probing question about participants' attitudes towards the concept of orchestration. Here, there were very varied responses, from the very concise to the widely verbose, which also hinted at aspects of the experts' conception of orchestration or, at least, the aspects they were more interested in. In general, attitudes were positive, since many participants considered it an important and unsolved problem in TEL/CSCL, that raised a novel awareness of the complexity of the teachers' work in authentic settings. There were also, however, critiquing voices in the group, such as those who considered orchestration a "fuzzy" concept, or a simple "buzzword" (see Table 3.6). Other participants highlighted how the term was sometimes used as a an "umbrella term" used to somehow hide the underlying complexity of TEL/CSCL practices. Finally, others raised doubts about the convenience of using a term of that nature in academia (as opposed to doing so in the industry or in the development of products). As we can see, in this group of researchers we can find a much more varied and intense set of attitudes, with a larger proportion of critical or dissident voices.

Completeness of the framework (T2) By analyzing the responses to the framework assessment questionnaire [RP2-Q2], we can extract the following findings regarding the topic of the completeness and coherence of the framework (see also Figure 3.15 and Table 3.7): the framework was deemed quite logical by experts, with quite a few of them stating that "it made sense" 
for them - although numeric results were lower than for the RP1 study (average of 4.78 vs. 5.09, in a 1-6 Likert scale), and with a wider variance (standard deviations of 1 vs. 0.53 in RP1). Some of the participants suggested different groupings of aspects in order to enhance the framework's logical structure, pointing out the existence of certain transversal elements (something that some researchers had also noted in RP1). Others highlighted the need for clearer relationships among elements of the framework.

The participants in general considered the framework very clear, both numerically an through their qualitative responses (even more so than their RP1 counterparts, see Figure 3.15 and Table 3.7). Those who did not find the framework entirely clear pointed out that the role of the Theory aspect was not clear, or proposed different terms and organizations of concepts. Interestingly, other experts found the provided notion of orchestration as fairly indistinguishable from teachers' routine practice and from the related concepts that, in fact, formed the framework.

Table 3.7: Selected findings and evidence from the RP2 panel, concerning the topic of the framework's completeness (T2)

\begin{tabular}{|c|c|}
\hline Findings & Selected supporting evidence \\
\hline $\begin{array}{l}\text { Participants thought the framework } \\
\text { fairly logical }\end{array}$ & $\begin{array}{l}\text { [to the question: explain why you (do not) find it logical] The aspects are } \\
\text { appealing and definitely relevant. I did not have the chance to review the } \\
\text { process of coming up with this selection (e.g. a literature survey, synthesis, } \\
\text { etc.) Otherwise it makes sense [RP2-Q2] } \\
\text { [to the question: explain why you (do not) find it logical] The framework has } \\
\text { an internal logic which seems clear to me.[RP2-Q2] } \\
\text { Only } 2 \text { out of } 24 \text { participants valued the framework in the negative part of } \\
\text { the "clear" question [RP2-Q2] } \\
\text { The framework was deemed "logical" by participants (avg=4.78, std=1 in a } \\
\text { 1-6 Likert scale) [RP2-Q2] }\end{array}$ \\
\hline $\begin{array}{l}\text { Logical structure: different grouping of } \\
\text { aspects }\end{array}$ & $\begin{array}{l}\text { [to the question: explain why you (do not) find it logical] }[. . .] \text { The } 5+3 \text { includes } \\
\text { "what" and "how" aspects. Perhaps the Actors issue should refer to a "who" } \\
\text { aspect? [...] [RP2-Q2] }\end{array}$ \\
\hline $\begin{array}{l}\text { Logical structure: need for clearer re- } \\
\text { lationships }\end{array}$ & $\begin{array}{l}\text { [to the question: explain why you (do not) find it logical] [...] I would be } \\
\text { tempted to treat pragmatism as a different kind of issue - more an "ap- } \\
\text { proach" than a conceptual issue in its own right. I also can see arguments for } \\
\text { both separating and combining theory and alignment - they are both "back- } \\
\text { ground influences" on the way that a teacher creates a design, and so could be } \\
\text { combined [...] I also prefer the second diagram which collapsed management, } \\
\text { adaptation and awareness together, as they seem more closely related to each } \\
\text { other than, say, the design stage, which is quite different [RP2-Q2] }\end{array}$ \\
\hline Logical structure: transversal elements & $\begin{array}{l}\text { [to the question: explain why you (do not) find it logical] Just an intuition } \\
\text { that the last dimensions could be integrated in the previous ones [RP2-Q2] }\end{array}$ \\
\hline $\begin{array}{l}\text { Participants thought the framework } \\
\text { clear }\end{array}$ & $\begin{array}{l}\text { [to the question: explain why you (do not) think that the framework's concepts } \\
\text { are clear] Aspects are well defined. Covers phenomena clearly [RP2-Q2] } \\
\text { [to the question: explain why you (do not) think that the framework's concepts } \\
\text { are clear] The building blocks as such are understandable and well-defined } \\
\text { [RP2-Q2] } \\
\text { Only } 1 \text { out of } 24 \text { participants valued the framework in the negative part of } \\
\text { the "clear" question [RP2-Q2] } \\
\text { The framework was deemed "clear" by participants (avg=5.04, std=0.76 in a } \\
1-6 \text { Likert scale) [RP2-Q2] }\end{array}$ \\
\hline $\begin{array}{l}\text { Lack of clarity: Alternative terms and } \\
\text { organization }\end{array}$ & $\begin{array}{l}\text { [to the question: explain why you (do not) think that the framework's con- } \\
\text { cepts are clear] "Alignment": A teacher always checks whether the learning } \\
\text { outcomes are reached, how far the learner is from these outcomes, etc. -i } \\
\text { this is part of Awareness ? I do not really understand the status of "Align- } \\
\text { ment" here. [...] "Pragmatism": refers to me to adaptation. Why putting it } \\
\text { as "specific" [...] "Management": I'd rather prefer "regulation" [...] [RP2-Q2] }\end{array}$ \\
\hline
\end{tabular}


Table 3.7 (continued from previous page)

\begin{tabular}{l}
\hline Findings \\
\hline Lack of clarity: Orchestration as syn- \\
onym with teaching practice \\
\\
\hline Lack of clarity: Orchestration as syn- \\
onym with teaching practice
\end{tabular}

Lack of clarity: Theory

\section{Selected supporting evidence}

[to the question: explain why you (do not) think that the framework's concepts are clear] The individual elements are clear - but it's not clear how orchestration as you define it can be distinguished from the routine practice of teachers in classrooms (almost all of whom, in the developed world, are currently supported by a range of technologies) [RP2-Q2]

[to the question: state any missing aspect or concept from the framework] What's missing is an attempt to differentiate it from lesson planning, scripting, authoring, formative assessment, learning design - all of which overlap with the definition [RP2-Q2]

[to the question: explain why you (do not) think that the framework's concepts are clear] The aspect of "Theory" is perhaps the one that is not completely clear [...] I'm not sure how to expect that a particular learning theory should "inform" the way a researcher might approach orchestration problems [...] I need an example in this part [RP2-Q2]

[to the question: explain why you (do not) find it logical] It seems very accurate to how I think of Orchestration. I would include all 5 and all 3 aspects, and no others [RP2-Q2]

[to the question: state any missing aspect or concept from the framework] [...] I think the most important aspects are covered [RP2-Q2]

[to the question: state any missing aspect or concept from the framework] It is really comprehensive, although it should put emphasis on the MOST important aspects [RP2-Q2]

Only 4 out of 24 participants valued the framework in the negative part of the "clear" question [RP2-Q2]

The framework was deemed "comprehensive" by participants (avg=4.52, std $=1.16$ in a 1-6 Likert scale) [RP2-Q2]

Missing aspects: beliefs, interactions
among aspects

[to the question: state any missing aspect or concept from the framework] Maybe [...] the concepts of "reflection" or "beliefs" could be integrated somewhere. [...] Also, there may be a lot of interaction between the 8 different points, so it could be a good idea to include that in the model [RP2-Q2]

Missing aspects: sharing [to the question: state any missing aspect or concept from the framework] For me, the most important reason for considering orchestration/Learning Design is the potential for teachers to share good ideas (ie, plans) with each other, so the concept of sharing (and related ideas, such as communities/repositories for sharing) is missing. [...] [RP2-Q2]

Missing aspects: technology

[to the question: explain why you (do not) think the definition is accurate] [...] Too much general pedagogy (good or bad), too little relation to computational/technology aspects [RP2-Q2]

[to the question: state any missing aspect or concept from the framework] [...] It dismisses computational aspects and opportunities [...] The framework could be read as a general pedagogical framework. Is this our business? [...] [RP2-Q2]

[to the question: state any missing aspect or concept from the framework] only one that jumped out at me was about the Actors, in orchestration.[...] but I add the technology. [...] I also include the environment as an actor, in an ambient sense [...] [RP2-Q2]

The framework as "too comprehensive" [to the question: explain why you (do not) find it logical] Very comprehensive, covers almost all the things I can think of [...]. On the other hand, if it covers so many aspects of TEL, what does it not cover? Comprehensiveness may be a strength but may also be a weakness [RP2-Q2]

[to the question: explain why you (do not) think the definition is accurate] [...] What I'm wondering now [...] is what's NOT orchestration. In other words, almost any TEL research line might claim to be focused on one aspect of the framework or another [...] [RP2-Q2]

[to the question: state any missing aspect or concept from the framework] Orchestration, according to your definition, is a very general notion, not very precise: fairly comprehensive at a general level, but lacks identification of precise issues. I propose an exercise: given your definition, how many researchers in TEL do not work on a topic that has something to do with orchestration? [RP2-Q2]

\begin{tabular}{ll}
\hline \hline $\begin{array}{l}\text { Participants thought the framework as } \\
\text { relevant to TEL/CSCL research }\end{array}$ & The framework was rated as very "relevant to TEL/CSCL research" by par- \\
ticipants (avg=5.13, std=1.14 in a 1-6 Likert scale) [RP2-Q2]
\end{tabular}


Table 3.7 (continued from previous page)

\begin{tabular}{l}
\hline Findings \\
\hline $\begin{array}{l}\text { Participants thought the definition of } \\
\text { orchestration was fairly accurate }\end{array}$ \\
\hline
\end{tabular}
Selected supporting evidence orchestra

The provided definition of orchestration was considered "accurate" by participants $(\mathrm{avg}=4.65, \mathrm{std}=1.15$ in a $1-6$ Likert scale) $[\mathrm{RP} 2-\mathrm{Q} 2]$

Definition: too complex [to the question: explain why you (do not) think the definition is accurate] At present, I think it's both too broad, and too narrow. Too broad in that it could apply to the everyday practices of teachers in classrooms [...] And it's too narrow in that it doesn't indicate the role of technology or collaborative learning in that process. It also doesn't indicate the opportunity for orchestration outside the classroom, or across formal and informal settings [...] maybe better to go for a definition that captures the gist of orchestration, accompanied by an exegesis, indicating its extensions and coordinations with other related concepts [...] [RP2-Q2]

[to the question: explain why you (do not) think the definition is accurate] Especially the part about "aligning... to achieve maximum learning.. informed by theory... while complying..." - it seems overly optimistic about what teacher's job is really like. It seems we are defining "orchestration" against an idealized teacher; but perhaps only 1

[to the question: explain why you (do not) think the definition is accurate] I agree, although it covers too many aspects [RP2-Q2]

[to the question: explain why you (do not) think the definition is accurate] The definition is somewhat awkwardly formulated, trying to include all key aspects. Personally I prefer more simple definitions [RP2-Q2]

Participants thought the representations were fairly helpful [to the question: state why you (do not) find the graphical representations useful] The circle is useful in a general sense for researchers, although it may still be too complex for teachers [...] I felt the process view was more useful for experts in the field, although again I'm not sure I'd agree with all of how it is constructed, but it does draw attention to many useful issues [RP2-Q2] [to the question: state why you (do not) find the graphical representations useful] Very nice representation. The example should accompany it, if possible, since it clarifies some abstract notions [RP2-Q2]

[to the question: state why you (do not) find the graphical representations useful] Yes, that helps in giving an overview of the model, with meaningful labels and layout [RP2-Q2]

The graphical representations were considered fairly "helpful" by participants (avg $=4.86, \mathrm{std}=1.17$ in a 1-6 Likert scale) $[\mathrm{RP} 2-\mathrm{Q} 2]$

Critics to graphical representations in general

to the question: state why you (do not) find the graphical representations useful] I have a personal issue with graphical representations, which I think tend to be overused in certain types of research to avoid deeper theoretical discussion and exploration [RP2-Q2]

[to the question: explain why you (do not) find it logical] [...] I miss an explanation why all this is part of "orchestration" (i.e. I do not see clearly the analogies to the musical domain). As the devil's advocate I would say that you have good names for all $5+3$ components, but why do you at all need that overarching 'orchestration' concept? [RP2-Q2]

[to the question: explain why you (do not) find it logical] As stated before, I do not believe that the term [orchestration] is a good basis at all. In the software technology perspective [...] configuration of tools and resources would be a strong implication. Teachers would not be central actors of [orchestration] in this perspective (but beneficiaries, of course!) [RP2-Q2]

[to the question: explain why you (do not) find it logical] Yes, its a nice organization of what people are talking about. It seems to leave out efficiency. My overall concern is that "orchestration" as a patch on badly designed, overly complex, basically unusable tools isn't going to work. Now let's suppose we have very nicely designed, pretty simple, highly usable tools that teachers like. I am not sure why we need orchestration on top of that. So either it fixes something that is too badly broken to be fixed or it adds little value to something that is already working quite nicely [...] [RP2-Q2]

Usefulness of the framework as a list [to the question: state why you (do not) find the graphical representations useful] The circle isn't much different from a list. Lists with 8 items are hard to remember. The process diagram doesn't seem quite right yet [...] [RP2-Q2] 


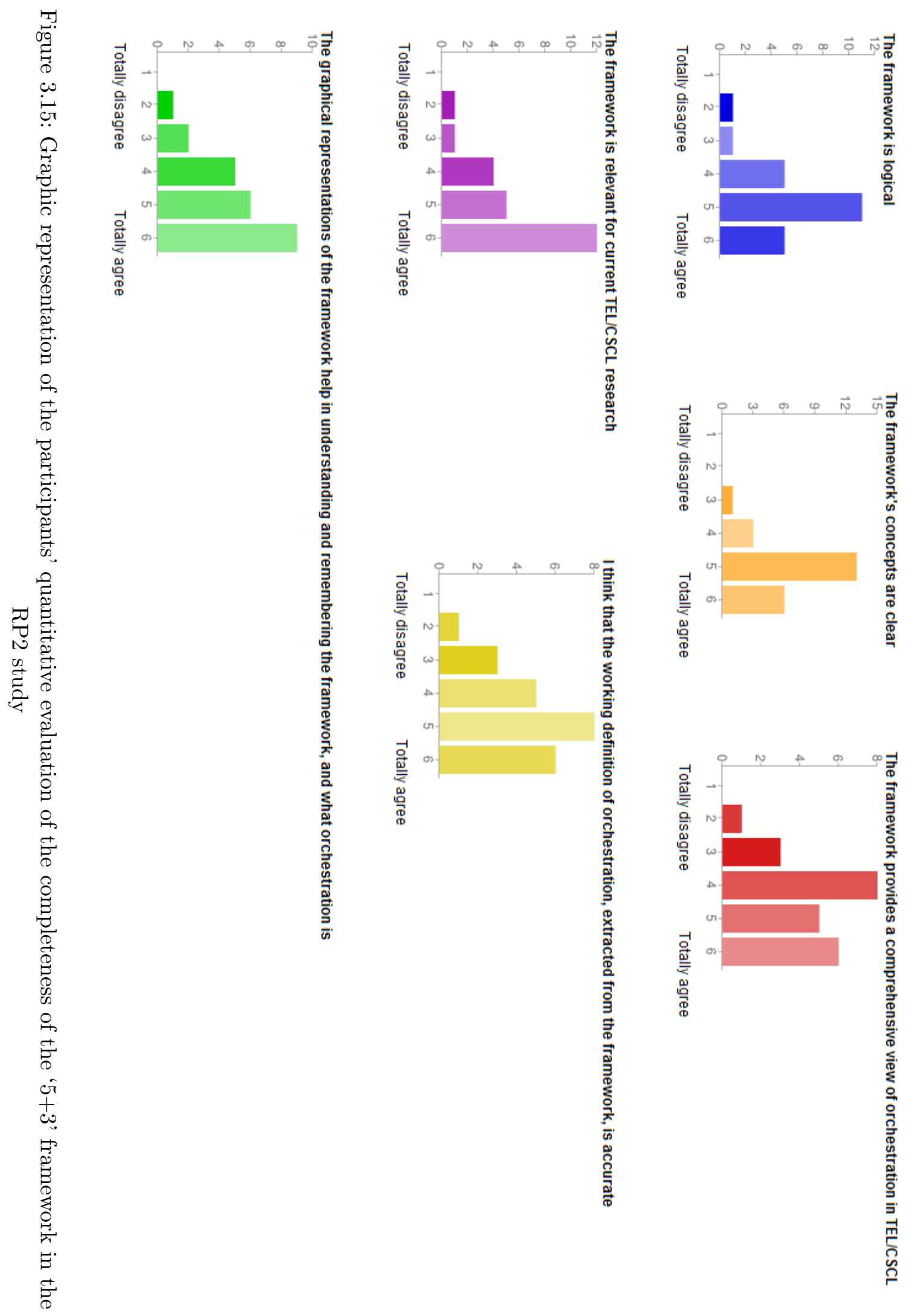


A majority of the expert researchers considered the presented framework fairly comprehensive (see Figure 3.15 and Table 3.7), although in this case the numerical values were both lower when compared with the RP1 study (average of 4.52 vs. 5.27 in RP1, in a 1-6 Likert scale), and also showed a wider variance (1.16 vs. 0.83 in RP1). Among the missing aspects, probably the most often referred to is technology (as it also happened in RP1), although others mentioned more abstract notions such as "beliefs", "interactions" or "sharing". Oddly enough, other participants found the framework "too comprehensive", leaving out very little of the TEL field from its definition. This is an interesting point, which raises the question of whether a framework synthesized from such a wide literature set, can become too broad and ineffective (an example of what is normally called "design by committee).

Moreover, the definition of orchestration and the graphical representations provided alongside the framework, were also rated favorably, although the ratings were not unanimous. Indeed, the definition was considered by a certain number of experts as too complex, encompassing too many of the framework's elements (thus favoring more minimalist definitions). There was not a clear consensus about which graphical representation was better (it seemed to be a matter of taste), or whether the representations added value to the textual explanations. Other participants expressed a general dislike for graphical representations in general (see Table 3.7).

However, in general participants thought the framework was very relevant to TEL/CSCL research. The general outlook of the experts' opinions was positive, although experts in this study showed more critical and more varied views of orchestration compared with the previous study (as it was to be expected from more mature scientists). As we can see in Table 3.7, sometimes these critiques are more directed towards the notion of orchestration as a whole, rather than to the framework itself. In any case, many of the results obtained were comparable.

Usefulness of the framework (T3) In this regard, the quantitative and qualitative responses to [RP2-Q2] indicate that participants, on average, saw the framework as moderately usable for their research practice in the future (see Figure 3.16), with 6 participants stating that they would surely use it in the future (that is, scoring it at 6 in the 1-6 Likert scale). The quantitative scores are comparable with those from RP1 (e.g. average score of 4.43, vs. 4.41 in RP1, on a 1-6 Likert scale). The qualitative answers to this question (Table 3.8 show that some of the experts found the framework useful as an integrative reference of the field, and to frame other more specific work. Also, quite a few experts stated that the reference could be useful to be given to (PhD) students, as a starting point on orchestration-related research, given its comprehensivenes: ${ }^{15}$. Other uses were also mentioned, such as the focusing of research data gathering, or as a simple checklist of factors that should be taken into account in the application of TEL/CSCL to practice in authentic settings. Many experts, however, stated that they were not sure about using the framework, either because it had not been tested in the "real world" (needing examples of real use in a research effort), or because it was too ambiguous (needing boundaries that distinguish it from other related concepts). Also, the concern was raised about its usage by teachers (which, let us remember, was not its primary goal), given its abstractness. Overall, looking at the wide

\footnotetext{
${ }^{15}$ As a curiosity, the quantitative self-assessment of orchestration knowledge after going through the materials showed that experts thought themselves, on average, slightly more knowledgeable about orchestration than they considered before the study (with an average of 4.65 vs. the original 4.46). Even if this has no statistical relevance, considered together with the qualitative responses, this hints at the didactic possibilities of the framework, especially for novel researchers or as an overview of the field.
} 
variance in the numeric scores and qualitative answers, we can only assert that some expert researchers (but not every one of them) found it useful and would use it. Moreover, this data confirmed the potential uses (e.g. as a pedagogical aid) and the improvement needs (e.g. with concrete examples) of the framework which already appeared in study RP1.

Regarding the framework's ability to spark new insights in orchestration research, responses were highly heterogeneous (see Table 3.8). The numeric score regarding this ability was only above average (see also Figure 3.16), probably indicating that none of the concepts in the framework is entirely novel (as some experts explicitly expressed). Other experts, however, remarked the framework's value as a holistic, integrative view of these well-known issues. This is not surprising, given that the participants were considered 'experts', and that some of their works had been used in the literature review that led to the framework. Other participants simply saw the framework as a confirmation of their own view of the field.

Table 3.8: Selected findings and evidence from the RP2 panel, concerning the topic of the framework's usefulness (T3)

\begin{tabular}{|c|c|}
\hline Findings & Selected supporting evidence \\
\hline $\begin{array}{l}\text { The framework is moderately useful for } \\
\text { research practice }\end{array}$ & $\begin{array}{l}6 \text { out of } 24(25 \%) \text { participants stated that they would surely use it in } \\
\text { the future (score of } 6 \text { in the } 1-6 \text { Likert scale) [RP2-Q2] } \\
\text { [to the question: explain why you would (not) use the framework in } \\
\text { your research practice] I hope that I can use it, or at least several of } \\
\text { the concepts and connections included in it [RP2-Q2] } \\
\text { [to the question: explain why you would (not) use the framework in } \\
\text { your research practice] It is a good summary. Definitely I will cite this } \\
\text { work in my lit review in future orchestration-related research [RP2- } \\
\text { Q2] } \\
\text { Participants saw the framework as mildly usable in their own research } \\
\text { practice in the future (avg }=4.43 \text {, std }=1.27 \text { in a } 1-6 \text { Likert scale) [RP2- } \\
\text { Q2] }\end{array}$ \\
\hline Pedagogical value of the framework & $\begin{array}{l}\text { [to the question: explain why you would (not) use the framework in } \\
\text { your research practice] I will give this to my students [RP2-Q2] } \\
\text { [to the question: explain why you see the framework as (not) useful } \\
\text { for practitioners] I think a teacher will find it a useful reflection of } \\
\text { what they do as practitioners. It might also give other educational } \\
\text { professionals (eg. designers who don't teach) a better idea of what } \\
\text { teaching actually encompasses [RP2-Q2] } \\
\text { [to the question: explain why you see the framework as (not) useful } \\
\text { for practitioners] Yes, if I had to teach this stuff, I would present this } \\
\text { framework to my students. It's a nice and easy-to-understand way to } \\
\text { introduce this perspective [RP2-Q2] }\end{array}$ \\
\hline Usefulness as a focus for data collection & $\begin{array}{l}\text { [to the question: explain why you would (not) use the framework in } \\
\text { your research practice] Maybe. I think I would be inclined to use it } \\
\text { as one of several starting points, but for the reasons given above, I } \\
\text { would shuffle a few things. After that, it might be a nice framework } \\
\text { for focusing/attuning data collection [RP2-Q2] }\end{array}$ \\
\hline Usefulness as integrative view & $\begin{array}{l}\text { [to the question: explain why you would (not) use the framework in } \\
\text { your research practice] Typically, the research questions I have are less } \\
\text { complex and cover maybe only one or two of your circles. However, } \\
\text { I think that the framework has integrative value and might well be } \\
\text { used for a synthesis of past research [RP2-Q2] } \\
\text { [to the question: state any insights sparked by the framework] The } \\
\text { categorization helped me in getting a more clear view of this fuzzy } \\
\text { field [RP2-Q2] } \\
\text { [to the question: explain why you would (not) use the framework in } \\
\text { your research practice] It is a good summary. Definitely I will cite this } \\
\text { work in my lit review in future orchestration-related research [RP2- } \\
\text { Q2] }\end{array}$ \\
\hline
\end{tabular}


Table 3.8 (continued from previous page)

Findings
Usefulness of the framework as a list
Selected supporting evidence

Usefulness of the framework as a list

[to the question: explain why you see the framework as (not) useful for practitioners] Yes, it may be good when developing new teaching/learning activities, in the sense that you can quickly check if you have given all the different aspects a thought [RP2-Q2]

[to the question: state why you (do not) find the graphical representations useful] The circle isn't much different from a list. Lists with 8 items are hard to remember. The process diagram doesn't seem quite right yet [...] [RP2-Q2]

Usefulness to frame other research work [to the question: state any insights sparked by the framework] Well it allows to position the own research in the field into the aspects covered by the framework. It may help to organize the links to related issues in the field [RP2-Q2]

Critique: Need of examples of use [to the question: explain why you would (not) use the framework in your research practice] It depends. I first want to see a successful real world test that demonstrates the usefulness of this thing [RP2-Q2]

Critique: Need for boundaries

[to the question: state any insights sparked by the framework] The definition of "orchestration" needs a boundary. Sometimes if we want to know "what is sth?" we need to be clear "what is not sth?". If it means everything then it means nothing [RP2-Q2]

[to the question: explain why you would (not) use the framework in your research practice] Not as it stands - as it isn't sufficiently precise to differentiate orchestration from related practices and theories [RP2Q2]

Critique: Usage by researchers vs. teachers [to the question: explain why you would (not) use the framework in your research practice] I am still searching for a similar kind of description, but would do it differently myself. I think I'd also separate out a "teacher oriented" view (simplified) vs an expert view (more complex, and more technical precision) [RP2-Q2]

The framework provides some insights $\quad$ Participants agreed mildly about the framework's ability to provide new insights (avg $=4.17$, std $=1.27$ in a 1-6 Likert scale) [RP2-Q2]

The framework's concepts are not new [to the question: state any insights sparked by the framework] Gives a nice synthetic presentation ... but of things I already knew about [...] [RP2-Q2]

[to the question: state any insights sparked by the framework] [...] the contents of the framework are everyday issues for me. What is new for me is stopping to really conceptualize orchestration per se. So the ideas are not especially new, but the packaging is [RP2-Q2]

[to the question: state any insights sparked by the framework] basi-

Confirmation of own views on orchestration cally, that someone else is thinking about this a whole lot like me, and that we both see the same basic patterns in the literature. Was reassuring $[\ldots]$ I'm going to try not to remember it too much [...] It seemed very sensible to me [RP2-Q2]

[to the question: state any insights sparked by the framework] It didn't provide insights so much as confirmation for me that this is a very broad term and encompasses a lot of research topics [RP2-Q2] [to the question: state any insights sparked by the framework] Once again confirms that the study of informal orchestration (or "selforchestration") is still a largely untouched topic [RP2-Q2]

Participants were not sure about the usefulness for practitioners Teachers assessed the usefulness of the framework for teachers as average $($ avg $=3.78$, std $=1.24$ in the 1-6 Likert scale) $[\mathrm{RP} 2-\mathrm{Q} 2]$

The framework is considered too conceptual [to the question: explain why you see the framework as (not) useful for practitioners] It's quite conceptual [RP2-Q2]

or complex for teachers

[to the question: explain why you see the framework as (not) useful for practitioners] May be still too complex, but has some use as is. [RP2-Q2]

Critique: Teachers need for normative guidelines

[to the question: explain why you see the framework as (not) useful for practitioners] As a basic set of things to consider, yes. But for usefulness to teaching practitioners it would require more extensive elaboration, particularly some guidelines and rules of thumb when considering the aspects in planning and executing educational activities. [RP2-Q2] 
Table 3.8 (continued from previous page)

\begin{tabular}{ll}
\hline Findings & Selected supporting evidence \\
\hline [to the question: explain why you see the framework as (not) useful \\
for practitioners] The current discussion is useful. But it is at the \\
theoretical level. The next step could be at more practical level - \\
pedagogy or strategies when orchestrating learning [RP2-Q2] \\
[to the question: explain why you see the framework as (not) useful \\
for practitioners] As a practitioner, the framework only gives me an \\
idea of the whole process of designing and enacting a TEL scenario \\
[... Thus, the model is a descriptive one, not a prescriptive one, which \\
could give me ideas on how to use the model in practice and deal with \\
the problems I may have concerning the different circles [RP2-Q2]
\end{tabular}

Finally, and although it was not a main issue in this study, our exploration of the usefulness of the framework for practitioners (which had yielded only average quantitative scores, see Table 3.8 and Figure 3.16 produced qualitative responses that were relatively homogeneous. While some experts saw the framework as useful for practitioners, as a checklist of things to consider (especially for non-teacher practitioners who e.g. design learning for the classroom), a relatively large number of experts saw the framework as too theoretical or too complex for teachers, adding that it did not give concrete advice or guidelines about how to solve "orchestration problems" (i.e. the normative value of the framework was low), which seemed to be a crucial goal of teacheroriented orchestration support. The descriptive (rather than prescriptive) value of the framework was already apparent to us from the outset, but it was not highlighted clearly in the materials of the study.

Other (emergent) general ideas (T4) The experts' panel also highlighted a number of hotly debated issues in the field of orchestration. Even if the ' $5+3$ Aspects' framework does not provide an answer to these issues, it did provide a target against which the researchers could voice these concerns. This also highlighted that, given the multiple disciplines and perspectives from which the field's researchers see the problem, these debates are bound to occur and a general consensus about how to go about them might not be reached.

One of these issues raised (see Table 3.9) is the role of the different actors (and especially, teachers) in the orchestration of a TEL scenario. The framework was presented in a way that led readers to think that teachers were the main actors, and that they were the ones that performed most of the framework's processes (design, adapt, manage, assess...). This bias was undoubtedly caused by the approach that the author's research group has taken in most of its recent research work (i.e. a "teachers as designers" approach). As some expert responses pointed out, this may not be the case, and will largely depend on the research and educational context (e.g. is this supported by local teacher culture and skills?). 


\section{Did reading the framework provide any insights about your current research related to orchestration?}

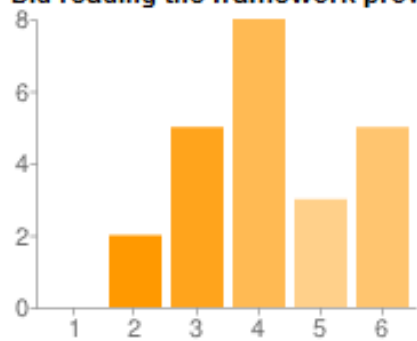

Not at allYes, it provided many insights

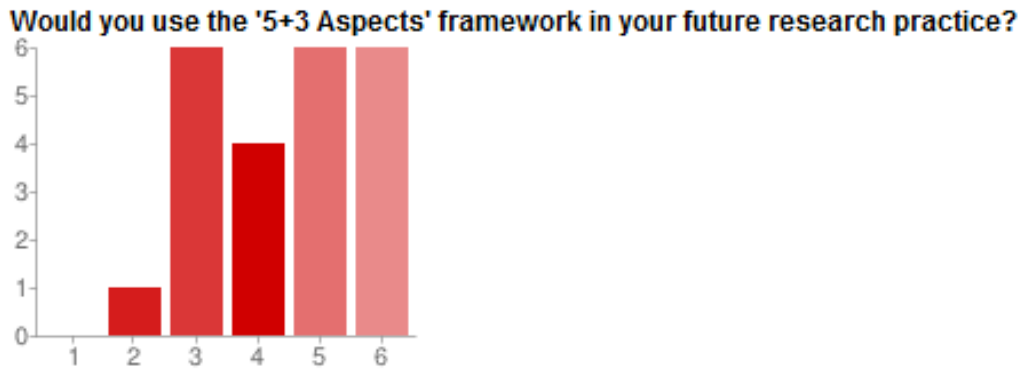

Not a chanceYes, I will surely use it in future orchestration-related research

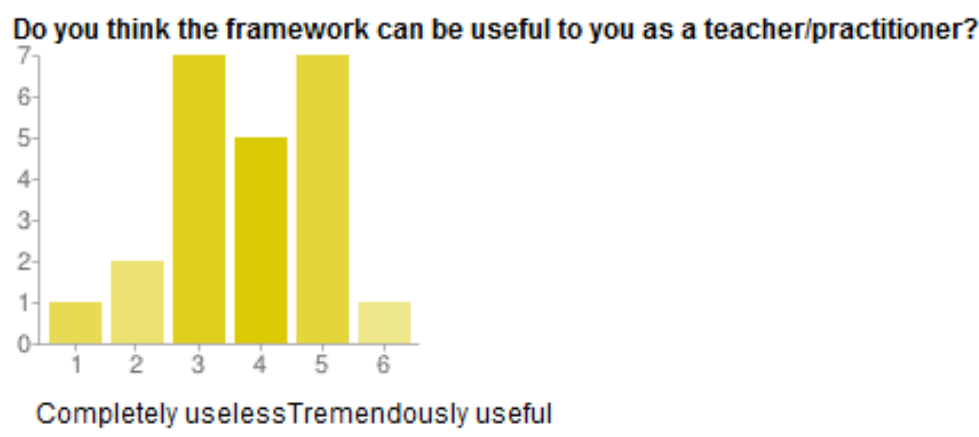

Figure 3.16: Graphic representation of the participants' quantitative evaluation of the usefulness of the ' $5+3$ ' framework in the RP2 study 
Table 3.9: Selected findings and evidence from the RP2 panel, concerning other general ideas emergent during the study (T4)

Findings
Debated issues: Role of the actors

Selected supporting evidence

[to the question: explain why you think the framework is (not) logical]

[...] The only thing I see is: there is an overemphasis on the teacher, thus it excludes informal learning (and other forms with social instruction). This is not required. [...] [RP2-Q2]

[to the question: explain why you think the framework's concepts are (not) clear] Seems to cover almost all aspects of planning and enacting a TEL scenario, except for the actual creation part that stands at the very beginning. Usually, it is not a teacher who develops a TEL scenario, but rather a programmer or a curriculum designer who does so. Only then, this scenario is taken up by a practitioner and adapted to her current needs [RP2-Q2]

[to the question: explain why you think the framework is (not) useful for teachers] My experience is scaling up TEL is that what teachers want is very nicely designed materials that are easy to use and work. I find very few teachers want to and are good at designing, adapting, aligning. Overall, I am just wary that the framework itself isn't going to help teachers much - and that it is going to suggest MORE COMPLEXITY to designers of curriculum materials, which will make the materials harder for teachers to use [RP2-Q2]

[to the question: explain why you think the framework's concepts are (not) clear] The reviews seems to be based on the work that use the term orchestration rather than the common meaning of orchestration. This approach may bring 2 issues 1 ) researchers mean different things when using orchestration because their different perspectives and contexts. Some researchers use the term but what they really mean could be different from what the mainstream researchers mean by orchestration, 2) some researchers use other terms (e.g., coordination, organization) but the meaning of the terms is similar to what the mainstream researchers mean by orchestration. This type of work is excluded in this review [RP2-Q2]

[to the question: explain why you think the framework is (not) relevant for TEL/CSCL] I doubt that you will get - nor will you want to get the other orchestrational researchers like myself, Dillenbourg, Fisher, Kollar, etc to adopt this framework. "Adopting" such a framework imposes structure that we will most likely want to avoid, given the nascent aspect of this domain. [...] Multiplexed meanings and applications aren't a bad thing. I get to talk about it as being "whatever I am treating it as" [...] But it will help many in the field as an overview [RP2-Q2]

[to the question: explain why you think the framework is (not) useful for practitioners] I think we (in our specialty of ${ }^{*} \mathrm{C}^{*} \mathrm{SCL}$ researchers) should address the potential functions of computational technologies in design and run-time usage around TEL scenarios more explicitly. [RP2-Q2]

[to the question: explain why you think the framework is (not) useful for practitioners] Designing and managing groupwork in/for the classroom has been an eternal issue of pedagogy [...] It cannot be our goal to "compete" on this level, we need to be more specific in the technology aspects [RP2-Q2]

It is also worth noting that a few of the experts posed quite valid critiques and opinions against the idea of the framework and how the study was performed. This suggests that further iterations of the study might be needed, and that, even then, there will be TEL and CSCL researchers that will consider it useless for their purposes (see Table 3.9). 


\subsubsection{Partial conclusions of the study}

Overall, this expert researcher panel garnered positive but highly heterogeneous feedback on the framework. Quite a few of the experts considered the framework relevant for the fields of TEL and CSCL, as one of the participants put it, "to increase the impact of TEL on educational systems" [RP2-Q2]. As we have seen, many experts agreed that it provided "an over-arching framework within which researchers could locate the focus of their work" [RP2-Q2], which could be used by $\mathrm{PhD}$ students or novel researchers first approaching the field of orchestration, "to define a general issue/perspective". Experts, however, considered it descriptive, "too general to guide effective work" [RP2-Q2], although, as one of the participants put it, "this does not mean one cannot define precise research questions from the framework, just that it requires an additional step" [RP2-Q2]. We can also summarize our partial conclusions around the different topics of interest of our evaluation:

T1 (participants' profile) As it happened in the RP1 study, in this panel it was found that the expert researchers' prior conceptions of orchestration of TEL/CSCL were coherent with the literature review presented in this chapter [RP2-T1-PC1], even if they were conceptually richer than in the RP1 study. Also, as it happened in the previous study, it was found that some of these researchers also shared our observation of the orchestration concept becoming something of a "buzzword" with indistinct boundaries [RP2-T1-PC2]. In general, many researchers saw orchestration as a relevant unsolved issue in TEL/CSCL research [RP2-T1-PC3], although others questioned its usefulness, given that it seemed to be a "fuzzy umbrella term" to hide underlying layers of complexity in TEL/CSCL practice [RP2-T1-PC4].

T2 (framework's completeness) As it happened in the previous study, the framework was generally considered logical, clear and comprehensive [RP2-T2-PC1], although responses were less enthusiastic and more heterogeneous than in the previous study. Indeed, some of the experts found that the framework could be too comprehensive, highlighting the need for orchestration to have a boundary [RP2-T2-PC2]. Regarding the missing elements, technology itself was one of the most commonly cited [RP2-T2-PC3] (as it happened also in RP1). Finally, it was clear from the experts' responses that, while definition and representations had a certain value, they needed further work to be really synthetic and useful complements to the framework [RP2-T2-PC4].

T3 (framework's usefulness) As it happened in the previous study, the framework was considered moderately useful for research practice [RP2-T3-PC1] (rather, indicating differences in opinion about its value for this purpose). The main usefulness of the framework was the integrative view that it provided of TEL/CSCL practice in authentic settings [RP1T3-PC2]. The framework's concepts, however, were not considered new nor revolutionary, but experts agreed on its potential pedagogical value [RP2-T3-PC3] (thus confirming the hints we found in study RP2). Finally, experts were not entirely sure about the usefulness of the framework for teachers, and they noted that it needs to provide more normative value in order to be used by teachers/practitioners [RP2-T3-PC4].

T4 (other general emergent ideas) The study also highlighted the lack of agreement about the respective roles of the different actors (e.g. teachers) in orchestration's aspects, a 


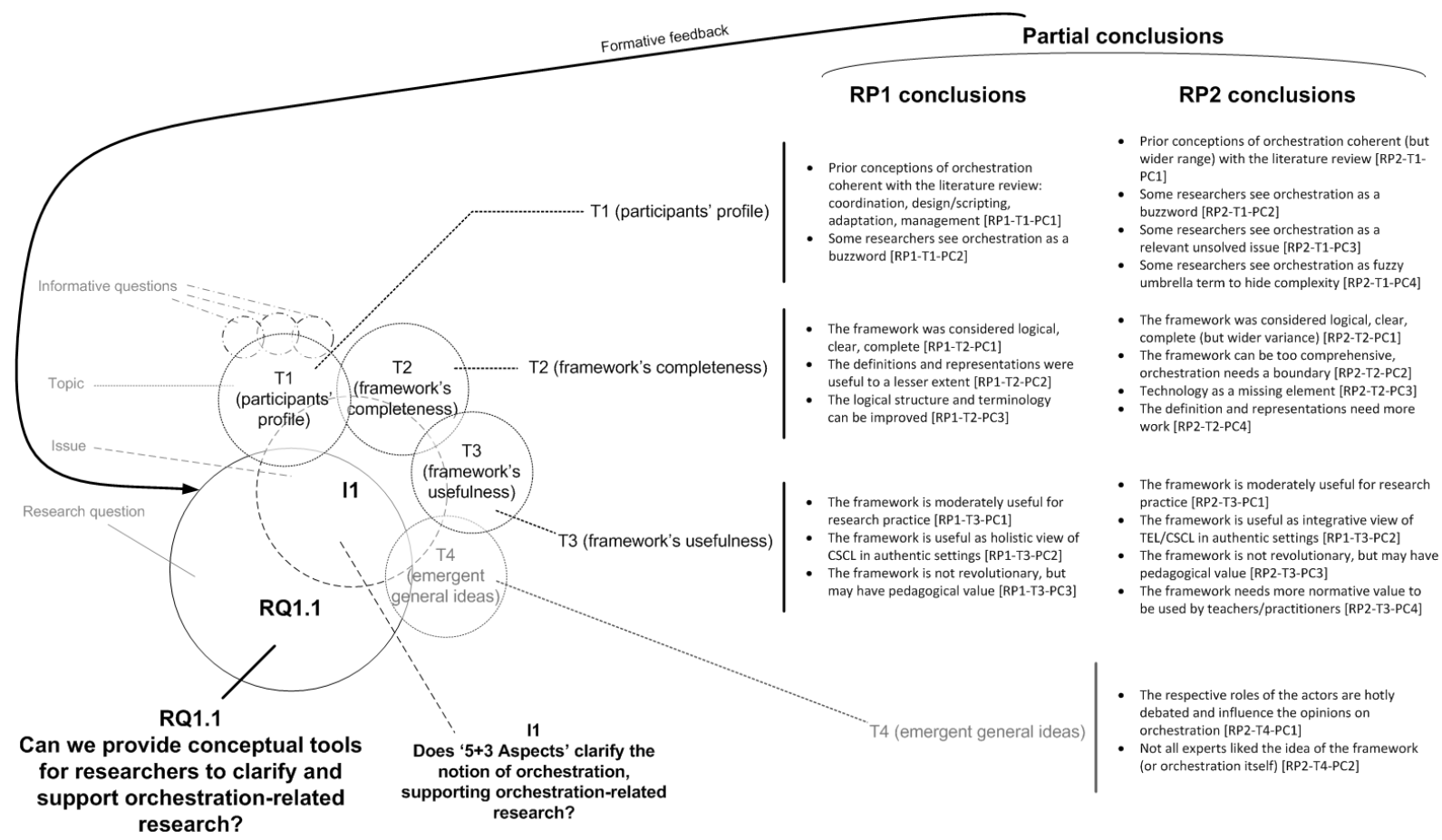

Figure 3.17: Graphic representation of the partial conclusions of the evaluations on the ' $5+3$

Aspects' conceptual framework

fact that influences the opinions of participants on orchestration and on the presented framework [RP2-T4-PC1]. Indeed, it was found that not all the experts liked the idea of the framework (or the idea of orchestration itself) [RP2-T4-PC2].

\subsubsection{Wrapping up the evaluation of ' $5+3$ Aspects'}

If we consider together the analyses from the two researcher panels (see Figure 3.17), and despite the heterogeneity of many of the responses (which is to be expected in this multidisciplinar field), we can reach a number of general conclusion of the evaluations performed so far. These conclusions help us illuminate each of the selected topics around our main evaluative tension (I1, "Does ' $5+3$ Aspects' clarify the notion of orchestration, supporting orchestrationrelated research?"), which in turn provide first answers on our main research question (RQ1.1, "Can we provide conceptual tools for researchers to clarify and support orchestration-related research?"), and suggest formative feedback for further iterations on such research (see Figure 3.18 .

- Regarding the topic of participants' prior knowledge and profile (T1), we can conclude that the researchers' opinions confirmed the general concepts of the literature review that made up the ' $5+3$ Aspects framework', and highlight orchestration as a relevant, unsolved research problem in the field of TEL/CSCL. There is however, a considerable danger of it becoming a "buzzword" if not clarified by the research community. [Supported 
by partial conclusions RP1-T1-PC1, RP2-T1-PC1, RP2-T1-PC3, RP1-T1-PC2, RP2-T1PC2]

- On the topic of the framework's completeness and coherence (T2), our main conclusion is that the framework was generally considered logical, clear and complete by participants (in varying degrees), although there exists a clear danger of making it too encompassing (thus losing part of its clarification potential). It was noteworthy that a portion of participants thought that the different supporting elements of the framework (definition, representations, terminology) should be refined, and especially that the role of technology should be included somehow in it. [Supported by partial conclusions RP1-T2-PC1, RP2T2-PC1, RP2-T2-PC2, RP1-T2-PC2, RP1-T2-PC3, RP2-T2-PC3, RP2-T2-PC4]

- The question of the usefulness of ' $5+3$ Aspects' for research practice (T3), a moderate number of our participants considered it useful for research practice, especially regarding the holistic view of TEL/CSCL practice in authentic settings that it provides. Although the framework's concepts are not considered novel, they provide a nice overview of the field with pedagogical value for younger researchers. [Supported by partial conclusions RP1-T3-PC1, RP2-T3-PC1, RP1-T3-PC2, RP2-T3-PC2, RP1-T3-PC3, RP2-T3-PC3]

- Finally, it also emerged the general idea (T4) that more thought should be given to the relative relevance and roles of the different actors, not in a normative way, but as an important conditioning factor in the discussions on orchestration. [Supported by partial conclusion RP2-T4-PC1]

Naturally, these conclusions have to be seen in the context of the situations were our data was gathered (e.g. the heavy participant biases in study RP1, and the limited richness of the data gathered in both studies), and thus statistical generalizability from this data is not possible (nor it was our goal). Nevertheless, given the breadth of the participants' origins and backgrounds, it is reasonable to view these conclusions as a portrayal of TEL and CSCL communities' "collective wisdom" [Moo05]. Further evaluations would be needed in order to provide more assertive conclusions, including studies with a higher number of researchers, both younger and more expert (e.g. large scale questionnaires, international research workshops where the framework is used), and in more authentic research settings (e.g. by using - and tracking the use of - the framework in concrete, real research efforts related to orchestration).

Also, these conclusions prompt us to develop and refine new research instruments inspired by the framework. Indeed, one such instrument has already been generated, and is described in the following section, along with preliminary evidences of usefulness.

\subsection{A research instrument derived from ' $5+3$ Aspects': An or- chestration interview/reflection guide}

Once the aforementioned " $5+3$ Aspects" framework for orchestration was in place, it was clear for the author that such framework could be used to structure and analyze certain elements of this dissertation. One example of this is the classification of the "orchestration atomic patterns catalogue" (OAPC, see Chapter 4 and Appendix B). Another is the analysis 


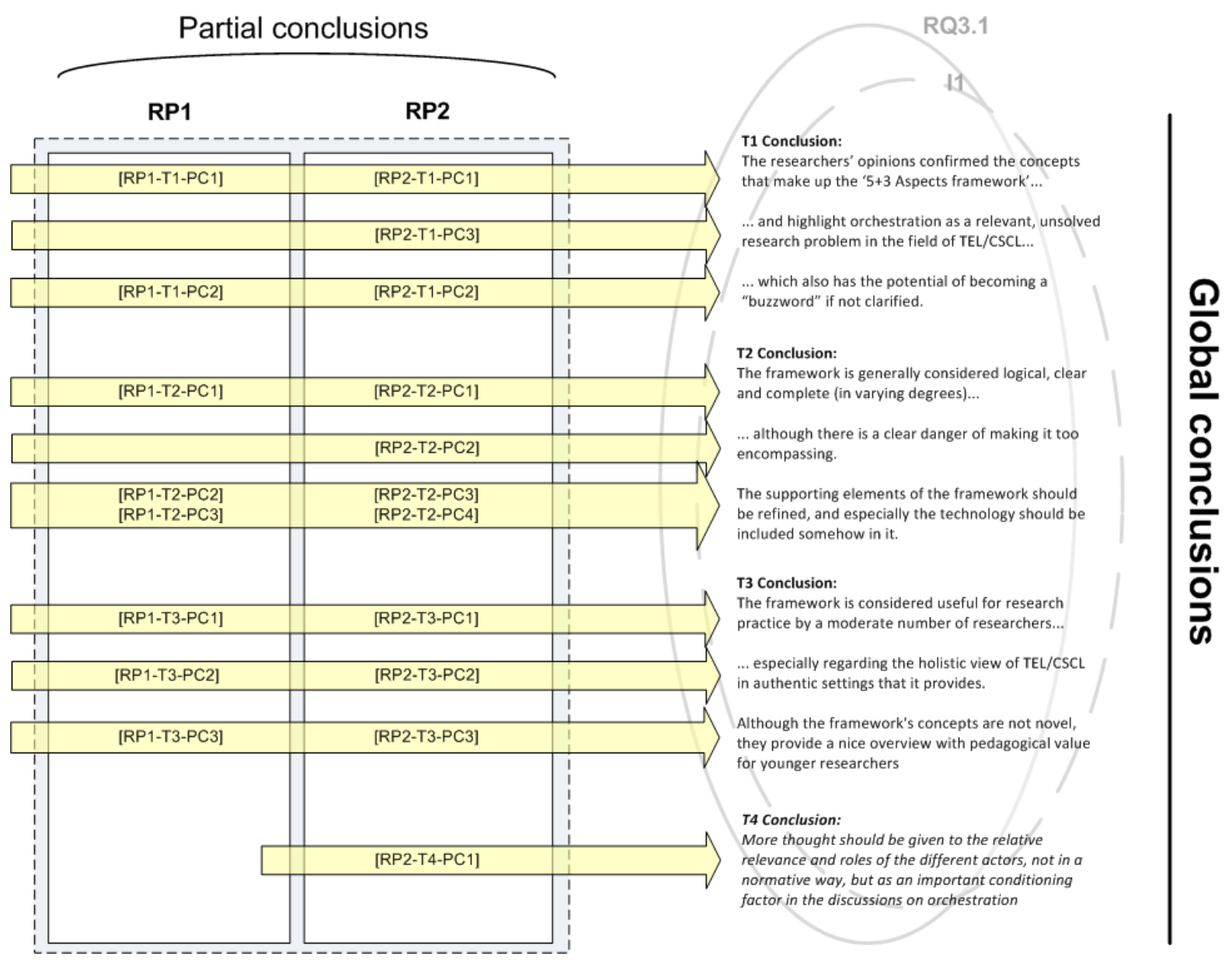

Figure 3.18: Graphic representation of the global conclusions of the evaluations on the ' $5+3$ Aspects' conceptual framework 
of the GLUE!-PS technological infrastructure (Section 5.3.1). However, even before that use, we detected the need of eliciting orchestration (recurrent) practices (see Section 4.3) in a new, previously unknown educational context. This kind of information, which we needed when first approaching the context of blended higher education courses that used Distributed Learning Environments (DLEs), was to be elicited by interviewing several teachers that regularly enacted CSCL activities in this kind of context (as a supplement to elicitation by direct observation in the classroom).

Thus, in order to help us, as researchers, in characterizing the practice of orchestration in a concrete educational setting, we elaborated an interview guide, with questions that addressed separately the different orchestration aspects (Section 3.2), asking the subjects (e.g. a teacher) about how such aspects were represented or performed in his/her concrete educational context (e.g. a course). However, this guide could also be used by teachers themselves (or by teacher-researchers), as a reflection guide to think about their orchestration practice, and propose (technological or conceptual) innovations that might improve such orchestration in their own setting.

\subsubsection{Structure of the guide}

The instrument had a simple structure, fit for a potential interviewer to use during an semistructured interview (normally with a teacher) of about 60 minutes. After a short summary of the instrument and its purpose, there was a preamble with fields to be filled in by the researcher (interviewee name, interviewer name, date, place of the interview and educational context to which the interview would be referred), and a number of general guidelines about the use of the instrument, regarding the priorization of questions, time management strategies during the interview, and so on.

After these introductory elements, a questionnaire followed, interspersed with indications of potential actions that could be taken during the interview (e.g. to log into the teacher's computer and show the interviewer how the design process took place, or the learning platform used in the course). The questionnaire has 42 questions, divided in sections for each of the aspects (9 questions for Design, 10 for Management, 8 for Adaptation, 3 for Awareness, 1 for Role of actors, 4 for Pragmatism, 4 for Synergy and 3 for Models). The complete guide is available in the appendixes of this dissertation (Appendix A), although below we can see two excerpts taken from the "Adaptation" and "Pragmatism" sections of the guide:

From Adaptation:

- Did the enactment of the learning activities occur exactly as it was designed? Which changes were necessary and why?

- (Here, direct the interviewee to the episodes/parts of flexibility mentioned)

- Did the learning design contemplate explicitly the possibility of changes during enactment? How was this expressed? Did the chance of change modify the choice or configuration of the technological support?

- Do the situations that provoked the changes occur frequently? How probable are they? 
- Were the changes made using the technological support, or did the change consist on dismissing the technological support in favor of e.g. pen and paper? Were these changes facilitated in any way by the chosen technologies' functionalities (i.e. do these technologies allow for changes with ease)? How does the teacher access those changes in the technology? Are there recurrent elements in these technological changes?

- $[\ldots]$

From Pragmatism:

- Were there contextual restrictions of the concrete educational setting that prompted for changes in the original activity design? And in its instantiation and deployment?

- Was the technology adapted to the learning activities to perform, or was it the other way around? Which form did those adaptations take? Are there any recurrent elements in that adaptation?

- Would the teacher have liked to enact the design or its technological implementation in a different way? What restrictions prompted him/her no to do it that way? Are there any recurrent restrictions?

- $[\ldots]$

\subsubsection{Preliminary evidence of usefulness}

As it happened with the conceptual framework itself, however useful this questionnaire had been for the author, a more objective evaluation of its usefulness for the TEL/CSCL researcher interested in TEL was in order. Given that the framework itself still had not been evaluated, we proposed a consensus-based evaluation very similar to the one for the " $5+3$ Aspects" framework. In fact, this instrument evaluation was adjoined to the framework evaluation, as an optional part of the same studies (Section 3.3). Thus, in studies RP1 and RP2, participants had the option to perform 2 further steps, after completing the framework evaluation questionnaire:

1. Participants were asked to think about a real, concrete TEL/CSCL context in which they were developing an orchestration-related research effort (or any concrete TEL/CSCL context that they knew in depth). Then, they would read the reflection guide (see Appendix A). If inclined to do so, participants were encouraged to write down (in a paper or in the document itself) any problems, solutions and other ideas that emerged as they answered the questions. The document could be then sent to the author.

2. Participants were to answer an instrument evaluation questionnaire ([RP1-Q3] for the first study among related researchers, [RP2-Q3] for the international experts' one) to assess the value of the reflection guide.

The presentation of this part as optional naturally led to a lower response rate among participants: only 8 out of the 22 participants that completed the framework part $(36,4 \%)$ answered to the instrument part of the study in RP1, and only 3 out of $24(17,4 \%)$ answered 
the optional questionnaire in RP2. Moreover, only 4 participants $(18,2 \%)$ submitted the filled-in questionnaire during RP1, while $3(17,4 \%)$ submitted it during RP2. This low response rate is the reason why we do not present the data in this section as a formal evaluation process, but rather as preliminary evidence of usefulness, a sort of "pilot study" that can be used to guide further iterations and stronger evaluations.

Whereas the results of the evaluations in RP1 and RP2 were quite similar with regard to the completeness and usefulness of the " $5+3$ Aspects" framework (see Section 3.3), this was not the case of the questionnaires about the reflection guide. Analyzing the (admittedly scarce) data provided by participants from a quantitative perspective gives us very marked differences between the responses of younger researchers from related research groups (RP1) and expert researchers (RP2), the latter being much more critical with the framework. For example, in RP1 researchers rated the guide's usefulness with a 5, on average (in a 1-6 Likert scale), while the 3 experts all rated it with a 4 . Similar score differences were found in the matter of whether the instrument enhanced their understanding of the " $5+3$ " framework (averages of 5 versus 3.67, in the same 1-6 scale), of orchestration in TEL/CSCL (5.38 vs 3.67) and, especially on the perspectives of future use for their own research (5.25 vs 2.67).

The qualitative data provided by participants of both studies cast additional light into the usefulness and defects of the reflection guide (see Table 3.10). In general, younger researchers found it useful as a guide for detecting orchestration problems in a TEL setting, although they highlighted that the instrument provided little help in proposing solutions (other than making you aware of the problems). They also noted that further practical examples could be useful in some parts of the questionnaire. Expert researchers, on the other hand, noted similar traits regarding the lack of solution proposal support, questioning what was the real goal of the instrument. Also, they provided proposals for re-structuring or otherwise improving the questionnaire.

Table 3.10: Preliminary findings and supporting evidence for the usefulness of the questionnaire/guide derived from the " $5+3$ Aspects" framework

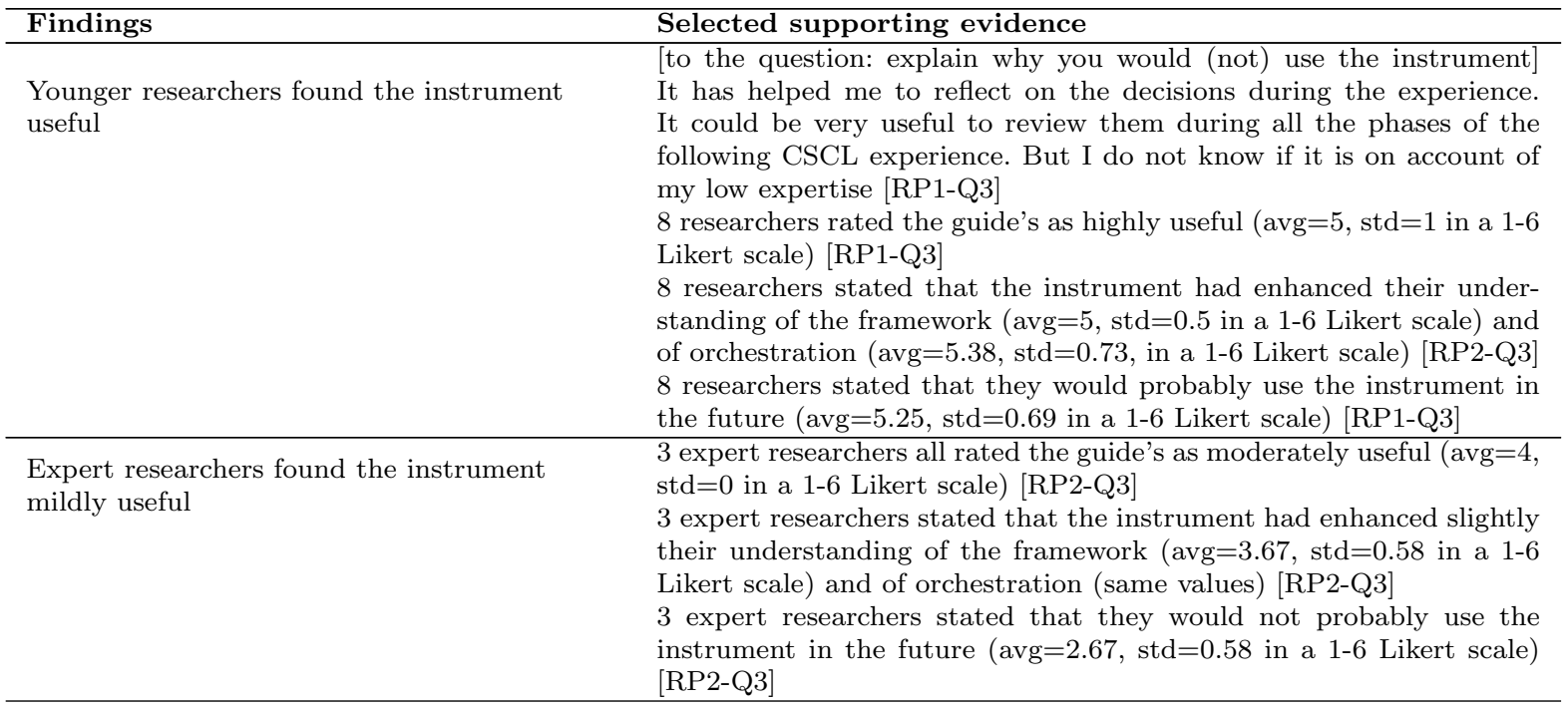


Table 3.10 (continued from previous page)

Findings
Usefulness as list of issues to consider
Usefulness for implementation of TEL/CSCL
in authentic settings
Selected supporting evidence

[to the question: explain why you would (not) use the instrument] $[\ldots]$ the instrument helped me to reflect on my own practice, showing new aspects I should consider while thinking in the best ways of orchestrating [RP1-Q3]

[to the question: explain your rating of the instrument's usefulness] USE [...] I think this provides some good starting points [...] I think it provides a lot of data - probably too broad [...] I think that connection between elements needs to be stronger [RP2-Q3]

[to the question: describe the insights that emerged from using the instrument] There were a few specifics that I don't always consider, and these were nice to see, e.g.: regulation of learning activities; reasons for specific combinations of resources used [RP2-Q3]

in authentic settings

[to the question: explain why you would (not) use the instrument] Provides clues to the development of research in the field of orchestration TEL/CSCL and the implementation of real-world scenarios [RP1-Q3]

Usefulness to clarify the elements of the framework

[to the question: describe the insights that emerged from using the instrument] It has helped me to clarify the scope of each aspect by means of thinking on my own experience [RP1-Q3]

Critique: The instrument was not specially useful for solution proposal

[to the question: explain why you would (not) use the instrument] I think it helps to find problems, but the solutions are left to the reader. [...] I don't distinguish scaffolding in the solution proposal [RP1-Q3] [to the question: explain your rating of the instrument's usefulness] [...] I would inventory problems and then probe each for orchestration issues [...] if this were to be used to propose something new, I would restructure the whole thing to first focus on areas that went well; second focus on areas that went poorly; thirdly to generate hypotheses about why things went poorly; fourthly to generate ideas about how to test those hypotheses; and lastly to propose new solutions [RP2-Q3]

Critique: Need for further examples

[to the question: explain why you would (not) use the instrument] [...] There are other issues in the framework that I would need something (a path with practical examples) to put it in practice in the context of my course (CSCL, teacher education, high ICT presence, high number of students...) [RP1-Q3]

Critique: Unclear purpose and target audience [to the question: explain why you would (not) use the instrument] I think it could be good for researchers to consider. It does seem to project a certain world view, but there's lots of interesting questions in there. I'm not really sure what its after [RP2-Q3]

\begin{tabular}{ll}
$\begin{array}{l}\text { The instrument provided insights about or- } \\
\text { chestration }\end{array}$ & $\begin{array}{l}11 \text { out of } 11 \text { respondents (100\%) stated that using the instrument had } \\
\text { provided some kind of insight [RP1-Q3] [RP2-Q3] }\end{array}$ \\
\hline Insights: Reflection about teaching practice & [to the question: describe the insights that emerged from using the in- \\
strument] It helped me understand the aspects involved in my "infor- \\
mal" orchestration. Actually, I was not aware on how I did orchestrate \\
my course [RP1-Q3] \\
[to the question: describe the insights that emerged from using the \\
instrument] For example, I had not thought of co-orchestration, that \\
is, how to guide students in these CSCL contexts so that they acquire \\
competences that enable them to solve problems and they are not so \\
dependent on the teacher. Also [...] about the importance of design \\
in enactment, and the need to take into account the affordances of \\
technology in design time [...] [RP1-Q3]
\end{tabular}

Oddly enough, despite these differences in opinion, all 11 participants who did this optional part asserted that the guide had provided some insight about orchestration or their research in the educational setting they used for the exercise. The qualitative answers were quite varied, going from reflections about own teaching practice to meta-reflections about the author's way of thinking of orchestration (see Table 3.10). 
As we can see, the evidence gathered is highly heterogeneous, but generally it suggests that further iterations of the instrument are needed before it can be considered a useful, reliable research instrument. Some partial conclusions from this data include hints that the reflection guide might provide greater value for younger rather than for expert researchers [Inst-PC1], or that its main usefulness is as a list of elements to consider when implementing TEL/CSCL [Inst-PC2]. In general, participants responses showed first evidences of potential as a source of insights/reflection, especially about TEL teaching practice in authentic settings [Inst-PC3]. But, in general, further development seems to be needed, especially regarding the solution proposal support it might provide [Inst-PC4].

Further steps in this direction could include re-thinking and stating more clearly the goal of the instrument (is it to characterize orchestration in a concrete setting? is it to detect problems? is it to scaffold the process of generating new solutions?). Taking this goal into account, the instrument can be improved by re-structuring it using some of the participants' suggestions. In fact, it should be considered to split this instrument into several different ones, depending on the particular goal they are aimed at.

The questions provided in this guide in its current form could also be seen as probes about smaller-scale orchestration aspects, and thus each question could in fact be the spark of a new orchestration-oriented research line. This questioning guide could thus be seen as a compendium, a guide of orchestration-related research questions which overlap with many existing TEL/CSCL research fields.

\subsection{Conclusions: Towards a new framework?}

In this chapter, we have provided a conceptual framework for orchestration, " $5+3$ Aspects", based on a review of the concept of "orchestrating learning", especially focused in the area of Technology-Enhanced Learning and Computer-Supported Collaborative Learning. Such review showed the variety of research contexts in which the term has been used. The proposed framework is composed of " $5+3$ " thematic groups: Design, Management, Adaptation and Awareness and Actors are relevant to the question "What is orchestrating learning?", while Theory, Pragmatism and Alignment are more related to the question "How can orchestration be achieved?". A first evaluation of the conceptual framework, by two panels of TEL/CSCL researchers has been presented, as well as a first research instrument (a reflection guide) based on the framework.

The results of the evaluations show that participant TEL/CSCL researchers, both at a local and at an international level, found it useful as an overview of the field of orchestration. It is important to note that " $5+3$ Aspects" is a rather abstract and flexible framework, which can be useful for descriptive purposes, e.g. to structure or focus the gathering of research data in a concrete setting, or as a checklist of aspects that have to be taken into account for TEL/CSCL in authentic settings, since it seems to describe a lot of what teachers (and other actors) already do in TEL scenarios. On the other hand, the framework and the derived instrument that we have proposed have little normative value, in the sense that they do not prescribe how orchestration should be achieved, or how orchestration problems should be solved (although directing the attention to existing problems can be considered the first step towards a solution). This is 
so by the very nature of the process involved, and the goals of our proposal: too many pedagogical aspects, in which the author is not an expert, influence such prescriptions; moreover, we hypothesize that the chance of providing adequate "orchestration advice" without carefully considering the concrete (authentic) setting of each case, is slim at best.

However, the evaluation also highlighted that the goal of the conceptual framework and its target audience have to be clearly marked, to avoid confusions when presenting it to multidisciplinar audiences such as TEL/CSCL are, with people coming from very different perspectives. In our own opinion, which is largely shared by the researchers that participated in the evaluation, the " $5+3$ " framework is better suited for researchers or other non-teacher practitioners, who want to understand or characterize orchestration in an educational setting, and want to have a first, holistic view of how a TEL innovation might impact such (authentic) setting. This might be especially useful for TEL and CSCL researchers, who often propose and develop innovations centered in just one educational aspect - having such holistic view in mind can be useful in order to consider how their innovations are impacting in other aspects of this ecosystem of elements, or when trying to evaluate the likeliness of adoption in a bigger scale.

Having clarified this aspect, we should note that the evaluation has prompted us to reflect on certain aspects of the framework and the instruments associated with it (definitions, graphical representations, reflection guide), in order to provide a third synthesis attempt. Although this kind of proposal will be done after the end of this dissertation, some of the suggested paths for modification are included here:

1. Even if some participants had the opinion that the framework largely related to teachers and teaching, after considering the feedback from different researchers we think that such assimilation would be restricting orchestration to the labor of teachers only, which is not necessarily the case. It is true that the framework as it has been proposed up to now tends to enforce a certain perspective of "teachers as the sole orchestrators", a world view that does not apply everywhere, or for every research effort related to orchestration. Thus, especially, the Actors aspect has to be re-thought, to open it up more clearly to the different research and educational flavors of orchestration: first, the variety of actors that should be considered should be broadened, to include multiple flavors of co-orchestration. Thus, not only teachers and students can be orchestrators in a certain setting, but also researchers, learning designers and other non-teacher practitioners, and even technology itself can be an actor (e.g. in the case of intelligent agents that adapt the lesson on-the-fly). This aspect also should be placed in a different category, somehow orthogonal to the other two (thus, the framework could be transformed into something like " $4+3+1$ " or, better yet, " $4 * 3 * 1$ ", which better suggests this transversality of some elements with respect to the others.

2. Also, the role of technology and other resources in the framework might be revised. Technology as an aspect was left out of the framework purposefully, since the author, as a technology developer, did not want to prescribe (and thus, restrict to) a specific role or relationship with other orchestration elements. The underlying assumption is that technology can be used in different ways within the four "what" elements (Design, Management, Adaptation, Awareness), in ways dictated by the tensions between the three "how" elements (Theory, Alignment, Pragmatism), by the different Actors involved (or it can even be an actor itself, see above). Thus, our framework tries to be technology-agnostic (does 
not dictate how technology is to be used, as long as it facilitates the whole "orchestration process"), in the same way that it tries to be pedagogy-agnostic (does not dictate which learning theories or pedagogic strategies are to be used to orchestrate). In this sense, we do not favor either "orchestrable" or "orchestrating" technologies (a distinction made by P. Tchounikine in [Tch11]).

3. The definition of orchestration, which was not very well-liked by a number of the expert researchers, could also be modified, although there is little chance (and it is doubtfully desirable) that a consensus can be agreed on it. For example, we could try a shorter definition followed by clarifications, which separate more clearly the different groups of aspects:

"Orchestration is the process of designing and managing in real-time (including awareness and adaptation mechanisms) the learning processes in a TEL authentic scenario. The responsibilities in this process are shared among a number of actors depending on the context (most often, teachers, but also students, researchers or technologies), trying to align resources pragmatically towards a maximum effect, considering their models/theories/beliefs."

This definition is hardly more elegant than the previous one, but it is (even) more open and takes into account the aforementioned modifications.

4. In the same way, the graphical representations might be modified to take these modifications into account, and to better separate the different kinds of elements in the framework. Figure 3.19 represents a possible alternative, which groups the different elements into three "planes": Actors, Activities and Background, all trying to achieve a certain Alignment of resources towards learning effects. Again, this representation is not simpler or more elegant than the previous ones, and its added value might be questionable.

All these changes could lead to a new proposal of the orchestration conceptual framework, which should be evaluated more thoroughly to assess whether it is more useful than its previous incarnations, using a wider array of techniques beyond the panels used here: case studies of researchers using it, more extensive surveys, workshops where researchers try to apply it to their own research, etc. Also, the range of instruments supporting the framework should be developed further, including examples and guidelines for researchers to use it, or its transformation for use by teachers/practitioners (instead of researchers). Finally, the didactic uses of the framework as a point of entry for novel researchers in the field is probably the 'low-hanging fruit' that could be most readily exploited. Several of the participant researchers asserted that they would use it with $\mathrm{PhD}$ students in their own research groups, and the other experts have been encouraged to do so. Immediate next steps in this direction could be to find a way of gathering data about this kind of use.

It is doubtful that we will arrive to some kind of "unified orchestration theory" agreed by all researchers, or that such a theory is desirable at all (Dillenbourg, for example, rather advocates arriving to design principles or guidelines for orchestration in [Dil11a]). However, we have tried in this chapter to come up with a way of organizing a fuzzy and confusing notion which is deemed to be highly relevant for a considerable part of the TEL and CSCL research communities. We believe that, regardless of the accuracy of the framework to model TEL approaches, at least 


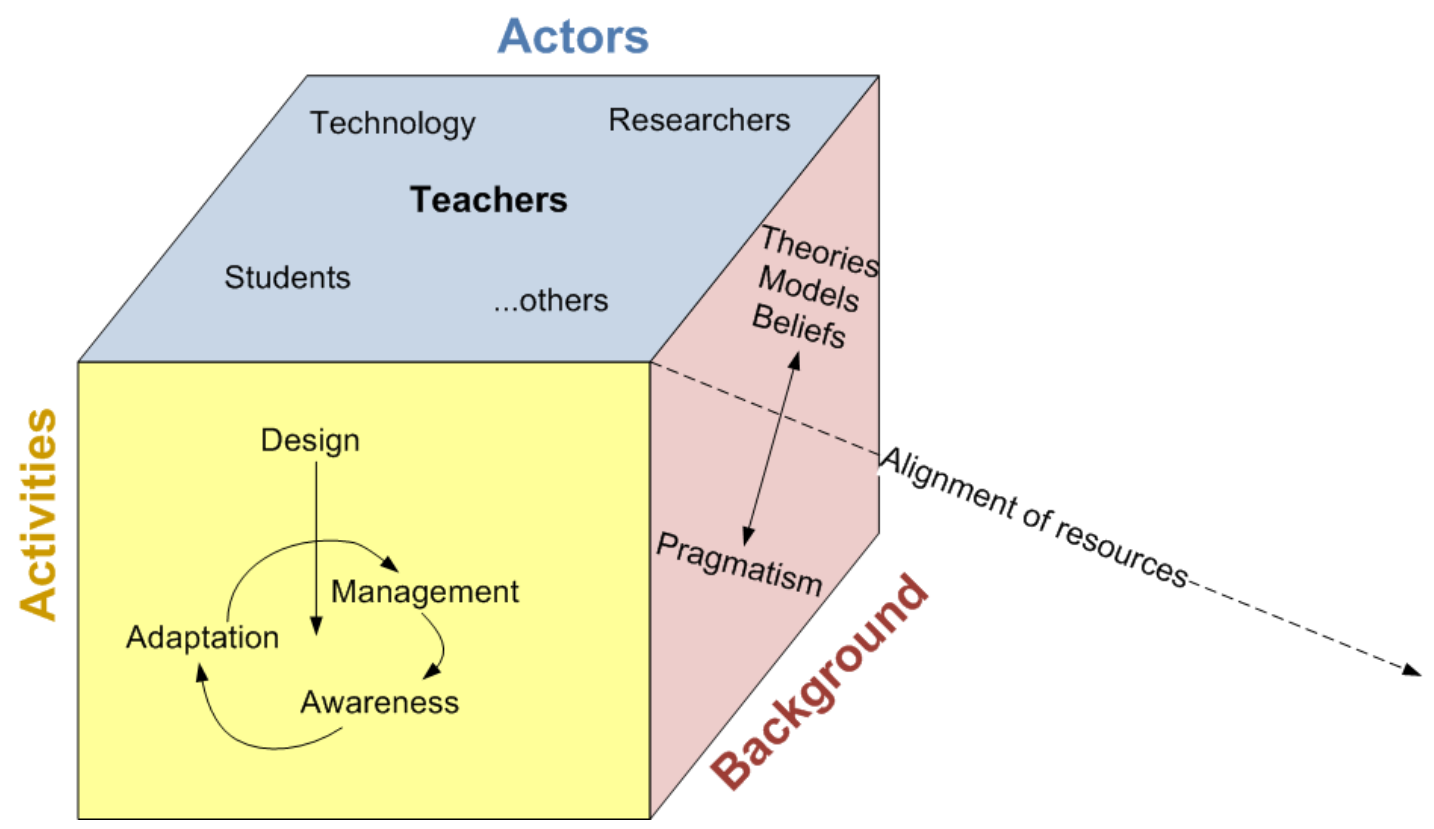

Figure 3.19: Proposal for the graphical representation of a new framework proposal that integrates some of the evaluation feedback

it will be valuable to communicate, to discuss (if nothing else) among the researchers who share a common concern about the increasing complexity of educational practice in authentic TEL settings. Even if it does not tell us how to orchestrate better, the conceptual framework presented here may help us to understand better these complexities so that the solutions we propose, however focused or minimalistic, can be more easily integrable with the rest of the classroom ecosystem and its constraints, where they will have to be used. This should lead to a greater (and more sustainable) impact of TEL research across our educational system. 


\title{
Chapter 4
}

\section{Atomic patterns as conceptual tools for orchestration}

\begin{abstract}
Summary: Design patterns (patterns, from now on) are a common way of communicating, creating and reflecting about practitioner knowledge in a number of fields, especially highly complex ones. During the course of the $\mathrm{PhD}$ work, we observed that a similar concept could be applied to the practice of orchestrating learning activities, first in primary school classrooms, then also in university-level CSCL practice involving DLEs. We propose these so-called "atomic patterns" as useful conceptual tools to orchestrate this complex ecosystem of technological tools and actors. This chapter describes how we extracted a catalogue of atomic patterns from the observation of authentic CSCL practice (first, in primary schools, and afterwards, in higher education), and then evaluated a subset of them through their iterative application in a series of professional development teacher workshops (in both educational contexts). The teachers reacted favorably to this kind of scaffolding, although interactions with several factors (teachers' beliefs and prior experience, or the workshops' own format) were hinted at by the mixed methods evaluation.
\end{abstract}

\subsection{Introduction: The role of patterns in orchestration}

Despite its thriving research community, CSCL has failed so far in influencing everyday educational practice on a large scale. The difficulties in transferring knowledge and findings from CSCL research to the classrooms in our schools can be traced back to several factors, including lack of presence in most pre-service and professional development programs. However, even with adequate training, the inherent complexity of adopting collaborative learning pedagogies (e.g. managing and subtly influencing learning processes of students doing a variety of activities in groups, individually or as a whole class) also poses a considerable challenge for practitioners. This is even more so if we consider the variety of tools (from pen and paper to laptops, interactive whiteboards or a myriad of web-based tools) that conform the current "classroom ecosystem" Luc08. TEL and CSCL researchers are increasingly appreciating the complexity of teachers' (and other actors, from students to researchers - see the previous chapter's conclusions) labor in authentic settings such as classrooms, where TEL/CSCL innovations are expected to be applied. As we saw in Section 2.3, orchestration has been recently coined as a term that encompasses this complexity.

If we look at the particular context of having to orchestrate blended CSCL activities (which involve face-to-face and online work, see Section 2.2) supported by Distributed Learning 
Environments (e.g. that include a VLE such as Moodle and external, "Web 2.0" tools, see Section 2.4.3), we can see how this complex task can prove problematic. This is especially true for educational contexts where teachers are in charge of most of the orchestration, such as universitylevel education, where teachers often enact courses designed by themselves. In this case, as we saw in Section 2.5, teachers often carry almost the totality of the orchestration work, and may experience a number of problems, including the technological complexity of implementing a learning design in such DLEs (what we called the 'deployment gap' in Section 2.5.3). Also, managing the learning activities in real-time (Section 2.5.4), or the lack of conceptual advice on how to combine and manage the resources at their disposal during the whole orchestration process (Section 2.5.2), can be problematic.

In the other common case of higher education institutions that also want to support CSCL practice in DLEs, but have different role structures in the orchestration of courses (e.g. they have specialized e-learning designers and support teams to design and deploy courses, with the courses being enacted by teachers/tutors), the design and deployment of the collaborative activities might not be such a problem (since specialized staff takes care of those issues). However, in these cases there might be a lack of advice or communication among the different teams, about how the created resources are to be used or combined in the course enactment, or what are the decision rules or actions that can be taken in the face of unexpected occurrences.

As we can see in Figure 4.1, this dissertation's global objective is to provide "technological and conceptual tools to support the orchestration". In this chapter we describe the second of these tools, that tries to tackle the latter aforementioned challenge, that is, to provide teachers (and, especially, teachers that are neither experts in CSCL or in the use of DLEs) with more conceptual advice about how to handle orchestration throughout the design and enactment of blended CSCL activities in DLEs.

In order to overcome this challenge, we propose to use a variant of the design patterns approach. As we saw in Section 2.5.2, design patterns are a way of documenting experience and knowledge about complex design problems that appear in a specific domain. More specifically, "[a] pattern describes a problem that occurs over and over again in our environment, and then describes the core of the solution to that problem, in such a way that you can use this solution a million times over, without ever doing it the same way twice" Ale77]. Design patterns (or variations of this approach) have been successfully applied before to complex practices as disparate as architecture [Ale77], software engineering Gam95] or learning design [HL06b. In this dissertation, we hypothesized that a similar approach could be taken to support non-expert teachers in the orchestration of blended CSCL activities across DLEs.

Thus, the second major contribution in this dissertation are "atomic patterns", a variation of the design patterns approach. As we will illustrate during this chapter, due to the peculiarities of orchestration practice (e.g. the fact that it involves not only design but also real-time management, awareness and adaptation), a slightly different (but complementary) flavor of design pattern was needed. These atomic patterns are heterogeneous, refer to concrete contextual features (e.g. a blackboard, a debate activity) and, very often, they are smaller-scale than typical, more abstract pedagogical patterns. Atomic patterns provide a way of aligning design, technology and teacher actions (embodied by some of these patterns) for effective orchestration. Additionally, this chapter also presents a multi-level pattern approach to the elicitation, development and use of such atomic patterns in combination with other pedagogical patterns 


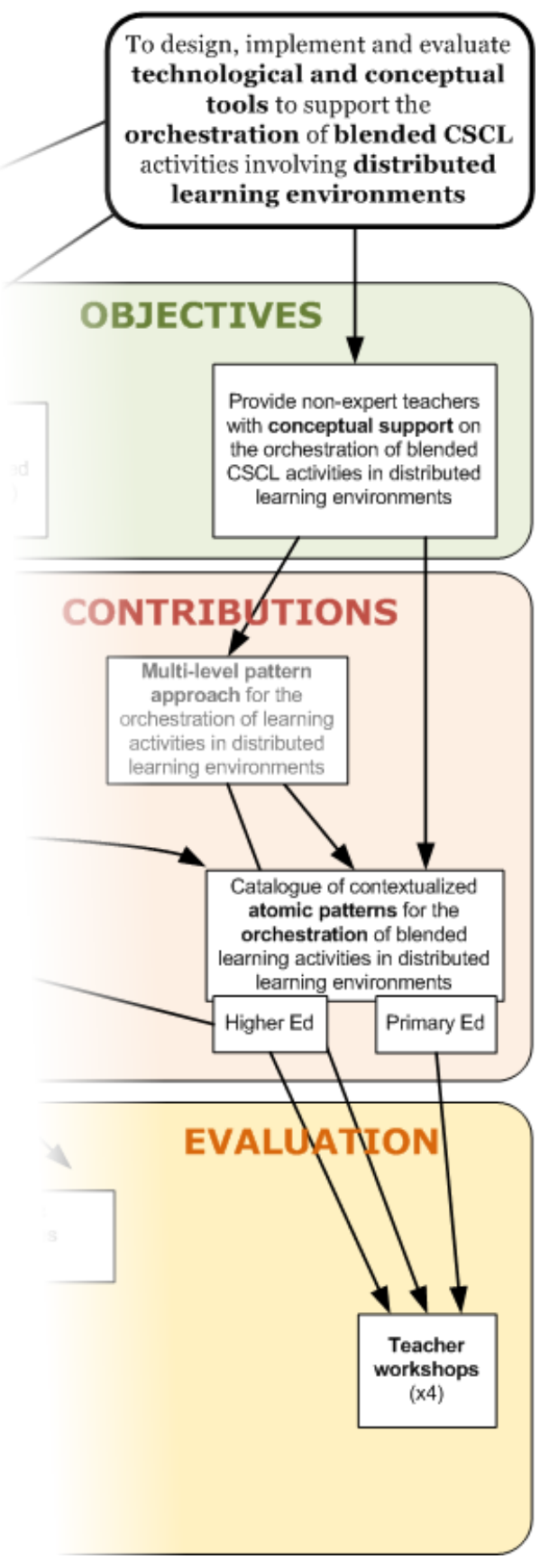

Figure 4.1: Parts of the thesis diagram concerned by chapter 4

traditionally used in the design phase of the orchestration, such as Collaborative Learning Flow Patterns (CLFPs, see HL09).

Although during this dissertation we have also explored the concept of atomic patterns as a useful analytical tool for researchers, in this chapter we will mostly refer to the role of such atomic patterns as a powerful mediating tool for teachers, and in the enhancement of professional 
development programs as well. Also, it is important to note that the work on atomic patterns was originated by joint research work undertaken in the context of primary school technologyenhanced classrooms (see Sara Villagrá's PhD thesis [VS12] and the author's own Master's thesis [Pri09]), where first evidence of their usefulness was gathered. As our research work progressively centered around DLEs and blended CSCL activities, this approach was then adapted and applied to higher education contexts.

During this chapter we will present this notion of "atomic patterns", and we will evaluate them with regard to the following research question: "Can we provide conceptual tools to support (non-expert) teachers in orchestrating learning? (sp. in blended CSCL using DLEs)" (RQ2.1).

This chapter is structured as follows: First, we can find a brief accounting of the methodology followed during the development of these contributions (Section 4.1.1). Afterwards, we present the notion of atomic patterns as conceptual tools to support non-expert teachers during orchestration, including comparisons and joint analysis work with related researchers from SRI International. A description of the multi-level pattern approach to the elicitation, development and use of atomic patterns can be found in Section 4.3. Then, we present other potential applications of atomic patterns (Section 4.4), such as our exploration of their usefulness in order to graphically represent orchestration, or hints about their use by technology designers. We close the chapter with an account of the iterative evaluation of atomic patterns through teacher workshops (Section 4.5), and concluding remarks about these contributions' relevance and future work (Section 4.6).

It is worth noting that parts of this chapter have already been published by the author and colleagues in international conferences and peer-reviewed TEL journals. More concretely, [Pri09] Pri10a Pri11g] describe the research work done in primary schools with atomic patterns ${ }^{1}$ while Pri11f] details the joint work and analysis done with SRI researchers. [Pri11c] depicts briefly the use of atomic patterns to represent orchestration, while [Dim11b] and [Pri12a describe the multi-level pattern approach.

\subsubsection{A note on methodology}

Again, as described in the introductory chapter, we have used the 'engineering method' Gla95 as the general methodological structure of the dissertation, which has also been used to investigate on each of the contributions. To develop the conceptual tools presented in this chapter, we have also followed cyclical iterations of the four phases of information, proposition, analysis and evaluation Adr93 that this method proposes. As we can see in Figure 4.2, during this dissertation we have completed four iterations, two of which were developed in a primary school context, while the other two took place in a higher education context. These iterations include:

1. Prior to the PhD work (i.e. in the Master's thesis [Pri09]), the author and colleagues explored the orchestration of face-to-face classrooms in primary education. In that research

\footnotetext{
${ }^{1}$ Note on terminology: in the first publications on the subject of atomic patterns, the term "routine" was used to refer to such patterns - due to the familiarity of the word for teachers. Afterwards, the term was changed to "atomic patterns", which is the one that we have used in this chapter, for clarity. Both terms, however, can be used interchangeably.
} 
work, a qualitative case study was conducted about the integration of a simple collaborative learning technology in authentic technology-enhanced lessons. This exploration led to the first proposal of "atomic patterns" as tools to support non-expert teachers in orchestrating lessons in TEL primary classrooms (Section 4.2). Using the observations and recordings of the lessons made during the aforementioned case study (especially from more expert teachers), a first set of atomic patterns was elicited, and evaluated in a professional development teacher workshop.

2. The promising evidence gathered in the first teacher workshop, along with a research stay at SRI Internationa $\left.\right|^{2}$ (in which similar findings on the value of small-scale patterns were compared), prompted the proposal of combining the existent set of bottom-up atomic patterns with other top-down (i.e. research-driven, as opposed to practice-driven) patterns. Afterwards, we analyzed the multiple levels of scaffolding that different kinds of patterns could provide, from the point of view of sociocultural activity theory [Eng99]. Also, further evaluation data was gathered on another teacher workshop, with an extended set of atomic patterns that integrated these different kinds of patterns.

3. As the dissertation work progressively focused around orchestration in the particular setting of blended CSCL activities across Distributed Learning environments, the need of conceptual advice, especially in the case of non-expert teachers was readily apparent, as it was the fact that atomic patterns were highly contextual. Thus, a number of observations and interviews with expert teachers that were already orchestrating blended CSCL in higher education, using VLEs and DLEs, were made. A new set of atomic patterns was elicited for this kind of educational contexts, and tested in a professional development teacher workshop with teachers coming primarily from technical backgrounds. This workshop provided us with preliminary evidences of usefulness in this new setting, and hinted at factors that might have influenced our results.

4. Finally, the multi-level approach for the elicitation and development of atomic patterns, and their combination with other researcher-driven design patterns was formally developed. The resulting integrated set of patterns for orchestration of blended CSCL in DLEs was then evaluated in a fourth teacher workshop, which garnered further positive evidence of their usefulness, and confirmed the need of technological support for such practices.

In the following section we present atomic patterns as the main dissertation contribution to support (non-expert) teachers conceptually in orchestrating TEL/CSCL. As we can gather from the previous discussion on the iterative process followed, this notion has progressively evolved during the dissertation. For clearness and brevity's sake, here we present atomic patterns in their current form, as opposed to providing a historical account of the evolution of this concept (although references to this evolution will be made while describing the iterative evaluation that took place - see Section 4.5).

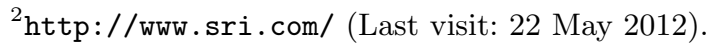




\section{"Supporting orchestration of blended CSCL scenarios in Distributed Learning Environments" Contribution \#2 - Atomic patterns for orchestration}

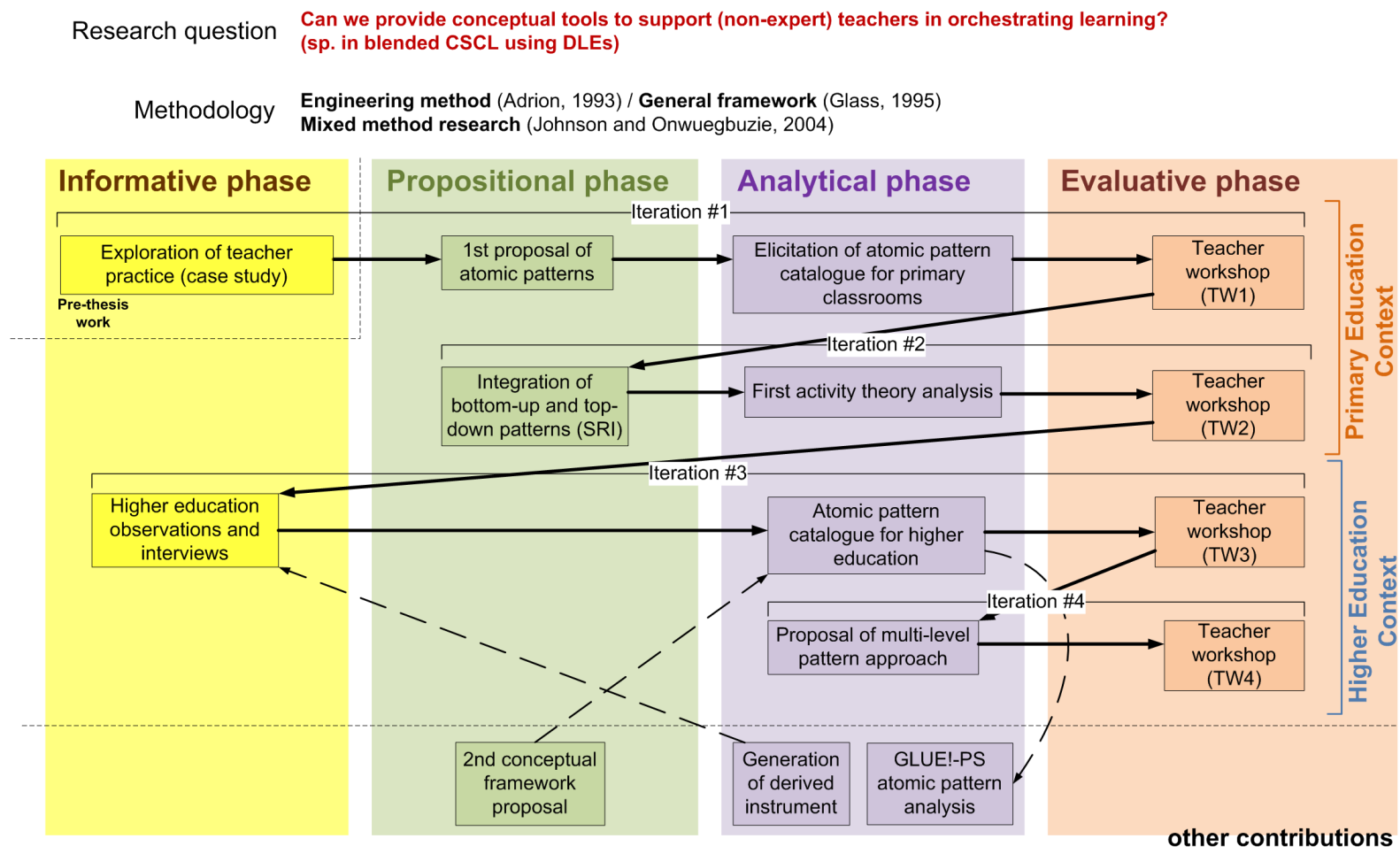

Figure 4.2: Detailed view of the engineering method followed during the research work around atomic patterns

\subsection{Atomic patterns for orchestration}

While there are evidences that pedagogical patterns are an effective strategy for CSCL designers to communicate their expertise and create novel activity designs [Mor08], it remains open to question whether such abstractions can be useful for teacher orchestration, since orchestration not only involves the calm, reflective activity of design, but also the enactment of the learning activities. As we have seen in throughout Chapters 2 and 3 , orchestrating CSCL activities is a very complex endeavor, which entails the design and real-time management of different groups of students, doing different kinds of tasks, using a variety of tools. Thereby, learning designs in general, and CSCL scripts in particular, can be seen as a way to relieve teachers from part of this burden. However, even if the teacher has carefully scripted the activity, classroom events can force (or make it recommendable) to deviate from the original design.

This section presents "atomic patterns", a notion related to Alexander's design patterns [Ale77], which were first uncovered by the analysis of activities designed and enacted by primary school teachers, as they tried to integrate a new collaborative technology in authentic classrooms where other technologies like tablet PCs, digital whiteboards, or web browsers, were already in use. This analysis showed that teachers designed and enacted activities with the new tool following a limited number of recurrent elements, or "routines". These recurrent elements were 
the seed of atomic patterns which, we hypothesized, could help non-expert teachers in taking recurrent decisions with respect to (low-level) pedagogical and technological problems, more easily.

\subsubsection{The origin of atomic patterns}

In an attempt to understand how teachers orchestrated TEL activities in authentic, computer-integrated primary classrooms, a qualitative case study [Sta05] was carried out in the educational setting of a primary school located in a village in Spain [Pri09]. The school, which is publicly funded and is located in a rural environment, had the peculiarity of having an exceptional amount of ICT resources, including digital whiteboards and tablet PCs. In this regard, the role of the school principal as a champion and promoter of ICT usage in the classroom cannot be understated. The main idea of our initial exploratory effort was to introduce a new CSCL technology in those classrooms, and observe the way teachers designed, enacted and even improvised with the new tool, alongside the other ICT and non-ICT tools in their classrooms ${ }^{3}$. For 6 months, the author and colleagues supported and observed the activities of three K6-7 and two K7-8 teachers in their first steps in the integration of GroupScribbles 4 (GS from now on) in their face-to-face classrooms. Each classroom had between 18 and 25 students of ages 6-8 (depending on the classroom and the day of the observation).

GS is a CSCL tool that was designed in order to allow social coordination of activities, having flexible activity enactment and improvisation in mind. This software is based on wellknown metaphors such as public and private boards, where ideas are shared, organized and improved, in the form of adhesive stickers (i.e. "post-its"), where teachers and students can draw and write text, using an ink-based interface. GS was designed with face-to-face scenarios in mind, and it is especially well suited to be used in classrooms with an electronic whiteboard and tablet PCs. The election of GS for our study was motivated by its affordances for flexibility and improvisation Ros07, including previous experience with GS by the author's own research group Dim07]. Figure 4.3 shows a screenshot of a GS activity, taken from the observations made during this period.

For this inquiry we collected data using a variety of qualitative techniques: three semistructured in depth interviews and one focus group with teachers, as well as 31 participant observations of classroom enactments. Screen, audio and video recordings, scripts of teacher's activities and other documentation, were analyzed in order to provide triangulated conclusions supported by enhanced evidence. The result was a set of emerging activity patterns or "routines", as we called them at the time [Pri10a], which were recurrent elements of practice in the design and enactment of the activities.

The observation of teachers designing with GS showed that teacher scripts tended to describe high-level tasks for the most part. Such a "high-level task description" means that large portions of the activity, spanning several minutes and involving teacher decisions and complex interactions between the teacher and the students, were taken care of with one small sentence in the written design, as it can be seen in the following excerpts from a teacher's notebook:

\footnotetext{
${ }^{3} \mathrm{~A}$ more detailed account of the school context and this exploratory research effort can be found in Vil09] and VS12.

${ }^{4}$ http://groupscribbles.sri.com/ (Last visit: 22 May 2012).
} 


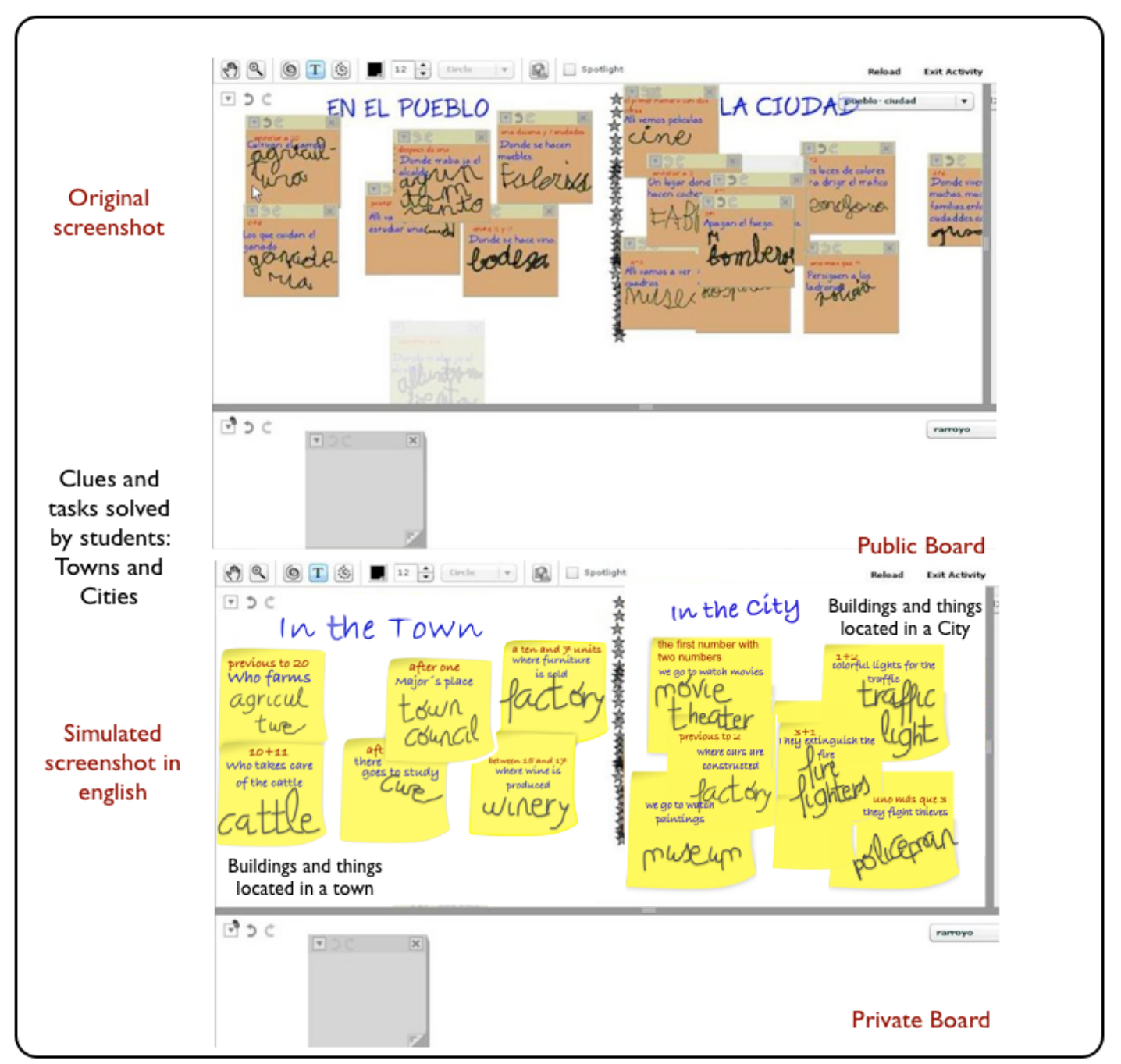

Figure 4.3: Screenshot of a Group Scribbles activity, taken from the observations at the primary school, both in its original Spanish form (top) and translated to English (bottom) 
Each student has to solve a simple arithmetical operation in order to know what is their task.

Write down buildings, places or services that are common in the cities and in the villages. Divide the class into two teams.

Team quiz. One point per correct answer / one point less for each duplicate answer.

The analysis of the designs uncovered a set of patterns (not as explicit, formal patterns used for designing, but rather, recurrent elements), such as those shown in Table 4.1. It is worth noticing that many of these routines had already been identified independently by the research team that developed the GS application, in their own field research work with GS in the United States [DeB11]. However, four of them were observed for the first time in our rural school context. This partial coincidence of design routines among very different contexts could be explained by GS's affordances, design and the metaphors it is based upon (e.g. "Where is on this image?" can be directly traced back to the ability to put background images in public boards in GS), but also by teachers finding creative ways of taking advantage of the tool's flexible design in activities with diverse student groupings (e.g. "Team Quiz").

Table 4.1: Catalogue of design atomic patterns encountered in activities of the Cigales School (taken from Pri10a $)$

\begin{tabular}{|lll|}
\hline Routines & Description & $\begin{array}{l}\text { \# appear- } \\
\text { ances }\end{array}$ \\
\hline \hline Representing Information/Questions & Students generate questions or ideas related to a certain topic & 22 \\
\hline Classification & $\begin{array}{l}\text { Organize stickers in a public board, according to certain hier- } \\
\text { archies or classification criteria }\end{array}$ & 15 \\
\hline Distributed Problem Solving & $\begin{array}{l}\text { Each student takes a sticker from the public board, represent- } \\
\text { ing a different task, solves it and puts it back to the public } \\
\text { space }\end{array}$ & 9 \\
\hline Clues & $\begin{array}{l}\text { Each student chooses a task, by solving a riddle (e.g. the con- } \\
\text { sonants presents in the student's name) }\end{array}$ & 9 \\
\hline Team Quiz & $\begin{array}{l}\text { Teams are formed inside the class, and points are awarded to } \\
\text { each team according to the resolution of the activity sub-tasks }\end{array}$ & 2 \\
\hline Poll & $\begin{array}{l}\text { Students vote which, among a set of options, is their favorite } \\
\text { (normally for later usage in the activity) }\end{array}$ & 1 \\
\hline Pipeline & $\begin{array}{l}\text { Each student takes a task from a public board, which repre- } \\
\text { sents a part of a bigger problem; after the student solves it, } \\
\text { another student uses it for solving his/her sub-task, and so on }\end{array}$ & 1 \\
\hline Self-Task & Students choose which is the next task to be done & 1 \\
\hline Where is on the image? & $\begin{array}{l}\text { The teacher poses a question, and students answer by marking } \\
\text { over a background image in the public board }\end{array}$ & 1 \\
\hline
\end{tabular}

An equivalent set of recurrent elements was encountered when we analyzed our observations of the activities as these designs were enacted (i.e. managed in real-time) in the classroom. As an example of how this analysis was performed, we present here a description and graphical representation (Figure 4.4) of how it was analyzed. These diagrams, which will be discussed more deeply in Section 4.4.1, show not only the design but also the enactment flow and the recurrent elements detected during such enactment, paying attention to the social planes in which they occurred (i.e whole-class activity, individual work, or small group work) and the recurrent elements that were associated to each phase of the activity. Please refer to Pri10a for further examples and materials about these analyses.

One of the observed activities was performed by a K6-7 teacher in a Natural Science class. The activity involved a group of 18 students working in pairs with tablet PCs. The main goal 


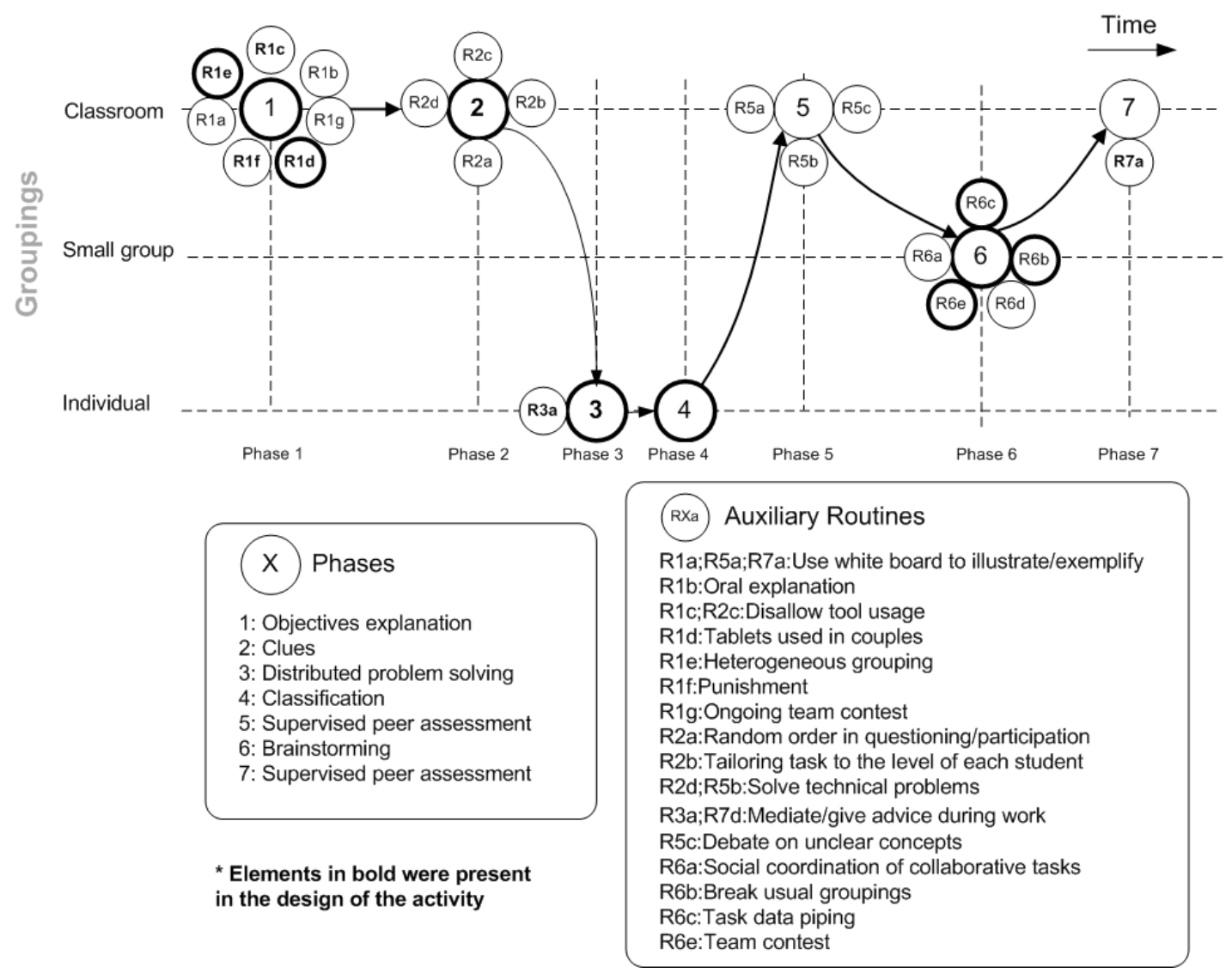

Figure 4.4: Graphical representation of the enactment analysis of an observed Group Scribbles activity

of the activity was for students to be able to recognize typical objects, buildings and professions that are more usual in rural contexts, in contrast with others commonly found in the cities. The activity flow consisted on each student taking a different task (from the public board of GS), solving a riddle related with the common theme of the lesson (e.g. "it is the place where you take ill people to", "it is the place where wine is made"), and classifying the results, according to whether the item could be found in villages or in urban areas. In order to distribute the tasks among the different students, each one was associated with a simple arithmetical operation (e.g. "one ten plus seven units", "twelve minus five", etc), whose solution was the list number of the corresponding student. The teacher coordinated the distribution of tasks socially, by asking students about these arithmetical operations randomly. Finally, the class was divided into two teams and there was a brainstorm in order to review more objects that could be found both in villages and in cities.

These diagrams distinguish the recurrent elements that were explicitly present in the teacher's design (in bold face, e.g. "Clues", marked as 2 in Figure 4.4), from those that emerged during the enactment of the activity (in regular typeface). Among these emergent elements, we can also distinguish two kinds: those which are necessary for the teacher to bridge the gap between the higher-level design of the activity and the concrete actions in the classroom (e.g. 
"Use whiteboard to illustrate/exemplify", marked as R1a), and those which were not part of the original plan at all, and thus can be considered improvisations. These improvisations arise from real-time decisions of the teacher in order to confront problems (e.g. "Disallow tool usage", marked as R1c) or take advantage of emergent opportunities.

During this kind of analysis it was noticed that the observed designs and enactments shared a surprising number of these recurrent elements, even though the topics of the activities spanned the whole curriculum. These recurrent elements sometimes could be traced back to the tools the teacher was using, and their affordances (e.g. "Use the white board to exemplify"), or to the kind of task that was being performed (e.g. "Random order in questioning/participation", "Debate on unclear concepts"), but also to unexpected occurrences in the classroom (e.g. "Punishment", "Disallow tool usage"). It is also worth noting that, even in the case of improvised parts of an activity, where the teacher lets the students choose the next task to be done, the choice was not completely free. Thus, the teacher remained within the scope of routines that were familiar to her (e.g. "Poll", "Brainstorming", "Classification" in Table 4.1).

It is also interesting to note what kind of reflections were made by teachers after enacting their classes (when this reflection is present at all), as seen in a teacher's notebook:

With this design, I have improved the development of the classroom. When I have to design an activity, I have to provide clear guidelines and show clearly what I expect of my students

As reported in [Pri09] [VS12], we can see that the evaluation is also high-level, scribbled in a few seconds on the teacher's notebook, and concentrating on aspects of classroom activity design and enactment (i.e. how the explanations are to be delivered), rather than on the pedagogical design of the activity. This hints at the importance that the issues of classroom enactment bear in teachers' minds. It also supports the idea that teachers' orchestration practice (especially, design and enactment with new technologies) is organized around a limited set of activity patterns.

From the analysis presented, it could be seen that the uncovered recurrent elements often appeared associated to other designed or improvised routines (e.g. R1a and R1b in Figure 4.4), or are alternatives to one another, towards similar goals (e.g. "Supervised peer assessment", versus "On-the-fly assessment"). This, coupled with the fact that in most of these elements we can see attempts to solve recurrent, specific educational problems, hinted at the possibility of finding what we could call an "orchestration pattern language", in the Alexandrian sense of "accepted solutions to recurrent problems" in the orchestration of CSCL activities. These patterns could be creatively combined to produce activity designs and enactments, and they could be used by practitioners (in this case, not learning designers, but rather the teachers that have to enact the activities) both to improve their practice and to communicate about it among themselves.

Still, it was arguable whether the invention of "yet another pattern language" would clarify or complicate our understanding of the orchestration of CSCL activities, both for us as researchers and more importantly, for their intended target users: teachers. Previous efforts in eliciting design pattern languages for education have found that teachers are not comfortable with such abstractions [Win09]. Moreover, how such a pattern language is formalized (e.g. for later usage in technological tools) could also prove problematic, since the more formalized the pattern is, more powerful can be the automation of such patterns and routines, but that will 
also make the tools more rigid. Since lack of flexibility has long been one of the main criticisms posed about enacting CSCL scenarios [Dil07d], the provision of enough "degrees of freedom" in the application of such patterns should also remain foremost in our minds when exploiting those patterns.

Thus, the question of what the exact nature of these patterns should be was still open, both regarding their level of complexity (since they had to be employed in a timely manner during enactment), and the level of formalization that those patterns would require in order to be an effective teaching and communication tool. Moreover, the scope of application of these atomic patterns had to be further delved into, to determine the relationship between these patterns and other contextual elements of the situation, such as the tools that are used, the pedagogical approach, or the subject matter and the goals of the lesson. For example, particular ways of orchestrating lessons (formulated as enactment routines) could be very useful when designing a collaborative course to foster creative thinking [Ret10], but not in other cases of collaborative learning. Most probably, no routine would be adequate to every educational context.

\subsubsection{What is an "atomic pattern"?}

As we have seen, by analyzing the designs of classroom activities and their enacted counterparts, a limited number of recurrent activity patterns were uncovered. The translation of abstract activity designs into concrete classroom enactments, and even the improvisation of parts of the activities due to unexpected occurrences (that is, the orchestration of such activities), were structured around creative combinations of these recurrent elements. In this dissertation we posit these elements, which we will call "atomic patterns" from now on, as a valuable conceptual tool for supporting teachers (especially non-experts) in the orchestration of TEL activities involving complex combinations of tools, activities and social levels (groups).

Atomic patterns is a variant of the design patterns approach, that aim at making a complex activity (the orchestration of technology-enhanced, collaborative learning activities) available and understandable for non-expert practitioners. We have chosen the word "atomic" as an expression of their granularity (they tend to be of a smaller scale, both time-wise and complexitywise, than other pedagogical design patterns, such as e.g. the Jigsaw strategy Aro92). This word is also appropriate because atomic patterns are often the smallest recurrent elements of teacher practice that can be elicited without going into micro-analysis of the discourse (i.e. utteranceby-utterance).

Taking into account the known properties of design patterns, we hypothesized that their problem-orientedness would allow for easy location of solutions when the practitioner faced a problem. Also, that they would allow practitioners to be more productive, as they are free to concentrate in the creative solution of unsolved problems (to which no patterns already exist). These patterns would share other design pattern advantages, such as making expert knowledge available to non-experts (in this case, teachers who are not experts in the pedagogical uses of ICT), as well as to serve as a tool for communication among practitioners in a field. Thus, teachers would be able to easily find solutions to recurrent problems in the orchestration of activities. Also, we hypothesized that a pattern approach would allow teachers to apply and combine these core solutions to lower-level problems when enacting activities with ICT, allowing them to concentrate on higher-order issues, such as creating an atmosphere of productive 
discussion and a sense of agency in the process of learning Mer07. These social and motivational aspects of the activities are emergent and largely contextual, and normally cannot be predicted in the design phase of an activity. Indeed, these latter aspects can very often be neglected as a result of teachers having to solve the myriad of minute technical and management problems that appear when ICT is present in learning activities.

As we mentioned in the previous section, teacher designs were rather abstract and highlevel, many of them being just a few phrases scribbled in a notebook Pri10a. However, if we compared the designs with the actual enactment of those designs, we could see two main differences, which could be mapped to the modes of improvisation described in Ker95. On the one hand, additional tasks or phases were added to the design, completing it as teachers realized that the enactment would not work adequately without them (e.g. an evaluation of the results of a classification task was added, so that students got feedback on their knowledge). Also, another kind of completion took place as teachers improvised in the face of unexpected problems and opportunities that emerged in the classroom (e.g. performing an improvised task when all the designed tasks were completed faster than expected). These completion processes could be seen as parallel to the "formulaic improvisation" in [Ker95]. On the other hand, even for the tasks that were explicit in the design, a concretization took place, since there are several ways in which such high-level tasks can be performed (e.g. the evaluation of a task can be done directly by the teacher, or through supervised peer assessment, or on-the-fly vs. after the task etc). This concretization process could be seen as "paraphrase improvisation". It is worth noting the parallelisms between these processes and the "kernel and rings" model of orchestration by Pierre Dillenbourg in Dil11a (where designed activities form the "kernel" and there are several "rings" of non-designed but necessary activities, see Section 2.5.4.

These parallel processes of completion of the task flow (parting from the abstract, incomplete designs) and concretization of the performance, can then be compared with the ones in other observed activities. From this comparison, recurrent elements begin to emerge. Continuing with the example of the assessment of a task, we observed that in many activities the teacher chose to perform the assessment verbally on her own, while in others the teacher chose a student and asked him/her to do the assessment using the digital whiteboard. These recurrent "ways of doing certain things with a certain purpose" is what we have termed atomic patterns. Here, the emphasis is in the purpose of the routine, making it a problem-oriented structure, in the same way that design patterns in architecture represent the core of a solution to recurrent problems Ale77.

Through this kind of analysis, both on the designs and the enactments of these designs, 42 atomic patterns were found ( 9 emerging from designs and 33 from enactment). Examples of these patterns can be seen in Table 4.1 (for design-time ones). Enactment-time atomic patterns include, e.g. "Use the digital whiteboard to exemplify" (the teacher performs the actions expected from students with the digital whiteboard, so that they understand better the task at hand), "Correct your own mistakes" (when the assessment shows that a student response is not correct, the teacher asks the student to think again and produce a new correct response to be shared with the class), or "On-the-fly assessment" (while a task is being done by students, the teacher monitors the progress, assessing their knowledge and barging in if needed, to point the students in the right direction). See also Table 4.2 for a longer list of enactment-time examples.

As we can see, at this point the form that these patterns take is very simple: a name, to identify the atomic pattern, and a short description (2-4 lines) of what the pattern looks like. 
Table 4.2: (Partial) Catalogue of enactment routines, classified by type of task

\begin{tabular}{|c|c|c|}
\hline Name & Short description & Task type \\
\hline Correct your own mistakes & $\begin{array}{l}\text { When an incorrect response is detected, the teacher asks the } \\
\text { author to revise the response and put it back again in the } \\
\text { public space }\end{array}$ & Assessment \\
\hline Democratic reward assignment & $\begin{array}{l}\text { The teacher helps the groups of students to reach a consensus } \\
\text { about how many rewards should be assigned to each group, in } \\
\text { light of their responses }\end{array}$ & Assessment \\
\hline Discuss selected results & $\begin{array}{l}\text { The teacher selects one or more of the student responses, to } \\
\text { discuss with the whole class whether they are correct or not, } \\
\text { and why }\end{array}$ & Assessment \\
\hline Probe with false information & $\begin{array}{l}\text { The teacher gives an incorrect response for a question or task, } \\
\text { so as to see if the students detect the error, or they take the } \\
\text { teacher's word for it }\end{array}$ & Assessment \\
\hline Students detect mistakes & $\begin{array}{l}\text { The teacher asks students to detect and manifest which pro- } \\
\text { posed results are incorrect (as opposed to pointing them her- } \\
\text { self) }\end{array}$ & Assessment \\
\hline $\begin{array}{l}\text { Student revises the results in } \\
\text { the whiteboard }\end{array}$ & $\begin{array}{l}\text { The teacher asks one student to use the whiteboard to assess } \\
\text { the responses, in front of the whole class }\end{array}$ & Assessment \\
\hline Disallow tool usage & $\begin{array}{l}\text { The teacher impedes the usage of tools (e.g. by taking away } \\
\text { the stylus of a Tablet PC, or disabling writing access in Group- } \\
\text { Scribbles), so that students pay attention to the assessment } \\
\text { or explanation in progress }\end{array}$ & $\begin{array}{l}\text { Assessment or Ex- } \\
\text { planation }\end{array}$ \\
\hline $\begin{array}{l}\text { Heterogeneous group forma- } \\
\text { tion }\end{array}$ & $\begin{array}{l}\text { Distribute students in small group work so that students with } \\
\text { disparate characteristics (e.g. levels of knowledge about the } \\
\text { subject matter) are put together }\end{array}$ & Explanation \\
\hline Prepare backup materials & $\begin{array}{l}\text { The teacher prepares additional (maybe identical) materials, } \\
\text { in prevision of emergent problems (e.g. if some of the tasks } \\
\text { are accidentally deleted) }\end{array}$ & Explanation \\
\hline Rules of the game & $\begin{array}{l}\text { The teacher explicits and emphasizes rules and routines to be } \\
\text { enacted during the activity (e.g. the order of tablet PC usage, } \\
\text { or the roles within each small group) }\end{array}$ & Explanation \\
\hline $\begin{array}{l}\text { Use digital whiteboard to ex- } \\
\text { emplify }\end{array}$ & $\begin{array}{l}\text { Using the digital whiteboard, the actions to be taken in a task } \\
\text { are exemplified by the teacher, so that students can reproduce } \\
\text { them later on }\end{array}$ & Explanation \\
\hline Use graphics to support a task & $\begin{array}{l}\text { The teacher uses drawings or images to represent the task or } \\
\text { parts of it in a visual manner }\end{array}$ & Explanation \\
\hline $\begin{array}{l}\text { Calibrate task difficulty ac- } \\
\text { cording to student level }\end{array}$ & $\begin{array}{l}\text { The teacher assigns the different individual or small group } \\
\text { tasks to each group, according to their knowledge level }\end{array}$ & Support/Task \\
\hline Disable idea copying & $\begin{array}{l}\text { The teacher hides the public space until a task is ended, so } \\
\text { that students do not copy the ideas from other students }\end{array}$ & Support/Task \\
\hline On-the-fly assessment & $\begin{array}{l}\text { The teacher monitors the progress and barges in if needed, to } \\
\text { point the students in the right direction }\end{array}$ & Support/Task \\
\hline On-the-fly peer assessment & $\begin{array}{l}\text { In a dyad of students, one of the students performs the task } \\
\text { while the other helps and/or regulates his partner. Then, the } \\
\text { roles are reversed }\end{array}$ & Support/Task \\
\hline $\begin{array}{l}\text { Use paper/tangibles to support } \\
\text { a task }\end{array}$ & $\begin{array}{l}\text { When performing an activity with ICT, the teacher also pro- } \\
\text { vides equivalent/complementary tangible materials (e.g. pen } \\
\text { and paper), so that students can manipulate them physically }\end{array}$ & Support/Task \\
\hline
\end{tabular}


Later on, a number of examples of use were added, to further clarify how the atomic could be put into practice in a concrete educational setting 5 . Finally, due to their high number, one or more classification criteria were added, to make their handling and location easier. These criteria for classification include: time (i.e. whether they are used at design-time, instantiation-time or enactment-time, see the notion of activity lifecycle in Section 2.2.2), kind of task that they are associated to (for example, explanations, support or assessment, see [Pri11g]), tools or other classroom elements that are used (e.g. "Use digital whiteboard to exemplify" clearly uses a digital whiteboard), or the orchestration aspect that they most notably refer to (see the " $5+3$ Aspects" framework in Section 3.2. None of these classifications, however, intends to be exhaustive since, for example, the assessment of a task can be performed on-the-fly while supporting that very same task, or a "Clues" task can be used in design-time, but also improvised in enactment-time. In Appendix B the reader can find the master catalogue of atomic patterns elicited during this dissertation, classified by primary and secondary orchestration aspects.

Thus, from the above explanation, we can gather several of the main characteristics of atomic patterns:

Heterogeneous As the reader may have noticed (especially if Appendix B has been looked at), under the label "atomic patterns" we have gathered very different kinds of recurring practices, from general "activity types" tied to a certain technology (e.g. Group Scribbles), to concrete "teacher moves" (e.g. when questioning students) or organizational tactics (e.g. such as dictating the use of computers in pairs to promote collaboration). This is in accordance with the heterogeneity of orchestration aspects themselves: if we look at the " $5+3$ Aspects" framework on orchestration (Section 3.2), we can find very different elements which make up orchestration: different activities, different ways of balancing up actors, tensions between theory and pragmatism. Thus, patterns to help e.g. in the design of activities are bound to be very different from patterns that help in making students feel a sense of agency. Moreover, patterns very often combine (or rather, align) more than one of the orchestration aspects, defying all attempts at a strict categorization.

Small scale It can also be seen that, albeit atomic patterns vary in their granularities, they tend to be of a smaller scale than other patterns, whose enactment sometimes spans an entire session (or several sessions). Let us think of the Jigsaw strategy Aro92, which can be expressed as a pattern, and normally requires one or more hours to be enacted in a classroom. In contrast, atomic patterns are at a lower level, and can be used in many ways as "flesh to fill the bones" of these larger patterns, to solve smaller-scale pedagogical problems, or to align different contextual elements within the general structure of such large-scale patterns.

Numerous As a result of their granularity, the amount of atomic patterns in a catalogue, even for a very concrete educational context, is often much larger than in other sets of design patterns (for example, 42 were elicited in our primary school observations with Group Scribbles, and more than a hundred in our application of the same method to higher

\footnotetext{
${ }^{5}$ This format was chosen over Alexander's more complex pattern description template because it was considered the bare minimum of information needed for teachers to handle atomic patterns. This decision was also influenced by their high number and relatively small granularity, and the fact that teachers had shown certain reluctance to such complex templates, both in our observations and in other researcher's Win09.
} 
education). In fact, from the analysis of just one 50-minute session we can often elicit a dozen or more of these atomic patterns, and even more in the case of expert teachers (who, as we observed, tended to use more complex combinations of these elements). Naturally, not all these atomic patterns are equally worthy or recurrent, and thus researchers (or other teachers) may select and refine the most useful among them. However, even if some of these elements are very recurrent, the classroom events are so varied that few "silver bullets" exist, and a teacher has to master a good number of them to orchestrate a classroom efficiently.

Informal As opposed to the Alexandrian design pattern formalism [Ale77], which dictates that a design pattern should be described using certain fields (a picture, a name, a context, the problem it solves, the forces in play, the solution and a diagram of the solution), atomic patterns are more informal, requiring only three fields (a name, a short description and a number of examples - the latter being optional). This a pragmatic consequence, mainly, of their big numbers (it would be extremely time-consuming to read hundreds of atomic patterns under Alexander's formalism).

Contextual/Concrete Finally, as opposed to most design patterns, which try to remain abstract in order to enhance their applicability to different contexts, atomic patterns are highly contextual, in the sense that very often they make explicit reference (even in their title) to concrete classroom features, be them either tools, activities, social features like groups, etc. This is to enhance their recognizability (especially during enactment, when timely reactions are crucial), as well as to address the indications by some authors that teachers tend to feel uncomfortable among such abstractions [Win09], which could be caused by their need of providing concrete performances in a concrete classroom. The real-practice examples in an atomic pattern play a big role in this "grounding" of the pattern in the concrete classroom.

\subsubsection{Atomic patterns vs. other pattern approaches}

Now that we have briefly described how atomic patterns were uncovered and elicited from teacher practice in authentic settings, and what are their main features, it is worth considering how these atomic patterns relate to other patterns that have been proposed in the past few years in the field of TEL, with somehow overlapping goals. Three main approaches will be compared, due to their close relationship with the labor of orchestration of CSCL: Collaborative Learning Flow Patterns, Adaptation Patterns and Teaching Routines:

CLFPs and the CSCL Script Pattern Language In her Doctoral Thesis, Hernández-Leo proposed a set of design patterns aimed at the design of effective CSCL scripts, called Collaborative Learning Flow Patterns (CLFPs) [HL07]. These patterns proposed different structures of collaborative learning activities that have been validated in the practice, such as the Jigsaw, Pyramid or Think-Pair-Share. Following an Alexander-like formalism, other complementary patterns were proposed (Activity patterns, Resource patterns, Roles and CL mechanisms patterns), thus forming a pattern language for the generation of CSCL macro-scripts [HL09], which could be translated into a computer-interpretable form using 
the IMS-LD specification IMS03a], through a dedicated authoring tool, called Collage HL06a.

The relationship of CLFPs and the resulting pattern language with atomic patterns is apparent, if we take into account that Design (and CSCL scripting is a form of learning design) is one of the main orchestration aspects. In general, CLFPs, which define a sequence of phases or activities within a script, are of a coarser granularity than atomic patterns, to the point that atomic patterns can be used to "fill in" the details of the orchestration of a learning design crafted using CLFPs. The same can be said of the "assessment patterns" portrayed in [VF11]: the granularity of those patterns (e.g. "written report review") is generally coarser than assessment-oriented atomic patterns (e.g. "onthe-fly assessment"). However, this general distinction begins to blur if we consider the other patterns in the CSCL Scripting Pattern Language: Activity patterns (e.g. "Introductory Activity" or "Preparing fruitful discussions using surveys") and Resource patterns (e.g. "Structured Space for Group Tasks"), begin to bear resemblance to equivalent atomic patterns, especially those used during design-time. Thus, we can see that these design patterns end up "blending" with atomic patterns as we go down in the pattern hierarchy, albeit the differences in the way the patterns are named and represented (formality and abstraction vs. informality and concreteness) still remain.

Overall, we consider the CLFP approach complementary to atomic patterns, and especially useful to structure the Design aspect of orchestrating CSCL (at a higher level). In fact, we have integrated these patterns in our multi-level pattern approach to orchestration (see Section 4.3), and we have successfully applied this combination in professional development teacher workshops in higher education (Section 4.5).

Adaptation Patterns According to Kar11], an "Adaptation Pattern" is "a pedagogically useful and well-targeted adjustment process that can be initiated by the teacher or the CSCL system, in order to foster an improved learning setting when specific conditions occur during the collaborative learning activity". These patterns were proposed to guide the design and operation of Adaptive Collaboration Systems (ACS), that is, systems to promote collaboration, whose behavior can adapt automatically (or semi-automatically) in the presence of certain events (e.g. student modeling unveiling certain preferences or breakdowns) Bru03]. Given the above definition, we could view adaptation patterns as a particular kind of orchestration patterns (given that Adaptation is one of the main orchestration aspects). However, it is very important to note that adaptation patterns are generally elicited with the explicit aim of implementing (adaptive) technological learning systems, either by automating the application of these patterns (in the vein of Intelligent Tutoring Systems [Sle82]), or by supporting teachers in performing such actions. While atomic patterns can be used with that aim, that is not their only possible application.

There are two ways in which these two concepts can be connected: one is to try to express "Adaptation Patterns" within the aspects of the orchestration conceptual framework presented in Chapter 3, adding them to the orchestration routines catalogue and trying to translate them to the technological context defined by the orchestration support to be presented in Chapter 5 . The complementary approach would be to express atomic patterns following the "Adaptation Pattern" formalism. Both paths have been followed in this dissertation: a) All nine "Adaptation Patterns" have been added to our catalogue of routines 
(see Appendix B), thus illustrating how the bottom-up elicited atomic patterns can be complemented with more research-driven elements; and b) Selected atomic patterns (especially those pertaining to the Adaptation aspect of orchestration) have been expressed using the Adaptation pattern template, as an exercise to illustrate how atomic patterns could be used as the seed of more formal patterns to design educational technologies (in this case, ACS systems, see Table 4.3.

Table 4.3: Example atomic pattern expressed using the Adaptation Pattern template

\begin{tabular}{|c|c|}
\hline Atomic pattern: & Reform groups in face of the current attendants \\
\hline Key idea: & $\begin{array}{l}\text { In a collaborative activity (sp. synchronous), when participants are unex- } \\
\text { pectedly absent, groups should be rearranged to enable the best collabo- } \\
\text { ration possible according to the design's pedagogical aims }\end{array}$ \\
\hline Activation conditions: & Absent participants are detected \\
\hline What to adapt: & $\begin{array}{l}\text { Group composition of current activity. Potentially, also group composition } \\
\text { of next activities }\end{array}$ \\
\hline Type of adaptivity: & Static \\
\hline Implementation (e.g. & $\begin{array}{l}\text { 1. Shuffere am und the participants in the groups of an activity. The algo- } \\
\text { see hhatter thit may be random, maximizing group size, maximizing group } \\
\text { number, or be dependent on the script (e.g. Pyramid) or other restric- } \\
\text { tions (e.g. heterogeneity). Thus, several plugins may be developed for this } \\
\text { purpose. } \\
\text { 2. Change the group composition of following activities. The same caveats } \\
\text { from step } 1 \text { above are applicable. }\end{array}$ \\
\hline
\end{tabular}

Teaching Routines A research effort that took a very similar approach to that of atomic patterns is SRI's Contingent Pedagogies project 6 . The aim of the Contingent Pedagogies project was to improve student science learning by integrating assessment activities into a widely used Earth systems science curriculum. The Contingent Pedagogies project developed activities-called interactive formative assessments (IFAs) - that use classroom network technology (clickers and Group Scribbles). The IFAs specify questions for teachers to pose; how classroom network technology will be used to support collection, aggregation, and display of data; and how teachers can use assessment information to organize instruction DeB11. To do that, researchers used a series of pedagogical patterns (called "teaching routines") that, in a similar vein to CLFPs, specified a sequence of (abstract) steps to be followed in the lesson, which had then to be adapted to the content subject of the lesson (e.g. by researchers/designers or by teachers themselves).

Even if these teaching routines, like CLFPs, are clearly related to the Design aspect of orchestration, it is even more striking that, in order to address certain teacher adoption problems, researchers defined a series of smaller-scale patterns aimed at helping during the enactment of the lessons. These mini-patterns included classroom norms (to promote the social practice of scientific argumentation, effective use of classroom network technology and promote students' willingness to reflect on and revise their ideas), discourse moves (to support teachers in scaffolding students' discussion of responses to questions posed by clicker technology, shifting responsibility for thinking to students and to encourage students to engage one another's ideas) and decision rules ("if-then" guidance to teachers about how to proceed, given a particular distribution of responses after students have had the chance to learn the material). These lower-level patterns, destined to be used during the enactment of a session, address several other aspects of or-

6 http://ctl.sri.com/projects/displayProject.jsp?Nick=contingent (Last visit: 27 Jun 2012). 
chestration, such as Management and Adaptation (with questions posed through clickers being the main Awareness agent in this case).

Given the similarity of these smaller patterns with our atomic patterns, and the contextual (relative) similarities between both projects (use of clickers and GS in middle schools vs. use of GS in primary schools), some of SRI's patterns were integrated into the atomic pattern catalogue that we developed, and a joint analysis about the kind of support that they provided for teachers was performed (see the following section). The reader can also refer to Pri11f] for a more detailed account of the relationship between both projects.

\subsubsection{Analyzing atomic patterns from an activity theory perspective}

As we can see, both SRI's Contingent Pedagogies project and our experiences in the primary school share a focus on smaller-scale, easily-actionable patterns. This kind of proposal was not done at random, and in both cases derived from the adoption problems that using (abstract) pedagogical patterns alone posed for teachers. As it is pointed out in DeB11] and VS12], in both research efforts teachers pointed out problems in adopting the presented pedagogical patterns in their everyday practice. These problems prompted both research teams, independently, to try a "smaller patterns" approach. This section analyzes why such problems might be arising, and why smaller-scale patterns might represent a good solution. This analysis was done jointly during a research stay of the author and another GSIC-EMIC researcher at SRI International in Fall 2010 (see also [Pri11f]).

The findings and evolution of these two research projects exemplify a common problem of CSCL research that tries to influence classroom practice in authentic settings: how to make the results of past research (often in the form of de-contextualized theories and principles or reified into new technological tools) available to practitioners in a way that they can appropriate them and, moreover, how to do it for practitioners who are not especially gifted or motivated or are not experts Dil09b]. Studies into the sustainability and scalability of research-based interventions Fis04 [Pen07] point to the importance of such factors as the professional development approach, its coherence with current reform ideas in the schools, and the challenges that teachers face in their daily practice.

Both the Contingent Pedagogies project and the GSIC experiences in primary schools initially focused on the idea of pedagogical patterns as a professional development approach that helps address everyday teaching challenges. As we have seen, design patterns are a common way of capturing theories and design principles in complex practice [Ale77, and had already been applied to the practice of teaching (pedagogical patterns, [Fin02]). Past research shows that the pattern approach offers several advantages: it serves as a means of communication between researchers and practitioners (and also among practitioners), it offers practitioners a number of building blocks that can be creatively combined into new solutions, and finally, it is suitable for non-experts because of its problem orientation. However, the evidence from the two projects showed that its application to everyday teaching practice is not without potential limitations. The findings of both research groups highlight two important tensions or gaps that often arise when researchers try to influence everyday practice in an authentic setting. These tensions are represented graphically in Figure 4.5 . 


\section{The Gaps}

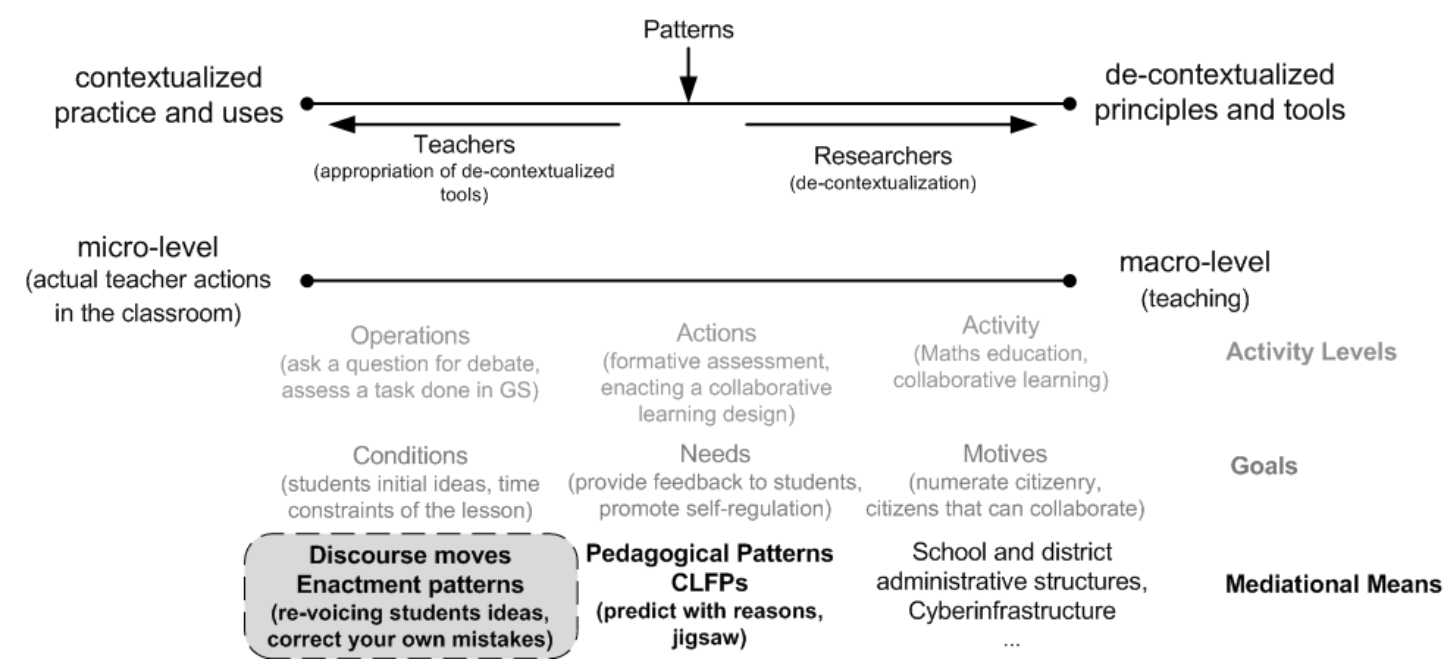

Figure 4.5: Graphical representation of the tensions found in the atomic pattern analysis from an activity theory perspective

The first of these tensions appears between researchers' efforts to de-contextualize empirical data to obtain widely applicable principles and teachers' application of those principles to concrete situations, which can be seen as an act of re-contextualization Goo92. Teaching practice, and especially innovative teaching practice, can be seen as the appropriation of the de-contextualized tools such as curriculum materials, classroom management techniques, as well as resources provided by researchers (e.g. theories, patterns, or even technological tools such as Group Scribbles). In this sense, pedagogical patterns provide de-contextualized advice on how to attain certain pedagogical goals. However, as noted by [Win09], dealing with de-contextualized tools can be difficult for teachers, even if they contain more elements of context than an abstract theory (e.g. they assume a certain kind of classroom, or they are provided along with a short narrative example of their application to other contexts). Instructional moves (such as SRI's classroom norms, discourse moves and decision rules) and orchestration atomic patterns elicited from actual teaching practice are also examples of this de-contextualization effort, but they originate from a different source than theory. Having more elements of a familiar context present in these patterns (e.g. assuming usage of the Group Scribbles tool and a whiteboard, or assuming a specific outcome of a previous task) enhances their mirroring properties and makes them more actionable (i.e. teachers recognize them as actions that they normally take in the classroom or as actions they may take in an easily recognizable situation).

There is also a second tension or gap between the macro-level designs and plans for instruction (provided by researchers, developed by teachers themselves or co-designed) and the emergent micro-level enactment of those plans by a specific teacher in his classroom. Such plans are incomplete by their very nature, since any representation of a practice is a simplification. Even if plans are designed by teachers thinking about their specific classroom context, plans cannot take into account all emergent occurrences or accurately predict students' notions and 
their evolution. If we look at this gap from the point of view of socio-cultural activity theory Eng87], lesson plans and pedagogical patterns at the macro-level (e.g. the CLFPs mentioned above) provide mediational tools for teachers at the action level that respond to needs such as providing feedback to students or promoting self-regulation. However, even with that scaffolding, teachers have still to make decisions on how to exactly enact the plans using specific instructional moves in their classroom context. These instructional moves (which correspond to operations in activity theory terminology) can be highly routinized and often vary based on teacher style. By also providing scaffolding at the operation level (e.g. Contingent Pedagogies' discourse moves, or GSIC's atomic patterns), more coherent pedagogical strategies can be enacted by teachers. Having a set of atomic, actionable patterns that are easy to call forth, tweak and recombine can empower teachers to creatively design and enact activities according to the theories and design principles of CSCL research [HL10a] and dialogic teaching research [Wel06] O'C07]. Moreover, this combination of patterns of different granularities is supported by Alexander's concept of a pattern language Ale77, that is, a set of related patterns that provide increasing detail on how to implement the higher-granularity patterns. In our case, norms, rules, moves, and atomic patterns can be seen as tools for goal-directed action that ideally become operationalized in ways that support their enactment of collaborative, dialogic activities.

Thus, it is important to note how, despite the differences in school context or even the overall research approach, researchers on both projects independently identified and analyzed several common issues that must be addressed. One is the gap between the de-contextualized theories and tools that researchers often produce and teachers' need to provide ad-hoc practice in their classroom situations (which can be seen as an act of re-contextualization). Another is the gap between the macro-level advice (e.g. in the form of pedagogical patterns or lesson plans) that is often given to teachers and the micro-level decisions and actions that teachers must take in their particular contexts. As we will see in Section 4.5, the use of more atomic, actionable teacher moves (coming from real practice and derived from literature) has shown promising results. The use of practice-derived atomic patterns, classroom norms, and decision rules seems to complement the advantages of macro-level pedagogical patterns and to enable a wider adoption and change of daily practice. Thus, we posit the combination of both kinds of patterns as a coherent mediational strategy for teachers to produce contextual, pedagogically-sound uses of technology, making the most of its affordances for enhancing teaching and learning. A concrete approach that uses such combination, e.g. to prepare teachers to become more proficient in orchestrating collaborative learning with ICT in real-world classrooms, is presented in the following section.

\subsection{A multi-level pattern approach to the orchestration of CSCL activities in DLEs}

As we have seen throughout this chapter, the de-contextualized support that design patterns offer for non-experts practitioners (be it in architecture, learning design, or teaching), however useful, has its limitations. In the complex practice of orchestrating CSCL, we have proposed atomic patterns as a conceptual tool to "bridge the gap" between the de-contextualized advice of design patterns and the contextualized performance that teachers have to deliver in the classroom. Nevertheless, we should not ignore the power of more abstract, high-level patterns in 
order to support the orchestration of pedagogically-sound scenarios at a higher level. Thus, this section proposes an approach to elicit, develop and combine atomic patterns with other kinds of patterns (an idea already advanced in Section 4.2.3), in order to produce an "enriched pattern set" that can be used to support non-expert practitioners in producing pedagogically-sound learning activities. This enriched set can then be used, e.g. in professional development actions, to help practitioners orchestrate scenarios that comply with the complex constraints and opportunities of authentic educational settings, such as available resources, or making deviations from the original plans. The reader can also refer to [Pri12a] for a more detailed depiction of the approach.

\subsubsection{Overview of the approach}

The approach is based on the combination of patterns of different kinds, at different levels of contextualization. We use existing pedagogical design patterns coming from different sources [Ret06], such as, e.g. the aforementioned CLFPs for CSCL script design. However, the main difference is that our approach relies on combining those patterns with atomic patterns (see Section 4.2 that have been extracted mainly from CSCL and TEL orchestration practice (i.e. from the design and enactment of learning activities) in authentic settings.

By combining these two kinds of patterns, learning designs take explicitly into account the particular contextual restrictions and opportunities of each classroom (e.g. specific tool affordances). The resulting designs provide a more complete view of the plans to be enacted in the concrete setting thus helping practitioners to "bridge the gap" between the de-contextualized advice of Alexandrian patterns and the contextualized performance that practitioners must deliver in the classroom (see the previous section, and [Pri11f]). Thus, using this approach, the elegant simplicity of the more de-contextualized design patterns is lost (or, rather, complemented), but part of the richness and complexity of the authentic contextual classroom is regained.

Although this approach has been used by the author and colleagues in the role of researchers trying to understand orchestration in authentic TEL settings Pri10a, the approach is motivated mainly by the needs of teachers and other actors in orchestrating a scenario (e.g. learning design practitioners). Thus, the approach described here is aimed at researchers, learning design experts or teacher trainers trying to foster orchestration practice within a particular focus of interest, in authentic settings. That said, nothing precludes teachers themselves from using the process to produce more contextualized learning designs and to orchestrate them.

As we can see in Figure 4.6, the approach follows four main phases. The first step is the elicitation of atomic patterns from authentic teacher practice (including the design of activities and their implementation and enactment in the classroom). Then, so that they can be used more easily later, this corpus of elicited atomic patterns is refined (e.g. are two atomic patterns slight variants of the same practice?) and categorized (e.g. in which moment is the atomic pattern used? Is it associated with a concrete ICT tool or task?). This classification can be guided by theoretical and pedagogical viewpoints (such as the conceptual framework presented in Chapter 3), by the aims of the research effort, as well as by practical limitations (e.g. the number of them that can be used in a specific professional development action such as a workshop). Afterwards, the atomic patterns can be combined with existing design patterns and pattern languages available for the target pedagogical approach (e.g. CSCL, Inquiry-based Learning, formative assessment 


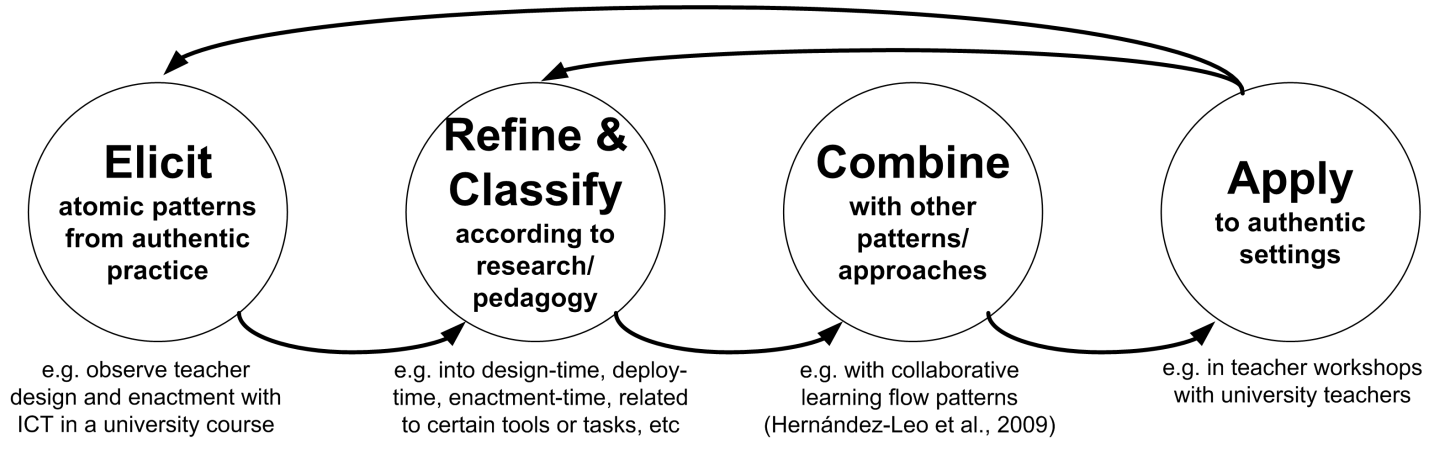

Figure 4.6: The four main phases of the multi-level pattern approach to support orchestration

and contingent teaching, etc.). This step aims to connect the overarching, pedagogically-sound structures that design patterns provide with the contextual elements of the authentic setting that atomic patterns represent. For example, we can take the aforementioned Pyramid design pattern as a base, and provide more richness and detail to our design by stating that in some phase students are to do a "conceptual mapping", or that we are going to "distribute participants physically to facilitate interaction" (both of which are atomic patterns, see Appendix B). Finally, this extended pattern set can be applied in the field (e.g. in teacher workshops, to design and enact learning experiences) to promote orchestration in teacher practice. Eventually, data can be gathered to further refine the combined set of patterns, or to guide further iterations of the research or professional development intervention.

\subsubsection{The approach in use}

In order for the reader to better understand the process to be followed when applying this approach, e.g. in authentic teacher development workshops to promote orchestration (see Section 4.5), we illustrate each phase with examples from our own use of the approach in the design of a teacher professional development workshop on the subject of designing and enacting (i.e. orchestrating) CSCL activities in higher education.

Phase 1: Eliciting atomic patterns from authentic practice The first step in this approach is to elicit recurrent orchestration elements from real teacher practice within our focus of interest. This includes gathering data through interviews about how teachers design their learning activities and also how they implement those activities with the available ICT and non-ICT resources, and how those designed activities are finally enacted in authentic settings (e.g. through observations). This kind of data is normally gathered from teachers who have previously applied the pedagogical approach of interest in their everyday practice (especially experienced/successful teachers).

In this example, we were trying to foster the design and enactment of CSCL activities in higher education in the context of the University of Valladolid (UVa), where blended learning and the use of Moodle and Web 2.0 tools are common. In order to elicit orchestration patterns, we observed 10 CSCL scenarios within UVa courses, taking audio recordings and/or observation 


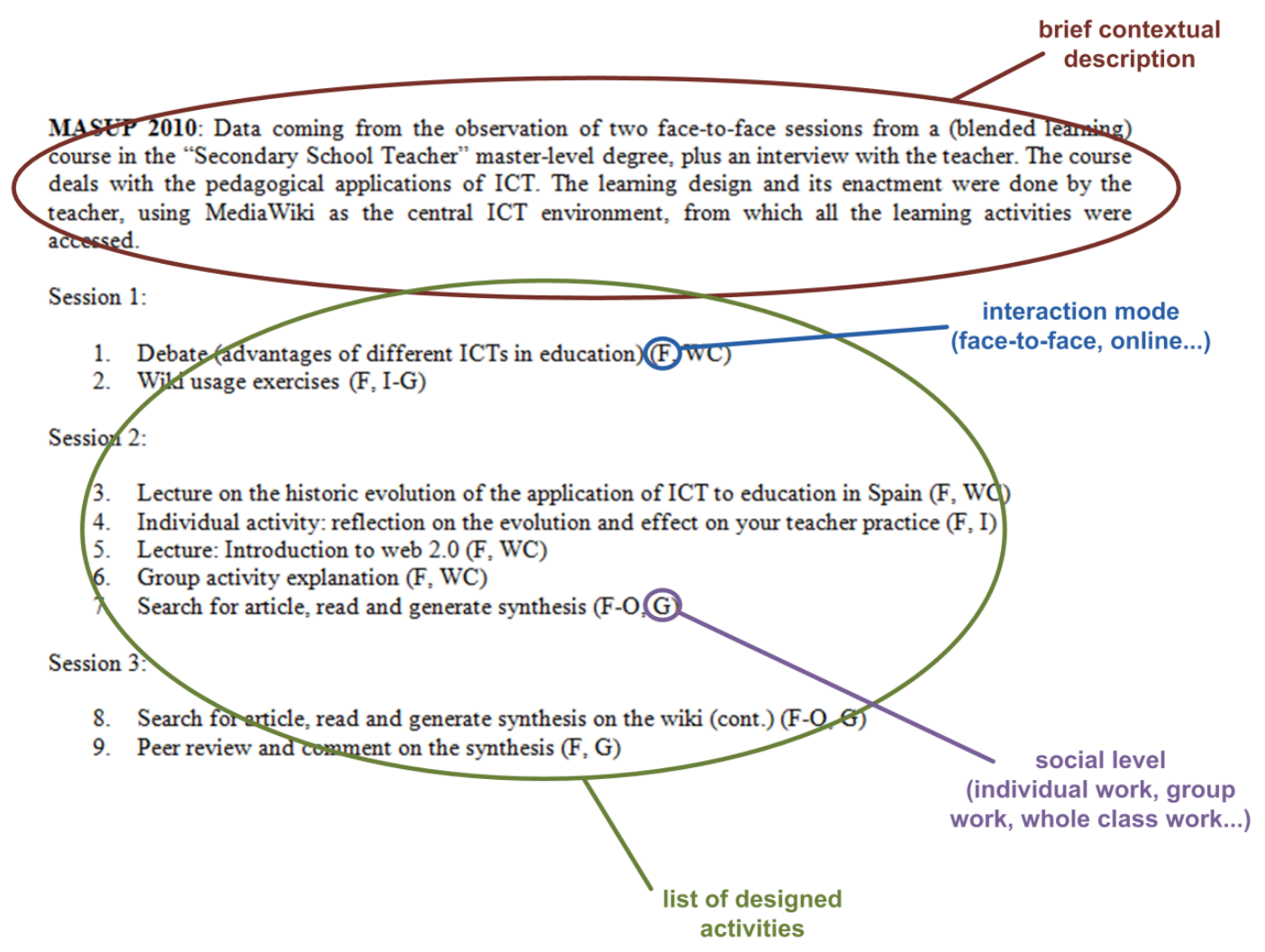

Figure 4.7: Example design analysis of a teacher's CSCL learning design

notes. We complemented this first-hand data with more indirect methods, such as discussions in a teacher workshop [Fer10], as well as three interviews with university teachers who included CSCL practice in their courses.

First, practitioners' learning designs in the authentic setting are analyzed, considering all data sources and formats at our disposal: teachers' notebooks, wikis, the design's implementation in a Learning Management System (LMS), like Moodle, and even non-written depictions of the designs (e.g. formal or informal interviews). This analysis aims at eliciting recurrent elements in teachers' learning designs, identifying typical activities and tasks, their flow in the overall plan, and how available ICT and non-ICT resources in the classroom are used. In our concrete case, we analyzed the designs of the aforementioned CSCL experiences. Figure 4.7 depicts the analysis of one of the studied teacher's designs of CSCL experiences, which was in the form of a wiki page. In Prieto et al. (2011c) we give an example from a primary school teacher's notebook.

After this first design analysis, similar steps are taken to analyze the deployment (i.e. how the design ideas are implemented concretely with the available ICT and non-ICT infrastructures of the classroom, and within other setting constraints): how does an activity get reflected into the LMS, how the group formation will be done, how learning materials will be delivered to students, how students will deliver their constructed artifacts to the teacher, and a long etcetera.

Finally, we analyze how each learning design considered was enacted in the classroom, paying special attention to deviations, improvisations and other emergent occurrences, and how 


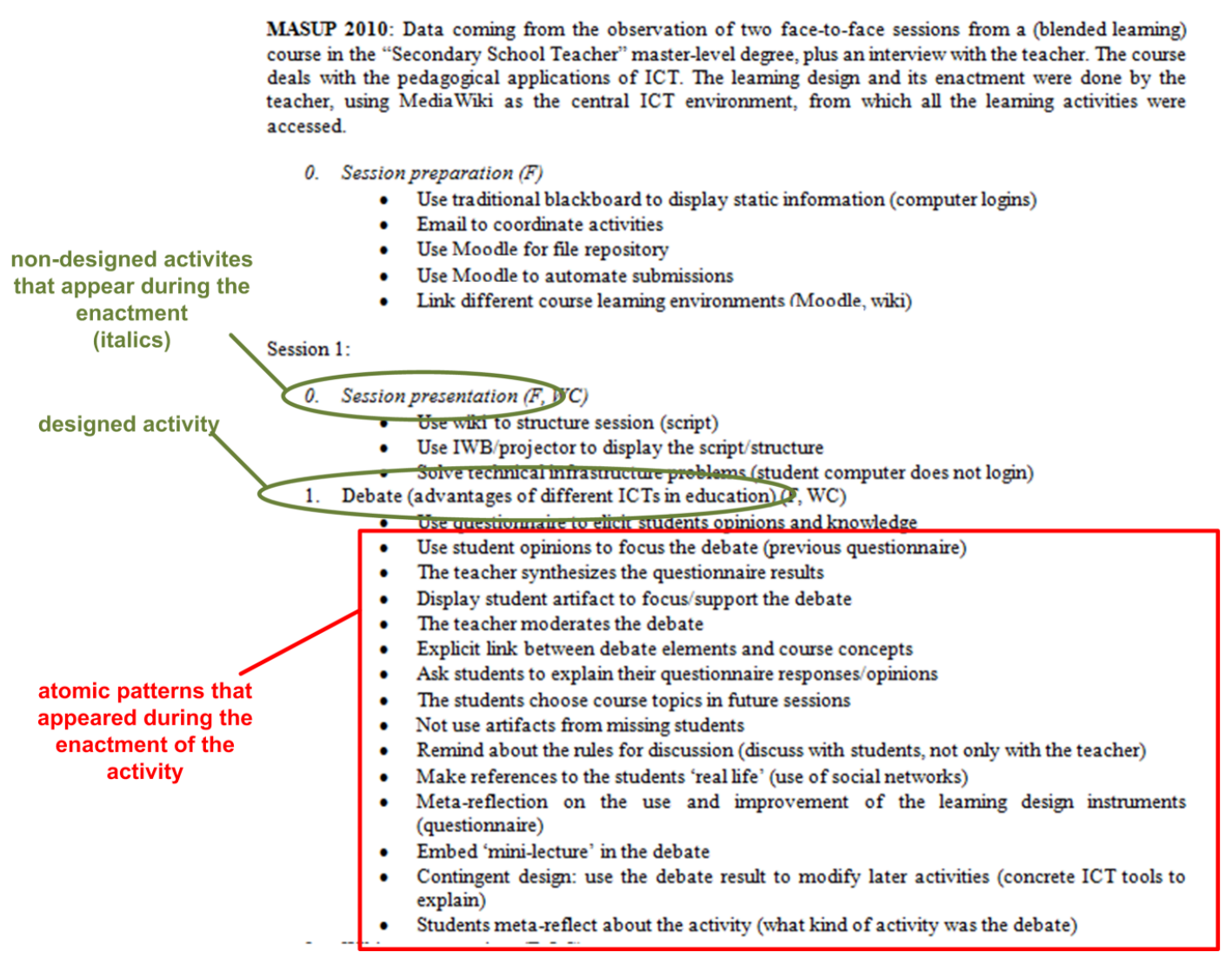

Figure 4.8: Example enactment analysis of a teacher's CSCL learning design

the teacher orchestrated them. Here again, it is very important to count on different data sources and techniques for triangulation (observation notes, computer system log files, access to the actual activities in the learning platform, audio and video recordings of the lesson, interviews, etc). Moreover, if at all possible, multiple researchers should perform this analysis in parallel, for analysis triangulation purposes [Sta05]. In our case, we analyzed the observation notes, audio recordings and post-experience. Our deployment and enactment analysis produced a synthesized description of the activity enactment, both in narrative/outline form (see Figure 4.8), as well as through graphical representations depicting the different activity phases, and the social levels and tools that were used at each moment, by teachers and students [Pri11c]. Additional examples from primary education are available in Pri11g.

Phase 2: Refine and classify From the design, deployment and enactment analyses we extract recurrent elements of teacher practice that have a certain pedagogical aim, or serve to help teachers to comply with the many restrictions of authentic settings (time restrictions, class management, discipline, etc). These recurrent elements are collected from the analyses and refined into "atomic patterns" (being "atomic" in this sense that they are not normally subdivided into smaller steps). As we have already mentioned, these atomic patterns do not follow the typical Alexandrian formalism of design patterns (problem, forces, solution, etc.), 
being at this stage formalized only through a title, a short description, and a number of examples of use in real practice. These atomic patterns are then gathered in a catalogue.

Moreover, since these catalogues may have a large number of atomic patterns, it is very important to classify them. Multiple classifications can emerge in this stage: according to the implementation phase in which they appeared (design time, deployment time or enactment time), specific tasks to which they are related (e.g. assessment, debates, teacher explanations, online work), specific technological elements that appear in them (e.g. related to the use of Moodle, Google Docs, the physical blackboard in the classroom), educational context in which they were elicited (e.g. primary vs. higher education), or other categorizations specific to the researchers' or designers' pedagogical focus. These classifications can ease the retrieval of patterns and the drawing of relationships with one another, and with contextual elements in the classroom situation.

In our example (see also Appendix B), we were able to extract 169 atomic patterns. In that Appendix we can find the patterns classified by primary and secondary orchestration aspects, although in the professional development workshops (see Section 4.5), they were often presented to teachers in a time-based classification (design-time, deployment-time or enactmenttime atomic pattern: 7 .

Phase 3: Combine patterns to foster learning design and orchestration Once a catalogue of categorized atomic patterns is in place, we can combine them with other available pedagogical patterns that have proved useful for learning design within our focus of interest (e.g. the pedagogical approach we wish to promote, such as CSCL). The pedagogical patterns may come from different origins: educational practice, pedagogical theories, or combinations of both Ret06. Aiming to foster the design and enactment of CSCL experiences, we combined the atomic patterns uncovered so far with the notion of Collaborative Learning Flow Patterns (CLFPs, see Section 4.2.3). In the following phase we show how these two kinds of patterns were combined into a concrete learning design for professional development.

Phase 4: Applying the patterns in the field: the design of a teacher workshop Once we have a clear idea of how to combine the elicited atomic patterns with other pedagogical patterns in our field of interest, it is finally time to apply this pattern set in authentic educational settings, to design activities, implement them and enact them (and, eventually, to evaluate and refine the pattern set itself, which may always be improved).

For example, we used the aforementioned approach combining CLFPs and atomic patterns in designing a professional development workshop for university teachers, to help them in designing and enacting non-trivial CSCL activities in their everyday practice. We designed the first part of the workshop using this very same approach that we were presenting to the teachers, so that they could experience a CSCL activity of the kind they were learning about.

Thus, the first 3-hour face-to-face session of the workshop was designed following the Pyramid CLFP as the basic structure. In this case, the Pyramid's activities tried to tackle the problem of designing a small CSCL activity (also using the Pyramid CLFP and a subset of the

\footnotetext{
${ }^{7}$ In the dissertation CD, an Excel spreadsheet is available with the complete catalogue, classified by orchestration dimensions and also by time-based categories and educational settings of origin.
} 
elicited atomic patterns printed in cards). This general session design was enriched using our atomic patterns catalogue, as it can be seen in Table 4.4 .

Finally, it is important to note that having elicited those atomic patterns from real practice can also help in facing unexpected occurrences, both by taking these patterns into account when designing, as well as a reminder during the enactment of available options to react on-the-fly. For example, knowing that very often you have to "change face-to-face activity to online" because of time limitations (a common enactment-time atomic pattern), the teacher may decide that it is better that the peer review is done using a wiki instead of pen and paper (which makes the change to online work more seamless). Also, for instance, knowing that you may want to do "on-the-fly assessment" (another enactment atomic pattern) may prompt you to open all groups' online documents in a browser during the activity, for easier monitoring of the groups' advances.

Table 4.4: Learning design of one of the workshop sessions in a teacher workshop, using a Pyramid CLFP as the general structure, and indicating the atomic patterns that were used to enrich it

\begin{tabular}{|c|c|c|}
\hline Activity & Grouping & (partial) List of used atomic patterns \\
\hline Session presentation & Whole class & $\begin{array}{l}\text { - Introduction to the subject/experience } \\
\text { - Anticipate script } \\
\text { - Centralized file repository } \\
\text { - Distribute participants physically to facilitate interaction } \\
\text { - Explicit connection between activity and learning objectives } \\
\text { - Use traditional blackboard to show static information } \\
\text { - Use wireless mouse as a computer remote }\end{array}$ \\
\hline $\begin{array}{l}\text { First design phase } \\
\text { (Pyramid Level 1) }\end{array}$ & $\begin{array}{l}8 \text { groups, } 2-3 \\
\text { people each }\end{array}$ & $\begin{array}{l}\text { - Conceptual mapping } \\
\text { - Form groups } \\
\text { - Teacher determines group formation } \\
\text { - Students choose their work topic } \\
\text { - Wander and solve doubts }\end{array}$ \\
\hline $\begin{array}{l}\text { Peer review (Pyramid } \\
\text { Level 1) }\end{array}$ & $\begin{array}{l}8 \text { groups, } 2-3 \\
\text { people each }\end{array}$ & $\begin{array}{l}\text { - Peer review } \\
\text { - Freeze intermediate artifact } \\
\text { - Reuse generated artifacts } \\
\text { - Use ICT for persistence of intermediate artifacts }\end{array}$ \\
\hline $\begin{array}{l}\text { Second design phase } \\
\text { (Pyramid Level 2) }\end{array}$ & $\begin{array}{l}4 \text { groups, } 5 \\
\text { people each }\end{array}$ & $\begin{array}{l}\text { - Conceptual mapping } \\
\text { - Group computer use } \\
\text { - Teacher determines group formation } \\
\text { - Make monitoring explicit } \\
\text { - Monitoring web browser } \\
\text { - Pen and paper plan B } \\
\text { - Teacher chooses tools } \\
\text { - Use ICT for persistence of intermediate artifacts } \\
\text { - Use ICTs for real-time monitoring } \\
\text { - Use open-access ICT to do peer review } \\
\text { - Use shared whiteboard to make student concepts explicit }\end{array}$ \\
\hline Break & Whole class & - Take a break during the session \\
\hline $\begin{array}{l}\text { Medium-group design } \\
\text { presentations (Pyramid } \\
\text { Level 3) }\end{array}$ & Whole class & - Oral presentation \\
\hline Debate (Pyramid Level 3) & Whole class & $\begin{array}{l}\text { - Debate/Discussion } \\
\text { - Synthesis / comparison among presentations } \\
\text { - Use IWB to trace conversation/debate }\end{array}$ \\
\hline Tool demo \& closure & Whole class & $\begin{array}{l}\text { - Meta-reflection } \\
\text { - Demonstration } \\
\text { - Discussion synthesis }\end{array}$ \\
\hline
\end{tabular}




\subsubsection{Advantages, disadvantages and challenges of the approach}

Thus, we hypothesize that this multi-level approach to Learning Design has the following advantages, which mainly stem from the notion and usage of atomic patterns:

- The atomic patterns are easy for practitioners to identify and to use, because they are simpler and only slightly formalized (as opposed to the typical Alexandrian pattern, which is much more complex and formalized). Also, the fact that these atomic patterns have been extracted from real practice and contain explicitly classroom contextual elements, make them easily identifiable for a practitioner.

- The atomic patterns allow practitioners to reflect not only about the design but also about the enactment of activities in the classroom, and its many aspects and restrictions. In this sense, atomic patterns could be a useful tool to guide practitioners in the orchestration of complex activities in TEL scenarios.

- Although this approach may also be useful for expert teachers, it is especially indicated in the case of novice (even pre-service) teachers and learning designers, and for those who have very limited experience with the use of ICT for education. Atomic patterns, rather than being normative rules about classroom practice, can provide nice starting points to bootstrap their practice with ICT in the classroom.

However, this method is not without its disadvantages:

- The first, most obvious disadvantage of this approach is that it is quite costly in terms of time and effort for the researchers and/or teacher trainers who apply the method, since it requires the observation and analysis of teacher practice in authentic settings, to extract atomic patterns. Naturally, if educational contexts are considered similar enough, researchers can also use existing catalogues (such as the one used in this dissertation) as a starting point.

- Related to the previous one, it is important to note that the application of these atomic patterns is strongly contextual, and thus atomic pattern catalogues may not be completely reusable across very different contexts. However, even if the complete catalogue of patterns is not entirely reusable, given their number, and following the principles of "naturalistic generalization" [Sta05], a part of the catalogue might probably be applicable to different contexts.

- Given that the atomic patterns are extracted from existing teacher practice, this approach has limited power in developing revolutionary designs, from a pedagogical point of view. This disadvantage might be alleviated, however, by using research-driven atomic patterns (or derivating atomic patterns from the practice of highly innovative teachers).

Among the main challenges that the application of this approach poses, the difficulty of abstracting, refining and classifying the atomic patterns is probably the most prominent (e.g. obtaining feedback from real contexts about the atomic patterns effectiveness, triangulating the 
design and enactment data and analyses, etc.). The proliferation of atomic patterns may also be problematic (tens or even hundreds of them may emerge from the application of this approach); the sheer number of available patterns may make it difficult for practitioners to manage and use them in practice. This section has outlined some possible criteria for classification and usage, although more formal databases, classification schemes and refining instruments can be very helpful in making atomic patterns and this overall approach more sustainable and easy to use. In fact, supporting the reusability of practices with value among practitioners is not a trivial issue, and it remains an open challenge in learning design in general. For instance, the design principles database (DBP) Kal08 as a pedagogical model that aims to assist graduate students in the educative design of technology-enhanced curriculum modules, provides a good example of this kind of system. Also, in the field of CSCL, Cha11 proposes a pattern ontology for the design of CSCL scripts, in order to assist practitioners in selecting a set of patterns for the design of meaningful CSCL scripts.

Overall, this approach tries to acknowledge and address the complexity of designing and enacting CSCL activities in a "messy classroom". To that end, we have considered the combination of "atomic patterns" (which are finer-grained, less-formal, and contextual) with existing pedagogical patterns (more abstract, formal and de-contextualized) to build enriched learning designs. Thus, by providing a process to elicit and combine these patterns (e.g. by researchers or teacher educators), we hope to eventually help practitioners in reflecting about the implementation and enactment of activities, the deviations that often take place, the inevitability (even the necessity) of improvisation and the non-linearity of the whole learning activity lifecycle.

\subsection{Other uses of atomic patterns}

Up to now, we have been discussing the usefulness of atomic patterns to support teachers in the orchestration of ICT-supported activities, such as CSCL. However, we have already hinted at the usefulness of this concept for other actors in the orchestration of a TEL/CSCL scenario. In these section we describe the two main alternative uses of atomic patterns, for researchers and for educational technology designers.

\subsubsection{Representing orchestration through atomic patterns}

Researchers and learning designers have tried to find new ways of conceptualizing their designs in a graphical way, both to communicate with others and for themselves to reflect and create new designs. However, few representations of a learning design take into account how the activities will actually unfold when the design is enacted in a classroom. As it has been already hinted in Section 4.2.1, this dissertation proposes a graphical representation for (CSCL) learning designs, which focuses on depicting how the activities are actually enacted by the teacher, using the notion of atomic patterns. By using this conceptual tool, researchers/designers can provide more detail into how the different elements of the classroom ecosystem can be used (as well as by teachers themselves to enrich their own learning designs).

Learning design practitioners have used a number of verbal, textual, visual and data-driven representations to depict the most salient aspects of the designed learning activities, at different 
levels of granularity (see Con10b for an overview). However, no matter how detailed a learning design representation is, it does not unequivocally depict what should happen in the classroom as that learning design is enacted, e.g. by a teacher. Moreover, the same learning design can be implemented in many ways, some of them successful, some of them utter failures, depending on a myriad of contextual factors, minute teacher actions and unexpected events that are bound to occur in the classroom's "messy environment". This often calls for teachers flexibly changing elements of the design and their own performance, in a "disciplined improvisation" [Saw04] that we have come to identify as one of the main aspects of orchestration (see Chapter 3).

As the number of elements to be taken into account when designing learning for a modern classroom increases (multiple ICT and non-ICT tools, educational resources, different kinds of activities, pedagogical approaches and theories, etc), the practice of Learning Design is becoming more complex for design practitioners (and teachers who often fulfill that role). Furthermore, it is also becoming more complex for the teachers that ultimately have to understand and enact those designs effectively, often on the basis of learning designs that do not take many contextual factors of that classroom into account.

Up to now, most learning design representations only considered a few of these contextual elements (for obvious practical reasons, such as time constraints and cognitive load). Following our observations of teacher practice in primary and higher education (see Dim11b), during this dissertation we have proposed a new form of representing learning designs which tries to take into account many of these elements of classroom orchestration. This kind of representation provides a more detailed view of a learning design's activities, focusing on the most prominent elements of classroom orchestration, such as the social level where the activities should occur, the tools and resources to be used, and especially how those tools should be used in the classroom in order to further the pedagogical aims of the design. This latter aspect is included in the design in the form of the already presented atomic patterns.

This kind of representation has been used by the author and colleagues as an analysis tool in order to understand how teachers' learning designs are transformed during classroom enactment (see Pri11g] and Section 4.2.1). This kind of representations, as presented here, share typical benefits with other learning design representations (e.g. making the learning design - and its enactment, in this case - explicit and shareable). Nevertheless, the representation could also be developed further, into more complete computational representations of learning designs, in the vein of, e.g., IMS-LD.

Basic template: activities, tools and social structures The most basic form of this representation is a variant of the "task swimlane" kind of representation, or the "link and node" representation, commonly used to provide a micro-level view of the learning design (e.g. spanning only a few hours of a lesson, see Con10b ). However, there are a number of interesting additional traits in this kind of diagram, which can be seen in Figure 4.9.

- In a similar vein as done in Dil08a and many other works on CSCL scripting, the representation depicts the different social planes where the learning activities may occur (individual, small group or class-wide), as horizontal lines where the different tasks are positioned, making the task flow jump from one plane to another. 


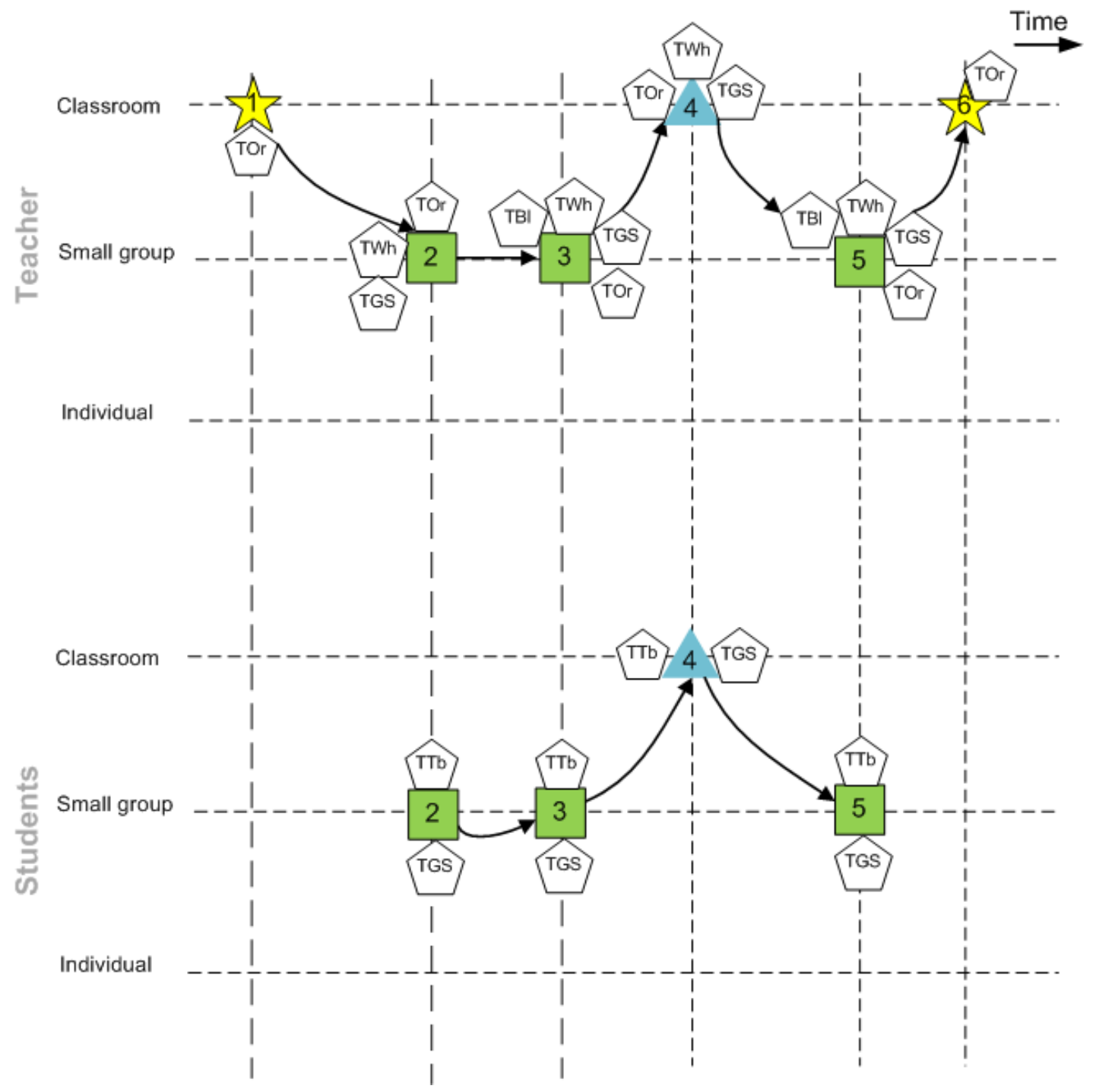

Figure 4.9: Example representation of a learning design. Basic template depicting roles (horizontal swimlanes), social levels (horizontal lines within each swimlane), tasks (colored forms) and tools (white polygons). 
- The different kinds of activities and tasks are depicted using colored, numbered nodes, to indicate different categories of tasks (e.g. explanation, support/student task, assessment), which are further explained in the legend (each number corresponding to one atomic design pattern, such as "Where is on the image?", see [Pri10a ).

- The different tools and resources available in the classroom, which should be used for each task, are depicted as white, pentagonal nodes (i.e. TTb indicates the use of a Tablet PC).

- Since teachers and students often play different roles and use different tools throughout the different phases of the learning design, separate "swimlanes" are provided for students and for the teacher to indicate their respective activities and the tools used.

Adding enactment elements (atomic patterns) The aforementioned template already provides a reasonably detailed description of many of the elements in the orchestration of a learning design. However, it still lacks one important element that constantly arose in our observation of teacher orchestration in authentic classrooms: the orchestration not only depends on which activities learners perform, or which tools they use; rather, the learning outcome is heavily influenced by how the teacher coordinates those elements through her concrete actions in the classroom $[$ Pri11d].

Our approach to representing learning design and classroom practice makes use of the notion of atomic patterns (Section 4.2), representing them through the white, rounded nodes that are attached to each of the different phases in the teacher's task swimlane in Figure 4.10. These nodes are further explained in the legend to the right of the figure (e.g. R2a indicates "Using a whiteboard to exemplify" a task so that learners understand the task and the usage of the related software tools).

It is worth noting that the graphical representation in Figure 4.10 is only one of the possible ways of depicting these orchestration elements in a diagram following the aforementioned principles. The reader may refer to [Pri10a] VS11] Pri11g for variations of the same representation, each one emphasizing different aspects of our analyses.

Practical representations: Using atomic patterns for design in teacher workshops As we have mentioned, the proposed representation is a useful analytical tool for researchers to investigate and describe what happened in the classroom, when compared to the original learning design. However, this idea of using atomic patterns in conjunction with other orchestration elements in representations also has other uses more directly related to the practice of learning design, either by teachers or by design practitioners. In fact, given that these atomic patterns are normally extracted from real classroom practice, and that they make direct reference to classroom objects, this kind of representations could be suitable as part of a "professional language to describe and communicate [teachers'] design ideas" [Law11].

As we have already mentioned, and will show further in Section 4.5, this idea of atomic patterns has been used in professional development workshops both in primary and higher education settings, to support orchestration of collaborative learning activities with ICT, as well as part of a learning design approach where different kinds of design patterns are utilized by teachers to design and reflect about the enactment of activities (Section 4.3). In those workshops, 


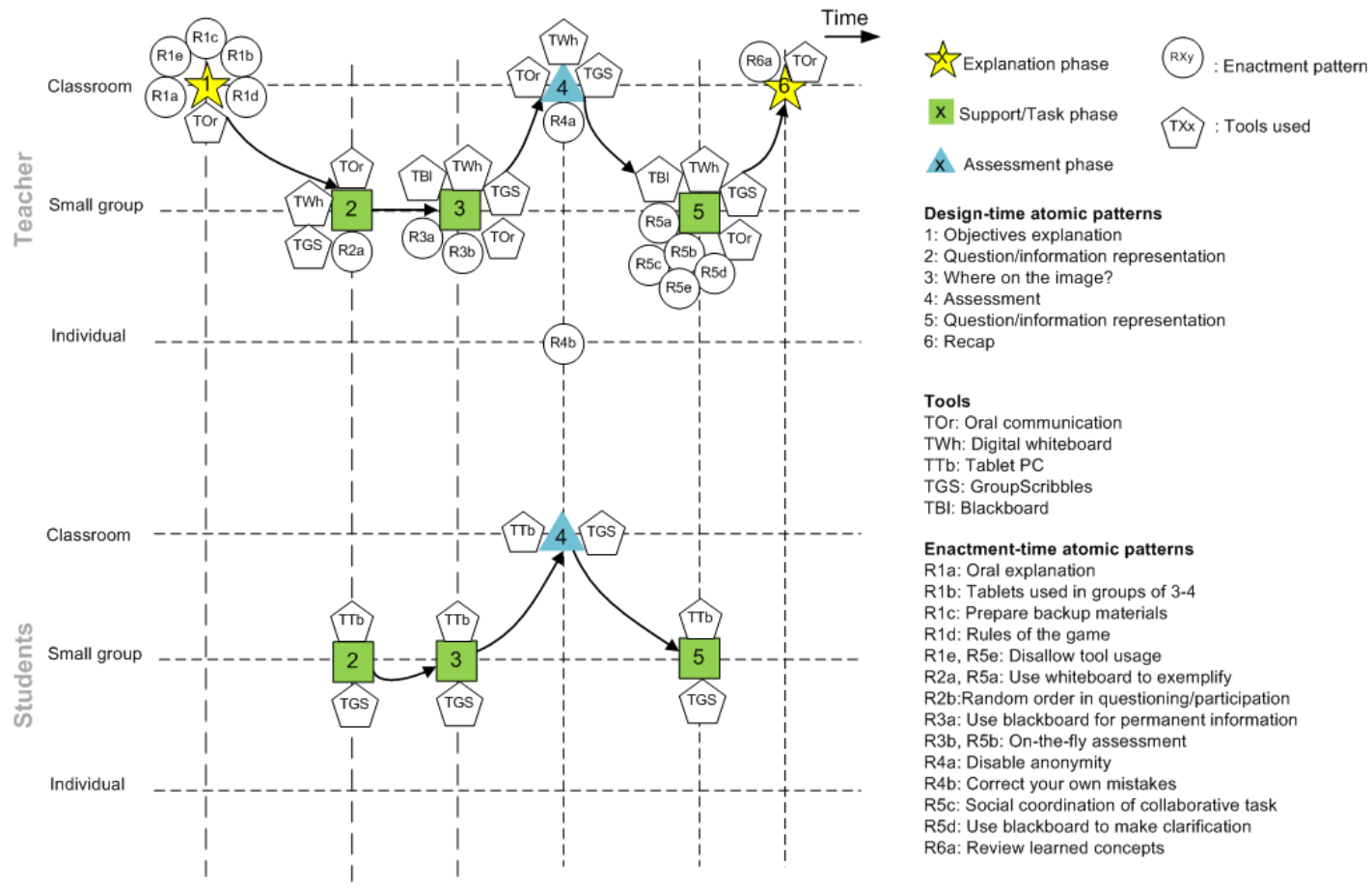

Figure 4.10: Example representation of a learning design. Completed template with the atomic patterns present in each phase (white circles).

teachers devised (normally using pen and paper) ad-hoc graphical and textual representations of their learning designs, further enriching them with atomic patterns. In several cases, teachers spontaneously used the atomic patterns (which were provided as paper cards) in such design representations, marking the phase in which they would use a certain atomic pattern. Figure 4.11 shows two examples of teacher-made designs that use representations of atomic patterns. This remarkable use of atomic patterns hints that teachers intuitively saw the usefulness of atomic patterns to flesh out the general structure of the learning activities, in a more detailed way.

\subsubsection{Using atomic patterns to develop technology}

As the reader may notice from the atomic pattern catalogue in Appendix B, among the heterogeneous set of atomic patterns that we elicited from primary and higher education practice, we can find examples of atomic patterns that are (or can be) enacted/automated using ICT tools (e.g. "Use results of a task in a different task", "Monitoring web browser", "Peer review"), while others relate more to social coordination of the orchestration (e.g. "Punishment", "Remind about debate rules"). Thus, as we developed the concept of atomic pattern, we wondered to which extent these atomic patterns, as recurrent elements of orchestration practice, can be taken as good indicators for the development of "orchestrating" or "orchestrable" technologies 8 . However,

\footnotetext{
${ }^{8}$ This distinction was recently proposed by P. Tchounikine Tch11, to separate technologies that "achieve or support the activity of orchestrating" (orchestrating) from technologies "which use can be decided or adapted [...] by the actors in charge of the orchestration [...] while orchestrating the setting" (orchestrable).
} 


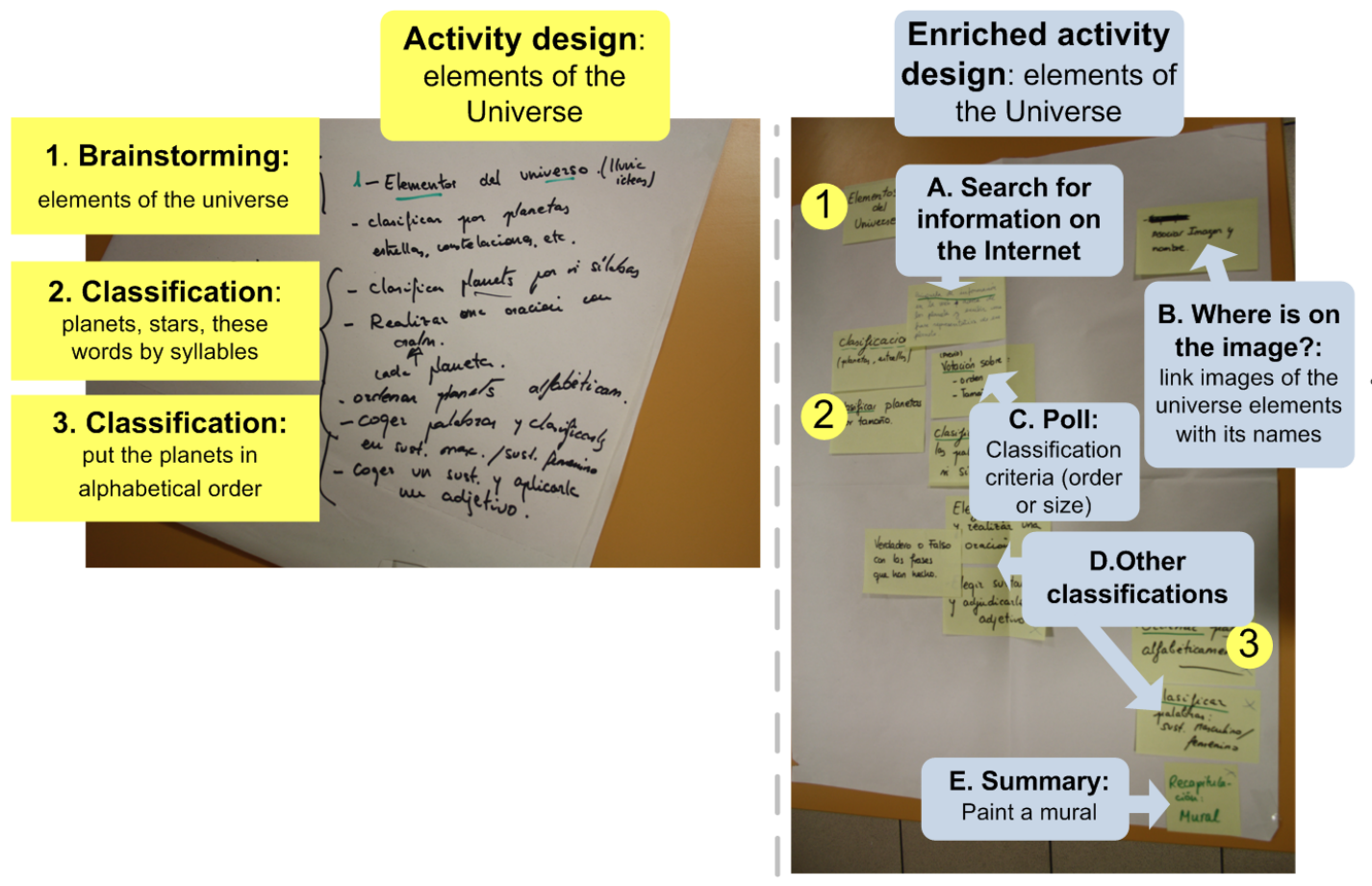

Figure 4.11: Example teacher-generated representation of a learning design enriched with atomic patterns, taken from one of the teacher workshops

we chose to keep this concern out of this dissertation's main research questions, which were about the usefulness of atomic patterns as conceptual tools for teachers as the main orchestration actors in the contexts we were to study.

In fact, one could see atomic patterns as a particular form of use cases [Jac92, a common entity used in software engineering for the elicitation of requirements for a software system. Use cases basically express a system's expected functionality in terms of a series of steps, in which an actor (e.g. a human agent or another system) interacts with a system, towards a concrete goal. Use cases, like design patterns, tend to be expressed in abstract terms, although additional contextual information can be added to them [Coc01]. Thus, given atomic patterns' higher contextuality, they might rather be compared with user stories [Coh04], a device used in agile software engineering processes Bec01] to guide development of a system. Although the difference between use cases and user stories has not been clearly set 9 generally speaking user stories tend to be more informal and contextual, but the main difference is the writer of the device: use cases are typically written by the development team, while the user stories are written by the different stakeholders of the system (e.g. a customer or and end-user). Also, use cases tend to focus more on the behavior of the system to be built (and thus, completeness and correctness are important in them), while user stories normally are about the user's needs (and clarity for end users is a crucial value in their description).

During this dissertation, we have experimented with the usage of atomic patterns to guide

\footnotetext{
${ }^{9}$ See the historical discussion within the agile and software engineering community, available at http://c2. com/cgi/wiki?UserStoryAndUseCaseComparison (Last visit: 30 May 2012).
} 
the development of technological tools to support orchestration, such as the GLUE!-PS architecture to be presented in Chapter 5. As it will be described in more detail in Section 5.3.2, we have chosen a subset of the atomic pattern catalogue from blended CSCL in DLEs (those that appeared more frequently and had a clearer technological role), and we have used them to build the GLUE!-PS implementation roadmap within the scope of this dissertation.

The adequacy of atomic patterns as requirement indicators for the development of educational technologies that support current orchestration practice, however, has not been directly evaluated within this dissertation, due to a lack of resources. We have indirect evidences of usefulness from the point of view of teachers as end users of the system: for example, in the last teacher workshop to evaluate the GLUE!-PS system (TW6, see Section 5.5), 24 teachers were confronted (in a role-played exercise that included the usage of GLUE!-PS) with three problematic situations that required flexible adaptation of the activities to face unexpected situations (e.g. students not showing up, or additional ICT resources being needed in enactment-time). These situations, based on atomic patterns, were rated as frequent by the 21 teachers that answered the questionnaire (average of 7.19 in a 1-8 Likert scale [TW6-Q2]), and the solutions provided by GLUE!-PS were rated as useful (average of 7.38 in the same Likert scale [TW6Q2]). Also, indirect evidences of usefulness can be gathered from the other positive feedback gathered in that same workshop, including the high usability ratings, or the fact that teachers' expressed concerns about the usage of the tools were more related to non-functional aspects such as reliability, technical support or the teachers' own beliefs about the adequacy of the CSCL approach in general (also explained in more detail in Section 5.5).

Apart from that, we only have the subjective evidence from our own experience as system developers during the dissertation, that atomic patterns (and their relative importances marked by the range of situations where they can be applied and their relative frequency of appearance in orchestration practice) can be useful to determine the roadmap of an educational technology such as the one presented in Chapter 5. The fact that these patterns indicate common moves and actions that teachers often take when orchestrating activities, may help system designers in supporting such actions, without prescribing the form such support should take (it may be by automating it, or by providing clear controls to enact it, indicators regarding when it could be applicable, etc.). Clearly, more evaluation would be needed in order to ascertain the usefulness of these atomic pattern catalogues for technology designers (e.g. by using them in educational technology design workshops, or by conducting a researcher/designer panel among educational technology experts). Also, further investigation about the best way of presenting those (numerous) sets of patterns to designers, integrating them in a more structured design process, would be in order.

\subsection{Evaluating atomic patterns: Four teacher professional de- velopment workshops}

As it has been described in the introductory chapter of this dissertation, and at the beginning of this chapter (Section 4.1, we have followed the engineering method Adr93 to structure our research towards providing conceptual tools that can help teachers with the lack of practical advice about how to orchestrate learning activities in their everyday practice. Throughout this chapter we have proposed the notion of "atomic patterns" as useful conceptual tools for 
teachers, and we have analyzed their properties and applications (including a multi-level pattern approach to their elicitation and use). However, it is in the evaluation phase of each iteration where the trustworthiness of the proposals is better demonstrated.

In the rest of this section we describe our evaluation of atomic patterns through four professional development teacher workshops, two of them held in a primary school in a rural area near Valladolid (Spain), and the other two in the context of higher education, as part of the University of Valladolid's "Centro Buendía" courses for professional development. These four workshops provided evaluation material for the iterative development of the atomic patterns and the multi-level pattern approach described throughout this Chapter. More concretely, they explored the value of these two contributions with regard to the following research question (also mentioned in Section 4.1): "Can we provide conceptual tools to support (non-expert) teachers in orchestrating learning? (sp. in blended CSCL using DLEs)" (RQ2.1).

In order to provide insights into this question, this Section is structured as follows: first, we outline the general context and method of the evaluation (4.5.1), and then we describe each of the four evaluation iterations, outlining their peculiarities, the findings and selected supporting evidence, closing with remarks about how those findings influenced the following iterations 4.5.2, 4.5.3 4.5.4 and 4.5.5). A global wrapping-up and discussion of the evaluation closes this Section (4.5.6).

\subsubsection{Context and method of the evaluation}

As it was outlined in Section 1.2, we have followed the CSCL-EREM framework JA09b to design the evaluation phases across the four iterations in the development of atomic patterns. Following the framework's emphasis on responsiveness, the evaluation presented here has evolved, progressively focusing [Sta10] on emergent issues uncovered in previous iterations. Also, it should be noted that this evaluation developed together with the research on the other thesis contributions, presented in the previous and following chapters.

This evolution prompted us, first, to study the role of atomic patterns in orchestration and the support they gave to teachers in a primary school classroom context (Iterations \#1 and \#2 in Figure 4.2, where our first exploratory studies on orchestration took place [Pri09]. As the PhD work unfolded and we centered on blended CSCL practice that used Distributed Learning Environments (DLEs), the proposed atomic pattern catalogue and the multi-level pattern approach described in this chapter were adapted and applied in such settings (Iterations \#3 and \#4 in Figure 4.2). This progressive focus provided us with a remarkable opportunity to to test the adaptability and applicability of the proposed contributions to an entirely different technological and educational context.

In order to evaluate our atomic pattern proposal in each of those iterations, we chose to perform professional development (PD) teacher workshops in which participants (teachers, in this case) joined with the main goal of acquiring new skills (as opposed to participation for the sole reason of providing data). The choice of such "situated experiments" ${ }^{10}$ was motivated by the emphasis of orchestration research in the needs and constraints of average teachers in authentic

\footnotetext{
${ }^{10}$ Throughout the dissertation we will use the term "experiment" in a general sense of "data-gathering event" (as opposed to denoting an adherence to experimental principles such as the control of variables). In previous works by the author's research group (e.g. HL07]), the term "experience" was used to denote data-gathering events,
} 
settings Dil10]. Data gathering in authentic classrooms (e.g. where an individual teacher orchestrates activities using atomic patterns) could have also been used for this purpose. However, teacher workshops had the advantage of letting us reach a higher number of teachers, from a wider variety of disciplines, and with a more "average" profile (i.e. normally, only enthusiasts or experts agree to introducing such novel devices in their classrooms right away, while receiving a short course does not force participants to commit to an innovative practice). Nevertheless, it should be noted that these PD workshops can be very powerful instruments in contacting teachers that could eventually agree to participate in more detailed authentic experiments - several workshop participants already showed interest in applying this approach in their classrooms.

Despite the fact that we have two different contexts where the evaluation has been performed (primary school and higher education) and that, consequently, each evaluation had certain different contextual elements (e.g. participants and their profiles, or the educational setting features), we will represent the evaluation design with a single diagram, which can be seen in Figure 4.12. As we can see, there are several elements which remain invariant throughout the evaluation process: most importantly, the Evaluand (i.e. atomic patterns), which determines this as being a single evaluation, but also the evaluators and their Perspective (goals, issues, etc.) remain the same throughout the whole evaluation process.

Thus, as we can see in the figure, our Perspective as evaluators can be described through our Goal, which is to support teachers in orchestrating learning activities, through the use of atomic patterns. The main Evaluator is the author of this dissertation, although at different points of the evaluation, he was supported by a multi-disciplinar team of up to 5 researchers from the GSIC-EMIC group, who had previous experience in the evaluation in this kind of contexts. The Issues in this evaluation evolved with time (progressive focusing, see [Sta95]) but, generally speaking, they revolved around our main research question (RQ2.1): "Can we provide conceptual tools to support (non-expert) teachers in orchestrating learning? (sp. in blended CSCL using DLEs)". Given the different evaluation contexts throughout the work on atomic patterns, the first two iterations had a global evaluative tension which we could formulate as: "Do atomic patterns support (non-expert) teachers in the orchestration of activities with ICT?" (I1a). Afterwards, in our re-focusing for higher education contexts, this issue was also re-focused ("Do atomic patterns support (non-expert) teachers in the orchestration of blended CSCL activities using DLEs?", I1b), and a new emergent but secondary issue appeared ("Is it useful to have atomic patterns embedded into technology, and in which form?", I2). Moreover, these tensions or issues were in turn explored through a series of topics, which were used to group our informative questions. This conceptual organization of the data from the evaluation is adapted from Huberman \& Miles's anticipated data reduction procedure [Hub94] (which is quite typical in qualitative data analysis), and it is graphically represented in Figure 4.13

The Ground of our evaluation is represented centrally by the notion of atomic patterns as they have been presented throughout this chapter. The main Stakeholders of the evaluation have been the 61 teachers participating in the different iterations of the study (16 in the primary school, and 45 in the higher education workshops), although other stakeholders can be mentioned, such as the primary school principal (very interested in the integration of ICT in the school) or the masters-level program director who promoted the third PD workshop among its

or "happenings" as the CSCL-EREM framework calls them. However, several native English researchers have advised us against this use of the word "experience", which seems to be incorrect. Therefore, in this dissertation we use the term "experiment" in this general sense of "event where data is gathered". 


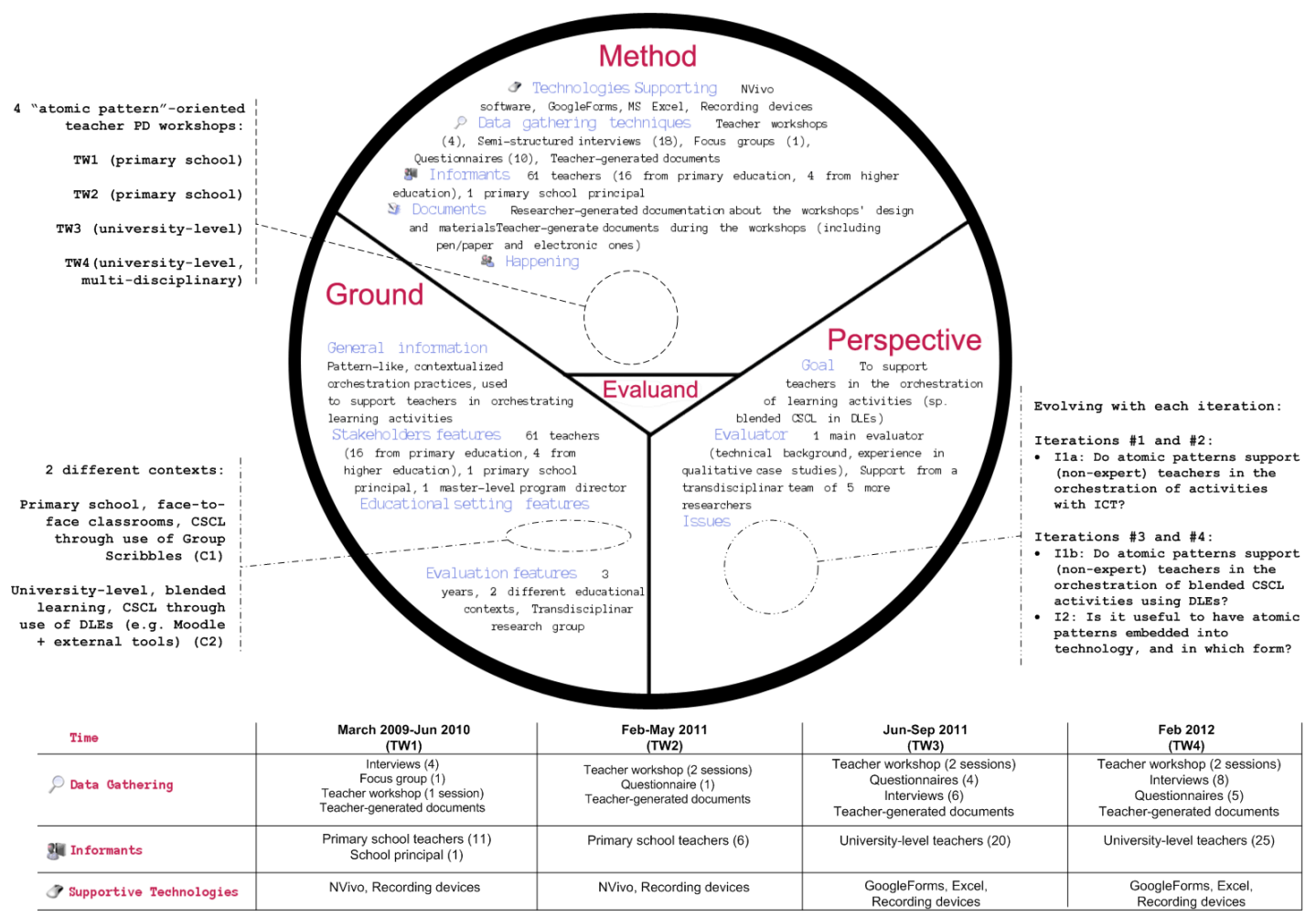

Figure 4.12: Graphic representation of the evaluation design of atomic patterns as a conceptual tool for orchestration 


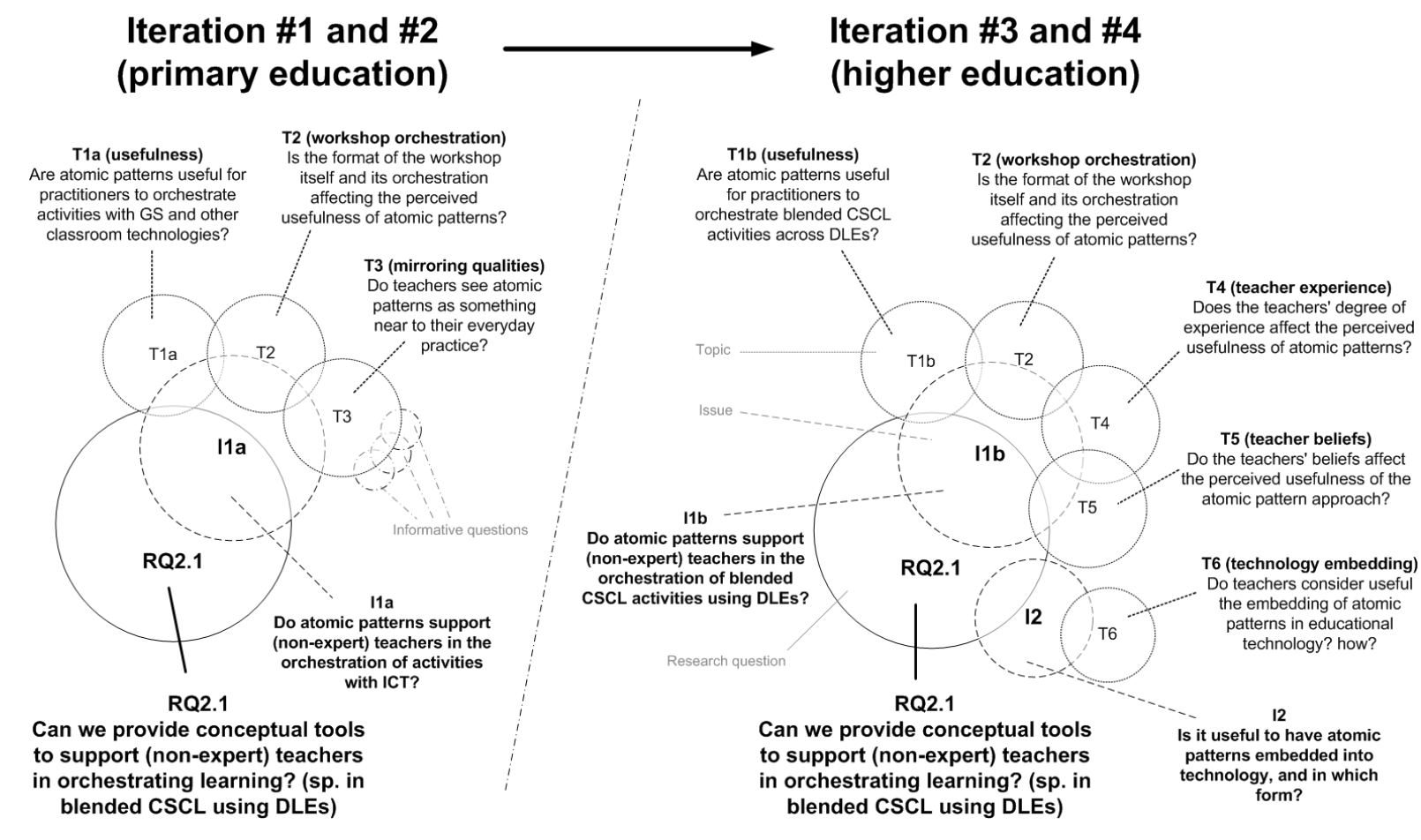

Figure 4.13: Graphical representation of the research questions, issues, topics and informative questions used during the evaluation of atomic patterns, as the research effort evolved 
professorship. This Evaluation took place at different points in time during 3 years (2009-2012), involving several members of a trans-disciplinar research group, and spanned two different $E d$ ucational settings: (C1) a primary school with high penetration of ICT resources and a focus on promoting CSCL in face-to-face classrooms through the use of the Group Scribbles tool, and $(\mathrm{C} 2)$ the professional development program of a university, in which the focus was on the promotion of blended learning using CSCL activities that made use of DLEs (especially those based on Moodle, the institutional platform).

Finally, the Method we have chosen in the evaluation included a variety of Data gathering techniques, such as observation and recording of face-to-face sessions, semi-structured interviews, paper- and web-based questionnaires, focus groups, and analysis of the teacher-produced artifacts (e.g. teacher designs done during the workshops). The Supporting technologies used during the evaluation included NVivd ${ }^{11}$ qualitative analysis software $\left[\right.$ Ric99], MS Exce ${ }^{12}$ for quantitative and qualitative analysis, PiratePad ${ }^{13}$ and GoogleForms ${ }^{14}$ for web-based questionnaires, or diverse recording devices. Our Informants were the aforementioned 61 teachers (plus the school principal), and the Documents that were available for analysis included teacher-generated artifacts and our own design and enactment materials to support the workshops. Nevertheless, we will provide more detail about the contextual and methodological peculiarities of the evaluation in each iteration, at the beginning of the following subsections 4.5.2, 4.5.3, 4.5.4, and 4.5.5).

It is also worth mentioning the way we have applied the mixed methods approach to the data gathering and analysis during these evaluations. As outlined in Section 1.2 , we have applied a variation of the mixed methods approach to studying CSCL, first proposed in Mar06b]. In the concrete case of the evaluation of atomic patterns, we have followed a slight variation of the flow depicted in Figure 1.5, to account for the contextual differences in each setting (such as the different availability of data sources). A particularized data gathering and analysis diagram can be seen in Figure 4.14. As we can see there, several data gathering techniques have been used (depending on their applicability to each context) to obtain triangulated evidence about the participant teacher's profiles, including their prior experience, background, technological and pedagogical beliefs, etc. During the different happenings (the workshops), participants were observed by at least one researcher, and photos, audio and video recordings of the sessions were made, whenever possible; in some cases, questionnaires were presented to the participants during the workshop, to better assess the evolution of their opinions and reflections. Finally, semistructured interviews and questionnaires were used after each experiment, to obtain triangulated feedback and reflections about the atomic patterns' usefulness. All this (mostly qualitative, but also quantitative) data was then analyzed, using qualitative and quantitative (descriptive statistical) analysis techniques, triangulating evidence where possible. This analysis led us to partial conclusions which were used to decide the issues to focus on in the following evaluations, as well as to generate our global conclusion about atomic patterns as a conceptual tool for teacher orchestration.

One interesting feature of this process, which cannot be fully appreciated in this graphic, is the fact that the first two iterations (happening in primary school) were exclusively qualitative in the data gathering and analysis performed (due to the exploratory nature of these iterations

\footnotetext{
11 http://www.qsrinternational.com/products_nvivo.aspx (Last visit: 6 Jun 2012).

12 http://office.microsoft.com/en-us/excel/ (Last visit: 6 Jun 2012).

13 http://piratepad.net (Last visit: 6 Jun 2012).

${ }^{14}$ http://www.google.com/google-d-s/forms/ (Last visit: 6 Jun 2012).
} 


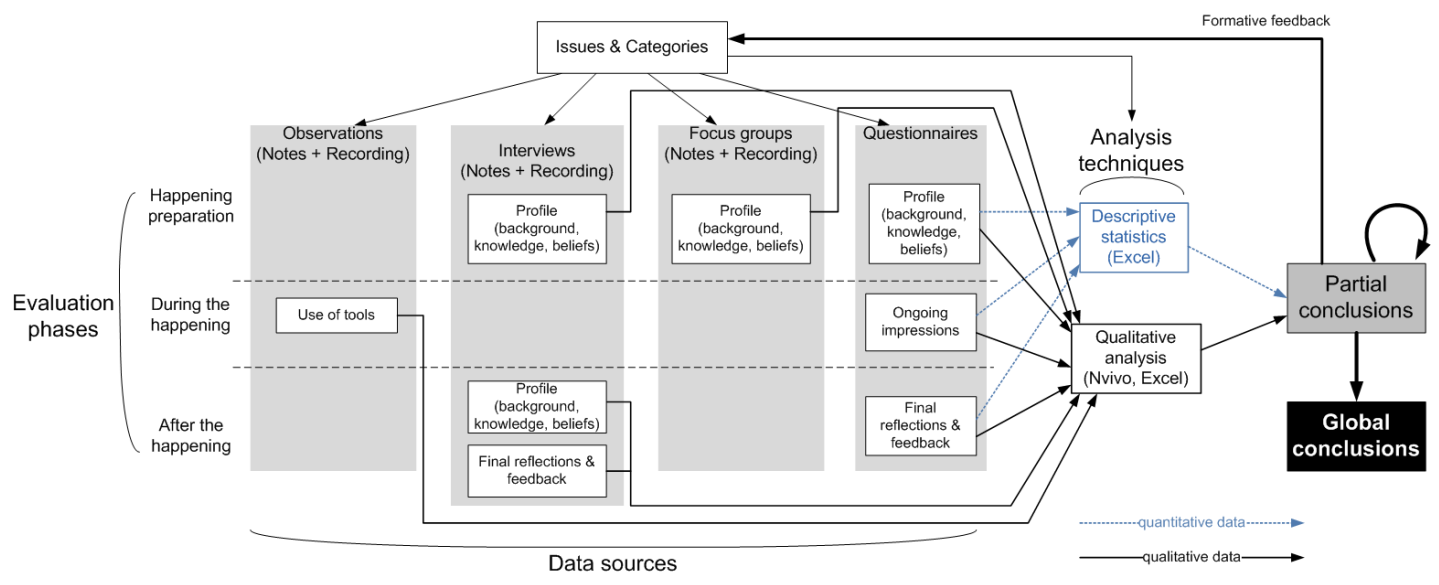

Figure 4.14: Graphic representation of the data gathering and analysis techniques flow during the evaluation of atomic patterns, adapted from Mar06b.

and the lack of prior theoretical frameworks specific to orchestration Cre03), while the third and fourth iterations also added quantitative data (in order to have first indications of the relative importance of the different issues and trends discovered during the study, and to further triangulate our evidence about atomic patterns' usefulness).

Finally, we should note that, as we did in the evaluation presented in Chapter 3 , in this section we have chosen the "findings/evidence tables + summarizing text" formula to present the evaluation findings and evidence. As described in Section 1.2, this is done to keep the dissertation's length at bay, while allowing readers to define their reading patterns more flexibly.

\subsubsection{Iteration \#1: First attempt at an atomic pattern teacher workshop in primary education (TW1)}

Before we describe the evaluation that took place in our first research iteration (i.e. after we first proposed atomic patterns as a useful tool for supporting orchestration of CSCL activities), we think it is important to go briefly over the research efforts that led to this proposa 15 . This will help the reader understand, not only our perspective as evaluators, but also will help in clarifying the origins of several prior assumptions and guiding issues that we used during the first stages of our research on atomic patterns.

As it has been already mentioned in Section 4.2.1, the author and colleagues had spent 6 months introducing a new CSCL tool in a primary school with high presence of ICT in the classrooms, to observe how teachers integrated this new tool in the existing classroom ecosystem Zha03 Luc08 and the mandatory curriculum, throughout the whole CSCL activity lifecycle Pri09 Vil09]. This exploration of teacher enactment already uncovered several factors that seemed to influence heavily the integration of new ICTs into the teachers' everyday practice: the concrete educational context of the school and its restrictions, the teachers' beliefs (e.g.

\footnotetext{
${ }^{15} \mathrm{~A}$ much more detailed account of these efforts, from a pedagogical and professional development point of view, can be found in Sara Villagrá's PhD thesis VS12. (only in Spanish).
} 
about pedagogy, about ICT, about collaboration), teachers' knowledge (e.g. background, prior training) and the concrete teacher practices typical of each teacher (exemplified by the designtime and enactment-time routines of each teacher - which later would crystallize into our notion of atomic patterns) Pri09].

After this first stage of exploratory research, in a face-to-face session with teachers to share the results of the study and discuss future lines of work, teachers expressed that their main interest was to "work in strategies to promote collaboration" (from the post-session poster shared with teachers, see VS12]). This led our research team to propose a teacher workshop based on the concept of Collaborative Learning Flow Pattern (CLFP, see HL06b] and Section 4.2.3), which had been successful with higher education teachers before [HL07].

Such a "CLFP-oriented" workshop was held at the primary school in November 2009. The feedback gathered from it, however, was less positive than the research team had expected: teachers saw CLFPs as something complex to put in practice in their immediate context, for different reasons such as them being rigid and artifical, or that children (6-8 years old) were considered too young to collaborate effectively. However, the research team also shared certain doubts that this rejection might be caused in part by the workshop's design and the scaffolding we provided, especially the lack of adequately contextualized examples of CLFP use [VS12].

After such a relative failure, the research team opted for trying to promote the sharing and reusing of collaborative learning designs among the involved teachers, and to reflect about their enactment. This was attempted through the development of a web-based tool where teachers could share (rather informal) designs and accounts of their enactment: ${ }^{16}$. As it is described elsewhere [VS12], albeit this platform was an idea proposed by the teachers and co-designed with them, the platform was rarely used by teachers, probably because of the lack of a clear added value and the lack of integration with the everyday practice of the school teachers, especially considering the tight time constraints that the everyday life in the school imposed on teachers. Other possible causes include the high turnover among the teacher staff (e.g. more than half of the teachers that helped in the design of the platform had to leave the school the next year).

All these subsequent research efforts into the promotion of CSCL activities that are integrated into the everyday practice of this concrete school, led to the study of atomic patterns as a tool to overcome the previous efforts' shortcomings. After the first elicitation and proposal of atomic patterns (Section 4.2.1), we proposed to study their usefulness through a new PD action: an atomic-pattern-oriented workshop, which we will denominate TW1 from now on.

\section{Context and method of the evaluation}

The Rural Grouped School (CRA in Spanish) "Ana de Austria" $[17$, in Cigales, is the head of a rural school spanning Cigales itself and three nearby villages (Mucientes, Fuensaldaña and Trigueros). The area of Cigales, located some 15 kilometers from Valladolid, is best known for its wine-making, the biggest industry in that zone. The school has 12 primary and 6 elementary classrooms, harboring a total of around 400 students (around 300 of them in Cigales) and 42

\footnotetext{
${ }^{16}$ This tool is called CReA-TIC, and it is still available online at http://www.gsic.uva.es/CReA-TIC/ (Last visit: 31 May 2012).

${ }^{17}$ Now renamed to "CRA Entreviñas", see http://craentrevinas.centros.educa.jcyl.es/sitio/ (Last visit: 27 Jun 2012).
} 
teachers. Even if the composition of the classrooms and their students is heterogeneous, all the villages share a Common Educative Project (PEC). This project establishes the main goals and principles of the school, derived from the ideal of "Educating for liberty, equality, solidarity, democracy and tolerance, respecting individuality and differences" [C. 09]. In most regards, this school does not differ greatly from other public primary schools in Spain.

One aspect, however, where this school is different from other rural primary schools is the ongoing effort (by its community and especially the directive staff) to gather technological resources to support education and other educative innovations. Even if most Spanish schools are immersed in several institutional projects and processes to introduce new technologies in the schools, the principals of the school have tried, for the past decade, to go further in this regard.

Thanks to public investments from the autonomic government, computers have been acquired, initially to populate the so-called "computer labs". The executive board of the school, however, decided later to progressively integrate the new computers in all ordinary classrooms (an innovative strategy that is still not widespread in Spain [Pla06]): in the first place, a single (fixed) computer was placed in each class, and later, Internet connections were added to them, allowing for a whole new set of capabilities, and converting the classroom into a more complex technological ecosystem. The process continued with the acquisition of whiteboards and projectors, and finally, of digital whiteboards and wireless tablet PCs, mostly through collaborations with educational hardware and software vendor projects. Most notably, in the last years a Moodle VLE for the school has been installed, and is now used by most of the teachers (mainly as a repository for storage of digital educational materials). The school had invested a lot of effort and money in obtaining ICT resources, and is still working on integrating them in their educational processes through a variety of projects. Even if several factors have hindered this integration (time constraints, staff turnover and the consequent lack of technical knowledge by new teachers), the school's teacher culture of high participation and peer-helping made any intervention to foster ICT usage more promising.

Within this research and educational context, our research team (formed by 5 transdisciplinar researchers including telecommunications engineers such as the author, but also pedagogists) designed and enacted a 2-hour teacher workshop for this primary school. The goal for participants was to share information about their designs and ICT practice, and to experiment with alternative ways to design and enact (i.e. to orchestrate) TEL activities. The session was to be structured as follows:

1. Teachers, in groups, outline the design of an activity around two curriculum items from different areas (e.g. Spanish language and Maths), one selected by researchers, the other proposed by teachers. The activity should make use of GS and other classroom tools. [15 minutes]

2. Design-time atomic patterns are presented, in the form of paper cards that included their name, a short description and 1-2 examples of use in the classroom context (see Figure 4.15). [10 minutes]

3. Teachers choose one of the activity outlines and try to "enrich" their activity ideas with the presented atomic patterns, using pen and paper (e.g. posters, cards, post-its). [15 minutes] 

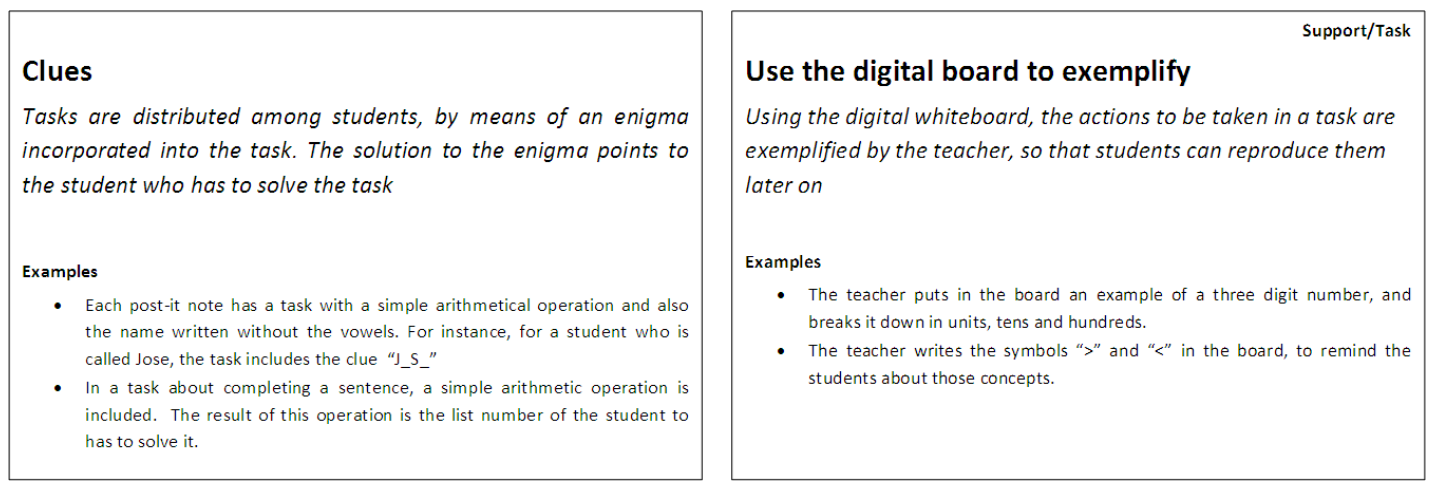

Figure 4.15: Example atomic pattern cards used in the workshop, both for design-time (left) and enactment-time (right) [Translated from the Spanish original]

4. Teachers answer a short online questionnaire about the presented atomic patterns. [10 minutes]

5. As a warm-up activity before focusing on enactment, teachers brainstorm common problems when enacting TEL activities in the classroom [5 minutes]

6. Teachers role-play the enactment of one of the activities they have designed, with one participant acting as the teacher, and the others acting as students. A number of problematic situations when enacting a TEL activity using GS (e.g. students stealing other students' virtual sticky notes) are role-played (see Figure 4.16). [20 minutes]

7. Teachers reflect about the problems that have emerged during the enactment, and possible solutions that may be used to solve them (i.e. to elicit new -or confirm existing- atomic patterns). [10 minutes]

8. Presentation of a selection of enactment-time atomic patterns, again printed in cards (see Figure 4.15]. [10 minutes]

9. Teachers answer a short questionnaire about the second set of presented atomic patterns. [10 minutes]

For the research team, the main evaluative tension has been outlined at the beginning of the evaluation section, and it was very similar to RQ2.1 above: "Do atomic patterns support (nonexpert) teachers in the orchestration of activities with ICT?" (I1a). This question (which was our main Issue, following the CSCL-EREM terminology), was explored through the answering of questions around three topics: "Are atomic patterns useful for practitioners to orchestrate activities with GS and other classroom technologies?" (T1a), "Is the format of the workshop itself and its orchestration affecting the perceived usefulness of atomic patterns?" (T2), and "Do teachers see atomic patterns as something near to their everyday practice?" (T3, i.e., the atomic patterns' mirroring properties). These issues are also represented in Figure 4.13. To answer these questions, the session was audio and video recorded, photos were taken, and two other researchers from the team acted as external observers. The short questionnaires that teachers filled in and 


\section{ROLE PLAYING}

\section{In order to complete this activity, try to represent the role of a student that steals the post-its with the task to some of his/her classmates.}

Figure 4.16: Example role card handed out to teachers during the role-playing in the workshop

[Translated from the Spanish original]

the artifacts generated by the teachers during the workshop (e.g. learning designs produced) were also collected. Moreover, although they were performed months before the workshop, the interviews and focus groups with school teachers done at the beginning of our research efforts in the school, could provide valuable evidence about the profiles of many of four of the participant teachers. Table 4.5 summarizes the main data sources used during this workshop, along with the codes that will be used to refer to them throughout the text.

Table 4.5: Main data sources used during the TW1 workshop

\begin{tabular}{|lll|}
\hline Source & Kind of evidence & Codes \\
\hline \hline Observation notes & Qualitative & TW1-O \\
\hline Session recording & Qualitative & TW1-R \\
\hline Teacher-generated documents & Qualitative & TW1-D \\
\hline Questionnaires answered during the workshop & Qualitative & TW1-Q \\
\hline Interviews with teachers & Qualitative & TW1-I \\
\hline Focus group with teachers & Qualitative & TW1-F \\
\hline
\end{tabular}

\section{Findings and evidence}

The aforementioned workshop session finally took place in June 2010, and 9 primary school teachers participated in it (four who had worked with us in the previous efforts with Group Scribbles, and five who had no prior experience). The session was dynamized by two researchers (the author and a fellow Pedagogy researcher), with two additional researchers acting as observers. The teachers were able to complete the tightly scheduled design of the workshop, and the atmosphere was relaxed but focused on the proposed tasks. Teachers created in groups several outlines of activities that used Group Scribbles to work around two different topics of the curriculum, and then chose one of them (about the Solar System, with parts of it also working on Spanish language) to enrich it with the design-time atomic patterns presented (a case of using atomic patterns to "fill in the bones" of skeleton designs, see Figure 4.11). Afterwards, teachers role-played the enactment of the situation, representing the problematic situations and solving them with a high degree of engagement.

Regarding the issues that concerned us, we uncovered the following findings: 


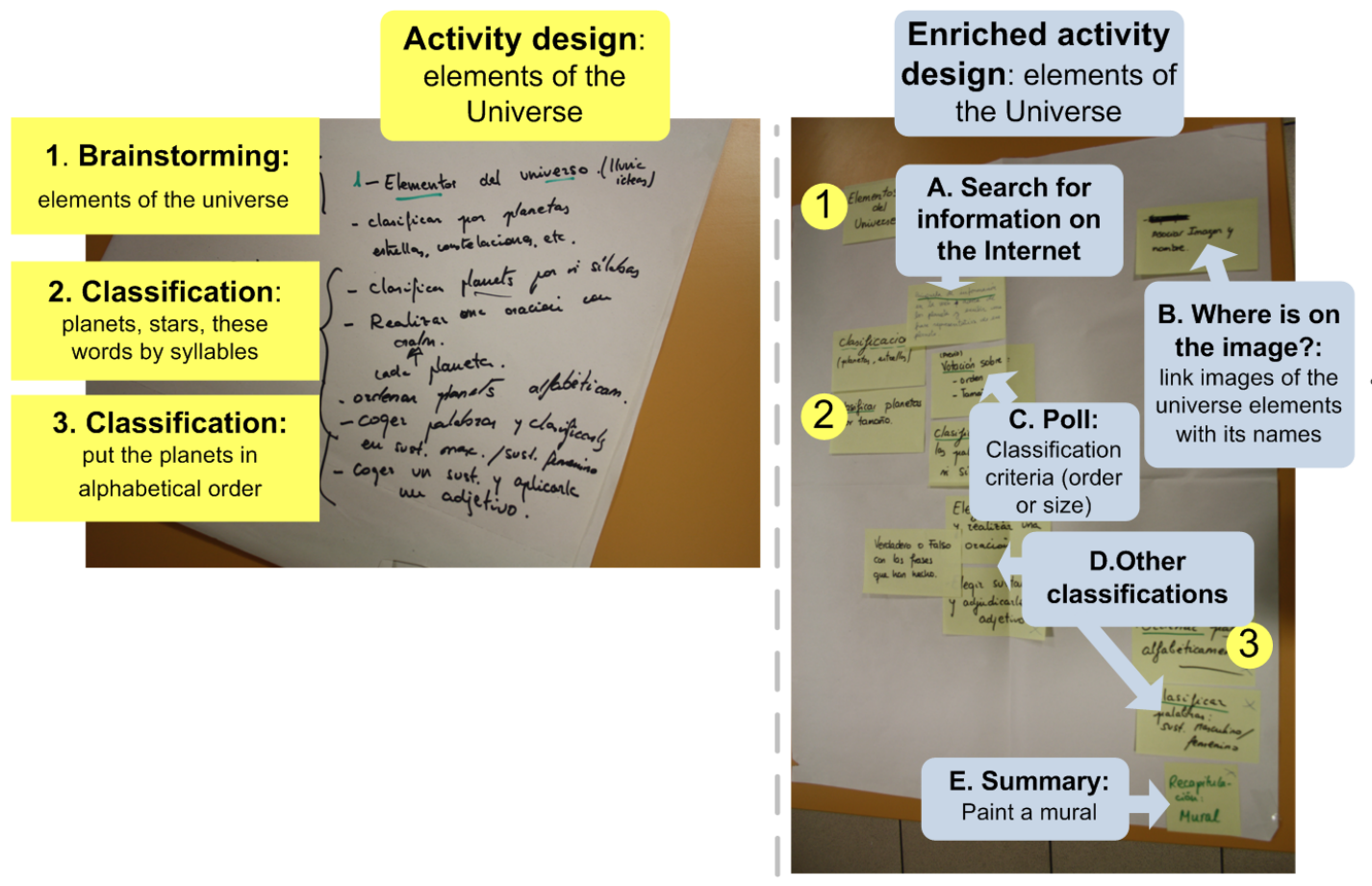

Figure 4.17: Original design draft done by teachers during the workshop (left). Design enriched with design-time atomic patterns (right)

On the atomic patterns' usefulness (T1a) As hinted by the qualitative evidence from the observation and the questionnaires during the session (see Table 4.6), teachers found atomic patterns useful as a way of enriching their initial ideas of activity designs, under tight time constraints (teachers were able to design and enrich the activities with atomic patterns, with no prior knowledge of them, in less than 30 minutes). The enactment-time atomic patterns were also considered useful for everyday practice. Teachers valued especially the patterns' ability to provide starting ideas on new things that can be done with the available ICT (mainly Group Scribbles, in this case). This is interesting given the fact that this set of atomic patterns had been elicited from many of those same teachers practice at that same school: atomic patterns seemed to serve as mediating artifacts in gaining new knowledge about their colleagues' existing orchestration practices with ICT. Moreover, some teachers already hint at the possibility of having a catalogue of atomic patterns, which could be useful, e.g. to use them as inspiration before designing or enacting learning activities.

Moreover, maybe due to their good mirroring qualities of atomic patterns (see below), these enriched activity designs were seen by teachers as readily applicable to their practice (when compared, e.g. with the CLFP-based approach that we had tried previously in the same school). However, teachers also detected a well-known danger of design pattern approaches, which is the tendency to over-use the provided set of patterns, trying to cram as many of them as possible in every design, thus producing unnecessarily complicated designs (especially given the time constraints of school lessons). 
Table 4.6: Selected findings and evidence from the TW1 workshop, on the issue of atomic patterns' usefulness (T1a). A, B, C, D denote utterances of participant teachers.

\begin{tabular}{|c|c|}
\hline Finding & Selected supporting evidence [Source] \\
\hline $\begin{array}{l}\text { Atomic patterns are applicable to enactment } \\
\text { using GS }\end{array}$ & $\begin{array}{l}\text { [to the question: Can any of these [enactment-time atomic patterns] } \\
\text { be applied to the role-played situation?] A: Yes, both the explanation } \\
\text { ones and the support ones. Although normally we use them sponta- } \\
\text { neously. D: Yes, I think that in the roleplay, most of the enactment- } \\
\text { time atomic patterns have appeared. It could be interesting to be } \\
\text { reminded of them before enacting an activity with Group Scribbles. } \\
\text { [TW1-Q] }\end{array}$ \\
\hline Atomic patterns are useful to design using GS & $\begin{array}{l}\text { Teachers were able to complete a design and enrichment of a complex } \\
\text { activity in less than } 30 \text { minutes [TW1-O, TW1-R, TW1-D] } \\
\text { [to the question: Did these atomic patterns help you in enriching the } \\
\text { activity design? Why?] A: Yes, because they offer new ideas about } \\
\text { how to work the different subjects in different ways. C: Yes, and also } \\
\text { to make it a bit more complicated. [TW1-Q] }\end{array}$ \\
\hline Dangers of pattern overuse & $\begin{array}{l}\text { Iván: Luis Pablo asks them, once the new enriched design has been } \\
\text { generated, if the design in its current state could be put in practice. } \\
\text { D [a teacher] says yes, but that time should be managed very well. E } \\
\text { [another teacher] says that the result of this Didactic Unit could be } \\
\text { transferred to a poster. [TW1-O] } \\
\text { [to the question: did these atomic patterns help you enrich the de- } \\
\text { sign of the activity? Why?] C: Yes, and also to make it a bit more } \\
\text { complicated. [TW1-Q] }\end{array}$ \\
\hline $\begin{array}{l}\text { Enactment-time atomic patterns are useful for } \\
\text { everyday practice }\end{array}$ & $\begin{array}{l}\text { [to the question: do you think that these atomic patterns are useful to } \\
\text { your teaching practice?] C: I believe that practice is completed with } \\
\text { a series of routines that we teachers apply every day, and thus I think } \\
\text { that the catalogue [of atomic patterns] can be useful. A: They can } \\
\text { help, probably to do things with ICT in a different way. [TW1-Q] }\end{array}$ \\
\hline $\begin{array}{l}\text { Having a catalogue of atomic patterns would } \\
\text { be useful }\end{array}$ & $\begin{array}{l}\text { D: (on atomic patterns as ideas for ways of doing things in the class- } \\
\text { room) There are times that we do things [in design and enactment] } \\
\text { in such an assuming, automatic way that we do not think any more } \\
\text { about other things that could help us [TW1-R] } \\
\text { [to the question: do you think that these atomic patterns are useful to } \\
\text { your teaching practice?] C: I believe that practice is completed with } \\
\text { a series of routines that we teachers apply every day, and thus I think } \\
\text { that the catalogue [of atomic patterns] can be useful. [TW1-Q] }\end{array}$ \\
\hline $\begin{array}{l}\text { The activities enriched with atomic patterns } \\
\text { are feasible to put in practice }\end{array}$ & $\begin{array}{l}\text { Iván [an observer]: Luis Pablo asks them, once the new enriched design } \\
\text { has been generated, if the design in its current state could be put in } \\
\text { practice. D [a teacher] says yes, but that time should be managed } \\
\text { very well. E [another teacher] says that the result of this Didactic } \\
\text { Unit could be transferred to a poster. [TW1-O] }\end{array}$ \\
\hline
\end{tabular}

On the influence of the workshop's orchestration (T2) Despite the very limited amount of time that the research team had available to gather evidence, and at the same time provide a useful professional development experience (which, let us not forget, was another primary goal of the session), the workshop format used during the session seemed to be quite successful (as opposed to, maybe, a more theoretical explanation of orchestration and/or atomic patterns). Observers noted that the pace that facilitators imposed was rather frenetic, but teachers seemed to adjust very well to the practical orientation of the proposed tasks (see Table 4.7). Not only were participant teachers able to design and enrich a complex activity (let us remember we tried to make it work on two different areas of the curriculum), but they were also able to role-play its enactment and reflect about the problems that arose (not to mention the additional questionnaires that had to be filled in). The teachers reacted especially well to the role-play of the activity enactment, being very engaged in the task while maintaining a positive, relaxed attitude. This kind of activity proved very useful in order to break the design-orientation that 
previous PD actions had, and which often neglected the enactment side, which is essential in orchestrating TEL activities.

Table 4.7: Selected findings and evidence from the TW1 workshop, on the influence of the workshop's own orchestration in the atomic patterns' usefulness (T2)

\begin{tabular}{|c|c|}
\hline Finding & Selected supporting evidence [Source] \\
\hline The level of the tasks was adequate & $\begin{array}{l}\text { Iván [an observer]: As this first part [the design-time part of the ses- } \\
\text { sion] unfolds I notice that the [short] explanation has been sufficient, } \\
\text { because all teachers do what they are asked for in a fast and focused } \\
\text { way. I imagine it may be related with the previous work and sessions } \\
\text { that Luis Pablo and Sara have done with them. The engagement of } \\
\text { participants is really good. [TW1-O] }\end{array}$ \\
\hline $\begin{array}{l}\text { The role-playing situation is adequate for this } \\
\text { kind of workshop }\end{array}$ & $\begin{array}{l}\text { Iván [an observer]: Participants assume their roles and engage abso- } \\
\text { lutely in the [role-playing] activity. D [a teacher] becomes a central } \\
\text { dynamizer, treating her colleagues as she would treat her students. } \\
\text { [TW1-O] } \\
\text { Yannis [an observer]: People play their roles well. Order is important. } \\
\text { She distributes tasks to the different groups (one task per group). } \\
\text { It is clear that they enjoy playing the student. It [the role-playing] } \\
\text { could be a great resource for our training. The teacher walks by the } \\
\text { [students'] computers to see what is being done, but does not look at } \\
\text { her computer or the blackboard. She also advices [students]. [TW1-O] }\end{array}$ \\
\hline The workshop design was tight & $\begin{array}{l}\text { Iván [an observer]: I wonder if, with such a quick explanation, they will } \\
\text { have understood what is being asked from them. Yannis [an observer], } \\
\text { in a later conversation, tells me he observed it too. [TW1-O] } \\
\text { Yannis [an observer]: Sara is explaining things quite fast. Do they } \\
\text { understand everything? [TW1-O] }\end{array}$ \\
\hline
\end{tabular}

On the atomic patterns' mirroring qualities (T3) As the qualitative evidence gathered during the session suggests, teachers readily recognized the presented atomic patterns (both in design-time and enactment-time) as applicable (or already part of) their everyday teaching practice. Moreover, these atomic patterns seem to be in many cases actions they take naturally in their practice. Thus, this evidence seemed to support our hypothesis that this kind of smallerscale, more informal patterns, could bridge the contextualization gap existing between more abstract research-generated advice and the concrete performance that teachers have to deliver in their everyday practice.

Table 4.8: Selected findings and evidence from the TW1 workshop, on the issue of atomic patterns' mirroring qualities (T3)

\begin{tabular}{ll}
\hline Finding & Selected supporting evidence [Source] \\
\hline $\begin{array}{l}\text { Design-time atomic patterns are recognized by } \\
\text { teachers as elements of their everyday practice }\end{array}$ & $\begin{array}{l}\text { [to the question: Have you ever used these [atomic patterns]? Which } \\
\text { ones?] A: Yes, most of them, Brainstorming, Classification, Where is } \\
\text { on the image, etc. B: Yes, I use them often when I use GS. [TW1-Q] }\end{array}$ \\
\hline $\begin{array}{ll}\text { Enactment-time atomic patterns are recog- } \\
\text { nized by teachers as elements of their everyday } \\
\text { practice }\end{array}$ & $\begin{array}{l}\text { [to the question: Can any of these [enactment-time atomic patterns] } \\
\text { be applied to the role-played situation?] A: Yes, both the explanation } \\
\text { ones and the support ones. Although normally we use them sponta- } \\
\text { neously. [TW1-Q] }\end{array}$ \\
\hline
\end{tabular}

\section{Conclusions of this iteration}

The findings of our evaluation of the TW1 workshop (described in the previous section), can be synthesized into the following partial conclusions (see Figure 4.14), which can begin to 
illuminate our main evaluation tension (I1a) through the focus on the three aforementioned topics (T1a, T2 and T3). These conclusions and the relationship with the aforementioned issues and questions are represented in Figure 4.18 .

- Atomic patterns (both design-time and enactment-time) are considered useful by teachers, in order to orchestrate activities using GS (both by enriching skeleton ideas of designs, and to reflect on the enactment of those designs). [TW1-T1a-PC1]

- There are first hints that having a (probably classified) catalogue of atomic patterns could be useful for teachers, not as a normative tool, but rather as an inspiration to look for new design elements, and to be reminded about useful enactment-time strategies. [TW1-T1a$\mathrm{PC} 2]$

- The activities that are built by enriching a skeleton idea of a design with atomic patterns are considered feasible by teachers, to be put in practice in the context of their classrooms. [TW1-T1a-PC3]

- There exists a danger of over-using atomic patterns, thus producing designs which are no longer feasible given the constraints of the setting (e.g. lesson duration). [TW1-T1a-PC4]

- The workshop format proposed (which basically consisted in a design task followed by a role-played enactment task) was both feasible and engaging for teachers in the primary school. [TW1-T2-PC1]

- However, it was also clear that, through the accumulation of up to 9 different tasks in a single 2-hour session, we ran the risk of making the workshops too short and busy to really assimilate the notion (and the number) of atomic patterns being presented. [TW1-T2-PC2]

- Our evidence hinted that atomic patterns, elicited both from design-time and enactmenttime orchestration of TEL activities using the Group Scribbles tool, had good mirroring qualities for orchestration practice (in the sense that teachers recognized the patterns as part of their practice, or applicable to it), at least within the same school and educational level. [TW1-T3-PC1]

However, it should be remarked that this first experience was rather limited in scope, and that all the aforementioned conclusions are not to be taken as generalized assertions about atomic patterns. Rather, they should be understood as always being tied to the particular context of the school where we conducted the study, and the participants that took part on it.

Thus, although the evaluation of this first application of atomic patterns to a professional development teacher workshop was rather satisfactory, providing first evidences of usefulness, further studies were needed to confirm these hints of usefulness, determine whether they could be applied to other contexts, or to refine the format of the workshop to increase the usefulness for the teachers.

Particularly, a number of modifications in our research focus and in the orchestration of the workshops themselves were suggested by this first evaluation (and by the analysis of alternative pattern approaches, see Section 4.2.3): to extend workshop duration (to favor teacher reflection the assimilation of more atomic patterns), or to integrate this practice-elicited pattern approach with other researcher-driven patterns (e.g. SRI's teacher moves to favor more productive science classes). 


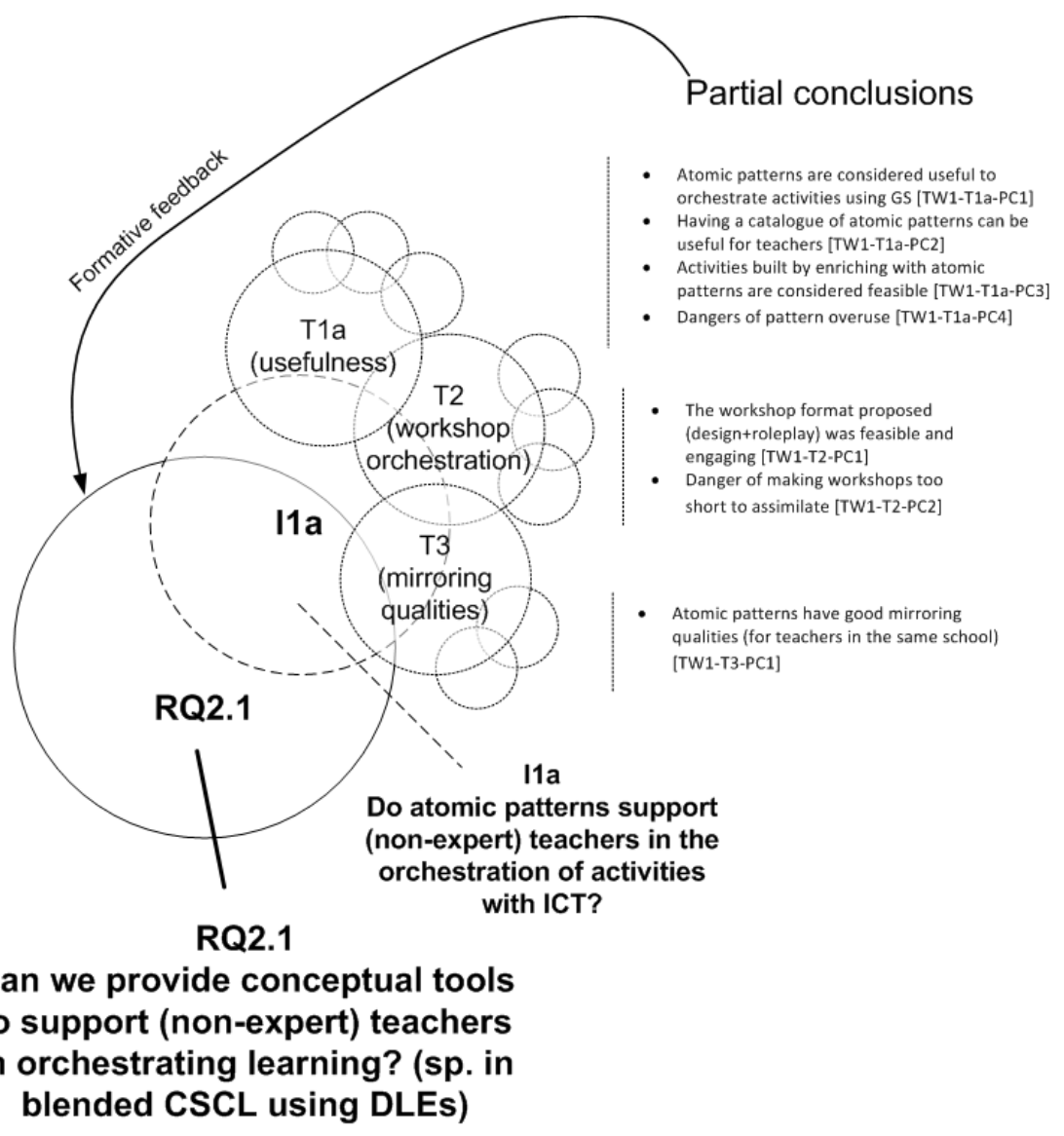

Figure 4.18: Graphical representation of the partial conclusions to Iteration \#1

\subsubsection{Iteration \#2: A second atomic pattern teacher workshop in primary education (TW2)}

After our first approach to using atomic patterns for orchestration in primary classroom, in the following academic year (2010-2011), two more training sessions with teachers were performed, with goals similar to the previous one (to ascertain whether atomic patterns could be useful tools for teachers). Following up on our partial conclusions from the previous iterations, we tried to lengthen the training sessions. It is important to note, however, that due to the school's tight schedule, our training was restricted to 2-hour slots once per week (and even that slot was not available every week, since the school also had other purposes for it in their everyday schedule, such as coordination meetings or other ongoing training efforts - e.g. about usage of other ICT tools available). Also, we intended to frame our training within one of the school's ongoing innovation projects (called "A, B, C, Science!"). In order to better cater for the needs of this project, which aimed at making children think more "like scientists", we proposed to blend some of SRI's teacher moves for improving science lessons (see 4.2.3) with our existing atomic pattern catalogue. Also, during meetings with the teachers of the school, it was decided attempt the design of a scientific experiment activity within these training sessions [VS12]. 


\section{Context and method}

The context of this evaluation was, in most regards, identical to the one described for the previous iteration (see Section 4.5.2). There was, however, an important contextual difference: in this new academic year, the primary school first cycle (children aged 6-8) staff, with whom we were working the previous years, was almost entirely replaced. Thus, we now counted with 6 teachers, of whom only 1 had previously worked with us. Even if this fact provided an opportunity to test atomic patterns with a new set of teachers, it is also true that (as described in [VS12]) the school's frantic activities and emphasis on ICT usage could create a challenging (even oppressive) atmosphere for newcomers to the school.

Regarding the method of this iteration's study, we proposed to use two 2-hour sessions to design, enrich and reflect on the enactment of the activities through a role-played situation. The focus of the orchestration would be, as mentioned above, that of a scientific experiment. More specifically, each classroom (i.e. each teacher) would perform a different experiment. Initially, the structure of the first 2-hour session was to be as follows:

1. Researchers would introduce the session, the goals and methods to be used during the workshop. [15 minutes]

2. As a warmup activity, teachers would brainstorm about problems that they had encountered when trying to make students think scientifically. This activity was to be performed with Group Scribbles. [10 minutes]

3. A second brainstorm would take place, to generate ideas about how to support students in thinking more like scientists. Again, the activity would be performed using Group Scribbles. [10 minutes]

4. The teachers would produce, using pen and paper, a first outline of the design of the experimental activity, including its goals, task sequence and assessment. [20 minutes]

5. Using the previous experiment idea as a base, two groups of 3 teachers, were to enrich the design with a set of design-time atomic patterns that were to be presented to them (including the ones elicited at this school, plus others extracted from literature). Teachers also were to agree to use one of the proposed enriched designs (or a blend of both). [30 minutes]

6. Finally, teachers would fill in a short online questionnaire about the session and the presented atomic patterns. [10 minutes]

However, we should highlight that, as described in |VS12|, this first 2-hour session could not be completed, since teachers were not able to agree on the general idea of the activity and the experiments that each classroom should perform (and the researchers had not prepared for this eventuality). Thus, no evidence could be gathered in this session regarding atomic patterns, and the design of the second session (which originally was intended to work on enactment-time atomic patterns) was modified to work on both design- and enactment-time atomic patterns. The modified structure for the second session was as follows: 
1. Introduction to the session, including the review of the experiment idea that had been finally agreed on the previous session ${ }^{18}$, [15 minutes]

2. Using the previous experiment idea as a base, two groups of 3 teachers, were to enrich the design with a set of design-time atomic patterns that were to be presented to them (including the ones elicited at this school, plus others extracted from literature). [20 minutes]

3. Sharing of the two enriched designs, and reaching of a consensus about which one (or which blend) to put in practice. [10 minutes]

4. Presentation of the notion of enactment-time atomic pattern and of a subset of the atomic patterns elicited the previous year, printed in cards (see Figure 4.15). [10 minutes]

5. Role-playing of the (enriched) experiment activity. In this case, one teacher performs the role of the teacher, and the researchers act as students, role-playing a number of problematic situations. The rest of the teachers, using the provided atomic pattern cards, try to help the teacher, suggesting atomic patterns that are applicable to the situation. [30 minutes]

6. Teachers fill in a short questionnaire about the session and the atomic patterns' usefulness. [5 minutes]

Again, in this round of evaluation, for the research team the main focusing Issue was very similar to the one in the previous iteration (and to RQ2.1 above): "Do atomic patterns support (non-expert) teachers in the orchestration of activities with ICT?" (I1a). So were the topics used to explore this issue: "Are atomic patterns useful for practitioners to orchestrate activities with GS and other classroom technologies?" (T1a). Apart from this question, two more related topics interested us: "Is the format of the workshop itself and its orchestration affecting the perceived usefulness of atomic patterns?" (T2), and "Do teachers see atomic patterns as something near to their everyday practice?" (T3, that is, the atomic patterns' mirroring properties). These issues are also represented in Figure 4.13. This session was dynamized by one researcher, while one other researcher acted as an external observer, taking notes. The session was audio recorded and teachers, at the end of the session, answered a short questionnaire about the usefulness of the presented atomic patterns. Please refer to Table 4.9 for the complete list and coding of our data sources.

Table 4.9: Main data sources used during the TW2 workshop

\begin{tabular}{|lll|}
\hline Source & Kind of evidence & Codes \\
\hline \hline Observation notes & Qualitative & TW2-O \\
\hline Session recording & Qualitative & TW2-R \\
\hline Teacher-generated documents & Qualitative & TW2-D \\
\hline Questionnaires answered during the workshop & Qualitative & TW2-Q \\
\hline
\end{tabular}

\footnotetext{
${ }^{18}$ This was especially important since, due to different (non-research-related) obligations that teachers and researchers had, three months had passed since the previous session.
} 


\section{Findings and evidence}

When the aforementioned second 2-hour session was finally enacted, in May 2011, another unexpected event came to disrupt the development of this evaluation: due to an unexpected teacher meeting in the school, the session started 50 minutes late, and could only count with four teachers instead of the expected six $\overline{V S 12}$. Also, the initial stages of the session were marked by the teachers lack of agreement with the experiment outline agreed in the previous session. All these events led to a hasty performance of the session design, especially regarding the use of atomic patterns. Also, the online questionnaires to be filled in at the end of the workshop, were answered asynchronously by teachers in the following days. Thus, the amount and quality of the evidence gathered in this iteration was somehow diminished.

Nevertheless, certain evidence could be gathered (mainly from the questionnaires) regarding the issues that concerned us, as we can follow from Table 4.10, which summarizes selected evidence from this session. The main findings can be summarized as:

On the atomic patterns' usefulness (T1a) Despite the diminished evidence of this iteration, the gathered pieces of evidence still confirm that teachers saw atomic patterns as useful for their everyday practice (both in design-time and in enactment-time). Also, we have hints of a further insight, which is the high occurrence of the orchestration problems that some of the atomic patterns (especially those used in the role-playing situations) try to solve. This initial evidence leads us to select some of these atomic patterns as more important, for example, in the case we desired to automate them (or otherwise support teachers in enacting them) through technology.

On the influence of the workshop's orchestration (T2) Probably the clearest finding uncovered during this session, precisely because of its relative failure, was the importance of the way atomic patterns were presented to teachers, which could easily render the workshops ineffective if not handled with care. We can note from the gathered evidence that it is of the utmost importance to be clear on the tasks and goals to be achieved during the session. Here, the researchers always try to strike a balance between closed, concrete (and probably less authentic) tasks, and open-ended tasks which are directly applicable to the teacher's immediate practice. As this evaluation showed, erring on the side of concreteness can be useful to avoid problems such as the one encountered in our first TW2 session. Also, the importance of time management in this kind of short professional development sessions (even if the second TW2 session went awry because of unforeseeable events) was highlighted by this evaluation.

As a side note, our inclusion of researcher-driven patterns among the atomic patterns (e.g. basic collaboration strategies extracted from literature, like "reciprocity", which were inserted as cards alongside the rest of the atomic patterns) did not seem to be very successful, as they were not used by teachers during the workshop. Although we did not gather any relevant evidence, we started to doubt at the time whether that way of combining top-down with bottom-up patterns (i.e. putting them at the same level) was adequate. This eventually led to the multi-level pattern approach described in Section 4.3 . 
Table 4.10: Topics, findings and selected supporting evidence from the TW2 workshop

\begin{tabular}{|c|c|c|}
\hline Topic & Finding & Selected supporting evidence [Source] \\
\hline \multirow[t]{3}{*}{$\begin{array}{l}\text { On the atomic } \\
\text { patterns' usefulness } \\
\text { (T1a) }\end{array}$} & $\begin{array}{l}\text { Atomic patterns are useful to } \\
\text { design using GS }\end{array}$ & $\begin{array}{l}\text { [to the question: Did the atomic patterns help you to enrich } \\
\text { the design?] A: Yes, at least to be conscious that their use } \\
\text { improves not only the design, but also guarantees the success } \\
\text { of the process and the attainment of the desired goals. E: We } \\
\text { have not done many experiments before, but there are atomic } \\
\text { patterns which are more adequate for the design of enactment } \\
\text { of the experiment (Poll, Recap, Brainstorming) [TW2-Q] }\end{array}$ \\
\hline & $\begin{array}{l}\text { Enactment-time atomic } \\
\text { patterns are useful for } \\
\text { everyday practice }\end{array}$ & $\begin{array}{l}\text { [to the question: Do you believe that the presented atomic pat- } \\
\text { terns helped the teacher to solve successfully the [problematic] } \\
\text { situations? Why?] E: Yes, because I have been able to harmo- } \\
\text { nize the students' differences and achieve the activity's goal. } \\
\text { [TW2-Q] }\end{array}$ \\
\hline & $\begin{array}{l}\text { Enactment-time atomic pat- } \\
\text { terns solve frequent problem- } \\
\text { atic situations }\end{array}$ & $\begin{array}{l}\text { to the question: Do you think that the problematic situations } \\
\text { presented were realistic?] A: Yes, they are the ones we habit- } \\
\text { ually have in the classroom. E: Even if they seem exaggerated } \\
\text { in their role-playing, in the classroom you find students with } \\
\text { that kind of behavior, and so we have to know how to solve } \\
\text { [those situations], which is not always easy. [TW2-Q] }\end{array}$ \\
\hline \multirow{2}{*}{$\begin{array}{l}\text { On the influence of the } \\
\text { workshop's } \\
\text { orchestration (T2) }\end{array}$} & $\begin{array}{l}\text { The workshop was poorly } \\
\text { timed and lacked clarity }\end{array}$ & $\begin{array}{l}\text { The first session did not gather any evidence about atomic } \\
\text { patterns. The two sessions were } 3 \text { months apart. [TW2-R, } \\
\text { TW2-O] }\end{array}$ \\
\hline & & $\begin{array}{l}\text { Only one design-time atomic pattern was used to enrich the } \\
\text { design [TW2-D] } \\
\text { [to the question: Score the sessions from } 1 \text { to } 7 \text {. Why?] A: } 4 \text {. } \\
\text { I would have liked that they had more continuity, and that } \\
\text { there were not only two of them, isolated. It is important that } \\
\text { everyone knows clearly the goal of the workshop, what we } \\
\text { intend to achieve, and I think that [in this workshop] this has } \\
\text { been very hard. [TW2-Q] } \\
\text { [to the question: Do you believe that the presented atomic pat- } \\
\text { terns helped the teacher to solve successfully the [problematic] } \\
\text { situations? Why?] A: It has been very hard. There were very } \\
\text { few of us, and everything happened very fast. [TW2-Q] }\end{array}$ \\
\hline $\begin{array}{l}\text { On the atomic } \\
\text { patterns' mirroring } \\
\text { qualities (T3) }\end{array}$ & $\begin{array}{l}\text { Design-time atomic patterns } \\
\text { are recognized by teachers as } \\
\text { elements of their everyday } \\
\text { practice }\end{array}$ & $\begin{array}{l}\text { [to the question: Do you see [design-time atomic patterns] as } \\
\text { something close to your current practice, or that you could use } \\
\text { in the future?] A: Many of them are very close to the everyday } \\
\text { [practice] and others, different or not often used [but] I will be } \\
\text { able to use them in the immediate future. E: Not all of them, } \\
\text { but most are part of the everyday practice. [TW2-Q] } \\
\text { [to the question: Have you ever used these [design-time } \\
\text { atomic patterns]? Which ones?] E: Classification, Poll, Re- } \\
\text { cap, Concept mapping, Explanation of objectives, Collabo- } \\
\text { rative enigma, Brainstorming, Task evaluation, Reciprocity, } \\
\text { Distributed problem solving. [TW2-Q] }\end{array}$ \\
\hline
\end{tabular}


On the atomic patterns' mirroring qualities (T3) In this iteration of the evaluation, we gathered new evidences that pointed towards the idea that atomic patterns have good mirroring qualities, even with a (mostly) different set of teachers at the same school, who asserted that they saw these atomic patterns (design-time ones, in this case) as elements from their everyday practice, or immediately applicable to it.

\section{Conclusions of this iteration}

This second iteration of our evaluation of the atomic patterns as a teacher support for orchestration in primary school classrooms was certainly less successful than the first one, mainly due to a series of unforeseen events which disrupted our data gathering. However, even the diminished evidence gathered in the second teacher workshop, provided us with interesting findings, which we can summarize in the following partial conclusions (see Figure 4.19), around the three topics that interested us (T1a, T2 and T3):

- As we did in the previous iteration, in this evaluation we gathered further evidences hinting that atomic patterns are considered useful to orchestrate activities using GS (in this iteration, mostly regarding the design-time atomic patterns). [TW2-T1a-PC1]

- Also, we obtained strands of evidence about a slightly different perspective of the usefulness of atomic patterns: do they solve problems that appear frequently? (the more frequent problems are, more useful atomic patterns will be, provided that the solutions are not trivial). Thus, in this workshop we also obtain evidences that the practice-elicited atomic patterns solve frequent orchestration conflicts, in this case in the use of ICTs (especially GS) in face-to-face primary school classrooms. [TW2-T1a-PC2]

- Probably the main conclusion that we could draw from this iteration of evaluation is the crucial importance of the workshop's own design and enactment in the usefulness that teachers may extract from the use of atomic patterns. In this case, we found that the clarity of the tasks and the time management of the sessions are crucial for the success of the training actions. This influence is to be expected from this kind of situated actions but, nevertheless, we should take it into account for following iterations of our study, since these extraneous events substract from the validity of our findings and conclusions. [TW2-T2-PC1]

- Finally, we obtained further evidence that highlighted atomic patterns' good mirroring qualities, not only for the teachers from whose practice they had been elicited, but also for different teachers in the same school (even for newcomers to the school). [TW2-T3-PC1]

These partial conclusions, together with the ones from the previous evaluation (TW1), served to further illuminate our main evaluative issue (I1a), about the usefulness of atomic patterns as a conceptual tool for teacher orchestration. Again, our findings and these conclusions are closely tied to the situated professional development action that took place in this concrete primary school. This iteration, however, did widen the scope of our findings (albeit in a small way), since almost all the teachers were newcomers to the specific context of this school. 


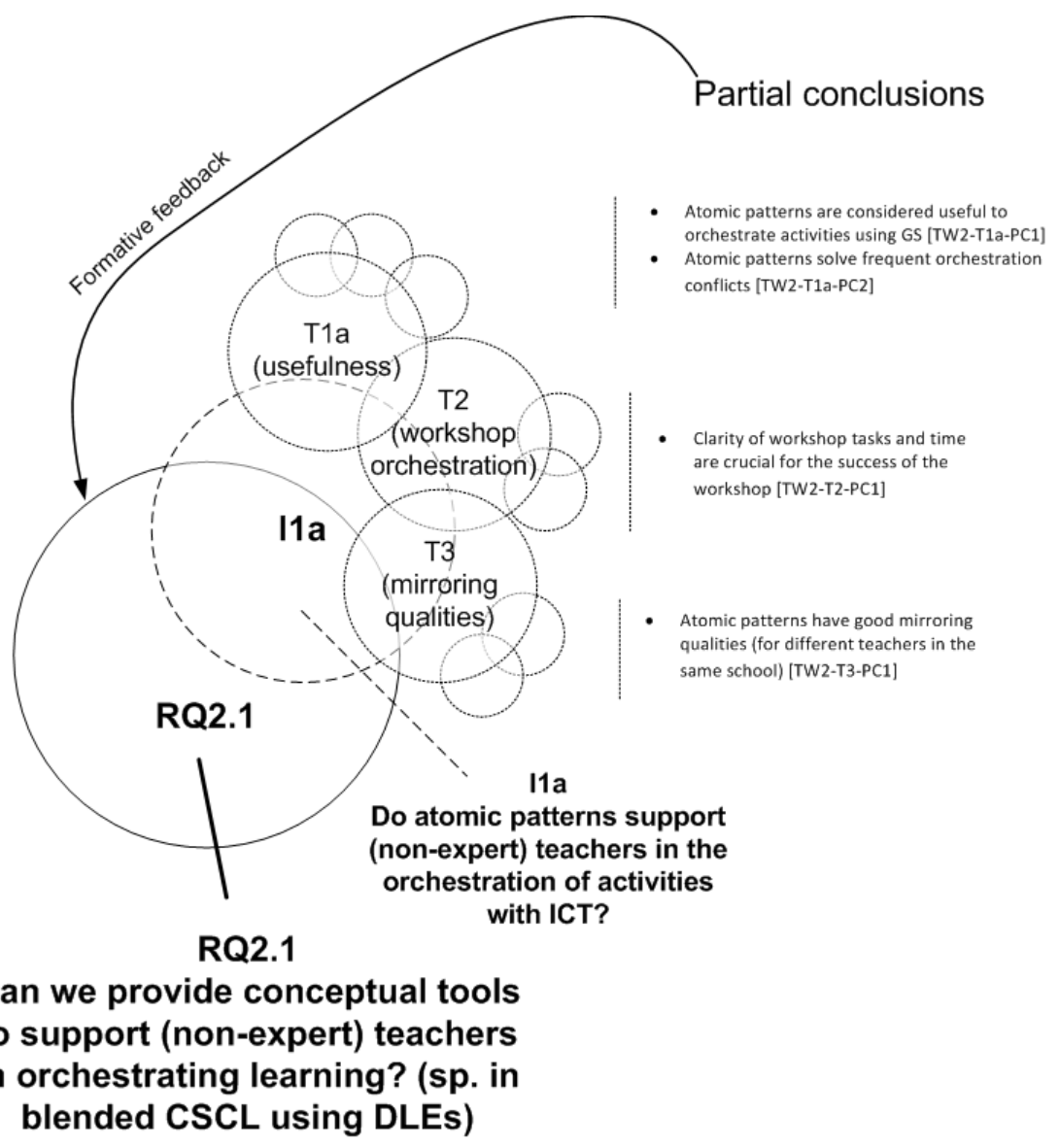

Figure 4.19: Graphical representation of the partial conclusions to Iteration \#2

The experience of this iteration also prompted certain formative feedback for following iterations: First of all, we were reminded that the preparation of the workshops should be really careful, and that the design should include concrete tasks for teachers to perform, but remaining flexible enough to cope as much as possible with unexpected events (i.e. orchestration workshops should be orchestrable - not surprisingly). Also, as we closed the second year of interventions in the school, we started to wonder how much of our efforts (including the atomic pattern catalogue, but also the general approach followed) could be applicable to other different educational contexts.

\subsubsection{Iteration \#3: Generation of a new atomic pattern catalogue, and first teacher workshop in higher education (TW3)}

After the first indications of usefulness provided in the previous two iterations, the question remained as to whether this atomic pattern set or, at least, the same atomic pattern "approach" (see Section 4.3 could also be useful in the main contextual setting of this PhD: blended learning using Distributed Learning Environments (DLEs), especially in higher education. Thus, the author set out to elicit an extended set of atomic patterns from practice in such higher education 
contexts, to see if we could confirm common atomic patterns with the primary school case, or to find new ones that were more suitable for this new context. The process followed and the resulting atomic pattern set, which spanned more than 150 atomic patterns, is described elsewhere (Section 4.3 , and Appendix B).

It is also worth noting that the study of this new educational and technological context also uncovered a number of orchestration challenges which had not been so apparent in the primary school. Namely, the challenge of deploying a teacher's learning design across the DLE, and that of enacting it in a flexible manner (see Section 2.5), had not been detected as very problematic (given the simplicity and inherent flexibility of Group Scribbles [Ros07, which enabled the primary school teachers, even those new to the technology, to deploy and change the activities on-the-fly easily [Pri09]). In this new context, the deployment of non-trivial CSCL designs, as part of a CSCL script lifecycle (Section 2.2.2), acquired a new importance, and led us to classify our recently-elicited catalogue into design-time, deployment-time and enactment-time atomic patterns.

Once this extended catalogue was in place, it was necessary to evaluate whether those atomic patterns would be useful for teachers in the context of blended CSCL using DLEs. Following the same rationale as in the case of the primary school, and in order to better compare our findings with the ones in the previous iterations, we decided to do this evaluation through professional development teacher workshops. Moreover, following our analysis of atomic patterns from an activity theory perspective (Section 4.2.4), we decided to investigate further and evaluate the combination of patterns at different levels, e.g. by combining atomic patterns with CLFPs (Section 4.2.3), which had proved quite successful with university-level teachers in the past HL10b.

In this new stage of our research, we had already identified our main evaluative question (issue) as it was presented at the beginning of Section 4.5 to assess the usefulness of atomic patterns for practitioners in the orchestration (design, deploy and enactment) of CSCL across DLEs (I1b), while extending our focus also to the perceived usefulness and form most adequate to embed atomic patterns in educational technologies (I2).

\section{Context and method}

To provide evidence about the aforementioned evaluative tensions, we proposed to conduct a professional development workshop for teachers at the University of Valladolid, whose topic would be the design and enactment (i.e. the orchestration) of advanced collaborative activitie: 19 in Distributed Learning Environments. This workshop would provide a first introduction to the design of collaborative activities using CLFPs (see 4.2.3), and structuring them further with a selection of the extended atomic pattern catalogue elicited from higher education, to obtain evidence about their usefulness for the orchestration of CSCL activities.

However, it is very important to understand that evaluating atomic patterns was not the only research goal of this workshop. In parallel, the work on the GLUE!-PS technological infrastructure for orchestration of DLEs (see Chapter 5.2 was being carried out, and this workshop was also intended to test one of the prototypes of this infrastructure with university teachers.

\footnotetext{
${ }^{19}$ In the sense of "non-trivial", going beyond a group report or discussions in a plain online forum.
} 
Even though in this section we will only describe the findings and evidence regarding atomic patterns, some references to the other goals and activities of the workshop (concerning GLUE!-PS, see Section 5.5.3 will be unavoidable.

Other interesting contextual data about the workshop include: the fact that the workshop was a professional development action done within the auspices of the Buendia center at the University of Valladolid 20 and it was open for university teachers with a basic knowledge of the involved ICT tools, up to 20 participants (in order for the workshop design to be manageable). Among these participants, teachers from the Master-level degree on ICT research (MUI-TIC from now on, from its Spanish initials) were given preference, since they already had performed a related workshop on the design of master-level TEL activities [Fer10]. This was done in order to minimize the problems related to the basic usage of the ICT tools involved, which could disrupt the course of the workshop and our data gathering itself (although this obviously produced a bias in our results, since most of the participants had a technical background as a result).

The workshop was supposed to involve teachers for a total of 10 hours in a blended learning format course (2 face-to-face sessions of 3 hours each, plus 4 hours of online work), which itself was designed using CLFPs and atomic patterns. In this workshop, participant teachers were to learn through individual and collaborative (practical) activities, how to design and enact CSCL activities using different ICTs, including the university's institutional platform (the Moodle VLE) and a variety of other ICT tools, thus conforming a DLE. During the workshop teachers would work collaboratively with other teachers in designing, deploying and enacting (role-playing) a hypothetical but realistic scenario, and they would work individually in the orchestration of a scenario relevant for their own practice (e.g. one of the courses each of them taught). The workshop's learning design involved the following coarse-grained phases (see Table 4.4 for a more detailed excerpt of this design and the patterns involved):

- Pre-session online activities, which included the reading of the sample scenario to be used during the workshop, and answering an online questionnaire with background and starting knowledge questions (TW3-Q1).

- First face-to-face session, where teachers had to design a CSCL experience for the proposed generic scenario, collaborating in groups (following the Pyramid CLFP, and using pen \& paper, but also ICT tools), and using design-time and deployment-time atomic patterns as aids during the process.

- Post session online activities, including answering a questionnaire about the perceived usefulness of the presented atomic patterns and the first session in general (TW3-Q2), and proposing a real CSCL experience, similar to the one in the first session, using the same CLFPs and atomic patterns, but this time to be done individually for a subject each teacher taught.

- Second face-to-face session, in which teachers individually tried to implement their designs using the WebCollage learning design tool, and trying to deploy it to Moodle using the GLUE!-PS system. Also, the teachers would role-play the enactment of parts of the

\footnotetext{
${ }^{20}$ Which, among other things, takes care of the in-service teacher professional development, more information at http://www . buendia.uva.es/ (Last visit: 3 Jun 2012).
} 
designed experiences, using a subset of the elicited enactment-time atomic patterns as a support to react and reflect on such simulated enactment.

- Post session online activities, including answering a questionnaire about the usefulness of the presented technological tools and atomic patterns (TW3-Q3). Additionally, participants had to answer another questionnaire about the professional development action, passed on by the Buendia center for their own purposes (but which was also analyzed in our research) (TW3-Q4).

Regarding our data sources (some of which have already been mentioned), we followed the general mixed method data gathering and analysis structure outlined in Figure 4.14. The face-to-face sessions of the workshop were audio and video recorded (TW3-R1, TW3-R2), and observation notes of the session were taken by two researchers acting as external observers (TW3O1, TW3-O2). Several questionnaires with quantitative and qualitative data were answered by the participants, before, during and after the workshop, to understand the participants' profile and prior knowledge (TW3-Q1), and to assess the perceived usefulness of the workshop and of the conceptual and technological tools presented in it (TW3-Q2, TW3-Q3, TW3-Q4). The artifacts (e.g. the learning designs) generated by the teachers during the workshops were also used as documentary evidence (TW3-D). Finally, six semi-structured interviews were conducted with volunteer participants, to gain further insights about their background and the perceived usefulness of the tools presented in the workshop (TW3-I). These data sources and their codes are also summarized in Table 4.11

Table 4.11: Main data sources used during the TW3 workshop

\begin{tabular}{|lll|}
\hline Source & Kind of evidence & Codes \\
\hline \hline Observation notes of the first face-to-face session & Qualitative & TW3-O1 \\
\hline $\begin{array}{l}\text { Observation notes of the second face-to-face ses- } \\
\text { sion }\end{array}$ & Qualitative & TW3-O2 \\
\hline First session's recording & Qualitative & TW3-R1 \\
\hline Second session's recording & Qualitative & TW3-R2 \\
\hline $\begin{array}{l}\text { Teacher-generated documents during the work- } \\
\text { shop }\end{array}$ & Qualitative & TW3-D \\
\hline First profiling questionnaire & Qualitative & TW3-Q1 \\
\hline $\begin{array}{l}\text { Second questionnaire, about the usefulness of the } \\
\text { first session elements }\end{array}$ & $\begin{array}{l}\text { Quantitative \& Qualita- } \\
\text { tive }\end{array}$ & TW3-Q2 \\
\hline $\begin{array}{l}\text { Third questionnaire, about the usefulness of the } \\
\text { online and second session elements }\end{array}$ & $\begin{array}{l}\text { Quantitative \& Qualita- } \\
\text { tive }\end{array}$ & TW3-Q3 \\
\hline $\begin{array}{l}\text { Fourth questionnaire, about the overall workshop } \\
\text { evaluation }\end{array}$ & $\begin{array}{l}\text { Quantitative \& Qualita- } \\
\text { tive }\end{array}$ & TW3-Q4 \\
\hline $\begin{array}{l}\text { Post-workshop semi-structured interviews (profil- } \\
\text { ing and evaluation) }\end{array}$ & Qualitative & TW3-I \\
\hline
\end{tabular}

Even if our evaluation had a clear goal, represented by the evaluative tensions marked above (I1b, I2), its findings can also be organized around the following topics (see Figure 4.13), some of which were already present in the previous evaluation iterations. However, other issues emerged from the data collected in this new context: "Are atomic patterns useful for practitioners to orchestrate blended CSCL activities across DLEs?" (T1b), "Is the format of the workshop itself and its orchestration (i.e. the learning design and its enactment) affecting the perceived usefulness of atomic patterns?" (T2), "Does the teachers' degree of experience (with CSCL and in general) affect the perceived usefulness of atomic patterns?" (T4), "Do the teachers' 
beliefs (e.g. about pedagogy) affect the perceived usefulness of the approach?" (T5), and "Do teachers consider useful the embedding of atomic patterns in educational technology?" (T6). The following subsection details the findings around these issues and topics, providing qualitative and quantitative evidence.

\section{Findings and evidence}

The workshop, as it has been mentioned, accounted for 10 hours of teacher work, distributed in two 3-hour face-to-face training sessions and 4 hours of online work. The face-to-face sessions were finally held in June and September 2011, respectively (in order to take advantage of less busy times in the university teacher's dynamics, and because of restrictions in the development of the GLUE!-PS prototype). The general phases and activities of the workshop design described above were followed, although many changes emerged, especially during the face-to-face sessions, where accumulated delays, technical failures and a too-optimistic time plan prompted for changes in the concrete activities that were done: for example, a face-toface debate to be held in the first workshop session was substituted by an online Moodle forum where teachers and participants commented on the learning designs done during the first session. Here, it is important to note that a moderate amount of improvisation was needed, but also having alternative plans and resources in case of failures or delays (which itself was an elicited orchestration atomic pattern) were very helpful in coping with these emergent occurrences.

A selection of 61 atomic patterns (plus the Pyramid CLFP ${ }^{21}$ ) was used in the workshop; the atomic patterns were divided in three groups: 19 design-time, 20 deployment-time and 22 enactment-time atomic patterns (albeit during the workshop they were generally labeled "routines", which is a more familiar term for teachers - thus, this term would appear repeatedly in the evidence presented below). Thus, the following findings in reality apply to this subset of atomic patterns applied in the workshop (although probably some 'naturalistic generalizations' could be made for the rest of the routine catalogue).

On the usefulness of atomic patterns for practitioners to orchestrate blended CSCL activities across DLEs (T1b) Regarding our first and most important issue, we gathered further evidence of the perceived usefulness of the presented atomic patterns. As it can be seen in Table 4.12 and Figure 4.20, teachers valued highly (quantitatively, but also with qualitative responses) the three different groups of atomic patterns presented (design-time, deploymenttime and enactment-time) regarding their usefulness as a conceptual tool during the different orchestration phases. Also, they were seen as very likely applicable to their teaching practice, and every participant teacher (who answered the questionnaire) asserted that they had used some of the presented atomic patterns in their practice (and thus, seemed to confirm the good mirroring qualities already observed in previous iterations). However, this closeness also had a flip side: teachers did not see them as really new or revolutionary, sometimes even calling them "obvious" (but this also depends on the participant's experience, see topic T4 below). Nevertheless, teachers expressed that they considered atomic patterns useful as a source of inspiration with different possible alternatives or, as some may put it, "reminders". Some teachers also warned against

${ }^{21} \mathrm{~A}$ detailed description of this pattern is available online, at http://www.gsic.uva.es/ daviniahl/clfp/ pyramid-en/ (Last visit: 3 Jun 2012). 


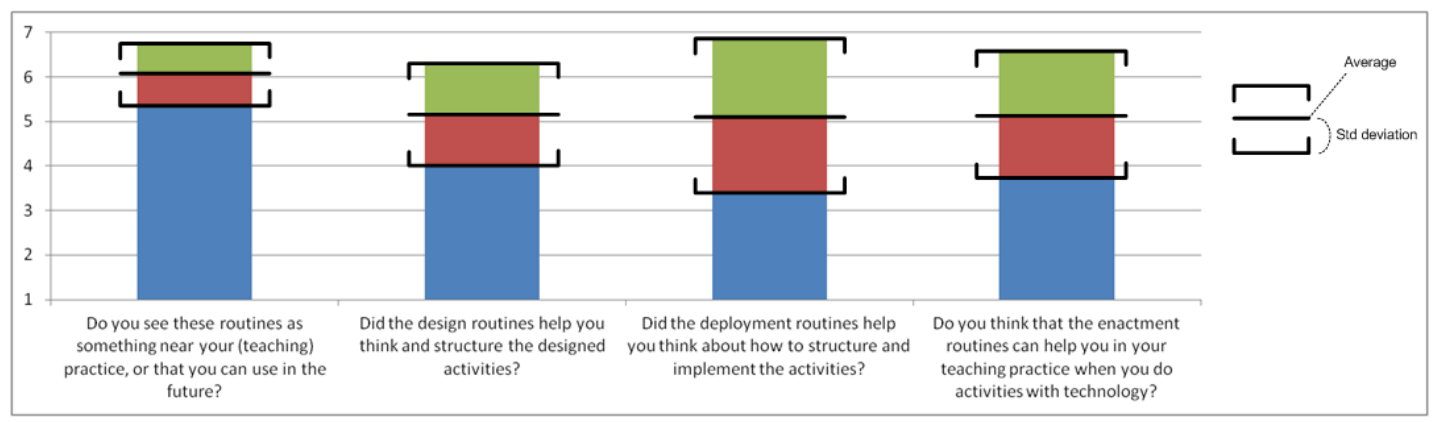

Figure 4.20: Graphical representation of selected quantitative evidence from the questionnaires in the evaluation of the third iteration (TW3)

the dangers of over-using patterns, making too complex designs that may no longer be feasible to put in practice.

Interestingly, some teachers saw enactment-time atomic patterns as useful even in designtime since, by providing solutions to common enactment problems, these atomic patterns allowed teachers to anticipate orchestration problems that could arise during enactment. The classification of atomic patterns into the aforementioned three phases (design, deployment and enactment) was considered adequate, but teachers already noticed that it was rather fuzzy, with some atomic patterns being usable in multiple phases. Also, some teachers seemed to confirm our hypothesis that the provision of concrete contextual elements in the atomic pattern (i.e. examples of use in concrete settings) was useful for them.

Table 4.12: Selected findings and evidence from the TW3 workshop, on the issue of atomic patterns' usefulness (T1b)

\begin{tabular}{|c|c|}
\hline Finding & Selected supporting evidence [Source] \\
\hline $\begin{array}{l}\text { Atomic patterns are seen as feasible, close to } \\
\text { everyday practice }\end{array}$ & $\begin{array}{l}\text { [why do you think [atomic patterns] are near your practice?] Because } \\
\text { they are very practical. [TW3-Q3] } \\
\text { [why do you think [atomic patterns] are near your practice?] Many of } \\
\text { them are common sense (others aren't, and it is good to be reminded } \\
\text { of them). [TW3-Q3] } \\
\text { Teachers valued closeness of atomic patterns to everyday practice very } \\
\text { highly (average of } 6.06 \text { in a 1-7 Likert scale) [TW3-Q2] } \\
17 \text { out of } 17 \text { teachers who answered the questionnaires (100\%), stated } \\
\text { that they had used in their practice some of the presented atomic } \\
\text { patterns [TW3-Q2] }\end{array}$ \\
\hline $\begin{array}{l}\text { Atomic patterns are useful as a source of } \\
\text { alternatives }\end{array}$ & $\begin{array}{l}\text { [to the question: Why did you find [design-time atomic patterns] use- } \\
\text { ful?] They make you think about different strategies to follow in the } \\
\text { classroom. [TW3-Q2] } \\
\text { [to the question: Why did you find [design-time atomic patterns] use- } \\
\text { ful?] Well, I could have done something without them... but they } \\
\text { helped to think about alternatives. [TW3-Q2] }\end{array}$ \\
\hline Atomic patterns are useful as reminders & $\begin{array}{l}\text { [about the novelty of atomic patterns] Some of them are more like } \\
\text { "reminders" [TW3-I] } \\
\text { [about the novelty of the atomic patterns] They look like common } \\
\text { sense once you're told them, but it is good having them because some- } \\
\text { times they may not occur to you. [TW3-I] }\end{array}$ \\
\hline
\end{tabular}


Table 4.12 (continued from previous page)

Finding
$\begin{aligned} & \text { Atomic patterns are useful during the design } \\ & \text { phase }\end{aligned}$
Selected supporting evidence [Source]

[about the enactment-time atomic patterns] They might be things that you do instinctively. But if you explicit them during the design, they're perfect $[\ldots]$ they help you follow a script for the lesson $[\ldots]$ to follow the guiding principle ["hilo conductor"] in a correct way [TW3-I]

[to the question: Why did you find [design-time atomic patterns] useful?] Because, as we were planning [the design], we could choose the one that most suited each moment. Many of them were previously unknown to me, and thus they also served me to know [new ones]. [TW3-Q2]

[to the question: Why did you find [design-time atomic patterns] useful?] Having the whole catalogue of possibilities in front of me helps to organize the ideas better at the beginning. Probably if you have lots of experience you already have them in mind and do not need the cards, but at the beginning it is very useful. [TW3-Q2]

[to the question: Why did you find [design-time atomic patterns] useful?] I had used used some of the routines already in my teaching. However, having them in front of me help me to plan the design faster. [TW3-Q2]

Teachers valued design-time atomic patterns' usefulness highly (average of 5.18 in a 1-7 Likert scale) [TW3-Q2]

Atomic patterns are useful in

deployment-time

[to the question: Why did you find [deployment-time atomic patterns] useful?] Because sometimes we have a clear idea of what we want, but not how to get it with our means. In this sense, the catalogue of recommendations can be an excellent guide to design activities. [TW3-Q2]

[to the question: Why did you find [deployment-time atomic patterns] useful?] They are, evidently, implementation aids. I think they help very much in determining how to do what you had designed. [TW3Q2]

Teachers valued deployment-time atomic patterns' usefulness highly (average of 5.12 in a 1-7 Likert scale) [TW3-Q2]

Dangers of atomic pattern overuse [about the novelty of the atomic patterns] [...] The bad side is that you wanted to use this, and this, and this... and you ended up with a host of routines. [TW3-I]

Enactment-time atomic patterns are useful to anticipate problems in TEL practice

[why did you find [enactment-time atomic patterns] useful?] [...] Because they anticipate solutions to problematic situations that you can find in your teaching practice. [TW3-Q3]

[why did you find [enactment-time atomic patterns] useful?] [...] I believe they cover a wide array of situations, and their application is adequate for activities with technology. [TW3-Q3]

Teachers valued enactment-time atomic patterns' usefulness highly (average of 5.17 in a 1-7 Likert scale) [TW3-Q3]

[about the novelty of atomic patterns] Maybe some of them I have never used them, but in general [...] they were more or less obvious [...] Anyway, I think they are useful because they are standard elements [of practice] [TW3-I]

[about the novelty of the atomic patterns] they are everyday things [...] but not all of them are obvious, some are very interesting, I liked the idea of routines [...]. [TW3-I]

[why did you find [enactment-time atomic patterns] useful?] [...] but they are a bit common sense. Some of them, however, may not be so obvious. [TW3-Q3]

\begin{tabular}{ll}
\hline The classification of atomic patterns is & [about the order in which atomic patterns were presented] It is logical \\
adequate, but fuzzy & {$[\ldots]$ but there are some that can be... yellow-greenish [reference to the } \\
& colors of the design and deployment routine cards - yellow and green \\
& respectively]. [TW3-I] \\
& [about the presented atomic patterns] the routines systematization \\
& was especially positive [...] it was a systematization of common sense, \\
but the systematization is needed, because having it prepared can get & you out of trouble. [TW3-I]
\end{tabular}


Table 4.12 (continued from previous page)

\begin{tabular}{ll}
\hline Finding & Selected supporting evidence [Source] \\
\hline $\begin{array}{l}\text { The provision of concrete contextual elements } \\
\text { (examples) in the atomic pattern is useful }\end{array}$ & $\begin{array}{l}\text { [about the novelty of the atomic patterns] some are very interesting, } \\
\text { I liked the idea of routines [...] I liked that you described the routines } \\
\text { and then you put some examples [...] The most helpful part were the } \\
\text { examples [...] [TW3-I] }\end{array}$ \\
\hline
\end{tabular}

Furthermore, it is worth noting that, during the realization of the workshop and especially during the analysis of the gathered data, it became apparent that the design of the workshop and the way we had orchestrated it was probably affecting the perceptions and opinions of teachers about them (see Issue TW3-I2, below), an aspect that had already appeared in previous iterations. That is why we tried to gather quantitative and qualitative data around this issue, which is detailed below.

On the the influence of the workshop's own orchestration in the perceived usefulness (T2) In general, the workshop itself was very well valued by participants as a training action (see Table 4.13), as they were the materials provided within the workshop (most notably the selection of the atomic pattern catalogue, both in electronic and paper card forms). Moreover, the proposed hypothetic scenario was considered significant and useful for teaching practice.

However, both by looking at the quantitative and qualitative responses to questionnaires, we can see that one aspect that many participant teachers considered lacking was the time allocated for the workshop and other time management issues, which highlighted that the pace and duration of the workshop had been miscalculated, given the proposed tasks. Also, participants were in some cases confused about what was the expected outcome of each task undertaken in the workshop. This was true both in the first face-to-face session (which dealt mostly with designand deployment-time atomic patterns) and in the second one (which dealt with enactment-time atomic patterns), which very often made teachers act instinctively instead of reflecting on the conceptual tools being provided. Also, we probably underestimated the total time load of the workshop, and spanning the workshop over 3 months was probably not a good idea.

However, even with these problems, the fact that teachers were in most cases able to provide designs that used the atomic patterns, and to role-play them in the simulated enactment, even under such time pressure, pointed at the potential of atomic patterns for this kind of teacher professional development actions. It is also worth highlighting that the practical nature of the workshop, and especially the usage in our workshop design of the same techniques that were being taught (CLFPs and atomic patterns), were considered a very positive aspect of the workshop.

From the evidence around this issue we can gather, as we did in previous iterations, that the situated nature of the workshop, and our ability as workshop facilitators to orchestrate the experiment adequately, had an important effect on what teachers learned through atomic patterns, and the usefulness perceived in consequence. The fact that this workshop tried at the same time to expose teachers to very different kinds of tools (atomic patterns and GLUE!-PS) in such a short time, had obvious shortcomings. Overall, we can say that probably a longer workshop, dedicated solely to atomic patterns, better planned time-wise, and with better-specified tasks, would have been more effective in communicating atomic patterns' usefulness. However, even this improvement would probably be limited by factors that we describe in the issues below. 
Table 4.13: Selected findings and evidence from the TW3 workshop, on the influence of the workshop's own orchestration in atomic patterns' perceived usefulness (T2)

Finding
The timeframe of the sessions was n
adequate
The workshop tasks sometimes were
not clear enough for participants
not clear enough for participants
Selected supporting evidence [Source]

[about the workshop activities regarding atomic patterns] Something that struck me was that you just handed the cards without saying anything [...] there was almost no time to read them. That is something I would change [...] separating the sessions [by three months] was not a good idea either. [TW3-I] [to the question: explain your scoring of the first part of the workshop] [...] Since time was limited, it would have been crucial to detail more concretely what was being asked to do in each phase. [TW3-Q2]

[to the question: explain your scoring of the first part of the workshop] Negative: $[\ldots]$ it is needed to convince participants of the benefits of the workshop $[\ldots]$ time was very scarce [...] It lacked a bit of detail about the tasks that we had to do- [TW3-Q2]

[while doing the design in small groups] Group 3 seems to have difficulties to plan the design. One component says "I'm quite basic at structuring tasks, I see this as very abstract". The ideas she exchanges with her partner are totally unrelated to the pyramid CLFP. [TW3-O1]

[to the question: explain your scoring of the first part of the workshop] [...] The idea of doing the proposal in groups and supergroups [i.e. the pyramid pattern] was productive [...] Having the cards in digital format helps a lot to work offline, but I find it more efficient to have them [physically] with you and being face-to-face to review the results. [TW3-Q2]

[to the question: explain your scoring of the first part of the workshop] I liked it very much because I could try the implementation of a real collaborative work [TW3-Q2]

[to the question: explain your scoring of the first part of the workshop] It is very positive to teach a learning-teaching methodology using that same methodology in the course. The preparation of the course was very good, and so were the media and materials chosen [...] [TW3-Q2]

[to the question: explain your scoring of the first part of the workshop] Positive: the practical structure of the workshop, the routines given are very useful to remember things to take into account when designing [...] [TW3-Q2]

The workshop was highly valued by teachers (average of 8.3 in a 10-point scale) [TW3-Q4]

The workshop materials were highly valued by teachers (average of 8 in a 10-point scale) [TW3-Q4]

The first part of the workshop (collaborative, design and deployment-oriented part) scored high on participant satisfaction (5.59 in a 1-7 Likert scale) [TW3Q2]

The proposed scenario was thought significant and useful to illustrate teaching practice (5.15 for significance, 5.4 for learning usefulness in a 1-7 Likert scale) [TW3-Q1]

The workshop's workload was miscalculated [general observations to the course] The workload of the online tasks has been bigger than defined. The course would have benefited from some more face-to-face session. [TW3-Q4]

[about the usage of atomic patterns in the role-played enactment] I think we acted instinctively [...] the problem was time, there was no time for proper reflection. [TW3-I]

Workshop's fast pace did not leave enough time for learning and reflection [about the usage of atomic patterns in the role-played enactment] I think we responded spontaneously [vs looking at the atomic pattern cards]

[about the workshop activities regarding atomic patterns] Something that struck me was that you just handed the cards without saying anything [...] there was almost no time to read them. That is something I would change [...] separating the sessions [by three months] was not a good idea either [TW3-I] [to the question: why did (not) you find deployment-time atomic patterns useful?] We had almost no time to read them. In the end, it was more like a puzzle where we tried to fit the cards in the script that we had [TW3-Q2] [to the question: why did (not) you find design-time atomic patterns useful?] Because of lack of time. [TW3-Q2]

[to the question: why did you find deployment-time atomic patterns useful?] [...] however, in the case of the [deployment] routines, there was less time to read and apply them. [TW3-Q2]

The time allocated for the workshop was valued considerably lower than the rest of workshop aspects (6.44 in a 10-point scale) [TW3-Q4] 
On the the influence of the participants' prior experience in the perceived usefulness (T4) An interesting emergent issue that we came across while analyzing data was the noticeable influence of participants' prior experience (e.g. years of teaching experience, past experience with collaborative learning) in how useful atomic patterns seemed to them. Most participants were not very experienced in teaching through collaborative learning (3.25 in a 1-7 Likert scale), and even less in doing CSCL (2.55 in a 1-7 Likert scale). However, most of them had already more than 6-8 years of teaching experience (which relates to the fact that a majority were teaching master-level courses). Their proficiency on the use of ICT was generally high (with a few outliers), but not so many of them used ICT frequently for teaching (3.75 in a 1-7 Likert scale).

As we can see in the evidence below (Table 4.14), in the case of participants that had already done collaborative learning teaching in the past, the outcome of those experiences shaped highly their attitude and motivation towards the whole collaborative approach to pedagogy (and, hence, towards the workshop contents). In many of the qualitative responses it can be seen that more experienced teachers dismiss the routines as "obvious" or "common sense" (albeit others also considered them useful as "reminders"), trusting more their own internal routines. Some pieces of evidence suggest that less experienced people may find them more interesting, and that some teachers see them as solutions that have worked for them in the past.

Thus, we may suggest that the atomic patterns presented in the workshop would be more useful for less-experienced teachers (especially regarding expertise in ICT usage for teaching). However, there seems to be a lower threshold of ICT knowledge and usage, below which teachers do not even try to learn these ICT uses represented by atomic patterns (as it happened with a few participants in the workshop, which abandoned it once they learnt the amount of ICT work that the workshop implied). In any case, this workshop only provided preliminary evidence on this influence of experience in atomic patterns, which we decided should be explored more deeply in following iterations.

On the influence of beliefs (about ICT, about collaboration) in the perceived usefulness (T5) There exists yet another factor which seems to have influenced the perceptions of teachers about the presented routines, which is their beliefs and attitudes towards ICT, teaching and collaborative learning in general. The participants of the workshop varied hugely in their attitudes towards CL (avg=4.5, $\mathrm{std}=1.54$ in a 1-7 Likert scale). As we can see in Table 4.15, these initial attitudes have a variety of origins, many of them pragmatic, often relating to orchestration problems inherent to collaborative learning in authentic settings (e.g. timing issues, difficulties for individual assessment, incompatibility with subject or tight curriculum constraints, etc).

As we can see from the qualitative evidence, even if many teachers used atomic patterns in the individual design they did as part of the workshop's online work, the workshop had very limited success in changing these prior attitudes (with a few exceptions of motivated teachers that embraced the newfound method, casually among the younger participants). In fact, the workshop's own collaborative design made apparent some of the common orchestration problems in CSCL (teacher increased workload, necessity of having alternatives in case of failures, etc) in a very practical way. On the other hand, it seems that the workshop did not discourage positive 
Table 4.14: Selected findings and evidence from the TW3 workshop, on the influence of participants' experience in the perceived usefulness of atomic patterns (T4)

\begin{tabular}{l}
\hline Finding \\
\hline Experienced teachers dismiss atomic \\
patterns as obvious
\end{tabular}

patterns as obvious
Selected supporting evidence [Source]

[about the novelty of routines] Maybe there were some that you would not think of but most of those things are already familiar to a teacher [...] I have identified them as things I use daily, and that is an added value [...] maybe because I work a lot with [ICT] and you are used to the problems it implies [...] I understand that people that do not know these things will find them useful. [TW3-I]

[to which extent you used the workshop materials for your design?] I used the provided design template [...] I did not read again the routines because I got the idea that [routines] were just a formalization of things we already do. [TW3-I]

Novel teachers see atomic patterns as useful

[about using routines in real practice] I asked you if I could take them home $[\ldots]$ Yes, I use them [...] This year, when I have designed some activity [with ICT] I have used some of them [...] yes, they give you ideas. Maybe it is because I am relatively novel [using ICT/CL]. [TW3-I]

Prior experience shaping perceptions and attitudes towards CSCL [about previous experience with CL] Last year I did a "kind of" jigsaw [...] students were very happy, engaged, motivated [...] with pen and paper. [TW3I]

[about previous experiences with collaborative work] It was difficult to measure student effort, great variability [...] many students (50\% or more) did not like it, especially the collaborative work [...] how do you assess? [...] The learning results were $[. .$.$] very uneven. [TW3-I]$

[about the applicability of collaborative learning] In the bachelor's course I've been teaching [...] the students are less motivated to be distracted by this sort of thing [...] In the master's courses I think it fits much better. [TW3-I]

Some experienced teachers see atomic patterns as useful reminders [why did (not) you find deployment-time atomic patterns useful?] [...] They serve to remember procedures or routines that, if you try to do this kind of activity, will emerge spontaneously, from your personal experience. [TW3-Q2] [why did you find enactment-time atomic patterns useful?] [...] I believe that most of them are known to teachers. They may be useful to remind us of problems that can emerge and to plan alternatives. [TW3-Q3]

Some teachers see atomic patterns as solutions that have worked for them in the past [why do you think [atomic patterns] are near your practice?] Because they are routines that, in part, I already use and I have checked that they work well. [TW3-Q2]

[why do you think [atomic patterns] are near your practice?] I have used the routines for years because I think there are subjects where they fit better, but it also depends on the teaching style. I had teachers that used this kind of practice 3-4 decades ago when these theories were not so 'in vogue'. [TW3-Q2] 
attitudes towards CSCL, rather providing a more realistic and practical view of how CSCL can (and does) happen in an authentic classroom setting.

This lack of attitude change (which is somewhat expected from such a short exposure to complex collaborative learning) also marks clearly that one of the main barriers for adoption of CSCL methods is the fact that these orchestration problems still stand out while approaching CSCL in a typical classroom. Providing tools that support teachers in overcoming these problems, as this dissertation does, is thus justified, prompting researchers to find solutions to them.

On the usefulness of embedding atomic patterns in educational technology (T6) Although this issue (and the research question it represents) was not as relevant for us as the previous ones, parts of the questionnaires and interviews were directly targeted at it, since it represents a way of merging two of the main contributions of the thesis (the technological and the conceptual tools for teachers).

As we can see in Table 4.16, the opinions of participants regarding the form that this embedding of atomic patterns into technology should take is quite varied: some of them would find useful to have recommendations of routines during design, although many participants are concerned about that kind of support being too disruptive (or not being "smart" enough). In fact, many advocate the form of atomic patterns as automated actions, as far as it is possible. The idea of having detailed mini-tutorials for implementing a routine/action with a concrete ICT tool is thought useful, but maybe not usable in real-time (e.g. in a face-to-face CSCL session). However, many teachers did not have a clear idea of which form this integration would take, although some of them point out that they should be integrated within the existing tooling for orchestration of CSCL (e.g. in authoring tools or in the VLEs themselves).

Another interesting outcome in this regard is that some participants marked a number of routines as most interesting, for their implementation with technology or otherwise. These routines include: Debate, Brainstorming, Introduce mini-lecture, Submission of artifact, Peer review, Rubrics, Prepare backup ICT, Make activity face-to-face to ensure interaction, Divide and conquer, Test task to exemplify tool, Regroup in light of attendants, Synthesis, Experience presentation, Formative assessment (give feedback), or Use questionnaire as a base for debate. This list in fact was cross-checked with the one the author elaborated to try to include them in the GLUE!-PS roadmap (Section 5.3.2). However, this listing of atomic patterns and our inquiries about the form in which they should be incorporated into technology, were very preliminary and had to be continued in next iterations.

\section{Conclusions and feedback for the next iteration}

Overall, the workshop garnered rather positive feedback from participants, who considered the atomic patterns useful reminders, and the workshop itself a very interesting teacher development action. The main partial conclusions that we obtained from analyzing the available evidence through the aforementioned issues were (see also Figure 4.21 .

- We obtained further confirmation that atomic patterns are useful conceptual tools for orchestration in the eyes of teachers, across the different stages of orchestration (design, deployment, enactment) [TW3-T1b-PC1] 
Table 4.15: Selected findings and evidence from the TW3 workshop, on the influence of participant beliefs in atomic patterns' perceived usefulness (T5)

\begin{tabular}{l} 
Finding \\
\hline Participants obtained a more \\
realistic/practical view of CSCL
\end{tabular}

Selected supporting evidence [Source]

[to the question: did the workshop change your views about collaborative learning?] Yes, but I realized that it is complex to put in practice, that it requires lots of time and I believe it would not be so easy with certain groups of students. [TW3-Q3]

[to the question: explain your scoring of the first part of the workshop] [...] I see that the same problems that emerge in the workshop (optimistic time plan, ICT failures, etc) make the teacher workload (and stress) much higher than using traditional methods. [TW3-Q2]

[about collaborative learning] I think it is not time-efficient, neither for the teacher or for the student [...] it very engaging, reinforces personal interactions

Prior beliefs and attitudes about [...] but time is not its strength [...] plus, also, the time required for learning collaborative learning it. [...] I think collaborative learning is better in subjects were you construct your own knowledge, reality [...] (e.g. literature, arts, design) [...] Plus, there are teachers in the grade that already develop those transversal competences, I develop others. [TW3-I]

[about collaborative learning] some things I agree, but others... I'm not such an enthusiast [...] our students are sometimes lacking in ability to collaborate, but I am more worried about the lack of individual work capability. [TW3-I] [about previous courses about project-based learning] I thought that it was interesting, but I did not see how you could put that into your classroom with reasonable dynamics, fulfilling your objectives and especially, measuring them. [TW3-I]

[to the question: explain your scoring of the first part of the workshop] I believe that learning collaboratively increments the reflecting capability, decision making [...] and other personal skills very needed in our everyday life [...] [TW3-Q2]

[to the question: why do you think [atomic patterns] are near your practice?] It depends on the course. In first [bachelor's] year I think it is too complex, but they might be ideal for master's because there are less students. [TW3-Q2] [about previous experience in the use of ICT] I have always liked ICTs, I use them whenever I can [...] I think that nowadays it is crucial to use them. [TW3-I]

Teachers used the presented atomic-
pattern approach in their individual
work
[to the question: to which extent you used the workshop materials for your design?] I had the table full of green and yellow cards [...] I used them all at the same time [vs. in phases like design and deploy] choosing one or another depending on my ideas. [TW3-I]

The workshop did not change already
positive attitudes towards CSCL

The workshop did not change the negative attitudes towards CSCL 
Table 4.16: Selected findings and evidence from the TW3 workshop, on the usefulness of embedding atomic patterns in educational technologies (T6)

\begin{tabular}{l}
\hline Finding \\
\hline Atomic pattern recommender systems \\
could be useful for online learning
\end{tabular}

Automation of certain atomic patterns would be useful
Selected supporting evidence [Source]

[about incorporating routines as more actionable advice] I think that probably we would not read them [in a face-to-face session] [...] but in distance work, I would use them [...] I think that would be good, if brief and simple. [TW3-I] [about incorporating atomic patterns as actions in the system] things that you have to do with these tools which are not immediate [e.g. changing groups in Moodle], automating them would be useful. [TW3-I]

[about incorporating routines as more actionable advice] I think it would be good [to have more detailed steps about executing a routine], but if the system could do it [to execute e.g. the re-grouping in an activity], that would be even better. [TW3-I]

[about incorporating routines as recommendations] a system that monitored what was happening and recommended [...] would simplify teacher's work. A recommender [...] is OK, but I think that the actions would be more useful. [TW3-I]
Doubts about the form of integrating atomic patterns into technology [about incorporating routines as advice during the design] I think that it would be useful, if we had it in a subtle way, optional, non-disruptive. [TW3-I] [about incorporating routines as more actionable advice] Rather, I would probably go for a totally different tool [e.g. pen and paper] [...] especially in big classrooms of 50 or so [e.g. bachelor's]. [TW3-I]

[to the question: do you think it would be useful to incorporate these routines into educational technology?] I am not sure of it. What would be really important is to teach teachers to use these routines. [TW3-Q3]

Integration of atomic patterns into existing tools would be useful to the question: do you think it would be useful to incorporate these routines into educational technology?] I would find it more intuitive if you could do it directly from Moodle. [TW3-Q3]

[to the question: do you think it would be useful to incorporate these routines into educational technology?] I would find it useful to incorporate them throughout the process, both for designing and for its implementation. [TW3Q3] 
- We also confirmed another of our conclusions from the primary school context, that highlighted atomic patterns as feasible and close to the everyday practice of teachers in the same institution, even across different disciplines (although the bias towards technical backgrounds in this workshop still left space for doubt). As a consequence of this closeness, teachers did not see atomic patterns as new or revolutionary [TW3-T1b-PC2]

- That many teachers saw atomic patterns as useful reminders of options and alternatives that they can put in practice in their teaching, more as starting points than as a normative guide [TW3-T1b-PC3]

- There was also evidence pointing at the fuzzy limits of our atomic pattern classification, especially time-wise: for example, enactment-time atomic patterns can also be useful in design-time, to anticipate problems and adjust the design accordingly [TW3-T1b-PC4]

- We gathered further confirming evidence that teachers appreciated the contextual elements (examples) present in the atomic patterns, which seemed to be useful for them to connect the advice to their own everyday practice [TW3-T1b-PC5]

- After several iterations, the workshop's design structure and materials proved adequate to show atomic patterns' usefulness in such a short period of time [TW3-T2-PC1]

- However, there were still complaints about the workshop's alloted time being still insufficient (as the reader may have noticed, the workshops involved a total of two hours in the first iteration, 3-4 hours in the second one, and around 7-8 in this third one) [TW3-T2-PC2]

- Even if the workshop design was quite adequate, our orchestration of it could be improved, not only by increasing the time dedicated to the different tasks, but also stating more clearly the expected task outcomes in every phase of the workshop [TW3-T2-PC3]

- We have preliminary evidences that experienced teachers (even if they are not CSCL experts) tend to dismiss atomic patterns as obvious, thus being more useful for targeting novel teachers [TW3-T4-PC1]

- That prior experiences with CSCL might affect the learning gains from atomic patterns [TW3-T4-PC2]

- As we already did in the first stages of our work in primary schools, we have confirmed again that prior beliefs (about teaching, ICT, but especially about collaborative learning) are hard to change through atomic patterns, or at least using this kind of limited training actions [TW3-T5-PC1]

- Even if the practical nature of the workshop showed clearly some of the orchestration problems typical of CSCL in DLEs, the workshop did not discourage already existing positive attitudes towards collaborative learning, rather giving those teachers a more realistic point of view on how to apply it to their classrooms [TW3-T5-PC2]

- Regarding the embedding of atomic patterns in educational technologies, most teachers prefer the automation of atomic patterns over atomic pattern recommender systems, although opinions are note unanimous in the least [TW3-T6-PC1] 
- Finally, we also gathered further evidence highlighting the importance for teachers of the integration of orchestration support, such as atomic patterns, with existing educational tooling (e.g. in the institutional VLE), emphasizing the non-disruptiveness of such embedding [TW3-T6-PC2]

Again, the usual caveats of the contextuality of our results apply to these partial conclusions, although most of them confirmed and expanded on the ones that had been obtained in a very different educational setting (face-to-face classes in a primary school). Nevertheless, at this point in our research, it was not clear that we had exploited the full potential of atomic patterns. Hence, it was considered that, during the time span of this $\mathrm{PhD}$ thesis, another teacher workshop would be necessary to further confirm these findings, following the general lines of this one, but making a number of modifications that emerged as formative feedback from this iteration:

- To reduce the amount of content in the workshop, to allow for proper reflection and learning about atomic patterns (e.g. leave out the technological side of orchestration through GLUE!-PS usage). For the same reason, the amount or length of face-to-face sessions, and the overall workshop time should be extended.

- To target a more varied group of teachers, especially those with not so much experience with collaborative learning and ICT, to further assess the applicability of atomic patterns to teachers across different disciplines.

- To modify the workshop materials in order to specify more clearly the tasks and expected outcomes, and modify the workshop dynamics to foster more reflection and usage of the presented atomic patterns, as well as the debate among participants and with workshop tutors.

- To gather further data about the prior experience and beliefs of the participants, to better assess their influence in the perceived usefulness of atomic patterns.

\subsubsection{Iteration \#4: A second higher education teacher workshop (TW4)}

As we have described in the previous section, a workshop had been held between June and September 2011 with University of Valladolid teachers (with a big proportion of teachers from technical backgrounds), in order to evaluate atomic patterns in higher education settings, as a support for teacher orchestration of CSCL using Distributed Learning Environments. Although the results were mostly positive on the usefulness of atomic patterns, several issues appeared to be influencing greatly the results: the design and enactment of the workshop itself (i.e. teachers considered the workshop format as very conducent to their learning, although they complained that its enactment was hurried and somewhat haphazard), the teachers' prior experience (i.e. novel teachers seemed to see routines as more useful than experienced ones) and the teachers' own beliefs and attitudes about collaborative learning and ICTs (positive-attitude teachers saw the routines as more useful than negative-attitude ones). Moreover, initial evidences had been gathered about the way in which these atomic patterns should be embedded into educational technologies. 


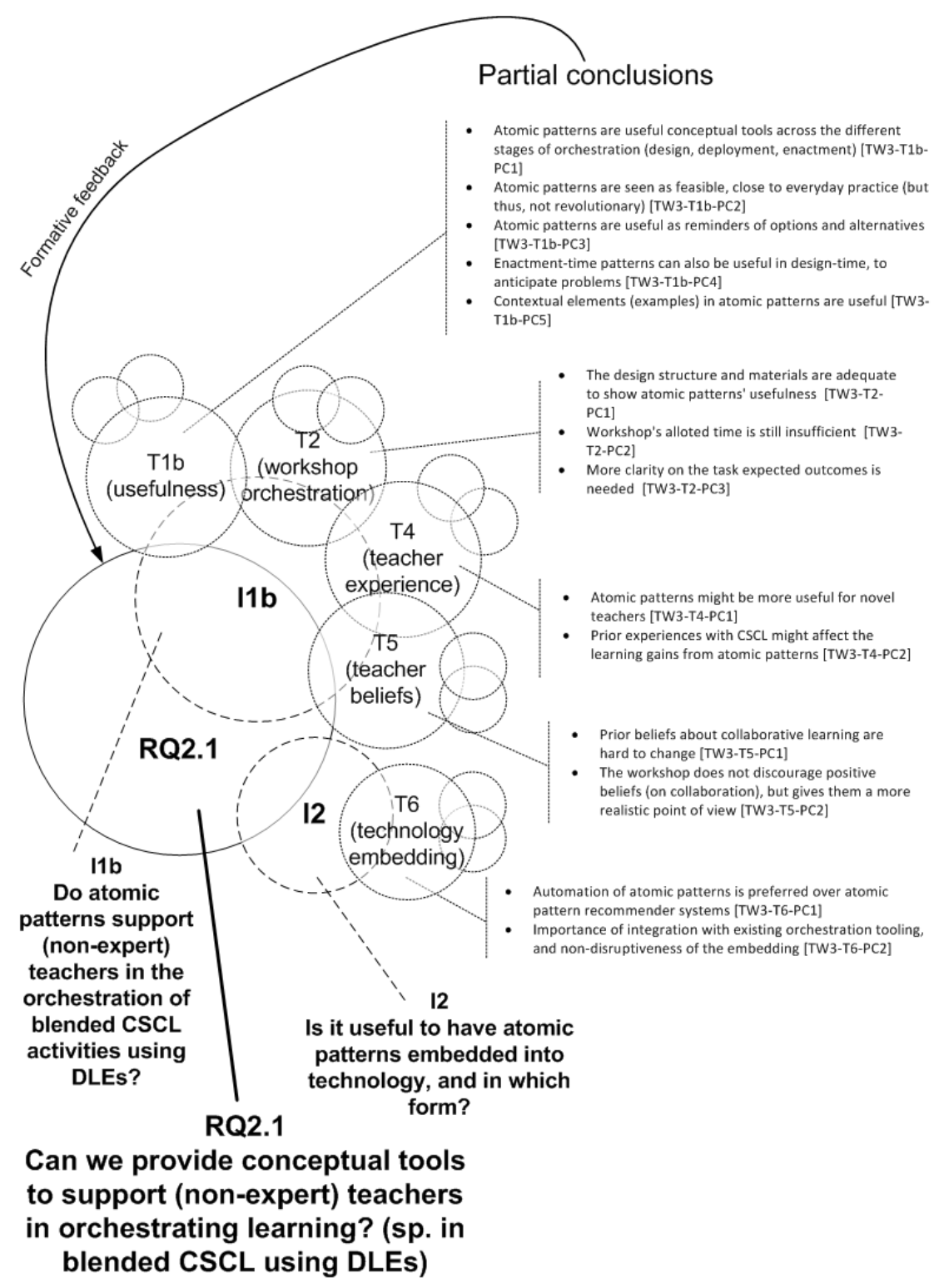

Figure 4.21: Graphical representation of the partial conclusions to Iteration \#3 
Since it was clear for us that further evidence on the usefulness of atomic patterns had to be gathered, with a less-biased group of teachers, in February 2012 we conducted another workshop experience, whose data gathering and workshop design had been modified slightly, this time widening the audience to address teachers from all disciplines. Our goal was to gather more focused data about the aforementioned issues and get a more definitive picture of the usefulness or routines and the multi-level pattern approach that had been recently proposed ([Pri12a $]$, see Section 4.3). Thus, we maintained our main research questions as they were presented in Section 4.5. to assess the usefulness of atomic patterns for practitioners in the orchestration (design, deploy and enactment) of CSCL across DLEs (RQ2.1), while extending our focus also to the perceived usefulness and form most adequate to embed atomic patterns in educational technologies (RQ2.2).

\section{Context and Method}

Following the rationale of previous iterations, we proposed to conduct a professional development workshop for teachers at the University of Valladolid, whose topic would be the design and enactment (i.e. the orchestration) of non-trivial collaborative activities in Distributed Learning Environments. As it happened with the one in the previous iteration, this workshop would provide a first introduction to the design of collaborative activities using collaborative learning flow patterns (see 4.2.3), and structuring them further with a selection of the extended atomic pattern catalogue elicited from higher education. However, in this case atomic patterns would be the sole focus of the workshop, leaving the use of technological tools for orchestration (that is, GLUE!-PS) to a different complementary PD action.

Most of the contextual characteristics of this workshop were similar to the previous one: the workshop was a professional development action done within the auspices of the Buendia center at the University of Valladolid, and it was open for university teachers with a basic knowledge of the involved ICT tools, up to 24 participants (in order for the workshop design to be manageable). However, in this case we imposed no other requirements or biases into the participants, in order to reach a more multi-disciplinar audience.

As it turned out, 25 university teachers participated in the workshop, from very different disciplines and departments ( 7 from Economics \& Business, 7 from Engineering and Computer Science, 4 from Humanities, 3 from Education \& Social sciences, plus people from Physics, Medicine or Architecture). The workshop was supposed to involve teachers for a total of 12 hours (2 face-to-face sessions of 4 hours each, plus 4 hours of online work). Again, participant teachers were to learn through individual and collaborative (practical) activities, how to design and enact CSCL activities using different ICTs, including the university's institutional platform (the Moodle VLE) and a variety of other ICT tools, thus conforming a DLE. During the workshop teachers would work collaboratively with other teachers in designing, deploying and enacting (role-playing) a hypothetical but realistic scenario, and they would work individually in the design of a scenario relevant for their own practice (e.g. one of the courses each of them taught). The workshop's learning design involved the following coarse-grained phases:

1. Pre-session activities that included reading a sample scenario to be used during the workshop, and answering an online questionnaire with background and starting knowledge questions (TW4-Q1). 
2. First face-to-face session, where teachers tried to design a CSCL experience for the proposed scenario, collaborating in groups (following the Pyramid CLFP, and using mostly pen \& paper, but also ICT tools), and using design and deployment routines as aids during the process. The session also included a small mid-session questionnaire about mainly to inquire about the first attempt to design activities using solely CLFP patterns (TW4-Q2).

3. Answering a questionnaire about the perceived usefulness of the presented routines and the first session in general (TW4-Q3).

4. Proposing a real CSCL experience, similar to the one in the first session, using the provided set of CLFPs and routines, but this time to be done individually in a subject the participants were teaching. Workshop teachers were to revise those designs to later give feedback to the participants.

5. Second face-to-face session, in which a small debate was to be held about the designs provided by participants (the debate was also structured as a Think-Pair-Share CLFP ${ }^{22}$. Also, the teachers would role-play the enactment of parts of the designed experiences, using some of the elicited enactment routines as a support to react and reflect on such simulated enactment.

6. Post-session activities, that involved mostly doing a second draft of the individual design for a real course of theirs, which was to be done online, and to which workshop teachers/researchers were to give feedback again. These activities also included answering a questionnaire about the usefulness of the presented technological tools and routines (TW4Q4), and another questionnaire about the professional development action itself, passed on by the Buendia center (but which was also analyzed in our research) (TW4-Q5).

Regarding our data sources (some of which have already been mentioned), we followed the general mixed method data gathering and analysis structure outlined in Figure 4.14. The face-to-face sessions of the workshop were audio and video recorded (TW4-R1, TW4-R2), and observation notes of the session were taken by two researchers acting as external observers (TW4O1, TW4-O2). Several questionnaires with quantitative and qualitative data were answered by the participants, before, during and after the workshop, to understand the participants' profile and prior knowledge (TW4-Q1), and to assess the perceived usefulness of the workshop and of the conceptual and technological tools presented in it (TW4-Q2, TW4-Q3, TW4-Q4, TW4-Q5). The artifacts (e.g. the learning designs) generated by the teachers during the workshops were also used as documentary evidence (TW4-D). Finally, eight semi-structured interviews were conducted with volunteer participants, to gain further insights about their background and the perceived usefulness of the tools presented in the workshop (TW4-I). These data sources and their codes are also summarized in Table 4.17.

In this evaluation we chose to maintain the same evaluation structure already explained for happening TW3, with the following guiding issues as conceptual organizers, and topics that helped us in focusing on the desired tensions of our evaluand [Sta05] (atomic patterns as a tool for orchestration). Figure 4.13 provides a graphical representation of their respective relationships:

\footnotetext{
${ }^{22}$ A description of the Think-Pair-Share (TPS) can be found in http://www.gsic.uva.es/ daviniahl/clfp/ tps-en/ (Last visit: 4 Jun 2012).
} 
Table 4.17: Main data sources used during the TW4 workshop

\begin{tabular}{|lll|}
\hline Source & Kind of evidence & Codes \\
\hline \hline Observation notes of the first face-to-face session & Qualitative & TW4-O1 \\
\hline $\begin{array}{l}\text { Observation notes of the second face-to-face ses- } \\
\text { sion }\end{array}$ & Qualitative & TW4-O2 \\
\hline First session's recording & Qualitative & TW4-R1 \\
\hline Second session's recording & Qualitative & TW4-R2 \\
\hline $\begin{array}{l}\text { Teacher-generated documents during the work- } \\
\text { shop }\end{array}$ & Qualitative & TW4-D \\
\hline First profiling questionnaire & $\begin{array}{l}\text { Qualitative \& Quantita- } \\
\text { tive }\end{array}$ & TW4-Q1 \\
\hline $\begin{array}{l}\text { Second questionnaire, about the perceived useful- } \\
\text { ness of CLFPs }\end{array}$ & $\begin{array}{l}\text { Qualitative \& Quantita- } \\
\text { tive }\end{array}$ & TW4-Q2 \\
\hline $\begin{array}{l}\text { Third questionnaire, about the usefulness of the } \\
\text { first session elements }\end{array}$ & $\begin{array}{l}\text { Qualitative \& Quantita- } \\
\text { tive }\end{array}$ & TW4-Q3 \\
\hline $\begin{array}{l}\text { Fourth questionnaire, about the usefulness of the } \\
\text { online and second session elements }\end{array}$ & $\begin{array}{l}\text { Qualitative \& Quantita- } \\
\text { tive }\end{array}$ & TW4-Q4 \\
\hline $\begin{array}{l}\text { Fifth questionnaire, about the overall workshop } \\
\text { evaluation }\end{array}$ & $\begin{array}{l}\text { Qualitative \& Quantita- } \\
\text { tive }\end{array}$ & TW4-Q5 \\
\hline $\begin{array}{l}\text { Post-workshop semi-structured interviews (profil- } \\
\text { ing and evaluation) }\end{array}$ & Qualitative & TW4-I \\
\hline
\end{tabular}

- I1b Are atomic patterns useful for practitioners in the orchestration of blended CSCL activities across DLEs?

- Are atomic patterns useful for practitioners to orchestrate blended CSCL activities across DLEs? (T1b)

- Is the format of the workshop itself and its orchestration (i.e. the learning design and its enactment) affecting the perceived usefulness of atomic patterns? (T2)

- Does the teachers' degree of experience (with CSCL and in general) affect the perceived usefulness of routines? (T4)

- Do the teachers' beliefs (e.g. about pedagogy) affect the perceived usefulness of the approach? (T5)

- I2 Is it useful to have atomic patterns embedded in the technology? in which form?

- Do teachers consider useful the embedding of atomic patterns in educational technology? (T6)

The following subsection details the findings around these questions and issues, providing qualitative and quantitative evidence.

\section{Findings and evidence}

The workshop, as it has been mentioned, accounted for 12 hours of teacher work, distributed in two 4-hour face-to-face training sessions and 4 hours of online work. The face-to-face sessions were finally held in February 2012, with both sessions separated by one week. The general phases and activities of the workshop design described above were followed, although many changes emerged, especially during the face-to-face sessions, where accumulated delays, 
and a modified but still too optimistic time plan prompted for changes in the concrete activities proposed and their timing.

In order to better compare our results with those of the previous workshop, the same selection of 61 atomic patterns (plus a selection of 4 CLFPs) was used in this workshop. Again, the atomic patterns were divided in three groups, and they were generally called "routines" for easier labeling. Thus, we had 19 "design routines", 20 "deployment routines" and 22 "enactment routines".

On the usefulness of atomic patterns for practitioners to orchestrate blended CSCL activities across DLEs (T1b) The evidence from this workshop confirmed the usefulness of the presented atomic patterns, already hinted at by previous workshops, but also add more depth to our findings. As we can see from Table 4.18, atomic patterns were seen as useful by teachers, but it is remarkable that the quantitative scores for usefulness of the different kinds of atomic patterns were noticeably lower in the case of design-time and enactment-time atomic patterns (4.30 and 4.13, respectively, in a 1-7 Likert scale, versus 5.18 and 5.12 in the previous workshop). The gathered qualitative evidence does not entirely clarify the reason for this difference in scores, although teachers expressed problems due to the high number of atomic patterns being dealt with, or the alloted time for their usage which, despite the increased workshop length, still was considered insufficient (see Issue TW4-I2 below). We can also gather indirect indications of usefulness of the atomic patterns, by comparing the participants self-perceived ability to orchestrate the design they were doing throughout the workshop, from an average of 4.83 (in a 1-7 Likert scale, with a standard deviation of 2.13) after the first round of design (just with CLFPs), to 5.23 ( $\mathrm{std}=1.50)$ after the workshop. This improvement, however, could also be due to the fact that, after the workshop, more design exercises/iterations had been carried out on the designs anyway (see Issue TW4-I2 below). Also, many (86.3\%) of the participant teachers used the provided atomic patterns when doing the individual design work about their own courses (using atomic patterns was not mandatory for that exercis $\ell^{23}$ ). Nevertheless, almost all interviewed participants said that they would use the workshop materials if they were to do collaborative activities in their teaching practice.

It is noteworthy that, from this workshop, we obtained further evidence regarding the added value that atomic patterns represented for the participant teachers, including their usefulness as starting points for bootstrapping the process of designing and implementing CSCL scenarios, the fact that they help teachers in organizing and making more concrete their abstract or implicit design ideas, or the new pedagogical affordances of ICTs that atomic patterns uncovered for them (see Table 4.18). It is also interesting the evidence found about the way some teachers used atomic patterns in the mental process of designing and implementing the scenario: it seems that, for them, the design was a two-way process going back and forth between their ideas and the ones provided by atomic patterns, which apparently were complementary.

On the other hand, the evidence gathered in this workshop confirmed previous findings about the good mirroring qualities of atomic patterns and their closeness with everyday practice

\footnotetext{
${ }^{23}$ Further reflections on the authenticity of the designs (and hence, the validity of this measure) can be found in $\mathrm{MC12b}$ : basically, the designs, despite being for authentic courses, were largely conditioned by the workshop instructions (which is to be expected, since the workshop tried to promote new practices).
} 
(albeit with lower numeric scores), and the fact that atomic patterns are not considered revolutionary or new, especially by more veteran teachers, who dismissed them in favor of acting by intuition (see topic T4 below). In fact, some participants pointed out their usefulness as "giving names and surnames" to existing practices, to communicate among practitioners, or as giving a structure and methodology to practices that happened spontaneously in the classroom. However, we can also find multiple assertions from participants that counter the arguments above (e.g. people that state that routines provided new practices, or participants using their own ideas and routines in a back-and-forth manner). Thus, we can see a wide variability in the perceptions of usefulness of the routines. The following issues will help us shed a certain light into the causes for this variability.

Table 4.18: Selected findings and evidence from the TW4 workshop, on the issue of atomic patterns' usefulness (T1b)

\begin{tabular}{|c|c|}
\hline Finding & Selected supporting evidence [Source] \\
\hline Atomic patterns are not new & $\begin{array}{l}\text { [when asked about why they think enactment-time atomic patterns } \\
\text { are useful] [they are useful] only to name what you are already doing. } \\
\text { [TW4-Q4] } \\
\text { [when asked about why they think enactment-time atomic patterns } \\
\text { are useful] They are very common sense [TW4-Q4] } \\
\text { [when asked about why they see atomic patterns as near to their } \\
\text { practice] I see that I was already using most of them, although I did } \\
\text { not know they had a concrete name, and some of them I was not } \\
\text { conscious that they were routines [TW4-Q3] }\end{array}$ \\
\hline $\begin{array}{l}\text { Atomic patterns are useful as starting points } \\
\text { of ICT usage }\end{array}$ & $\begin{array}{l}\text { [when asked about good things that the workshop had] the fact that } \\
\text { you have opened our minds about new methods and possibilities of } \\
\text { using ICTs [TW4-I] } \\
\text { [when asked about the added value of atomic patterns] It is the found- } \\
\text { ing, the structure, the skeleton [...] you may have some ideas, but if } \\
\text { you don't know anything about what to do [...] it is a seed, more than } \\
\text { a seed it is like... like LEGO blocks [TW4-I] } \\
\text { [when asked about the main value of the workshop] the catalogue of } \\
\text { patterns and the catalogue of routines [...] as a reference guide or a } \\
\text { skeleton to structure activities, to begin making things [TW4-I] } \\
\text { [when asked about why they think enactment-time atomic patterns } \\
\text { are useful] I think they are, because I am not used to ICTs, and they } \\
\text { would help me get started [TW4-Q4] }\end{array}$ \\
\hline $\begin{array}{l}\text { Atomic patterns are useful for teachers } \\
\text { during the design phase }\end{array}$ & $\begin{array}{l}\text { [during the first design exercise in the first session] 11:09 the intense } \\
\text { murmur in the classroom and my walking around the groups denotes } \\
\text { that they are working intensely [...] [during the first usage of design- } \\
\text { time atomic patterns in the first session] 11:50 group } 8 \text { are enriching } \\
\text { their design with the yellow design-time atomic patterns } 4.22 \text { [TW4- } \\
\text { O1] } \\
\text { Design-time and deployment-time atomic patterns are scored above } \\
\text { average on their usefulness (averages of } 4.30 \text { and } 4.13 \text { in a } 1-7 \text { Likert } \\
\text { scale) [TW4-Q3] }\end{array}$ \\
\hline $\begin{array}{l}\text { Atomic patterns are useful to concrete and } \\
\text { organize abstract teacher ideas }\end{array}$ & $\begin{array}{l}\text { [when asked about why they think design-time atomic patterns are } \\
\text { useful] They enable you to concrete much more the tasks and organize } \\
\text { better the teacher's and the students' work [TW4-Q3] } \\
\text { [when talking about the usage of enactment-time atomic patterns in } \\
\text { the individual design] Yes, I used them [...] to see how the idea is } \\
\text { concreted, focused, but also reading them to extract ideas for some } \\
\text { situations [TW4-I] } \\
\text { [when asked about why they think design-time atomic patterns are } \\
\text { useful] They help clarify and explicit more clearly the implicit ideas } \\
\text { that are present when you develop the activity. [TW4-Q3] }\end{array}$ \\
\hline
\end{tabular}


Table 4.18 (continued from previous page)

Atomic patterns as relatable and complementary with teacher ideas [when asked about why they think design-time atomic patterns are useful] The fact of seeing concrete examples with classroom usage, allows you to see more easily how these strategies are applied. Initially, when planning the task we were proposing, we were a bit lost, but design routines clarified enormously the initial concepts. [TW4-Q3]

[when asked about the usage of enactment-time atomic patterns cards during the roleplay] I think we first used common sense, and then we looked for the card that matched our ideas [TW4-I]

[when talking about the usage of atomic patterns during the workshop] What happened to me during the workshop is that you have ideas [...] you see those ideas reflected in the routines, and the routines give you new ideas $[. .$.$] the process is not only "routines to give you ideas", but$ rather ideas-routines-ideas, a bit like a complement [TW4-I]

Deployment-time atomic patterns are used less that design-time ones

[during the second usage of deployment routines in the first session] In general it looks like the groups have used less deployment-time atomic patterns than design-time ones. [TW4-O1]

Deployment-time atomic patterns' usefulness is rated lower than design-time ones (averages of 4.14 vs 4.30 in a 1-7 Likert scale) [TW4Q3]

Enactment-time atomic patterns are seen as useful

[when asked about whether enactment-time atomic patterns helped in the roleplaying] Without the cards [the problematic situations] would have also been solved, although possibly we wouldn't have considered other alternatives. [TW4-Q4]

[when asked about whether enactment-time atomic patterns helped in the roleplaying] The ideas exposed previously [i.e. design-time and deployment-tim ones] I think they were more helpful. The enactment routines clarify some issues, but in some cases their content is repetitive. [TW4-Q4]

Enactment-time atomic patterns are valued as highly useful, quantitatively speaking (average of 5.31 in a 1-7 Likert scale) [TW4-Q4]

Participants discovered new pedagogical affordances of ICT through atomic patterns

Problems due to the quantity of atomic patterns [when asked about the usage of atomic patterns in the individual design] I grew more conscious that we could use the ICTs to monitor the students work [...] it saves time [TW4-I]

[when asked about things that the workshop had taught her] to change an activity from face-to-face to online is easy to say, but the way to do this concretely is what I learned, that you have GoogleDocs, wikis [...] [TW4-I]

[when asked about why they think deployment-time atomic patterns are useful] Of course green cards [deployment-time atomic patterns] helped us in accompanying the concrete activities, both face-to-face and online, with a physical way of doing them. [...] Other options were clearly related with ICT and they clarified things more, since they were the physical medium with which the [task and work that we had designed] was submitted, the ways teacher interacted with students. These options were very useful because they showed [...] [the potential] ICTs have when applied to our practice. [TW4-Q3] [when asked about why they think design-time atomic patterns are useful] Yes, they helped me organize and verbalize already known processes. [...]. It could be interesting [...] to try and cluster them according to some criteria, I don't know: some are for generating information, others to present it, others to discuss it... [TW4-Q3] [when asked about why they think deployment-time atomic patterns are useful] They weren't either, we were overwhelmed with so many cards, and how to place them, we had more ideas without them. [TW4Q3]

[when asked about why they think design-time atomic patterns are useful] There are too many, there is no time to decide among so many. [TW4-Q3] 
Table 4.18 (continued from previous page)

Finding

Teachers found the provided atomic pattern approach useful (or not) for their individual practice

\section{Selected supporting evidence [Source]}

[when talking about the usage of routines in the individual design] The truth is I didn't [use them] [...] people want to appease the teacher who ordered the exercise [...] if you taught 15 things, they think they should introduce at least 10 of them [...] in the design in the face-toface session, there were six steps and 20 routines! [TW4-I]

[to the question: Did you use deployment-time atomic patterns in your individual design?] Yes. I have tried to see which ICTs, mainly Internet resources such as GoogleDocs, I can integrate in the Moodle I use currently, in order to favor collaborative work among students, which currently is done through discussion fora. [TW4-Q4]

[when asked about the process followed in doing the individual design] [...] the pattern first, then I used the design [routines] [when asked if he actually went through the routines] Yes, yes... I opened the documents and so on $[\ldots]$ [TW4-I]

19 out of 22 respondents ( $86.3 \%$ ) stated that they had used the provided atomic patterns during their individual work 


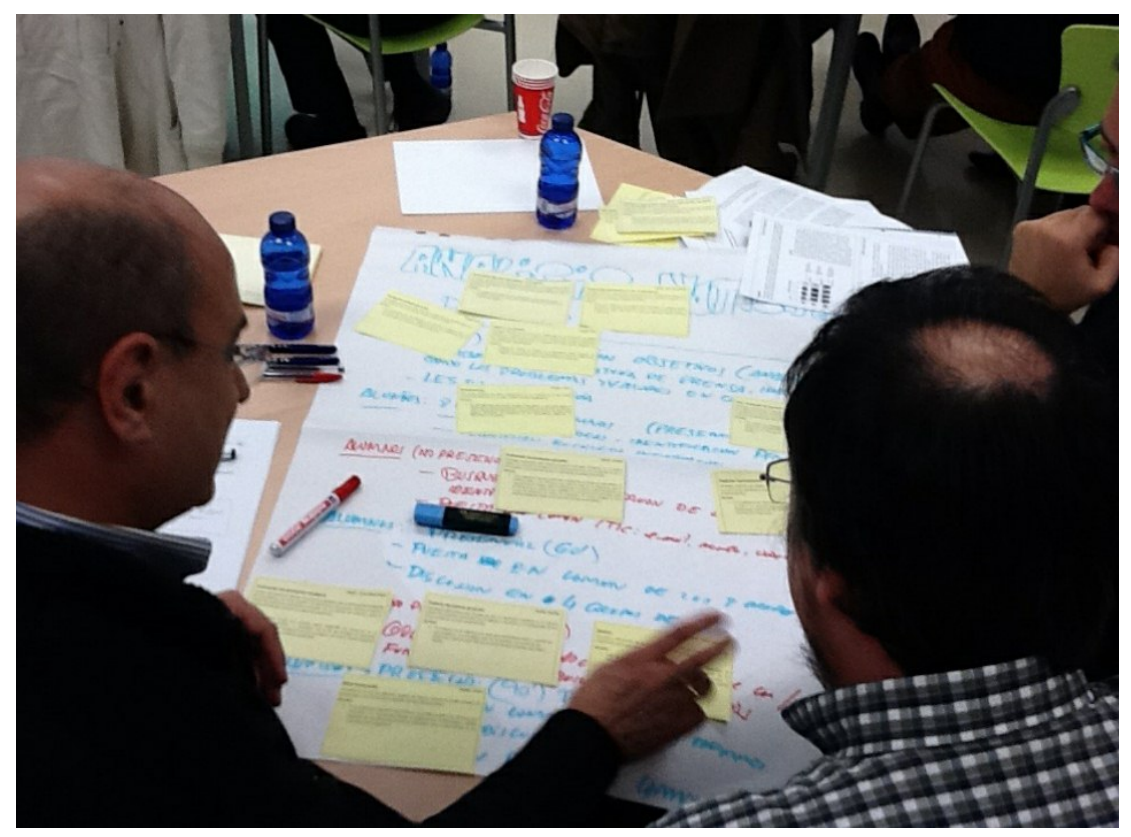

Figure 4.22: Photograph taken during the TW4 workshop

Table 4.18 (continued from previous page)

\begin{tabular}{ll}
\hline Finding & Selected supporting evidence [Source] \\
\hline Teachers see atomic patterns as usable in & [when asked about the eventual usage of atomic patterns in real prac- \\
their everyday practice & tice] Yes, yes... in fact, the next day I tried, not this [the individual \\
& design] but I did a pyramid in class [...] With pen and paper [TW4-I] \\
& [when asked about using the patterns/routines when doing a CL de- \\
& sign in future teaching practice] Sure. Now that I have them, I would \\
& take a look at them [...] I would use this [method] I know [TW4-I] \\
Teachers scored atomic patterns as close to their everyday practice & (average of 5.22 in a 1-7 Likert scale] (TW4-Q3) \\
\hline
\end{tabular}

Regarding the apparent lower perceived usefulness of atomic patterns in this workshop, there is little we can infer from these data, given the myriad of factors that could influence a professional development action of this kind. We could hypothesize that the wider range of participants and the fact that the atomic patterns catalogue was mainly extracted from engineering and education courses, might be connected to give this lower perception of value in the routines. It is striking how the scores of enactment-time atomic patterns were in this case much higher than for the other kinds of atomic patterns (contrary to what happened in the previous workshops), but this may be connected with the fact that in this workshop the roleplaying activity was modified so that teachers had more time to read, handle and familiarize with enactment-time atomic patterns (see also topic T2 below).

On the the influence of the workshop's own orchestration in the perceived usefulness (T2) As the reader may remember, in previous workshops it had been detected that the way 
the atomic patterns had been presented (due to the workshop's own design as a formative action) had a considerable influence in the perceived usefulness of atomic patterns. The design of the workshop was modified slightly to address the main objections posed by teachers in past workshops (e.g. making it longer, and taking out of the workshop the usage of specific ICT tools for orchestration such as GLUE!-PS), and such design, along with the materials provided (e.g. the atomic pattern cards) were well-valued by teachers. Still, we found that qualitative evidence in this workshop (see Table 4.19) still points towards this influence, both on the negative side (e.g. teachers felt too "rushed" by the workshop design) and on the positive side (e.g. teachers valued highly the practical nature of the workshop, or the fact that the workshop itself was an example of collaboration and ICT usage, as much as they valued the atomic patterns themselves). In fact, some teachers stated that, in doing their individual CSCL designs, they had mimicked the workshop method and activities as they had experienced them, rather than using the provided atomic patterns.

Apart from these general positive and negative influences of the workshop's orchestration in the usefulness of atomic patterns, some teachers provided evidence about other, more concrete problems, such as certain problems they had with basic usage of the ICTs used in the workshop (even if such usage ability was stated as one of the workshop's prerequisites). Oddly enough, some teachers stated that the second face-to-face session was too slow or "weak", highlighting the delicate balance between stress and boredom that the workshop facilitators have to strike when orchestrating it.

Thus, any positive effects and results deriving from this formative action cannot be said to be caused solely by the usage of atomic patterns in it, but also by the way the workshop was designed and enacted in this concrete instance. This influence is unavoidable given the fact that we chose to evaluate the atomic patterns in a realistic, situated action such as this one. This kind of evaluation does not allow us to provide proof of the validity of atomic patterns per se, separated from all other variables. However, our evaluation has the added value of demonstrating by example how a professional development action can be devised using this kind of patterns, and how teachers valued highly the new knowledge that had been gained through them. The following issues will delve deeper into potential causes and influencing factors for these results, which may help us in finding the best target audience for this kind of training actions in the future.

On the the influence of the participants' prior experience in the perceived usefulness (T4) Another factor that had emerged in previous workshops as an influencing factor in the atomic patterns' perceived usefulness was the amount of prior teaching experience: more experienced teachers tended to see less value in atomic patterns than novel teachers. In this workshop, it was striking that most of the teachers were quite experienced in teaching (only $10 \%$ of the teachers had 5 or less years of experience, while $72 \%$ had 15 or more years of experience). This, together with the noticeable (inverse) correlation between the quantitative scores of usefulness and the years of teaching experience, supports the hypothesis that experienced teachers tend to value more their own experience and routines than the atomic patterns provided. The qualitative evidence from this experience (see also Table 4.20) seems to confirm this hypothesis : many experienced teachers saw the routines as "just naming what they already did", and reported using their teaching experience or "sixth sense" to do the individual design exercise. However, 
Table 4.19: Selected findings and evidence from the TW4 workshop, on the influence of the workshop's own orchestration in atomic patterns' perceived usefulness (T2)

Finding
Problems with basic usage of certain ICTs

Selected supporting evidence [Source]

[when asked to provide general feedback about the workshop] I expected that more ICT resources would be used [TW4-Q5]

[when talking about downsides of the workshop] It assumed people knew what we were talking about $[\ldots]$ we have seen ICTs that many people around me do not know they even exist! [...] for example, the case of GoogleDocs [TW4-I]

Some teachers mimic the workshop orchestration (rather than using atomic patterns) [did you use deployment-time atomic patterns in you individual design?] No, I used what I learned in the workshop, without focusing in the cards [TW4-Q4]

[when asked about the usage of routines in the workshop] We did not use them that much because there was so little time [...] we were not so conscious of what we had in our hands [the routine cards] [...] but at home [in the individual design] I was more conscious of what they implied and what were their implications [TW4-I]

The practical orientation to design and enactment is seen as useful

[did the workshop change your view of collaborative learning?] I have seen activities that I did not know well, and which can enrich the tasks, the way of working in the designed tasks. Maybe the most interesting [aspect] is the preparation of collaborative activities and then seeing the problems that they entail [TW4-Q4]

[when asked about the workshop's usefulness] There was very little time, that was a problem [...] Discovering all the routine terminology, and tasks that I did not know of, was very interesting [...] Very useful, playing the "guinea pigs" like students in class, enables you to see points of view that you had forgotten. [TW4-Q3]

The second face-to-face session was too slow/weak for some teachers

[when asked to provide general feedback about the workshop] I would make the second [face-to-face] session faster [TW4-Q5]

[when talking about the enactment routines used in the roleplaying] I think the second session was weaker [...] the roleplaying part was weak $[\ldots]$ the first routine we used I remember because we were playing the students, that we had to go out of the classroom [TW4-I]

[when asked about the workshop's usefulness] I think the workshop has been very useful so far, I have learned many things, and the way of working, I think it is very good. [TW4-Q4]

The workshop's design and materials were adequate [when asked about the workshop's usefulness] It is very well organized. The contents are very interesting. [TW4-Q3]

[when talking about the design process in the second individual design draft] In this one I was less rigid [...] [the researcher's] feedback was very useful [...] Instead of following the steps, I tried to think in each activity, what was the most coherent option [TW4-I]

[when the interviewer said that only a subset of the elicited routines had been used in the workshop] I want more of them! Where are they? [TW4-I]

Teachers scored the workshop very highly as a training action (8.42 in a 10-point scale) [TW4-Q5]

[when asked about the workshop's usefulness] [...] However, I think the pace has been too high, the time was too structured, and there was too little time to complete the multiple activities. Overall, very stressful. [TW4-Q4]

[when asked about why they think design-time atomic patterns are useful] There were too many cards in too little time. In our case, [cards representing atomic patterns] have not been fundamental. [TW4-Q3] [when asked to provide general feedback about the workshop] The estimated workload and the real workload are not as aligned as they could have been. The flow of the course sometimes is hasty (there is no time to read and understand what has to be applied to the task). [TW4-Q5]

6 out of 21 respondents (28.6\%) stated that the pace of the workshop was "too fast", and none of them stated that it had been "too slow" [TW4-Q5] 
even with this triangulated evidence, the correlation was far from perfect, and we could find experienced teachers who stated that they had learnt new practices from atomic patterns, as well as relatively novel teachers that preferred to rely on intuition rather than on atomic patterns.

Table 4.20: Selected findings and evidence from the TW4 workshop, on the influence of participants' experience in the perceived usefulness of atomic patterns (T4), including the teaching experience of the participants providing the evidence

\begin{tabular}{|c|c|}
\hline Finding & Selected supporting evidence [Source] [Years of experience] \\
\hline $\begin{array}{l}\text { Atomic patterns as putting names to existing } \\
\text { expert practice }\end{array}$ & $\begin{array}{l}\text { [to the question: Did the workshop change your view of collaborative } \\
\text { learning?] No. Just putting names to concepts that I already use in } \\
\text { my teaching practice. [TW4-Q4] [13 years] }\end{array}$ \\
\hline $\begin{array}{l}\text { Even experienced teachers may learn new } \\
\text { practice from atomic patterns }\end{array}$ & $\begin{array}{l}\text { [when asked about why they see atomic patterns as near to their } \\
\text { practice] Yes, I have used them without knowing that they were rou- } \\
\text { tines, after so many years of teaching you progressively apply them, } \\
\text { especially when they work. Maybe I discovered other [routines, in the } \\
\text { workshop], or how the ones that I used were named. [TW4-Q3] [20 } \\
\text { years] } \\
\text { [when talking about the usage of enactment-time atomic patterns dur- } \\
\text { ing roleplaying] I think we went directly to common sense [...] I also } \\
\text { did the same when doing the individual [design], I used common sense } \\
\text { and then asked myself "how is this called in the routines?" [...] and, } \\
\text { as you look through them, you also see other [routines] and you open } \\
\text { your mind a bit [...] expand common sense. [TW4-I] [18 years] }\end{array}$ \\
\hline $\begin{array}{l}\text { More experienced teachers tend to rely on } \\
\text { intuition or common sense rather than on } \\
\text { provided atomic patterns (with exceptions) }\end{array}$ & $\begin{array}{l}\text { Correlation of quantitative scores for atomic patterns vs. years of } \\
\text { teaching experience is noticeable, but far from perfect (values of - } \\
0.27,-0.25 \text { and }-0.19 \text { for the design-, deployment- and enactment-time } \\
\text { atomic patterns, respectively) [TW4-Q3] [TW4-Q4] } \\
\text { [to the question: Did you use deployment-time atomic patterns in your } \\
\text { individual design?] No, I used my sixth sense as a teacher. [TW4-Q4] } \\
\text { [ } 5 \text { years] } \\
\text { [when asked about whether enactment-time atomic patterns helped } \\
\text { in the roleplaying] No, I believe all of us would use our teaching ex- } \\
\text { perience, more than guiding us with routines. [TW4-Q4] [20 years] } \\
\text { [when asked about whether enactment-time atomic patterns helped } \\
\text { in the roleplaying] What helps is experience, logic and common sense. } \\
\text { It is impossible to foresee all that can happen. [TW4-Q4] [24 years] }\end{array}$ \\
\hline
\end{tabular}

Taking this slight correlation into account, the experienced profile of most participant teachers might partially explain the lower values of usefulness perceived in the routines (when compared with previous workshops). However, it is important to note that this is only a general tendency, and that factors other than the teaching experience seem to have an effect on the opinions provided by teachers. Topic T5 below delves into some of these factors.

On the influence of beliefs (about ICT, about collaboration) in the perceived usefulness (T5) To close the issues related to atomic patterns' usefulness, a recurring factor in the evaluations during the author's $\mathrm{PhD}$ thesis has been the influence of teachers' beliefs and prior attitudes towards ICT usage in teaching or collaborative learning in general, in shaping the usefulness perceived of these patterns. As we can see in Table 4.21, the qualitative evidence obtained in the questionnaires and interviews also raises this issue, not by direct questioning about them, but rather by teachers voicing these beliefs and attitudes when asked about other issues such as atomic patterns' or the workshop's usefulness. Some teachers hold that collaborative learning is beneficial for the learning of students, while others believe it is unfeasible, or that individual work capability is more important. Finally, others state that certain constraints (e.g. subject matter, or the number of students) make it more difficult to attempt this kind 
of collaborative learning. As it happened in the previous workshop, certain perceived "orchestration problems" in CSCL (the number of students, the management of all the involved ICT infrastructure) seemed to be affecting the perceived usefulness of atomic patterns in particular and of CSCL in general. This again encourages us to investigate in tools to support CSCL orchestration, as a way of overcoming this adoption barrier.

To which extent the workshop and the atomic patterns modified these beliefs? In this regard, although in many cases the workshop did not change them, teachers did assert that their knowledge about CSCL and how it worked in practice was greatly improved, enabling them to make a more informed decision about whether they could use it in their classroom (or to which extent). Thus, positive attitudes to CSCL seemed to be reinforced. Among the evidence, however, we can find very varied responses, including teachers whose beliefs on CSCL seemed to be changed to a more positive attitude, and others for whom their (negative) vision of CSCL was not modified.

The importance of intrinsic motivation and the alignment of teacher beliefs and the proposed innovations is already well known in educational literature (see, e.g. [Sch99]), and these results only come to confirm them again. However, it is important to note that when we refer to "beliefs", this should not be taken as some sort of derogatory term analog to "superstition". Many teachers hold these beliefs from past experience with collaborative learning or ICT usage, and thus they are entitled to those beliefs until us researchers show them empirical proof of usefulness and feasibility of such active approaches within their classroom's constraints. That is why we believe that tools that try to improve orchestration (such as GLUE!-PS or these atomic patterns presented here) have an importance if we want CSCL to be adopted in classrooms.

On the usefulness of embedding atomic patterns in educational technology (T6) Regarding our secondary research question, parts of the questionnaires and interviews were directed at targeting it. As it happened in previous workshops, when confronted with the question of embedding atomic patterns within technology, the answers were rather heterogeneous, and in some cases not very informative. As we can see from Table 4.22, it is interesting that some participants equated the word "technology" with the Moodle VLE, and noting some problems they have in, we could say, "orchestrating CSCL through Moodle". The promotion that the University of Valladolid is doing about Moodle as the main technology to use in its courses is probably the cause of this unexpected effect.

In the interviews, most of the teachers manifested that embedding atomic patterns in technology could be useful. The opinions regarding the form that this embedding should take, was generally more veering towards automated actions. Others, however, also saw usefulness in having recommendations of context-dependent routines during design or enactment (trying to mitigate the problem of a too numerous routine catalogue), although many participants were concerned about the degree of "intelligence" that such a system could achieve (to provide timely recommendations). The idea of having detailed mini-tutorials or other atomic pattern reference is thought useful by some, but maybe not usable in real-time (e.g. in a face-to-face session). Other teachers, plainly, did not have an opinion or the issue, or dismissed the idea as not very useful.

Another interesting outcome in this regard is the responses of participants when asked about the concrete routines that were most interesting for their implementation with technol- 
Table 4.21: Selected findings and evidence from the TW4 workshop, on the influence of participant beliefs in atomic patterns' perceived usefulness (T5)

\begin{tabular}{l} 
Finding \\
\hline Atomic patterns reinforce positive \\
attitudes towards CSCL, by giving \\
concrete advice
\end{tabular}
the perceived usefulness of atomic patterns
Selected supporting evidence [Source]

[to the question: Did the workshop change your view of collaborative learning?] Now I have a much clearer idea of what is a collaborative activity and, especially, which patterns to use to encourage that those activities are really collaborative. I extract many lessons from the course [...] how to complement this learned [patterns] with the use of ICT, the ICTs that can be used and some of their affordances. [TW4-Q4]

[to the question: Did the workshop change your view of collaborative learning?] The most important aspect is that real cases are presented and, from them, it is easier to gather ideas that are easily transferable to my courses. I came out with the impression that I can put in practice a collaborative activity, really; up to now it did not occur to me anything other than ordering a report and making students present it in class. [TW4-Q4]

[when asked about the changes brought about by the workshop] now I'm more convinced [...] because now I know how to put it in practice [...] up to know you never saw concrete examples, tasks. [TW4-I]

[when asked about the feasibility of using CLFPs] It demands a time that I am not sure I have, and a big effort in collaborating, all to reach uncertain results. [TW4-Q2]

[when asked about why they see atomic patterns as near to their practice] I believe the main problems are the high number of students and adjusting the timeframe of the course [...] [TW4-Q3]

[when talking about prior beliefs about (computer-supported) collaborative learning] It is a cost-benefit analysis [...] what you win, I do not know if it compensates what you loose [...] either you have very little content to go through, or you sacrifice content in favor of other objectives [like collaborating]. [TW4-I]

Beliefs about students affecting the perceived applicability of atomic patterns Medicine I do not know anyone who has put collaborative learning in practice, students are very competitive and individualistic. [TW4-Q3]

[when talking about the applicability of the individual design to everyday practice] I think that collaboration, today, in my class, with all the people collaborating at the same time [...] with 28-30 students [...] I see it unfeasible [...] they are very heterogeneous [...] to keep everyone aligned, motivated. [TW4-I]

Beliefs about teaching affecting the perceived usefulness of atomic patterns [when talking about the usage of enactment routines during roleplaying] in my opinion, 25 years of experience give you many things [...] a novel teacher, no matter how many routines and papers you give them... I believe there is people that are valid [for teaching] and other aren't. [TW4-I]

Orchestration problems in CSCL affecting the perceived usefulness [did the workshop change your view of collaborative learning?] My vision is still favorable, but I am concerned by the complexity of managing it via ICT. In fact, without ICT, collaboration is equally complex, but the problem is less explicit. [TW4-Q4]

[when talking about doing CSCL in a DLE as in the example course shown] When I see all those links, folders, etc [...] I think it is too much work, nonpedagogical work involved [...] does it all compensate in order to reach what? To make a summary? [...] I'm a skeptic. [TW4-I]

The workshop changed beliefs about CSCL [to the question: Did the workshop change your view of collaborative learning?] Totally. I had always reduced collaborative work to working in dyads, and essentially, I thought they were worthless. The success of this course is that I have seen that they can be really useful - if well designed - for the learning processes. [TW4-Q4]

[when asked about usage and knowledge about CL prior to the workshop] I thought collaborative [learning] was worthless, [...] in my mind it was reduced to dyad work, that you had to do because it is an innovation [...] I have seen that, if you do it well, you can learn a lot with it [...] designing it with time, thoughtfully, and applying these patterns, you can get more outcomes from a subject. [TW4-I]

[to the question: Did the workshop change your view of collaborative learning?] That ICTs are not so difficult as I thought, and that they match perfectly my idea of teaching how to work in groups from a more modern perspective that students will like. It is also applicable to the industry. [TW4-Q4] 
Table 4.22: Selected findings and evidence from the TW4 workshop, on the usefulness of embedding atomic patterns in educational technologies (T6)

\begin{tabular}{l}
\hline Finding \\
Atomic patterns considered more useful to be \\
embedded
\end{tabular}

Selected supporting evidence [Source]

[when asked about whether embedding routines in the technology would be useful, and which ones] Yes. Publish group decisions, etc. [TW4-Q4]

[when asked about whether embedding routines in the technology would be useful, and which ones] Some of them. [...] Peer review, Example task to exemplify tool, [...] Reform groups in face of the current attendants, Display artifact during the discussion. [TW4-Q4] [when asked about routines that were used repeatedly in the design] Monitoring during the activity [...] Change places [possibly refers to re-form groups] [...] change activity from face-to-face to online $[\ldots]$ the one about random evaluation [of group work] [...] introduce minilecture in the debate [...] remind debate rules [...] take notes of the debate $[\ldots]$ freeze intermediate artifact $[\ldots]$ peer review. [TW4-I]

[when asked about ICT-related routines that would be more useful to have automated] the one about freezing the intermediate artifact $[\ldots]$ the creation of artifacts ... the preparation of the whole activity. [TW4-I]

Teacher preferring atomic patterns embedded as automated actions

[when asked about the "routine suggester"] I would see more usefulness in the other option [routines as embedded actions] [...] it is very difficult to have a program that makes adequate suggestions. [TW4-I] [when asked about routines as actions embedded in technology] everything that is clicking a button and it does a number of things that you would have to do by yourself [...] go for it. If you had [...] a button that says "re-shuffle the pyramid" and it rearranges the groups, that would be a miracle. [TW4-I]

[when asked about embedding routines as actions in the learning technologies] Yes, that would be ideal [...] for people like me, that are not so comfortable with technology[...] [when asked about routines suggestions embedded in technology] I'm not sure to which extent this could be useful [...] it depends on how "intelligent" they would be. [TW4-I]

Teacher preferring atomic patterns embedded as recommender systems [when talking about embedding routines in technology] If I had to choose, the actions [...] maybe I could do it myself. But to suggest routines, that I cannot do myself, you have to know them all. [TW4-I]

Teachers preferring an atomic pattern reference

[when asked about whether embedding routines in the technology would be useful, and which ones] It would be very useful to have them available inside a "Help" tool. [TW4-Q4]

[when asked about the value that routines had for him] to have a list of things, which may or may not occur to you [...] a catalogue, easy to see, well-explained, with examples [...]. [TW4-I]

Teachers thinking of the VLE (Moodle) as the go-to place for controlling the course activities

[when asked about whether embedding routines in the technology would be useful, and which ones] Some things that are missing in Moodle 1) If I want to practice or try something in Moodle, I have to make a real group, there is not a sandbox. 2) Groupings within groups do not work well or are not intuitive. Is it possible to put a link [in Moodle] and that only one subgroup sees it? [...] I do not know how to do it. [TW4-Q4]

Teachers undecided, or not seeing usefulness [when asked about whether embedding routines in the technology would be useful, and which ones] I don't know. [TW4-Q4] [when asked about whether embedding routines in the technology would be useful, and which ones] No. [TW4-Q4] 
ogy. These routines include: Publish group decisions, Peer review, Example task to exemplify tool, Reform groups in face of the current attendants, Display artifact during the discussion, Monitoring during the activity, Change activity from face-to-face to online, Random evaluation of group work, Introduce mini-lecture in the debate, Remind debate rules, Take notes of the debate, Freeze intermediate artifact or Peer review. Unfortunately, these responses provided too scattered data, and there are no clear trends that might help us in further prioritizing our atomic pattern catalogue regarding their relative usefulness.

\section{Conclusions and feedback for subsequent iterations}

Overall, the workshop garnered rather positive feedback from participants, even despite the fact that they were a very heterogeneous group, with lots of very experienced teachers. Teachers seemed to find the atomic patterns useful in structuring their designs and reflecting about the enactment in the classroom. They also found the workshop itself a very interesting teacher development action. We can further summarize the findings during this evaluation iteration into the following partial conclusions (see also Figure 4.23):

- We have accumulated further evidence that, on average, atomic patterns are a useful conceptual tool for teachers in the different orchestration phases of design, deployment and enactment. However, this assertion has to be moderated with a number of caveats, since teachers show a wide variety of responses to them. These caveats are represented by some of the other conclusions below [TW4-T1b-PC1]

- The most common benefits of atomic patterns, as expressed by participants, include: being starting points for inspiration, serving to discover educational affordances of concrete ICTs, and helping in making teacher ideas more explicit and concrete [TW4-T1b-PC2]

- There is a high variability in the ways teachers think, use and appreciate atomic patterns, which may be linked to the different ways in which they design and, in the end, orchestrate learning activities in their classrooms [TW4-T1b-PC3]

- There is evidence supporting the need of further classification and refinement of the catalogue of atomic patterns, either to make their number smaller, or to find easier ways of navigating through their high numbers [TW4-T1b-PC4]

- The workshop's general structure and materials are very appreciated by most of the teachers, due to their practical nature and concreteness. Given that a big part of those materials are the atomic pattern cards, we can further infer atomic patterns' usefulness from this conclusion [TW4-T2-PC1]

- As we have progressively seen throughout the different evaluations, the pace of the workshop has to be carefully controlled for teachers to appreciate atomic patterns fully, being especially careful to provide enough time for teachers to read and familiarize with the atomic pattern set [TW4-T2-PC2]

- The workshop's practical nature and the fact that it is a CSCL scenario using DLEs in itself, is the most appreciated aspect by some teachers, which gives them the opportunity to experience a real example of this kind of learning situation [TW4-T2-PC3] 
- We have gained further evidence about the fact that experienced teachers tend to dismiss atomic patterns as too obvious, in favor of intuition, especially during enactment-time of a situation, in the face of unexpected events [TW4-T4-PC1]

- We have also seen again how beliefs about CSCL (and very especially its typical orchestration problems) seem to affect the perceived usefulness of atomic patterns, with negative attitudes bringing a lower perception of usefulness [TW4-T5-PC1]

- We have also gathered evidence about the limited power that atomic patterns (or their application in such a short amount of time) have in changing these prior beliefs, although some change seems to be brought about, mostly by providing concrete advice about practice of orchestrating CSCL [TW4-T5-PC2]

- Regarding the way of embedding atomic patterns into technology, teachers seem to prefer atomic patterns that are embedded as automated actions in the technology, over other options like recommender systems or reference materials [TW4-T6-PC1]

- We have also gathered a list of the atomic patterns that are considered more useful to be embedded into technology, although the names on this list are too scattered to show guiding trends [TW4-T6-PC2]

Given the situated nature of the evaluation, these conclusions should be regarded with the usual reservation, taking into account that they apply to the concrete context of this workshop. Thus, the conclusions in reality apply only to the subset of atomic patterns presented in the workshop, rather than to the concept of atomic pattern as a whole. However, since we have already completed four iterations in two different contexts, it is possible to think that some "naturalistic generalizations" could be made for other educational contexts, provided that a similar process of elicitation and refinement takes place.

The noticeable coincidence of most conclusions of this workshop with the previous ones, leads us to think that we are reaching a point of "diminishing returns" by going along this kind of evaluation happening and approach. That does not mean, however, that we cannot learn more about orchestration or the role that atomic patterns have in it. In the following section we outline several lines for future work suggested by the evidence in this and previous workshops.

\subsubsection{Wrapping up the evaluation of atomic patterns}

During these four iterations of evaluation, atomic patterns elicited from authentic teacher practice, and a multi-level pattern approach to elicit and apply such patterns, have been evaluated through situated professional development actions in the form of workshops. As it was mentioned at the beginning of this Section, we had one main research question (RQ2.1, "Can we provide conceptual tools to support (non-expert) teachers in orchestrating learning? (sp. in blended CSCL using DLEs)"), which was explored for atomic patterns through two issues one which was present from the beginning of our effort (albeit in a slightly different form), and another one that emerged as our efforts to support orchestration developed:

- I1a/I1b Are atomic patterns useful for practitioners in orchestration? 


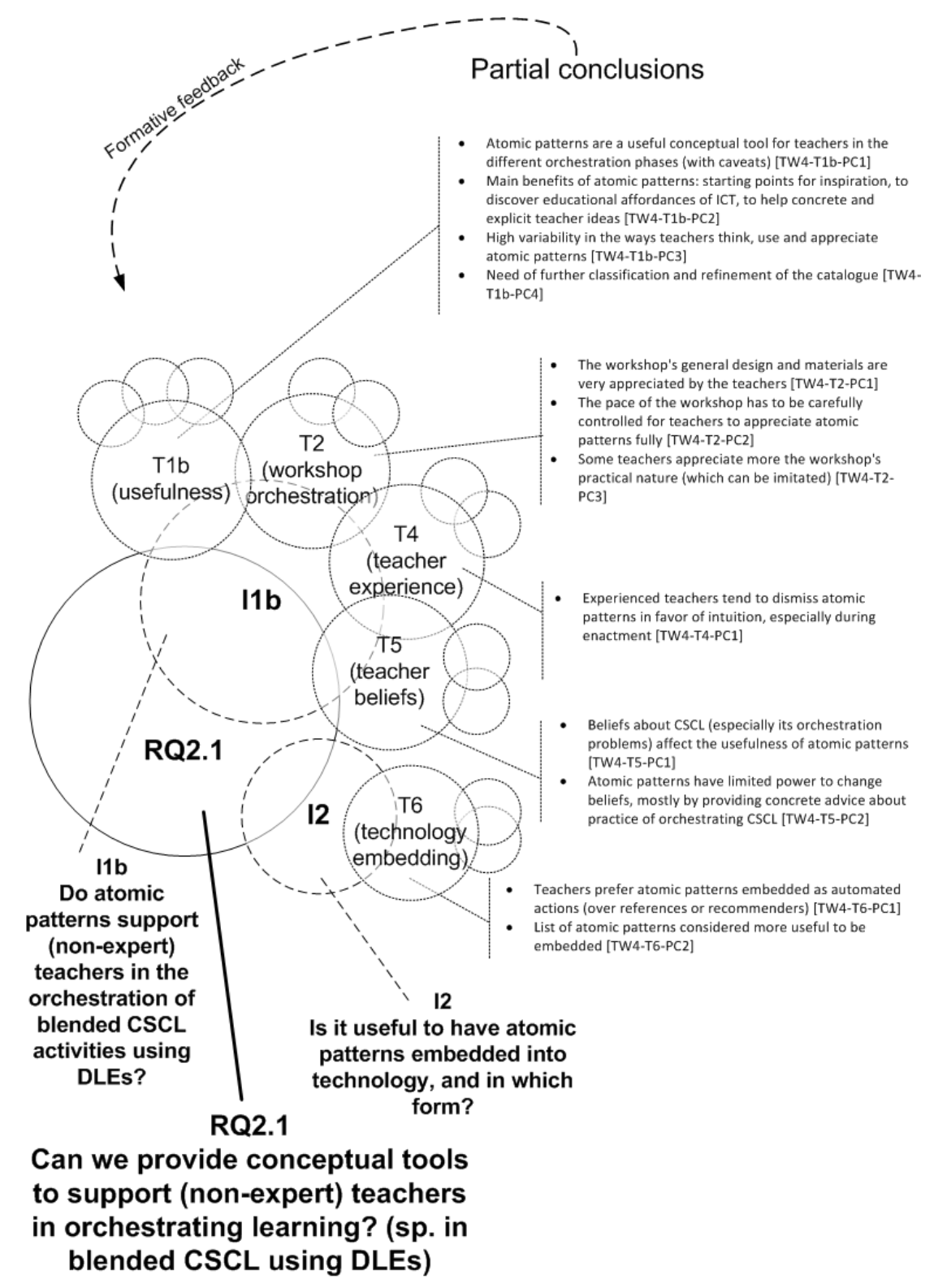

Figure 4.23: Graphical representation of the partial conclusions to Iteration \#4 
- I2 Is it useful to have atomic patterns embedded in the technology? in which form?

In order to provide first answers to these questions, at least in the concrete situated contexts where the evaluations would be performed, we chose to focus on different Issues, conceptual organizers which evolved iteratively until we reached our final iteration. By gathering evidence around these issues, taking into account the partial conclusions of each iteration in a process akin to a cross-case analysis [Sta06b], we can reach five global conclusions for our evaluation, which illuminate each of those issues, as represented in Figure 4.24:

Conclusion 1: Atomic patterns are considered useful and close to everyday practice, across the different orchestration phases. Our main research concern, and the one for which more supporting evidence has been gathered, is the question of whether our atomic patterns elicited from practice were useful or not for teachers, in designing, deploying and enacting blended CSCL activities across DLEs. In all four iterations of the evaluation we have reached a majoritarily positive answer to this question, with separate positive conclusions about the usefulness of design-time and deployment-time atomic patterns in the preparation of CSCL activities in the workshops, and of enactment-time atomic patterns in role-played simulations of the enactment of such activities. However, not all of the 61 teachers who informed us considered atomic patterns equally useful, and we came to notice that several factors such as prior experience and beliefs about collaborative learning were affecting this perceived usefulness. These factors were further explored in other issues, and conclusions concerning them are provided below.

Furthermore, despite being abstractions of concrete orchestration practices observed, atomic patterns still retain enough contextual elements (through the inclusion of examples of their use in real practice) so that teachers easily recognize them as part of their practice, or as elements that they could put in practice immediately, producing designs and feasible implementations of CSCL activities.

Moreover, not only we have reached this "yes, with caveats" answer to our question on atomic patterns' usefulness and mirroring properties. We have also gained further insights about what aspects of atomic patterns are more useful, and how teachers conceive their use: the catalogue of atomic patterns is considered useful by teachers as a reminder of different alternatives in the design of activities and the use of ICTs for CSCL, starting points for inspiration and reflection tools to anticipate problems that may emerge in the enactment of the activities. We have also evidences that hint at the main usefulness of atomic patterns to help concrete and explicit teacher ideas, albeit the way they think about the orchestration process and use our patters, is highly varied. [Supported by partial conclusions TW1-T1a-PC1, TW1-T1a-PC2, TW1-T1a-PC3, TW1-T3-PC1, TW2-T1a-PC1, TW2-T1a-PC2, TW2-T3-PC1, TW3-T1b-PC1, TW3-T1b-PC2, TW3-T1b-PC3, TW3T1b-PC4, TW3-T1b-PC5, TW4-T1b-PC1, TW4-T1b-PC2, and TW4-T1b-PC3]

Conclusion 2: The workshop design and orchestration were adequate to show atomic patterns' potential, but require more time. In the evaluation of tools which happen in situated, authentic settings, taking the concrete context of the experiment into account is of the utmost importance, since it can greatly shape the results of the evaluation. In our case, the context was marked by the fact that these workshops were professional development 
actions in which participant teachers had certain learning goals which we had to cater for, as we gathered data on the usefulness of atomic patterns. By designing and orchestrating the workshop using our own pattern catalogue, and iterating four times across two different contexts, we gradually polished the design of the workshop to avoid any shortcomings on the participants' learning but also on our gathering of data. The evidence from the different iterations (excepting the second iteration, which was thwarted by a host of unforeseeable events) showed that participant teachers were in general very satisfied with the learning experience and the materials provided (based mostly on elicited atomic patterns). There were, however, chronic time management issues which prompt us to explore in the future other formats that might be less time-constrained.

This success of the formative actions has the downside that it is impossible to separate atomic patterns from their use in the workshop itself, which some researchers might see as problematic, desiring to evaluate atomic patterns' power in an isolated way. However, we believe that these evaluations provided direct evidence about the potentialities of using atomic patterns in PD actions, which is equally valuable. [Supported by partial conclusions TW1-T2-PC1, TW1-T2-PC2, TW3-T2-PC1, TW3-T2-PC2, TW4-T2-PC1, TW4T2-PC2, and TW4-T2-PC3]

Conclusion 3: Atomic patterns seem more useful for novel teachers, although experienced ones may appreciate them. The issue of teaching experience affecting the teachers' perceived usefulness of atomic patterns emerged as we evaluated them in the higher education setting. This influence, which had already been spotted in previous work by the author Pri09 became very apparent as we gathered more data around it. Through our third and fourth iteration we found that many of the more veteran teachers dismissed atomic patterns as obvious, and relied more on their own instincts, while many of the more novel ones found them good as starting points to bootstrap their orchestration process. Still, this trend is far from being unanimous, and several experienced teachers (especially those who had positive attitudes about collaborative learning, see Conclusion 4) were enthusiastic about atomic patterns and the proposed approach. In any case, we have not tried atomic patterns on a population of uniformly novel (even pre-service) teachers yet, and thus this trend has yet to be further proved. [Supported by partial conclusions, TW3-T4-PC1, TW3-T4-PC2, and $\left.T W_{4}-T_{4}-P C 1\right]$

Conclusion 4: Atomic patterns have limited power in changing beliefs, but provide concrete advice. As we had also seen in our first research efforts in the primary school [Pri09], and literature confirmed [Sch99], teacher beliefs are a very important part in integrating innovative practices and technologies in the classroom. Our PD workshops were no exception, and many of the more skeptic teachers (regarding collaborative learning) were the ones that found atomic patterns less useful. Atomic patterns had only limited results in changing these beliefs, and in those cases where prior conceptions of CSCL were changed, it was mostly because of the concrete advice that atomic patterns and the workshop experience provided, thus demystifying a practice that seemed esoteric or unfeasible for them. [Supported by partial conclusions TW3-T1b-PC5, TW3-T5-PC1, TW3-T5-PC2, TW4-T5-PC2, TW4-T2-PC3, TW4-T5-PC2]

Conclusion 5: Teachers seem to prefer automation over other forms of embedding. As we grew more interested in the technological support of teacher orchestration in blended CSCL 


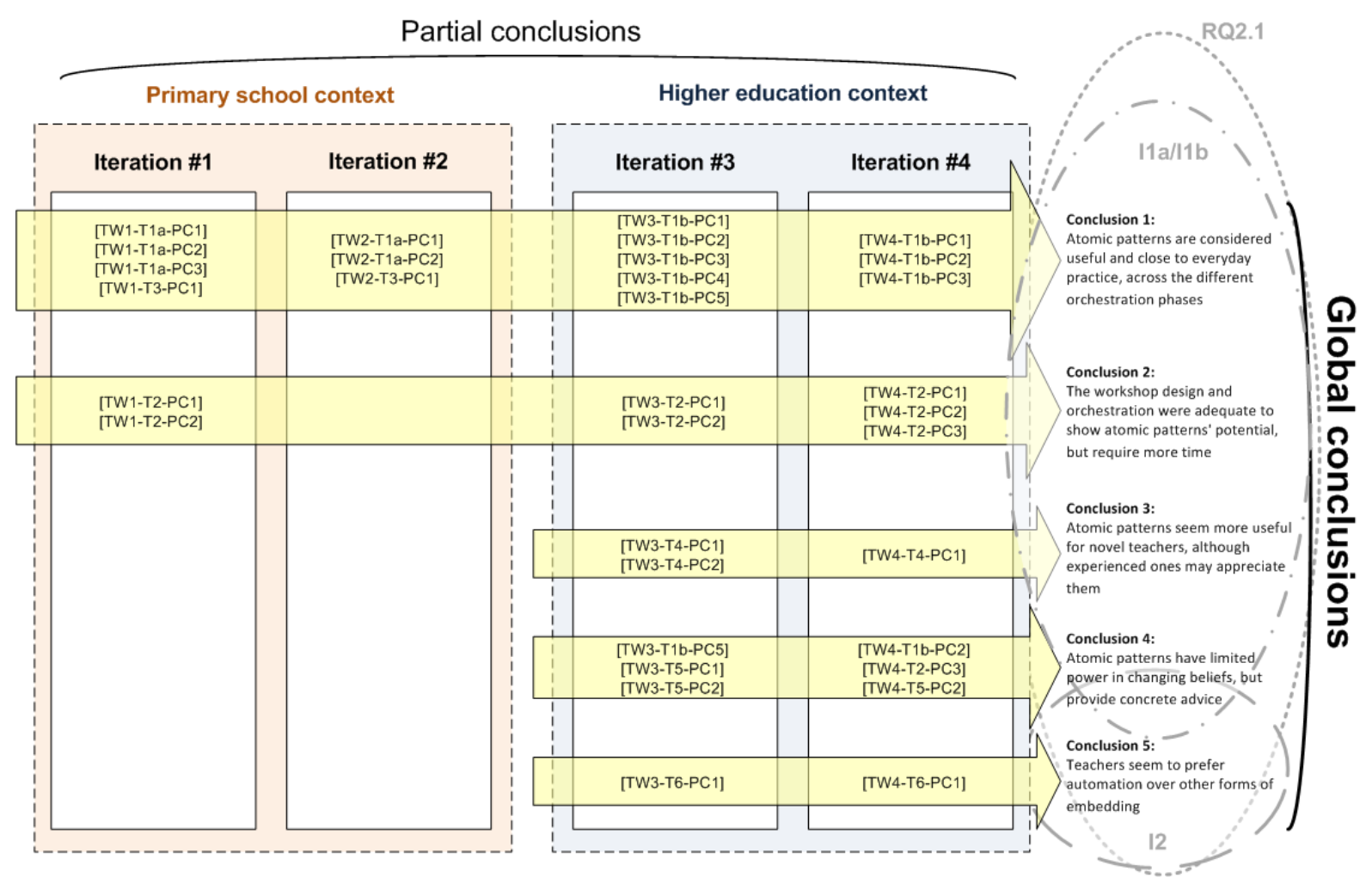

Figure 4.24: Graphical representation of the partial conclusions leading to the global evaluation conclusions on atomic patterns as a conceptual tool for orchestration

across DLEs (see Chapter 5), we started to wonder what would be the best way of incorporating the conceptual support of atomic patterns into educational technologies. Several options are possible, including the automation of those atomic patterns which are prone to such automation, or the context-dependent recommendation of atomic patterns that might be applicable to a certain situation. In this regard, although results are not conclusive, teachers seemed to veer towards the automation of tasks, rather than the other options. Also, we gathered evidence about concrete atomic patterns which might be useful to be embedded/automated, although results so far are not clear enough to guide the roadmap of any technological system. [Supported by partial conclusions TW3-T6-PC1, and $T W 4-T 6-P C 1]$

Overall, we can say that teachers appreciated the capability of atomic patterns to make concrete their practices or ideas that were implicit or hazy before. As one of the participant teachers put it:

When we planned the general design of the activities, we did it first without the [atomic pattern] cards. I think cards helped us, not so much to have new ideas, but to flesh out the ones we already had and materialize some proposals we had in mind but did not know how to put in practice. In that sense, cards were very useful because 
sometimes we thought about what we wanted students to get out of the activity, or what we wanted as the result, but we did not know how to structure the activity. However, I think we were not very conscious at that moment, because of the hurry, I'm noticing it right now. [TW4-Q3, translated from Spanish]

\subsection{Conclusions, relevance and future work on atomic patterns}

The increasing presence of ICT in all areas of our daily life highlights the need to provide students with learning experiences that reflect this change. However, through observation of TEL practice in a number of primary and higher education contexts, we have come to believe that providing technological endowments is not enough to enhance the learning of students. Nowadays, teachers are called to orchestrate ICT and non-ICT tools in a creative and effective way that leads to technology-enhanced learning. However, very little support is given with regard to how teachers should do it. In this chapter we have described one way for researchers to analyze everyday teacher practices in authentic settings, describing how teachers develop complex TEL and CSCL activities through the use of what we have called "atomic patterns".

These atomic patterns represent recurrent ways of orchestrating TEL/CSCL activities, with a certain purpose. Atomic patterns share common traits with other design patterns [Ale77, although they differ in several aspects, being more informal, heterogeneous, small-scale, numerous, and less abstract than most design patterns. The fact that we have extracted them from real teaching practice in authentic settings, make them both pragmatic and not totally decontextualized, which helps teachers in retrieving, understanding and applying them to their everyday practice. The differences with other kinds of (pedagogical) design patterns, however, does not mean that they cannot be used in conjunction - rather, they are complementary and provide advice at different activity levels, helping in bridge the gap between the de-contextualized advice of most pedagogical patterns and the contextualized performance that teachers have to deliver in their practice. In fact, in this chapter we have proposed a multi-level pattern approach to the elicitation, development and combination of atomic patterns with other patterns for use, e.g. in professional development actions. This multi-level pattern approach can be seen as a first attempt in better understanding teacher orchestration practice and promoting design strategies for professional development that help teachers in orchestrating TEL scenarios. Throughout this chapter we have also showed other uses of atomic patterns for different orchestration actors, through examples of use made during the dissertation (e.g. using atomic patterns in graphical representations to understand orchestration as researchers, or using atomic patterns as indicators for orchestration/orchestrable educational technologies). We have, however, focused most of our efforts in analyzing and evaluating the value of atomic patterns for teachers, since they are the main orchestration actors in the educational contexts that we analyzed.

Since currently the vast majority of teachers are not experts in the pedagogical application of ICT to education (as noted, e.g. in Pla06 for the Spanish educational system), we believe that efforts towards making educational practices with ICT easier to grasp for non-expert teachers would represent a very worthy contribution. The main advantages and usefulness of this contribution are, for the most part, the same that can be encountered in design patterns literature for the usage of design patterns in other practices [Ale77] [Gam95]: this vocabulary of atomic patterns can serve as a tool for communication of experiences and best practices among 
practitioners and with researchers, and they can also serve as a tool for reflection and design for practitioners (by making practice, and the problems it aims to solve, explicit). Moreover, we hypothesize that these routines could have an important role in making teaching practice with ICT more agile, by internalizing/automating certain aspects of the practice to which solutions already exist (thus avoiding "reinventing the wheel"). This would allow teachers to concentrate on other issues, such as the social and motivational aspects of the activity, which are much more dependent on context, and require high levels of attention and creativity from the teacher to be successfully solved.

Our evaluation of atomic patterns and the multi-level pattern approach, through four iterations of professional development workshops in two different contexts (a primary school and a university in Spain), yielded rather positive results. A majority of the 61 teachers that participated in these workshops considered atomic patterns useful and close to their everyday practice, throughout different stages of the orchestration process (design, deployment and in simulated enactments). The situated nature of the workshops made this usefulness hard to separate from the design and orchestration that we did of the workshops, which were very appreciated due to their practical orientation (despite certain time management issues due to the workshop's short duration). Our evaluation also showed a high variability of responses to atomic patterns among teachers, which seemed to be due to several other factors such as teachers' degree of experience and prior beliefs and attitudes towards the workshop subject matter. Albeit the workshops had a limited power in changing these attitudes, most teachers valued the practical, realistic perspective on the practice of CSCL that they provided. Regarding the embedding of atomic patterns into technology, teachers seemed to prefer the automation of atomic patterns over other ways, although results were not conclusive.

However, our evaluations and the whole atomic pattern approach taken in this dissertation can be criticized from a number of standpoints. First of all, and given the means that have been chosen for the data gathering (mostly through qualitative studies in one concrete educational context), one may wonder to what extent the atomic patterns uncovered in one context are generalizable to other contexts. Given our experience transferring the approach from primary schools to universities, we can say that the generalizability of the routines will be low if educational and technological contexts are radically different. Other dangers and tensions in this approach come from its relationship with the design patterns field of study, and some of its well-known issues. For example, the fact that atomic patterns are derived from actual practice makes practice based on atomic patterns less innovative (something that the most veteran teachers in our evaluations also detected). However, we can argue that by keeping the teacher on the safe side in some aspects of their practice (e.g. technology usage), we allow them to be more creative in other areas (e.g. the social/motivational ones). Another possible criticism is that it may make practice too rigid, if teaching is restricted to just using these atomic patterns. To this, our own point of view, backed up by the evaluation with teachers, is that atomic patterns are not a "complete catalogue of the possible actions for teachers", but rather they offer possibilities, starting points to bootstrap the creative process of designing and orchestrating the learning activities. Thus, atomic patterns can be taken as-is, or combined in new forms, or serve as guides for making orchestration practice more creative. Finally, there is always the risk of "pattern-itis" (that is, to use as many patterns as possible in over-complex designs, see [Gam02]). The answer to this is also simple: teachers should use these atomic patterns in good measure, since there is no claim that the quality of the learning experience will correlate with the number of atomic patterns 
used in the orchestration.

One last criticism will serve us to close this discussion and as a link to our future work: our set of atomic patterns, as they have been elicited so far, represent the practices of teachers with ICT in the observed classrooms. As such, we do not assert that these concrete atomic patterns, and the associated professional development initiatives described in Section 4.5, are applicable to all primary or higher education classrooms. Also, we do not claim that the proposed set of routines are a complete solution, but rather that this sort of initiatives contribute to minimize current uncertainty in the effective use of ICT in education. Indeed, the set of useful patterns will be dependent on the socio cultural context of each institution and classroom, and on the intent of each professional development initiative. Moreover, we cannot assert that all of these practices can be labeled as best practices. In fact, there is a heated debate in the area of pedagogy as to the existence of such thing as best practices. This way, orchestration atomic patterns can be seen as good or bad depending on the objectives and values that teachers have in mind. Thus, the same activity design can be enacted by different teachers in totally different ways, according to their teaching styles. In this sense, the works of [Mer07] and [Vas10], from a socio cultural perspective, have provided ample evidence that high levels of learning achievement do not depend solely on how teachers design and enact their activities. In those studies, classrooms differed in terms of their participation structures, and the quality of children's talk (e.g. whether pupils are taught how to talk productively or not, pupils' sense of agency on the process of learning, etc.) showed a strong impact on the quality of learning.

However, we have provided in this chapter initial evidence that the general idea of atomic patterns and its reflection back to teachers in professional development, has great potential as a way to help teachers in understanding and improving their orchestration practice, especially with ICT. Furthermore, the use of these atomic pattens in TEL and CSCL research could prove useful not only to disentangle how teachers design their lessons, but also how those lessons are transformed into the actual classroom performance. Thus, to understand orchestration better.

In order to address some of the shortcomings outlined above, several lines of research work on atomic patterns could be followed. In fact, the evidence from the professional development workshops that have taken place so far provides formative feedback for this purpose, hinting at possible directions for future iterations of this research line:

- The apparent link between teaching experience and perceived value of the routines makes targeting more novel teachers the most obvious next step. In fact, it could be hypothesized that this kind of practical advice could be very beneficial even for pre-service teachers. In such a context, probably the influences of prior experience and beliefs would be less noticeable, and maybe a more experimental approach could be taken, if desired (e.g. teaching students to design collaborative learning with ICT with and without patterns).

- The current workshop format and materials were devised for blended CSCL practice in distributed learning environments. Another obvious path for research would be to try to apply this same approach to other TEL contexts (e.g. IBL with mobile devices). This would probably require another cycle of elicitation of routines (which is rather costly). However, it is possible that comparing the routines extracted from a different setting and pedagogical approach, commonalities could arise, and studying them could be a worthy line of work. 
- Although our evaluation experience showed that it is dangerous to combine conceptual tools and technological tools in a very reduced timeframe (as we did in workshop TW3), we believe that this combination of the technological and the pedagogical support is rather powerful, as some theories on professional development propose (e.g. TPCK, see [Mis06]). We have already taken first steps in this direction, by proposing the last professional development workshop in the evaluation of GLUE!-PS as a follow-up course to the TW4 workshop presented in this chapter (with very positive results, see Chapter 5). Thus, linking this dissertation's pedagogical and technological tools for teachers into a unified approach to professional development, and studying the interdependencies among the different aspects, is another promising way forward (e.g. what new atomic patterns appear when the using GLUE!-PS in a DLE?).

- Also regarding the technological support for orchestration, teachers' apparent preference for automation, among the different forms of technological support to atomic patterns, should influence (and, in fact, has influenced, see Section 5.3.2) our efforts to provide technological support for the orchestration of blended CSCL using Distributed Learning Environments, which will be described in the next chapter. Thus, any kind of automation support provided to commonly-used atomic patterns will probably have greater chances of adoption by teachers (especially those that have been exposed to our set of atomic patterns).

- Last, but not least, it is important to note that all these PD actions are rather useless unless the tools they present are finally used in authentic classroom practice, especially by "average teachers" (as opposed to experts, or teacher-researchers). As we have seen in the interviews conducted, some of the workshop participants have already begun to try some of the workshop elements in their classes, and others have already contacted us in order to try the atomic patterns and the GLUE!-PS in their courses in the next academic year. Thus, further evaluation of atomic patterns in authentic experiments within the university curriculum are probably the main "low-hanging fruit" to provide further evidences of the use of atomic patterns in everyday orchestration practice. 


\title{
Chapter 5
}

\section{GLUE!-PS: An architecture and data model for the orchestration of learning designs in Distributed Learning Environments}

\begin{abstract}
Summary: Orchestrating CSCL in Distributed Learning Environments is a daunting task for practitioners. Considering the many aspects of orchestration, we can see multiple problems, just from the technological point of view. The first problems to be encountered are the lack of technological support to deploy teachers' learning designs into the DLEs, and to flexibly manage the deployed activities in enactment-time. This chapter presents the main technological contribution of the dissertation: the Group Learning Unified Environment - Pedagogical Scripting (GLUE!-PS). This service-based architecture and data model were extracted from a review of learning design tools and languages, and mainstream VLEs, in order to allow for the deployment, management and adaptation of CSCL activities (expressed in multiple learning design languages) across DLEs (based on multiple learning platforms). We also present here the iterative development and evaluation of the GLUE!-PS proposal through analytical and authentic experiments, and teacher workshops. Finally, we highlight how the GLUE!-PS is not only a powerful support in orchestrating learning, but also enables the development of more advanced orchestration support technologies targeting DLEs.
\end{abstract}

\subsection{Introduction}

As we saw in Section 2.5, orchestrating CSCL activities in the particular technological context of Distributed Learning Environments poses several problems to teachers (or whoever is the main orchestrating actor in the educational setting). First of all, the deployment of learning designs (i.e. the transformation of the design ideas into an ICT infrastructure that reifies those ideas) into our choice of DLE is severely undersupported (what we have called the "deployment gap", see Section 2.5.3). This is especially true considering that we can express learning designs using multiple different languages/specifications, and that our DLE will probably include an institutionally-mandated VLE and several external tools in various domains.. Second, currently there is no way of flexibly modifying in run-time a CSCL design that is deployed across a DLE (especially those based on widespread VLEs), without recurring to tedious, error-prone manual 
steps (Section 2.5.4). Given orchestration's emphasis on going from the design to the enactment, and in adapting that enactment flexibly in the face of unexpected events, we can conclude that current implementations of Distributed Learning Environments are hardly "orchestrable", at least for average teachers under typical restrictions in authentic educational settings.

Given this dissertation's main goal (to design, implement and evaluate technological and conceptual tools to support the orchestration of blended CSCL activities involving DLEs, see Figure 5.1], it follows that one worthy partial objective would be to provide technological tools that support orchestration in that context. From our definition and conceptual framework on orchestration (described in Chapter 3), we can assume that this support should affect one or more of the " $5+3$ Aspects" that orchestration entails. Moreover, from our research on the role of atomic patterns in orchestration (Chapter 4), we can also anticipate that such technological help should support teachers in performing such recurrent practices (or, at least, not hampering them unless they cause the emergence of other, more efficient patterns).

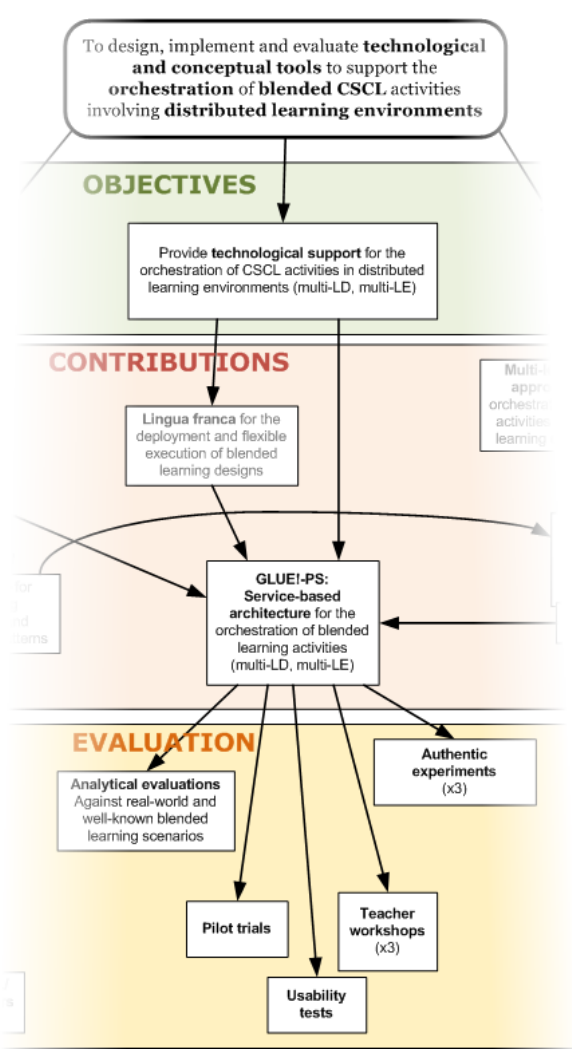

Figure 5.1: Parts of the thesis diagram concerned by chapter 5

This chapter proposes the main technological contributions of this dissertation, namely a tool to orchestrate CSCL activities across DLEs: the GLUE!-PS architecture. This serviceoriented architecture [Pap03 uses two series of Adapters [Gam95] to enable the deployment of learning designs expressed in one of multiple computerized learning design languages (such as the IMS-LD specification IMS03a]), across DLEs based on mainstream learning platforms 
(e.g. Virtual Learning Environments such as the Moodle VLE ${ }^{1}$ ) and external tools (e.g. "Web 2.0" tools such as Google Docs ${ }^{2}$ or Wookie Widgets 3 ). In order to provide this many-to-many interoperability (many LD languages, many learning platforms), the GLUE!-PS architecture uses a common intermediate data model (a sort of lingua franca, which we will name GLUE!-PS LF), which emerged from a study of main LD languages and learning platforms. This data model has been devised in order to maximize community acceptance from both teachers/practitioners and educational system developers: its aim is to be expressive enough to enable the translation of concepts from LD languages to DLEs, maintaining the design's essential features, but being simple enough to encourage third parties into developing the necessary adapters for making such translations possible. Moreover, the GLUE!-PS architecture can also be used to adapt the designs deployed in these multiple flavors of DLE in enactment-time, thus enabling the kind of flexibility that is necessary when enacting complex pedagogies such as blended CSCL, in authentic educational settings.

It is worth noting that, albeit many actors may be involved in the orchestration of this kind of scenarios (teachers, students, specialized learning designers, educational systems developers...), throughout this chapter we have chosen to take, for the most part, the point of view of the main end user of the GLUE!-PS system: a teacher that designs, deploys and enacts a series of blended CSCL activities through the DLE. Especially during the evaluation of our proposal, the teacher's point of view will be majoritarily portrayed, although the perspective of the system developer or the students might be mentioned throughout this chapter. Nevertheless, in certain particular contexts (e.g. certain universities), the aforementioned actions might be split among several actors (e.g. specialized technical staff that designs the courses, or that deploys them in the learning platform). We hypothesize that the kind of technological support provided by GLUE!-PS can be equally useful for those actors, although further research should be conducted in order to assess that assertion, and the consequences of such a separation of roles throughout the orchestration process.

Thus, in this chapter we will present, analyze and evaluate the GLUE!-PS architecture and its underlying data model (GLUE!-PS LF), with respect to the following research question:

RQ3.1 Can we provide technological tools to deploy and flexibly manage in run-time blended CSCL activities across DLEs?

The structure of the chapter is as follows: after we introduce the concrete form of the engineering methodology taken to research about GLUE!-PS, we present GLUE!-PS itself, including its requirements, architecture, data model and functionality (Section 5.2). We then analyze the GLUE!-PS with respect to the other two contributions of the dissertation (the " $5+3$ Aspects" framework and the atomic patterns) in Section 5.3. Later on, we describe the GLUE!-PS prototype implementation that has been developed throughout the dissertation (Section 5.4), including the technologies involved, its evolution, current features and limitations. Afterwards, the iterative evaluation of the GLUE!-PS system in supporting teacher orchestration in higher education settings is depicted in Section 5.5. Finally, the chapter ends with several remarks about the relevance of this contribution and future lines of related research work.

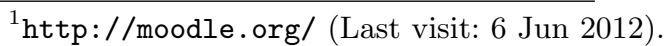

2 https://docs.google.com (Last visit: 6 Jun 2012).

${ }^{3}$ http://getwookie.org/ (Last visit: 6 Jun 2012).
} 
It is worth noting that several pieces of the discussion presented in this Chapter have already been published in TEL-related international conferences and workshops, by the author and several colleagues from the same research group. For instance, the proposal of the GLUE!-PS architecture and data model first appeared in [Pri11b], including the first analytical validation of deployment in VLEs. A demonstration of the use of the GLUE!-PS reference implementation to deploy learning designs is depicted in [Pri12b]. AH12b] describes more thoroughly how GLUE!PS utilizes the GLUE! architecture AH10] to deploy learning designs across DLEs (especially Moodle-based ones). Finally, $\mathrm{MC12b}$ details experiments done during the dissertation in order to assess the loss of information when deploying learning designs using GLUE!-PS.

\subsubsection{A note on methodology}

In the research work around GLUE!-PS, as described in the introductory chapter, we have used the 'engineering method' Gla95 as the general methodological structure of the dissertation, and of the research on each of the contributions. To develop the technological support presented in this chapter, we have also followed cyclical iterations of the four phases of information, proposition, analysis and evaluation Adr93 that this method proposes. As we can see in Figure 5.2, during this dissertation we have completed four iterations, one evaluated analytically (and thus, de-contextualized), and the other three evaluated in the context of higher education (more concretely, at the University of Valladolid, in Spain). These iterations are described below:

1. Once the "deployment gap" had been detected in the orchestration of learning designs across VLEs and DLEs, we set out to analyze the most widespread learning platforms and learning design languages to assess this gap. This analysis led to the first proposal of the GLUE!-PS architecture and its internal data model, which were validated analytically to provide a preliminary assessment of the advantages and disadvantages of the solution to deploy learning designs across different learning platforms (e.g. VLEs).

2. Given the positive results of the analytical evaluation, an initial prototype of GLUE!PS was implemented, which supported only translations from the most widespread LD language (IMS-LD) to the most widespread VLE (Moodle). Then, the prototype was validated, first in a pilot trial where researchers (the author and colleagues) deployed an authentic situation (a collaborative teacher workshop), and afterwards in two teacher workshops, one with university teachers, and another with teachers-researchers from primary-, secondary-, and university-level education.

3. Afterwards, the GLUE!-PS reference implementation was extended to support additional LD languages and VLEs, and it was tested in an authentic experiment, where a university teacher-researcher used it to deploy a wiki-based CSCL learning design, developed with the WebCollag $\biguplus^{4}$ IMS-LD-compliant LD tool.

4. Once the other contributions of the thesis (the conceptual framework on orchestration and the atomic patterns) produced significant outcomes, they were used to analyze our

${ }^{4}$ An evolution of the pattern-based CSCL authoring tool Collage HL06a. Available online at http://pandora. tel.uva.es/wic2/ (Last visit: 7 Jun 2012). 
GLUE!-PS proposal, to describe the orchestration support in terms of orchestration-aspectclassified atomic patterns. This analysis was also used to set the roadmap for the implementation of the GLUE!-PS prototype. Since the results of the previous iterations had highlighted several usability issues, the GLUE!-PS graphical user interface (GUI) was reimplemented, and GLUE!-PS was expanded to allow for certain run-time flexibility. This new prototype was then tested in two authentic experiments with teacher-researchers (using wiki-based DLEs), and a teacher workshop with non-expert university teachers from multiple disciplines.

\section{"Supporting orchestration of blended CSCL scenarios in Distributed Learning Environments" Contribution \#3 - GLUE!-PS as technological support for orchestration of CSCL in DLEs}

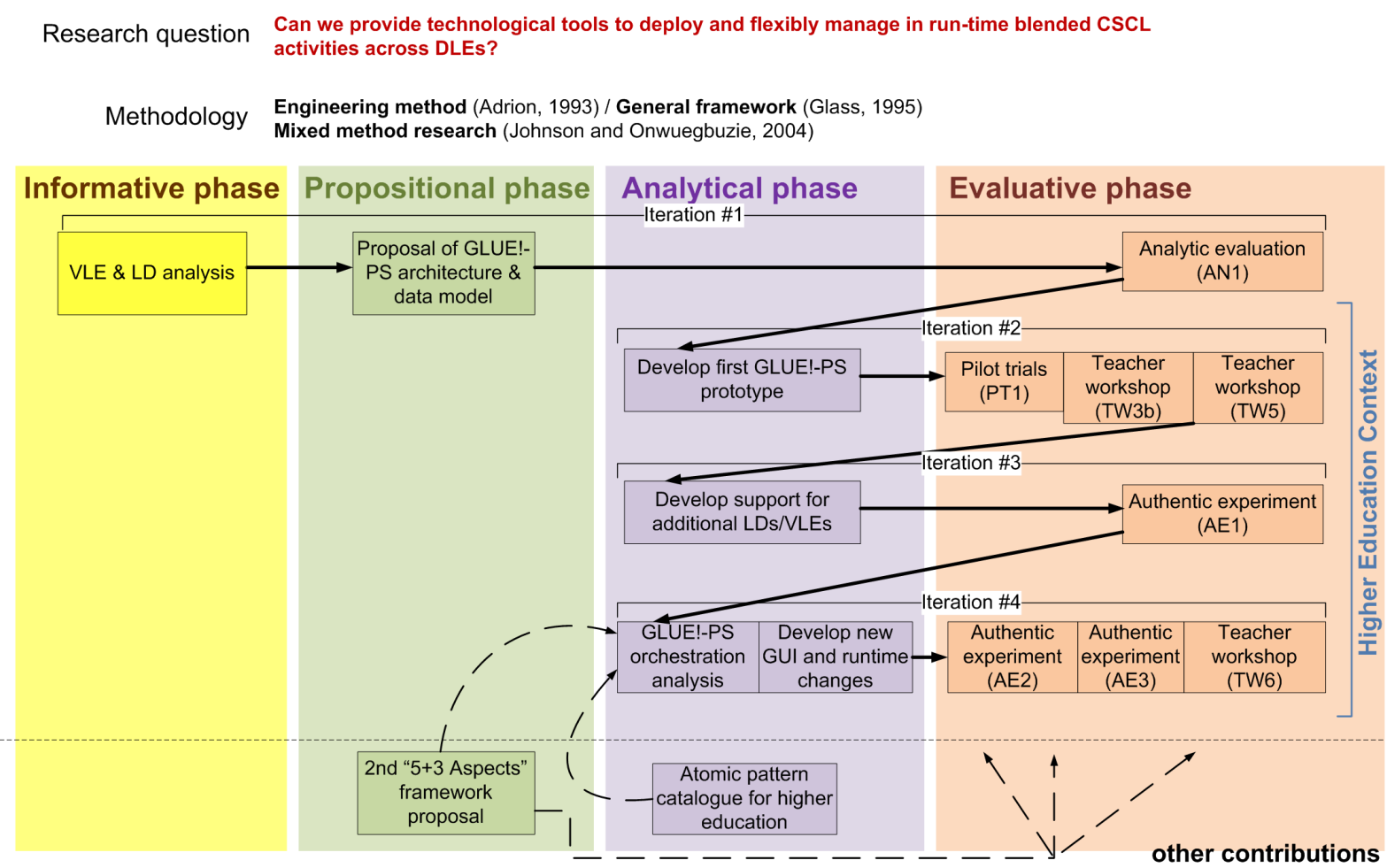

Figure 5.2: Detailed view of the engineering method followed during the research work around GLUE!-PS

Leaving aside the research methodology itself, as a technological contribution, GLUE!-PS can also be seen as a software engineering project and thus, we have also followed a more specific process for its design and implementation. Specifically, we have followed a lightweight variation of the Unified Process (UP, [Lar02]), more akin to the Agile Unified Process (AUP, [Amb02]), coupled with Agile software development methods [Bec01] such as Scrum [Sch02]. The rationale for using this hybrid software engineering approach is that, albeit the UP/AUP methodology is in some aspects appropriate to our problem and research methodology (due to its iterativeness, architecture-centrism and its emphasis on use cases), we also considered that our development should be able to cope with the adaptive planning and time-boxed iterative approach that a truly iterative research effort following the engineering method requires. That is, rapid and 
flexible response to change should be possible as a consequence of the successive results of our evaluation phases. Furthermore, the use of a well-known framework like Scrum (coupled with the iterative evaluation of the system using a variety of techniques - see Section 5.5 promoted a better identification of priorities depending on the expected research outcomes and evaluation results, reduced overtime, and facilitated the coordination among researchers and the members of the development team.

In the following section we present GLUE!-PS as the main dissertation contribution to support (non-expert) teachers technologically in orchestrating TEL/CSCL scenarios that make use of Distributed Learning Environments. As we can gather from the previous discussion on the iterative processes followed, the architecture, data model and especially its reference implementation, have evolved progressively during the dissertation. For clearness and brevity's sake, here we present GLUE!-PS in its current form, as opposed to providing a historical account of its evolution (although references to this evolution will inevitably be made while describing the iterative development and evaluation that took place - see Section 5.5.

\subsection{GLUE! Pedagogical Scripting (GLUE!-PS)}

Despite the increasing use of VLEs, PLEs and other "Web 2.0" in education, we saw in Section 2.5 how all these web learning environments and tools are not easily managed by practitioners, since they are not centrally controlled, each one forming in practice a "walled garden". Thus, learning designs that try to utilize the affordances of several of them to the advantage of the learning experience, often end up with complex schemes and ways of accessing the different learning activities, resulting in confusion for both students and teachers (see, for example, the experience in $[$ Fer10 $]$. This complexity and confusion can be experienced throughout the learning activity lifecycle:

1. The effort of designing activities is greater (e.g. there is a need to search for and select the best web tool for the purpose of each task in the activity)

2. The instantiation and deployment of the web infrastructure (e.g. VLEs and external web tools) normally has to be done manually by someone with technical expertise, and so it is time-consuming and prone to errors

3. The enactment of learning activities is more complex, since different tools and environments are used (e.g. raising the need for a unified access point to all the resources and tools involved in the design, see for example [Fer10]). Making changes to that deployed design in run-time, throughout the distributed ICT infrastructure, is also difficult

4. Also, the assessment and evaluation gets more complex, since the web infrastructure provides heterogeneous and distributed sources of information about the activities

Here we present a technological system that aims to support practitioners in the second and third of the aforementioned problems, i.e. of deploying and coordinating learning activities that include learning environments (especially the so-called VLEs, but not only) and external web tools. This problem can also be seen as the challenge of translating a learning design into 
the ICT infrastructure that will be used for its enactment, and making and translating changes to those designs in runtime, across multiple (web) learning environments and tools. This technological system proposed has been named the Group Learning Unified Environment - Pedagogical Scripting (GLUE!-PS from now on).

A note on the GLUE!-PS name The name GLUE!-PS (related but not to be mistaken with GLUE!, the architecture for the integration of VLEs and external tools described in [AH10]) was selected mainly for historical reasons, since both systems were intended to be part of a larger architecture for the orchestration of DLEs. The GLUE!-PS proposal, however, is independent of GLUE! in the sense that it can exist separately, as an architecture to deploy and manage learning designs in VLEs and other learning platforms. Nevertheless GLUE!-PS and GLUE! are related, since they follow similar design principles (see below), and GLUE!-PS utilizes the integration capabilities of GLUE! to manage the external tools to the VLEs (and thus, address DLEs), as described in AH12b.

\subsubsection{Requirements and guiding principles of GLUE!-PS}

As we have mentioned in previous paragraphs, the technological problem we were meaning to tackle with GLUE!-PS has mainly two aspects: one of interoperability between multiple LD languages and specifications, and multiple already widespread VLEs and learning platforms (as the central point of a variety of DLEs); another of coordinating the learning design (in deploytime or in run-time) across the different platforms and tools that conform the DLE. Since the GLUE! architecture provided a solution to the integration of external tools in learning platforms (providing most of the functionality needed for the coordination across the DLE), GLUE!-PS was concerned mostly with solving the LD-VLE interoperability problem (and, of course, of using GLUE!-PS to keep the resulting VLE infrastructure coordinated with the rest of the DLE according to the learning design).

As we saw in Section 2.5.3, despite the efforts towards the standardization of pedagogical design (embodied mainly in the IMS Learning Design specification [IMS03a), those standards are not widely used, and today there exist multiple learning design tools which produce designs in a number of different formats (see Appendix C). A similar situation is found in the learning platforms arena, where different major players (with user bases of thousands of institutions and millions of users) provide execution environments for learning activities which are not interoperable among each other. Against this fractured background, rather than trying to provide its own authoring tool, learning design model or execution platform, the GLUE!-PS architecture aimed to make learning designs deployable and executable using any VLE and external tools (i.e. DLEs) supported by the architecture, regardless of the authoring tool and platform used. In this regard, certain trends and design principles (maximize community acceptance, minimizing development efforts through code reuse and simple service contracts, non-modification of external tools, see below) could be used as guidelines for implementing the GLUE!-PS.

Guiding principles Given the aforementioned panorama of LD languages and learning platforms (Figure 5.3), and the focus of this dissertation in supporting teacher orchestration (with its emphasis on addressing the contextual constraints of average practitioners and other involved 


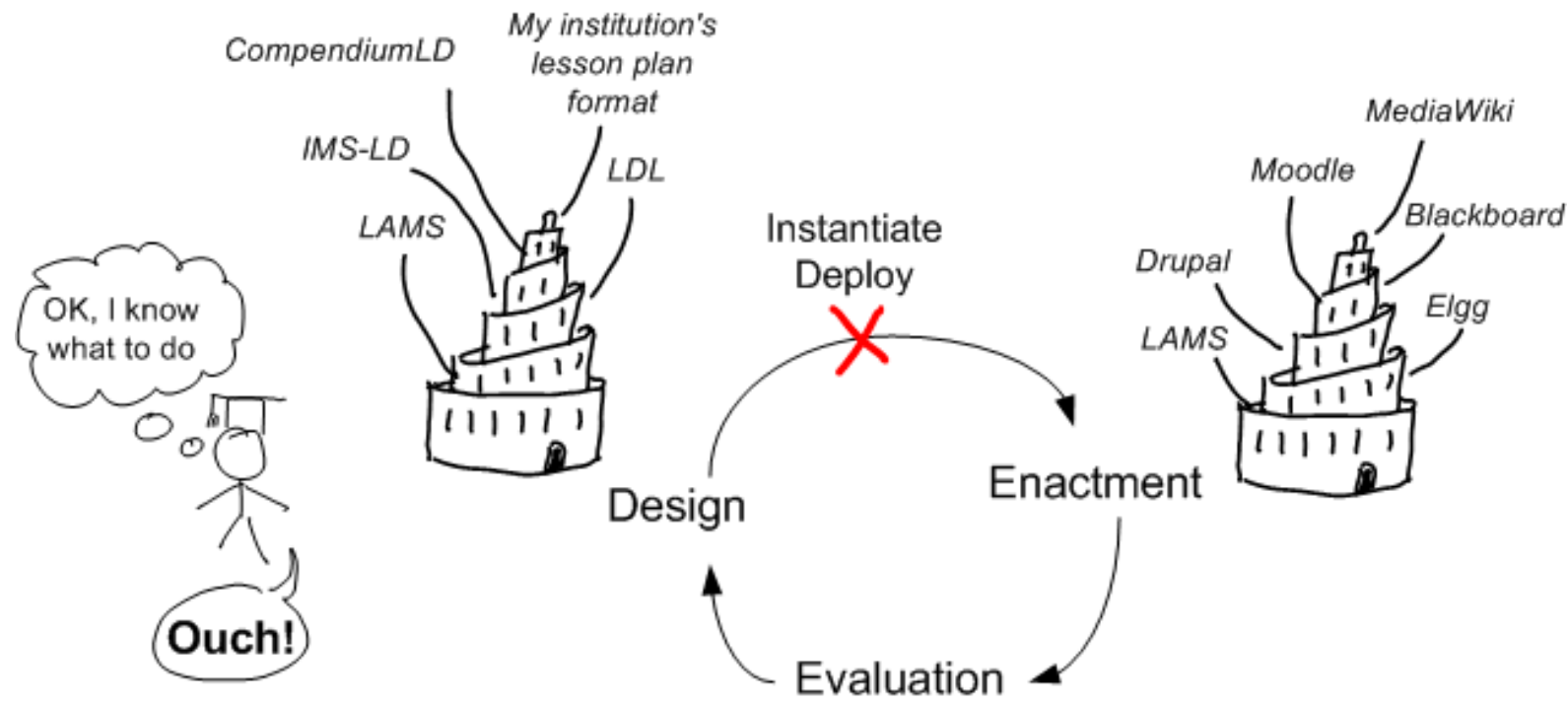

Figure 5.3: Cartoon representation of the "deployment gap" between computer-interpretable LD languages and the variety of widespread learning platforms

actors Dil09b]), our main design principle was to maximize community acceptance. This means that we aimed to addressing the needs of actors in an "average" blended CSCL scenario which, for our purposes, meant mainly a non-expert teacher who had to orchestrate activities across a DLE (often using an institution-chosen VLE), but also a software developer that had limited resources in order to develop the system to be used by such a teacher 5 . This main principle of community acceptance could be further concreted into five guidelines (please refer to AH12a for a more detailed rationale about how these guidelines emanate from a highly-fragmented landscape of existing systems to interoperate):

1. To impose a low number of restrictions on the systems involved (in this case, LD tools and learning platforms). Given the large user bases involved currently in the different VLEs (and, to a lesser extent, authoring tools), and the limited power that our initiatives would have to convince these large vendors to comply with the GLUE!-PS requisites, lowering the restrictions necessary to be compliant with GLUE!-PS would increase the number of potentially integrable systems, thus increasing the number of practitioners that could have access to the functionalities GLUE!-PS provides.

2. To impose only restrictions that current systems already fulfill. As a corollary to the previous one, lowering the restrictions for GLUE!-PS compliance until most existing systems (e.g. LD tools, learning platforms) already comply with them natively, would greatly increase the chance of adoption since no development effort would be needed by the tool/platform providers.

\footnotetext{
${ }^{5}$ While this last argument about the lower average development effort is critical for the GLUE! architecture, due to the myriad of available external learning tools, it is less important in our case, since the number of LD tools is comparatively lower. Thus, our evaluations will emphasize more the "teacher-institution" side of the problem, rather than the "software development" side
} 
3. Enable a many-to-many integration. Given the current variety of learning platforms and authoring tools, finding an integration approach that keeps development efforts to integrate new LD tool/platform combinations low, would increase the chance of thirdparty (including institution-hired) software developers extending this integration's scope to new systems. This again would increase the number of practitioners that have access to GLUE!-PS.

4. To promote a loose integration scheme. Since the degree of software coupling also has an effect on the development effort required to integrate each LD tool with a new learning platform, promoting a loose integration scheme would lower the barrier of entry for developers desiring to integrate new systems.

From these four design guidelines we can already extract a number of (non-functional) requirements for the GLUE!-PS system:

- R1: Avoid modifications to the source code and APIs (application programming interfaces) of external authoring/learning tools and VLEs. Using a VLE or tool extension capabilities (e.g. plugins) is permitted.

- R2: Minimize development efforts on the part of integration agents.

- R3: Foster code reuse between integrations.

- R4: Provide simple contracts for the intermediate layer between the authoring tools and the VLEs (this can be seen as a consequence of R2 and R3). This basically means that the GLUE!-PS programming interface should remain as simple as possible.

- R5: Extensibility of the design languages and VLEs supported (e.g. in case a new specification or VLE is made available and becomes popular). This can be achieved by using plug-ins or adapters and, to a lesser extent, by maintaining the GLUE!-PS contract simple.

- R6: Where possible, provide interoperability and compatibility with existing standards and specifications.

Main functional requirements Despite nomenclature used so far, in which GLUE!-PS is a single system, the GLUE!-PS architecture has to fulfill several related but different functionalities, all connected with the deployment and run-time execution of learning designs. This includes, mainly:

- The contextualization of abstract learning designs, which generally specify a pedagogical method (e.g. a sequence of activities), but not the concrete students, groups or tools that will be used to execute it. This process is often referred to as operationalization or instantiation (see Section 2.2.2).

- The deployment of a (contextualized) design in the (de-centralized, in this case) learning environment that will be used for its execution. This includes creating the content and activity structure in the VLE based on the learning design, and creating the necessary (external and internal) tool instances for each of the users and groups involved in the (contextualized) design. 
- The run-time execution of the (contextualized) design, which itself is an ill-defined concept, and can be implemented in a variety of ways, with different levels of regulation (from the mere display of the design activity structure, which can be freely navigated through, to the enforcement of a sequence by only displaying certain activities at certain times). An example of this kind of functionality can be found in IMS-LD players such as CopperCor 6 or VLEs that support scripting, such as LAMS7. The requirements of teachers in this regard are highly contextual, and may be even variable with time (in what some authors have called the "fading" of scripts Wec07]).

One of the main difficulties in the specification and implementation of these functionalities lies in the fact that different authoring tools and VLEs support different parts of this feature set. For example, some authoring tools provide group instantiation of designs (e.g. InstanceCollage HG08), while most others don't. Many VLEs do not support scripting natively (e.g. Moodle), and some of the learning environments considered do not even support clearly the concept of activity, mixing it with the tool used to support it. In an ideal case, the GLUE!-PS would provide the functionalities that are lacking in the learning design tool-learning environment combination considered by the user (or, even if a functionality is supported natively, the user may choose to use the support provided by GLUE!-PS). This fact hinted at a modular design, where different functionalities may be available on a case-per-case basis. Thus, we can define a series of functional requirements for GLUE!-PS:

- FR0: Modular functionality. The functions provided by the GLUE!-PS should be variable depending on the authoring tool and VLE used, and also depending on the teacher's preference.

- FR1: Allow for the instantiation of people and groups (i.e. the assignment of the concrete users that will participate in the learning experience, to the different groups, roles, activities) of an abstract design, in case the used design tool/language does not support such functionality. This implies communicating with the VLE (or any other entity that manages users and groups) to obtain such information.

- FR2: Allow for the instantiation of tools 8 of an abstract design, in case the used design tool/language does not support such functionality.

- FR3: Deployment of instantiated learning designs into a learning platform, including the associated learning contents, tool instances and sequence of activities.

- FR4: Support the execution of scripts, in the sense of being able to access (i.e. browse) the activity structure in the learning design, including its contents and tools (e.g. external tool instances). The elements visualized should be dependent on the role of the user accessing it.

- FR5: Support the execution of scripts, in the sense of enforcing the activity sequence defined by the script, if any (to decrease students' cognitive load). This may be done by

\footnotetext{
6 http://coppercore.sourceforge.net/

7 http://www.lamsinternational.com/index.html

${ }^{8}$ In the sense of the assignment of the concrete external or VLE tool instances to the users that will use them. For the external tools, the GLUE! architecture provides functionality to manage the lifecycle of tool instances.
} 
hiding the non-active activities, or by disabling them in any other way. This requirement is optional, although desirable.

- FR6: Flexibility in the modification of the instantiated learning design (which may also imply changes to the underlying abstract design), before it is deployed into the learning environment.

- FR7: Flexibility in the modification of the instantiated learning design (which may also imply changes to the underlying abstract design), during the run-time of the activities. This kind of functionality is highly dependent on the features of each learning environment, and thus the support for this requirement will likely be variable.

- FR8: Translation of instantiated (and potentially modified, see FR6 and FR7) designs back to their original format in an (abstract) learning design language, for its reuse by practitioners. This requirement is optional, if other means of cross-platform reuse is provided.

- FR9: Being sensitive (as far as the other requirements and design principles allow it, e.g. without modifying the VLE code base) to the changes made by the different actors directly in the web environment or the external learning tools (e.g. in the activity and group structure, permissions, etc). This requirement is also optional.

Orchestration requirements On top of those guiding and functional requirements, we can also extract a number of requirements from the literature review on orchestration (see Section 2.3), especially from some of the latest works of Dillenbourg [Dil09b [Dil10 [Dil11b] which propose several requirements and guiding principles when designing for orchestration 9 ;

- OR1: To be usable by teachers, as the main orchestrators of activities in many learning situations. Albeit this is mostly a user interface requirement, making the GLUE!-PS model and concepts intuitive for non-technology experts is a desirable feature (to keep the UI development effort low). Orchestration and usage by students themselves should not be dismissed either.

- OR2: Functional minimalism, i.e. providing only the bare minimum functionality that is useful for the orchestration of activities (to avoid cognitive overload of the orchestrator). This requisite is optional (and ill-defined).

- OR3: The service should be usable by most skilled teachers, not just by exceptional teachers with high improvisational abilities. Again, this is mostly a user interface requirement, but may influence design decisions in the internal structure of GLUE!-PS.

- OR4: Support for individual, group and whole class activities, since these are the most common social levels at which orchestration occurs.

\footnotetext{
${ }^{9}$ As the reader may notice, following the findings and discussion on what is orchestration in Chapter 3 , these and many other requirements could be derived from the " $5+3$ Aspects" framework. However, since that framework had not been developed when this requirements analysis was done, we have chosen not to reformulate the GLUE!-PS requirements in those terms.
} 
- OR5: Support for shared, reused artifacts (e.g. intermediate outputs of previous activities), another common feature of orchestrated scenarios.

- OR6: Pedagogical neutrality, that is, supporting a wide range of pedagogical approaches, such as collaborative learning, project-based learning, etc.

- OR7: Performing basic operations through the GLUE!-PS should be time-efficient, since in the thesis we are considering not only distance/asynchronous activities but rather blended learning activities which may happen face-to face (with the temporal constraints that this carries).

- OR8: Since awareness of the evolution of the activities is one of the basic orchestration axioms (so that corrections can be made in their coordination), the system should support this kind of awareness. This does not include the implementation of the awareness mechanisms themselves, but rather providing "hooks" for these awareness mechanisms to be made available to the users.

- OR9: Support for legacy tools already present in the learning situation, including non-ICT tools. This requisite is optional (and currently ill-defined).

- OR10: Making the workflow tangible, that is, making it both visible and modifiable in some way. This requisite is optional (and currently ill-defined).

\subsubsection{GLUE!-PS architecture}

\section{Overall architecture description}

GLUE!-PS tries to address some of the problems in the orchestration of blended CSCL scenarios (expressed through LD authoring tools) that combine VLEs and external tools (i.e. DLEs), outlined at the beginning of this section (the deployment and run-time management of such designs). Following from the design principles and requirements outlined above, we propose a loose-coupling integration model, embodied by the proposed GLUE!-PS architecture (see Figure 5.4 . GLUE!-PS is a three-tier service-oriented architecture (SOA, [Pap03]) with loosely-coupled distributed services, where $\mathrm{m} \mathrm{LD}$ authoring tools and specifications, and $\mathrm{n}$ learning platforms are adapted to each other through an intermediate software layer and a set of adapters. This use of the Adapter pattern [Gam95] [Mon03] aims at reducing the development effort, since the required integration code is partially assumed by this common intermediate software layer. The architecture defines a simple Representational State Transfer (REST, see Fie02 ) contract, and uses learning platforms' native mechanisms and APIs to deploy learning designs and to manage them in run-time.

These two sets of adapters are used to wrap the varying contracts and data models used by both LD authoring tools and learning platforms, reducing them to just two generic, homogeneous contracts: a GLUE!-PS contract for LD tools and a GLUE!-PS contract for learning environments. This structure enables the integration of LD tools and learning platforms without modifying their respective implementations. The intermediate software layer (GLUE!-PS Manager) offers these two integration contracts, and is used to decouple LD and LE adapters, thus allowing for their independent development, and implementing most of the common integration 


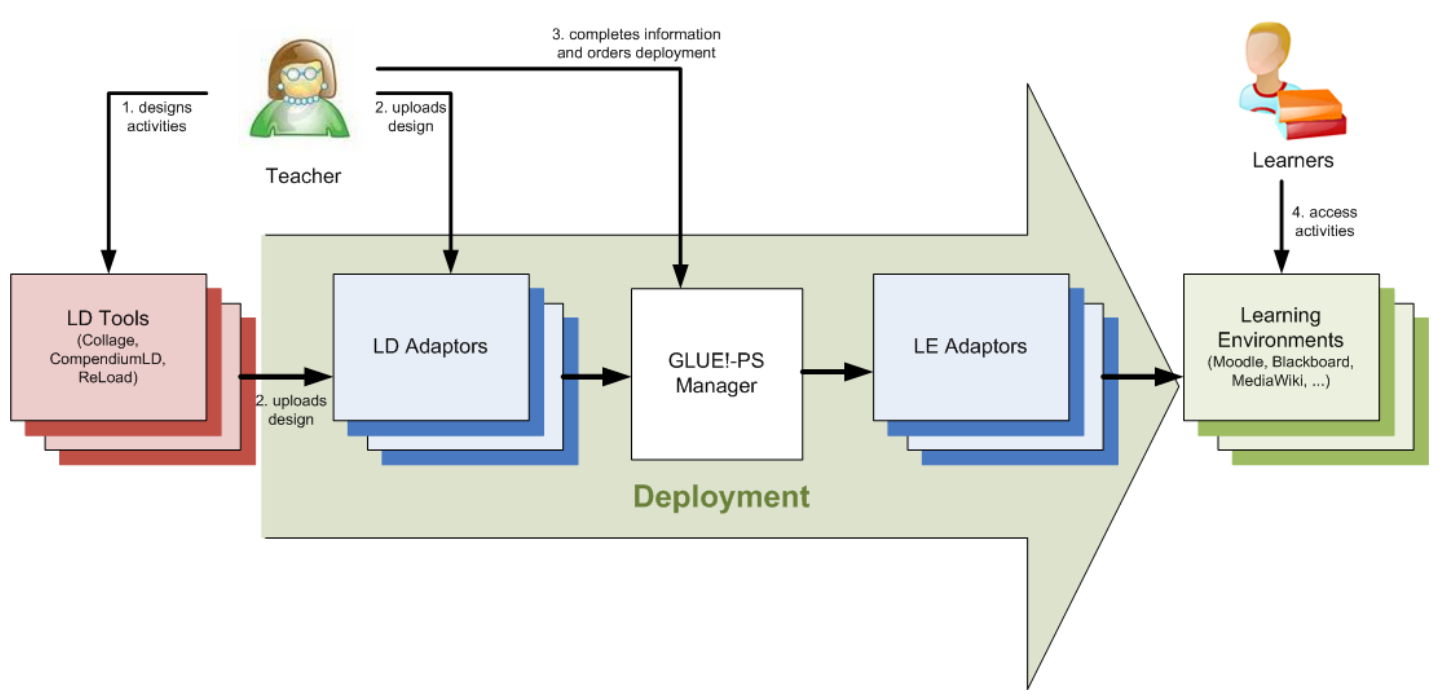

Figure 5.4: GLUE!-PS simplified architecture

functionality (thus lowering the development effort of new integrations, i.e. new adapters). In this way, a many-to-many integration is enabled, since every new LD adapter developed for a learning design language/specification automatically enables its integration in all the learning environments for which there exists an LE adapter, and vice-versa.

Our approach tries to find a compromise between the different degrees of expressiveness of LD languages and the varying capabilities of currently widespread learning environments. Taking into account the current fragmented state of both the learning design and learning environment landscapes, as well as past experiences (such as IMS-LD not getting widely adopted), our main design principle has been to maximize community acceptance, by lowering the barrier of entry for all involved stakeholders (teachers and learners, institutions, learning environment and other software developers). Thus, our solution aims at integrating as many LD tools/languages and learning environments as possible, while avoiding modifications to the source code of existing systems, minimizing the average development effort for the integration to take place, and allowing institutions or practitioners that have opted for specific LD tools or learning environments to keep their choices. Moreover, the solution tries to provide a more time-efficient deployment alternative for practitioners (vs. current manual approaches to deploying across a DLE).

Coarsely speaking, the architecture would translate from the learning designs in the different formats to a common data model native to the GLUE!-PS using the different LD adapters. Then, the design information in this common data model is completed with instantiation information about the specific context, provided by the teacher (e.g. number and composition of groups, specific tools to be used, etc.) and finally translated automatically to the concepts and data format of the target learning environment using the LE adapters, which deploy the instantiated/particularized design to the target environment. It is important to highlight the importance of this common set of concepts, which is essential for the architecture to be effective. This data model for the central GLUE!-PS service serves as a "lingua franca" for the translation between learning designs on one side, and learning environments on the other. As we will see in Section 5.2.3, in order to minimize the effort of developing these adapters, this common set of concepts should be as close as possible to the concepts being translated to and from. 
This kind of service-oriented architecture poses several well-known advantages: a) it allows third parties (authoring tool developers, VLE providers and other interested parties like institutions) to develop the adapters, as long as they comply with the GLUE!-PS contracts; b) it allows implementors of the different components to use any desired technology internally; c) using the Adapter pattern allows for extension of the architecture to new languages and environments, maximizing the code reuse among adaptations and reducing the average development effort by the developers (e.g. once an LD adapter is implemented, that LD language can be used with all the supported learning environments). Moreover, the architecture also complies with aforementioned design principles such as not requiring modifications to either design authoring tools or existing learning environments. Finally, it is worth mentioning that, due to the existence of a central element (the GLUE!-PS Manager service), a way is provided to introduce instantiation information in a unified way, regardless of the original LD language or the target learning environment.

However, up to now we have only described how GLUE!-PS would make LD authoring tools and specifications interoperable with VLEs and other learning platforms. However, GLUE!PS was posed to support orchestration of learning environments that included not only learning platforms but also external tools (i.e. Distributed Learning Environments - DLEs). In order to support the deployment and real-time management of activities in DLEs, GLUE!-PS utilizes the functionalities of the GLUE! architecture AH10. As we saw in Section 2.4.3. GLUE! is another service architecture intended for the integration of VLEs and external tools, allowing for the management of the external tool instances from inside a VLE (thus, we could say that GLUE! enables the management of DLEs). Given that GLUE!-PS uses the GLUE! architecture to manage the external tools involved in a DLE, it is important to note that, although GLUE!PS can interoperate between multiple LD authoring tools and learning platforms imposing very few constraints, the GLUE!-PS solution is only able to support orchestration in DLEs that are based on the GLUE! architecture.

Thus, as we can see in Figure 5.5, the deployment (and also the run-time management) of a learning design using GLUE!-PS would follow a "forked path": the learning designs coming from any GLUE!-PS compliant authoring tool are translated into GLUE!-PS's common data format; through GLUE!-PS, the learning design is particularized, modified or extended to include concrete information about groups, activities and resources (in this regard, GLUE!-PS overcomes the limitations of many authoring tools and LD language: ${ }^{10}$. Then, before deploying a learning design in a particular DLE, GLUE!-PS can import (through the corresponding GLUE!-PS LE adapter) the list of participants registered in a certain course or lesson, as well as the list of built-in tools provided by such VLE, allowing teachers to populate the groups and to select the specific tools supporting each learning activity. Moreover, thanks to the use of the GLUE! architecture, when teachers set the tools intended to support the learning activities within the GLUE!-PS interface, they may select any of the tools in the DLE (both VLE internal ones, and GLUE-supported external ones). The deployment of the learning design in the DLE then done across the different domains, using GLUE! to manage the external tools, and then integrating all the internal and external resources in the VLE (e.g. in the form of a course that allows users to access both built-in and external tools selected by the teacher). Thus, the GLUE! GLUE!-PS partnership allows for the deployment and management of learning designs (created

\footnotetext{
${ }^{10}$ The designs generated with many authoring tools do not consider the concept of groups, or concrete participants, which learning platforms do consider.
} 
with multiple authoring tools) in multiple flavors of DLEs, which include multiple VLEs and multiple external tools.

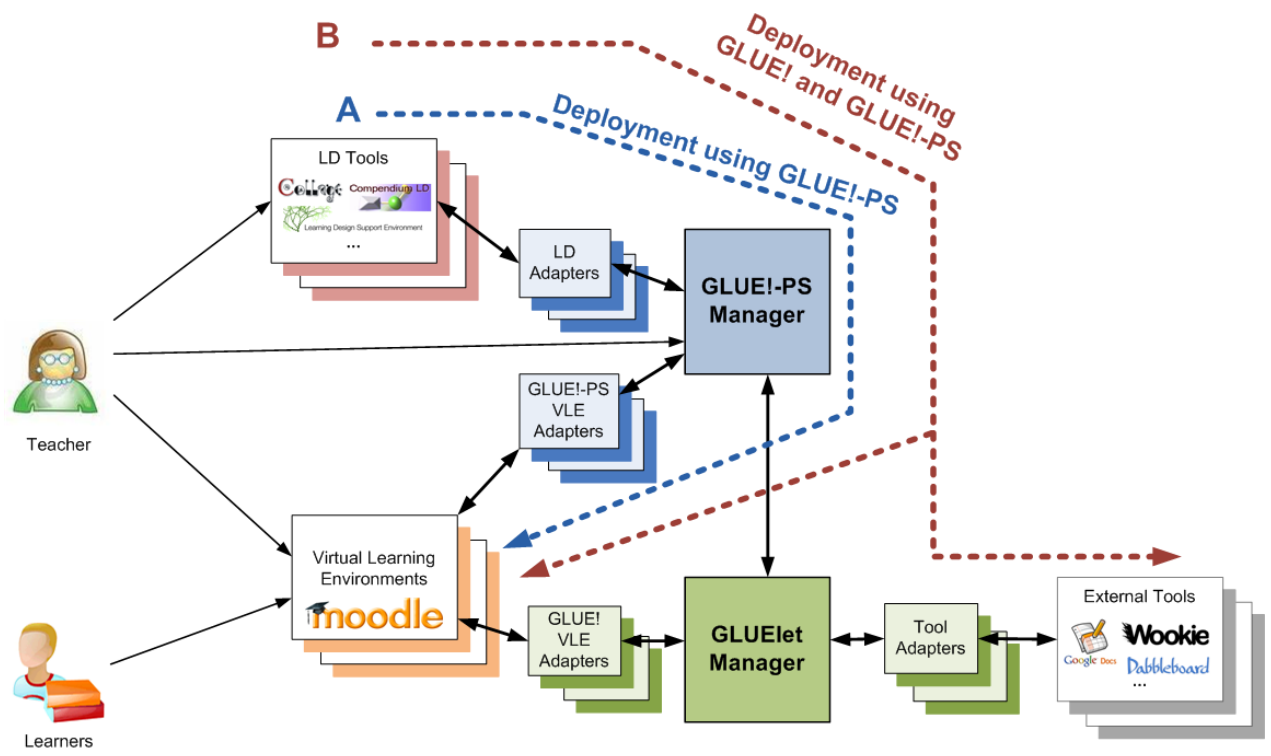

Figure 5.5: GLUE!-PS simplified architecture, including the use of the GLUE! architecture to address DLEs

\section{Integration contracts}

Following the design principles detailed in Section 5.2.1, we have chosen integration contracts that try to facilitate adoption, by imposing few restrictions, using broadly accepted technologies, and defining a minimum set of expected functionality from the LD/LE adapters (which in turn may impose certain requirements from the systems they try to adapt). These contracts are as follows:

LD authoring tool integration contract GLUE!-PS imposes only one mandatory condition on $L D$ authoring tools: that the learning designs they produce can be expressed in a computerinterpretable format. This includes, for example, XML bindings such as the one defined in the IMS-LD specification IMS03a, or any other binary form as long as its format is well-defined. This restriction, in fact, is fulfilled by all the LD authoring tools that we have notice of, and it might even apply to other more informal learning design languages (e.g. institutional lesson plans), as long as structured binary or text-based representation of it can be produced. Although other requirements might be useful to facilitate the development of adapters (e.g. that the tool has a web-based API to access to such design representations), the fact that many existing LD tools do not comply with such restriction (e.g. many are desktop applications which run on the end users' machines) made us reject them for the integration contract.

The GLUE!-PS integration contract also details the minimum overall behavior that LD tool adapters (not the LD tools themselves) should display, when invoked from the GLUE!-PS central element. Basically, this behavior can be summarized as: 
1. Translation: Receive an invocation specifying the learning design to be translated, it its original form (e.g. in IMS-LD format if the LD authoring tool is IMS-LD compliant). This learning design can be encapsulated in the invocation (e.g. an HTTP call with the IMSLD Unit of Learning as the body of the message), or an identifier can be provided that univocally represents the learning design (e.g. the URL where the learning design can be downloaded from). Internally, the adapter translates the learning design from its original format into the GLUE!-PS common data format (see Section 5.2.3). Finally, it responds to the invocation by providing the same learning design translated to the GLUE!-PS data format.

2. Other optional functionalities are possible, such as the storing of the original learning design and/or the translated design in the GLUE!-PS data format, for later retrieval.

Other restrictions that the LD tool adapters have to fulfill, from a technological point of view, is that they have to offer their functionality to the GLUE!-PS. Ideally, this would be done as services, more concretely, as REST services, which offer very simple resource-based services (e.g. an HTTP POST invocation to the /design resource), and respond with the learning designs expressed in an XML or JSON bindings of the GLUE!-PS data format. However, even these relatively easy technological restrictions are optional, and adapters could be implemented, for example, as binary libraries 11 . However, their implementation as services allows for the advantages that service orientation provides (independence of the underlying implementation technology, development by third parties, etc.).

LE integration contract As it did in the case of the integration of LD tools, the GLUE!-PS imposes few restrictions on the learning platform that will serve as the center of our Distributed Learning Environment. Only four of these restrictions are mandatory. First, the learning platform must be able to render web contents, so that the external tools can be easily embedded in the learning platform, either as IFrames, or HTML Objects (this is a restriction required by the GLUE! architecture, since most existing external tools are developed as web applications, see AH12a]).

A second restriction is that the learning platform has to support the concept of activity, or tool (or other concepts which can be somehow assimilable to those). As we will see in the following section, both tools and activities are central concepts in the GLUE!-PS "lingua franca", as they appear both on most LD languages and on most VLEs. However, this apparently strong requirement is in practice quite flexible, since non-VLE learning platforms (e.g. a wiki) normally have a basic concept that can be easily assimilable to an activity or a tool (e.g. a wiki page can represent an activity, an IFrame embedded in a wiki page can represent a tool).

A third restriction is that the learning platform has to support the concept of user, separate from other users. Albeit this concept is currently so widely supported that we barely notice it anymore, the separation between one user and other users is essential to the notion of instantiation, especially in CSCL (since very often different users may perform different activities or take different roles within a CSCL design), and thus has to be taken into account.

\footnotetext{
${ }^{11}$ In fact, the current prototype of the GLUE!-PS reference implementation (see Section 5.4 invokes LD adapters as Java libraries.
} 
The other mandatory restriction is that it has to have a means of modifying the learning environment's structure of activities (or whichever similar concept exists in the platform). For example, many VLEs have the notion of "course", as a container for a set of related activities and tools that are used when enacting such course. However, the different ways in which this modification may take place depending on the learning platform, leads us into two possible variants of the deployment (and later management) of the activities, which do not exclude each other:

Dynamic deployment If the learning platform has some kind of API that can be invoked from outside in order to modify the course structure, then GLUE!-PS (by means of the corresponding LE adapter) can invoke that API in run-time to deploy or manage the course. For example, the MediaWiki wiki engine provides an API for the creation of wiki pages (which are assimilable to activities and tools) - thus, GLUE!-PS can make dynamic deployments to MediaWiki.

Static deployment If the learning platform provides some way of modifying the course structure at the user's request (e.g. restoring a backed up course), GLUE!-PS can provide the teachers with the means for deploying the learning design in a format that the target learning platform understands. A typical example of this kind of deployment is the Moodle VLE v1.9. GLUE!-PS can generate a "Moodle course backup" with all the internal and external tool information embedded into it, that the teacher can download and restore into her Moodle VLE course.

Other desirable, but non-mandatory requirements that learning platforms could implement include the offering of an extension interface (e.g. so that dynamic deployment means can be implemented without modifying the platform's code), that they understand the concept of group and the concept of role (which is very interesting for CSCL since it involves activities at different social levels). It is important to highlight how most mainstream VLEs and other non-VLE learning platforms comply with these mandatory and optional requirements in one form or another.

The GLUE!-PS integration contract also details the minimum overall behavior that LE adapters (not the learning platforms themselves) should display, when invoked from the GLUE!PS central element. Basically, this behavior can be summarized as having the following functionalities:

1. In the case of an "static deployment" learning platform (see above), the adapter should be able to accept an invocation that encapsulates or refers to a particularized learning design in the GLUE!-PS data format, and it should translate it into a LE-dependent static representation of the particularized design (e.g. a zip file with the Moodle course backup).

2. In the case of a "dynamic deployment" learning platform (see above), the adapter should be able to accept an invocation that encapsulates or refers to a particularized learning design in the GLUE!-PS data format, and it should translate it into LE-dependent concepts, which the adapter will dynamically create through the platform's programmatic interface, in order to produce a course (or equivalent notion) that reflects the particularized design (including both internal tools and embedded external tools created through the GLUE! 
architecture). Then, the adapter will respond with a reference to the platform where the deployed design can be found.

3. Also in the case of "dynamic deployment", in order to allow the updating of the learning design's activities (e.g. for run-time adaptations of the design), the adapter should be able to accept an invocation to update the deployed tools and activities to comply with a new GLUE!-PS data format representation which is encapsulated or referred to in the invocation. After using the target platform's API to do this updating, the adapter should respond with a reference to the location of the updated design within the platform.

4. Also in the case of "dynamic deployment", the adapter should accept an invocation in order to delete the particularized design's activities and tools, when they are not needed anymore.

5. To allow for the retrieval of the available courses in a learning platform (or any other concept that can be assimilated to this one), so that the teacher can select which portion of the learning platform's structure she wants to affect when deploying.

6. To allow for the retrieval of user information from the learning platform, either as a list of all the platform's users or, more desirably, in smaller-grained portions, such as the users enrolled in a course.

7. Other optional functionalities are also desirable, such as the retrieval of already existing groups in the learning platform (or in the selected course), the storage and retrieval of "static deploy" representations (in the case of static deployment platforms), and so on.

Other restrictions that the LE adapters have to fulfill, from a technological point of view, is that they have to offer their functionality to the GLUE!-PS. Again, this would be ideally done as simple REST services offering simple resource-based services using, e.g., XML or JSON bindings of the GLUE!-PS data format. However, even these relatively easy technological restrictions are optional, and LE adapters can be implemented, for example, as binary libraries ${ }^{12}$. However, their implementation as services allows for the advantages that service orientation provides (independence of the underlying implementation technology, development by third parties, etc.).

As we have seen throughout the description of the GLUE!-PS architecture, a crucial element in its operation, as well as a determining factor in the development effort that implementing the adapters requires, is the GLUE!-PS common data model to express the particularized learning designs to be deployed and managed in run-time. This data model is described in the following section.

\section{Behavior expected by GLUE!-PS Manager clients}

As it has been mentioned, the GLUE!-PS central element is a service that, following the REST service style [Fie02] [Ric07], defines a series of "resources" that clients of the service (such as practitioners, using a kind of user interface) can use to access the main functionalities of the GLUE!-PS architecture. These functionalities include:

\footnotetext{
${ }^{12}$ The current prototype of the GLUE!-PS reference implementation (see Section 5.4 also invokes LE adapters as Java libraries.
} 
1. The creation, retrieval, and deletion of learning designs. Especially in the creation of designs, diverse formats and LD languages can be used as part of the request for creation (including GLUE!-PS own data model), since the GLUE!-PS Manager will use the different LD adapters to transform them to its common data model.

2. The creation, retrieval, update and deletion of particularized learning designs to be deployed/managed (also called "deploys"). Again, the creation of this kind of data structures can be done using GLUE!-PS own data model or other data models supported by LD authoring tools which do support the particularization of designs, such as InstanceCollage HG08 or WebCollage 13 .

3. The retrieval of the available learning platform installations (also called "learning environments") in which this GLUE!-PS Manager service can deploy and manage designs. The information about learning environments should include also information about the courses (or equivalent notion) available in the platform, and the available tools, both built-in and external (available through the GLUE! architecture).

4. The retrieval of information about the configuration data needed to create instances of the different built-in and external tools available in a certain distributed learning environment.

5. The retrieval of users (e.g. students) available in a certain learning platform installation and course. This information can be used, e.g., to particularize a design before it can be deployed in a learning platform.

6. The creation, retrieval, update and deletion of individual (built-in or external) tool instances that are used in a particularization of a learning design (e.g. a Google Docs document that is to be used by a concrete group of students to produce a report, within a learning design where a report is elaborated using the Jigsaw strategy Aro92).

7. The deployment, re-deployment (e.g. adaptations of a design in run-time) and undeployment (i.e. deletion of the activities and tools that represent the learning design in the platform and in the external tools) of a particularized learning design (deploy). Depending on the deployment modality supported by the learning platform (static or dynamic), these actions will be performed different ways (see above).

Thus, using these generic functionalities, GLUE!-PS Manager clients (e.g. a graphical user interfaces that can communicate with the service) can attain the desired outcomes that we set out at the beginning of this chapter: to deploy and manage in run-time a learning design, across a distributed learning environment. Table 5.1 provides a resource-oriented version of these functionalities, assuming that HTTP requests (GET, PUT, POST and DELETE) on those resources are used to implement the invocations to GLUE!-PS. Section 5.2.4 provides further details into the behavior of the architecture, by detailing the basic use cases and how the different GLUE!-PS architecture elements interact in them.

\footnotetext{
${ }^{13}$ http://pandora.tel.uva.es/wic2/ (Last visit: 9 Jun 2012).
} 
Table 5.1: Behavior of the GLUE!-PS Manager service, represented as REST resources

\begin{tabular}{|c|c|c|c|}
\hline REST resource offered & $\begin{array}{l}\text { HTTP } \\
\text { methods } \\
\text { offered }\end{array}$ & Coarse-grained actions & $\begin{array}{l}\text { Interactions } \\
\text { with other } \\
\text { elements }\end{array}$ \\
\hline /designs & $\begin{array}{l}\text { GET POST } \\
\text { DELETE }\end{array}$ & $\begin{array}{l}\text { Creation, retrieval, and deletion of learning de- } \\
\text { signs. Accepts diverse formats and LD languages } \\
\text { for creation. Responds with GLUE!-PS LF data } \\
\text { on retrieval. }\end{array}$ & LD adapter \\
\hline /deploys & $\begin{array}{l}\text { GET } \\
\text { POST PUT } \\
\text { DELETE }\end{array}$ & $\begin{array}{l}\text { The creation, retrieval, update and deletion } \\
\text { of particularized learning designs to be de- } \\
\text { ployed/managed. Accepts diverse formats for cre- } \\
\text { ation. Responds with GLUE!-PS LF data on re- } \\
\text { trieval }\end{array}$ & $\begin{array}{l}\text { LD adapter } \\
\& \text { LE } \\
\text { adapter }\end{array}$ \\
\hline /learningEnvironments & GET & $\begin{array}{l}\text { Retrieval of the available learning platform instal- } \\
\text { lations in which this GLUE!-PS Manager service } \\
\text { can deploy and manage designs. Responds with } \\
\text { GLUE!-PS LF data including information about } \\
\text { the courses and tools available, both built-in and } \\
\text { external. }\end{array}$ & $\begin{array}{l}\text { LE adapter } \\
\& \text { GLUElet } \\
\text { Manager }\end{array}$ \\
\hline $\begin{array}{l}\text { /learningEnvironments/ } \\
\text { \{learningEnvironmentId }\} / \\
\text { tools/\{toolId }\} / \text { configuration }\end{array}$ & GET & $\begin{array}{l}\text { Retrieval of information about the configuration } \\
\text { data needed to create instances of built-in and } \\
\text { external tools available in a certain distributed } \\
\text { learning environment. Responds with XForms } \\
\text { Wor09 data. }\end{array}$ & $\begin{array}{l}\text { LE adapter } \\
\text { or GLUElet } \\
\text { Manager }\end{array}$ \\
\hline $\begin{array}{l}\text { /learningEnvironments/ } \\
\{\text { learningEnvironmentId }\} / \\
\text { courses } /\{\text { courseId }\}\end{array}$ & GET & $\begin{array}{l}\text { Retrieval of users (e.g. students) available in a } \\
\text { certain learning platform installation and course. } \\
\text { Responds with GLUE!-PS LF data. }\end{array}$ & LE adapter \\
\hline /deploys/deployId/toolInstances & $\begin{array}{l}\text { GET POST } \\
\text { DELETE }\end{array}$ & $\begin{array}{l}\text { Creation, retrieval, and deletion of individual } \\
\text { (built-in or external) tool instances that are used } \\
\text { in a particularization of a learning design. }\end{array}$ & $\begin{array}{l}\text { LE adapter } \\
\text { or GLUElet } \\
\text { Manager }\end{array}$ \\
\hline /deploys/deployId/live & $\begin{array}{l}\text { GET PUT } \\
\text { DELETE }\end{array}$ & $\begin{array}{l}\text { Deployment, } \text { re-deployment and un- } \\
\text { deployment of a particularized learning de- } \\
\text { sign across a concrete DLE that supports } \\
\text { dynamic deployments. Performs the deploy- } \\
\text { ment/redeployment/undeployment through the } \\
\text { LE adapter and responds with a reference to the } \\
\text { location of the deployed/updated particularized } \\
\text { learning design in the learning platform. }\end{array}$ & $\begin{array}{l}\text { LE adapter } \\
\& \text { GLUElet } \\
\text { Manager }\end{array}$ \\
\hline /deploys/deployId/static & $\begin{array}{l}\text { GET PUT } \\
\text { DELETE }\end{array}$ & $\begin{array}{l}\text { Deployment, re-deployment and un-deployment } \\
\text { of a particularized learning design across a con- } \\
\text { crete DLE that supports static deployments. Re- } \\
\text { sponds with a static representation of the de- } \\
\text { ployed/updated particularized learning design.. }\end{array}$ & $\begin{array}{l}\text { LE adapter } \\
\& \text { GLUElet } \\
\text { Manager }\end{array}$ \\
\hline
\end{tabular}

\subsubsection{GLUE!-PS Lingua Franca (GLUE!-PS LF): A data model to deploy and manage learning designs in DLEs}

During the inception of the GLUE!-PS service architecture, it became readily clear for us that the data model used in the GLUE!-PS central element, as an intermediate language to translate from multiple LD languages (with widely varying degrees of expressiveness) to multiple learning platforms (with different ways of expressing their native concepts) would be one of the cornerstones that could decide the success or failure of the GLUE!-PS proposal. The development effort needed to implement both the LD and LE adapters above (i.e. the barrier of entry for software developers) would depend mostly on the complexity of the GLUE!-PS integration contracts and the underlying data model of such service (thus favoring the selection of a simpler model). However, a too simple model could lose too much of the original learning design's expressivity, producing deployed infrastructures that no longer complied with the design's essential 
characteristics.

Since this data model had to integrate languages and concepts with opposing goals (e.g. de-contextualizing for reuse in LD languages, contextualizing for execution in learning environments), we analyzed and compared several languages and sets of concepts from both sides, in order to determine the most commonly supported deployable characteristics of computerized learning designs. These learning design traits have been extracted from the review of several scripting conceptual frameworks available in literature [Kol06] [Kob07] [Wei09], and include aspects like the presence of sequencing features, multiple roles participating in a single activity, etc. The results of our analysis are summarized in Figure 5.6.

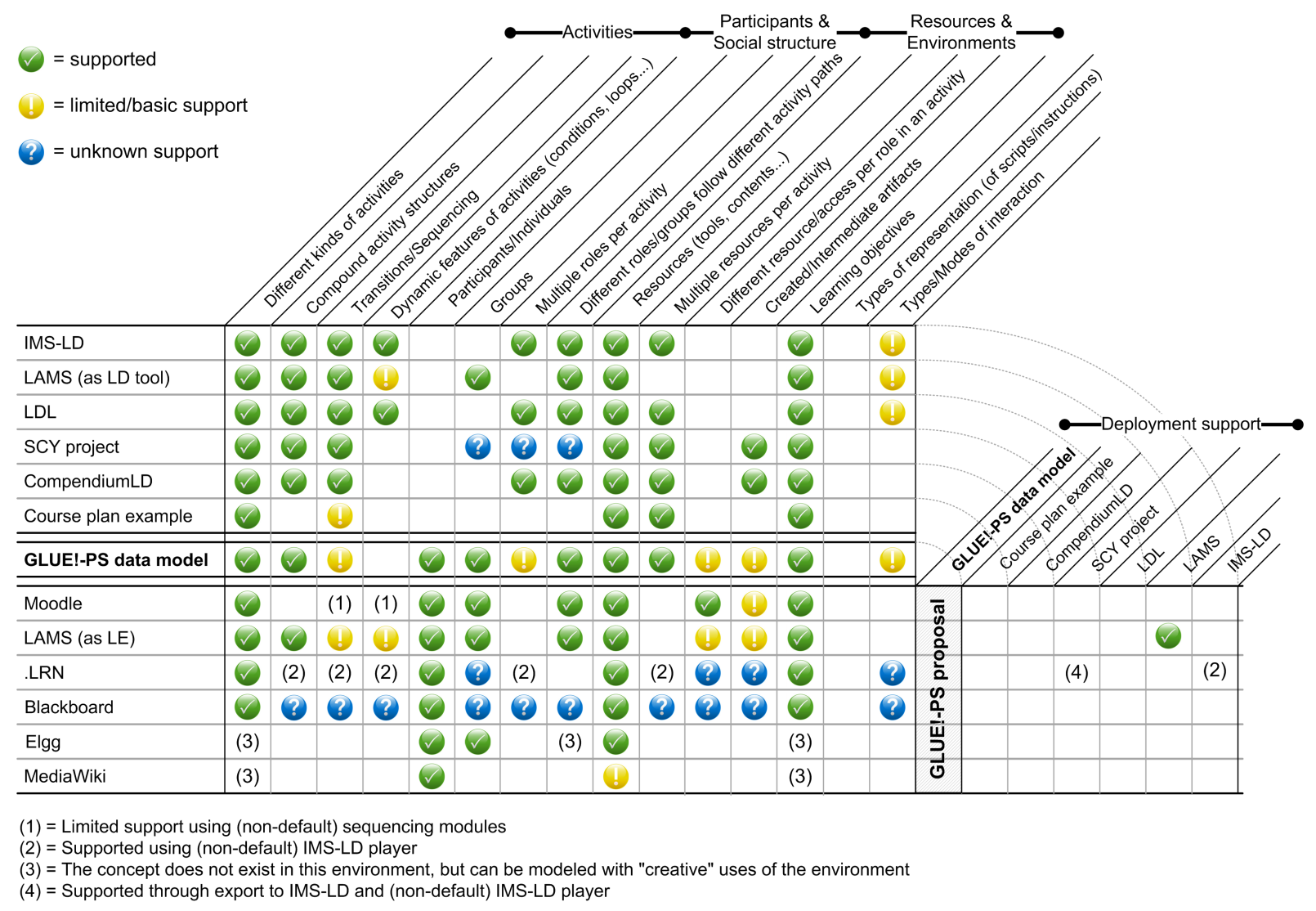

Figure 5.6: Summary of the main scripting characteristics supported by learning design languages and learning environments, extracted from [Kol06 Kob07] Wei09

Our analysis of LD languages (upper rows in Figure 5.6) included the aforementioned IMSLD IMS03a, plus LAMS Dal03, LDL Fer08 and the SCY project design approach Lej09b. In order to get a wider view and include current LD practice, we also included two languages not intended for automated deployment, such as the language of CompendiumLD Bra08 and the implicit language used in course plans in an authentic setting (at University of Valladolid). As we can see, they are all activity-based ${ }^{14}$ and provide some kind of structuring of the learning

\footnotetext{
${ }^{14}$ Even if LDL puts more emphasis on single interactions, the notion of activity exists and is a basic organizing concept Fer08.
} 
activities. Often, they also model learning objectives and resources, as well as the social structure of the design, often in the form of roles (but not individuals).

When analyzing learning environments (lower rows in Figure 5.6), we chose several of the main players in the VLE arena (like Moodle or .LRN), but also other web 2.0 tools that are being used as learning environments, such as the MediaWiki ${ }^{15}$ wiki engine or the Elgg ${ }^{16}$ social networking software. These environments always recognize individual participants, but invariably have poorer activity sequencing and dynamics (if any) than those offered in LD languages. Many of them define groups of participants (but not functional roles in activities), and all provide modeling of resources. We have also represented the current LD deployment support in the different environments, which is currently very scarce - precisely the gap that the GLUE!-PS proposal intends to cover.

This section proposes a model based on that analysis (a more detailed version of this analysis can be found in Appendix C), which can be thought of as a sort of "lingua franca" 17 between LD authoring tools and languages and learning platforms. Thus, we will refer to this model as GLUE!-PS LF from now on.

With the results of the aforementioned analysis, we have constructed a data model that represents the scripting properties available in LD languages, to the extent that learning environments support them, pondering at the same time the relative acceptance of the different conceptual sets (e.g. the concepts of Moodle or IMS-LD had more weight in our design decisions than those of LAMS or LDL). The proposed model appears in Figure 5.7; each learning design is composed of a number of activities, which can be structured in an arbitrarily deep tree structure, and can be sequenced (even if just for presentation purposes). Each activity can be performed by different functional roles, and is mediated by one or more resources (which can be static documents in the sense of its location being known at design-time, or tools that can be instantiated so that each group accesses a different instance of the tool, e.g. a shared drawing board for each learner team). A learning design can be particularized to be deployed, and each deploy is a contextualization of that learning design for a specific learning-environment. In such a deploy, the concrete participants (i.e. users in that learning environment) that will take part on the activities have to be specified. For each activity in the design, a number of instanced-activities will be created, one per group. Finally, each group performing an activity that requires the use of (instantiable) tools will be assigned an instanced-tool so that they can work independently from other groups if needed. The scripting properties of this model can also be seen in Figure 5.6 (middle row).

It is important to note that this data model does not intend to be "yet another" LD language or conceptual framework. Rather, it strives to represent deployable characteristics of learning designs, taking into account the practitioners' desire for contextualization, since they "are more interested in effectively and efficiently bringing these proposals in the real classroom with certain guarantees for sustainability and scalability" Wei09.

\footnotetext{
${ }^{15}$ http://mediawiki.org

${ }^{16}$ http://www.elgg.org

${ }^{17}$ A lingua franca, also referred to as working language, bridge language, or vehicular language, is a language systematically used to make communication possible between people not sharing a mother tongue, in particular when it is a third language, distinct from both mother tongues Chi08.
} 


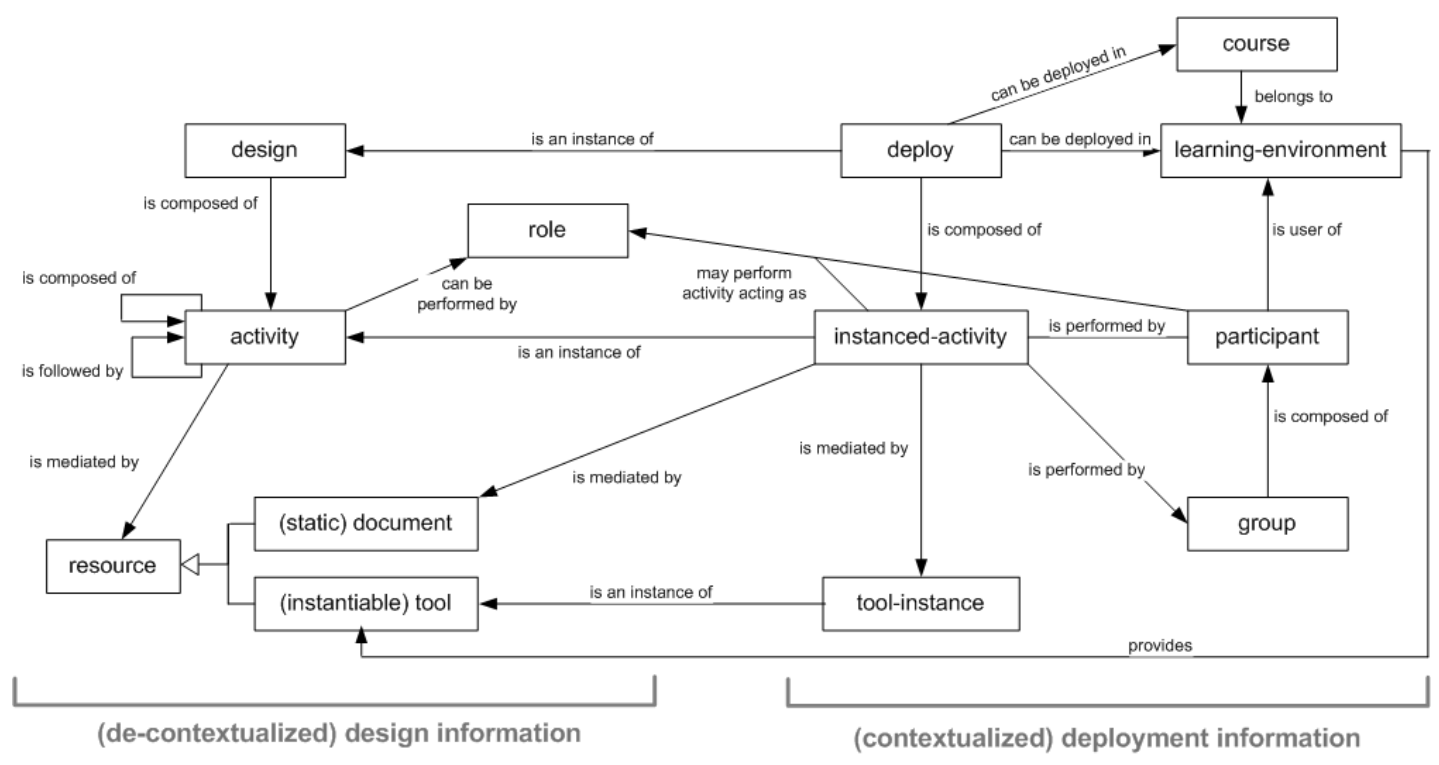

Figure 5.7: GLUE!-PS data model

\subsubsection{GLUE!-PS functionality: Main use cases}

Section 5.2 .2 provided a general overview of the elements in the GLUE!-PS architecture, and summarized the respective contracts that each of the elements should fulfill, and the restrictions imposed on the different elements to be integrated. Furthermore, Section 5.2 .3 described the data model that is used in most of the interactions within and without the GLUE!-PS architecture (the GLUE!-PS LF). Now we will describe how all these elements come together to address the needs of the end users (e.g. a practitioner), expressed through the two most typical use cases: The particularization and deployment of a learning design across a DLE, and its adaptation in run-time, once it has already been deployed. Each use case has two variations, for static- and dynamic-deployment platforms. These use cases also highlight the need of a graphical user interface for GLUE!-PS so that the end-users (e.g. teachers) can access the functionalities of the GLUE!-PS manager and the rest of the GLUE!-PS architecture.

It should be noted that these are just the most illustrative examples of behavior of the architecture, and other use cases (or other variations of these ones) are possible, within the loose restrictions of the different integration contracts 18 . In order to make the use case more easily understandable, each one will be described with a short narrative describing an instance of the use case, followed by a general module interactions diagram, plus a more detailed sequence diagram explaining the internal behavior of the architecture.

\footnotetext{
${ }^{18}$ For example, a LD authoring tool could request tools or users information to GLUE!-PS in order to make the particularization within the LD tool. This case has been implemented in the aforementioned WebCollage authoring tool, and used extensively in our evaluations.
} 


\section{Use Case I: particularization and deployment of a learning design in a DLE (dynamic deployment)}

Sara is a university teacher, delivering a course on the applicability of ICTs (especially "Web 2.0") to education, in University of Valladolid's Faculty of Education. As part of this "ICTs 2.0" course, where she teaches two 24-student groups, she intends to conduct a collaborative learning activity, spanning three face-to-face sessions and a few hours of online work, using the course's main learning platform (which is a wik: $\left.{ }^{19}\right)$, Google Documents ${ }^{20}$ and Google Presentations ${ }^{21}$, to support an inquiry about the educational affordances of three kinds of ICT tools: blogs, social networks and wikis. She has the idea of structuring the activity using the Jigsaw strategy: first, dyads of students will investigate on one of the proposed technologies and produce a wiki page with their findings; second, the students who researched about the same technology ("experts") will agree and produce a structured document (using Google Docs) with the main affordances of that technology. Finally, dyads of the different technologies will join in "jigsaw groups" and produce a presentation with their overall conclusions about the affordances of "Web 2.0" technologies.

With this structure in mind, Sara uses the WebCollage tool to design such a jigsaw scenario. However, she notices that WebCollage does not allow for the creation of dyads of students in the first phase of a jigsaw (it assumes that it is individual work). Since she knows that this limitation can be solved in GLUE!-PS, she goes on and saves her learning design as an IMS-LD Unit of Learning (a zip file), wishing there would be a 'magic button' that would let her deploy those ideas directly into her course's wiki22.

Sara then accesses the GLUE!-PS graphical user interface (a web application), where she uploads her IMS-LD zip file with the activities and resources that she intends to use for the scenario. Through the web interface, she states that she wants to particularize the design for the first 24-student group, to be deployed in a DLE where the central point is her wiki-based learning platform (which already is GLUE!-enabled). Then, she is presented with a representation of the activities in her jigsaw learning design. With the help of the GLUE!-PS user interface she defines the different groups of concrete course students that will conduct each activity (dyads, expert groups, jigsaw groups), and the resources that each group will use (wiki pages, Google Documents and Google Presentations). Finally, she hits on the "Deploy" button and, after some moments, the application states that her deployment has been completed.

In order to test it, she clicks on a link that appears on screen, which takes her to the course's wiki, where a wiki page with the activity structure she defined had been created. Navigating through that structure, she accesses the different resources of

\footnotetext{
${ }^{19}$ This scenario is based on a real course, whose learning platform is located in http://gsic.uva.es/TIC2 (Last visit: 9 Jun 2012).

20 http://www.google.com/google-d-s/documents/ (Last visit: 9 Jun 2012)

${ }^{21}$ http://www.google.com/google-d-s/presentations/ (Last visit: 9 Jun 2012)

${ }^{22}$ This kind of integration between an LD authoring tool and the GLUE!-PS architecture has been, in fact, implemented (see, for example, http://youtu.be/nd2bcaIMrpU). However, it requires the modification of the LD authoring tool, and thus is not part of the basic use cases, in which it is easier to understand the role and inner workings of the GLUE!-PS elements.
} 
the different groups and checks that everything has been created according to her ideas.

Then, Sara comes back to GLUE!-PS to deploy the activities for the second 24student group...

The aforementioned narrative depicts the typical workflow of a teacher designing a series of learning activities using a LD authoring tool (WebCollage, in this case), and then deploying it across a DLE in which her course's wiki plays the role of the central VLE. This workflow is also represented in Figure 5.8 .

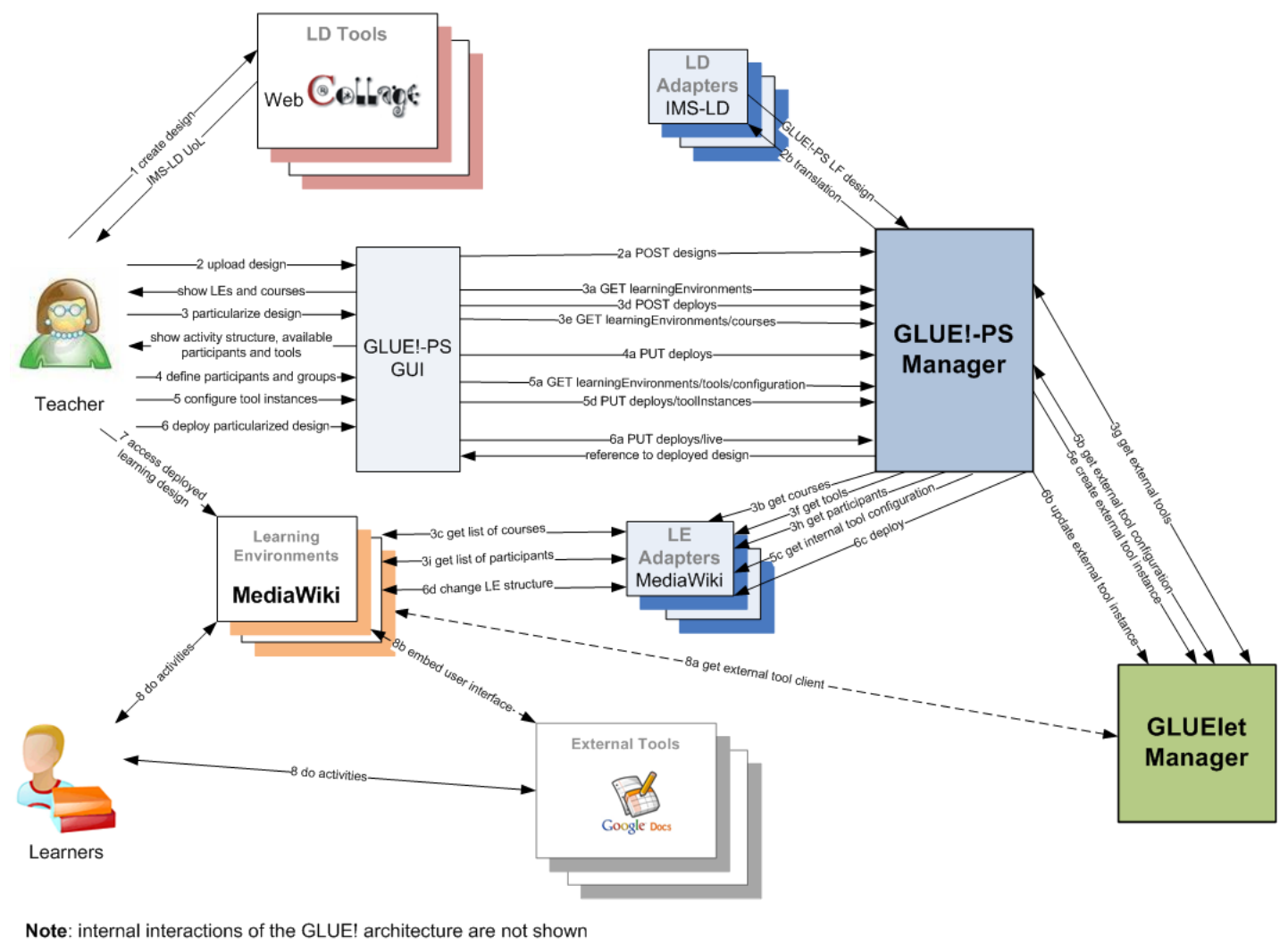

Figure 5.8: GLUE!-PS block interactions diagram for Use Case I (dynamic deployment of a IMS-LD design done with WebCollage, into a MediaWiki-based DLE)

In that figure we have chosen not to represent the interactions among the GLUE! architecture's internal elements, in order to make our diagram more readable (please refer to AH12a for a more detailed account of these interactions). As we can see in Figure 5.8, this external behavior that Sara, our end-user, sees, in turn causes the following sequence of events between the different elements of the GLUE!-PS architecture:

1. The teacher creates a design using a LD authoring tool of her choice, and afterwards downloads a computer-interpretable representation of the design. In this phase normally GLUE!-PS is not involved (unless the LD tool has been modified to interact with GLUE!PS, e.g. to extract the available tools to add them to the design description, or to upload the design automatically). 
2. The teacher uses GLUE!-PS's user interface to upload the design to the GLUE!-PS Manager service (2a, in Figure 5.8). This service, in turn, invokes the corresponding LD tool adapter, to perform the translation of the design from its original format into the GLUE!PS LF data model that represents that design (2b).

3. Once this is done, the user states that she wants to particularize a design for deployment. The GLUE!-PS GUI asks the GLUE!-PS Manager for the list of available learning platform installations where designs can be deployed (3a) and, once an installation has been selected, the GUI shows a list of the available courses $(3 \mathrm{~b}, 3 \mathrm{c})$ so that the teacher can select one of them. Once these selections are done, the particularized design (or "deploy") is created (3d). In order to show the activity structure to the teacher to begin the particularization of the design, the GLUE!-PS GUI asks for several pieces of relevant information for such particularization (3e), including the available built-in and external tools (3f, $3 \mathrm{~g}$ ), and the participants in the course $(3 \mathrm{~h}, 3 \mathrm{i})$.

4. The teacher particularizes the learning design to be deployed, adding the concrete group structure, participants and the distribution of concrete (built-in or external) tool instances that each group has at their disposal. All these particularizations generate successive requests to the GLUE!-PS Manager in order to update the particularized design data model (4a).

5. During the particularization process the teacher can configure the particular instances of external or built-in tools (e.g. create the particular Google Documents of one of the groups in deployment time, in order to customize its contents) (5a, 5b, 5c) and, eventually, create them $(5 \mathrm{~d}, 5 \mathrm{e})$.

6. Once the teacher has finished fine-tuning her design for its enactment in this particular environment and for this particular set of students and tools, she can order the deployment of the particularized design across the DLE (6a). This will set off an invocation to the GLUElet Manager (6b) and the corresponding LE adapter (6c), which will be in charge of modifying the structure of the learning platform installation to suit the design (e.g. creating activities, linking them with built-in tools, embedding external tools, etc.) (6d). Once these modifications are finished, the teacher is provided with the location of the newly deployed design.

7. The teacher can access this location in the learning platform, and navigate the different activities and resources created reflecting the particularized design.

8. Conversely, using the learning platform's interface students can access the activities and resources set up by the teacher, and enact them. The access to the external tools is normally provided by retrieval and embedding of the external tool's client interface within the learning platform's user interface (8a, 8b).

Figure 5.9 represents the detailed sequence diagram with the invocations to the different services' resources in a typical REST services implementation of the scenario. Whenever possible, the same labels used in Figure 5.8 have been maintained. Also, in order to simplify the diagram, the resources ' $\mathrm{X}$ ' and 'list of $\mathrm{X}$ ' (e.g. designs and list of designs), which are normally treated as different resources in REST service descriptions [Ric07], have been treated here as a single entity. 


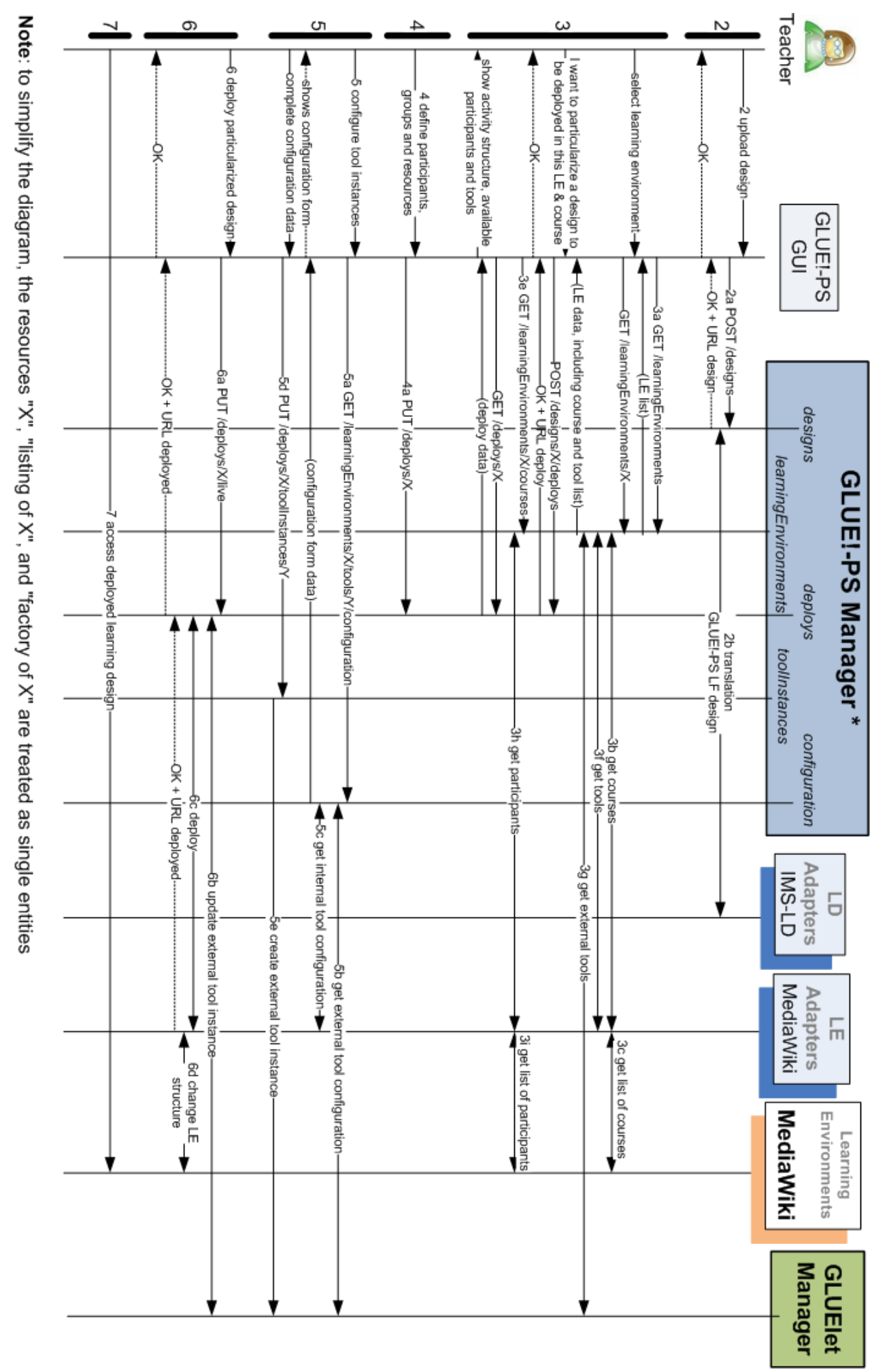

Figure 5.9: GLUE!-PS sequence diagram for Use Case I (dynamic deployment of a IMS-LD design done with WebCollage, into a MediaWiki-based DLE) 


\section{Use Case Ib: particularization and deployment of a learning design in a DLE (static deployment)}

If we now re-envision the previous use case in the situation of deploying a particularized design into a learning platform installation of the "static deployment" kind (e.g. Moodle version 1.9, where the deployment is currently done through a static file with a Moodle course backup), the use case would be mostly identical to Use Case I in its worflow and sequence of invocations, with the following differences:

- In the narrative depiction of the use case, when she hits on the "Deploy" button, she is presented with a link which lets her download a zip file (the Moodle backup course) (6d). Sara then would have to access her Moodle course, and use the "Restore" option in Moodle (7, in Figure 5.10) to upload and restore such zip file representing the particularized design including the participants, groups and (built-in and external) tools she chose. Once this "pseudo-course" is restored, she can access the course normally in Moodle (8), accessing both internal and external tools from the VLE interface.

- In the structure diagram (see Figure 5.10), everything would be identical to Use Case I, except that in phase 6 , the LE adapter does not directly communicate with the Moodle platform to modify the course's structure. Rather, a static file with the deployable course structure is generated, and in phase 7 the teacher deploys the "pseudo-course" using Moodle's native "Restore" function. Afterwards, the access both for her and for the students would be similar as in Use Case I.

- Figure 5.11 depicts the complete sequence of invocations for this use case. The only differences, again, can be found in the interactions of phases 6 and 7 , which reflect the aforementioned different way of modifying the course's structure.

\section{Use Case II: run-time adaptation of a deployed design (dynamic deployment)}

For the description of this use case, let us assume that Use Case I (the deployment of the particularized learning design) has already been executed. Thus, the main pre-requisite for this use case is to have a design already deployed across a DLE whose center is a "dynamic deployment" learning platform, e.g. MediaWiki.

Following the deployment of her jigsaw scenario on the educative affordances of "Web 2.0" technologies, Sara arrives to her classroom for the first face-to-face session of the jigsaw. Unfortunately, just as she arrived to the classroom, a student tells her that two of her classmates will not be able to come to today's session. Since she does not think it fair to let some of the students work alone in this first phase, she decides to change the groupings so that the absent students are in one dyad, allowing their respective workmates to work in dyads in this face-to-face session. Thus, the absent ones can complete this part of the activity at home, asynchronously.

While the students finish a previous assignment, Sara enters the GLUE!-PS interface and selects the deployed design for this particular student group. Once she is 


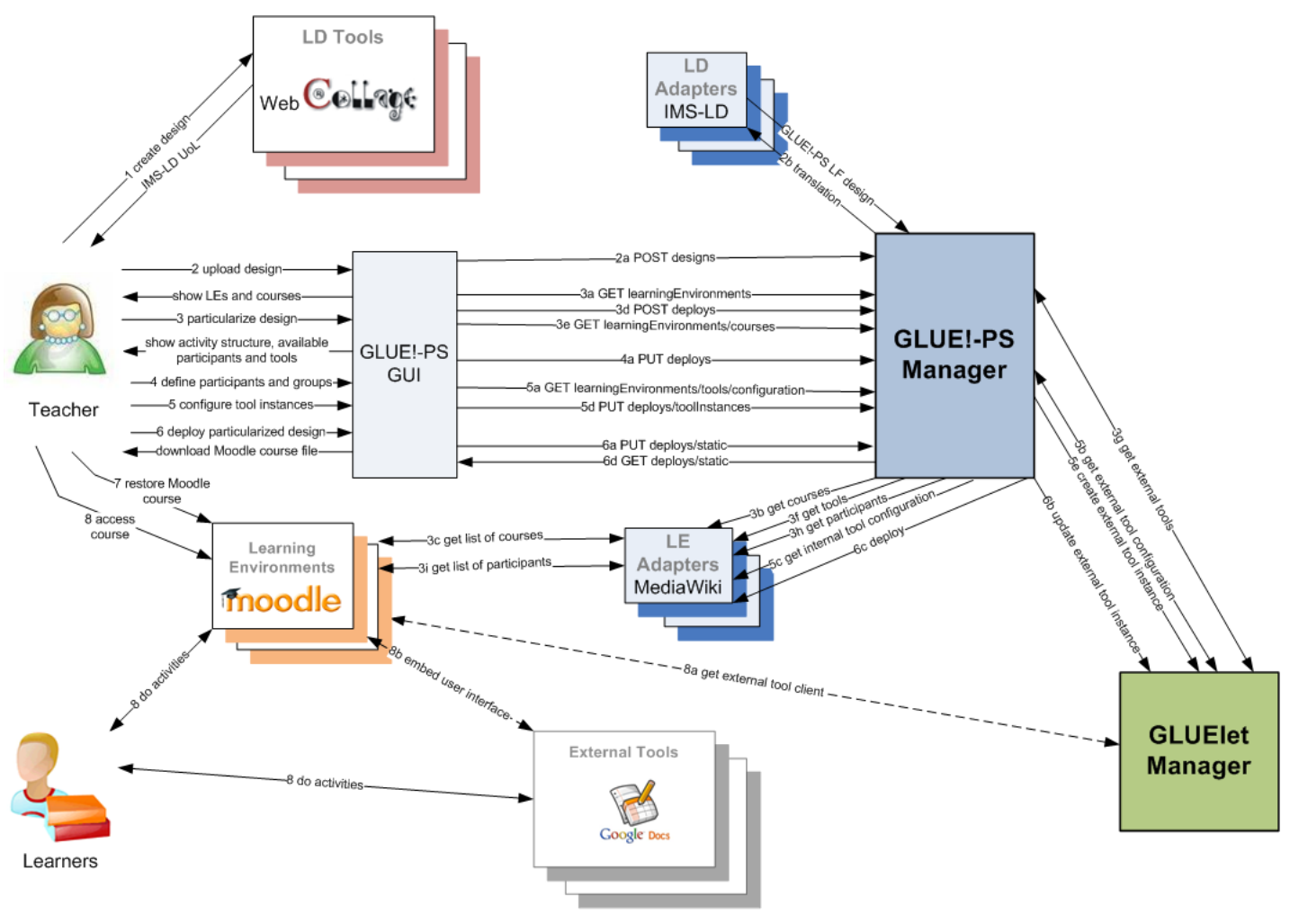

Note: internal interactions of the GLUE! architecture are not shown

Figure 5.10: GLUE!-PS block interactions diagram for Use Case Ib (static deployment of a IMS-LD design done with WebCollage, into a Moodle-based DLE)

presented with the activity, group and resources structure that she had configured in GLUE!-PS, she re-arranges the dyads in the first activity, as well as the resulting expert and jigsaw groups in the later phases. Once she has finished the rearrangement, she hits on the same "Deploy" button to make the changes be effective in the course's wiki-based platform. Then, she starts explaining the purpose and structure of the jigsaw scenario to the students...

... however, it seems that students are not understanding the expected outcomes of this first dyad phase. After a few confused questions by students, she decides to add a few examples of the work done by students in the previous academic years, as a source material for the dyad work activity. She accesses again the GLUE!-PS user interface, and creates several new resources linking to examples in the previous year's section of the wiki. Then, she assigns those resources to all groups in this first phase, and clicks the "Deploy" button again. Afterwards, she tells the students to refresh the activity wiki page, where the new resources are now available.

Hoping that nothing else will go wrong, Sara continues the lesson...

The aforementioned narrative depicts the typical workflow of a teacher modifying in runtime a particularized design that she had already deployed across a DLE. In this case, the LD authoring tools are not necessary, and the changes can be done directly through GLUE!-PS's user 
CAP. 5

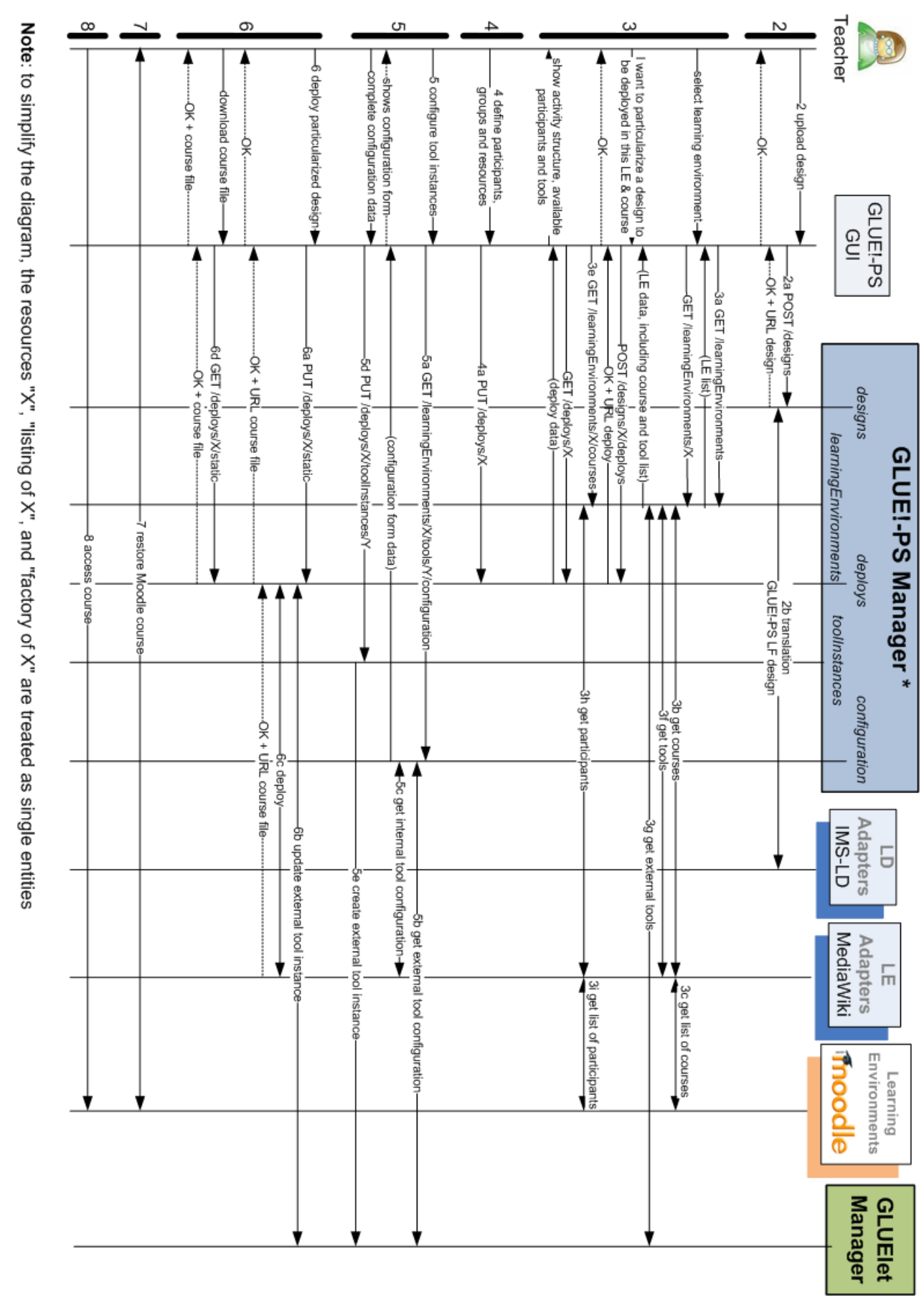

Figure 5.11: GLUE!-PS sequence diagram for Use Case Ib (static deployment of a IMS-LD design done with WebCollage, into a Moodle-based DLE) 
interface. The changes depicted in this use case are two of the most common ones, according to our observations: a re-structuring of the groups and a modification of the resources to be available in a certain activity. The workflow depicted in this narrative is also represented in Figure 5.12 .

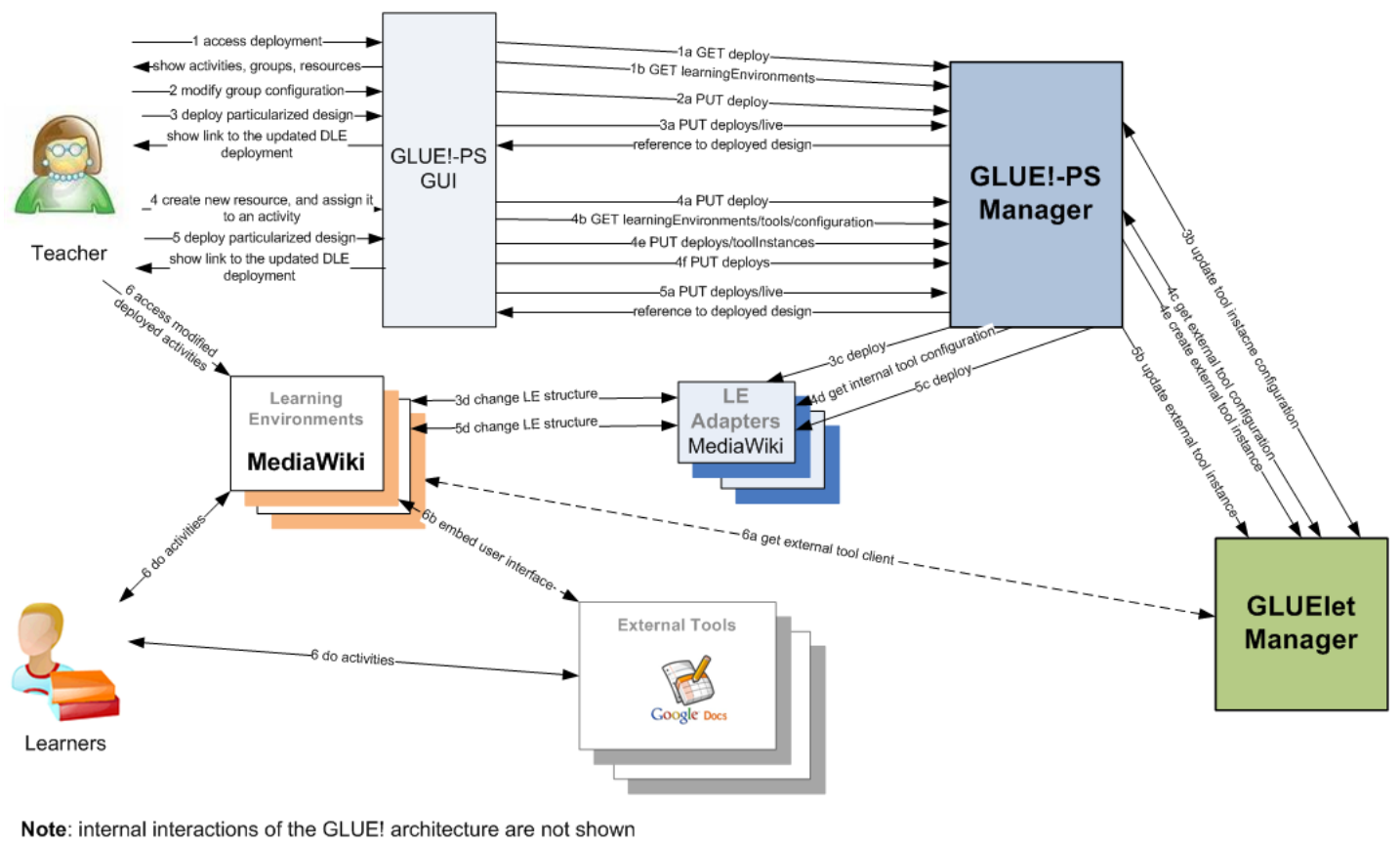

Figure 5.12: GLUE!-PS block interactions diagram for Use Case II (dynamic run-time modifications of a deployed design, across a MediaWiki-based DLE)

In that figure, again, we have chosen not to represent the interactions among the GLUE! architecture's internal elements, in order to make our diagram more readable (please refer to AH12a for a more detailed account of these interactions). As we can see in Figure 5.12, the external behavior that Sara experiences, in turn causes the following sequence of events between the different elements of the GLUE!-PS architecture:

1. The teacher accesses the particularized design that has already been deployed, using the GLUE!-PS GUI, which in turn asks the GLUE!-PS manager for the data representing such deployed design (1a). In the background, other requests are also performed to gather relevant pieces of information such as the (built-in and external) tools available in the DLE (1b).

2. In order to re-arrange the group structure of the activities, the teacher uses GLUE!-PS's user interface, which in turn generates one or more requests to the GLUE!-PS Manager, to update the group structure of the particularized design accordingly (2a). It is important to note that these changes are not automatically reflected in the DLE, to avoid overburdening the LE adapters and the learning platform itself in the case that the user makes mistakes or is simply "playing around" (since the deployment of a particularized learning design is often a costly operation). 
3. Once the necessary group rearrangements are done, the teacher re-deploys the newly particularized learning design, by a chain of requests similar to the ones in the deployment use case: from the GUI to the GLUE!-PS Manager (3a), from the GLUE!-PS Manager to both the GLUElet Manager (to update the access information of the tool instances concerned by the group changes) (3b) and to the LE adapter (to change the learning platform's structure to comply with the new groupings) (3c, 3d).

4. In a similar way, in order to change the resources to be used by one or more groups of participants in certain activities, the teacher uses the GLUE!-PS interface to create, change or delete the resources as she sees fit. This is done by the GUI invoking the GLUElet Manager, either to update the particularized design's specification to include the new resources (4a), and/or eventually to create (or delete) one or more built-in or external tool instances to be used by the different groups (e.g. through calls to the GLUElet Manager, in the case of external tools) (4b, 4c, 4d, $4 \mathrm{e})$. In any case, at the end of the operations, the GUI updates the model with the newly created tool instance information (4f).

5. Again, once the necessary resource rearrangements have been done, the teacher re-deploys the newly particularized learning design, by a chain of requests similar to the ones in the deployment use case: from the GUI to the GLUE!-PS Manager (5a), from the GLUE!-PS Manager to both the GLUElet Manager (to update the access information of the tool instances concerned by the changes) (5b) and to the LE adapter (to change the learning platform's structure to comply with the new configuration) $(5 \mathrm{c}, 5 \mathrm{~d})$.

6. Once the deploy is completed, the teacher and the students can access the newly deployed design using the learning platform's interface $(6 \mathrm{a}, 6 \mathrm{~b})$. This normally may require the refreshing of the browser window by the end-users, to acknowledge the new activity/resource/group structure.

Figure 5.13 represents the detailed sequence diagram with the invocations to the different services' resources in a typical REST services implementation of the scenario. Whenever possible, the same labels used in Figure 5.12 have been maintained. It is also important to note that the LE adapter is responsible for maintaining the coherence of the course and the user experience when this kind of run-time changes are performed (e.g. determining what happens to the data of deleted built-in tools, or when groupings change restricting access to previously allowed resources), to the extent that the LE adapter implementors consider adequate. This is done because the mechanisms for maintaining the coherence and the access to the activities and resources is highly dependent on each learning platform and the way it is implemented.

\section{Use Case IIb: run-time adaptation of a deployed design (static deployment)}

Translating the above Use Case II to the situation of run-time changes in a learning platform installation of the "static deployment" kind (e.g. Moodle version 1.9, where the deployment is done through a static file with a Moodle course backup), the use case would be mostly identical except, again, with regard of the means that are used to synchronize the state of the particularized design in the GLUE!-PS Manager with the structure of the course in the learning platform. Thus, the narratives and diagrams of Use Case IIb would be similar to those in Use Case II, with the following differences: 


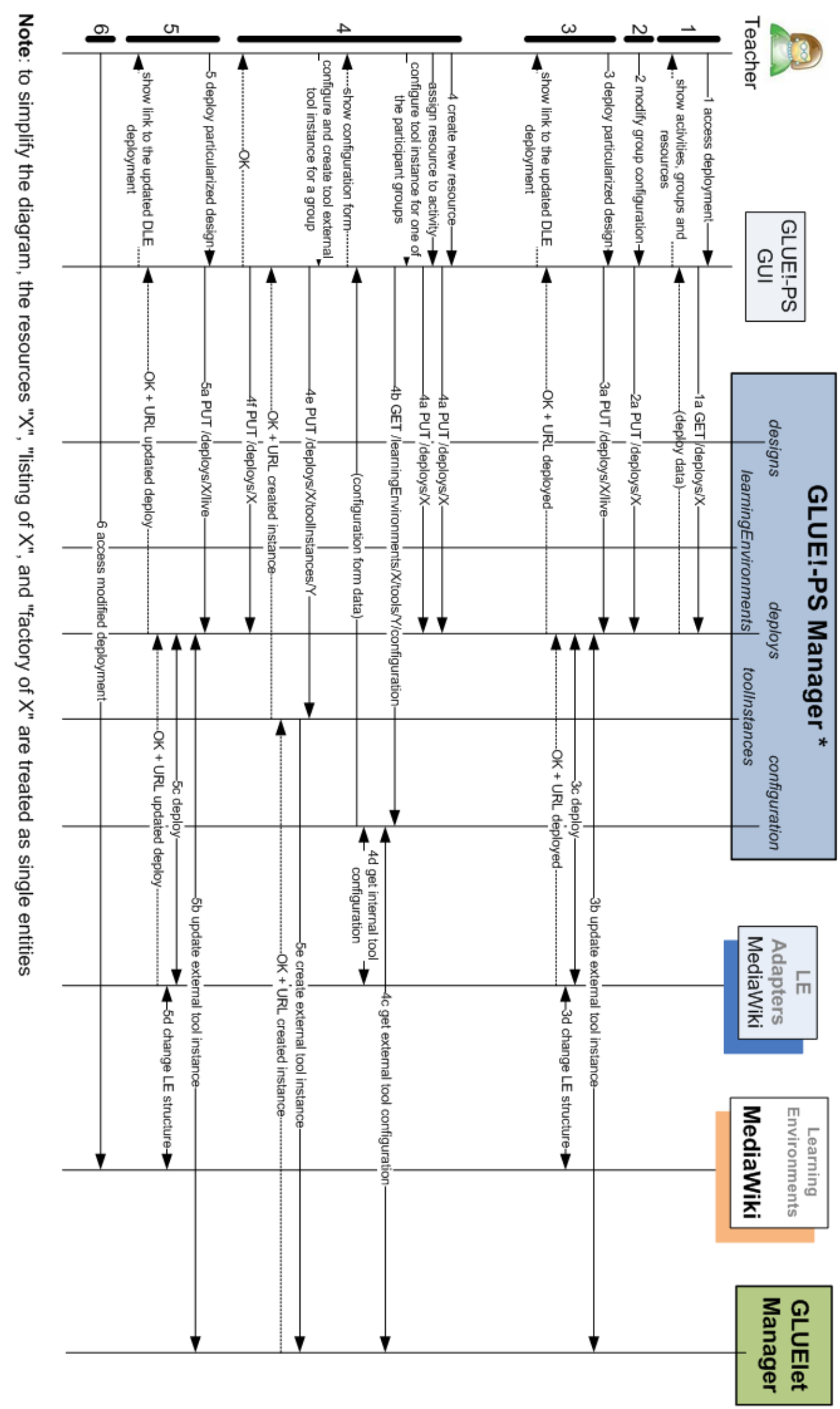

Figure 5.13: GLUE!-PS sequence diagram for Use Case II (dynamic run-time modifications of a deployed design, across a MediaWiki-based DLE) 
- In the narrative depiction of the use case, when Sara hits on the "Deploy" button, she is presented with a link which lets her download a zip file (with the updated Moodle backup course, for example) (5d). She would then have to access her Moodle course, and use the "Restore" option in Moodle (6, see Figure 5.14) to upload and restore such zip file representing the particularized design including the new participants, groups and (builtin and external) tools structure. Once this "pseudo-course" is restored, she can access the course normally in Moodle, accessing both internal and external tools from the VLE interface (7).

- In the structure diagram (see Figure 5.14), everything would be identical to Use Case II, except that in phase 5, the LE adapter would not directly communicate with the Moodle platform to modify the course's structure. Rather, a static file with the new deployable course structure would be generated, and in phase 6 the teacher would deploy the "pseudocourse" using Moodle's native "Restore" function. Afterwards, the access both for her and for the students would be similar as in Use Case II.

- Figure 5.15 depicts the complete sequence of invocations for this use case. The only differences, again, can be found in the interactions of phases 5 and 6 , which reflect the aforementioned different way of modifying the course's structure.

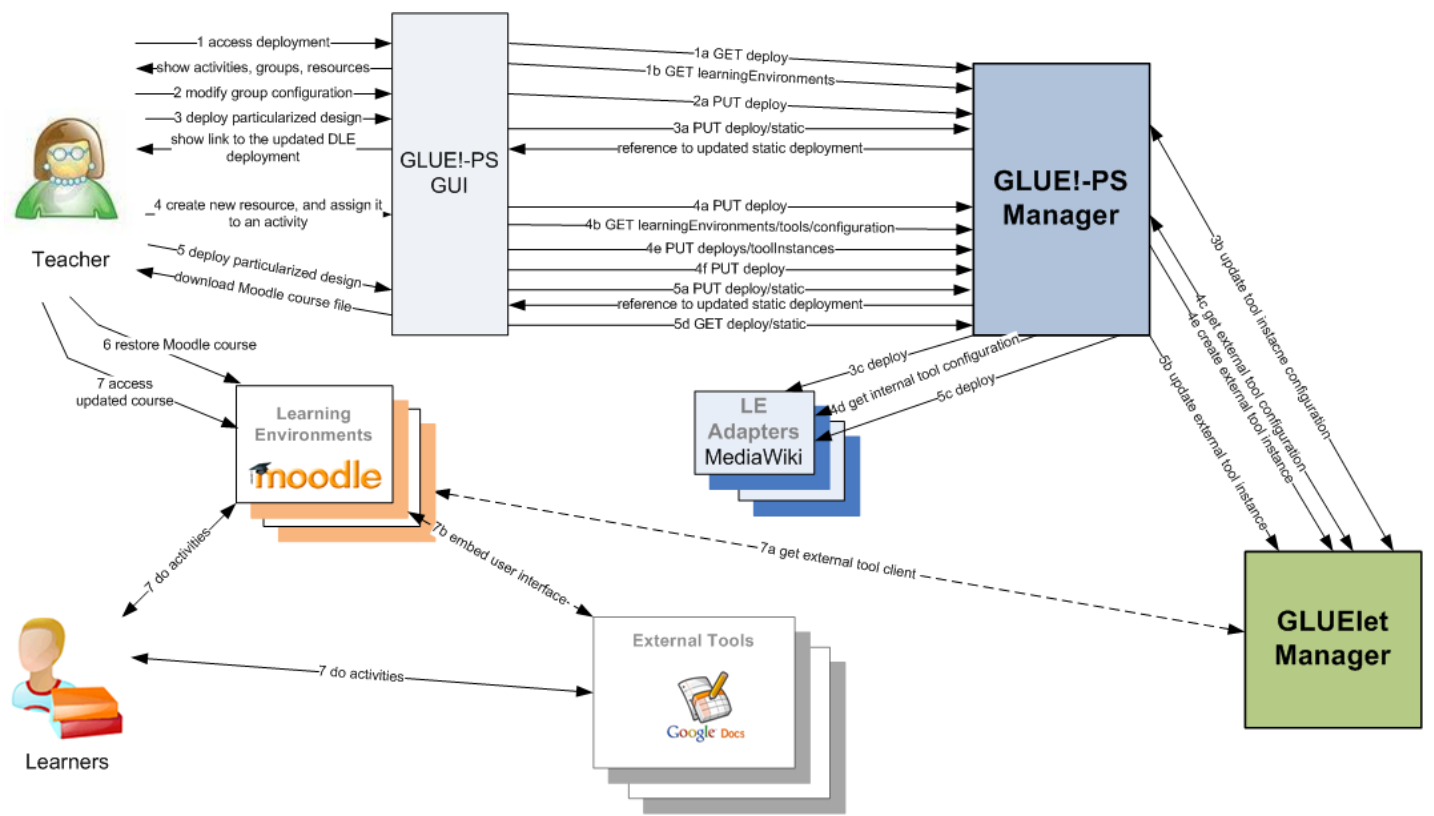

Note: internal interactions of the GLUE! architecture are not shown

Figure 5.14: GLUE!-PS block interactions diagram for Use Case IIb (static run-time modifications of a design deployed into a Moodle-based DLE)

There is an important caveat to be made in this case. Parallel to what was said in Use Case II, the responsibility of maintaining the course structure usable across these changes belongs to the LE adapter, and the mechanisms that the learning platform offers for making on-the-fly changes and maintaining the coherence of the user experience. For example, this is a current limitation of the Moodle 1.9 implementation of the (see Section 5.4), since the restoring of the 


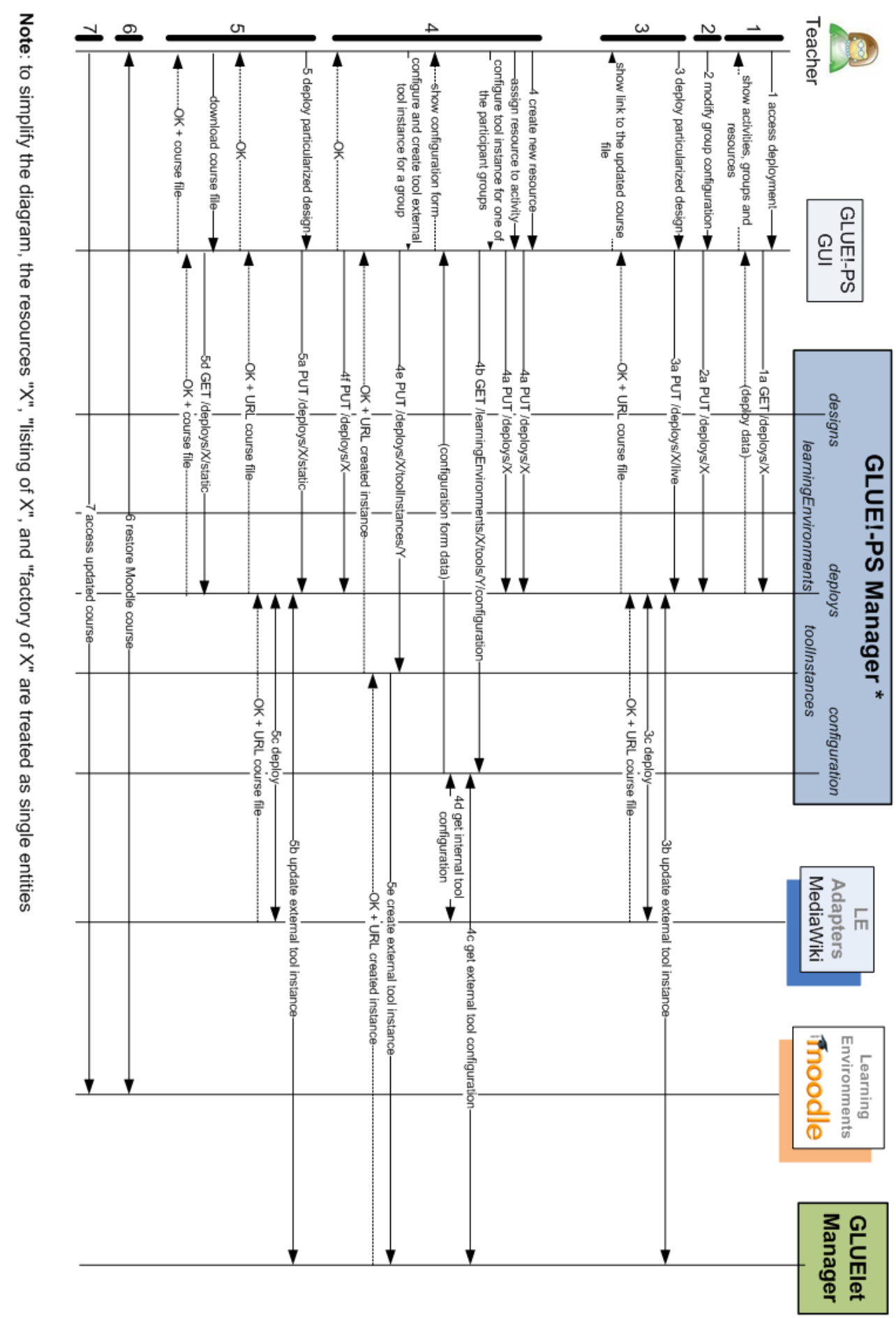

Figure 5.15: GLUE!-PS sequence diagram for Use Case IIb (static run-time modifications of a design deployed across a Moodle-based DLE) 
aforementioned "pseudo-course backups" over an existing course forces to either delete previous data (thus potentially destroying existing student and teacher information in the built-in tools) or to create new activities (thus creating duplicates of the existing activities).

\subsection{Analyzing GLUE!-PS as a tool for orchestration}

Despite the fact that this dissertation presents three main contributions (the ' $5+3$ Aspects' framework, the atomic patterns and the GLUE!-PS), which are to be understood mostly as three independent approaches to support different aspects of orchestration, there are different ways in which these contributions can inform each other. In this section we present two ways in which the contributions presented in Chapters 3 and 4 have informed the research on GLUE!-PS during the dissertation. More concretely, it presents analyses of the GLUE!-PS technological infrastructure support in terms of the conceptual framework presented in Chapter 3, and of the atomic patterns for orchestration (Chapter 4). Not only these analyses were useful for us in developing (Section 5.4) and evaluating (Section 5.5 the GLUE!-PS solution, but also they provide much-needed examples of how the previous contributions can be used as an "analytical lens" to conceptualize other research efforts on orchestration.

\subsubsection{GLUE!-PS within the '5+3 Aspects' conceptual framework for orches- tration}

As described in Section 3.2, the different TEL and CSCL literature sources that delve into the concept of 'orchestration', tackle very different aspects from one another, although there are a number of common trends and concepts that can be clustered into 8 dimensions, which we have denominated "aspects" of orchestration: Design, Management, Awareness, Adaptation, Role of actors (in the orchestration), Pragmatism, Theory and Synergy.

On the other hand, the GLUE!-PS infrastructure, as described in Section 5.2, has been posed as a technological tool to support orchestration of blended CSCL activities across Distributed Learning Environments (DLEs). Does this mean that it intends to solve all the orchestration problems and tackle all the orchestration aspects equally? Certainly not. In order to better understand the support for orchestration that GLUE!-PS provides, we have analyzed GLUE!-PS against the aforementioned 8 dimensions.

As we can see in Table 5.2, GLUE!-PS provides support primarily in four of the orchestration aspects: Design, Management, Adaptation and Pragmatism. The other four aspects are only barely considered in the orchestration support provided by GLUE!-PS, although other systems (or extensions of the GLUE!-PS architecture) could be enabled by GLUE!-PS to provide higher support for these aspects (e.g. a monitoring system that displays awareness information through the GLUE!-PS user interface).

As we will see when we evaluate the GLUE!-PS architecture and data model (Section 5.5), this classification of aspects could be used as a guidance in assessing to which extent this technological tool supports each of the different aspects, and what kind of support is provided for each of them (e.g. by gathering information related to each of those aspects). This kind of "orchestration aspect analysis", if used extensively by the TEL/CSCL community, could help 
Table 5.2: Analysis of the orchestration support provided by GLUE!-PS, according to the different aspects in the " $5+3$ " framework

\begin{tabular}{|c|c|c|}
\hline Orchestration aspect & $\begin{array}{l}\text { Amount of } \\
\text { support }\end{array}$ & Description of support \\
\hline Design & High & $\begin{array}{l}\text { GLUE!-PS enables teachers and other actors to transform a } \\
\text { wide range of learning designs into DLE-supported ICT in- } \\
\text { frastructures, thus greatly facilitating the preparation of the } \\
\text { learning scenarios. GLUE!-PS even could be used to modify } \\
\text { original learning designs, as a sort of "primitive LD authoring } \\
\text { tool". }\end{array}$ \\
\hline Management & Medium & $\begin{array}{l}\text { GLUE!-PS enables certain management operations on the ac- } \\
\text { tivities and resources across the DLE (management of groups, } \\
\text { management of resources, management of learning activities). } \\
\text { However, it defers responsibility of many management aspects } \\
\text { to the learning platform that acts as a center of the DLE (e.g. } \\
\text { enforcing an activity sequence, time limits, etc.) }\end{array}$ \\
\hline Adaptation & $\begin{array}{l}\text { Medium- } \\
\text { High }\end{array}$ & $\begin{array}{l}\text { GLUE!-PS makes it easier to adapt a learning design in run- } \\
\text { time, since it allows the changing of the particularized learn- } \\
\text { ing design and the application of those changes across the } \\
\text { distributed ICT infrastructure. }\end{array}$ \\
\hline Awareness & Low & $\begin{array}{l}\text { GLUE!-PS does not provide special awareness mechanisms, } \\
\text { either for teachers or among students (this aspect is also de- } \\
\text { ferred to the learning platform). GLUE!-PS does, however, } \\
\text { provide a graphical user interface that can be used as central } \\
\text { point of access to all the resources being used by the differ- } \\
\text { ent participants and groups, allowing for real-time (if a little } \\
\text { primitive) monitorization. }\end{array}$ \\
\hline Role of actors & Low & $\begin{array}{l}\text { GLUE!-PS does not provide special support for managing the } \\
\text { balance in the orchestration burden among the different possi- } \\
\text { ble actors (e.g. teachers, students). In fact, it tends to assume } \\
\text { a certain distribution of roles in which a teacher (or similar } \\
\text { actor) is the main manager of the activities. }\end{array}$ \\
\hline Pragmatism & High & $\begin{array}{l}\text { Due to its design principles of maximizing community accep- } \\
\text { tance, GLUE!-PS is intended to be a highly pragmatic support } \\
\text { for the orchestration, addressing current concerns regarding } \\
\text { existing ICT infrastructures, time constraints and needed ex- } \\
\text { pertise to perform orchestration. One of its main aims are to } \\
\text { make current orchestration practice of CSCL in DLEs, which } \\
\text { is unfeasible for most practitioners, available to the "average } \\
\text { teacher". }\end{array}$ \\
\hline Theory & Low & $\begin{array}{l}\text { GLUE!-PS does not provide special support in applying a cer- } \\
\text { tain theoretical, pedagogical or cognitive approach. It tends to } \\
\text { assume a certain pedagogical approach (that of CSCL), which } \\
\text { is facilitated explicitly (e.g. by allowing flexible group forma- } \\
\text { tion), but it does not preclude the use of other pedagogical } \\
\text { approaches. }\end{array}$ \\
\hline Synergy & Low & $\begin{array}{l}\text { GLUE!-PS does not provide especial support to better align } \\
\text { the different elements to be orchestrated, other than letting } \\
\text { practitioners choose among a wide variety of tools when partic- } \\
\text { ularizing the design, and transferring that design to the DLE, } \\
\text { offering integrated access to the DLE through the learning } \\
\text { platform (which could be thought of as a synergical element). }\end{array}$ \\
\hline
\end{tabular}


TEL/CSCL researchers to frame their respective efforts and compare them with each other, thus facilitating the understanding and communication of the currently heterogeneous research community that is working in this emergent research area.

We should note that this kind of analysis should not be taken in a quantitative manner (i.e. a tool that supports 4 aspects is better than one that supports only one). As noted by Tch11, there is a difference between "orchestration" (which tend to play the central role in the orchestration and often cover many of these aspects) and "orchestrable" technologies (which are easily managed by humans or other technologies, and often are more minimalist, covering fewer aspects). One category is not inherently better than the other, and we believe that the final test is whether practitioners are able (and willing) to integrate those technologies in their everyday life.

\subsubsection{Analyzing GLUE!-PS support for orchestration through atomic pat- terns}

Once the GLUE!-PS orchestration support had been characterized using the " $5+3$ Aspects" framework, it became obvious for us that the other conceptual tool of the dissertation (the atomic patterns for orchestration) could also be used in a similar way. Thus, we could analyze which ones of the 169 atomic patterns uncovered in primary and higher education were supported by GLUE!-PS, and in what form was that support provided. Moreover, we could also think about the implementation roadmap of orchestration tools such as GLUE!-PS in terms of atomic patterns, by determining which atomic patterns are most frequently used, and implementing first the GLUE!-PS functionalities that enable the support for such recurrent practices. This would also help in extending the initial support for orchestration for the 4 main target aspects described in the previous section, and even into supporting new aspects (represented by atomic patterns that have that have those new aspects as their primary characteristic - see Appendix B).

It should be noted that, due to the atomic patterns' heterogeneity (see Section 4.2), and the kind of orchestration support provided by GLUE!-PS, not all the uncovered atomic patterns can be expressed in terms of the GLUE!-PS architecture and data model (e.g. some atomic patterns, such as "Random evaluation of group work" describe purely social orchestration practices, where the technology has little or no role). Thus, from the original catalogue of 169 atomic patterns, 21 were selected for a deeper implementation analysis, according to the following criteria: a) to have at least one or two atomic patterns that represent each orchestration aspect; b) that ICTs have a direct role in the application of the atomic pattern; and c) that we have evidence (even if its preliminary) that the atomic pattern is frequently used or highly needed by practitioners. Once this basic set of atomic patterns was selected, we analyzed it from different perspectives.

Implementation advantages First, we compared how the atomic pattern is applied in current practice in blended CSCL using DLEs (i.e. without GLUE!-PS support), with the potential support that GLUE!-PS could provide to such atomic pattern, in order to assess the potential advantages of its implementation in GLUE!-PS (e.g. better efficiency, enabling previously impossible practice, not impeding current practice, etc.). In Table 5.3 we can see an excerpt of the 
analysis performed, for different atomic patterns. These atomic patterns were classified according to the general orchestration phase (design-time vs. deployment-time vs. enactment-time) in which they normally appear, and the primary orchestration aspect that they represent.

From this first step in our atomic pattern analysis, we could observe a number of trends:

- That GLUE!-PS seemed to enable very few new practices (represented by atomic patterns) that were previously impossible to apply in the current use of DLEs (without GLUE!-PS). This is to be expected since most of the atomic patterns describe current practice with DLEs. In fact, further observations of the orchestration of DLEs using GLUE!-PS would be necessary in order to elicit new atomic patterns (if any emerged), which could in turn be used in professional development actions to promote the use of GLUE!-PS as a tool to orchestrate CSCL in DLEs, as it was done in Chapter 4.

- That, for each atomic pattern, GLUE!-PS can provide different levels of support and automation. During this dissertation we have implemented the most basic support to many of these atomic patterns, but it should be noted that a system like GLUE!-PS has an important role in enabling further atomic pattern support by providing "orchestration hooks" that more complex systems (e.g. advanced monitoring systems such as the one proposed in RT12]) can use, via GLUE!-PS's information and management capabilities.

- In many cases, the implementation of the atomic pattern through GLUE!-PS would require changing current practices that are coordinated socially and dynamically, to a practice where all the elements (e.g. group formation, resource allocation) are formalized up front. There is a clear tradeoff between formalizing these learning design traits beforehand or not: often, GLUE!-PS support is more efficient (depending on participant and resource numbers), more clearly labeled, easier to reuse and provides more advanced functions (e.g. monitoring the students' work in real time)... but such support is also less flexible than the socially-coordinated version, and puts an arguably bigger workload and responsibility on teachers (when compared to a more de-centralized, socially-coordinated variant of the routine), even if GLUE!-PS helps in optimizing those chores (resource allocation, group formation and labelling, etc.). This could potentially make teachers prefer not to use GLUE!-PS, especially for face-to-face sessions (where flexibility and timeliness are paramount) and smaller groups of students (where manual management of groups and resources is more feasible).

- It is also interesting to compare GLUE!-PS operation with that of other existing approaches to DLEs (such as the ones presented in Section 2.4.3). The main difference is that in most other approaches the orchestration is done through direct operation in the learning platform and the web 2.0 tools (with more or less help, as in the case of using the GLUE! architecture), versus using a computer-interpretable design to define this orchestration and deploy it semi-automatically to the VLE. Here, the benefits of using GLUE!-PS are evident as long as the teacher is willing to define for herself the particularization data (concrete ICT tool instances, groups, participants) in advance, especially when a practitioner wants to reuse an existing learning design. 
Table 5.3: Atomic pattern support analysis for GLUE!-PS (excerpt). First stage: implementation advantages

\begin{tabular}{|c|c|c|c|}
\hline $\begin{array}{l}\text { Atomic pattern } \\
\text { [Phase] [Aspect] }\end{array}$ & Current practice with DLEs & GLUE!-PS implementation & $\begin{array}{l}\text { Implementation } \\
\text { advantage }\end{array}$ \\
\hline \begin{tabular}{lr} 
Assign & \multicolumn{2}{r}{ manage- } \\
ment & roles to \\
students $\quad[$ Depl $]$ \\
{$[$ Rol $]$}
\end{tabular} & $\begin{array}{l}\text { Currently this is done mostly socially, } \\
\text { as long as the access to resources does } \\
\text { not pose a problem to the manage- } \\
\text { ment by students (e.g. free access by } \\
\text { everyone in a wiki). Also can be done } \\
\text { directly in the VLE, if it allows to } \\
\text { set management roles to students (as } \\
\text { Moodle does) }\end{array}$ & $\begin{array}{l}\text { The teacher would select an activ- } \\
\text { ity and state that some students are } \\
\text { to act as a managers. Clone activi- } \\
\text { ties and (empty) groups are created } \\
\text { for the management task, and the } \\
\text { teacher will select who acts as man- } \\
\text { ager within each group, thus pop- } \\
\text { ulating this newly created manage- } \\
\text { ment activity. This routine can be } \\
\text { implemented in deploy-time (1) or in } \\
\text { enactment-time }(2)\end{array}$ & $\begin{array}{l}\text { More efficient / } \\
\text { Less flexible (if } \\
\text { compared with } \\
\text { the social man- } \\
\text { agement of the } \\
\text { artifact flow) }\end{array}$ \\
\hline $\begin{array}{l}\text { Monitoring the } \\
\text { task [Enact] [Awa] }\end{array}$ & $\begin{array}{l}\text { The teacher goes through all the par- } \\
\text { ticipant resources (if possible, e.g. re- } \\
\text { sources determined by the teacher- } \\
\text { otherwise, it is not possible). The } \\
\text { VLE serves as "hub" to access the re- } \\
\text { sources to monitor. If face-to-face ses- } \\
\text { sions, the teacher walks around and } \\
\text { does more "social" monitoring. }\end{array}$ & $\begin{array}{l}\text { GLUE!-PS enforces resource deter- } \\
\text { mination by the teacher beforehand. } \\
\text { GLUE!-PS currently enables auto- } \\
\text { matically the access of the teacher to } \\
\text { the resources, which may not be im- } \\
\text { mediate if the deployment was done } \\
\text { by hand (again, at the price of de- } \\
\text { termining resources beforehand). So, } \\
\text { it makes activities more easily trace- } \\
\text { able. It provides a unified entry point } \\
\text { for advanced monitoring systems. }\end{array}$ & $\begin{array}{l}\text { Does not im- } \\
\text { pede routine. } \\
\text { Slightly more effi- } \\
\text { cient in enabling } \\
\text { teacher access to } \\
\text { resources. Can } \\
\text { increase teacher } \\
\text { load. Enables fur- } \\
\text { ther monitoring } \\
\text { systems. }\end{array}$ \\
\hline $\begin{array}{l}\text { Reform groups in } \\
\text { face of the current } \\
\text { attendants [Enact] } \\
\text { [Adap] }\end{array}$ & $\begin{array}{l}\text { Currently this is done either by } \\
\text { changing the groups in the VLE man- } \\
\text { ually (which is often unfeasible), or in } \\
\text { a purely social manner if the names } \\
\text { do not appear explicitly in the VLE }\end{array}$ & $\begin{array}{l}\text { (1) by implementing successive de- } \\
\text { ployments and deploying each activ- } \\
\text { ity at the beginning of its enactment; } \\
\text { (2) by using GLUE!-PS as a "master" } \\
\text { to the DLE, reflecting any changes } \\
\text { immediately (requires some kind of } \\
\text { GLUE!-PS-LE synchronism) }\end{array}$ & $\begin{array}{l}\text { Depending on the } \\
\text { level of implemen- } \\
\text { tation and the } \\
\text { current practice } \\
\text { to be compared } \\
\text { with: May impede } \\
\text { practice (cannot } \\
\text { do it socially } \\
\text { anymore) / More } \\
\text { efficient (auto- } \\
\text { mates new group } \\
\text { creation/instance } \\
\text { association) }\end{array}$ \\
\hline $\begin{array}{lr}\begin{array}{l}\text { Students } \\
\text { termine }\end{array} & \text { de- } \\
\text { formation } & \text { [Enact }] \\
{[\text { Rol }]} & \end{array}$ & $\begin{array}{l}\text { This is often done socially. However, } \\
\text { complex group flows like jigsaw re- } \\
\text { quire some kind of algorithm or care- } \\
\text { ful explanation to be operative by } \\
\text { students (and the chances of error are } \\
\text { high anyway) }\end{array}$ & $\begin{array}{l}\text { This can be implemented by pro- } \\
\text { viding a student view of GLUE!-PS, } \\
\text { where teacher defines empty groups } \\
\text { and each student can choose which } \\
\text { group to join. }\end{array}$ & $\begin{array}{l}\text { Does not impede } \\
\text { routine / Slightly } \\
\text { more efficient (au- } \\
\text { tomates creation } \\
\text { of instances and } \\
\text { linking from the } \\
\text { VLE and group } \\
\text { assignment) }\end{array}$ \\
\hline $\begin{array}{l}\text { Successive deploy- } \\
\text { ment of activities } \\
\text { [Depl] [Adap] }\end{array}$ & $\begin{array}{l}\text { Currently this can be done directly } \\
\text { in the VLE, by deploying manually } \\
\text { the activities, one at a time (or de- } \\
\text { ploying all and hiding them until the } \\
\text { time comes) }\end{array}$ & $\begin{array}{l}\text { The teacher could select the activi- } \\
\text { ties one by one to be deployed on a } \\
\text { per-activity basis (including instance } \\
\text { creation and group assignment) }\end{array}$ & More efficient \\
\hline $\begin{array}{l}\text { Use results of a } \\
\text { task in a different } \\
\text { task / Reuse gen- } \\
\text { erated artifacts } \\
\text { [Depl] [Des] }\end{array}$ & $\begin{array}{l}\text { The teachers simply link in the later } \\
\text { activity the previous resources, or } \\
\text { just refer to them socially. }\end{array}$ & $\begin{array}{l}\text { It is done by telling the system to } \\
\text { reuse tool instances in later activities } \\
\text { (1). There is an alternative way to } \\
\text { implement this kind of artifact reuse } \\
\text { through tool instance reuse, which is } \\
\text { (2) "freezing" the outcome of the pre- } \\
\text { vious activity (e.g. convert a white- } \\
\text { board into jpg) and using it as an in- } \\
\text { put resource to the review activity, } \\
\text { making each available to the correct } \\
\text { participants }\end{array}$ & $\begin{array}{l}\text { Slightly more effi- } \\
\text { cient (provides a } \\
\text { GUI for determin- } \\
\text { ing reuse) }\end{array}$ \\
\hline
\end{tabular}


Technological and user-perspective issues In a second phase of our analysis, we delved deeper into some of the technological and user-perspective issues that emanate from the implementation of each of the atomic patterns described in the previous analysis. We can see an excerpt of the results in Table 5.4. This second stage in our analysis would help us in identifying potential challenges of implementing the different atomic patterns, and suggest modifications or extensions to the GLUE!-PS architecture and data model (e.g. to guide future implementations), as well as new opportunities for the use of the system in authentic settings.

From this second analysis stage, we can see that the implementation of the atomic patterns depicted above posed a series of architectural challenges to the GLUE!-PS system and data model:

- In general, supporting the 21 routines analyzed would require little or no architectural modifications to the GLUE!-PS system, at a high level (in fact, 13 of those routines were supported by design by the prototype implementation that was in place when the analysis was done). Leaving the necessary GUI changes aside, the main architectural tension was the requirements that those atomic pattern implementations would impose to the learning platform and/or the LE adapters (thus, varying the integration contracts defined in Section 5.2 . Those requirements could be grouped in two main areas:

- Many enactment-time atomic patterns, especially those pertaining to the Adaptation aspect, require GLUE!-PS to allow changes in the activities after the initial deployment. Although the data model itself does not preclude this, implementing such features would require the LE adapter to have ways of modifying the deployed activities in real-time. Two issues arose here: a) the fact that this in practice required the VLE to have an API for such modifications; and b) the problem of maintaining the coherence between the GLUE!-PS data model and the learning platform structures. The first requirement is quite strong, as not all VLEs provide APIs natively (or easy ways of developing them, see for example Moodle 1.9). The second requirement can be implemented in a variety of ways by LE adapters, depending on how complex it is in the learning to merge the real-time modifications with existing activities (sp. regarding activities that already have student-generated data associated to it).

- Making partial/successive deployments is itself a deployment-time atomic pattern that can also be used in the implementation of many of the aforementioned enactmenttime adaptations (as long as the activities to change have not yet been deployed), as a sort of "intermediate implementation" of the flexibility that is required of the GLUE!-PS system. The main requirement it imposes to the LE adapters is to provide ways to deploy single activities (as opposed to the full learning design). This, in practice, often requires the LE to have some kind of API for the creation of activities, groups and tools from the outside. It also imposes slight data model modifications in GLUE!-PS, to make activities (and instanced-activities) "deployable resources" on their own.

- Regarding the GLUE!-PS LF data model, the main modifications that the implementation of atomic patterns would require, fall into two areas:

- As we have seen, both the implementation of enactment-time changes and successive deployments needs the activities to be first-class, deployable entities in themselves. 
Table 5.4: Atomic pattern support analysis for GLUE!-PS (excerpt). Second stage: technological and user-perspective issues

\begin{tabular}{|c|c|c|}
\hline $\begin{array}{l}\text { Atomic pattern } \\
\text { [Phase] [Aspect] }\end{array}$ & Architecture and data model issues & User perspective issues \\
\hline $\begin{array}{l}\text { Assign } \text { management } \\
\text { roles to students [Depl] } \\
{[\text { Rol] }}\end{array}$ & $\begin{array}{l}\text { Its implementation would require a series } \\
\text { of GUI changes (intelligent creation of ac- } \\
\text { tivities and groups and resources according } \\
\text { to the user-introduced parameters), but LF } \\
\text { and the architecture support it by design } \\
\text { in the case of (1) [see same routine in Table } \\
5.3 \text {. For implementation of (2) [see same } \\
\text { routine in Table } 5.3 \text {, the aforementioned } \\
\text { LE-GLUE!-PS sync issues apply. This kind } \\
\text { of activity also raises the issue of differ- } \\
\text { ent roles performing the same activity (are } \\
\text { they the same activity or not? does the } \\
\text { group comprise the two participants, or is } \\
\text { it two one-person groups?), which was not } \\
\text { entirely clear when defining LF: currently, } \\
\text { they would probably look like two simulta- } \\
\text { neous (but different) activities. }\end{array}$ & $\begin{array}{l}\text { Again, implementing this in GLUE!-PS has } \\
\text { the advantage of automating to a cer- } \\
\text { tain extent the creation of new activities, } \\
\text { assigning resources (more efficiency), but } \\
\text { again this forces us to set the partici- } \\
\text { pants, groups and flow beforehand (and be- } \\
\text { ing less flexible in the face of enactment- } \\
\text { time changes, unless sync mechanisms are } \\
\text { implemented between GLUE!-PS and LE). } \\
\text { However, in many cases teachers will pre- } \\
\text { fer the flexibility of social coordination } \\
\text { of management (especially if the num- } \\
\text { ber of groups/participants is low, and the } \\
\text { VLE/resources allow for it, e.g. in and open } \\
\text { access kind of scenario) to this upfront or- } \\
\text { ganization }\end{array}$ \\
\hline $\begin{array}{l}\text { Monitoring the task [En- } \\
\text { act] [Awa] }\end{array}$ & $\begin{array}{l}\text { To do basic monitoring, no architec- } \\
\text { tural/LF modifications are needed. To do } \\
\text { more advanced monitoring, additions will } \\
\text { be needed to LF and GLUE!-PS architec- } \\
\text { ture (see e.g. } \text { RT12]) }\end{array}$ & $\begin{array}{l}\text { Forcing resource determination beforehand } \\
\text { enables more complex monitoring features, } \\
\text { but it is unflexible and increases teacher } \\
\text { load (many teachers prefer to let the stu- } \\
\text { dents choose and manage their resources). }\end{array}$ \\
\hline $\begin{array}{l}\text { Reform groups in face } \\
\text { of the current attendants } \\
\text { [Enact] [Adap] }\end{array}$ & $\begin{array}{l}\text { In (1) [see same routine in Table [5.3], it } \\
\text { requires additional flags in LF and meth- } \\
\text { ods in the GLUE!-PS and LE adapters } \\
\text { to do one-activity deploys (this is better } \\
\text { done if the VLE has an activity-creation } \\
\text { API, such as mediawiki). For (2) [see } \\
\text { same routine in Table 5.3], synchronism be- } \\
\text { tween the LE and GLUE!-PS is required, } \\
\text { which in turn requires VLE APIs for cre- } \\
\text { ating/reforming groups; also, ways for de- } \\
\text { tecting VLE changes in groups (triggers?) } \\
\text { would help in the case of teachers changing } \\
\text { groups directly in the VLE. }\end{array}$ & $\begin{array}{l}\text { Managing groups through the GLUE!-PS } \\
\text { can be seen as problematic, independently } \\
\text { of the level of implementation. In face- } \\
\text { to-face enactments, teachers may consider } \\
\text { more natural to drop back to social proto- } \\
\text { cols for grouping. However, using GLUE!- } \\
\text { PS might prevent this social protocol from } \\
\text { working correctly (e.g. students would not } \\
\text { have access to the new groups' instances). } \\
\text { Using open access policies to resources } \\
\text { (e.g. wiki-like - everybody sees everything) } \\
\text { might help in this kind of situation. }\end{array}$ \\
\hline 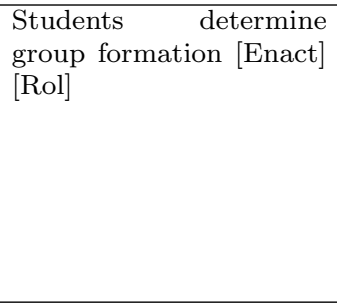 & $\begin{array}{l}\text { Big GUI changes would be needed, to have } \\
\text { this student view of GLUE!-PS, and to al- } \\
\text { low for the creation of "empty groups" with } \\
\text { their instances (ala LAMS?). The architec- } \\
\text { ture and LF support this by design. Also, } \\
\text { if we allow the choosing of groups after } \\
\text { deploy-time (which makes all the sense), } \\
\text { the aforementioned issue of GLUE!-PS-LE } \\
\text { syncing would apply. }\end{array}$ & $\begin{array}{l}\text { This kind of practice is quite common } \\
\text { (see post-workshop interviews) but cur- } \\
\text { rently not supported by Sofocles tools. I } \\
\text { think this is one of the most promising } \\
\text { paths of future research (co-orchestration } \\
\text { between students and teacher). }\end{array}$ \\
\hline $\begin{array}{l}\text { Successive deployment } \\
\text { of activities } \\
\text { [Adap] }\end{array}$ & $\begin{array}{l}\text { This would require extending LF to make } \\
\text { activities a deployable resource on its own } \\
\text { (basically, having a flag and location for de- } \\
\text { ployment), which would require extension } \\
\text { of the GLUE!-PS API and the LE adapter } \\
\text { functionality (apart from the GUI changes) }\end{array}$ & $\begin{array}{l}\text { Some teachers prefer to have the design de- } \\
\text { ployed completely at the beginning of the } \\
\text { experience, in order to explain it (but then, } \\
\text { they would probably not use this routine) }\end{array}$ \\
\hline $\begin{array}{l}\text { Use results of a task } \\
\text { in a different task / } \\
\text { Reuse generated arti- } \\
\text { facts [Depl] [Des] }\end{array}$ & $\begin{array}{l}\text { (1) [see same routine in Table [5.3] was } \\
\text { supported by GLUE!-PS / LF by design. } \\
\text { GLUE!-PS automates it to some extent, } \\
\text { but further automations could be thought } \\
\text { of (GUI issue, not architectural). Imple- } \\
\text { menting (2) [see same routine in Table } \\
5.3 \text {, which itself is another routine, would } \\
\text { pose an additional requirement to each tool } \\
\text { adapter (VLE internal or external), which } \\
\text { is the ability to invoke the "freeze" func- } \\
\text { tion (apart from creating, configuring and } \\
\text { deleting instances). }\end{array}$ & $\begin{array}{l}\text { Reusing instances has proved to be the sin- } \\
\text { gle most problematic feature of GLUE!-PS } \\
\text { from the GUI point of view. Even seasoned } \\
\text { CSCL teachers have problems (or just for- } \\
\text { get) with this instance reuse concept. Even } \\
\text { with further automations, the implemen- } \\
\text { tation of this routine is a problem (and } \\
\text { GLUE!-PS does not solve it fully) }\end{array}$ \\
\hline
\end{tabular}


This fact would require a small extension of the current GLUE!-PS LF model, in the concept of "activities" and "instanced-activities" (i.e. the activity performed by one concrete group).

- Another issue that emerged from atomic patterns such as "Assign moderators" or "Assign management roles to students", is the issue of whether different participants can perform the same activity under different roles (e.g. a group forum-based collaboration, in which one group member acts as moderator). In the current state of GLUE!-PS LF, an activity can have different roles, but there is no connection between those roles and the participants/groups performing the activity . Supporting this kind of routine would thus require a small extension of the "instanced-activity" entity, to assign group participants to different roles if needed. There is, however, the added problem of how this feature is supported in each learning platform and in the built-in and external tools, which is not straightforward. It could be argued that this is a relatively rare practice, which is probably best coordinated socially (rather than by implementing it and making the integration contracts more complex).

- There is mention in several routines to further implementation by more complex systems (e.g. for monitoring, or for intelligent/automatic adaptation). Implementing those systems would probably require entirely different architectures and data models, but GLUE!-PS and LF concepts could provide very valuable information and a unified point of entry for actions that those systems would require to be done across the DLE.

Also, the implementation through GLUE!-PS of these atomic patterns uncovered a series of user-perspective issues (including issues in usability, pedagogy and teacher/student practice). These issues can be grouped into 5 main areas:

- One of the key limitations of the current GLUE!-PS system is its limited ability to cope with enactment-time changes, which require the teacher to stop (even if only for some minutes) and change the the particularized design to address the emergent events (i.e. a certain loss of flexibility). In the face of this limitation, some teachers will probably prefer the flexibility of social coordination, unless there are huge efficiency gains (e.g. for large classrooms, high number of tool instances and complex groupings). Some of the underlying problems in providing this flexibility have been outlined above. While implementing realtime flexibility would overcome one of the most important barriers for adoption of GLUE!PS, we should be aware that implementing run-time changes can make the development of LE adapters more costly (i.e. it makes LE adapter contracts much more complex), and may not even be feasible for all learning platforms.

- Another key assumption that GLUE!-PS makes is that the teacher is willing to specify the particularization of the design (especially in the sense of creating groups, resources, etc) all by herself, and to do it before the enactment of the activities. This is however, not always the case (as it was observed in our authentic experiments and noted in several interviews with university teachers), which is an important limitation of the current GLUE!-PS system. Even if the aforementioned problems of synchronizing GLUE!-PS and learning platform structures in run-time are solved, the load that this imposes on the teacher could also hamper adoption of the system. However, there is nothing in the data 
model itself that enforces this approach and thus, having more distributed approaches to orchestration can be considered as one of the most interesting paths for future research.

Roadmap Taking into account the aforementioned analyses and the issues extracted from them, and the relative importance of the different atomic patterns (which can be elicited from observation or asking teachers directly - as we did in teacher workshops, see Section 4.5), we can then propose to follow a certain implementation roadmap, by ordering the atomic patterns to implement and clustering/abstracting which features need to be implemented to support them (e.g. allowing group formation from the GLUE!-PS GUI would be a necessary step for the implementation of more than one atomic pattern). As we can see in Table 5.5, 13 atomic patterns are supported by the prototype implementation of GLUE!-PS developed during this dissertation (see Section 5.4), and 5 have been selected for future implementation. Others (3) have not been selected for implementation at all, mostly because they are not recurrent enough.

Table 5.5: Atomic pattern-oriented roadmap for GLUE!-PS RI

\begin{tabular}{|l|l|l|}
\hline Implemented currently in GLUE!-PS & Future implementation & Disregarded \\
\hline RI & & \\
On -the- fly assessment (Monitoring + For- & Students determine group formation & Peer review \\
mative Assessment) & Backup ICT infrastructure \\
Reform groups in face of the current atten- & Assign management roles to students & Reciprocity \\
dants & & \\
Spontaneous use of additional ICT & Assign Moderator \\
Strategies to group formation / Form & Students choose their tools & \\
groups & & \\
Successive deployment of activities & & \\
Teacher as participant in student groups & & \\
Teacher chooses tools & & \\
Use Moodle to automate submissions & & \\
Use pre-existing groups & \\
Use results of a task in a different task / & & \\
Reuse generated artifacts & & \\
Use wiki to do collaborative writing & & \\
Use wiki to structure activities & & \\
\hline
\end{tabular}

\subsection{GLUE!-PS reference implementation (GLUE!-PS RI)}

In order to evaluate the usefulness of the GLUE!-PS proposal, and to facilitate the work of potential implementors of the architecture, a prototype of the architecture and data model has been implemented iteratively throughout the dissertation. This reference implementation of GLUE!-PS (GLUE!-PS RI from now on) includes a first implementation of the GLUE!-PS Manager service, as well as two minimal sets of LD and LE adapters, thus providing a usable implementation of the architecture and data model in a moderately-sized problem, available to everyone intended to study, develop, use or evaluate the architecture. Not only this implementation is advisable to show the feasibility of developing such architecture and to be used in our evaluations of GLUE!-PS's orchestration support for practitioners. Rather, this implementation can also be used as a the seed to start growing an ecosystem of new LD and LE adapters through the contribution of external developers who may share their implementations in order to increase the number of integrated systems, thus helping practitioners and their institutions 
in managing new flavors of Distributed Learning Environments without having to change their learning platform infrastructures.

It is important to note that this implementation is only one possibility, and that other implementations of each of the elements (GLUE!-PS Manager, LD and LE adapters) are feasible too, either by extending the functionality of the reference implementation presented here, or starting from scratch, using different technologies. Given the low amount of strictly mandatory requirements, and the different ways in which those requirements can be implemented for each LD authoring tool and learning platform (e.g. in the way of deploying the learning designs into platform structures), multiple variants of the architecture elements could exist, each one providing a slightly different set of functionalities (within the GLUE!-PS contracts), among which the practitioners can choose depending on their contextual restrictions.

As mentioned in 5.1.1, the overall methodology employed in developing this reference implementation was a lightweight version of the Unified Process Amb02, incrementally and iteratively developing new functionality into the prototype, both into the GLUE!-PS Manager and by developing new LD and LE adapters. This development was managed using a variant of the the Scrum framework for small teams [Kni06] [Ris00], since this development has been undertaken by a variable development team of $2-3$ people (including the author).

\subsubsection{Technologies involved}

The GLUE!-PS Manager in the GLUE!-PS RI has been designed and implemented as a REST web service, which exposes a set of "resources" through uniform interfaces, more concretely, HTTP operations such as GET, PUT, POST and DELETE [Fie99. This web service (and the rest of the architecture) has been implemented mostly using Java ${ }^{23}$. In order to develop this service, a RESTful web services framework for Java, called Restlet ${ }^{24}$ has been used Lou12]. Other Java libraries and frameworks used in the implementation of the GLUE!-PS Manager include: $\mathrm{JAXB}^{25}$ (to manipulate XML representations of the GLUE!-PS LF and to convert them to/from their object representations), GSON ${ }^{26}$ (to manipulate and convert between Java object representations of GLUE!-PS LF and its JSON 27 binding), JPA ${ }^{28}$ (to persist the service's resources into a relational database) or the Apache Commons ${ }^{29}$ set of libraries (to perform various file and input/output operations). The GLUE!-PS service also relies in a MySQL database management system 30 for persistence of the entities it manages.

On the other hand, the LD and LE adapters are currently implemented as Java libraries (not as separate services) that the GLUE!-PS Manager service invokes when needed. Thus, in its current implementation the communication between the central service and the adapters is done through standard Java calls that often encapsulate Java object representations of the service

\footnotetext{
${ }^{23}$ http://www.java.com/ (Last visit: 11 Jun 2012).

${ }^{24}$ http://www.restlet.org/ (Last visit: 11 Jun 2012).

${ }^{25}$ http://jaxb.java.net/(Last visit: 11 Jun 2012).

${ }^{26}$ http://code.google.com/p/google-gson/ (Last visit: 11 Jun 2012).

${ }^{27}$ JavaScript Object Notation, see http://www.json.org/ (Last visit: 11 June 2012).

${ }^{28}$ Java Persistence API, see http://jcp.org/aboutJava/communityprocess/final/jsr317/index.html (Last visit: 11 Jun 2012).

26 http://commons. apache .org/ (Last visit: 11 Jun 2012).

30 http://www .mysql.com/ (Last visit: 11 Jun 2012).
} 
resources following the GLUE!-PS LF model. This was a compromise solution for the initial implementation of the adapters, and their conversion to independent services to produce a truly distributed service-oriented architecture is currently being implemented.

The GLUE!-PS graphical user interface (GUI) in GLUE!-PS RI has been implemented using HTML, CSS and Javascript, but more specifically the Dojo toolkit ${ }^{31}$ for developing rich web applications, has been used. The communication between the GUI and the GLUE!-PS manager is mostly done through Ajax ${ }^{32}$ interactions that use either an XML binding of the GLUE!-PS LF data model, or a JSON binding of such data model.

However, it is important to note that all the aforementioned elements of the GLUE!-PS architecture could be implemented using other technologies and frameworks, as long as they all comply with the different contracts and behaviors described in Section 5.2.

\subsubsection{Evolution of the GLUE!-PS RI}

The development of the GLUE!-PS RI prototype has evolved incrementally from its original conception, adapting its roadmap to the feedback of the successive evaluation happenings and the needs of the following iteration's evaluations. Thus, we could divide the implementation of this prototype into roughly five phases (which match the 5 iterations of the engineering method described in Section 5.1.1). The main features of the GLUE!-PS RI along these phases is summarized in Table 5.6 .

Table 5.6: Summary of the roadmap followed in the implementation of the GLUE!-PS RI

\begin{tabular}{|l|llll|l|}
\hline Phase & $\begin{array}{l}\text { New LD adapter } \\
\text { features }\end{array}$ & New LE adapter features & $\begin{array}{l}\text { New GLUE!-PS core fea- } \\
\text { tures }\end{array}$ & $\begin{array}{l}\text { (approximate) } \\
\text { Release date }\end{array}$ \\
\hline \hline 1 & - & - & - & April 2011 \\
\hline 2 & IMS-LD adapter & $\begin{array}{l}\text { Moodle adapter v1 (deploy } \\
\text { only) }\end{array}$ & $\begin{array}{l}\text { GLUE!-PS Manager v1 (de- } \\
\text { ploy only), GLUE!-PS GUI v1 }\end{array}$ & October 2011 \\
\hline 3 & - & $\begin{array}{l}\text { MediaWiki adapter v1 (deploy } \\
\text { only) }\end{array}$ & February 2012 \\
\hline 4 & $\begin{array}{l}\text { Pattern } \\
\text { adapter }\end{array}$ & Collector & $\begin{array}{l}\text { MediaWiki adapter v2 (run- } \\
\text { time changes) }\end{array}$ & $\begin{array}{l}\text { GLUE!-PS Manager v2 (run- } \\
\text { time changes), GLUE!-PS GUI } \\
\text { v2 }\end{array}$ & April 2012 \\
\hline 5 & - & $\begin{array}{l}\text { Moodle adapter v2 (run-time } \\
\text { changes) }\end{array}$ & $\begin{array}{l}\text { GLUE!-PS Manager v3 (se- } \\
\text { lective deployment, additional } \\
\text { atomic pattern support) }\end{array}$ & June 2012 \\
\hline
\end{tabular}

\subsubsection{Current state of the GLUE!-PS RI}

In order to provide the reader with a rough idea of the functionalities and limitations of the GLUE!-PS RI prototype at the end of this dissertation, we provide in this section an overview of each of the implemented elements of the architecture. Currently, the GLUE!-PS RI provides an implementation of the GLUE!-PS Manager service and the GLUE!-PS GUI (end-user graphical interface), plus a minimal set of two LD authoring tool/language adapters (LD adapters, for the IMS-LD specification IMS03a and for the Pedagogical Pattern Collector authoring too 33

\footnotetext{
31 http://dojotoolkit.org/ (Last visit: 11 Jun 2012).

${ }^{32}$ Asynchronous JavaScript and XML, a group of interrelated web development techniques used on the clientside of web applications (i.e. the browser) to create asynchronous web applications Gar05.

${ }^{33}$ See Lau11, can be accessed at http://tinyurl.com/ppcollector3 (Last visit: 11 Jun 2012).
} 
and two learning platform adapters (or LE adapters, for Moodle and for the MediaWiki wiki engin ${ }^{34}$, implemented as Java libraries included with the GLUE!-PS Manager distribution. Moreover, due to its usage of the GLUE! architecture, a total of 9 kinds of external tools can be added to those learning platforms to form Distributed Learning Environments (DLEs): Google Docs (Documents, Spreadsheets and Presentations), MediaWiki, Dabbleboard, W3C widgets deployed in Apache Wookie servers, Doodle, Facebook Live Stream, Kaltura, Noteflight and a generic "URL representing a web content" AH12a.

The GLUE!-PS RI will be licensed under the GNU General Public License ${ }^{35}$ (GPL) for non-commercial uses. Therefore, the reference implementations of the available elements can be redistributed and modified under the terms established in the GPL license. Those interested in employing some of these elements for commercial purposes should contact the Intelligent \& Cooperative Systems Research Groun ${ }^{36}$ (GSIC), which holds the intellectual proprietary of the GLUE!-PS RI.

IMS-LD LD adapter The first LD authoring tool/language adapter to be developed for the GLUE!-PS architecture to be implemented was the one that adapts learning designs specified using the IMS-LD specification [IMS03a]. This was the most obvious choice of LD adapter, since IMS-LD is the most widely cited LD language or specification, and good number of existing authoring tools (CopperAuthor, Reload LD, ASK LDT, Collage, ReCourse, ... see Appendix C) support IMS-LD natively or, at least, can export their designs to such a data format. An example IMS-LD compliant authoring tool that is specialized in CSCL designs is WebCollag€37. whose user interface is shown in Figure 5.16.

The current implementation of the IMS-LD adapter uses the IMS-LD reference implementation (Coppercore ${ }^{38}$ to parse the IMS-LD learning design before translating it to GLUE!-PS LF concepts. The Coppercore source code (Coppercore is open source) was modified in order to access the IMS-LD items necessary for the translation and, thus, a version of such code is distributed as part of this LD adapter. IMS-LD defines several levels of compliance with the specification (A, B, or $\mathrm{C}$ ), depending mostly on the complexity and expressivity that the authoring or execution tool can handle. In this regard, due to GLUE!-PS LF's simplicity and (purposefully) limited expressivity, the implemented GLUE!-PS IMS-LD adapter provides only Level A compliance with the specification (e.g. it does not handle conditions and other advanced scripting concepts). This choice reflects mostly the current features of widespread learning platforms, which barely support all the concepts featured in Level A IMS-LD.

It is also important to note that this adapter also supports the instantiation (i.e. particularization) data format used by the WebCollage authoring tool. Albeit this format is not standardized in the IMS-LD specification, it builds on the data from the IMS-LD de-contextualized design format. WebCollage uses the notion of CLFPs (see Section 4.2 .3 ) to facilitate the particularization regarding groups and participants and thus, this information can be used by GLUE!-PS to provide an initial particularization of the learning design which the practitioner can then complete through the GLUE!-PS GUI.

\footnotetext{
${ }^{34}$ http://mediawiki.org (Last visit: 11 Jun 2012).

35 http://www.gnu.org/licenses/gpl.html (Last visit: 11 Jun 2012).

${ }^{36}$ http://gsic.uva.es

${ }^{37}$ http://pandora.tel.uva.es/wic2/ (Last visit: 7 Jun 2012).

38 http://coppercore.sourceforge.net/ (Last visit: 11 Jun 2012).
} 


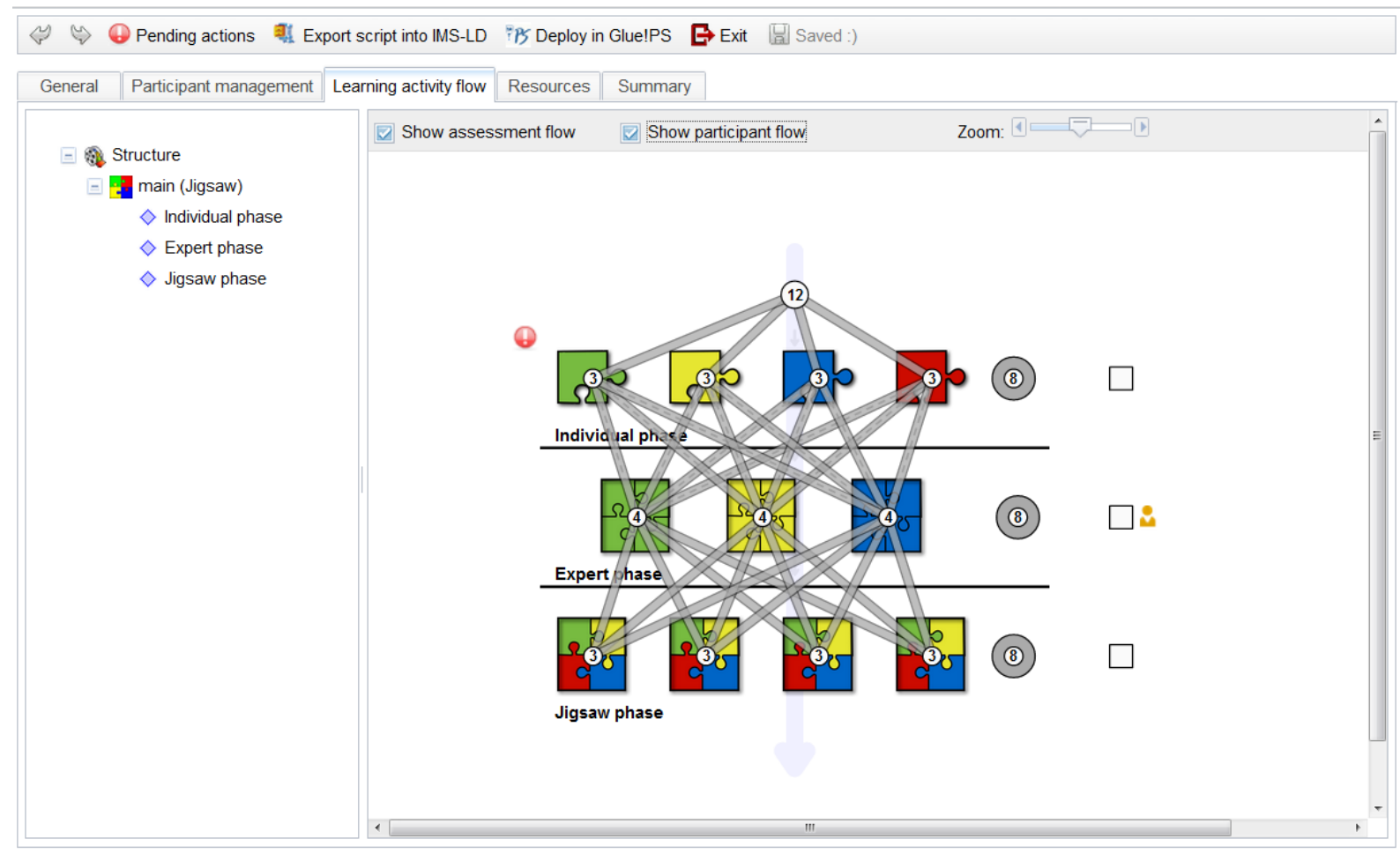

Figure 5.16: Screenshot of the WebCollage LD authoring tool, showing a learning design based on the Jigsaw pattern and an initial group particularization 


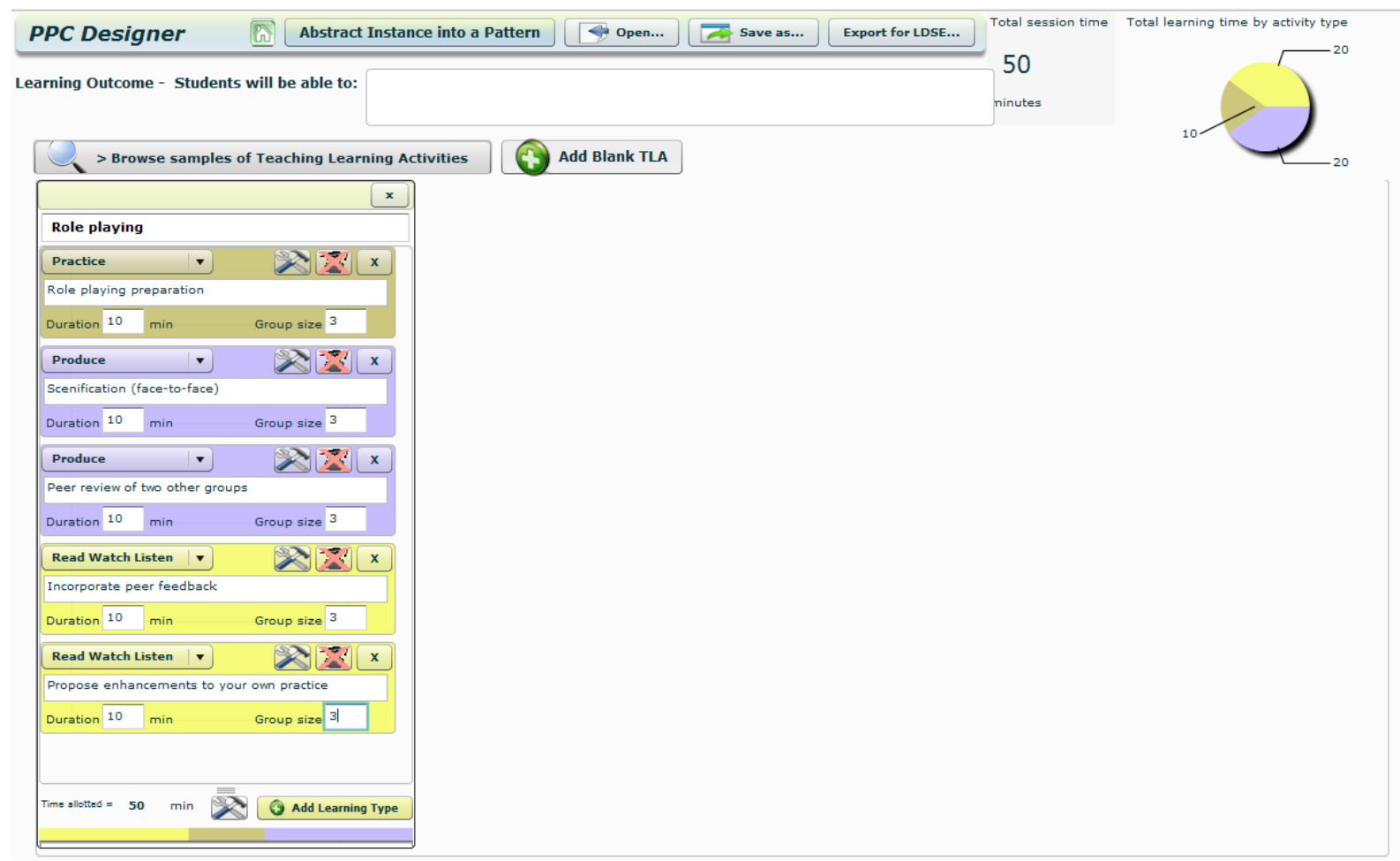

Figure 5.17: Screenshot of the Pedagogical Pattern Collector LD authoring tool, showing a simple role-playing collaborative scenario, taken from a (currently ongoing) authentic experiment

Pedagogical Pattern Collector LD adapter As a second example of LD authoring tool that has been integrated with the GLUE!-PS architecture, we chose the London Knowledge Lab's (LKL ${ }^{39}$ Pedagogical Pattern Collector (PPC) [Lau11. This simple LD authoring tool was developed for teachers, in order to help them in designing their lessons and, more importantly, help them in extracting design patterns from them so that they can be reused (thus, an effort related to our "atomic patterns" in Chapter 4). As a LD authoring tool, the PPC allows teachers to define learning activities at two different granularity levels, including information of the kind of activity that is performed, the group size and duration, etc. (see Figure 5.17). The main reason for choosing this authoring tool were the simplicity of its operation, designed to be used by teachers and which lends itself to fast specification of simple-to-medium complexity designs, which we believe is more adequate for our main target user (non-expert practitioners) than other more advanced LD tools.

The current implementation of the PPC adapter takes the XML representation of a learning design that PPC uses to save its design data (which follows a proprietary but simple format), and transforms it to a Java object representation of the learning design following the GLUE!-PS LF model. This can then be particularized through the GLUE!-PS GUI and deployed using the available LE adapters.

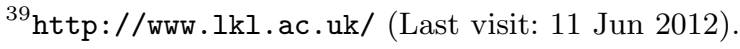




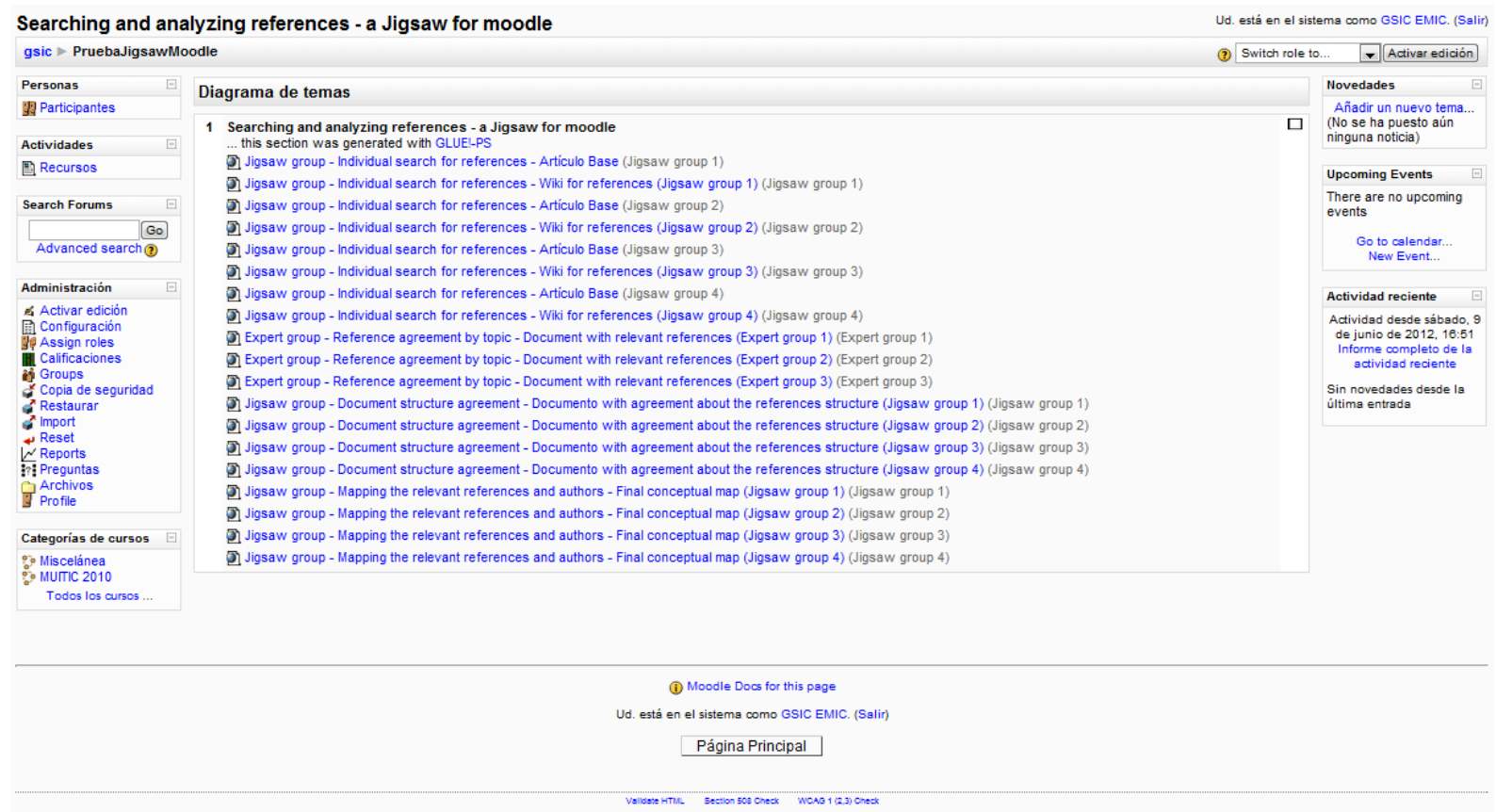

Figure 5.18: Screenshot of a Moodle VLE where a Jigsaw-based learning design (see Figure 5.16 has been deployed using GLUE!-PS

Moodle LE adapter The first and most obvious choice of a learning platform that would be useful to be integrated in GLUE!-PS is the Moodle VLE, which spans more than 65,000 installations and over 58 million users worldwide 40 , being by far the most widespread open-source VLE. Moodle provides an easy to understand user interface to manage courses, classrooms, groups and it offers a number of built-in learning tools (see Appendix C for more information on the Moodle VLE main concepts).

The current implementation of the Moodle LE adapter is developed as a Java library invoked from within the GLUE!-PS Manager. This implementation, which was developed for Moodle version 1.9, relies on what we have termed "static deployment" of learning designs. In practice, this means that the Moodle LE adapter transforms the particularized design data (in GLUE!-PS LF format) to a "Moodle pseudo-course" using Moodle 1.9's course backup format, which can then be restored into the Moodle installation (using Moodle's native user interface and functionality) to provide a course that reflects the particularized learning design (see, e.g., Figure 5.18). This method of deployment has the advantage of not requiring any modification to the target Moodle installation and thus, can interoperate as-is with any of the thousands of Moodle 1.9 installations available worldwide.

Albeit the deployment of the courses (i.e. the particularized designs) does not need any modifications to the Moodle installation, there is another aspect that does need the inclusion of an extension which is not available in Moodle by default. We have used a Moodle plug-in, called QAPI 1 , which is used to provide the GLUE!-PS Manager with the course, participant

\footnotetext{
${ }^{40}$ According to Moodle's own statistics, available from http://moodle.org/stats/ (Last visit: 11 Jun 2012 ).

${ }^{41}$ Standing for Quick API, see http://code.google.com/p/qapi/ (Last visit: 11 Jun 2012).
} 
and group information that the GLUE!-PS Manager needs to let the teacher particularize the learning design (e.g. to know which students are enrolled in the course where we want our design to be set) Thus, we could say that currently the Moodle LE adapter is distributed: part of it is executed within the GLUE!-PS Manager as a Java library, but also part of it is implemented through these QAPI calls, which basically are PHP code executed within the Moodle installation.

This LE adapter also supports the selective deployment of activities (i.e. the teacher can choose to deploy only the first activity of a design, or just the second and the third, etc.) in order to make the "Successive deployment" of a learning design (one of the Adaptation atomic patterns detected in Chapter 4 possible. However, as it was mentioned in Section 5.3.2, the main limitations of the current "static" implementation of the Moodle LE adapter concerns the adaptation in run-time of already-deployed learning designs: even if the re-deployment of the modified learning activities is technically possible, the way Moodle manages the restoration of backups leads to duplication of the activities when re-deploying. Also, this method poses problems in the preservation of student-generated data within Moodle's built-in tools (the redeployment of GLUE!-integrated external tools is safer in this regard, as long as the teacher does not delete the external tool instances through the GLUE!-PS GUI).

MediaWiki LE adapter In order to provide a second LE adapter that completed a multiLD, multi-LE implementation of the GLUE!-PS architecture, we chose to explore the frontiers of the learning platform landscape, by choosing a platform that does not strictly qualify as a VLE: wikis. MediaWik 43 is an open-source wiki engine, currently used to power large and wellknown wikis such as the Wikipedia 4 . This platform is also known to be used to support learning activities (see, for example, JA07] [MM08]) where MediaWiki's open access to information and capabilities for collaborative asynchronous writing and general accumulation of knowledge are valuable. MediaWiki, however, was not intended originally as a learning platform, and thus it does not include several typical VLE concepts such as courses, activities, tools, or groups practitioners that use wikis for learning often mimic those concepts by using wiki pages (which are basic HTML pages, flexible enough to represent any of those concepts).

Given the fact that MediaWiki provides an API to create wiki pages remotely, the current implementation of the MediaWiki LE adapter provides "dynamic deployment" of activities (i.e. automatic, run-time deployment at the end user's request). Thus, when the particularized design is complete, this LE adapter is invoked, which in turn uses this API to create a structure of linked wiki pages (see Figure 5.19 that represent the overall design, each (abstract) activity, the activity of each of the involved groups of participants, as well as the built-in (i.e. wiki pages) and external tools (embedded in wiki pages thanks to the GLUE! architecture). Albeit we have developed five different implementations of this adapter, to be used in the different evaluation experiences, these implementations mostly differ in aesthetic aspects of how the pages (e.g. with activity information) are presented. In fact, all 5 implementations provide "open access" to all participants (in the sense that any student can navigate to any wiki page, following MediaWiki's default access policy), which was a feature that the teachers using it expected. Other implementations with more closed policies would also be possible, although

\footnotetext{
${ }^{42}$ This kind of functionality is now natively supported by Moodle version 2 .

43 http://mediawiki.org (Last visit: 11 Jun 2012).

${ }^{44}$ http://wikipedia.org (Last visit: 11 Jun 2012).
} 


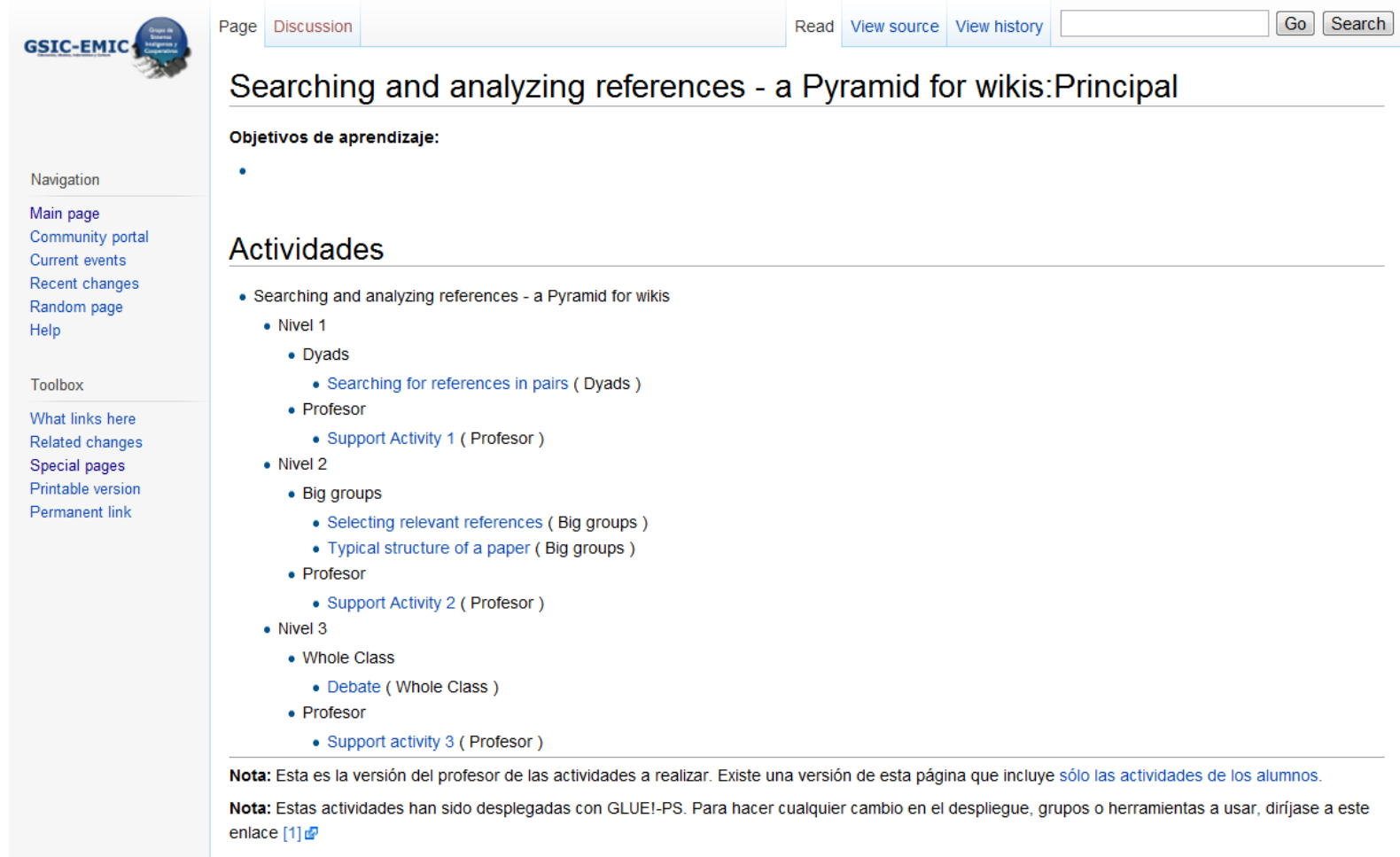

Figure 5.19: Screenshot of a MediaWiki installation where a collaborative learning design has been deployed using GLUE!-PS

MediaWiki's access control capabilities are rather limited. This LE adapter also supports the live re-deployment of activities (i.e. run-time modifications of the deployed designs), with the only caveat that manual modifications to the activity pages will be overwritten (this normally does not imply a loss of student data since these activity pages can only be written to by the teacher).

The main restriction of the current implementation on the MediaWiki installations so that they can be used as target learning platforms for GLUE!-PS is that GLUE!'s LE adapter (which takes a form of a standard MediaWiki extension) is installed in the platform, in order to embed the external tools within wiki pages.

GLUE!-PS Manager The GLUE!-PS RI also incorporates a first implementation of the GLUE!-PS Manager service, which allow teachers to upload designs in the two aforementioned LD formats (IMS-LD and PPC), particularize them, deploy them to the two different kinds of DLEs currently supported (Moodle-based and MediaWiki-based, through the use of the GLUE! architecture), and to adapt them in run-time. This service is implemented in Java using the aforementioned Restlet framework.

The GLUE!-PS Manager currently implements its functionalities as "resources", which include the serving of the static files of the GLUE!-PS GUI (see below) to the end users' web browsers, as well as the REST operations detailed in Table 5.1. The GLUE!-PS persistent data 


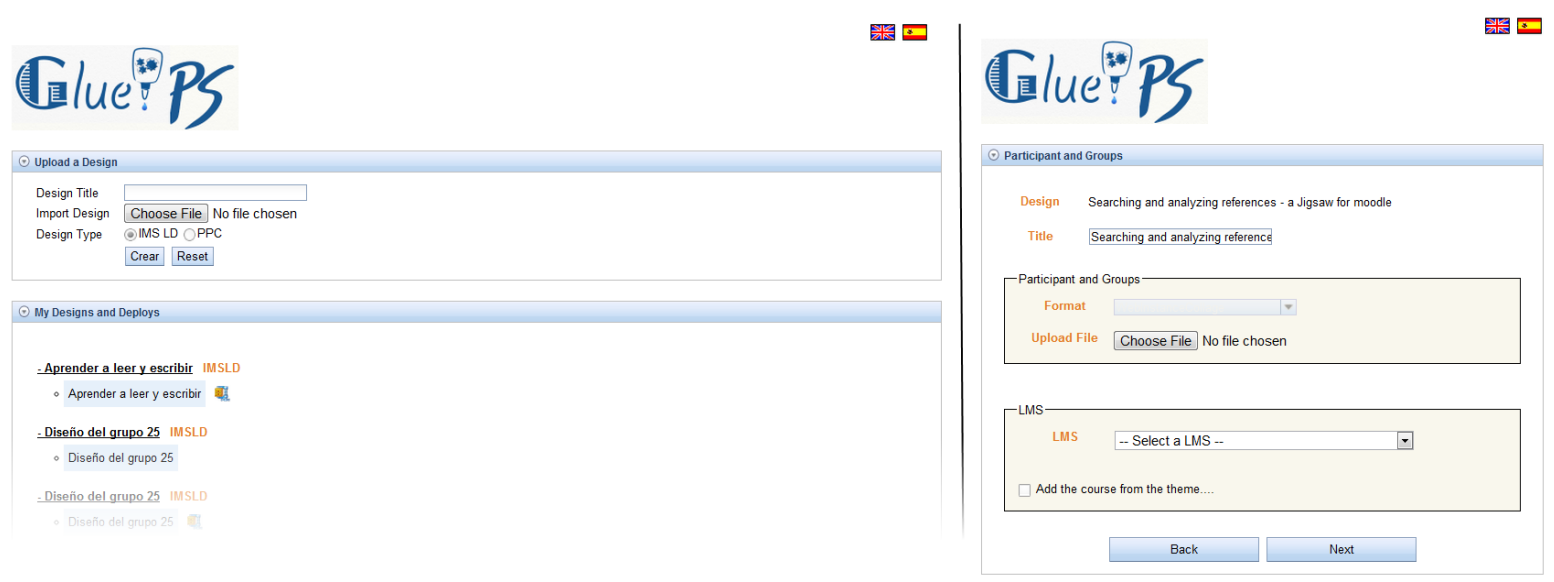

Figure 5.20: Screenshot of the GLUE!-PS RI user interface, showing the initial page to upload new designs (left) and the page to create a new particularization of a design (right)

(stored in a MySQL database) includes basically GLUE!-PS users data, the (de-contextualized) learning designs uploaded by users (in GLUE!-PS LF format), the particularizations done to those designs (also in GLUE!-PS LF format), as well as the information about the different learning environments (i.e. learning platform installations) available for deployment and management. Please refer to Appendix $\mathrm{D}$ for further information about the data model underneath this implementation.

GLUE!-PS GUI Finally, the GLUE!-PS RI also includes an implementation of a rich web user interface, developed using Dojo, which lets users interact with the GLUE!-PS Manager resources, thus manipulating designs, particularizing them, deploying them across the different DLEs and, eventually, adapting them in run-time or deleting them when no longer needed. Examples of the current GLUE!-PS user interface are provided in Figures 5.20 and 5.21 .

Roughly speaking, apart from the initial screens that let the users upload designs and create new particularizations of them for different target learning platforms (including GLUE!integrated tools), the main user interface screen (Figure 5.21) provides a graphical view of the learning design activities (on the left side), including the different groups of participants that are to enact each activity, and the particular resources that each of those groups will use (using treelike representations). The right side of the screen provides information and controls to manipulate resources (top part) and groups of participants (lower part) in more detail. When finished, the user clicks a button to deploy the particularized learning design to the DLE. A running prototype of this implementation in currently running at http://pandora.tel.uva.es/GLUEPSManager/ gui/glueps/ (Last visit: 21 Jun 2012). Credentials and basic user documentation (e.g. in the form of worksheets such as those used in our evaluations, see the following Section) are available upon request. 


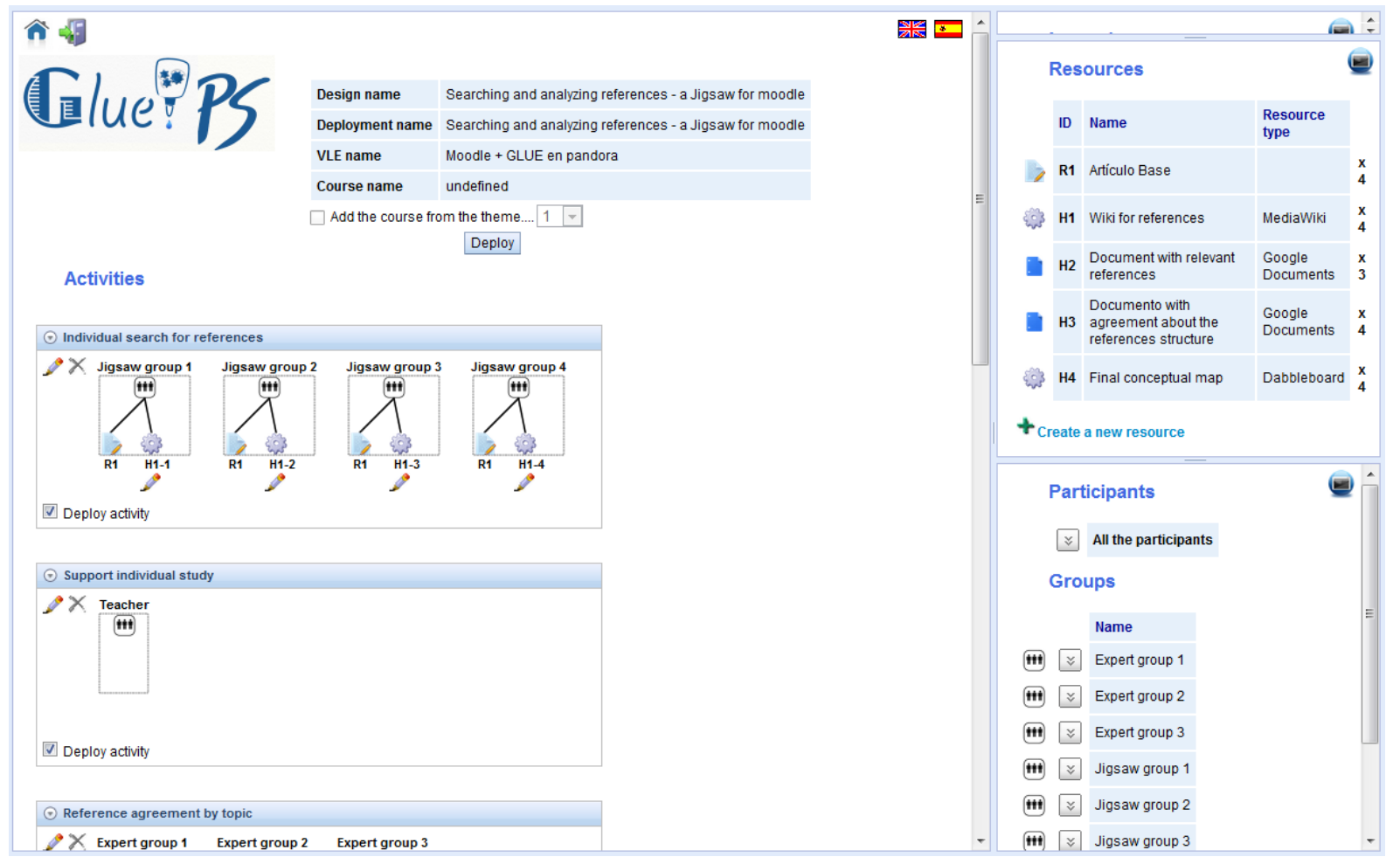

Figure 5.21: Screenshot of the GLUE!-PS RI user interface, showing the main interface for the particularization of learning designs 


\subsection{Evaluating GLUE!-PS}

As it has been described in the introductory chapter of this dissertation, and at the beginning of this chapter (Section 5.1), we have followed the engineering method Adr93 to structure our research towards providing a technological tool to support teachers in the orchestration of blended CSCL scenarios across Distributed Learning Environments. Throughout this chapter we have proposed the GLUE!-PS architecture and data model as a technological infrastructure for such orchestration, we have analyzed its properties according to the previous dissertation contributions, and we have described the development of a prototype implementation. However, it is in the evaluation phase of each iteration where the trustworthiness of this proposal should be demonstrated.

In the rest of this section we describe our iterative evaluation of GLUE!-PS through multiple different techniques, including an analytical evaluation, three teacher workshops and four authentic experiments, developed (except the analytical evaluation) in the context of higher education, as part of the University of Valladolid's activities, either in professional development activities, research project meetings and in undergraduate- and master-level courses. These "happenings" provided evidence for the iterative evaluation of GLUE!-PS, exploring the value of this contribution with regard to the following research question (also mentioned in Section 5.1):

RQ3.1 Can we provide technological tools to deploy and flexibly manage in run-time blended CSCL activities across DLEs?

In order to provide insights into this question, this Section is structured as follows: first, we outline the general context and method of the evaluation (5.5.1), and then we describe each of the four evaluation iterations, outlining their peculiarities, the findings and selected supporting evidence, closing with remarks about how those findings influenced the following iterations (in Sections 5.5.2, 5.5.3, 5.5.4, and 5.5.5, respectively). A global wrapping-up and discussion of the evaluation closes this Section 5.5 .6 .

\subsubsection{Context and method of the evaluation}

As outlined in Section 1.2, we have used the CSCL-EREM framework [JA09b] to design the evaluation phases across the four iterations conducted so far in the research on GLUE!PS. Following the framework's emphasis on responsiveness, the evaluation presented here has evolved, progressively focusing [Sta10 on emergent issues and topics uncovered in previous iterations. Also, it should be noted that this evaluation evolved together with the research on the other thesis contributions, presented in the previous Chapters. Especially important is the influence of the conceptual framework for orchestration presented in Chapter 3 : the " $5+3$ Aspects" framework has been used, not only to analyze theoretically the orchestration support provided by GLUE!-PS (Section 5.3.1), but it has also been used to focus and organize the empirical evidence gathered during the evaluations.

Thus, even if our evaluations have been constantly trying to explore the same evaluative tension (or Issue, in CSCL-EREM terminology): "Does GLUE!-PS support teachers in the orchestration of blended CSCL across DLEs?", each iteration focused on a different set of topics, 
matching one or more of the four main orchestration aspects supported by GLUE!-PS (see Section 5.3.1 opportunistically, depending on the kinds of data available and the affordances of the GLUE!-PS prototype to explore each of those aspects. These topics can be exemplified by the following questions:

T1 (deployment) Is GLUE!-PS able to deploy designs to DLEs preserving the design's essential qualities? (representing the Design aspect of orchestration)

T2 (time efficiency) Is GLUE!-PS time efficient (from the point of view of the teacher)? (which is one of the defining Management tensions when we look at the orchestration of an scenario)

T3 (real practice use) Would teachers use GLUE!-PS in their everyday practice? (which represents the Pragmatism aspect of complying with the multiple restrictions of real practice in authentic settings)

T4 (run-time changes) Is GLUE!-PS able to support useful runtime changes to a deployed design? (clearly linking to the Adaptation aspect of orchestration)

Each of those topics is in turn informed by several informative questions that try to probe for information. The other orchestration aspects, which GLUE!-PS does not support strongly, were considered our evaluation's "invariants". This conceptual organization of the data from the evaluation, adapted from Huberman \& Miles's anticipated data reduction procedure (typical in qualitative data analysis (Hub94]) is graphically represented in Figure 5.22"

The aforementioned evolution of the available means for evaluation (in the form of prototypes or the opportunity for an evaluation happening) prompted us, first of all, to study GLUE!-PS's ability to deploy learning designs (T1) analytically, when no prototype was yet available (Iteration \#1). Then, after the first prototype was developed, we tried to explore not only this deploying ability (T1), but also gather first evidences about its time-efficiency in doing so (T2) and the potential usefulness that teachers saw in using GLUE!-PS in their everyday practice (T3); to do this we did some first pilot trials and we tested the GLUE!-PS prototype in two teacher workshops, both with non-expert and with more experienced teachers/researchers (Iteration \#2). Afterwards, in order to obtain evidence that explored the aforementioned topics (T1, T2, T3) in an environment closer to real teaching practice, a first authentic experiment of the use of GLUE!-PS in a real course was done by an experienced CSCL teacher/researcher (Iteration \#3). Once the GLUE!-PS prototype was able to provide certain adaptation capabilities, we expanded our focus to explore also the usefulness of the run-time change features of GLUE!-PS (T4), and we evaluated the prototype through two more authentic experiments in real university courses by teacher/researchers, plus an additional teacher workshop for non-expert teachers from multiple disciplines (Iteration \#4). Finally, the GLUE!-PS prototype in its state at the end of the dissertation was evaluated through an authentic experiment in an undergraduate-level course, to provide further evidence around the four topics of interest (Iteration \#5).

\footnotetext{
${ }^{45}$ It is worth noting that this kind of " $5+3$ Aspects"-oriented focusing of the evaluation was possible for the GLUE!-PS contribution since the framework existed already when the first GLUE!-PS evaluations were taking place. We chose not to re-focus our evaluation of atomic patterns (Section 4.5) because of the large quantities of evaluation data and reports that had already been produced for that contribution.
} 


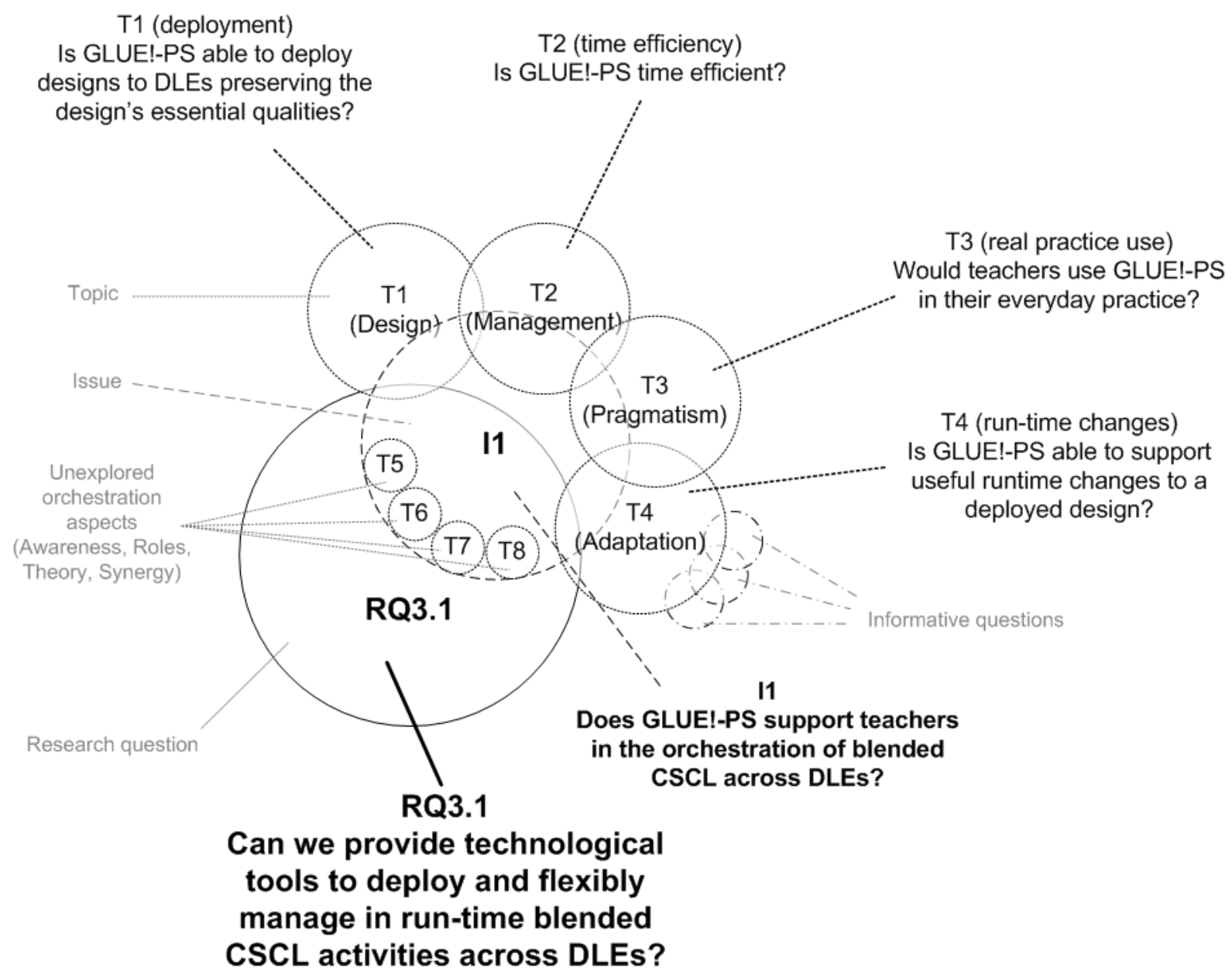

Figure 5.22: Graphical representation of the research questions, issues, topics and informative questions used during the evaluation of GLUE!-PS 
As we have mentioned, we chose to perform different kinds of data gathering events in order to evaluate our proposal, with a preference of authentic experiments (i.e. during the orchestration of a real course, where the GLUE!-PS orchestrated activities are part of the course's curriculum) and teacher workshops (especially professional development ones where participants intend to acquire new skills - not just provide data to researchers). As in the case of the evaluation of atomic patterns, this choice of "situated experiments" was motivated by the emphasis of orchestration research in the needs and constraints of average (i.e. non-expert) teachers in authentic settings Dil10. Given that the GLUE!-PS prototype was in an experimental stage (reliability-wise) throughout most of the dissertation's timespan, we decided to combine the usage of GLUE!-PS in authentic settings by expert teachers (or teachers who had a certain expertise on CSCL practice), with the usage by non-expert practitioners in close-to-real situations (practical workshops). This combination allowed us to have evidences from real situations, and at the same time reach a wide range and number of teachers that could provide feedback on the use of the technology. Moreover, these PD workshops can be very powerful instruments in contacting non-expert teachers that could eventually agree to participate in more detailed authentic experiments - indeed, several workshop participants already showed interest in using GLUE!-PS in their classrooms.

With all of the above in mind, we can represent our evaluation design for the GLUE!-PS contribution through the diagrammatic view provided by the CSCL-EREM framework (Figure 5.23 . As we can see, our Evaluand in this case is the GLUE!-PS system, as a tool to support orchestration of blended CSCL scenarios across DLEs. Our Perspective as evaluators can be described through our Goal, which is to support teachers in orchestrating learning activities, through the use of the GLUE!-PS infrastructure. The main Evaluator was the author of this dissertation, although at different points of the evaluation, he was supported by a multi-disciplinar team of up to 5 researchers from the GSIC-EMIC group, most of whom had previous experience in the evaluation in this kind of technological and educational contexts. The central Issue in this evaluation, that we have mentioned, was the tension represented by the I1 question in Figure 5.22 ("Does GLUE!-PS support teachers in the orchestration of blended CSCL across DLEs?"). However, this tension was resolved through the exploration of the aforementioned four topics that explore different orchestration aspects (T1 through T4).

The Ground of our evaluation is represented centrally by the GLUE!-PS architecture and data model as they have been presented throughout this chapter. The main Stakeholders of the evaluation have been the 57 teachers participating in the different iterations of the study, although other stakeholders can be mentioned, such as the responsible for the professional development program or the research project where the workshops were framed, or the students that enacted the activities that were orchestrated using GLUE!-PS in the authentic experiments. This Evaluation took place at different points in time during one year and a half (2011-2012), involving several members of a trans-disciplinar research group, in the general Educational setting of the University of Valladolid (although in different contexts, from undergraduate-level courses in Education to Master-level courses in Computer Science, or PD actions, in which the focus was on the promotion of blended learning using CSCL activities that made use of DLEs).

Finally, the Method we have chosen in the evaluation included a variety of Data gathering techniques, such as observation and recording of face-to-face sessions, semi-structured interviews with participant teachers, paper- and web-based questionnaires, and the analysis of teacher-produced artifacts (e.g. teacher designs and deployments done during the workshops). 


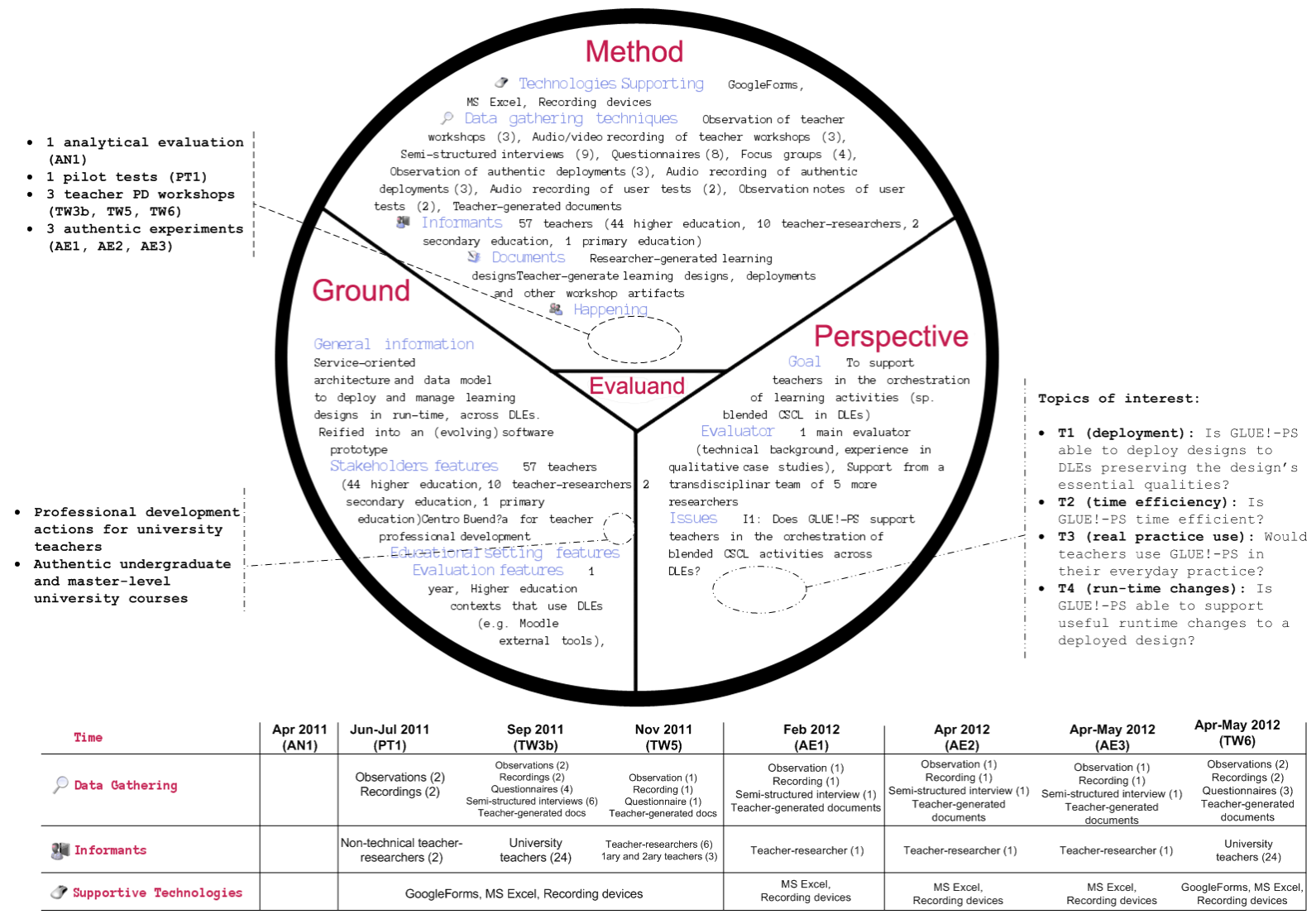

Figure 5.23: Graphic representation of the evaluation design of GLUE!-PS as a technological tool for orchestration 
The Supporting technologies used during the evaluation included MS Exce 46 for quantitative and qualitative analysis, GoogleForms 47 for web-based questionnaires, or diverse recording devices. Our Informants were the aforementioned 57 teachers (including teacher-researchers in the authentic experiments and teachers and teacher-researchers from multiple disciplines in the workshops), and the Documents that were available for analysis included teacher-generated artifacts and our own design and enactment materials to support the workshops. Nevertheless, we will provide more detail about the contextual and methodological peculiarities of the evaluation in each iteration, at the beginning of the following subsections 5.5 .2 through 5.5.5).

It is also worth mentioning the way we have applied the mixed methods approach to the data gathering and analysis during these evaluations. As outlined in Section 1.2, we have applied a variation of the mixed methods approach to studying CSCL, first proposed in [Mar06b]. In the concrete case of the evaluation of GLUE!-PS, we have followed a slight variation of the flow depicted in Figure 1.5, to account for the contextual differences in each setting (such as the different availability of data sources). A particularized data gathering and analysis diagram can be seen in Figure 5.24. As we can see there, several data gathering techniques have been used (depending on their applicability to each context) to obtain triangulated evidence about the participant teacher's profiles, including their prior experience, background, technological and pedagogical beliefs, etc. During the different happenings (the workshops and authentic experiments), participants were observed by at least one researcher, and photos, audio and video recordings of the sessions were made, whenever possible; in some cases, questionnaires were presented to the participants during the workshop, to better assess the evolution of their opinions and reflections. Finally, semi-structured interviews and questionnaires were used after each experiment, to obtain triangulated feedback and reflections about the usage of GLUE!-PS. All this (mostly qualitative, but also quantitative) data was then analyzed, using qualitative and quantitative (descriptive statistical) analysis techniques, triangulating evidence where possible. This analysis led us to partial conclusions which were used to decide the changes to focus to be made in the following evaluations, as well as to generate our global conclusions about GLUE!-PS as a technological tool for teacher orchestration.

Finally, we should note that, as we did in the evaluation presented in Chapters 3 and 4 , in this section we have also chosen the "findings/evidence tables + summarizing text" formula to present the evaluation findings and evidence, whenever possible, to keep the evaluation's length under control, while allowing readers to define their reading patterns more flexibly.

\subsubsection{Iteration \#1: A first analytic evaluation of GLUE!-PS}

Once the GLUE!-PS architecture had been proposed, and its data model had been defined (see Section 5.2), we decided to do a first analytical evaluation of our proposal (AN1, in Figure 5.2 , even before a prototype was available. The method and findings of the study are explained below.

46 http://office.microsoft.com/en-us/excel/ (Last visit: 6 Jun 2012).

47 http://www.google.com/google-d-s/forms/ (Last visit: 6 Jun 2012). 


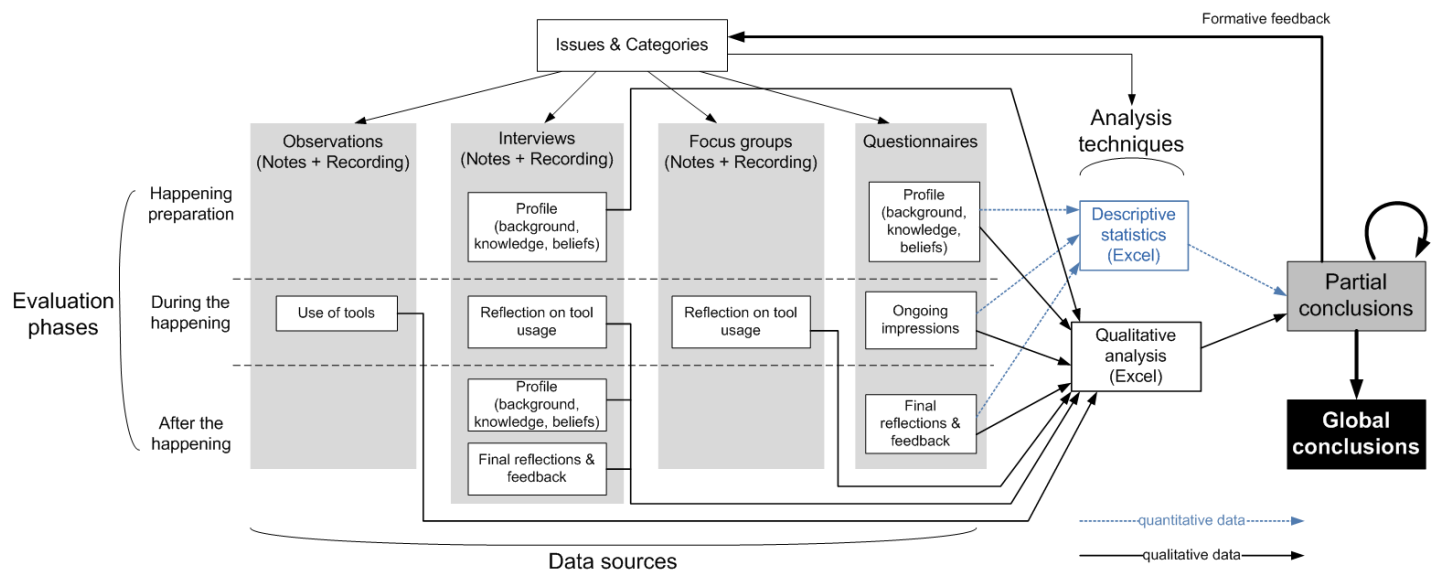

Figure 5.24: Graphic representation of the data gathering and analysis techniques flow during the evaluation of GLUE!-PS, adapted from Mar06b

\section{Happening AN1: Analytical evaluation of the PlanetGame scenario}

For this first theoretical validation of the proposed infrastructure and its data model, we simulated manually the process of translation, particularization and deployment of a well-known learning design scenario (following the different steps and elements that GLUE!-PS specifies), from its expression in several LD languages to various target learning environments not supported originally. Then, we analyzed the original scripts and their deployed counterparts, to ascertain whether the original scenario's essential pedagogical qualities (which some authors have termed "intrinsic constraints" Dil07d]) were maintained or not throughout the process. This analytical evaluation provided first evidences of the orchestration support provided by the GLUE!-PS proposal, even if only from the Design aspect of orchestration (T1, in Figure 5.22).

Context and method of the study The Planet Game scenario Vig08b is a game-based collaborative learning scenario which is part of a real lifelong learning situation in Astronomy. Its main goal is for learners to acquire knowledge about the planets in the Solar System, classifying them with respect to their distance from the Sun. In the scenario, learners are grouped into two teams, and each team is given part of the needed information, so that they must collaborate to succeed (although they also compete to be the winners of the game). The scenario is structured as follows: Team A is given expert interviews which contain some planets' properties from which they can deduce the planets' order, but not their names. Team B is given another set of clues that contain the planets' names and a few properties so that the order cannot be deduced from them alone. In order to collaborate, each team will have a private chat room, and all the participants can communicate through a forum. The teacher may add new clues to the interviews of both teams, and see how the activity progresses through the forum. When the teacher deems so, the collaborative part ends, and each learner takes an individual questionnaire to assess their knowledge about the planets' distance from the Sun. In light of the results, the teacher finally announces who is the winner of the game.

This scenario had been used by the learning design community as a benchmark to compare 
and explore the advantages and disadvantages of different LD languages and approaches. It was the object of a LD languages workshop in 2006 and a follow-up special issue at the Journal of Interactive Media in Education ${ }^{48}$ in 2008. At the time, the scenario had been modeled using different LD languages and approaches (e.g. various forms of IMS-LD, LAMS, LDL), including also the operationalization and execution of the generated computer-specified scripts in the supported learning environments (CopperCore, LAMS, LDI, etc.).

In order to optimize our efforts and learning gains from this kind of analysis, we decided to explore the limits of the deployment of the Planet Game scenario using GLUE!-PS, by means of four different translations, trying to cover both commonplace and extreme cases of this translation and deployment process. Two of the original LD formalizations were considered: the PlanetGame IMS-LD script described in [HL08] (as an example of formalization using a pedagogy-agnostic, widespread LD language) and the LDL model detailed in Fer08 (an extreme example, since LDL is a much more expressive language, specifically designed for collaborative learning). As target learning environments, we chose Moodle (currently the most widely used VLE) and MediaWiki (as an extreme case, due to its limited feature set, see Figure 5.6 .

Findings Since the full description of the four mentioned translations, and the decisions that are to be taken by the LD and LE adapters to translate from the original LD language to the target learning environment, would take up too much space, we will describe here some of the most noteworthy aspects found in our analysis. As mentioned in Pri11b, interesting limitations were found in the most commonplace (IMS-LD to Moodle) and most extreme (LDL to MediaWiki) translation cases 49 .

The transformation of the concepts of roles and groups, from the IMS-LD formalization HL08 to its deployment in Moodle (using Moodle's course backup data format), is depicted in Figure 5.25. The two teams in the Planet Game scenario are modeled in IMS-LD as roles of the "jigsaw group" type. The IMS-LD adapter would then transform those roles to role entities in the GLUE!-PS data model. However, nowhere in the original script it is specified that there are two teams, or which learners are in each team (to increase reusability, e.g. in the case that we want three teams). It is the teacher who has to complete this "instantiation" (i.e. particularization) data, defining how many group entities should exist. In the original specification of the scenario in IMS-LD this kind of information would have been completed manually by the teacher in the execution environment's (e.g. CopperCore's) administrative interface, and is done in an ad-hoc way for each execution environment. With GLUE!-PS, once the instantiation information is in place, the conversion from the GLUE!-PS data model to the Moodle concepts of "group" and "grouping" $[50$ is easily automatable (even if it is time-consuming and error-prone to do it manually). Moreover, using GLUE!-PS has the advantage of letting the teacher introduce this kind of particularization data at a unified point and in a unified manner, no matter what the target learning environment is.

Let us now take a look to the translation of the concepts related to activities, in the extreme case of deploying the LDL formalization of Planet Game Fer08 in MediaWiki. LDL

\footnotetext{
48 http://jime.open.ac.uk/

${ }^{49}$ Complete diagrams and data from the translations are available at http://www.gsic.uva.es/ lprisan/ Prieto2011-ECTEL-AdditionalData.zip

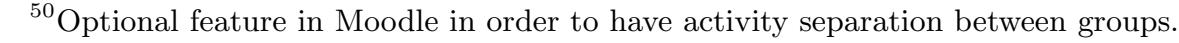




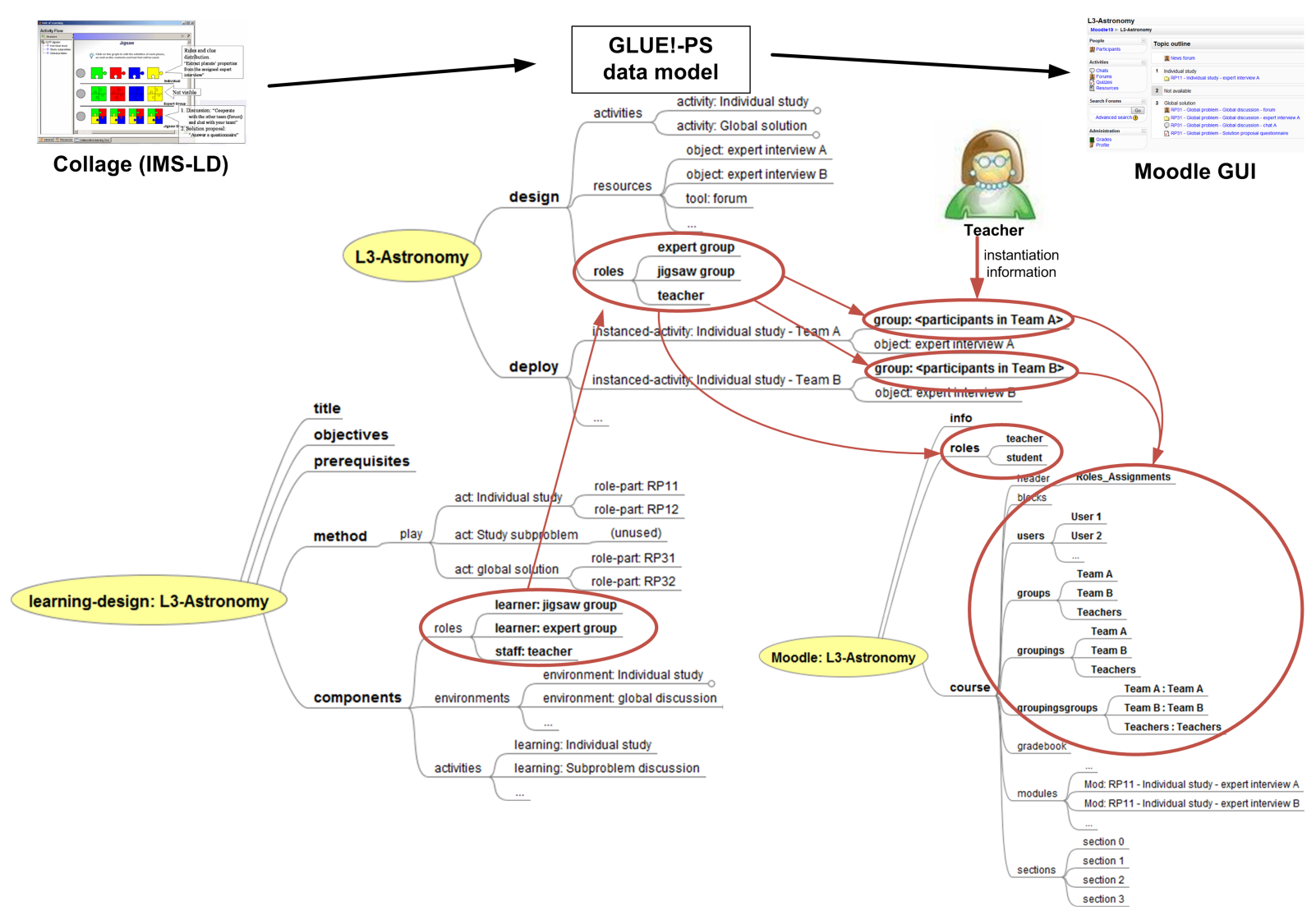

Figure 5.25: Converting roles and groups in GLUE!-PS: from IMS-LD to Moodle through the GLUE!-PS data model

allows for more detail than IMS-LD, and allows for a high degree of expressiveness regarding collaborative activities. Albeit it supports the concept of activity, its central emphasis is in a more fine-grained element: the interaction (i.e. for each activity, several interactions among participants are defined). We have made the LDL adapter to convert interactions and activities to a hierarchy of activities. The resulting activity structure is much more verbose than the one coming from the IMS-LD specification (making the instantiation process more time-consuming). The activity tree would then be converted to wiki pages by the MediaWiki adapter. However, there is an important limitation in the deployment of the design in MediaWiki: due to its default open access policy, it is very difficult to implement a hard separation of resources among teams (e.g. nothing precludes team A from accessing team B's expert interview), making the game's success dependent on the participants' good will. This is a consequence of our general approach, which promotes loose coupling and tries not to modify the elements to be integrated (i.e. LD tools and learning platforms): the basic nature of the learning environment will not be modified, and so the teachers should consider whether the selected learning environment is adequate for the design or not (e.g. MediaWiki may not be the best option for a scenario that requires separation of who sees what).

Figure 5.26 summarizes the analysis of the Planet Game scenario described in Vig08b, and how those properties were preserved throughout the different translations. The script as- 


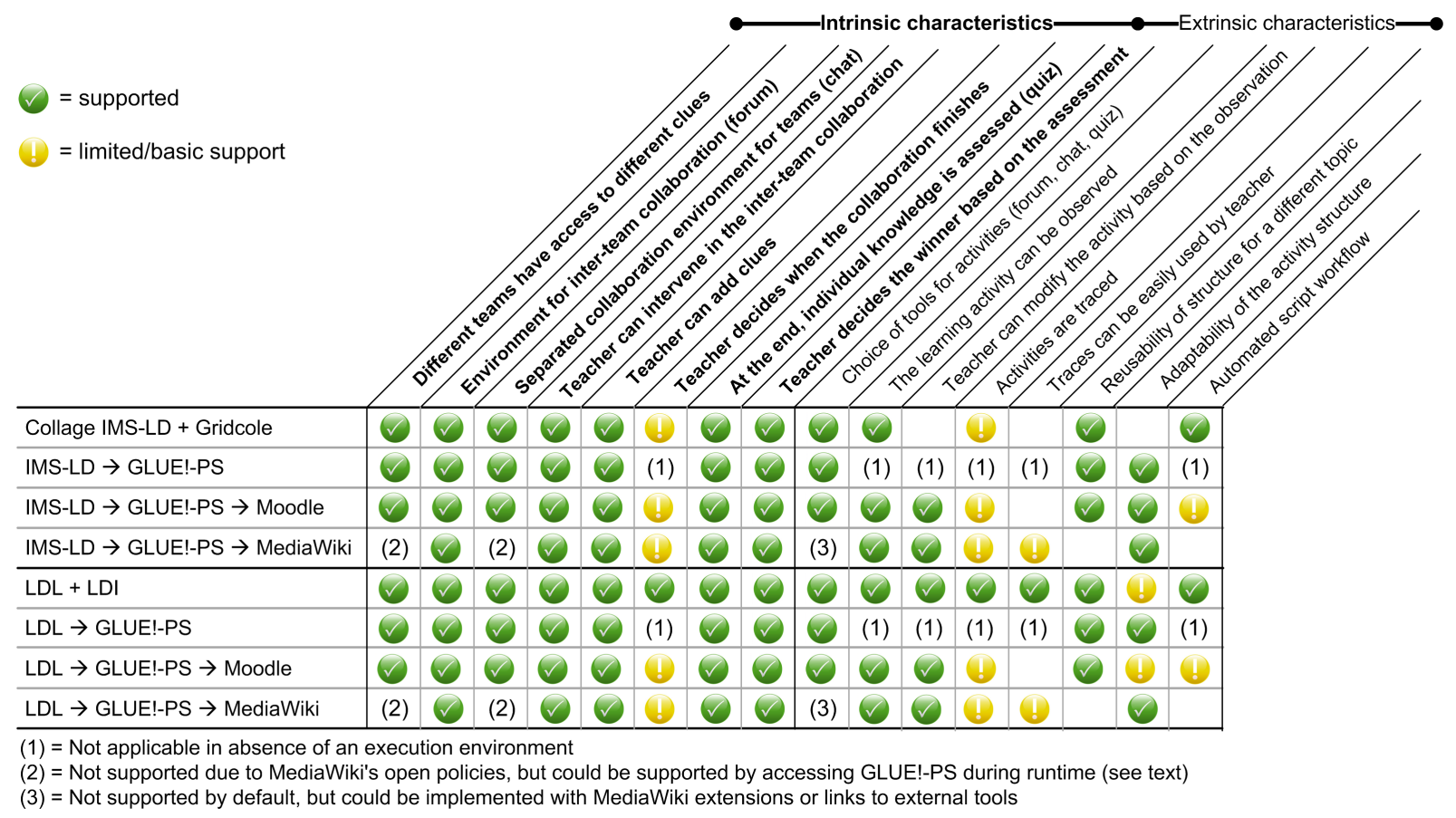

Figure 5.26: Summary of the intrinsic and extrinsic characteristics of the Planet Game script extracted from [Vig08b], and how they are preserved throughout the translations

pects have been classified as intrinsic (essential for the pedagogic intent) or extrinsic (due to implementation restrictions, such as the specific technologies used), following the categorization from Dil07d. As we can see, the Moodle deployment preserves all intrinsic characteristics, and most extrinsic ones, regardless of the original LD language. The MediaWiki deployment preserves most aspects, although it breaches the "separation of teams" constraint, due to its lack of group-based access control. Nevertheless, several possible solutions for this learning environment limitation exist, from integrating advice mechanisms into GLUE!-PS (so as to warn teachers of these breaches in the script principles), to implementing such group-based access control in GLUE!-PS (to whom MediaWiki would delegate such functions).

\section{Partial conclusions and feedback for the next iteration}

This kind of theoretical validation has obvious limitations, not only with regard of its completeness (only four translations of one specific scenario/design were tested) and scope (this analysis only explores one aspect of orchestration, the Design one), but especially because of its de-contextualization: it does not take into account any contextual constraints, e.g. those regarding the expertise of teachers (e.g. can an "average teacher" do an LDL modeling of the PlanetGame scenario?), integration in the curriculum and timeframe of the courses (although the scenario is taken from real practice), and so on. However, this gathering of data illustrates clearly some interesting insights and limitations about the GLUE!-PS approach. These partial conclusions and the formative feedback that they provide to the next iteration of research are represented graphically in Figure 5.27. 


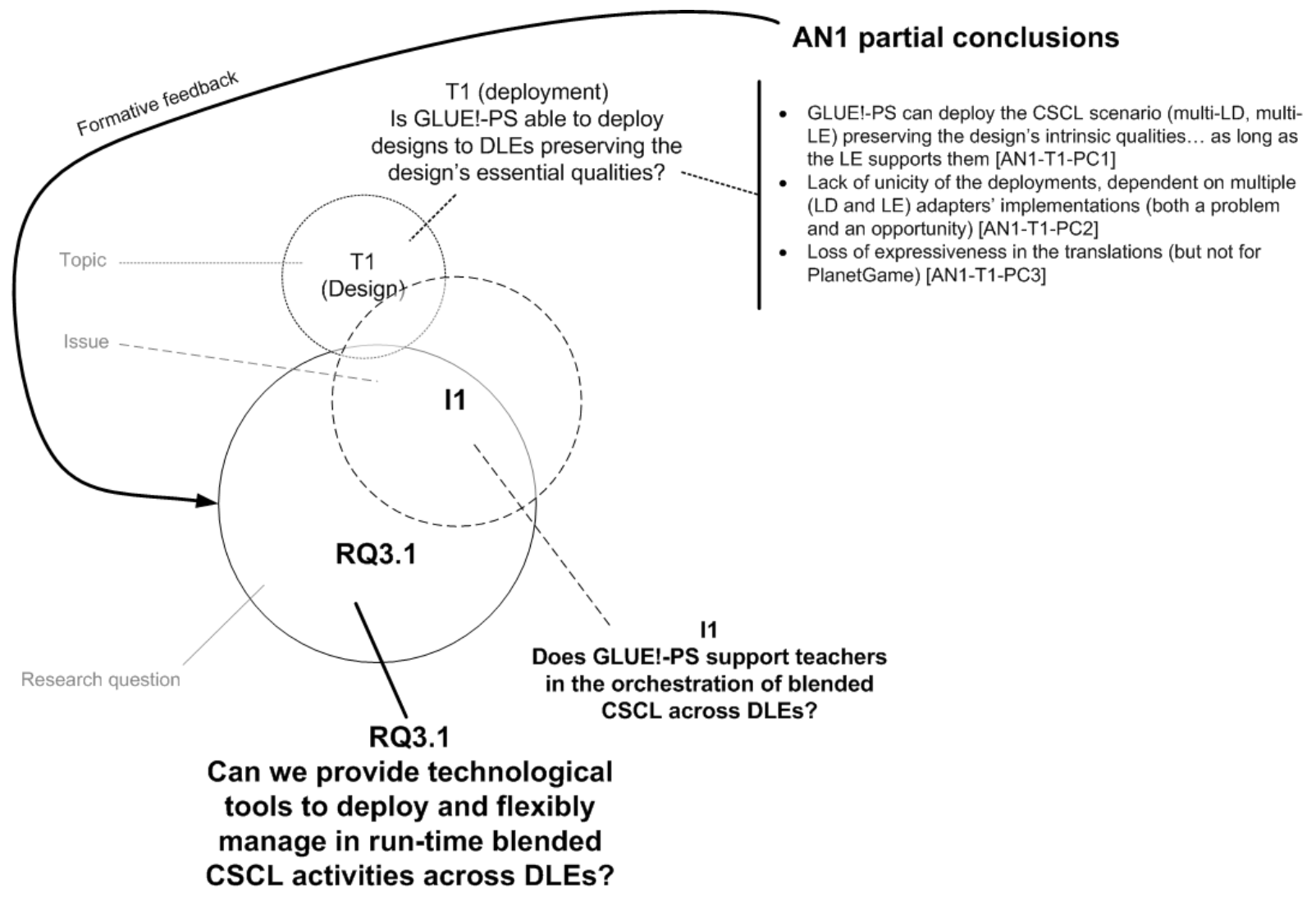

Figure 5.27: Graphical representation of the research questions, issues and partial conclusions of Iteration \#1 in the GLUE!-PS evaluation

The proposed GLUE!-PS architecture and data model proved to be capable of deploying the chosen TEL design (PlanetGame), expressed in two different LD languages, to multiple execution infrastructures, while preserving the intrinsic characteristics of the original script, as long as the target learning environment supports those characteristics. Also, due to the use of service orientation and adapters in the architecture, this can be achieved for a large number of combinations of LD languages and learning environments, at a reduced development cost, with minimal modifications to installed infrastructures (LD tools, VLEs, etc.). If, using this approach, we can provide a time-efficient way of deploying learning designs in existing learning environments, this system can greatly help in the orchestration of complex TEL scenarios (at least, in the Design aspect of orchestration). However, this approach is not without limitations.

The comparison of the LDL and IMS-LD examples above show a lack of unicity in the resulting deployments of a learning scenario (even for a same target learning environment). This variability not only depends on the available concepts in the source LD language (and the fact that designs were conceptualized by different designers), but also on the decisions taken by the implementors of the different $L D$ and $L E$ adapters (e.g. converting each interaction in LDL to one GLUE!-PS activity vs transforming it to instructions in the description of a high-level GLUE!-PS activity). The adequacy of mappings is very subjective, and largely depend on the practitioners' pedagogical beliefs. Indeed, the service-oriented nature of the proposed architecture favors the 
appearance of multiple implementations of the adapters, rather than enforcing a single view of how such transformations should be done. Thus, practitioners can potentially choose, among different implementations of an adapter, the one that better suits their needs.

Still, the most important limitation of our approach is the loss of expressiveness that is bound to appear in the two translations involved (first from LD language to the GLUE!-PS LF, and then to the learning environment concepts). As the application to the PlanetGame scenario showed, the proposed data model is expressive enough to capture most essential structural elements of a script (e.g. activities, resources, groups, roles, sequencing, see Figure 5.6), to the extent that the learning environments themselves support them. In this sense, the biggest losses are in the area of complex sequencing and other dynamic properties of activities (conditions, loops and features related to advanced activity flow automation). Given that the implementation of those features varies wildly between one LD language and another, and that few learning environments support them anyway, we have chosen to leave most of these features out of our data model. Thus, even if the loss in translations will be higher for complex designs that exploit the full capabilities of a highly expressive LD language, we contend that the support given by our proposal matches the support given by current learning environments, with a comparatively low development effort 51 Furthermore, the provision of a single point for adding instantiation data (the GLUE!-PS service) would allow teachers to check the translated script model so that such loss can be remedied.

This first favorable validation of the GLUE!-PS approach (which exemplified how it could support the deployment common learning design scenarios taken from a real course, from multiple LD languages to multiple learning platforms), prompted us to implement a first prototype of the architecture, and to test this apparent support to the Design aspect of orchestration in more authentic scenarios. These first results also hinted at two additional ways in which we could expand our inquiries in more authentic settings: to evaluate the time efficiency of the proposal, and whether teachers would use such orchestration support in their everyday practice (and why).

\subsubsection{Iteration \#2: Evaluating the first GLUE!-PS prototype in workshops}

After the first analytical validation, the first operative prototype of the GLUE!-PS architecture and data model was implemented, with the development of the central service (GLUE!-PS Manager), one LD adapter (for the IMS-LD specification) and one LE adapter (for the Moodle VLE). With this minimal prototype, which did not even feature a proper graphical user interface, first pilot trials of the deployment of learning designs were made (PT1 in Figure 5.2) by the author to deploy an authentic learning situation: a collaborative learning workshop session on the design and enactment of CSCL using atomic patterns (TW3, see Section 4.5.4). This first deployment of an authentic learning situation was complemented by end-user trials of incomplete versions of the GLUE!-PS GUI, in controlled usability tests (also encompassed in happening PT1). Once a fully functional prototype of the GLUE!-PS infrastructure (including the GUI) was completed, it was tested in teacher workshops: the second half of the teacher workshop

\footnotetext{
${ }^{51}$ As a first-level approximation and example, IMS-LD support in Moodle was considered for months by an external group of researchers and teachers using Moodle (see [Ber05]), and finally dismissed as not worth the effort. On the other hand, developing an IMS-LD adapter and a Moodle adapter for the GLUE!-PS architecture has taken 45 days for two developers working part-time on the project.
} 
on the orchestration of CSCL (TW3b, coincident with the TW3 workshop depicted in Section 4.5.4), where non-expert (but mostly ICT-oriented) teachers were to deploy their designs using GLUE!-PS; and a teacher-researcher workshop framed within a research project (TW5), where a set of more expert teacher-researchers from primary, secondary and university education used the GLUE!-PS infrastructure along with other project-developed tools for the orchestration of CSCL. The findings and evidence of these three evaluation happenings are detailed below.

\section{Pilot trials (PT1)}

Context and method Within this evaluation happening we have chosen to accumulate the preliminary evidence gathered in the diverse user trials performed before the first fully functional prototype of the GLUE!-PS infrastructure was completed. These trials include several points of data gathering:

- A first trial where the author deployed a IMS-LD learning design of a blended collaborative workshop, and deployed it to a Moodle VLE where the workshop was then enacted (PT1-1). The design of the session, which followed a Pyramid CLFP, is described in more detail in Section 4.5.4, and was formalized using the WebCollage LD authoring tool. The IMS-LD Unit of Learning thus generated was translated to the GLUE!-PS data model automatically by the corresponding LD adapter. Afterwards, the author added the rest of the particularization data (e.g. the documents that each of the groups was to use) manually, and then the resulting particularized design was deployed automatically to Moodle using the GLUE!-PS LE adapter for Moodle. This trial was not formally recorded, although the author took observation and reflection notes, including the time it took to complete the deployment process, the problems encountered, and so on (PT1-1-O).

- A trial with a teacher-researcher from the author's research group (with teacher education background), in which a set of animated mockups representing the GLUE!-PS GUI (see Figure 5.28 were used to gain first evidences about the usability and potential problems of using GLUE!-PS through a graphical user interface (PT1-2), by mimicking the deployment of a learning design. This session was audio recorded (PT1-2-R), and observation notes were taken (PT1-2-O).

- A second animated mockup trial with another teacher-researcher (also with teacher education background) was conducted several days later (PT1-3), which was also recorded (PT1-3-R) and observed (PT1-3-O).

Findings Given the preliminary stage of these first user trials (which have limited relevance compared to the following happenings), and for brevity's sake, we will not provide detailed evidence taken during the trials. Rather, we will briefly report which were the main findings and conclusions that the evaluators took from them.

Regarding the topic of GLUE!-PS deployment abilities (T1), the PT1-1 trial exemplified again that GLUE!-PS was able to cope with medium-complexity ${ }^{52}$ CSCL designs that used the

\footnotetext{
${ }^{52}$ In the sense that they used a single CLFP, instead of more complex combinations of collaborative flow patterns.
} 


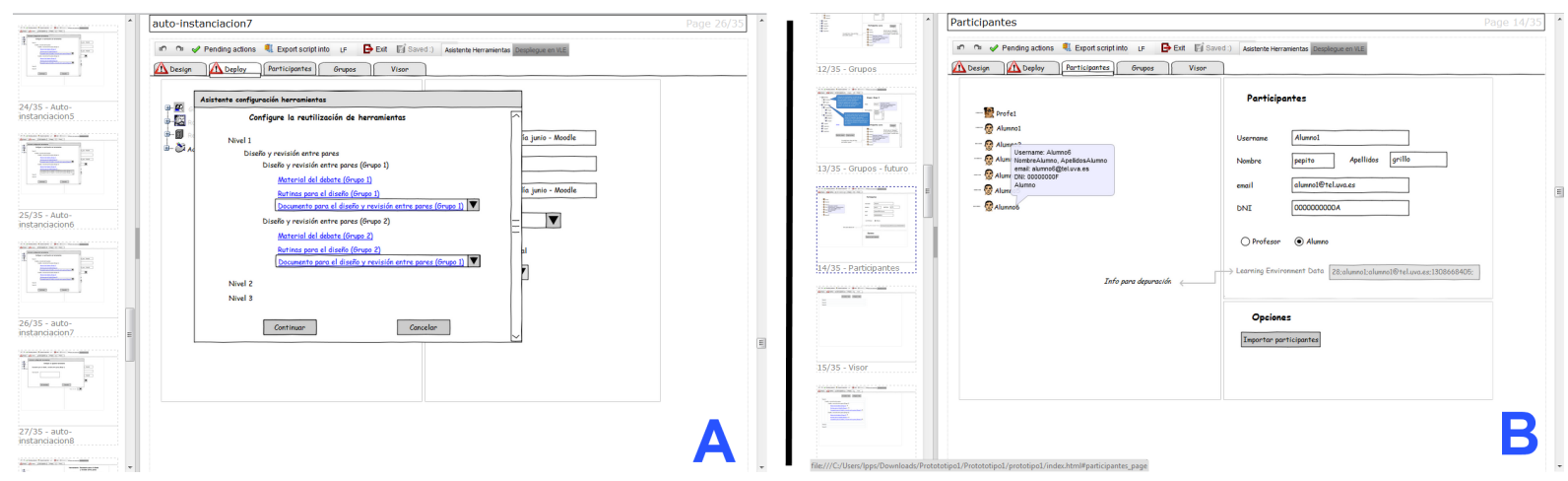

Figure 5.28: Example mockup screens used in the first usability tests of the GLUE!-PS GUI (PT1)

IMS-LD specification for formalization. The resulting deployed course in Moodle complied with the pedagogic restrictions of the Pyramid CLFP (separate groups of increasing size, reusability of artifacts generated by smaller groups, etc.) and it was, in fact, enacted during the TW3 workshop. Since they did not imply the deployment of a real learning design, no further evidence was gathered by the other trials (PT1-2, PT1-3) regarding this aspect.

The time efficiency aspect of GLUE!-PS's support (T2) was not a central aspect in these trials, albeit it provided first hints of evidence. In the PT1-1 trial, one learning design for 20 participants was developed with the WebCollage LD tool, and deployed by a researcher-developer (the author), performing the tool instantiation by directly manipulating the GLUE!-PS LF model. Even with these limitations the subjects were able to design and deploy the design in Moodle in under 30 minutes. Implementing the same design directly in Moodle has taken around 47 minutes (39 minutes if we use GLUE! to manage the external tools). Obviously, doing this required considerable knowledge about the system, and it is not at all suitable for teachers in authentic settings. However, this small experience served to hint at the potential time-efficiency gains of the system, once it is made usable through a GUI. Again, the other two trials did not delve into the time-efficiency of GLUE!-PS operation.

Finally, regarding the usage of GLUE!-PS in real practice (T3), some first evidences can also be gathered from the trials In PT1-1, a learning design was deployed in a time-efficient fashion, although the lack of a graphical user interface made the prototype usable by none except the most ICT-expert teachers. The usage of such deployed design during the workshop, was rather revealing, especially regarding the need for modifications in run-time (e.g. one of the external tools used, Dabbleboard, was down during the session, and the debate activity had to be moved to an asynchronous forum after the session). These extraneous events highlighted one weakness and one strength of the GLUE!-PS approach. The weakness was that the GLUE!PS prototype did not support (yet) run-time changes, which proved to be a very much needed feature. The strength is that, due to GLUE!-PS's loose coupling integration with VLEs, the run-time changes could be made manually anyway using Moodle's GUI (i.e. GLUE!-PS does not impede the VLE's natural flexibility features). In the other two trials (PT1-2, PT1-3), even though no real design was implemented, we obtained certain evidence from the two teacher educators (not ICT-experts although used to a certain degree of ICT usage): First, that some aspects of the GUI could be improved in order for a teacher to find it easy to use in real practice: 
a) improving the vocabulary used in the system's user interface; b) including contextual help in the system's user interface, with examples and explanations about its main concepts.

Especially difficult in this regard was the way of conceptualizing and depicting the artifact reuse across groups (e.g. using in one activity a document that other group had generated in a previous activity, which is in fact a recurrent "atomic pattern" in many CSCL designs, see Chapter 4). For example, one of the teacher educators stated "[When presented with the tool reuse screen in GLUE!-PS GUI] I have no idea what this is about [...] some kind of explanatory note is needed" (PT1-3-R), while the other said that "this task is not clear ... [once the task is explained, facilitators ask user to label it] ... maybe description of activities ... [several minutes later, gives up in finding words to label this task] ... it is not clear at all what this step is about $[\ldots]$ the problem is that it is not visually clear where can I manipulate stuff [...] with icons this would be much easier" (PT1-2-R).

Also, in this happening, we gathered evidence that hinted at GLUE!-PS and its deployment abilities as an important enabler technology for LD tools, which are currently disregarded in favor of designing CSCL using pen and paper: "[When discussing the first GLUE!-PS prototype's functionality] So, it can only be used with WebCollage? [...] then I don't know if I would use it [...] because I can do a design in WC in 4 hours, while I can do it with pen and paper in 10 minutes [...] but maybe [with GLUE!-PS] it would be useful [...] if I can get in the [learning] platform all the groups and everything configured [...] and I can reuse the design later, so the time I spend the first time its saved for the [following ones]" (PT1-2-R).

All these findings served to further guide the development of the GLUE!-PS prototype, which was being finished for its usage in the first formal evaluation, through a teacher workshop (see below).

\section{A workshop with university teachers (TW3b)}

In order to provide first formal evidence about our research question on the orchestration support provided by the GLUE!-PS infrastructure, we proposed to conduct a professional development workshop for teachers at the University of Valladolid, whose topic would be the design and enactment (i.e. the orchestration) of advanced collaborative activities 53 in Distributed Learning Environments. This workshop would provide a first conceptual (but practical) introduction to the design of collaborative activities using CLFPs (see 4.2.3), and structuring them further with a selection of the extended atomic pattern catalogue elicited from higher education. Finally such designs would be implemented with ICT tools, including the aforementioned WebCollage LD authoring tool, the GLUE!-PS infrastructure and Moodle-based DLEs.

Context and method As the reader may have noticed, this workshop is actually the same event that has already been described in the evaluation of atomic patterns for orchestration (TW3, in Section 4.5.4). As it was mentioned there, this workshop spanned two sessions separated by three months. While the first of these sessions concerned mostly the conceptual part of atomic patterns (and its ICT support was, in fact, implemented using GLUE!-PS in our first pilot trial, PT1-1 in the previous section), the second session that took place three months afterwards

\footnotetext{
${ }^{53}$ In the sense of "non-trivial", going beyond a group report or discussions in a plain online forum.
} 
was targeted mostly at the evaluation of the GLUE!-PS technological proposal. Thus, it is very important to understand that evaluating GLUE!-PS was not the only research goal of this workshop. We have chosen to label this happening as TW3b, to denote that it is the same event, but in this case the evaluation perspective is different, since the Evaluand is GLUE!-PS. In this section we will only describe the findings and evidence regarding GLUE!-PS, although some references to the other goals and activities of the workshop concerning atomic patterns will be unavoidable.

Other interesting contextual data that should be remembered include the fact that the workshop was a professional development action done within the auspices of the Buendia center at the University of Valladolid, and that it was open for university teachers with a basic knowledge of the involved ICT tools, up to 20 participants (in order for the workshop design to be manageable). Among these participants, teachers from the Master-level degree on ICT research (MUI-TIC from now on, from its Spanish initials) were given preference, since they already had performed a related workshop on the design of master-level TEL activities Fer10. This was done in order to minimize the problems related to the basic usage of the ICT tools involved, which could disrupt the course of the workshop and our data gathering itself (although this obviously produced a bias in our results, since most of the participants had a technical background as a result).

As mentioned in Section 4.5.4 the workshop was intended for a total of 10 hours in a blended learning format course ( 2 face-to-face sessions of 3 hours each, plus 4 hours of online work), which itself was designed using CLFPs and atomic patterns. The goal for participants was to learn how to design and enact CSCL activities using different ICTs, including the university's institutional platform (the Moodle VLE) and a variety of other ICT tools, thus conforming a DLE. During the workshop teachers would work collaboratively with other teachers in designing, deploying and enacting (role-playing) a hypothetical but realistic scenario, and they would work individually in the orchestration of a scenario relevant for their own practice. The workshop's learning design involved the following coarse-grained phases:

- Pre-session online activities, which included the reading of the sample scenario to be used during the workshop, and answering an online questionnaire with background and starting knowledge questions (TW3-Q1).

- First face-to-face session, where teachers had to design a CSCL experience for the proposed generic scenario, collaborating in groups (following the Pyramid CLFP, and using pen \& paper, but also ICT tools), and using atomic patterns as aids during the process.

- Post session online activities, including answering a questionnaire about the perceived usefulness of the presented atomic patterns (TW3-Q2), and proposing a real CSCL experience, similar to the one in the first session, using the same CLFPs and routines, but this time to be done individually for a subject each teacher taught.

- Second face-to-face session, in which teachers individually tried to implement their designs using the WebCollage learning design tool, and trying to deploy it to Moodle using the GLUE!-PS system. Also, the teachers would role-play the enactment of parts of the designed experiences, using a subset of the elicited enactment-time atomic patterns as a support to react and reflect on such simulated enactment. 
- Post session online activities, including answering a questionnaire about the usefulness of the presented technological tools and atomic patterns (TW3-Q3), and the professional development action (TW3-Q4).

Since in this happening we obtained evidences mostly from the second face-to-face session, where teachers were to use the GLUE!-PS infrastructure themselves, it is interesting that we describe the activities of this session in more detail. After a short introduction and reminder about the workshop's goals and activities, teachers were to implement the learning design that they had done individually over the past three months, using the WebCollage LD tool and a worksheet that glossed over the basic design process with the tool (in 30 minutes). Afterwards, they would particularize their designs with concrete participants and groups (in WebCollage) and tools (using GLUE!-PS), using a similar worksheet (in 15 minutes). Afterwards, they were to use GLUE!-PS to deploy the particularized designs in Moodle (in another 15 minutes). Afterwards, some representative problematic situations were to be role-played in order to use the enactmenttime atomic patterns (in around 1 hour). A debate and final reflections were to take up the last 30 minutes of the session.

Regarding our data sources (which were already detailed in Section 4.5.4), we have chosen not to modify the labels of the sources, in order to avoid further confusion. Nevertheless, we reproduce the summary table of data sources in Table 5.7. In this evaluation, it is interesting to note that most of the evidences come from the observation and recording of the second face-toface session, but also from the six semi-structured interviews conducted after the sessions with volunteer participants, which covered both the usage of atomic patterns and GLUE!-PS. Also, the (non-formalized) individual learning designs generated by the teachers were formalized using the WebCollage authoring tool, and implemented (using GLUE!-PS) by a researcher during the three-month "interlude", in order to obtain further evidences of the ability of GLUE!-PS to deploy learning designs (TW3-LD).

Table 5.7: Main data sources used during the TW3b workshop

\begin{tabular}{|lll|}
\hline Source & Kind of evidence & Codes \\
\hline \hline Observation notes of the first face-to-face session & Qualitative & TW3-O1 \\
\hline $\begin{array}{l}\text { Observation notes of the second face-to-face ses- } \\
\text { sion }\end{array}$ & Qualitative & TW3-O2 \\
\hline First session's recording & Qualitative & TW3-R1 \\
\hline Second session's recording & Qualitative & TW3-R2 \\
\hline $\begin{array}{l}\text { Teacher-generated documents during the work- } \\
\text { shop }\end{array}$ & Qualitative & TW3-D \\
\hline First profiling questionnaire & Qualitative & TW3-Q1 \\
\hline $\begin{array}{l}\text { Second questionnaire, about the usefulness of the } \\
\text { first session elements }\end{array}$ & $\begin{array}{l}\text { Quantitative \& Qualita- } \\
\text { tive }\end{array}$ & TW3-Q2 \\
\hline $\begin{array}{l}\text { Third questionnaire, about the usefulness of the } \\
\text { online and second session elements }\end{array}$ & $\begin{array}{l}\text { Quantitative \& Qualita- } \\
\text { tive }\end{array}$ & TW3-Q3 \\
\hline $\begin{array}{l}\text { Fourth questionnaire, about the overall workshop } \\
\text { evaluation }\end{array}$ & $\begin{array}{l}\text { Quantitative \& Qualita- } \\
\text { tive }\end{array}$ & TW3-Q4 \\
\hline $\begin{array}{l}\text { Post-workshop semi-structured interviews (profil- } \\
\text { ing and evaluation) }\end{array}$ & Qualitative & TW3-I \\
\hline $\begin{array}{l}\text { Researcher-generated formalized designs and de- } \\
\text { ployments using the teacher's learning designs }\end{array}$ & Qualitative & TW3-LD \\
\hline
\end{tabular}

Thus, as we have have noted at the beginning of this section, we intended to address our main research question through exploring the issue "Does GLUE!-PS support teachers in 
the orchestration of blended CSCL across DLEs?" (I1), around three main areas: the ability of GLUE!-PS in deploying learning designs preserving the design's essential qualities (T1), its time-efficiency in doing so (T2) and the feasibility of using GLUE!-PS in everyday teaching practice (T3).
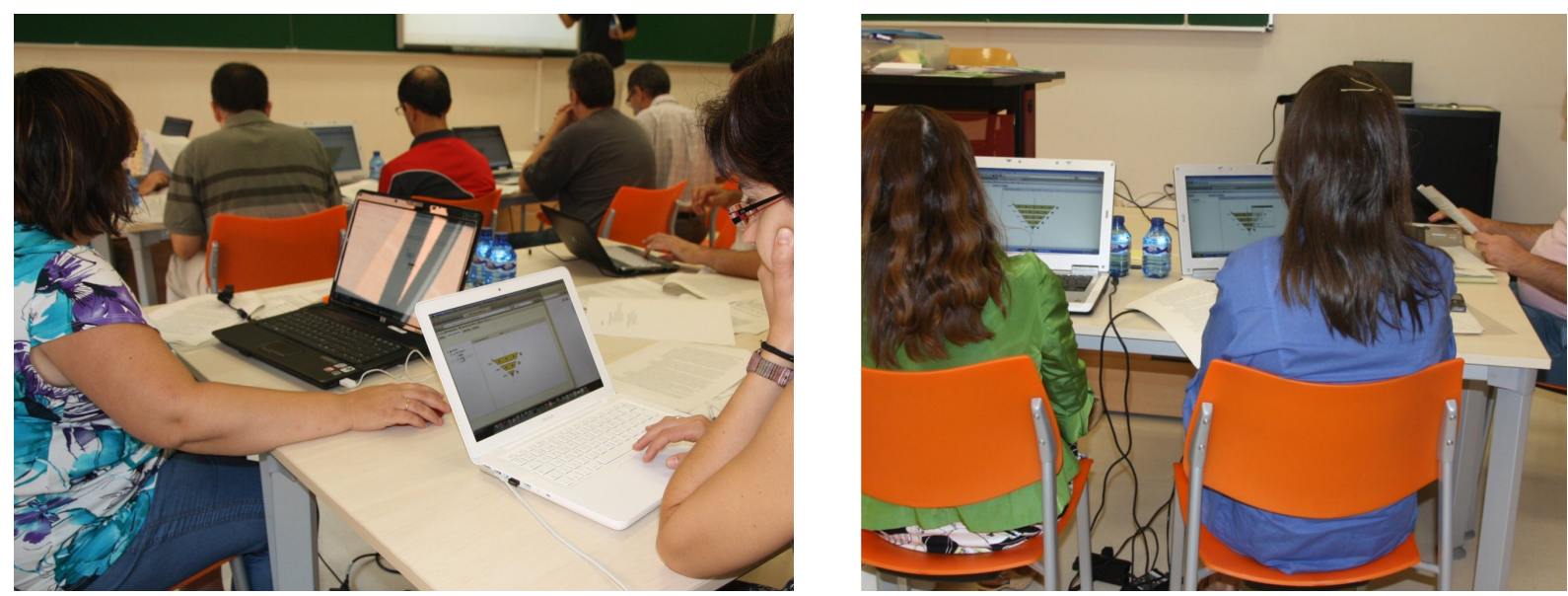

Figure 5.29: Photos taken during the TW3b evaluation happening

However, before we dive into the findings and evidence obtained in this workshop, it is worth noting that, as many other authentic learning situations, this workshop did not go by exactly as designed. First, only 14 of the 21 teachers which were doing the workshop were able to attend this second session, and inform us around the usage of GLUE!-PS (see Figure 5.29). Unfortunately, that was not the biggest problem, since a technical problem with the WebCollage tool in the particularization of the learning designs prevented users from completing the deployment using GLUE!-PS. Albeit participants were shown the whole deployment process live by the researcher team, the lack of direct user experience with the tool substracts from the validity and credibility of the evidences from this workshop session. This unexpected problem was afterwards mitigated in the six follow-up interviews (TW3-I), where the usage of GLUE!-PS was again exemplified and, in some cases, performed directly by participants.

Findings and evidence During this evaluation happening, and despite the aforementioned problems which hampered our gathering of evaluation data, we uncovered several findings about the orchestration topics that concerned us (see also the concrete supporting evidence in Table 5.8):

Table 5.8: Topics, findings and selected supported evidence from evaluation happening TW3b

\begin{tabular}{lll}
\hline Topic & Finding & Selected supporting evidence \\
\hline T1 (deploy ability) & $\begin{array}{l}\text { Dangers of dependency on ex- } \\
\text { ternal services }\end{array}$ & $\begin{array}{l}\text { Teachers could not deploy the learning designs, due to failures } \\
\text { in non-GLUE!-PS elements [TW3-O2] }\end{array}$ \\
\cline { 2 - 3 } & $\begin{array}{l}\text { GLUE!-PS is able to deploy } \\
\text { learning designs from IMS-LD } \\
\text { to Moodle }\end{array}$ & $\begin{array}{l}\text { A teacher-researcher was able to deploy 13 out of 13 teacher- } \\
\text { generated learning designs [TW3-LD] }\end{array}$ \\
& \\
\hline
\end{tabular}


Table 5.8 (continued from previous page)

\begin{tabular}{ll}
\hline Topic & Finding \\
\hline & $\begin{array}{l}\text { GLUE!-PS can be efficient } \\
\text { and fast when reusing }\end{array}$
\end{tabular}

Selected supporting evidence

[Do you think the time used to define the design is worth the and fast when reusing obtained result?] I don't think it is trivial to do the design... however, I think that with a little practice fast and efficient results are possible (because of reusability). I think these are T2 (time-efficiency) promising tools, but very specific and requiring considerable learning/practice time [TW3-Q3]

[Do you think the time used to define the design is worth the obtained result?] I think reusability is especially important (and, even if just for one use, the possibility of having the design prepared to deploy it in a concrete platform) [TW3Q3]

[Do you think the time used to define the design is worth the obtained result?] Of course, especially if you have big groups or you consider that you can reuse it for other activities [TW3Q3]

[Do you think the time used to define the design is worth the obtained result?] Yes. If you have a clear design idea, it can be reused easily [TW3-Q3]

GLUE!-PS as too time con- [Would you use GLUE!-PS in your real teaching practice?] No, suming $\quad$ I think it takes too much time putting this in practice, and I do not see it as crucial for the students' learning [TW3-Q3]

More adequate for large [Do you think the time used to define the design is worth the groups of students obtained result?] Of course, especially if you have big groups or you consider that you can reuse it for other activities [TW3Q3]

[Do you think the time used to define the design is worth the obtained result?] Sure in other designs it is worth it, but in mine I think it takes more time than doing it by hand or at home. I think that with fewer students it is not worth it. [TW3-Q3]

The lack of time and real use prevented the understanding of the deployment process [Do you think the time used to define the design is worth the obtained result?] [...] given my lack of knowledge [about CSCL] I would have needed more time to assimilate the contents [TW3-Q3]

[Do you think the time used to define the design is worth the obtained result?] It seems that it is [worth it], but since I did not do it myself I do not have a strong opinion [TW3-Q3]

GLUE!-PS as helpful in addressing CSCL [Would you use GLUE!-PS in your real teaching practice?] Yes, that is my goal. I think they make teaching easier, taking into account the way new curricula are structured. [TW3-Q3] [Would you use GLUE!-PS in your real teaching practice?]

Influence of pedagogic and content beliefs Not at the moment, because I think it does not adapt to the kind of courses I teach (the kind of content and the extensive curriculum are not adequate for collaborative learning). [TW3-Q3]

[Would you use GLUE!-PS in your real teaching practice?] I am not sure. I think you must know the techniques and practice them before taking them to the classroom, especially for bigger groups [i.e. classrooms]. For smaller groups, I could consider using it, but my main problem is that I am not convinced of the usage of these [collaborative] techniques in undergraduate-level courses. [TW3-Q3]

[Would you use GLUE!-PS in your real teaching practice?] No, I think it takes too much time putting this in practice, and I do not see it as crucial for the students' learning [TW3-Q3]

Low reliability of data due to technical failures during the test

[Would you use GLUE!-PS in your real teaching practice?] First I would try to do [collaborative learning] without [WIC \& GLUE!-PS], to get the hang of Moodle. Once I'm familiar with Moodle, I can consider these tools. The main obstacle is that today, it is not a stable product [TW3-Q3]

Comparatively low usability $\quad$ The GLUE!-PS obtained a usability score of 3.83 in a 1-7 score 
Table 5.8 (continued from previous page)

\begin{tabular}{|c|c|c|}
\hline \multirow[t]{5}{*}{ Topic } & Finding & Selected supporting evidence \\
\hline & $\begin{array}{l}\text { More adequate for large groups } \\
\text { of students }\end{array}$ & $\begin{array}{l}\text { TWould you use GLUE!-PS in your real teaching practice?] I'd } \\
\text { like to. It depends on the number of students per classroom. } \\
\text { [...] [TW3-Q3] }\end{array}$ \\
\hline & $\begin{array}{l}\text { Need for online help in GLUE!- } \\
\text { PS }\end{array}$ & $\begin{array}{l}\text { [Explain your GLUE!-PS usability score] The [user] interface } \\
\text { needs a bit more of help for non-advanced users. But the pre- } \\
\text { sented documentation [i.e. the worksheet] explained its usage } \\
\text { step by step [TW3-Q3] }\end{array}$ \\
\hline & $\begin{array}{l}\text { The lack of time and real use } \\
\text { prevented the understanding } \\
\text { of the deployment process }\end{array}$ & $\begin{array}{l}\text { [Explain your GLUE!-PS usability score] We had little time } \\
\text { to understand the applicability and use of this tool [TW3-Q3] } \\
\text { [Explain your GLUE!-PS usability score] I cannot say much } \\
\text { about GLUE!-PS, because we did not get to use it [TW3-Q3] }\end{array}$ \\
\hline & Unclear role of GLUE!-PS & $\begin{array}{l}\text { [Explain your GLUE!-PS usability score] [...] Regarding } \\
\text { GLUE!-PS, technical problems aside, I think it is more com- } \\
\text { plicated [than WebCollage] and its real usefulness is not very } \\
\text { clear [TW3-Q3] }\end{array}$ \\
\hline
\end{tabular}

Regarding the ability of GLUE!-PS to deploy CSCL designs preserving its essential qualities (T1), the aforementioned problems in the design/instantiation phase prevented teachers from reaching the phase of GLUE!-PS usage and to deploy the designs themselves. However, in this iteration an alternative (albeit less authentic) form of evidence was gathered, since one researcher-developer was able to design, instantiate and deploy the 13 submitted teacher designs (TW3-LD), all supposedly following the Pyramid CLFP (as mentioned in [MC12b]).

In order to do these deployments, interpretations of the designs were made to be able to implement them using the WebCollage authoring tool (which generally forced designers to comply with a limited set of patterns - in fact, CLFPs - to design the scenarios), since the teacher-generated designs were originally expressed in "pen and paper" (i.e. not formalized) and sometimes they were incomplete, or did not follow the CLFP. Thus, it is arguable whether the deployed designs really complied (or not) with the intrinsic design constraints in the mind of their original authors. Also, further analysis would be needed about the amount of change which is due to WIC's (strict) implementation of the patterns, rather than due to GLUE!-PS data mode 54

Overall, it is rather obvious that the software prototype's unreliability had prevented us from gathering useful validation data about the GLUE!-PS system. However, it is especially important to note that many of these unreliabilities were not due to the GLUE!-PS software itself, but rather by the fact that GLUE!-PS relies in many other external services for its operation, which itself is part of a cycle where other tools are also used (LD authoring tools, VLEs and external learning tools). In any case, this dependence on external elements is worth highlighting, as it illustrates an inherent risk of our "loose-integration" approach.

The opinions of the 14 informing teachers with regard to the time-efficiency of the GLUE!PS prototype (T2) were highly heterogeneous (see Table 5.8), probably due to the fact that only a vicarious experience of the deploy was provided (something that some of the participants manifested explicitly). Many of them mentioned the advantage of the reusability of the designs, which compensated for the extra time that formalizing the tasks through a LD authoring tool

\footnotetext{
${ }^{54}$ Actually, this kind of study has been conducted and recently presented at a TEL conference $\mathrm{MC} 12 \mathrm{~b}$, and some of its evidences have been added to our evaluation in later iterations (see Section 5.5.5).
} 
took. Others noted that the automated deployment through GLUE!-PS is time efficient in cases where the number of groups/participants is higher. We can also observe how several teachers alluded to the time-intensity of enacting CSCL (independently of using GLUE!-PS or not). These and other pedagogical (or orchestration) beliefs would prevent the usage of the tool regardless of its time-efficiency, and would only be countered by convincing teachers that these techniques are really applicable in the classroom, and that real, time-efficient learning gains will be obtained from them (which somehow points towards the use of other conceptual tools like atomic patterns as a complement to systems like GLUE!-PS).

Finally, regarding its use in real practice (T3), again the general lack of workshop time and the technological failures prevented us to gather more useful data. In general, GLUE!-PS was not considered as very usable (see Table 5.8), although most participants noted the lack of time and real usage as the main problem for the low quantitative usability score. However, some evidence also hints at another reason: the functionality of GLUE!-PS (i.e. to deploy learning designs) is not so clear for some teachers, along the script life-cycle. This might be due to the fact that the whole learning design approach followed in the GLUE!-PS proposal (using a LD-specific tool to do the design, including external web tools, and then deploying it semi-automatically in the DLE) was new for them, and its advantage over other approaches (such as the "bricoleur" approach taken by most Moodle teachers [Ber05]) is unclear. Oddly enough, there was a wide range of responses to the question of its potential use in everyday practice, with external factors (e.g. pedagogical beliefs or the number of students in the classroom) cited as the main reasons for not doing so (rather than the system's usability or reliability). This, once again, reminds us that just providing technological tools is not enough for changing teaching practice; also conceptual change has to occur [Ert99].

Finally, this workshop also provided some hints to the ongoing implementation efforts of GLUE!-PS. Not only the usability of the prototype had to be improved, but also we found from the WebCollage technical problem and the role-playing of problematic situations (see Section 4.5.4), that the ability to perform participant/group particularization of a learning design (which was not implemented at this stage) was a very needed functionality in GLUE!-PS (to avoid dependency on WebCollage on this aspect), but also that group re-formation was a very commonly appearing atomic pattern, which should be highly prioritized in the GLUE!-PS roadmap.

\section{A project-oriented teacher-researcher workshop (TW5)}

Two months after the TW3b teacher workshop, in November 2011, a new opportunity was available to gather further data about the usefulness of GLUE!-PS to support orchestration of CSCL across Distributed Learning Environments. A 1-day workshop was to be held in Valladolid, as a closure event for Sofocles55, a Spanish research project undertaken by the GSIC-EMIC research group, aiming to provide tools to support CSCL throughout the whole CSCL life-cycle, and which encompassed the efforts of the aforementioned GLUE! architecture AH12b] as well as the GLUE!-PS proposal. In this workshop, among other activities, expert teachers and teacherresearchers were to try the usage of the project's technological tools in a 3-hour hands-on session. Despite the limited length of the event, this new evaluation happening would provide us with

\footnotetext{
${ }^{55}$ SOFOCLES (Service-Oriented, Flexible, Collaborative LEarning Scripting), Spanish Ministry of Science and Innovation Project TIN2008-03-23.
} 
GLUE!-PS usage evidence from a slightly different perspective: that of more expert teachers and fellow teacher-researchers, both from our own research group and from external groups and educational institutions (e.g. primary and secondary teachers expert in the didactically applications of ICT).

Context and method As it has been mentioned, the session's main aim was to show the workshop participants the usage of the project's tools (including GLUE!-PS) through practical exercises in the implementation of a collaborative learning design using a Moodle-based DLE (very similar to the one used as a base scenario for workshop TW3). Thus, teachers were to deploy a CSCL design based on the Pyramid CLFP individually, using two alternative paths with project-proposed tools: 1) Using the GLUE! architecture to implement the learning design directly through Moodle's user interface; and 2) Using the WebCollage authoring tool and deploying the resulting formalization in the Moodle-based DLE with GLUE!-PS. Thus, roughly less than 1 hour would be spent using the GLUE!-PS system. In order to support participants in the task, a worksheet was provided that guided participants through the different steps in the formalization of the design and the deployment in Moodle. A photo from the hands-on session can be found in Figure 5.30

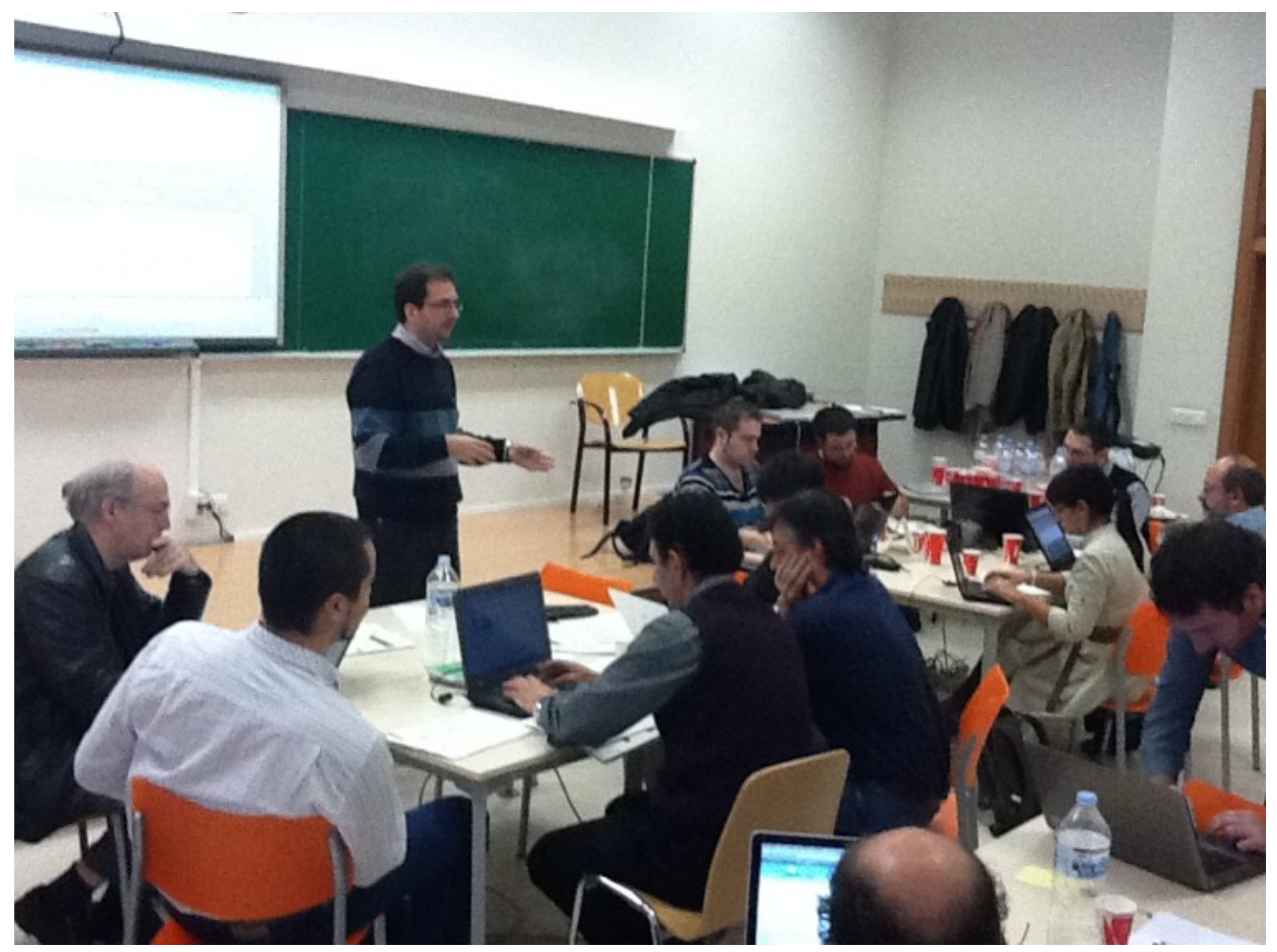

Figure 5.30: Photo taken during the TW5 evaluation happening 
Apart from the GSIC-EMIC researchers present (either as observers, facilitators or participants), 3 expert teachers were present ( 2 from secondary schools, 1 from a primary school), as well as 7 teacher-researchers from other Spanish and international research groups (with varying levels of research expertise, from $\mathrm{PhD}$ students to very experienced CSCL researchers). Because of unanticipated events, the session finally was 2.5 hours long, and it was video and audio recorded (TW5-R), and one researcher acted as external observer while two other researchers (including the author) acted as observer-facilitators (TW5-O). At the end of the session, participants answered to an online questionnaire with quantitative and qualitative questions about the session and the tools used (TW5-Q). Finally, the participant-generated artifacts (learning design formalizations, deployed courses) were used as documentary evidence for the evaluation (TW5-D). These data sources are summarized in Table 5.9.

Table 5.9: Main data sources used during the TW5 workshop

\begin{tabular}{|lll|}
\hline Source & Kind of evidence & Codes \\
\hline \hline Observation notes of the face-to-face session & Qualitative & TW5-O \\
\hline Session's video/audio recording & Qualitative & TW5-R \\
\hline $\begin{array}{l}\text { Participant-generated documents during the ses- } \\
\text { sion }\end{array}$ & Qualitative & TW5-D \\
\hline Evaluation questionnaire after the session & $\begin{array}{l}\text { Qualitative \& Quantita- } \\
\text { tive }\end{array}$ & TW5-Q \\
\hline
\end{tabular}

It is also worth highlighting that, although the session and the researchers conducting it had its own aim as evaluation of the research project's artifacts, here we will analyze the data from the point of view of this dissertation, oriented to the GLUE!-PS system as Evaluand, in a similar way as it was done for the TW3b happening. Thus, we will explore the issue "Does GLUE!-PS support teachers in the orchestration of blended CSCL across DLEs?" (I1), around the topics of the ability of GLUE!-PS in deploying learning designs preserving the design's essential qualities (T1), its time-efficiency in doing so (T2) and the feasibility of using GLUE!PS in everyday teaching practice (T3).

Similarly to workshop TW3b, this session was riddled with technical failures, including network outages, failure of several external tool services, or in the Moodle server that was being used for the tests and, in certain cases, bugs in the GLUE!-PS prototype. Several re-organizations of the steps described in the worksheet were made on-the-fly to address these problems, but finally some of the participants were able to finalize the deployment using GLUE!-PS. In any case, again the reliability of the ICT prototypes used (not necessarily of the GLUE!-PS system itself) make the following findings and evidence not as credible as we would have hoped.

Findings and evidence Regarding our first topic, the ability of GLUE!-PS to deploy CSCL designs across DLEs (T1), the ability of GLUE!-PS to deploy the proposed kind of scenario (a Pyramid-based design) had long been proven in previous experiments. However, it is interesting that, out of the external workshop participants, and despite the technical difficulties, an ample majority of the questionnaire respondents ( 7 out of $9,77.7 \%$ ) was able to deploy the learning design, and considered such deployment to support the pedagogical aims of the scenario adequately (see Table 5.10). However, these responses are not to be taken as representative of non-expert teachers' responses, since participants were more tech-savvy than the average teacher (ICT-expert teachers, teacher-researchers, etc.). This may explain why, even with all the technical problems and the lack of real hands-on experience with the system, the questionnaire results 
were rather positive. Let us remember that one of our requisites for orchestration support was that the support should be usable by an "average teacher" Dil09b.

Regarding our second topic (the time-efficiency of GLUE!-PS, which we have labeled T2), some initial evidence can be extracted from the fact that most of the external participants, with no prior knowledge of the tools, were able to design and deploy the proposed scenario in under 60 minutes. Also, a great majority of the external respondents also considered that the time employed in the design and deployment of the scenario was worth the final result, especially taking into account that the alternative implied configuring tools and groups by hand in Moodle ${ }^{56}$ (see Table 5.10), indicating they saw its potential in speeding up the current alternatives for the "design process" (i.e. the CSCL script life-cycle we saw in Section 2.2.2.

Our observation data, however also hinted that the real deployment process (the creation of the final course with the particularized design data) did not feel very "responsive" (e.g. the automated process of creating all the external tools' like shared documents for each group, etc. took around 2 minutes). This is in fact a limitation of the current prototype implementation and it could be easily replaced with other alternatives, either in the GLUE!-PS Manager service or in the LE adapters (e.g. by using a "lazy" instantiation such as the one in LAMS, where the resources are instantiated in run-time, when the student first accesses them).

We also gathered evidence that confirmed our assumption that time constraints are one of the most critical factors when adopting this kind of technologies (thus making time-efficiency a good way to measure orchestration gains, at least in the Management aspect). However, yet again the lack of more extended session time to explore the tools and the deployment process proposed substract slightly from these finding's credibility.

The applicability of the GLUE!-PS system and the rest of the Sofocles tools to everyday teacher practice (T3) was also given considerable attention in the hands-on session, which included a short debate about the subject after the tools' usage. Although the data gathered indicates that most of the external participants would use GLUE!-PS in real practice (especially due to the time-efficiency gains that it implies, see Table 5.10, several aspects were mentioned in which the prototype was lacking: the need for a more adequate support that includes contextualized examples of use in everyday practice, several usability problems that arose during the session, as well as the need for run-time flexibility. Other unexpected problems to the adoption of GLUE!-PS were hinted at, such as the large amount of different tools (built-in or external) that DLEs seem to imply, which may deter non-expert teachers who have not used them before.

Nevertheless, this evidence again has been shaped by the technical difficulties experienced during the session, which may have provoked unknown biases in the responses, which should be addressed in later iterations. Also, due to the session's design (in which the Sofocles tools, and specially the WebCollage-GLUE!-PS tandem, were used in a single exercise), it is difficult to separate the results that refer to GLUE!-PS from those that refer to the complete designinstantiation-deployment process.

Furthermore, this evaluation happening also prompted the reflection that, even if all the aforementioned enhancements to GLUE!-PS were implemented, the fact that implementing our approach relies on many external services and tools might hamper its widespread adoption (since

\footnotetext{
${ }^{56}$ They could make such comparations because, as we have mentioned, in the same session they had tried an alternative way of deploying the same design to a Moodle-based DLE, through the GLUE! architecture.
} 
Table 5.10: Topics, findings and selected supported evidence from evaluation happening TW5

\begin{tabular}{|c|c|}
\hline Topic & Finding \\
\hline \multirow[t]{3}{*}{ T1 (deploy ability) } & $\begin{array}{l}\text { GLUE!-PS deployments } \\
\text { support the design's essential } \\
\text { qualities }\end{array}$ \\
\hline & $\begin{array}{l}\text { ICT-expert teachers were able } \\
\text { to deploy a LD using GLUE!- } \\
\text { PS }\end{array}$ \\
\hline & $\begin{array}{l}\text { Deployment is not "respon- } \\
\text { sive" }\end{array}$ \\
\hline
\end{tabular}
Selected supporting evidence

8 out of $9(89 \%)$ external respondents said that the deployed result supported adequately the realization of the proposed scenario [TW5-Q]

7 out of $9(78 \%)$ external respondents said that the result complied with the pedagogical restrictions of the scenario (the rest did not respond negatively either) [TW5-Q]

$\mathrm{T} 2$ (time-efficiency)
GLUE!-PS can speed up the design process, compared to the alternatives
7 out of $9(78 \%)$ external respondents asserted that they were able to translate the design into the ICT infrastructure using GLUE!-PS [TW5-Q]

[During the hands-on session, while users were creating the Moodle course] Going from WebCollage to GLUE!-PS worked OK. Now we wait for GLUE!-PS to create the instances [i.e. each of the external tool documents for each group]. It takes almost 2 minutes. It is too much! [TW5-O]

7 out of $9(78 \%)$ external respondents with no prior knowledge of the tools, were able to deploy the design in under 60 minutes, with the sole help of a worksheet [TW5-R]

8 out of $9(89 \%)$ external respondents answered that the time employed was worth the final result of the deployment [TW5Q]

[Why do you think the time used to design and deploy was worth it?] As said before the tool(s) can greatly speed up the CSCL design process and can become a useful teacher designkit (maybe in an improved interface format being somehow more intuitive at certain points [...] [TW5-Q]

[Why do you think the time used to design and deploy was worth it?] because the alternative in terms of time is not suitable [TW5-Q]

[Why do you think the time used to design and deploy was worth it?] Because the needed time to create all the tools and permissions makes its usage worth it [TW5-Q]

Importance of time constraints [About the applicability collaborative LD tools in primary and time-efficiency compared classrooms] teachers normally say 'it is complex, it takes time, to learning gains and we do not have time' [...] you have to convince them that this can lead to learning [TW5-R]

Session time was not sufficient (Why do you think the time used to design and deploy was to grasp the deployment pro- (not) worth it?] We would have needed some more time to get cess

ICT-expert teachers would use GLUE!-PS in real practice

T3 (use in real practice)
Low reliability of data due to technical failures during the test

Need of contextualized exam-
ples of use

Need of further flexibility and usability

Non-expert teachers may have problems using DLEs given the amount of different tools

Time-efficiency of GLUE!-PS as a favorable factor for real practice use to know the whole process [TW5-Q]

7 out of $9(78 \%)$ external respondents said that they would use WebCollage and GLUE!-PS in real practice [TW5-Q]

[During the hands-on session, when the session ended and participants were asked to fill up the questionnaire] The questionnaire is not prepared for a session where [external] technological tools fail... it can damage the perception [of our tools] due to external factors [TW5-O]

[When asked about the applicability of collaborative tools like WC/GLUE!-PS] if I do not have models [examples] of use in reality, teachers are not going to use it [TW5-R]

[When asked about the general impression about WC and GLUE!-PS] the automation of the flow beforehand is nice, but it is not so flexible [...] I want to change [the design], move things [i.e. drag-and-drop] [TW5-R]

[Why you would use GLUE!-PS in real practice?] In courses where you have lot of groups it could be an easy way to perform the orchestration. However, the use of this quantity of different services could restrict the use of the whole architecture to many teachers [TW5-Q]

[Why you would use GLUE!-PS in real practice?] In order to avoid great amounts of useless time managing groups in Moodle, and the configuration of the external tools. [TW5-Q] 
that introduces multiple points of failure, and technological unreliability is one of the greatest fears of teachers when dealing with ICT [Vil09]. This fact also points toward the hypothesis that this approach will not be adopted until it is flexible enough to cope with all these "things that might go wrong".

\section{Partial conclusions and formative feedback}

Several partial conclusions can be extracted from the evaluation happenings conducted in this second iteration of GLUE!-PS evaluation (see Figure 5.31). If we analyze these conclusions from the point of view of the three focusing topics we had chosen for this iteration, we can observe that:

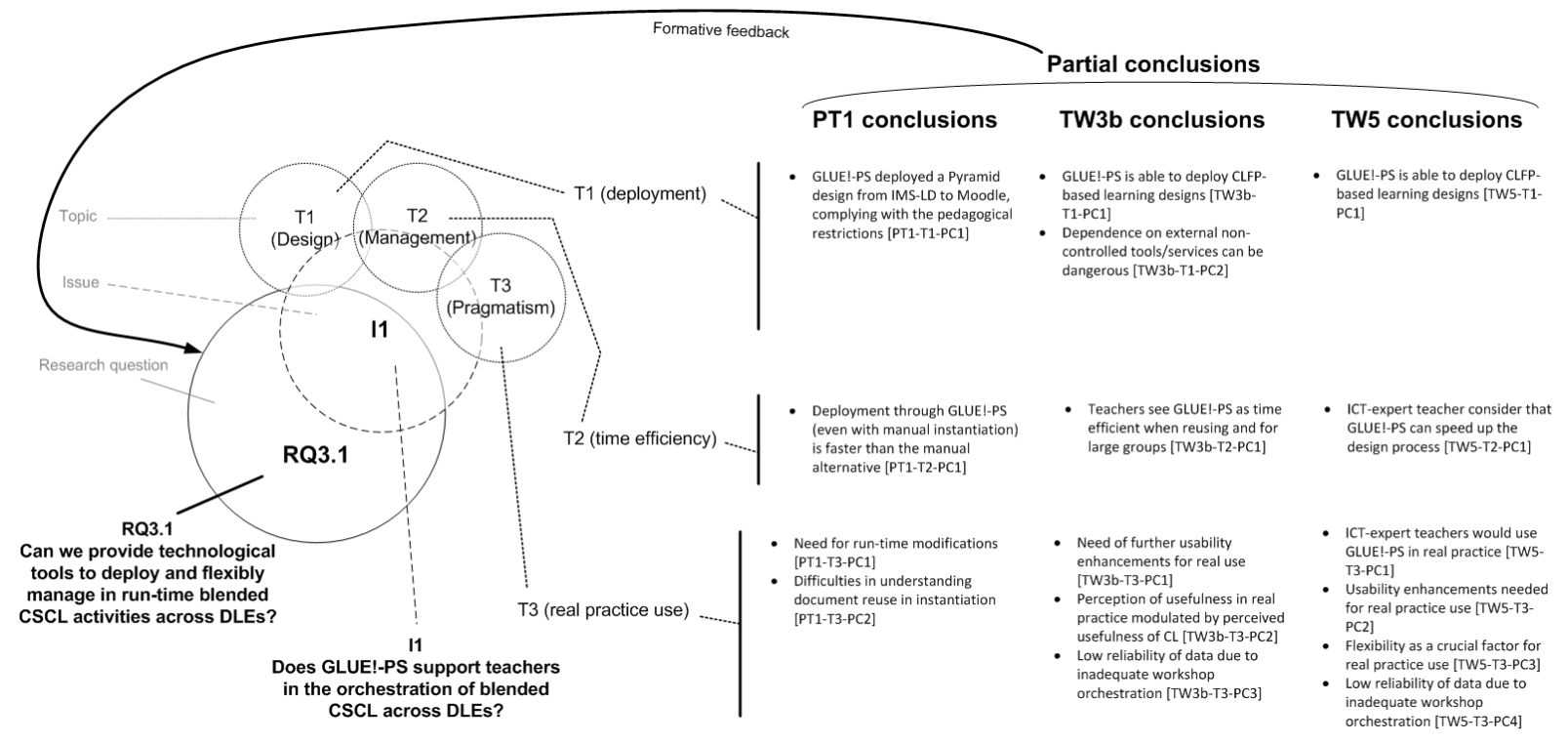

Figure 5.31: Graphical representation of the issues, topics and partial conclusions of Iteration $\# 2$

T1 (deployment ability) GLUE!-PS had showed good capability for supporting the deployment of Pyramid-based (PT1-T1-PC1) and other CLFP-based collaborative learning designs (TW3b-T1-PC1, TW5-T1-PC1), expressed in IMS-LD with the WebCollage authoring tool, into a Moodle-based DLE, in a way that complied with the pedagogical essential qualities of the designs. Also, the different technical problems experienced during the evaluations, many of which were not caused by the GLUE!-PS prototype itself but rather by other elements, hint at the dangers of the distributed, integrative approach of DLEs in general, and of the GLUE!-PS proposal in particular (which relies on multiple tools to complete the CSCL life-cycle), highlighting the need for flexibility and other "graceful degradation" mechanisms.

T2 (time-efficiency) GLUE!-PS showed first evidences of time-efficiency when compared with the manual deployment of CSCL designs to DLEs, even in absence of a graphical interface (PT1-T2-PC1). Expert teachers and teacher-researchers saw the potential of GLUE!-PS to 
speed up the CSCL lifecycle (TW5-T2-PC1), although non-expert teachers (with a mostly technical average profile) were not as enthusiastic, and considered GLUE!-PS especially useful only in certain cases, when reusing learning designs, and for orchestrating large groups of students working in a DLE (TW3b-T2-PC1).

T3 (use in real practice) Finally, despite the limitations of the conducted experiments, this iteration also provided us with first evidence about the usefulness and challenges of usage of the GLUE!-PS proposal in real practice. While ICT-expert teachers and teacherresearchers asserted that they would use GLUE!-PS in real practice (TW5-T3-PC1), many problems were detected by the different non-expert teachers that used the system in one way or another: aside from usability problems that needed to be addressed in later prototypes (TW3b-T3-PC1, TW5-T3-PC2), the lack of run-time flexibility was the most widely acknowledged shortcoming of the GLUE!-PS prototype for real practice use (PT1-T3PC1, TW5-T3-PC3). Other, more conceptual obstacles to the use of GLUE!-PS in real practice were also hinted at by the collected evidence: the notion of document reuse in a particularized learning design, despite being a common CSCL practice, proved difficult to understand for teachers (as it was to design a GUI for managing it) (PT1-T3-PC2). Also, we gathered evidence that the pedagogical beliefs of teachers (especially regarding the applicability of the CSCL approach to everyday practice) were affecting the perceived usefulness of the presented technologies (as they did for the other dissertation contributions) (TW3b-T3-PC2).

However, all these preliminary conclusions have been greatly modulated by the scope limitations of some of the trials, the insufficient time dedicated to the usage of the tools in the different workshops, and the unexpected technical problems experienced during the different workshop sessions. All these aspects make the gathered data not as credible as it was expected (TW3b-T3-PC3, TW5-T3-PC4). Also, it should be noted that in this iteration there were some clear biases in the population of participants, which should be acknowledged and taken into account when considering the aforementioned conclusions: a) most of the participants in the happenings had a technical background, or were considered ICT-expert teachers, thus making them much more accustomed to ICT usage than the "average teacher"; b) Most of the participants, especially in the PT1 and TW5 happenings, had a positive attitude towards the usage of CSCL in education, which probably made them "take CSCL for granted", a factor that can heavily affect whether you accept to use an ICT tool intended for the orchestration of CSCL activities.

Overall, this evaluation iteration provided us with certain evidences of the usefulness of GLUE!-PS, as well as illustrating potential problems for its adoption in real practice. The credibility of these findings, however, is rather weak (due to the limited "real use" of the system by teachers). This evaluation did, however, provide valuable formative feedback to the following research iterations, both regarding the kind of evaluation happenings that should be conducted (towards more authentic settings), the implementation of the evaluations (more emphasis on the reliability of the systems involved, availability of "B plans", and so on) and to the implementation of the GLUE!-PS prototype itself (usability enhancements, need of run-time flexibility features). Moreover, we also concluded that the aforementioned biases in the evaluations' informants should also be addressed, to the extent that our limited resources made it possible. 


\subsubsection{Iteration \#3: Extending VLE support in GLUE!-PS - a wiki-based experiment}

After the first round of empirical validations that used the GLUE!-PS prototype to gather evidences about the orchestration support that the proposed architecture and data model provided for teachers, it was time to test the prototype in a more authentic situation (e.g. for the deployment of a real learning design integrated in the usual curriculum of a course). Also, it was clear that most of our data so far had been focused on Moodle-based Distributed Learning Environments, a fact which might have slanted our data in unascertained ways. Thus, we set out to develop a second LE adapter: the MediaWiki LE adapter, which translates learning designs to linked structures of wiki pages (including external tools) representing a course, or a part of a course. This choice was motivated by the need of exploring the frontiers of the "VLE area" (MediaWiki cannot be strictly considered a VLE, albeit it is one of those "Web 2.0" applications which have been used in a similar way to a VLE), to assess the extent to which our approach to orchestrate DLEs is applicable outside the VLE (Personal Learning Environments could have been another possibility in this regard). Another, more pragmatic motive for choosing MediaWiki was the fact that several teacher-researchers at the multi-disciplinar GSICEMIC research group used this environment as the VLE-like center of the ICT support for their courses, which would be likely subjects for the kind of authentic experiments we were aiming at. This new development within the GLUE!-PS prototype, together with a number of usability and reliability enhancements, led us to proposing a new evaluation iteration through an authentic experiment in a master-level course on learning-teaching techniques (AE1), in February-March 2012. The findings and evidence of this evaluation happening are detailed below.

\section{First authentic deployment experiment (AE1)}

Context and method This authentic experiment was set to take place within a course in the master-level degree on Secondary Education, Vocational and Language Teaching at the University of Valladolid $[57$. The course's contents were aimed at future "Technology and Computer Science" teachers in high schools, and they dealt with learning and teaching approaches.

The teacher that was going to design, deploy and enact the activities in this authentic experiment (an expert teacher belonging to the GSIC-EMIC group, with prior CSCL experience) was one of the three teachers that shared (sequentially) the teaching of this course. This teacher had volunteered to try the WebCollage authoring tool and GLUE!-PS to generate the ICT support for a collaborative scenario she intended to enact as part of the course: the collaborative inquiry and generation of a poster summarizing the main teaching-learning strategies seen in the course, following the Jigsaw CLFP (see Section 4.2.3 based on the works by Aronson and others Aro92]). The students were 14 bachelor-level graduates, mostly with Engineering and Computer Science backgrounds.

The CSCL design that the teacher had in mind followed the typical structure of a Jigsaw, with a peer review added in the third phase of the scenario. It was intended to be enacted in a blended learning format, comprising the following phases that spanned almost 3 weeks and

\footnotetext{
${ }^{57}$ http://master.uva.es/profesor-de-educacion-secundaria-obligatoria-y-bachillerato-formacion-profesional-y-ensen (Last visit: 13 Jun 2012).
} 
three face-to-face sessions (the structure of the design is also graphically represented in Figure 5.32 , using the WebCollage authoring tool):

1. An individual study phase, in which each student researched, studied and produced a summary (in a wiki page) of the main features of two of the six teaching-learning approaches to be considered during the course. This activity was to be started in a face-to-face session, and completed online.

2. An "experts" phase, in which the students that had worked on the same techniques had to meet, review the generated material and agree to a graphical schema that summarized the main features of the studied techniques. This graphical representation was to be generated with a shared whiteboard tool called Dabbleboard 58 (which is supported by the GLUE! architecture). Also, students were to answer a brief questionnaire about the collaborative work done (for assessment purposes, and to be used in a parallel research effort, see below). This phase also spanned part of a face-to-face session, plus an amount of online work time.

3. A "jigsaw" phase, in which students were joined with others that had studied different techniques, and they were to agree a selection of the techniques they thought most adequate to teach about technology (the selection was to be published through an online Google Forms questionnaire). Then, they were to elaborate a poster (using whichever tool they liked, but uploading the final result to the wiki) summarizing the advantages and shortcomings of such selected techniques. These posters were then to be revised by their peers (by editing the wiki page where the posters had been embedded), prior to their presentation in a face-to-face session. After this presentation session, students were to answer another online questionnaire about the collaborative groupwork, and they were to peer-review their classmates' poster presentations through another online questionnaire. This phase spanned several hours of online work, both before and after the face-to-face presentation session itself.

During this authentic experiment, we gathered data in a number of ways, including the recording and observing of the co-design (and co-deploy) sessions with the teacher (AE1-R and AE1-O, respectively), as well as by using the different artifacts generated by the teacher (the design done in WebCollage, the particularization data in GLUE!-PS and the deployed infrastructure generated across the DLE, which the students used during the experiment) (AE1D). Furthermore, a semi-structured interview was conducted after the experiment (AE1-I), to gather the teacher's reflections on the usage of the GLUE!-PS system. Given the lack of run-time flexibility properties of the GLUE!-PS prototype at the time, we decided not to record or observe the enactment's face-to-face sessions (since our perspective was to analyze orchestration support for the teacher, and to avoid further data overload). All these data sources are summarized in Table 5.11 .

It is also important to note that this authentic experiment was also used in a parallel research effort on a design-informed monitoring process [RT12. Although this somehow substracts from the authenticity of the design (some of the aforementioned student questionnaires were conditioned by that parallel effort), we can safely assert that the teacher would have enacted a very similar design in absence of these research efforts. Furthermore, the data gathered

\footnotetext{
${ }^{58}$ http://www.dabbleboard.com/ (Last visit: 13 Jun 2012).
} 

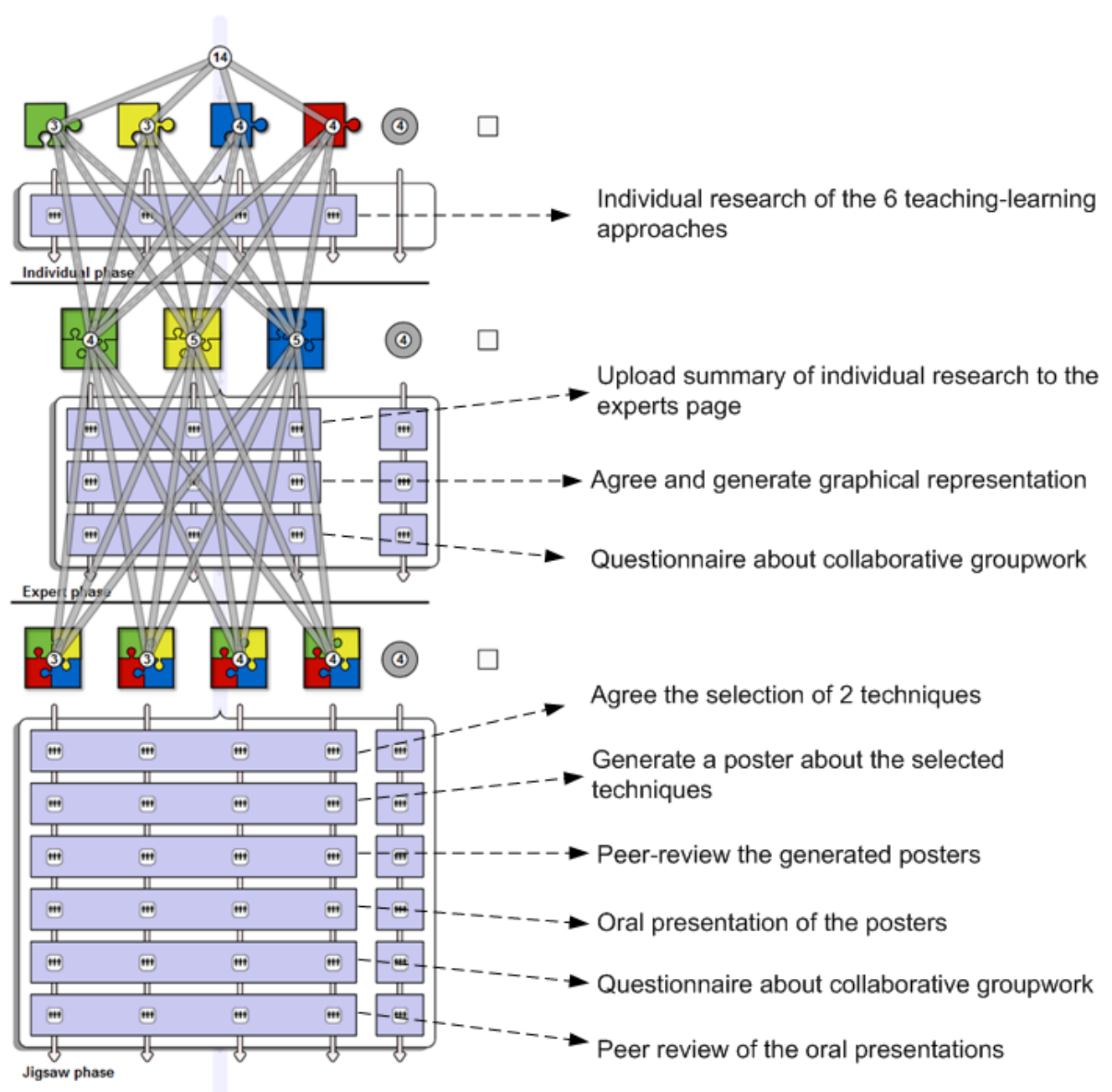

Figure 5.32: Graphical representation of the design done by the teacher in happening AE1

around this "slightly more complex than normal" learning design orchestration will provide equally valuable data about GLUE!-PS's capacity to support orchestration across DLEs. Regarding our own evaluation focus, here we will analyze the authentic experiment data from the point of view of this dissertation, oriented to the GLUE!-PS system as Evaluand, exploring the issue "Does GLUE!-PS support teachers in the orchestration of blended CSCL across DLEs?" (I1), around the topics of the ability of GLUE!-PS in deploying learning designs preserving the design's essential qualities (T1), its time-efficiency in doing so (T2) and the feasibility of using GLUE!-PS in everyday teaching practice (T3). Moreover, even if we did not study GLUE!-PS support for Adaptation (since it had not been implemented at the time), we gained first insights on the run-time flexibility (T4) that is most often needed in this kind of authentic situations.

Findings and evidence Before detailing the evidence gathered around these topics, it would be interesting to describe how the co-design (and co-deployment, we should say) process took 
Table 5.11: Main data sources used during the AE1 evaluation happening

\begin{tabular}{|llc|}
\hline Source & Kind of evidence & Code \\
\hline \hline $\begin{array}{l}\text { Observation notes of the design and deployment } \\
\text { sessions }\end{array}$ & Qualitative & AE1-O \\
\hline $\begin{array}{l}\text { Design and deployment session's video/audio } \\
\text { recording }\end{array}$ & Qualitative & AE1-R \\
\hline $\begin{array}{l}\text { Participant-generated artifacts along the process } \\
\text { (WebCollage, GLUE!-PS, MediaWiki data) }\end{array}$ & Qualitative & AE1-D \\
\hline Post-experiment semi-structured interview & Qualitative & AE1-I \\
\hline
\end{tabular}

place. Several 1-2 hour face-to-face meetings with the teacher took place in the weeks leading to the enactment of the designed CSCL scenario. In the first session, the teacher explained her (abstract) learning design ideas with two researchers (the main researcher in the parallel research effort and the author), checking the technologies that could be used for the different activities and whether that particularization complied with the research goals of both efforts. In this sense, although the general idea of the design was clear, the details (especially the concrete materials and tools that were needed for the enactment) emerged as the teacher and researchers discussed.

After that, a second session dedicated to the design formalization (using WebCollage) and deployment with GLUE!-PS took place (see Figure 5.33). In this session, the teacher successfully formalized the design in WebCollage (despite certain usability glitches) in 75 minutes, and did a first deployment of the activities in the wiki, in 44 minutes. However, during the particularization process it became clear for the teacher that the resulting infrastructure would not be adequate (either because of the way the activities were presented, or due to user errors in the formalization with WebCollag $\AA^{59}$ ). Thus, it was decided to make modifications to the GLUE!-PS prototype (e.g. to provide clearer labeling and awareness about group components) and re-formalize the script in a later session.

In the third session, the formalization of the learning design with WebCollage was modified to address the previous session's problems (which, given the ability of WebCollage to provide certain particularization data, also impacted greatly the way the design was transformed into a wiki page structure by GLUE!-PS). In a fourth session, this formalization was completed with the concrete group and participant information (in WebCollage), and deployed again with GLUE!PS. However, the resulting wiki structure was not entirely satisfactory, especially taking into account that a few errors were detected in the formalization of the design and its particularization (e.g. in the definition of the documents to be reused in the peer review task).

Finally, the teacher did the definitive WebCollage modeling online, and met with the researchers for a fifth time, in order to perform the final deployment of the activities in the wikibased DLE. Several unanticipated bugs in the software tools used appeared during this session, and prevented the teacher from completing the deployment herselfor. After this final deployment,

\footnotetext{
${ }^{59}$ As an example of this, the teacher wanted the individual work of phase 1 to be written to a wiki page by topic/expert group (for easier reference during phase 2 - the expert groups' work). However, WebCollage structures the IMS-LD model in phase 1 grouping students in the same "jigsaw groups" of phase 3. As a workaround, the teacher re-modeled phase 1 in WebCollage, which was re-defined as the "inquiry work", and the writing of such inquiry into the wiki was modeled as a first task in phase 2, so that the resources (the wiki pages) were correctly grouped by expert's topics.

${ }^{60}$ The deployment was actually completed by the author, 10 minutes after the deployment session finished, once the bugs were taken care of.
} 


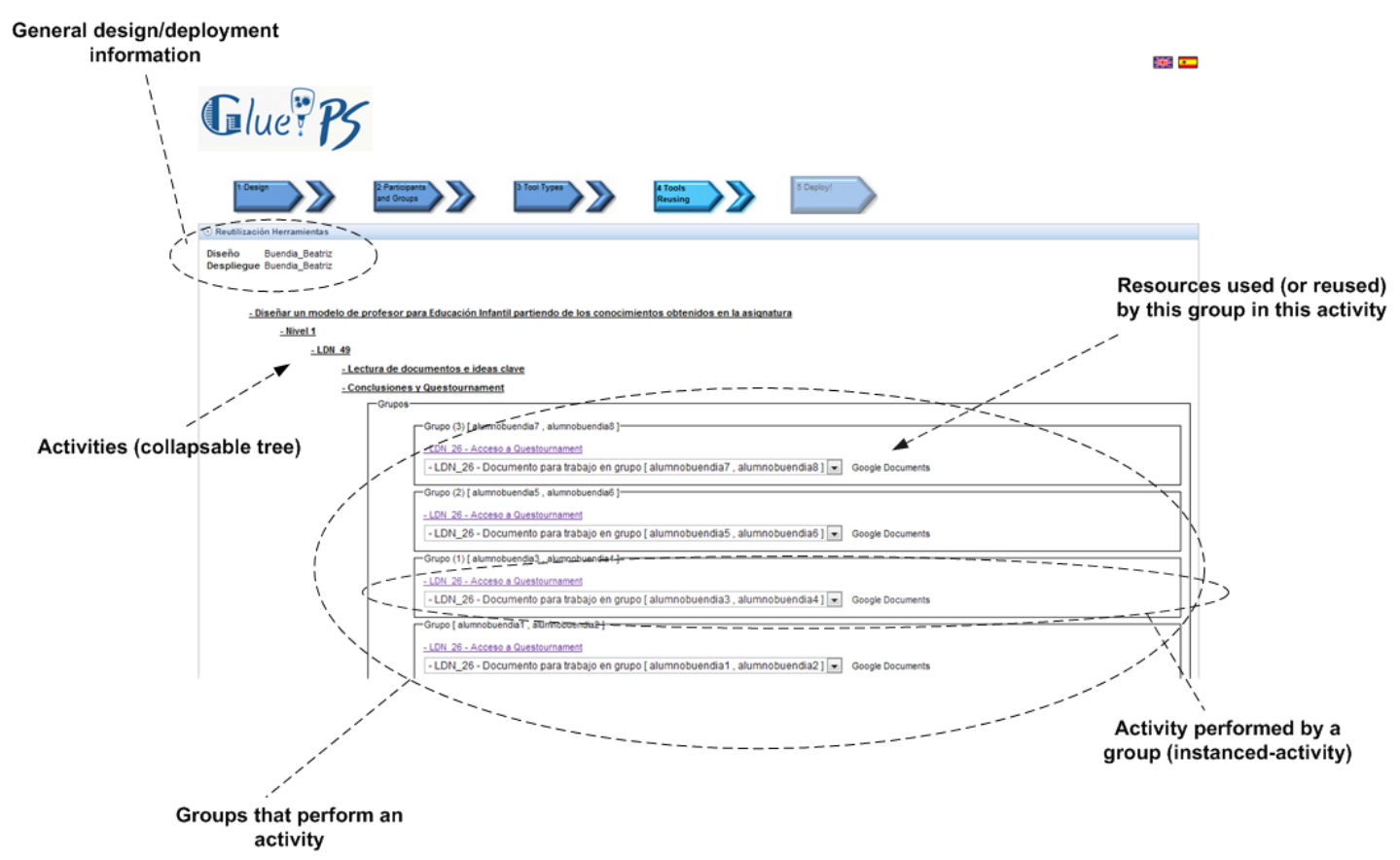

Figure 5.33: Screen capture of the (outdated) first version of the GLUE!-PS GUI. Includes basic explanation of the interface parts.

the enactment was done by the teacher with no major problems (see also the evidence below). Let us now look at the evidence from the point of view of our four topics around the evaluation issue we had defined.

Regarding GLUE!-PS's ability to deploy blended CSCL designs (T1), in this case to a different kind of DLE (based on a wiki), again GLUE!-PS demonstrated that it was able to deploy a CLFP-based learning design specified in IMS-LD, while complying with what the teacher considered the design's essential qualities (see Table 5.12). This last aspect is worth further commentary since, as we saw in our analytical evaluations (AN1, see Section 5.5.2), MediaWiki was not able to perform a strong access control on the resources (which might be seen as essential to the design or not, depending on the teacher style and the design itself). In this case, it seems that the possibility, e.g., of students copying one another, was not considered a relevant danger, while the open access to the learning community-generated artifacts was important.

Interestingly, the use of GLUE!-PS on a real learning situation also prompted the teacher to propose a few extensions to the system, regarding information that might be useful to detail while doing the particularization of the design (thus meaning minor extensions to the GLUE!-PS LF model, see Section 5.2.3): one is to add a description to the activity that each group performs, within an abstract activity in the design (the concept of "instanced activity" in GLUE!-PS LF), in order to provide group-specific activity instructions. The other extension would be to add timing information (e.g. deadlines) to the activities. Even if this timing information may not be enforced by the target learning platform (as in the case of MediaWiki), it can be used for presentation purposes, i.e., a management tip to remind students of the expected timing. Moreover, a stronger enforcement of this timing could be possible, just by implementing an LE 
adapter that implements such mechanisms.

Regarding the topic of GLUE!-PS time efficiency, the iterative design-deploy process followed by the teacher does not help to see clearly that advantage. However, in the interviews (see Table 5.12) the teacher expressed that she considered the deployment part of the process fast (i.e. the part that is performed with GLUE!-PS). Also, the teacher highlighted that, despite the long total time dedicated to the multiple iterations, the use of GLUE!-PS (and the rest of the LD process) was worth the effort, in the case of designs that included student-generated artifact reuse and resource access control. Moreover, the teacher also remarked that reusing the learning design the following years would be even faster.

Table 5.12: Topics, findings and selected supported evidence from evaluation happening AE1, around topics T1 (deployment ability), T2 (time-efficiency) and T4 (run-time changes)

\begin{tabular}{|c|c|c|}
\hline Topic & Finding & Selected supporting evidence \\
\hline \multirow[t]{3}{*}{ T1 (deploy ability) } & $\begin{array}{l}\text { GLUE!-PS deployment in } \\
\text { wikis support the design's } \\
\text { essential qualities }\end{array}$ & $\begin{array}{l}\text { [to the question: does the deployed wiki reflect your idea of } \\
\text { the design?] the positive side is the structuration of activities } \\
\text { in pages [the activity tree depicted as links to activity wiki } \\
\text { pages, see Figure } 5.34 \text {, is nice, and helps [...] the goals I had } \\
\text { to edit them [...] but that's secondary [AE1-I] }\end{array}$ \\
\hline & $\begin{array}{l}\text { ICT-expert teachers are able to } \\
\text { deploy a LD using GLUE!-PS }\end{array}$ & $\begin{array}{l}\text { GLUE!-PS successfully deployed the teacher's learning design } \\
\text { (several times) [AE1-O] }\end{array}$ \\
\hline & $\begin{array}{l}\text { Suggested expansions to } \\
\text { GLUE!-PS LF }\end{array}$ & $\begin{array}{l}\text { [in the second deployment session, reviewing the resulting } \\
\text { wiki] A [the teacher] notes that it would be useful to pro- } \\
\text { vide a differentiated description in each activity per-group } \\
\text { [i.e. GLUE!-PS LF's concept of instancedActivity] - possible } \\
\text { needed expansion of LF [AE1-O] } \\
\text { [when asked about the deployed design's shortcomings] I had } \\
\text { to add the deadlines for the contributions [AE1-I] }\end{array}$ \\
\hline \multirow[t]{2}{*}{ T2 (time-efficiency) } & $\begin{array}{l}\text { GLUE!-PS is efficient for cases } \\
\text { of artifact reuse, access control } \\
\text { to resources and LD reuse }\end{array}$ & 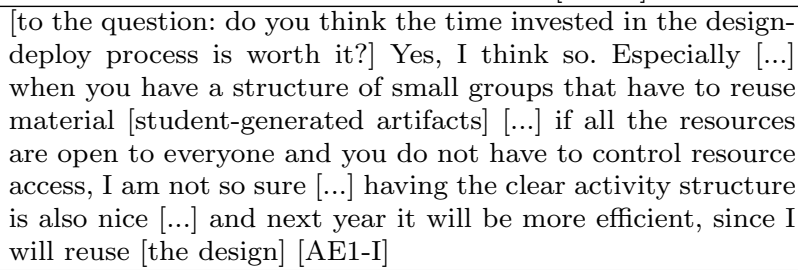 \\
\hline & $\begin{array}{l}\text { The teacher considered the de- } \\
\text { ployment fast (compared to } \\
\text { the design) }\end{array}$ & $\begin{array}{l}\text { [when asked to estimate the time that had taken the whole } \\
\text { design-deploy process] I think the design is the most costly } \\
\text { part [...] the design was fast, although we had some technical } \\
\text { difficulties }[\ldots] \text { and the fine-tuning in the wiki was minimal }\end{array}$ \\
\hline T4 (run-time changes) & Run-time changes needs & $\begin{array}{l}\text { [when asked about changes in the design/deployment that } \\
\text { were needed during run-time] I had to do changes in the dead- } \\
\text { lines [...] I had to re-structure some things in the wiki }[\ldots] \\
\text { some submissions were done in the wrong place }[\ldots] \text { I would } \\
\text { have liked to add or remove documents }[\ldots] \text { we had to substi- } \\
\text { tute one of the Google Forms that failed, for another Google } \\
\text { Forms [AE1-I] } \\
\text { [when asked about useful run-time changes that were not } \\
\text { needed during the enactment of the scenario] I think group } \\
\text { re-configuration is the clearest one }[\ldots] \text { changes in the group } \\
\text { configuration }[\text { AE1-I] }\end{array}$ \\
\hline
\end{tabular}

In this evaluation happening we first obtained first-hand information about the use of GLUE!-PS in real everyday practice (T3), even if by a teacher-researcher that was experienced in CSCL (thus, not a really "average teacher"). In this regard, probably the most striking finding of this experiment was the iterative nature of the design and deployment process using a LD authoring tool and GLUE!-PS, which took 5 face-to-face sessions. Although the typical depictions of the CSCL lifecycle (see Section 2.2.2 normally propose a cyclical but linear process, the fact 


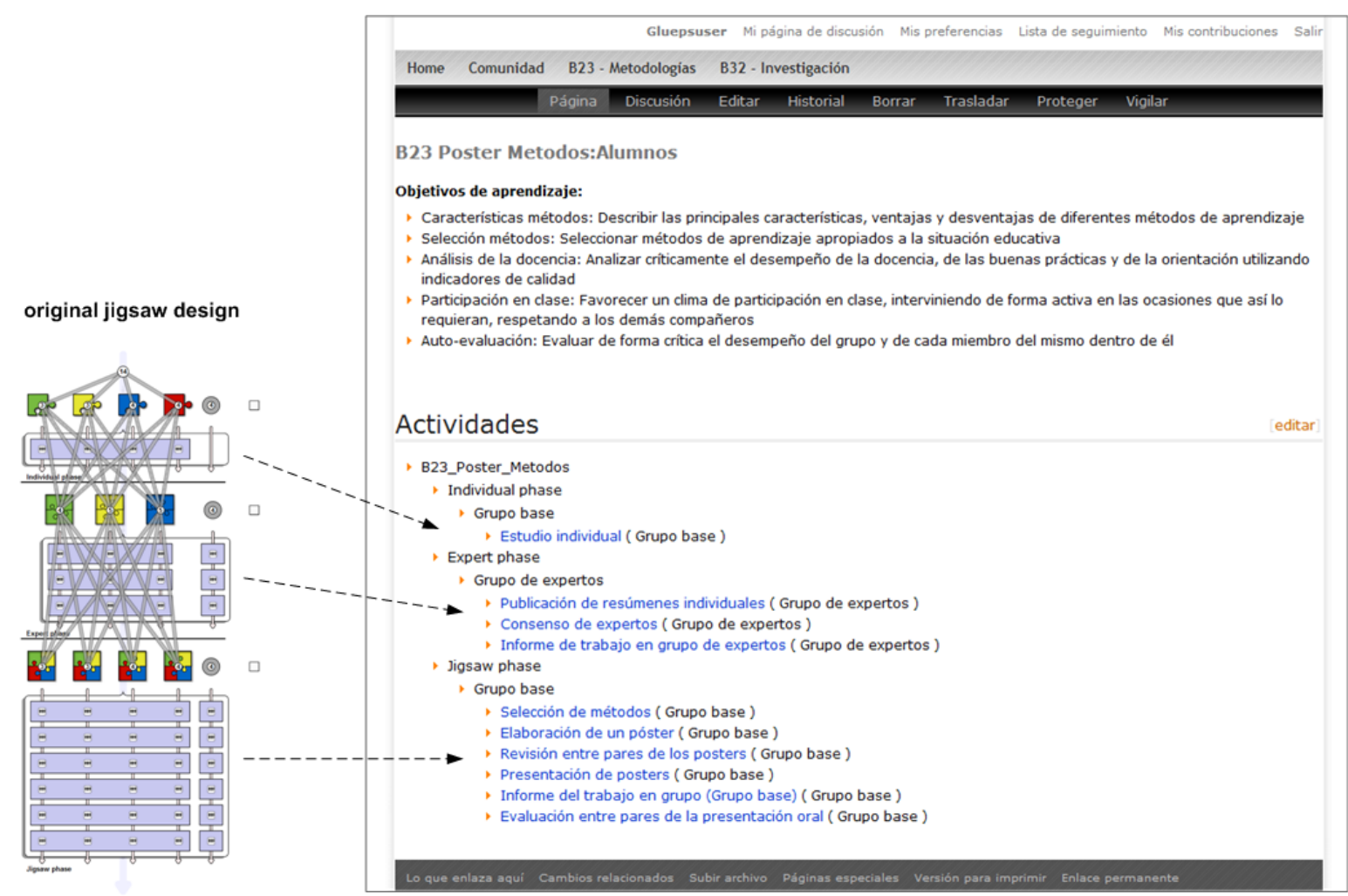

Figure 5.34: Screen capture of the Jigsaw learning design used in happening AE1, once it was deployed in a wiki by GLUE!-PS. Includes also the equivalence with the original learning design depicted in Figure 5.32 .

that the deployment is automated makes it very important to specify the LD formalization in such a way that the final presentation will fulfill the teacher's expectations. Thus, even an experienced teacher normally has to go back and forth a number of times between the different phases until satisfied by the results. This is in accordance with research that states that many teachers act as "bricoleurs" when implementing learning activities with ICT (e.g. in Moodle, see [Ber05]), trying different options until satisfied with the result. As noted by Ber05, the separation of design and implementation imposed by LD approaches somehow clashes with this way of working. Indeed, by trying to close the "deployment gap", GLUE!-PS can make this going back and forth more agile, thus enabling this kind of "bricolage". However, the GLUE!-PS prototype using during the experience was still lacking in this regard, since it did not allow users to modify all aspects of the particularized design (e.g. activity descriptions, resources), a fact that forced the teacher to go all the way back to the authoring tool (WebCollage). Also, the batch creation of the whole DLE infrastructure (including the external tools), which takes a few minutes, somehow hampers this iterativeness. Thus, alternative ways of providing direct feedback on the final result (or rather, how it might look like) would be a worthy addition to support this kind of behavior (what the teacher calls the "WYSIWYG" - What You See Is What You Get- editor).

Other factor that became obvious throughout this happening was the low reliability of the GLUE!-PS prototype itself, and of the different elements with which it interacted (especially, the 
GLUElet Manager service in the GLUE! architecture). This lack of reliability and the dependence on (also experimental) external services certainly hampered the user experience when deploying, albeit the teacher, a researcher herself, was used to this kind of failures. Although these reliability issues did not impact the rest of the orchestration (e.g., students did not have too many problems enacting the activities), they confirmed our reluctance to use these early prototypes with nonexpert external teachers that may have been frustrated by them.

This real practice usage also highlighted other teacher needs when particularizing and deploying learning designs across DLEs, especially concerning the need of tweaking all aspects of the particularized design in the GLUE!-PS LF model (something that the GLUE!-PS GUI did not yet allow in this prototype), as part of the aforementioned iterative process of "getting the result right", but also to fix shortcomings in the formalization that the LD tool does of the design ideas (e.g. in WebCollage, the way it "understands the jigsaw pattern"). Also, the need for alternative LE adapter implementations (which decide the way the particularized design is presented in the learning platform) appeared prominently, and in fact different variations of the MediaWiki LE adapter were implemented during the experiment, to cater for the teacher's needs. This fact also highlights the power of the GLUE!-PS adapter architecture, which has the potential to address the different needs that different teachers or different designs might have (even when working with the same learning platform).

Table 5.13: Findings and selected supporting evidence from evaluation happening AE1, around topic T3 (usage in real practice)

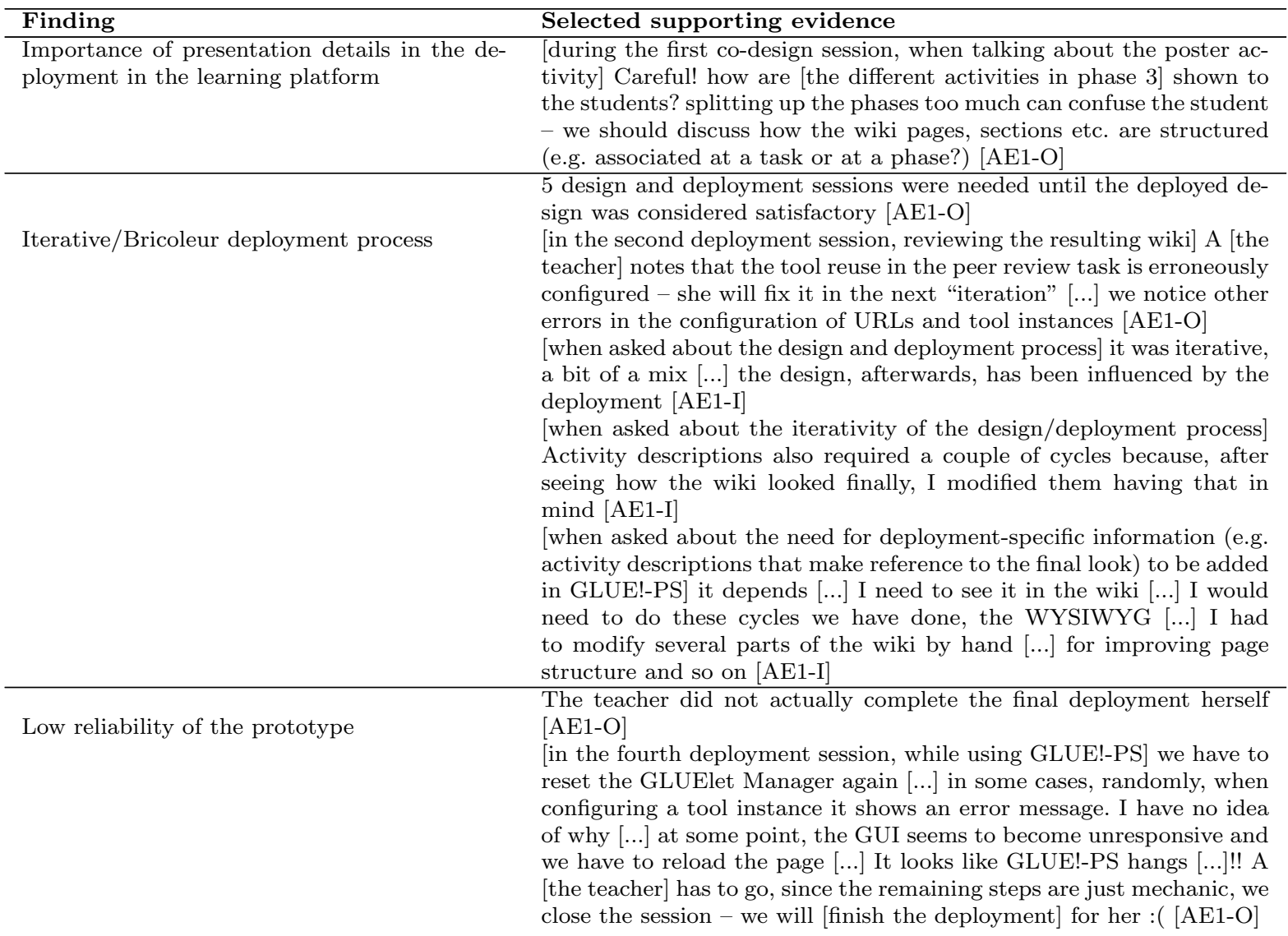


Table 5.13 (continued from previous page)

\begin{tabular}{l}
\hline Finding \\
\hline \\
\hline $\begin{array}{l}\text { Need for alternative LE adapter } \\
\text { implementations }\end{array}$
\end{tabular}
Selected supporting evidence

[to the question: were you able to complete the deployment of the design?] well, I required some help [...] to identify the different tool types $[. .$.$] the difference between document and tool [the two kinds of$ resources possible in WebCollage and the GLUE!-PS prototype] was not clear $[. .$.$] the concept of reusing was useful, but took some time$ to get used to [AE1-I]

implementations

[in the second deployment session, reviewing the resulting wiki] A [the teacher] notes that the non-leaf activities [in GLUE!-PS LF's modeling activities of different granularities are implemented in logical trees] are not very useful - we should implement a variant of the deployer [...] she still thinks that a "student view" (without the teacher activities) and a "teacher view" (complete) would be very useful [AE1-O]

[when asked about the deployed design's shortcomings] there are too many links [...] you have to click too many times to reach the activity resources $[\ldots]$ other interesting thing would be $[\ldots]$ some kind of portfolio of each person/group [...] that is very useful when assessing [AE1-I]

Need for fixes around the LD authoring tool [in the second deployment session, reviewing the resulting wiki] the formalization fact that phase 1 is structured around the jigsaw group [the way WebCollage models the individual study phase is with a jigsaw group, not individually] can confuse students [AE1-O]

[in the second deployment session, reviewing the resulting wiki] A [the teacher] notes that very often the activity instructions are written taking into account the final look [in the learning platform], which now implies going back to the beginning [WebCollage] - need of altering the design in GLUE!-PS [AE1-O]

[in the second deployment session, when the researcher asks the teacher about general impressions of the process] the dependencies between the different tools [WebCollage, GLUE!-PS, MediaWiki] and phases are complex, non-linear... GLUE!-PS could be very useful to do re-designs taking into account the final implementation [...] a "WYSIWYG deployment" would be great! [AE1-O]

[when asked about designing with WebCollage] at the beginning it was confusing, because of the idea WebCollage has of implementing the group structures [...] made things look, in the deployment, differently than what I had in mind [...] that is one of the reasons why we had to follow several cycles [...] the final design reflects what I wanted to do, but we had to go around a little bit to obtain it [AE1-I]

\begin{tabular}{ll}
\hline Need for particularization editing in GLUE!- & $\begin{array}{l}\text { [during the first deployment session, while using GLUE!-PS] when } \\
\text { we reach step } 3 \text { [deciding the tool types for the resources defined }\end{array}$ \\
& $\begin{array}{l}\text { in WebCollage], A [the teacher] asks whether you can change the } \\
\text { resources you had assigned to the activity - clear need of changing } \\
\text { the instantiation in GLUE!-PS [AE1-O] }\end{array}$ \\
\hline $\begin{array}{l}\text { No important orchestration problems from the } \\
\text { student perspective }\end{array}$ & $\begin{array}{l}\text { [when asked about problems that students had during the enactment] } \\
\text { some complained about the aesthetic of the wiki [...] other were due to } \\
\text { my handling of Google Forms [...] there were problems about knowing } \\
\text { where to put their submissions [...] some of them were a bit lost [...] } \\
\text { they did not like Dabbleboard at all }\end{array}$ \\
\hline $\begin{array}{l}\text { Risks of GLUE!-PS's dependence on external } \\
\text { services }\end{array}$ & $\begin{array}{l}\text { [in the fourth deployment session, while using GLUE!-PS] (10:57) } \\
\text { while we configure the reuse, GLUE!-PS stops responding, and we } \\
\text { reboot the GLUElet Manager [it had been known to hang up from } \\
\text { time to time] [...] (11:05) we have to reset the GLUElet Manager } \\
\text { again [AE1-O] }\end{array}$ \\
\hline
\end{tabular}

Finally, from the enactment of a GLUE!-PS-deployed learning design in an authentic situation, we obtained several hints about the run-time flexibility features (T4) that would be more useful to implement in GLUE!-PS. The teacher asserted that during run-time she would 
have liked to change the resources (e.g. source material, documents) for certain activities ${ }^{61}$. Also, she stated that, even if it was not needed in this enactment, the ability to make changes to the group formation would also be interesting. Albeit run-time changes could have been done manually through the wiki user interface by the teacher, having a centralized place to make such changes (especially when they involve external tools in the DLE) would be extremely useful.

\section{Partial conclusions and formative feedback}

In this iteration a new learning platform was added to the GLUE!-PS architecture, and we have tested a design and deployment process in a real learning situation, gathering data not only from the deployment process itself, but also about potential needs of the teachers during run-time. From the evidence gathered, we can obtain various conclusions, illuminating the four topics that we had defined around our evaluation issue: "Does GLUE!-PS support teachers in the orchestration of blended CSCL across DLEs?" (I1). These conclusions are summarized in Figure 5.35 .

T1 (deployment ability) We can conclude from this authentic experiment's data that GLUE!-PS, once again, is able to deploy CLFP-based learning designs (this time, based on a Jigsaw pattern), in a different DLE based on wikis, while complying with the teacher's pedagogical requirements (AE1-T1-PC1). Although this conclusion does not add very much to the previous data, it is interesting to note that in this case the data have been taken in a real situation, thus increasing the credibility of this conclusion. Moreover, this use of GLUE!-PS in a real situation also uncovered certain details that could be added to the GLUE!-PS LF model: group-specific activity descriptions and time management information (AE1-T1-PC2). Thus, we gained further evidence that the data model may be adequate for the task at hand (deploying learning designs across DLEs), with only minor modifications.

T2 (time-efficiency) The AE1 happening showed us first evidence that the GLUE!-PS system (and the CSCL life-cycle that it helps traverse) might not be as simply defined as expected (see the iterative deployment conclusions on T3 below), thus making time-efficiency harder to assess. The teacher, however, did see the benefit of spending time using GLUE!-PS, especially when defining student-generated artifact reuse, and when the teacher reuses a learning design (which is one of the main known benefits of computer-interpretable LD approaches) (AE1-T2-PC1).

\footnotetext{
${ }^{61}$ Regarding this need of run-time changes in the activity resources, it is interesting to note that there are two main ways of implementing such changes in GLUE!-PS, which prompt variations in the deployment process and in the sequence of invocations among the architecture elements. One way (which is used in the current prototype) is to include in the course structure created in the learning platform, a reference to the GLUElet Manager resource of the external tool (thus, during enactment, the wiki pages embed GLUElet Manager resources). In this case, a run-time change in the deployed resources would require a re-deployment of the new particularized learning design. On the other hand, the course structure could include references to the GLUE!-PS manager resources instead, adding a level of indirection to the process of accessing the external resource embedded in the learning platform (i.e. GLUE!-PS acts as a run-time "gatekeeper" for the resource). This level of indirection would allow us to make changes in the external tool (by changing the GLUE!-PS indirection) without having to modify the deployment. In any case, the current implementation of the GLUE! VLE adapter for MediaWiki (which is used for embedding the external tools in the wiki) only allows the mode of working that references the GLUElet Manager service.
} 


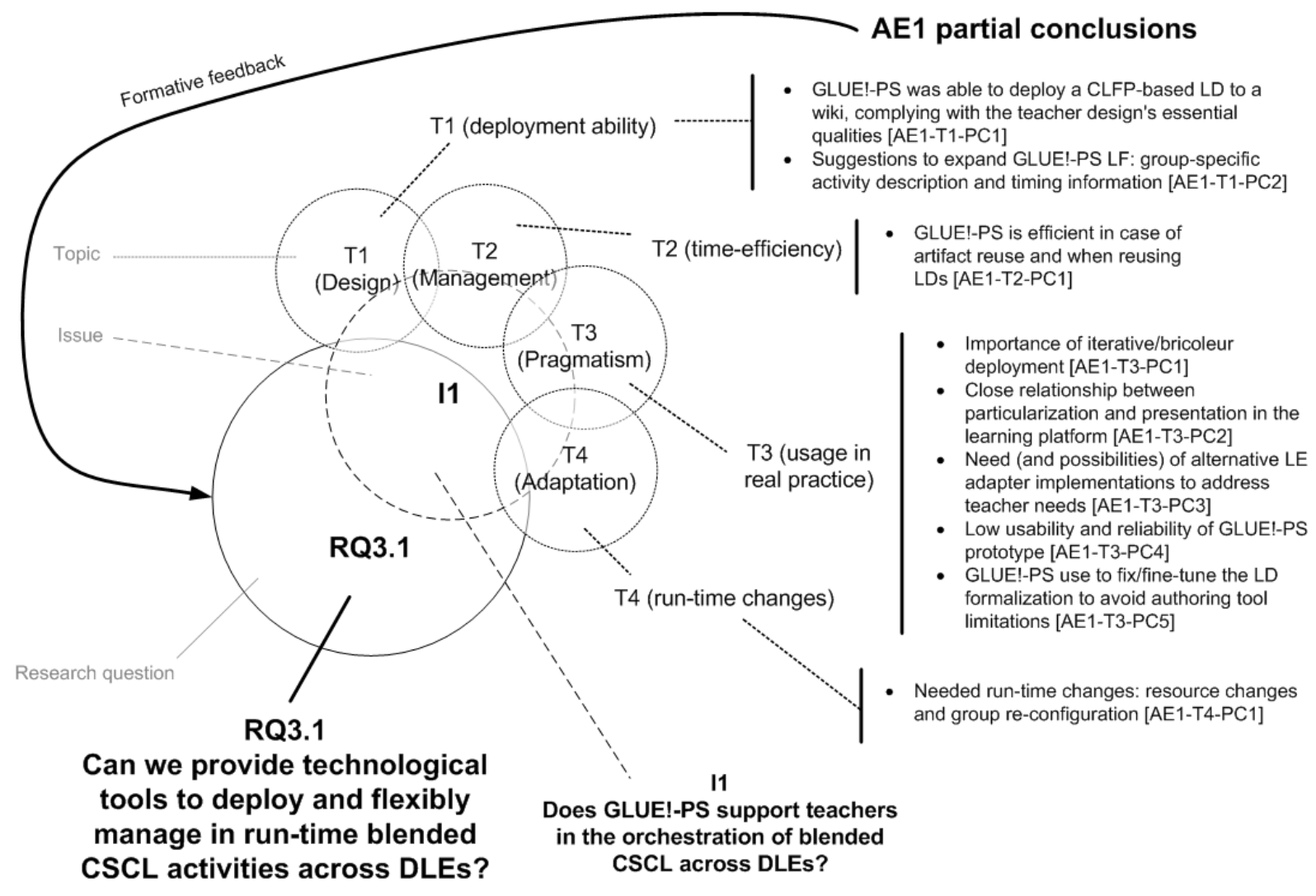

Figure 5.35: Graphical representation of the issues, topics and partial conclusions of the Iteration \#3

T3 (use in real practice) This first use of the system in a real situation provided us with several insights about the orchestration support that GLUE!-PS offered to teachers, from a pragmatic point of view. The most striking conclusion we can draw is the importance of iterativeness in the deployment process, also when enacting activities through a LD approach (AE1-T3-PC1). Even if GLUE!-PS somehow speeds up the implementation of formalized learning designs, the close relationship between the particularization of the design (and sometimes, also, of the "de-contextualized" LD modeling that lies underneath) and the semi-automated DLE implementation with GLUE!-PS (especially its graphical representation in the learning platform) make it necessary to visit alternatively different parts of the "script life-cycle" in a fluid way (AE1-T3-PC2). In this regard, GLUE!-PS provides a nice point of entry to fine-tune multiple aspects of the particularized design, including those that are defined in previous LD authoring phases (AE1-T3-PC5). However, the going back and forth among different steps and tools was still not seamless enough to support real "bricolage" while deploying the learning designs.

This iteration also highlighted the multiple usability and reliability problems that the prototypes had at the time (AE1-T3-PC4), although the successive implementations of multiple LE adapters in order to fine-tune the final aspect of the deployments (at a reduced development time, since they only took a few hours to modify and create new implementations), hinted at the extensibility and pragmatism of the GLUE!-PS adapter 
architecture. This extensibility leads us to anticipate an ecosystem of different LD and LE adapter implementations, that address the styles and contextual needs of different teachers (AE1-T3-PC3).

T4 (run-time changes) Finally, we obtained first evidence about run-time features that were most needed by teachers when enacting a learning design, namely the re-configuration of resources and tools used by the various groups along the learning activities, and a similar re-configuration of the participants and the groups they formed throughout the (particularized) learning design (AE1-T4-PC1). This evidence was not surprising, and coincided roughly with the atomic-pattern-defined GLUE!-PS implementation roadmap; having this kind of confirming support, however, encouraged us to promptly follow the roadmap into such implementations.

Again, all these conclusions are limited by the methods and context in which the evidence was gathered. For example, these conclusions can mostly said to apply to teachers that are already expert with teaching using CSCL techniques and a moderate use of ICT (as our volunteer teacher was). However, we believe that other uncovered aspects, such as the iterative nature of deployment, might be more generalizable. Also, the adjustment or not of GLUE!-PS-deployed DLE implementations to the pedagogical "essential qualities" seem to be highly subjective depending on each teacher and the teaching-learning techniques used (e.g. some put more emphasis on resource access control to prevent copying, while others like to encourage free circulation of student-generated knowledge), thus making GLUE!-PS's ability to deploy useful designs variable. However, the adapter architecture of GLUE!-PS makes it possible to adapt to most of these requirements by implementing and adequate LE adapter (as long as the GLUE!-PS LF model and the target learning platform support those requirements).

The evidence gathered during this iteration (especially the usability and reliability issues) advised us to work more thoroughly on the implementation side of the contribution, before we attempted further validations in more authentic learning situations. These situations should also try to avoid the participant biases that had characterized our evaluation so far, in order to increase the credibility of our findings.

\subsubsection{Iteration \#4: Adding run-time changes support and evaluation in workshops and wiki-based experiments}

After our third iteration of the evaluation of GLUE!-PS, and taking into account first contributions that had been made in the other two areas of the dissertation (mainly, publication of the " $5+3$ Aspects" proposal described in Section 3.2 and the elicitation of the atomic patterns catalogue from higher education practice with DLEs mentioned in Chapter 4), our architecture and data model proposals underwent an analysis from the point of view of those contributions. This analyses, which appear for the most part in Section 5.3, served to re-focus our contribution within the wider notion of orchestration, and to devise new evaluation happenings that would help us in the inquiry about the orchestration support that GLUE!-PS provided for non-expert teachers.

Also, the events and data gathered from the previous iterations prompted us to iterate further on the implementation of the GLUE!-PS prototype, and very especially on its graphical 
user interface. This GLUE!-PS GUI, the data had shown, had to expand its functionality to be able to modify almost all aspects of the GLUE!-PS LF model of a particularized design, while maintaining the simplicity and time-efficiency that were needed if the system was to be used by non-expert teachers in authentic learning situations. Thus, a series of paper prototyping sessions (see Figure 5.36) were conducted with non-technical teachers, and the GLUE!-PS GUI underwent several changes until a user interface very similar to the one presented in Section 5.4 , was implemented incrementally. In parallel, enhancements were made to the GLUE!-PS services themselves, not only to increase the reliability of the prototype, but also to provide the ability to be able to change the GLUE!-PS particularization and re-deploy it during run-time, thus providing first flexible adaptation features.

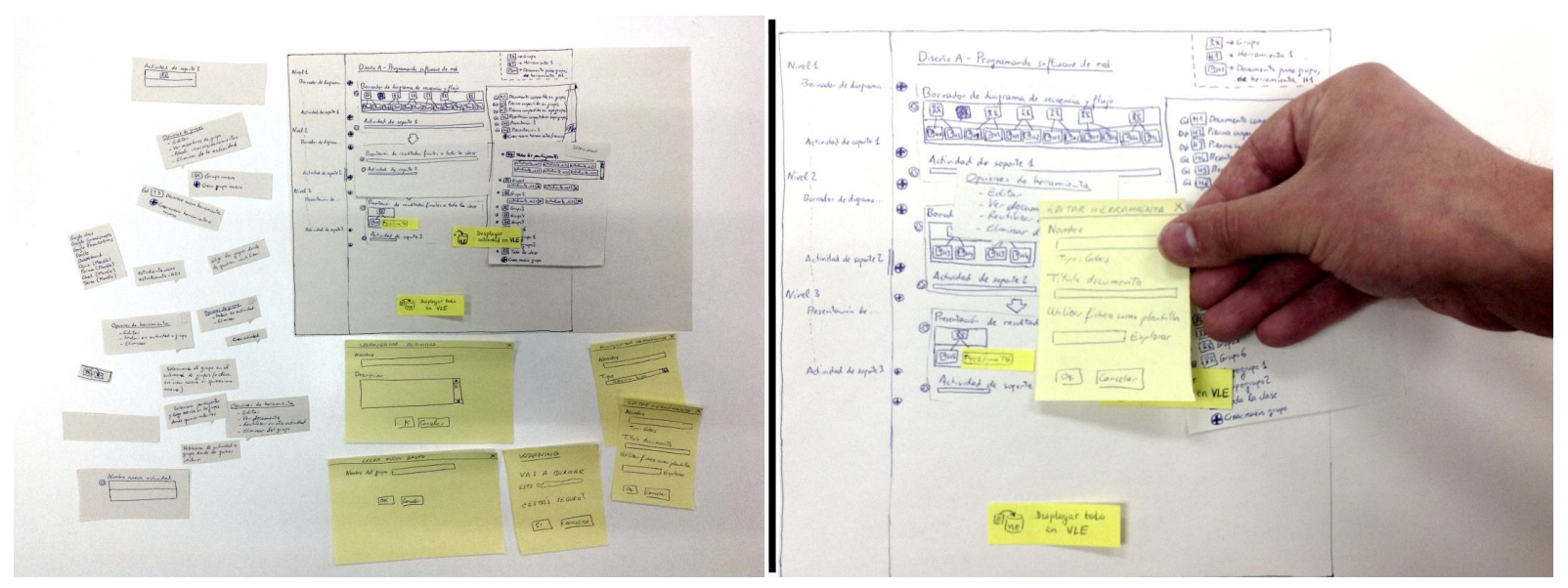

Figure 5.36: Examples of the paper prototypes of the GLUE!-GUI used during Iteration \#4

Thus, for the following evaluation iteration, we set out to expand the width and depth of our experiments, and thus we performed another authentic experiment (AE2) with the same teacher that had used GLUE!-PS in AE1 (to provide first feedback about the new graphical user interface in an authentic learning situation, when compared with the old one). Afterwards, we performed a third wiki-based authentic experiment (AE3), but with a non-technical, more novice CSCL teacher-researcher (to observe usage and usefulness differences for teachers with less teaching and ICT expertise). And finally, we performed a teacher workshop (TW6) where teachers from multiple disciplines and expertises used the GLUE!-PS system to deploy and simulate the enactment of blended CSCL activities (in order to gather feedback from a wide spectrum of teachers that were not CSCL experts).

\section{A second wiki-based authentic deployment experiment (AE2)}

Context and method This second authentic experiment of a teacher designing a CSCL activity and deploying it through the GLUE!-PS system was, in fact, conducted on a very similar context to that of the first experiment (see Section 5.5.4). The learning activities were framed within the same master-level degree on secondary education at the University of Valladolid, and the teacher was indeed the same one (let us remember, an expert teacher with previous experience with CSCL and a technical background). The course, in this case, delved into the 
research processes that can be performed as secondary teachers, and especially Action Research [Mil00]. The students were, in fact, the same 14 students as in the AE1 happening.

The learning design that the teacher had in mind was also based on CLFPs (see Section 4.2.3), but in this case on a Pyramid, which aims to reach progressive consensus by several phases of group work with groups of increasing size. The main goal of the scenario was to elaborate a research plan collaboratively, in groups of 4-5 students. The activities would be supported by the same wiki-based DLE already used in happening AE1, and the structure of the learning activities was as follows (see also Figure 5.37):

1. Individually, each student makes the proposal of a research problem to be studied, using a shared online document (Google Documents) to do it. This activity was to be completed online, asynchronously.

2. In groups of 2-3 people, students were to agree a common research question based on the proposals of the group members (thus, reusing the shared documents from the previous phase), and they were to elaborate further the context and methodology to tackle the chosen research problem. This second elaboration was also to be done through a different shared document (Google Documents was also used for this purpose).

3. In groups of 4-5 people, students were to agree to a common research purpose (to be described in a wiki page), and then elaborate the research plan to investigate such research problem through an Action Research effort (this would be detailed in a different wiki page). These three "big group proposals" were to be peer-reviewed by the other two groups (by making comments in the proposal wiki page), and then refined again by their original authors based on the peers' feedback (in the same wiki page).

4. Finally, the enhanced research proposals were to be presented before the whole group of students. The three wiki pages with the refined proposals would be used to support such presentations. Also, students were to fill in two questionnaires: one critiquing their peers' presentations, and another one about the groupwork performed (for assessment purposes).

This scenario was finally enacted in the month of April 2012, and spanned 3 weeks of online and face-to-face work. During this authentic experiment, as it happened in AE1, we gathered data by recording and observing the co-design (and co-deploy) sessions with the teacher (AE2-R and AE2-O, respectively), as well as by using the different artifacts generated by the teacher (AE2-D). Furthermore, a semi-structured interview was conducted after the experiment (AE2I), to gather the teacher's reflections on the usage of the GLUE!-PS system. Although run-time flexibility features were being implemented, they were considered still too unreliable for authentic use and thus, we decided not to record or observe the enactment's face-to-face sessions. All these data sources are summarized in Table 5.14.

Furthermore, this experiment was also used for the parallel research effort on the designinformed monitoring process [RT12], as AE1 did. Our main goal with this experience was to test the new version of the GLUE!-PS GUI for the deployment of a learning design, and to assess how this new prototype with (supposedly) enhanced usability and capabilities for deeper modification of the particularized design (GLUE!-PS LF) model, supported everyday orchestration practice. Thus, again we explored the issue "Does GLUE!-PS support teachers in the orchestration of 


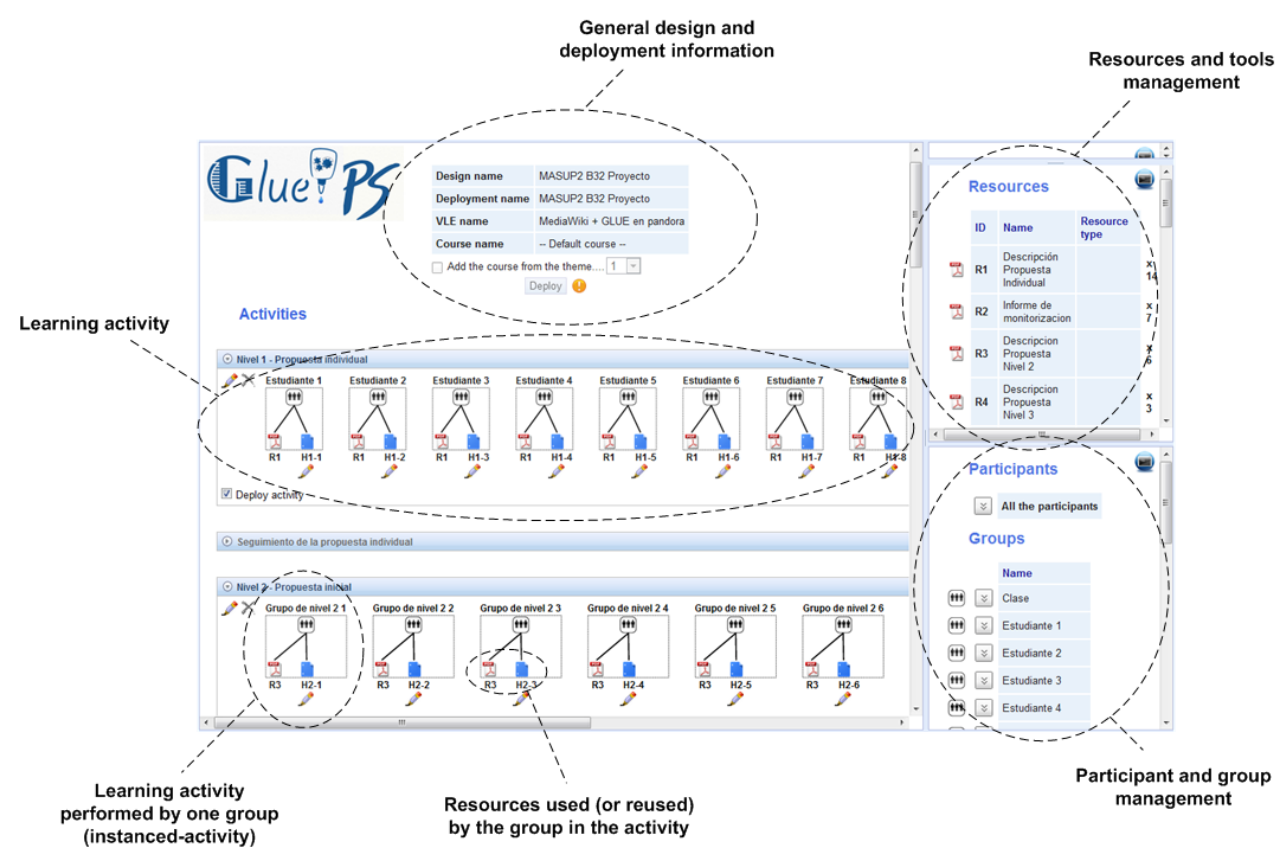

Figure 5.37: Screen capture of the Pyramid-based learning design used in happening AE2, as represented by the new version of the GLUE!-PS GUI. Includes basic explanation of the interface parts.

Table 5.14: Main data sources used during the AE2 evaluation happening

\begin{tabular}{|llc|}
\hline Source & Kind of evidence & Code \\
\hline \hline $\begin{array}{l}\text { Observation notes of the design and deployment } \\
\text { sessions }\end{array}$ & Qualitative & AE2-O \\
\hline $\begin{array}{l}\text { Design and deployment session's video/audio } \\
\text { recording }\end{array}$ & Qualitative & AE2-R \\
\hline $\begin{array}{l}\text { Participant-generated artifacts along the process } \\
\text { (WebCollage, GLUE!-PS, MediaWiki data) }\end{array}$ & Qualitative & AE2-D \\
\hline Post-experiment semi-structured interview & Qualitative & AE2-I \\
\hline
\end{tabular}

blended CSCL across DLEs?" (I1), around the topics of the ability of GLUE!-PS in deploying learning designs preserving the design's essential qualities (T1), its time-efficiency in doing so (T2) and the feasibility of using GLUE!-PS in everyday teaching practice (T3). We would also gather further evidence about needed run-time flexibility features (T4).

Findings and evidence As we did in the previous authentic experiment, we will briefly describe the co-design and co-deployment process followed by the teacher, before detailing the evidence gathered around these topics. When implementing the aforementioned learning scenario, the teacher followed a similar iterative approach, although in this case only two iterations were needed to achieve the final result. Thus, the teacher and two researchers met in a 1-hour session to discuss the design and make a preliminary attempt to formalize it using WebCollage. Afterwards, in another 1-hour session, the teacher was presented with the new GLUE!-PS GUI and the first attempt to learning design was deployed using GLUE!-PS. This first iteration served to uncover a number of usability and reliability issues of the system (i.e. bugs), as well 
as to elicit possible enhancements in the way the particularized design was transferred to the (wiki-based) learning platform.

After this first iteration, the teacher completed the formalization of the learning design on her own, using WebCollage (e.g. to insert the activity description/instructions in a way that, during the enactment, would be clear for students). Afterwards, in another 1-hour session the final deployment was done to the wiki-based DLE. These activities deployed into the wiki were then enacted by the teacher and students without considerable modifications (apart from changing deadlines and adding some links to resources that the teacher had forgotten to add the teacher did these changes manually, since the GLUE!-PS prototype did not allow for run-time changes yet at the time).

Regarding GLUE!-PS ability to deploy blended CSCL designs (T1), we found that the teacher was able to deploy the CLFP-based learning design using GLUE!-PS and, more importantly, that the ICT infrastructure created across the wiki-based DLE complied with the learning design's essential features (see selected evidence in Table 5.15).

Table 5.15: Topics, findings and selected supported evidence from evaluation happening AE2

\begin{tabular}{|c|c|c|}
\hline Topic & Finding & Selected supporting evidence \\
\hline \multirow[t]{2}{*}{ T1 (deploy ability) } & $\begin{array}{l}\text { GLUE!-PS deploy complies } \\
\text { with the learning design's } \\
\text { essential qualities }\end{array}$ & $\begin{array}{l}\text { (when asked whether the deployment in the wiki reflects her } \\
\text { learning design) It is good, it shows clearly the design and the } \\
\text { steps to follow [...] I cannot think of much better ways of doing } \\
\text { it, even if you did it manually [...] I don't think I could have } \\
\text { done it better [AE2-I] }\end{array}$ \\
\hline & $\begin{array}{l}\text { GLUE!-PS is able to deploy } \\
\text { a CLFP-based learning design } \\
\text { into a wiki }\end{array}$ & $\begin{array}{l}\text { The teacher was able to deploy her learning design to the wiki } \\
\text { (twice) [AE2-O] }\end{array}$ \\
\hline \multirow[t]{2}{*}{ T2 (time-efficiency) } & $\begin{array}{l}\text { The first-time usage of GLUE!- } \\
\text { PS is not time-efficient }\end{array}$ & $\begin{array}{l}\text { In the first deployment iteration, it took } 40 \text { minutes to de- } \\
\text { sign in WebCollage and } 40 \text { minutes to deploy with GLUE!-PS } \\
\text { [AE2-O] }\end{array}$ \\
\hline & $\begin{array}{l}\text { The time spent is worth when } \\
\text { groups change and student } \\
\text { documents get reused }\end{array}$ & $\begin{array}{l}\text { (when asked whether the time spent doing the design and } \\
\text { deployment is worth the result) for me, the main advantage } \\
\text { is when you have a flow of documents, tools [...] the fact that } \\
\text { each group has the link to the documents they have to peer- } \\
\text { review makes it worthy. If [the design] did not have that, then } \\
{[\ldots] \text { I would be tempted to do it [manually] from scratch. When }} \\
\text { you have complex interactions between groups, which change } \\
\text { in granularity, reuse documents [...] then it would surely be } \\
\text { worth it [AE2-I] }\end{array}$ \\
\hline \multirow{5}{*}{$\begin{array}{l}\mathrm{T} 3 \text { (use in real } \\
\text { practice) }\end{array}$} & $\begin{array}{l}\text { Activity vs. Time-based views } \\
\text { of the course }\end{array}$ & $\begin{array}{l}\text { The teacher did (manually) a "temporal view" of the course, } \\
\text { with links to the deployed activities [AE2-D] }\end{array}$ \\
\hline & $\begin{array}{l}\text { Importance of temporal as- } \\
\text { pects in orchestration }\end{array}$ & $\begin{array}{l}\text { (when asked about manual changes she did to the wiki after } \\
\text { the deployment) I used it as-is [...] except maybe introducing } \\
\text { some things which I forgot to put in the design [...] deadlines } \\
{[\ldots][\text { AE2-I] }}\end{array}$ \\
\hline & $\begin{array}{l}\text { Improved usability of the } \\
\text { GLUE!-PS GUI }\end{array}$ & $\begin{array}{l}\text { (when asked whether she used the new GLUE!-PS GUI to } \\
\text { deploy her design) Yes, this one was the clearer one [AE2-I] }\end{array}$ \\
\hline & $\begin{array}{l}\text { Need for alternative } \mathrm{LE} \\
\text { adapter implementations }\end{array}$ & $\begin{array}{l}\text { (when asked whether the deployment in the wiki reflects her } \\
\text { learning design) ... but you had to do a lot of clicks to reach } \\
\text { the [contributions] [AE2-I] }\end{array}$ \\
\hline & $\begin{array}{l}\text { Need for further usability } \\
\text { enhancements }\end{array}$ & $\begin{array}{l}\text { (when asked about problems in the deployment using GLUE!- } \\
\text { PS) there was a problem with the identification of [documents] } \\
\text { when reusing... it was not intuitive [...] everything else I un- } \\
\text { derstood it quickly, and it was useful [AE2-I] }\end{array}$ \\
\hline
\end{tabular}


Table 5.15 (continued from previous page)

\begin{tabular}{|c|c|c|}
\hline \multirow[t]{5}{*}{ Topic } & Finding & Selected supporting evidence \\
\hline & & $\begin{array}{l}\text { (when asked about problems in the deployment using GLUE!- } \\
\text { PS) ... also, there was this thing with the [resources], whether } \\
\text { they were tools or documents, which also has connotations } \\
\text { with what you can monitor [i.e. using the other researcher's } \\
\text { proposal which was tried in parallel] [...] from the point of } \\
\text { view of the teacher, it is a bit unclear [...] the implications and } \\
\text { subtleties are not expressed in the language you use normally } \\
\text { [AE2-I] }\end{array}$ \\
\hline & $\begin{array}{l}\text { Students had problems with } \\
\text { the technical support to } \\
\text { activities (not related to } \\
\text { GLUE!-PS) }\end{array}$ & $\begin{array}{l}\text { (when asked about difficulties experienced during the enact- } \\
\text { ment, from the student point of view) technically [...] they had } \\
\text { difficulties accessing the GoogleDocs [...] they were not very } \\
\text { happy with the kind of interaction [AE2-I] } \\
\text { (when asked about the learning platforms her students are } \\
\text { used to) maybe they were more used to Moodle }[\ldots] \text { the kind } \\
\text { of interaction you have in wikis is different }[\ldots] \text { my experience } \\
\text { with wikis is that [students] end up complaining }[\ldots] \text { they do } \\
\text { not know where to put their contributions }[\ldots] \text { but this is not } \\
\text { new }[\text { AE2-I] }\end{array}$ \\
\hline & $\begin{array}{l}\text { The teacher had a clearer idea } \\
\text { of the deployment process }\end{array}$ & $\begin{array}{l}\text { Only two deployment iterations were needed to achieve the } \\
\text { desired result in the DLE [AE2-O] }\end{array}$ \\
\hline & $\begin{array}{l}\text { The teacher would use it in real } \\
\text { practice for complex CSCL sce- } \\
\text { narios }\end{array}$ & $\begin{array}{l}\text { (when asked whether she would use GLUE!-PS in her real } \\
\text { practice, if it were not part of her research group) In these } \\
\text { courses which have a strong collaborative component, using } \\
\text { more-or-less complex interaction patterns, I would use it [...] } \\
\text { for smaller, simpler activities, maybe it would not occur to me } \\
\text { [AE2-I] }\end{array}$ \\
\hline T4 (run-time changes) & Run-time changes needs & $\begin{array}{l}\text { (when asked about manual changes she did to the wiki after } \\
\text { the deployment) I used it as-is [...] except maybe introducing } \\
\text { some things which I forgot to put in the design [...] deadlines } \\
{[\ldots] \text { and some resource which I did not have available in design- }} \\
\text { time [AE2-I] }\end{array}$ \\
\hline
\end{tabular}

Regarding the time-efficiency of the deployment process (T2) (let us remember that the run-time changes were not tested in this evaluation happening), we found out that, albeit the teacher needed fewer iterations to achieve the desired deployment result (compared with the previous authentic experiment - AE1), still we were observing a case of first-time implementation of a learning design and thus, it was not very time-efficient. Part of this time was employed in getting used to the new GLUE!-PS GUI and due to reliability issues (i.e. bugs) but, in any case, the teacher had to spend between 2 and 3 hours using the WebCollage and GLUE!-PS tools. The teacher, however, asserted that this time spent was worth the obtained result, and hinted that in the case of complex sequences of activities with groups of different granularities and re-using of student-generated artifacts (such as the peer review that the teacher proposed), the formal learning design and implementation with GLUE!-PS is more advantageous than its manual counterpart (see Table 5.15).

Regarding GLUE!-PS's potential use in everyday orchestration practice (T3), the gathered evidence confirms that the new GLUE!-PS GUI improved the usability of the system and was clearer for the teacher than the old one, although there were still aspects that needed more work (see Table 5.15). The evidence also confirmed the need for multiple implementations of the LE adapters in order to better adapt to the different needs and preferences of teachers regarding the final structure of the deployed activities in the DLE. 
From the students' perspective, the teacher also noticed certain technical problems with the access to certain resources across the DLE (e.g. interactions between Google Documents' user interface and the way such external tools are embedded into the wiki) and, especially, with using wikis in general. These problems, however, seem to be general and not GLUE!-PS-specific.

An interesting finding that also emerged from this authentic experiment was the importance for the teacher of temporal aspects, such as deadlines for tasks and the activities that are to be enacted in each face-to-face session. This importance manifested in the form of a "temporal view" of the course, which the teacher implemented by hand, detailing both the contents of each face-to-face session and the deadlines for all the submissions that students should make. This time-based representation of the activities (as opposed to the activity-based representation that GLUE!-PS LE adapters generally offer) seemed to be complementary. The fact that this kind of time management information is also often an aspect that needs to be modified in run-time (as it happened in this experiment), makes us think that multiple interlinked visualizations of the activities (e.g. a session-based one and an activity-based one) might be a useful addition to the GLUE!-PS system. Although this feature is theoretically possible just by modifying the LE adapter, it would also require an extension of the GLUE!-PS LF model, which currently disregards this kind of timing information.

Overall, the evidence hinted that the teacher had a clearer idea of how the deployment process worked and the amount of effort that it entailed. Thus, she was certain that she would use the GLUE!-PS LD approach again in real practice, but only in the case of learning designs of a certain complexity, where groups of different granularities have group-based access to different resources, especially when reusing student-generated ones.

Finally, regarding the needs of run-time flexibility, in this authentic experiment only a few changes were needed, regarding the addition of resources to activities which were not available (or were forgotten) in design-time. Other changes required involve the aforementioned timing information (i.e. deadlines), which, in the deployed design, was simply modeled as a remark within the activity descriptions.

\section{A wiki-based authentic experiment with run-time support (AE3)}

Context and method For the third authentic experiment using GLUE!-PS to support orchestration of blended CSCL activities, we chose a slightly more challenging educational context. In this case the experiment was framed within a course about "ICTs applied to education", in the undergraduate-level degree for kindergarten teachers at the University of Valladolid ${ }^{62}$, The teacher, in this case, was a relatively novel teacher-researcher (this was her third year teaching at the university) belonging to the GSIC-EMIC group, with an educational background. She agreed to use GLUE!-PS to orchestrate a practical, collaborative part of the course (that had already been performed in previous years, but manually deploying the design over the wiki-based learning platform), with two different groups of students. Thus, the experiment would consist on two parallel enactments of the same basic design, particularized for two different groups of students. We can see that, in this authentic experiment, the educational context (with education undergraduate students), the scale of the experiment (2 groups totaling 50 students) and the

\footnotetext{
${ }^{62}$ Descriptions of the degree (in Spanish) are available at http://www.uva.es/consultas/asignaturas.php? codigo_plan=398\&ano_academico=1112 (Last visit: 15 Jun 2012).
} 
teacher profile (novel teacher with non-ICT background) varied considerably with respect of the previous ones (AE1, AE2).

The design of the learning scenario to use for the experiment had been agreed among the five teachers in charge of the course ${ }^{63}$ and consisted on the inquiry about pedagogical uses of different ICTs, especially "Web 2.0" ones. The design was structured using a Jigsaw CLFP (see Section 4.2.3), and spanned 2 weeks of blended learning with three face-to-face sessions of one or two hours. The ICT support for this scenario was normally deployed manually by the teachers (who spent several hours creating the wiki page structure for students to follow). The scenario consisted of the following coarse-grained phases (see Figure 5.38 for a graphical representation of the design, and Figure 5.39 for examples of the wiki-based learning platform deployed with GLUE!-PS):

1. In dyads, students were to investigate about three basic questions concerning one of three "Web 2.0" technologies (each of the dyads would do it for only one of the three technologies): blogs, wikis and social networks. They were to do this answering in a wiki page (one for each dyad), both during a face-to-face session, and complemented with online work.

2. In groups of 4-5 students which had studied the same technology in phase 1 , students were to delve deeper into the educative uses, advantages and disadvantages of the studied technology, and produce a conceptual map (using a simple concept mapping web application called Text2Mindman ${ }^{64}$ and uploading the resulting image to their respective expert groups' wiki page). Again, this activity was to be started in a face-to-face session and finished online.

3. Finally, students were to join into "jigsaw groups" which had studied different technologies, and produce a multimedia artifact (presentation, concept map, video, blog, wiki, social network... using any technology they desired) that showed the different possible educational uses of all three studied technologies, which was to be uploaded to the jigsaw groups' wiki pages. This task was started face-to-face, included a considerable amount of non-presential work, and the products were presented in a later face-to-face session.

It is worth noting that, albeit this learning design is supported by a Distributed Learning Environment (since it involves a wiki learning platform and external tools such as Text2Mindmap), it cannot be said to be a GLUE!-based DLE (since neither Text2Mindmap or the multimedia tools used in phase 3 are supported by the current GLUE! implementation). Thus, the kind of orchestration that GLUE!-PS could do in this scenario was limited to the learning platform (i.e. the wiki pages used). We chose to study this scenario as it was (vs. making the teacher choose different tools or different tasks to comply with a GLUE!-supported DLE) in order to maintain authenticity (one of orchestration's core requirements) as much as possible, and to explore the frontiers of the GLUE!-PS support to different modes of implementing DLEs, trying to "maximize our learning gains" [Sta95]. Moreover, we did not consider it ethical to subvert the pedagogical goals and values of the teachers, leading to a poorer learning experience in favor of an "optimal testbed" for GLUE!-PS (e.g. the only concept mapping tool that GLUE!

\footnotetext{
${ }^{63}$ Every year, this course is taken by more than 400 students, divided in 7 groups of 50-60 students, each of which is divided in two groups for the practical activities, to which this scenario belonged.

${ }^{64}$ http://www.text2mindmap.com/ (Last visit: 15 Jun 2012).
} 


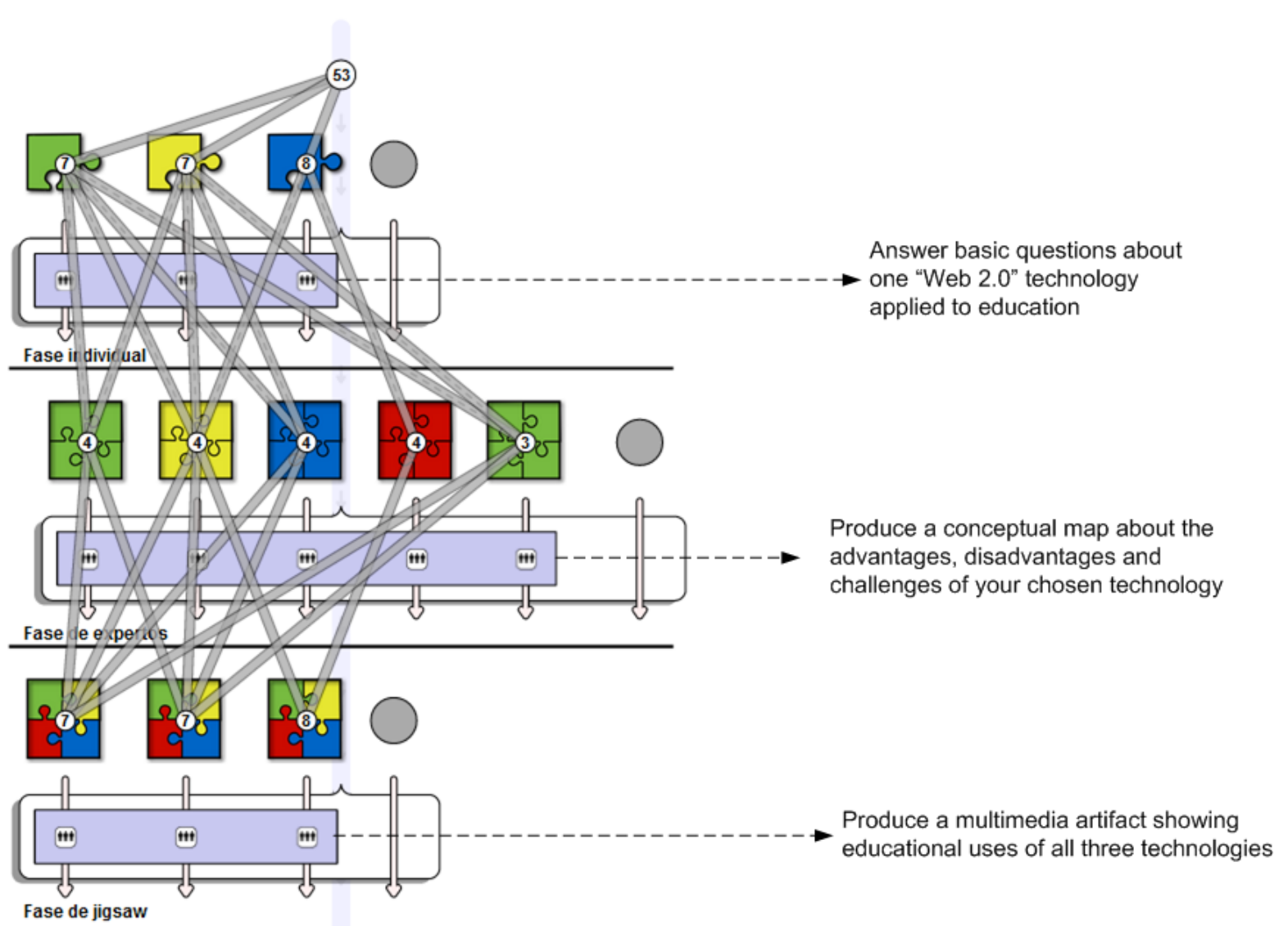

Figure 5.38: Graphical representation of the design done by the teacher in happening AE3

currently supports, Dabbleboard, had shown reliability issues, and was known to be difficult to use by students in previous authentic experiments - hence the use of Text2Mindmap, which teachers found ideal for their purposes).
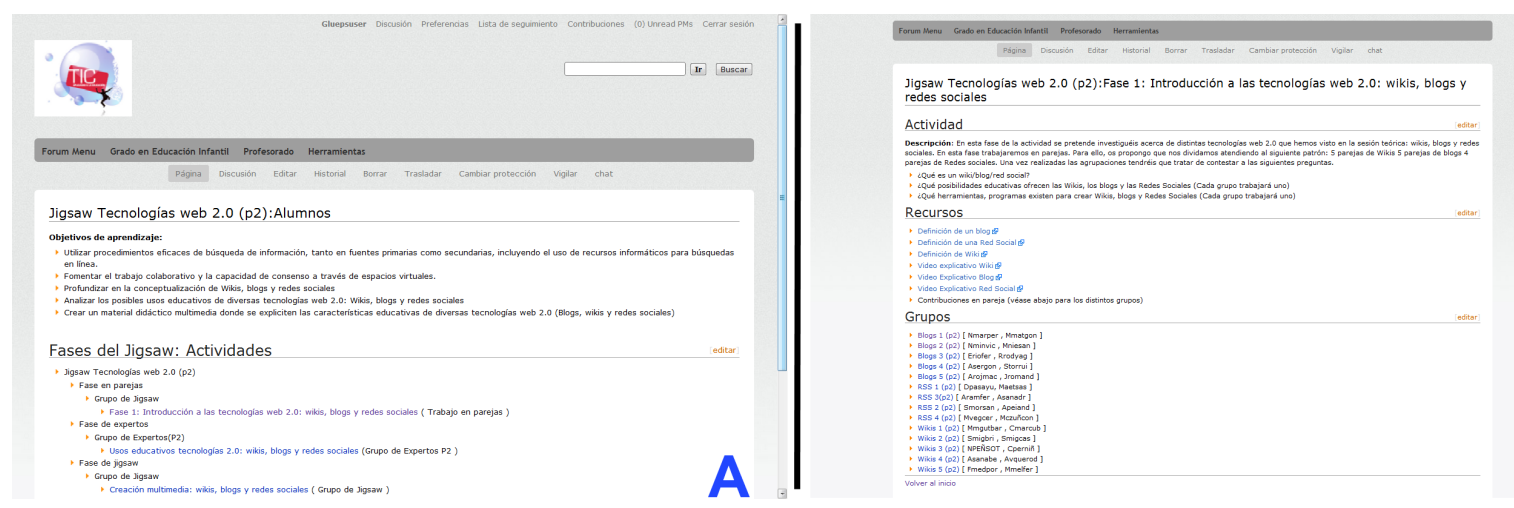

Figure 5.39: Screen captures of the Jigsaw learning design used in happening AE3, once it was deployed in a wiki by GLUE!-PS 
This scenario was finally enacted between the months of April and May 2012. During that time span, we gathered data by recording and observing the co-design (and co-deploy) sessions with the teacher (AE3-R1 and AE3-O1, respectively), as well as by using the different artifacts generated by the teacher (AE3-D). One researcher (the author) also observed several of the face-to-face sessions of the enactment (AE3-O2). Furthermore, a semi-structured interview was conducted after the experiment (AE3-I), to gather the teacher's reflections on the usage of the GLUE!-PS system. All these data sources are summarized in Table 5.16 .

Table 5.16: Main data sources used during the AE3 evaluation happening

\begin{tabular}{|lll|}
\hline Source & Kind of evidence & Code \\
\hline \hline $\begin{array}{l}\text { Observation notes of the design and deployment } \\
\text { sessions }\end{array}$ & Qualitative & AE3-O1 \\
\hline $\begin{array}{l}\text { Design and deployment session's video/audio } \\
\text { recording }\end{array}$ & Qualitative & AE3-R1 \\
\hline $\begin{array}{l}\text { Participant-generated artifacts along the process } \\
\text { (WebCollage, GLUE!-PS, MediaWiki data) }\end{array}$ & Qualitative & AE3-D \\
\hline $\begin{array}{l}\text { Observation notes of the enactment of face-to-face } \\
\text { sessions }\end{array}$ & Qualitative & AE3-O2 \\
\hline Post-experiment semi-structured interview & Qualitative & AE3-I \\
\hline
\end{tabular}

Our main goal with this experience was to see the usage of GLUE!-PS in an authentic learning situation, focusing especially on the new version of the GLUE!-PS GUI, as well as the newly-implemented run-time changes features. Thus, again we explored the issue "Does GLUE!PS support teachers in the orchestration of blended CSCL across DLEs?" (I1), around the four main topics of the ability of GLUE!-PS in deploying learning designs preserving the design's essential qualities (T1), its time-efficiency in doing so (T2), the feasibility of using GLUE!-PS in everyday teaching practice (T3), and the usefulness of GLUE!-PS's run-time flexibility features (T4).

Findings and evidence In order to better understand the findings and evidence gathered in this evaluation happening, it is important to understand how the design and deployment process developed, as well as how the activities were then enacted in the classroom. In a first interview with the teacher, the design of the scenario, as it had been implemented in previous years, was discussed with researchers, in order to ascertain whether the current GLUE!-PS prototype would be able to implement an acceptable wiki-based infrastructure for it. In this session it readily emerged the fact that it was not possible to reproduce the aforementioned group structure with WebCollage, due to the dyad grouping in phase 1 (which was one of the reasons teachers had implemented it manually so far). It was decided that phase 1 would be re-configured in GLUE!-PS (which now did support this kind of changes to the particularized design model) to comply with the teacher's design (working in dyads instead of in jigsaw groups). Another important emergent point was the fact that in other years' enactments of the design, teachers let students choose their own groups (e.g. the dyads), and thus the manually-deployed wiki structure sported "generic names" for the scenario ("wiki dyad 3", "blog dyad 2"), hardwiring the flow of participants across the different groupings (e.g. "jigsaw group 1 is made of wiki dyad 3 and blog dyad 3..."). These generic names were filled in with real student names in enactment-time. Since this way of operating was not easily supported by the current LE adapter in the GLUE!-PS prototype, and that aspect was not considered crucial, the teacher decided 
to distribute the teams beforehand using WebCollage and GLUE!-P\$65. After this first contact, the teacher modeled the Jigsaw using WebCollage on her own, and later met with a researcher (the author) for a second co-design/co-deploy session. In this session, which lasted two and a half hours, the particularization and deployment of the two groups of students was finalized.

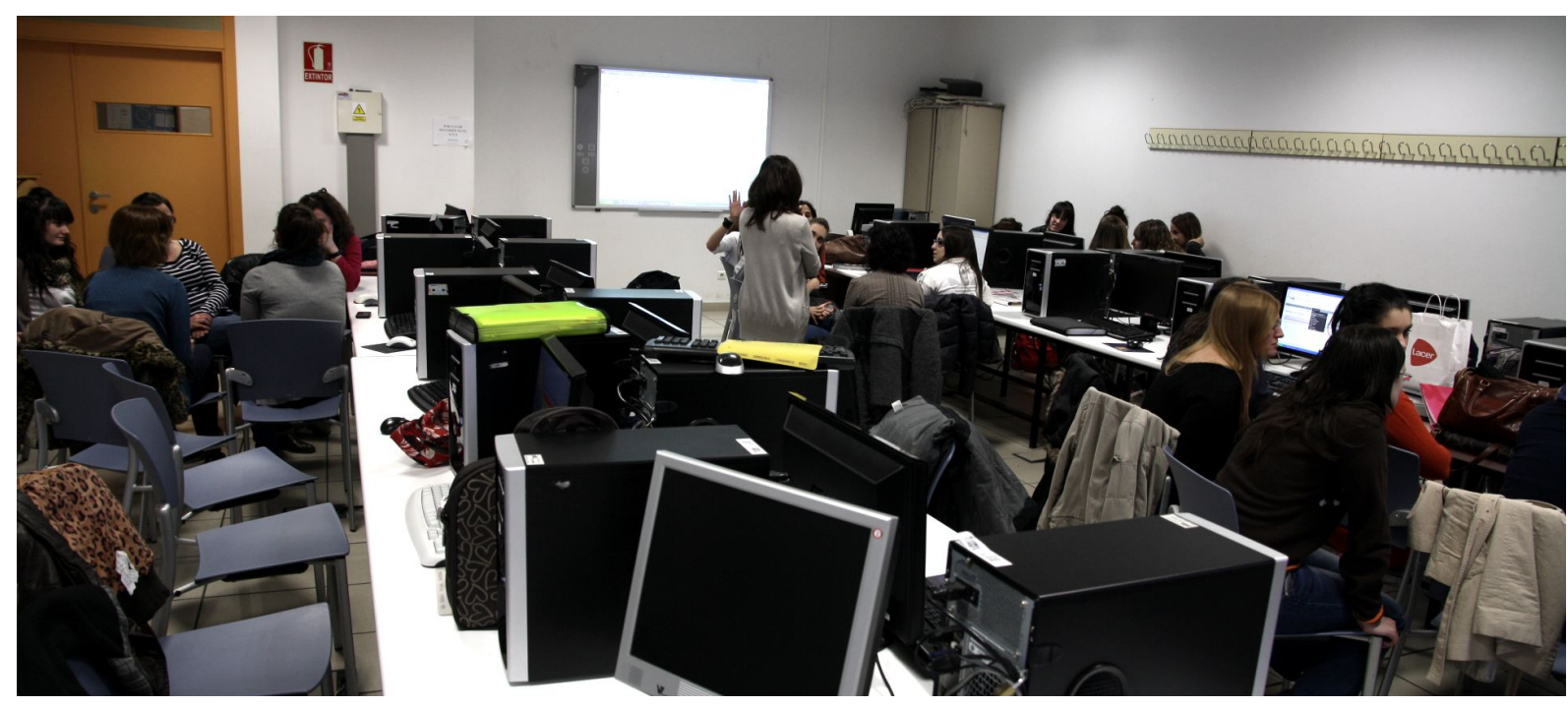

Figure 5.40: Photo of the enactment of one of the face-to-face sessions in evaluation happening AE3

The enactment of the learning activities (see Figure 5.40) with the two groups was warped by multiple extraneous events, especially regarding the students' assistance to the face-to-face sessions: several students showed up at the wrong student group, or did not show up at all; there were also latecomers, as well as other students who had to work individually at first and then were incorporated to the collaborative activities in later stages. The researcher that was observing the enactment (the author) spent a good part of the first face-to-face sessions helping the teacher to re-adjust the groupings and changing the wiki structure accordingly, by hand (since the teacher had made manual changes to the wiki that did not wish to be overwritten by the re-deployment with GLUE!-PS). Although these changes were tedious and error-prone (not only because of them being manual, but also because of the inherent difficulty of making group changes while complying with the Jigsaw strategy's constraints, see Figure 5.41, the difficulties were overcome and the students were able to complete the learning activities.

The evidence gathered during this happening regarding our first topic of interest, the ability of GLUE!-PS to deploy learning designs to a DLE preserving the design's essential qualities (T1), is summarized in Table 5.17. GLUE!-PS, once again, was able to deploy the teacher's learning design from the WebCollage-generated formalization to the wiki-based learning platform, preserving its essential qualities. Moreover, GLUE!-PS was used in this case to overcome some of the LD authoring tool's limitations (regarding the dyad groupings in phase 1). This shows that a novel teacher with non-technical background (albeit she was a teacher-researcher

\footnotetext{
${ }^{65}$ In theory, it would have been possible to make the particularization and deployment on-the-fly during the first face-to-face session. However, it was considered too risky (in case of technical failures in WebCollage or GLUE!-PS) and, as we saw later, the conceptual part of particularizing a customized jigsaw such as this one took too much time to be done on-the-fly.
} 


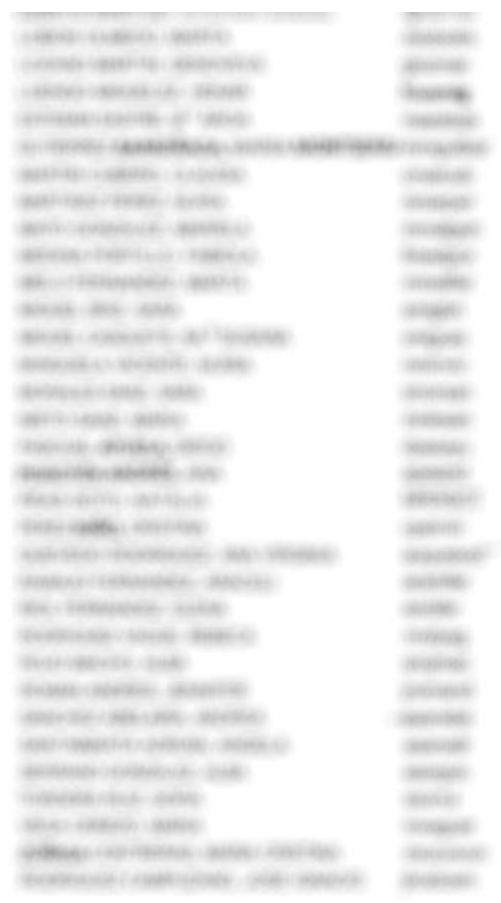

\begin{tabular}{|c|c|c|c|}
\hline ' & וuטu & inue & uv \\
\hline P1 & RSS3 & RSS2 & $\sqrt{3}$ \\
\hline P1 & BLOG4 & BLOG & J3 \\
\hline P1 & BLOG4 & BLOG & J3 \\
\hline $\mathrm{P}_{2}-$ & RSS1 & RSS1 & J1 \\
\hline P2 & WIKI1 & WIKI1 & $\mathrm{J} 1$ \\
\hline P2 & WIKI1 & WIKI1 & J1 \\
\hline P2 & BLOG1 & BLOG1 & J1 \\
\hline P2 & BLOG1 & BLOG1 & J1 \\
\hline P2 & WIKI5 & WIKI2 & \# \\
\hline P2 & WIKI5 & WIKI2 & J2 \\
\hline P2 & WIKI2 & WIKI1 & J2 \\
\hline P2 & WIKI2 & WIKI1 & J2 \\
\hline P2 & BLOG2 & BLOG1 & J2 \\
\hline P2 & RSS2 & RSS1 & J2 \\
\hline P2 & BLOG2 & BLOG1 & $\mathrm{J} 2$ \\
\hline P2 & RSS1 & RSS1 & J1 \\
\hline P2 & RSS2 & RSS1 & J2 \\
\hline P2 & WIKI3 & WIKI & J3 \\
\hline P2 & $\begin{array}{l}\text { WIKI: win't } \\
\text { RSs3-wits }\end{array}$ & $\begin{array}{l}\text { WHA+2M } \\
\text { RSS2 IIKI }\end{array}$ & -13 \\
\hline P2 & RSS3 & \begin{tabular}{l|l} 
RSS2 &
\end{tabular} & J3 \\
\hline P2 & BLOG3 & BLOG1 & J3 \\
\hline P2 & BLOG3 & BLOG2 & $\mathrm{J} 3$ \\
\hline P2 & BLOG5 & BLOG2 & $\mathrm{J} 3$ \\
\hline P2 & $\begin{array}{l}\text { BLOG5 } \\
\text { WIKI4 }\end{array}$ & $\begin{array}{l}\text { BLOG2 } \\
\text { W4K+2 }\end{array}$ & \\
\hline P2 & 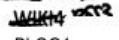 & WHAtz Ess & $480 \mathrm{din}$ \\
\hline P2 & BLOG4 & BLOG2 & $\mathrm{J4}$ \\
\hline P2 & BLOG4 & BLOG2 & $\mathrm{J} 4$ \\
\hline P2 & RSS4 & RSS2 & J4 \\
\hline P2 & RSS4 & RSS2 & J4 \\
\hline
\end{tabular}

Figure 5.41: Student list used to conceptualize the run-time regrouping of students for the Jigsaw design in AE3

with good knowledge of CSCL strategies) can perform this kind of deployment, at least when supported by researchers (the author, in this case). It is also remarkable that the teacher's original ideas regarding the way of distributing groups was changed due to the usage of GLUE!-PS (i.e. pre-defined the groupings instead of letting the students choose them) and the still-unreliable state of the prototype: as we saw during the enactment, making the groups on-the-fly in the first session would have avoided some of the problems that emerged (which is probably the reason why teachers did it that way in previous enactments of the design).

Table 5.17: Topics, findings and selected supported evidence from evaluation happening AE3, around topics T1 (deployment ability), T2 (time-efficiency) and T4 (run-time changes)

\begin{tabular}{|c|c|c|}
\hline Topic & Finding & Selected supporting evidence \\
\hline \multirow[t]{3}{*}{ T1 (deploy ability) } & $\begin{array}{l}\text { GLUE!-PS was able to deploy } \\
\text { the teacher's LD, preserving } \\
\text { the design's essential qualities }\end{array}$ & $\begin{array}{l}\text { (when asked whether the deployed wiki matches her idea of } \\
\text { the design) It matches perfectly [AE3-I] }\end{array}$ \\
\hline & $\begin{array}{l}\text { Non-ICT-expert teachers are } \\
\text { able to deploy designs using } \\
\text { GLUE!-PS }\end{array}$ & $\begin{array}{l}\text { The teacher deployed the learning design from WebCollage to } \\
\text { Mediawiki (with researcher support) [AE3-O1] }\end{array}$ \\
\hline & $\begin{array}{l}\text { Pre-definition of groups was } \\
\text { not part of the original design }\end{array}$ & $\begin{array}{l}\text { (when talking about changes in the orchestration caused by } \\
\text { the use of GLUE!-PS) the fact that you have to define groups } \\
\text { beforehand }[\ldots] \text { it is something you have to take into account } \\
{[\text { AE } 3-I]}\end{array}$ \\
\hline T2 (time-efficiency) & $\begin{array}{l}\text { Making manual run-time } \\
\text { changes to the deployment is } \\
\text { tedious and error-prone }\end{array}$ & $\begin{array}{l}\text { Doing the manual run-time changes in the first session of one } \\
\text { of the groups took the researcher around } 60 \text { minutes [AE3-O2] } \\
\text { The process of re-arranging the groups is supported by spread- } \\
\text { sheets and paper, with the help of (the author) [AE3-O2] }\end{array}$ \\
\hline
\end{tabular}


Table 5.17 (continued from previous page)

\begin{tabular}{|c|c|c|}
\hline \multirow[t]{5}{*}{ Topic } & Finding & Selected supporting evidence \\
\hline & $\begin{array}{l}\text { Making run-time changes with } \\
\text { GLUE!-PS is faster }\end{array}$ & $\begin{array}{l}\text { (when re-enacting some of the run-time manual changes using } \\
\text { GLUE!-PS) we have spent very little time [...] (when asked } \\
\text { to compare with the manual run-time changes done) that was } \\
\text { more time, much more [...] (when reminded that the manual } \\
\text { aesthetic changes would have been overwritten) if you are care- } \\
\text { ful when deploying, then [using GLUE!-PS] it is much more } \\
\text { advantageous [...] I see the advantages very clearly [AE3-I] }\end{array}$ \\
\hline & $\begin{array}{l}\text { The first-time usage of GLUE!- } \\
\text { PS is not time-efficient }\end{array}$ & $\begin{array}{l}\text { (when asked whether the time employed to deploy -around } 2.5 \\
\text { hours for each student group-, was a efficient use of her time) } \\
\text { I think that, if we did it now, we would spend less time }[\ldots] \\
\text { plus, this design could be reused [AE3-I] }\end{array}$ \\
\hline & $\begin{array}{l}\text { The used GLUE!-PS LD } \\
\text { approach is not much faster } \\
\text { than an (optimized) manual } \\
\text { deployment in a wiki }\end{array}$ & $\begin{array}{l}\text { It took around } 50 \text { minutes for the teacher to design in WebCol- } \\
\text { lage, and } 30 \text { minutes to particularize groups in WebCollage, } \\
26 \text { minutes to fix phase } 1 \text { groups in GLUE!-PS and } 10 \text { minutes } \\
\text { to finish particularizing resources and to deploy to the wiki, } \\
\text { for one student group of } 28 \text { students [AE3-O1] } \\
\text { (to the question: how much time did you invest in deploying } \\
\text { the jigsaw in the wiki by hand?) around } 2 \text { hours [...] } 1 \text { hour } \\
\text { for understanding the jigsaw, and [ } 1 \text { hour] creating the wiki } \\
\text { pages [...] the other way needed less wiki pages [AE3-R1] }\end{array}$ \\
\hline & $\begin{array}{l}\text { Time-efficiency gains start } \\
\text { when reusing designs }\end{array}$ & $\begin{array}{l}\text { When reusing the design for the second group, it took } 18 \text { min- } \\
\text { utes to re-particularize the groups in WebCollage, } 18 \text { minutes } \\
\text { to fix phase } 1 \text { groups in GLUE!-PS, and } 8 \text { minutes to fin- } \\
\text { ish particularizing resources and to deploy to the wiki for the } \\
\text { other group of students ( } 22 \text { students) [AE3-O1] }\end{array}$ \\
\hline \multirow[t]{3}{*}{ T4 (run-time) } & $\begin{array}{l}\text { GLUE!-PS does not solve the } \\
\text { conceptual problems of run- } \\
\text { time group changes in complex } \\
\text { CSCL designs }\end{array}$ & $\begin{array}{l}\text { (when asked about how time was spent when doing the manual } \\
\text { run-time changes) we wasted a lot of time looking at: "does } \\
\text { this jigsaw group have enough wiki experts?" and so on [...] } \\
\text { thinking the [implications] of the changes is complex... but } \\
\text { GLUE!-PS lets you execute them easily [AE3-I] }\end{array}$ \\
\hline & $\begin{array}{l}\text { Most run-time change needs } \\
\text { are covered by the GLUE!-PS } \\
\text { prototype }\end{array}$ & $\begin{array}{l}\text { (when asked about other useful run-time change needs) Apart } \\
\text { from the re-grouping and modifying the resources... I cannot } \\
\text { think of other ones [AE3-I] }\end{array}$ \\
\hline & $\begin{array}{l}\text { Unusual amounts of extrane- } \\
\text { ous events }\end{array}$ & $\begin{array}{l}\text { A (the teacher), referring to the amount of extraneous events: } \\
\text { this year... this had not ever happened to me before [AE3-O2] }\end{array}$ \\
\hline
\end{tabular}

With respect to the time-efficiency of GLUE!-PS (T2), the proposed learning design (a customized Jigsaw strategy to be enacted with a larger number of students) showed that the particularization process (especially if the LD formalization has to be modified through GLUE!PS) is not very time-efficient during the first-time usage of a learning design. The teacher, however, did see the advantages of reusing such learning design afterwards (see Table 5.17).

Regarding the time-efficiency of the run-time changes (as opposed to the deployment, which is the aspect that we have mostly analyzed so far), in this experiment we experienced how making changes to these larger-group collaborative designs can be quite tedious and error-prone. Much of this time, however, was not employed making the changes to the wiki structure, but rather in conceptualizing those changes over the pre-defined group formation. Although making these run-time changes was faster and easier using GLUE!-PS (as the teacher could see during the interview, when some of those changes were re-enacted using GLUE!-PS), this technological tool does not provide special support in the conceptual part of making a group re-formation that complies with the multiple constraints that, e.g., the Jigsaw strategy imposes. Other tools such as WebCollage, which exploits these strategies explicitly, might offer help in this conceptual part. The problem is that, since they are design tools, they normally are not available to offer help in run-time. 
Regarding the usage of GLUE!-PS in everyday practice (T3), this authentic experiment naturally provided us with several interesting pieces of evidence (Table 5.18). The fact that a novel teacher with non-technical background was able to deploy her learning design using GLUE!PS is encouraging (even if she was not exactly an "average teacher"). Although she mentions the increased usability of the new GLUE!-PS prototype, there are certain parts of the deployment process (such as using GLUE!-PS to "fix a design" done with another LD authoring tool, or managing the document reuse between different activities) still prove challenging, especially without adequate help mechanisms (be it online help, tutorials, or worksheets). Indeed, it seems that there is still space for improvement in the usability of the GLUE!-PS GUI.

Table 5.18: Findings and selected supporting evidence from evaluation happening AE3, around topic T3 (usage in real practice)

\begin{tabular}{l}
\hline Finding \\
\hline A non-technical teacher was able to deploy her \\
learning design using GLUE!-PS \\
Activity- vs. time-based views of the course \\
Importance of preserving manual changes in \\
the LE
\end{tabular}

Selected supporting evidence

(to the question: were you able to deploy the design with GLUE!-PS?) [nods] [AE3-I]

The teacher did (manually) a "temporal view" of the course, with links to the deployed activities [AE3-D]

the LE

(when asked about the reason for not using GLUE!-PS to re-deploy the wiki with the run-time changes) Since I had spent my time fixing the labels [manually] [...] I did not want to throw away that work I'd done [the LE adapter would have overwritten those manual changes] [AE3-I]

(during the enactment of the second face-to-face session, when a latecomer appears) A (the teacher) prefers to do the run-time changes manually because she had edited the wiki manually already [AE3-O2]

Increased usability of the GLUE!-PS prototype (when talking about the use of GLUE!-PS) [...] the usability with respect to what we had [the old prototype] is so much better [AE3$\mathrm{R} 1]$

Need for a deployment preview $\quad$ (after deploying two groups of students, when talking about problems in following the whole design-deploy cycle) the names of [wiki] pages [...] I do not have in mind how the final structure will be [...] this name that I establish in this step, I have to put it this way so that in the end it appears in this other way [AE3-R1]

Need for alternative LE adapter implementations (when asked whether the deployed wiki matches her idea of the design) It matches perfectly... although, now that I know how it has been deployed, maybe I would change a few things [...] that the contributions can be added in the group activity page [instead of in a separate wiki page] [AE3-I]

(when talking about the way the deployment in the wiki is structured) Now it is perfectly structured, orderly [...] but there are too many pages that are not strictly necessary [AE3-I]

Need for further usability enhancements

to the question: which parts of the GLUE!-PS application were more difficult?) the [tool] reuse it is not very intuitive, the [user] interface $[\ldots]$ there are too many icons, you end up messing things [...] (when asked about the possibility of a drag-and-drop use) yes, that would be easier $[\ldots]$ less time, and simpler $[\mathrm{AE} 3-\mathrm{I}]$

(when re-enacting some of the run-time manual changes using GLUE!PS) can't I go back [i.e. undo]? [AE3-I]

No important orchestration problems from the student's perspective (when asked whether students had any problem in doing activities through the deployed wiki) No [...] they already know how to edit, to link [...] I had to remind them that the contributions wiki page was down here $[\ldots]$ Also, when co-editing the same page [...] by design we had said that all the experts had to contribute to the same page [...] there were some editing conflicts [...] when we did the deployments manually, we created the sections for them [AE3-I]

(when talking about the usability of the GLUE!-PS prototype) it is complex $[. .$.$] the only problem I see is that... there is too much in-$ formation [...] if you practice a bit and make it a couple of times for classes, you would be autonomous, but till then [...] it is not for an average teacher [AE3-R1] 
Table 5.18 (continued from previous page)

\begin{tabular}{|c|c|}
\hline Finding & Selected supporting evidence \\
\hline & $\begin{array}{l}\text { (after deploying two groups of students) If I had to do it all alone, I } \\
\text { would have some problems [...] [AE3-R1] }\end{array}$ \\
\hline $\begin{array}{l}\text { The design was not adequate for using with } \\
\text { the GLUE!-PS approach }\end{array}$ & $\begin{array}{l}\text { (when asked whether the time employed to deploy -around } 2.5 \text { hours } \\
\text { for each student group-, was a efficient use of her time) we would have } \\
\text { to do again all the group distributions... I don't know [...] (when asked } \\
\text { to compare it with the manual deployment) the error was trying to } \\
\text { imitate a design that we had quite optimized already, and we did not } \\
\text { exploit all of GLUE!-PS's potential with respect to the tools that can } \\
\text { be used [...] in that sense, maybe it was not worth it for this concrete } \\
\text { design [...] with some changes it would have been worth it [AE3-I] }\end{array}$ \\
\hline $\begin{array}{l}\text { The GLUE!-PS advantage is clearer when } \\
\text { orchestrating DLEs }\end{array}$ & $\begin{array}{l}\text { (when talking about the design-deploy process with GLUE!-PS) this } \\
\text { would be very interesting if we used Google Docs [...] our design was } \\
\text { very optimized to our limitations when doing it by hand [AE3-R1] } \\
\text { (when asked whether she would use GLUE!-PS again the next aca- } \\
\text { demic year) I would use GLUE!-PS, but I would change things in the } \\
\text { learning design [...] to extract more potential from the system [...] our } \\
\text { design as it is now does not take advantage of GLUE!-PS [...] different } \\
\text { tools [...] it could help with preventing plagiarism [...] this year I have } \\
\text { detected several cases, copying among themselves and from previous } \\
\text { years' work }[\ldots] \text { to separate the resources from one another [AE3-I] }\end{array}$ \\
\hline $\begin{array}{l}\text { The importance of particularization details in } \\
\text { the deployment's aspect is not obvious for the } \\
\text { teacher at first }\end{array}$ & $\begin{array}{l}\text { (after deploying two groups of students, when talking about problems } \\
\text { in following the whole design-deploy cycle) the names of [wiki] pages } \\
{[\ldots . . .] \text { I do not have in mind how the final structure will be [...] this name }} \\
\text { that I establish in this step, I have to put it this way so that in the } \\
\text { end it appears in this other way [AE3-R1] } \\
\text { (when shown the deployed wiki) I spent some time re-touching [man- } \\
\text { ually] the labels, parentheses [...] if you are not careful in GLUE!-PS, } \\
\text { then [the resulting wiki] is a mess [...] you have to notice that those } \\
\text { names influence the presentation in the wiki [AE3-I] } \\
\text { (when talking about the deployed wiki) when you are [using GLUE!- } \\
\text { PS] you do not know how it is going to be deployed, visually, aesthet- } \\
\text { ically [AE3-I] }\end{array}$ \\
\hline $\begin{array}{l}\text { The teacher thinks she could deploy learning } \\
\text { designs unassisted }\end{array}$ & $\begin{array}{l}\text { (to the question: would you have been able to deploy the design on } \\
\text { your own?) I think I would have been able, yes }[\ldots] \text { the first time it } \\
\text { would have taken more time }[\ldots] \text { once you know how to do it, it's } \\
\text { automatic }[\text { AE3-I] }\end{array}$ \\
\hline $\begin{array}{l}\text { The teacher thinks she would need assistance } \\
\text { to do the LD modeling workarounds }\end{array}$ & $\begin{array}{l}\text { (when asked about the process of re-creating phase } 1 \text { groups in } \\
\text { GLUE!-PS) I don't think I would've been able without your help... or } \\
\text { without a worksheet [...] with a worksheet, no problem [AE3-I] }\end{array}$ \\
\hline
\end{tabular}

This evaluation happening also offered further evidence on the need for different implementations of the LE adapter, especially in order to produce different ways of structuring/presenting the deployed designs in the target learning platforms. Related to this issue, it became clear in this authentic experiment that we had underestimated the importance of preserving manual changes done to the wiki pages after the deployment. The current MediaWiki LE adapter simply overwrites those changes (since most of the changes, e.g. in the activity descriptions, can be done through the GLUE!-PS anyway). However, the loss of these manual changes was, in this authentic situation, enough to make the teacher opt for performing further run-time changes by hand (in order not to lose the previous manual work). This preservation of manual changes is possible by implementing a different, more "intelligent" LE adapter that is able to merge GLUE!-PS and manual changes (although the implementation of such an adapter would also be more costly in terms of development effort).

The gathered evidence also confirms previous preliminary conclusions, for example the fact that students do not have too much trouble in following the GLUE!-PS-deployed learning 
activities, or the need of some kind of "deployment preview" that helps teachers in envisioning how the particularized design activities will look like once deployed into the learning platform. This last issue seems especially relevant, since the influence of the particularized design into the final deployed result may not be obvious for the teacher (since she does not know the kind of decisions that the LE adapter implementors have taken to do the translation of concepts), especially in first-time usages of GLUE!-PS. Improving the user's idea of what the GLUE!-PS system does (in terms of translating the GLUE!-PS LF concepts to learning platform concepts) might be another way of helping teachers to attain the desired results more efficiently, without so much going back and forth between GLUE!-PS and the learning platform (see the discussion on the "bricoleur/iterative deployment" in previous authentic experiments).

Overall, it was clear both for the researchers and for the teacher that the learning design, which tried to be authentic by mimicking the one that had been enacted other years, was not ideal for its deployment and enactment with GLUE!-PS (since it did not really use a DLE, and the tweaking of the LD formalization coming from WebCollage took quite a long time). Indeed, the teacher asserted that, she would use GLUE!-PS for the following years, but that she would modify the design to take advantage of GLUE!-PS's features for supporting orchestration of DLEs.

Regarding our last topic of interest, the ability of GLUE!-PS to support useful run-time adaptations in the deployed learning designs (T4), we did not collect as much evidence due to the teacher not really using the run-time features during the enactment. Nevertheless, we have gathered evidence highlighting that GLUE!-PS's current capabilities for adaptation (which allow for the re-forming of groups and the modifications to the resources to be used in the learning activities), cover the most common run-time change needs of the teacher. However, the unusual amount of unexpected events that forced changes in the deployment also showed that this technological support to adaptation, however important, does not solve other conceptual problems of making these run-time changes, especially in the case of large groups of students and relatively complex grouping structures such as the Jigsaw.

\section{A workshop with teachers from multiple disciplines (TW6)}

In order to obtain evidence around the usefulness of the GLUE!-PS proposal from a wide variety of teachers that were neither teacher-researchers, nor ICT experts, we decided to implement a teacher professional development workshop, along the lines of the ones performed in previous iterations (see Section 5.5.3), but extended in depth and timeframe in order to address some of the shortcomings of those previous workshops.

Context and method As it happened with workshop TW3, this workshop was held within the framework the the Buendia center for professional development at the University of Valladolid. The workshop was advertised as a practical course in "ICT support for the design and implementation of collaborative learning" 66 , and it was open for university teachers with a basic knowledge of the involved ICT tools, up to 24 participants. Since our research goal was to evaluate the GLUE!-PS system as a support for CSCL practice, and not to promote such practice

\footnotetext{
${ }^{66}$ http://www.buendia.uva.es/forProfesoradoFicha.asp?IdForContinuaFicha=678 (Last visit: 18 Jun 2012).
} 
at the conceptual level, we prioritized the applications from participants of any of the previous teacher workshops done during this dissertation (e.g. TW3, TW4, see Section 4.5). This would ensure that most participants would have a minimal conceptual grasp of what designing and implementing CSCL entails, without further background biases (since those workshops, especially TW4, had been open for teachers of any discipline to participate), thus making it possible to evaluate the technological support for such a complex practice as CSCL in such a short amount of time with minimal participant biases.

The workshop was designed to involve teachers in a blended learning format for 12 hours, spanning two 4-hour face-to-face sessions and a certain amount of online work. The goal of the workshop for participants was to learn how to use specific ICT tools such as the WebCollage authoring tool and the GLUE!-PS system to design, implement and enact blended CSCL activities involving multiple ICT learning tools (thus conforming a DLE). During the workshop teachers would work collaboratively and individually in the design, deployment and (emulated) enactment of both a hypothetical but realistic CSCL scenario, and a real CSCL scenario relevant to the practice of each teacher (i.e. one of their actual courses). The workshop's own learning design involved the following coarse-grained phases:

1. Pre-session online activities, including the reading of the sample CSCL scenario to be used during the workshop (a blended CSCL design aimed at fostering competences on information search, critical thinking and structured report writing, which involved a Pyramid CLFP and a variety of ICT tools in a DLE, see Figure 5.42. Also, participants would answer an online questionnaire about their background and starting knowledge (TW6-Q1).

2. A first face-to-face session, in which teachers working in dyads would use the WebCollage authoring tool to formalize the proposed design, and implement it in a Moodle-based DLE using the GLUE!-PS system. The usage of both WebCollage and GLUE!-PS would be scaffolded by a detailed worksheet guiding the teachers through the process of the design and deployment.

3. Teachers would make, individually, a CSCL design for their own practice, using the tools and methods learned in the first session (mostly, the WebCollage authoring tool). Participants were encouraged to reuse parts of the design they had already done (in order to keep the participant workload under control, which was one of the typical complaints of previous workshops). The workshop's facilitators (i.e. the research team) would review those designs in order to give feedback to the participants regarding their feasibility and clarity.

4. In a second face-to-face session, participants would deploy their individual designs using GLUE!-PS, either into a Moodle-based DLE or into a wiki-based DLE (the two LE adapters implemented at the time). Afterwards, three "problematic situations" that required run-time changes (changes in group formation, as well as changes in the resources associated with activities) would be presented, and participants were asked to find solutions to those problems by using GLUE!-PS to implement those changes. In this case, participants were not scaffolded with worksheets during the session (albeit worksheets of the run-time changes were provided afterwards, as learning materials for their reference). Finally, participants would be engaged in four parallel mini-debates (similar to focus groups) 
regarding the use and applicability of the workshop's contents to their everyday practice, whose conclusions would be shared with the rest of the participants.

5. After the second session, participants were asked to fill in a questionnaire assessing the workshop and the presented ICT tools (TW6-Q2), as well as a general assessment questionnaire about the form and value of the professional development action (TW6-Q3).

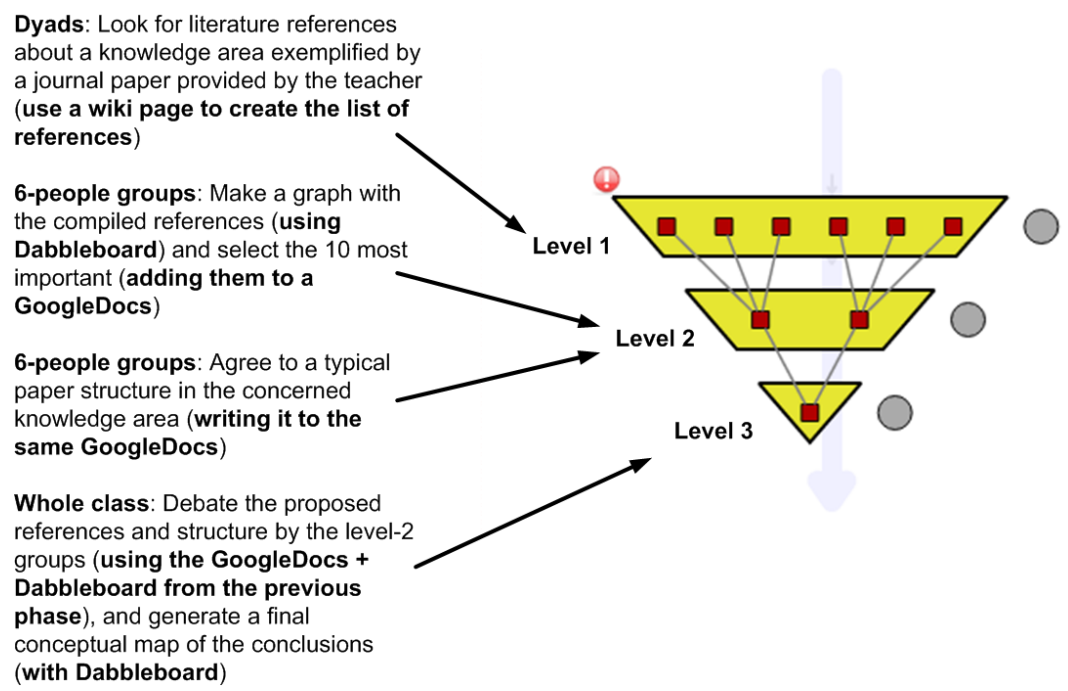

Figure 5.42: Graphical representation of the example learning design that participants in workshop TW6 had to implement using WebCollage and GLUE!-PS

This scenario was finally enacted at the end of April 2012, in two sessions separated by one week (see Figure 5.43). 24 teachers participated in the workshop, of which 21 had already participated in previous workshops on the design and enactment of CSCL. These teachers came from a variety of backgrounds, including Engineering (6 teachers), Computer Science (3 teachers), Education and Social Work (3 teachers), Medicine (2 teachers), Arts and Humanities (2 teachers) and several others ranging from Mathematics to Law. During the workshop, we gathered data by recording and observing (by at least two researchers) the face-to-face workshop sessions (TW6-R and TW6-O), as well as by analyzing the different artifacts generated by the participants during the workshop, such as WebCollage's learning designs and GLUE!-PS's deployments (TW6-D). We also recorded separately each of the focus groups that have been mentioned at the end of the second face-to-face session (TW6-FG). Furthermore, the three aforementioned questionnaires filled in by participants (TW6-Q1, TW6-Q2 and TW6-Q3) were also used in our analysis. All these data sources are summarized in Table 5.19.

Our main evaluation goal with this "situated action" was to observe how multiple teachers from different disciplines used the GLUE!-PS prototype to deploy CSCL scenarios and emulate run-time changes to such scenario. Thus, we would explore the participant teachers' perceptions of our main evaluation tension or issue ("Does GLUE!-PS support teachers in the orchestration of blended CSCL across DLEs?" - I1), around the four main topics that we have used so far: the ability of GLUE!-PS in deploying learning designs preserving the design's essential qualities (T1), its time-efficiency in doing so (T2), the feasibility of using GLUE!-PS in everyday teaching practice (T3), and the usefulness of GLUE!-PS prototype's run-time flexibility features (T4). 

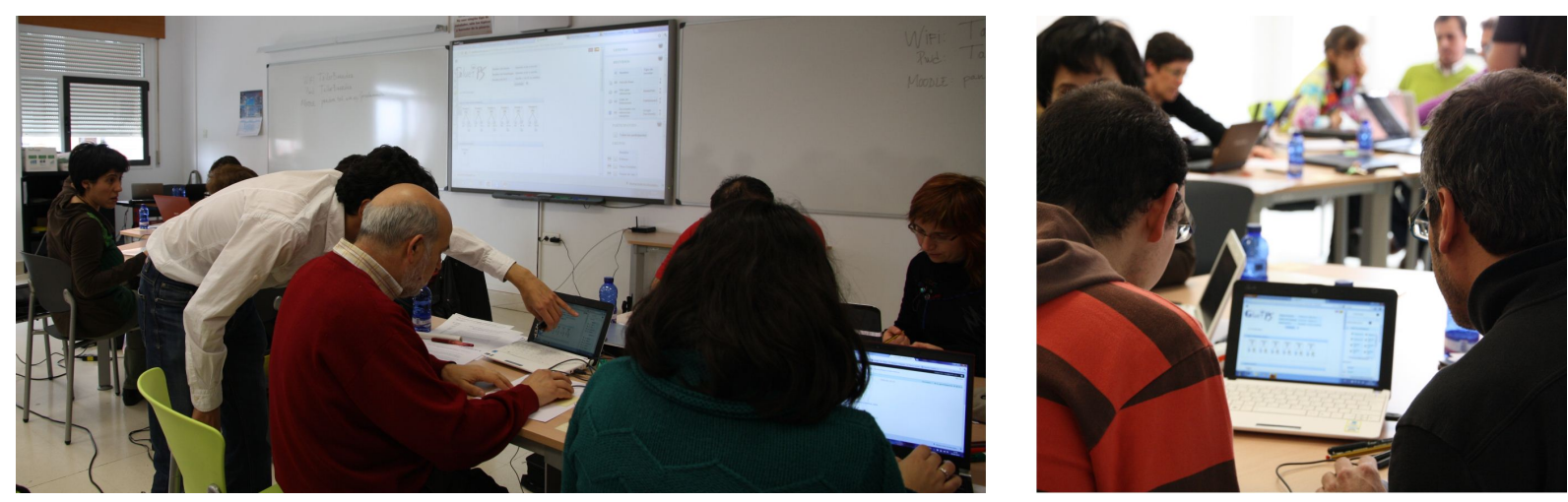

Figure 5.43: Photos taken during the face-to-face session of evaluation happening TW6

Table 5.19: Main data sources used during the TW6 evaluation happening

\begin{tabular}{|lll|}
\hline Source & Kind of evidence & Code \\
\hline \hline $\begin{array}{l}\text { Observation notes of the face-to-face workshop } \\
\text { sessions }\end{array}$ & Qualitative & TW6-O \\
\hline $\begin{array}{l}\text { Video/audio recording of the face-to-face work- } \\
\text { shop sessions }\end{array}$ & Qualitative & TW6-R \\
\hline $\begin{array}{l}\text { Participant-generated artifacts along the process } \\
\text { (WebCollage, GLUE!-PS, MediaWiki data) }\end{array}$ & Qualitative & TW6-D \\
\hline $\begin{array}{l}\text { Audio recording of the four parallel focus groups } \\
\text { in the second workshop session }\end{array}$ & Qualitative & TW6-FG \\
\hline $\begin{array}{l}\text { First questionnaire about participants' profiles } \\
\text { and prior knowledge }\end{array}$ & $\begin{array}{l}\text { Qualitative \& Quantita- } \\
\text { tive }\end{array}$ & TW6-Q1 \\
\hline $\begin{array}{l}\text { Second questionnaire assessing the presented } \\
\text { tools and the workshop }\end{array}$ & $\begin{array}{l}\text { Qualitative \& Quantita- } \\
\text { tive }\end{array}$ & TW6-Q2 \\
\hline $\begin{array}{l}\text { Third questionnaire assessing the workshop as a } \\
\text { professional development action }\end{array}$ & $\begin{array}{l}\text { Qualitative \& Quantita- } \\
\text { tive }\end{array}$ & TW6-Q3 \\
\hline
\end{tabular}

Findings and evidence The enactment of the TW6 workshop took place with only minimal technical failures, and all the designed activities were completed by the participants (with the exception of one participant who dropped out of the workshop). In fact, participants were very positive and optimistic regarding the workshop as a formative action: the workshop scored over 9 in a 1-10 scale, in average, in most of the parameters of the Buendía center's PD action questionnaire (e.g. the global assessment of the workshop as a formative action indicated an average score of 9.23, with a standard deviation of 0.70 [TW6-Q3]).

Regarding our first topic of interest, GLUE!-PS's ability to deploy learning designs preserving essential qualities (T1), we found out that a great majority of this set of trans-disciplinar, non-CSCL-expert teachers were able to deploy not only the provided example learning design, but also a the semi-authentic variation of the design they did for their own teaching practice (see Table 5.20). Moreover, they majoritarily agreed that the deployed courses in Moodle- or MediaWiki-based DLEs reflected their learning design's essential qualities, and that the way of presenting the DLE (e.g. integrated into the learning platform's interface) would be useful for the enactment of the activities. It is also interesting to look at the qualitative responses of those participants who could not complete the deployment of the learning design for their own practice, since none of them describe problems with the GLUE!-PS system itself, but rather allude to lack of time to do the online re-design task or because of the inability of the LD authoring 
tool to model the participant's ideas.

Table 5.20: Findings and selected supporting evidence from evaluation happening TW6, around topics T1 (deployment ability) and T2 (time-efficiency)

\begin{tabular}{|c|c|c|}
\hline Topic & Finding & Selected supporting evidence \\
\hline \multirow[t]{5}{*}{ T1 (deploy ability) } & $\begin{array}{l}\text { Non-expert teachers consider } \\
\text { the final format of the deploy- } \\
\text { ment adequate }\end{array}$ & $\begin{array}{l}\text { Teachers almost completely agreed with the fact that having } \\
\text { the DLE resources centralized under the learning platform fa- } \\
\text { cilitates the enactment of the activities (in a 1-8 Likert scale, } \\
\text { avg }=7.28, \text { std }=0.84 \text { ) [TW6-Q2] }\end{array}$ \\
\hline & $\begin{array}{l}\text { Non-expert teachers consid- } \\
\text { ered that the deployed designs } \\
\text { reflected their design's essen- } \\
\text { tial qualities }\end{array}$ & $\begin{array}{l}\text { Respondent teachers agreed strongly that their individual de- } \\
\text { ployed design reflected their design's ideas (in a 1-8 Likert } \\
\text { scale, avg }=6.62, \text { std }=1.60 \text { ) [TW6-Q2] }\end{array}$ \\
\hline & $\begin{array}{l}\text { Non-expert teachers were able } \\
\text { to deploy } \\
\text { moderately-authentic }\end{array}$ & $\begin{array}{l}\text { (when deploying the example scenario to Moodle) group } 8 \\
\text { sees their design deployed in Moodle [...] All deployments have } \\
\text { worked! [TW6-O] }\end{array}$ \\
\hline & CLFP-based learning designs & $\begin{array}{l}18 \text { out of } 21 \text { respondents }(85.7 \%) \text { were able to deploy their } \\
\text { individual learning designs in the second session [TW6-Q2] }\end{array}$ \\
\hline & $\begin{array}{l}\text { Teachers that did not deploy } \\
\text { due to causes unrelated to } \\
\text { GLUE!-PS }\end{array}$ & $\begin{array}{l}\text { (to the question: why were you not able to deploy the learning } \\
\text { design in Moodle?) I deployed it with GLUE!-PS but did not } \\
\text { restore it in Moodle [TW6-Q2] } \\
\text { (to the question: why were you not able to deploy the learning } \\
\text { design in Moodle?) I had not enough time to do the real course } \\
\text { design. With the indications of the workshop and the practice } \\
\text { we did it looked feasible to be done [TW6-Q2] } \\
\text { (to the question: why were you not able to deploy the learning } \\
\text { design in Moodle?) the real case I wanted to work on does not } \\
\text { adjust to the pyramid structure and, since I do not know other } \\
\text { techniques, I had trouble from the start [TW6-Q2] }\end{array}$ \\
\hline \multirow{6}{*}{ T2 (time-efficiency) } & $\begin{array}{l}\text { Non-expert teachers consid- } \\
\text { ered the design-deployment } \\
\text { process time-efficient }\end{array}$ & $\begin{array}{l}\text { The design-deployment process was considered time-efficient } \\
\text { by teachers (in a } 1-8 \text { Likert scale, avg }=7.14, \text { std }=0.72 \text { ) [TW6- } \\
\text { Q2] }\end{array}$ \\
\hline & $\begin{array}{l}\text { Potential features to improve } \\
\text { time-efficiency }\end{array}$ & $\begin{array}{l}\text { (to the question: if you do not think the deployment process } \\
\text { is time-efficient, indicate how would you like it to be) Maybe } \\
\text { the resource reuse, which does not translate from WebCollage, } \\
\text { could be automated when you deploy in GLUE!-PS [TW6-Q2] }\end{array}$ \\
\hline & $\begin{array}{l}\text { With adequate support, } \\
\text { non-expert teachers were able } \\
\text { to design and deploy } \\
\text { CLFP-based scenarios in less }\end{array}$ & $\begin{array}{l}\text { (when designing the example scenario with WebCollage) group } \\
2 \text { is only half-using the worksheet. They are doing the design } \\
\text { faster than we ourselves did in the testing session on Tuesday } \\
\text { [TW6-O] }\end{array}$ \\
\hline & time than they estimated & $\begin{array}{l}18 \text { out of } 21 \text { respondents }(85.7 \%) \text { were able to deploy their } \\
\text { individual learning designs in the second session (in under } 1 \\
\text { hour of GLUE!-PS usage) [TW6-Q2] }\end{array}$ \\
\hline & & $\begin{array}{l}20 \text { out of } 21 \text { respondents }(95.2 \%) \text { asserted they finished de- } \\
\text { signing and deploying the proposed scenario (in under } 2 \text { hours } \\
\text { of tool usage) [TW6-Q2] }\end{array}$ \\
\hline & & $\begin{array}{l}\text { Before the workshop, teachers estimated the time to imple- } \\
\text { ment the scenario at around } 9 \text { hours }(\text { avg }=8.94 \text {, std }=8.15 \text { ) } \\
\text { [TW6-Q1] }\end{array}$ \\
\hline
\end{tabular}

Regarding our second topic, the time-effectiveness of the GLUE!-PS approach (T2), our participant teachers valued the whole design-deployment process with WebCollage and GLUE!PS as time-efficient, although they also suggested a potential feature to facilitate the tool reuse (which does not get expressed adequately in the IMS-LD modeling of the learning designs, and thus is not captured by GLUE!-PS). As we can see from the evidences in Table 5.20, our set of trans-disciplinar teachers were able to design and deploy the example learning design (and the designs they had produced on their own) in less time than they had estimated prior to the workshop. We should remark, however, that the participants had the scaffolding of a detailed worksheet during the first face-to-face session, and that the research team was available during 
the face-to-face sessions to provide further technical support to the participants.

Concerning the question of whether teachers would use the GLUE!-PS approach in real everyday practice (T3), our participant teachers, who were not CSCL experts, said that they would likely use GLUE!-PS (and the GLUE!-PS-generated courses done during the workshop) in their own practice in the immediate future (see Table 5.21). This generally positive outlook in the questionnaire responses was moderated during the focus groups, where teachers expressed several problems that may arise in the usage of this kind of approach in real practice. However, from all the voiced concerns, only two aspects can be said to be concerning the GLUE!-PS directly: its lack of production-level reliability of the system (i.e. the fact that there are still bugs in the application), and the lack of further help and technical support for using the full functionality of the system (e.g. apart from the worksheets used in the workshop, tutorials and even human resources for technical support would be crucial, in the opinion of some participants). The rest of the problems expressed by participants, which may hamper the adoption of this kind of CSCL approaches, were more general in nature, and independent of the GLUE!-PS system: the ability of LD tools to model the teachers' ideas, general attitudes and beliefs concerning ICT use or collaborative learning, lack of perceived ability, lack of adequate ICT infrastructures or attitude problems on the side of students and fellow teachers.

Table 5.21: Findings and selected supporting evidence from evaluation happening TW6, around topic T3 (usage in real practice)

\begin{tabular}{|c|c|}
\hline Finding & Selected supporting evidence \\
\hline $\begin{array}{l}\text { GLUE!-PS is considered very usable by non- } \\
\text { expert teachers }\end{array}$ & $\begin{array}{l}\text { GLUE!-PS was highly valued for usability (in a 1-8 Likert scale, } \\
\text { avg }=7, \mathrm{std}=1 \text { ), compared with WebCollage (avg }=6.76, \mathrm{std}=1.09 \text { ) or } \\
\text { Moodle }(\mathrm{avg}=6.48, \mathrm{std}=1.36) \text { [TW6-Q2] }\end{array}$ \\
\hline Need for further usability enhancements & $\begin{array}{l}\text { (when designing the example scenario with WebCollage) group } 24 \\
\text { asks what LMS means. I explain briefly what they are doing when } \\
\text { they connect WebCollage with Moodle through GLUE!-PS [...] Yannis } \\
\text { explains the same issue to group } 12 \text { [TW6-O] } \\
\text { Throughout the [first face-to-face session] some ideas to enhance our } \\
\text { tools have emerged: Allow the editing of the titles and activity levels } \\
\text { in GLUE!-PS [the prototype did not allow that at the time]. Allow } \\
\text { the selection of multiple tools and documents to reuse. Re-think the } \\
\text { term "reuse" in GLUE!-PS [...] [TW6-O] }\end{array}$ \\
\hline $\begin{array}{l}\text { Non-expert teachers would likely use GLUE!- } \\
\text { PS for their own practice }\end{array}$ & $\begin{array}{l}\text { Teachers asserted they would likely use the proposed tools in their } \\
\text { everyday practice in the immediate future (in a } 1-8 \text { Likert scale, } \\
\text { avg }=6.19, \mathrm{std}=1.63 \text { ) [TW6-Q2] }\end{array}$ \\
\hline $\begin{array}{l}\text { Non-expert teachers would likely use the } \\
\text { workshop-generated courses for their own } \\
\text { practice }\end{array}$ & $\begin{array}{l}\text { Teachers asserted they would be moderately likely to use their de- } \\
\text { ployed designs in Moodle in the immediate future (in a } 1-8 \text { Likert } \\
\text { scale, avg }=6, \text { std }=1.64 \text { ) [TW6-Q2] }\end{array}$ \\
\hline $\begin{array}{l}\text { Reasons for not using GLUE!-PS: ability of LD } \\
\text { authoring tools }\end{array}$ & $\begin{array}{l}\text { (in the focus-groups, when discussing other run-time implementation } \\
\text { problems of blended CSCL) D (a participant) [says] [...] it is difficult } \\
\text { to translate his design ideas to the tool, and that he probably would } \\
\text { have to change his activity ideas [TW6-O] }\end{array}$ \\
\hline $\begin{array}{l}\text { Reasons for not using GLUE!-PS: attitudes to- } \\
\text { wards ICT use }\end{array}$ & $\begin{array}{l}\text { (when outlining one focus group's conclusions about whether they } \\
\text { would use WebCollage/GLUE!-PS in real practice) some of us would } \\
\text { not use it because they think [using ICTs] is complex and not neces- } \\
\text { sary, versus other simpler ways of doing collaborative work [TW6-FG] }\end{array}$ \\
\hline $\begin{array}{l}\text { Reasons for not using GLUE!-PS: beliefs and } \\
\text { attitudes towards collaborative learning }\end{array}$ & $\begin{array}{l}\text { (when outlining one focus group's conclusions about other run-time } \\
\text { problems in blended CSCL) we have also considered whether these } \\
\text { collaborative activities may substract from the [content] learning [...] } \\
\text { we should not mistake the means with the goals [TW6-FG] }\end{array}$ \\
\hline $\begin{array}{l}\text { Reasons for not using GLUE!-PS: circumstan- } \\
\text { tial }\end{array}$ & $\begin{array}{l}\text { (to the question: why would you not use the deployed course in your } \\
\text { practice?) I would use it, but [my design] is missing some more phases, } \\
\text { and I would have to review the [workshop] materials [TW6-Q2] }\end{array}$ \\
\hline
\end{tabular}


Table 5.21 (continued from previous page)

\begin{tabular}{|c|c|}
\hline Finding & Selected supporting evidence \\
\hline $\begin{array}{l}\text { Reasons for not using GLUE!-PS: general } \\
\text { student and teacher issues }\end{array}$ & $\begin{array}{l}\text { (to the question: if you will not use the presented tools in the future, } \\
\text { explain why) I wish I can. I'm missing: "community" (I must be the } \\
\text { freak in my department) [...] that the students are willing to be led } \\
\text { to the collaborative work easily [TW6-Q2] } \\
\text { (when outlining one focus group's conclusions about other run-time } \\
\text { problems in blended CSCL) another problem can be the attitude of } \\
\text { students [...] there are some students that want to work alone [TW6- } \\
\text { FG] }\end{array}$ \\
\hline $\begin{array}{l}\text { Reasons for not using GLUE!-PS: integration } \\
\text { into institutional ICTs }\end{array}$ & $\begin{array}{l}\text { (when outlining one focus group's conclusions about whether they } \\
\text { would use WebCollage/GLUE!-PS in real practice) also there is a } \\
\text { need of integrating this kind of scenarios in a larger [Moodle] course } \\
\text { [TW6-FG] }\end{array}$ \\
\hline $\begin{array}{l}\text { Reasons for not using GLUE!-PS: lack of in- } \\
\text { frastructure }\end{array}$ & $\begin{array}{l}\text { (in the focus-groups, when discussing whether they would use the } \\
\text { presented ICT tools) they see problems of classroom implementation, } \\
\text { because of the [ICT] infrastructure [TW6-O] }\end{array}$ \\
\hline $\begin{array}{l}\text { Reasons for not using GLUE!-PS: lack of own } \\
\text { perceived ability }\end{array}$ & $\begin{array}{l}\text { (to the question: if you do not consider GLUE!-PS easy to use, indicate } \\
\text { which parts are more problematic or tedious) It is the first time I } \\
\text { approach the application, I need more time to practice [TW6-Q2] }\end{array}$ \\
\hline $\begin{array}{l}\text { Reasons for not using GLUE!-PS: lack of reli- } \\
\text { ability }\end{array}$ & $\begin{array}{l}\text { (when outlining one focus group's conclusions about whether they } \\
\text { would use WebCollage/GLUE!-PS in real practice) we think this use } \\
\text { is feasible but }[\ldots] \text { there is a need of further training and }[\ldots . \text { the tool is } \\
\text { not completely debugged }[\ldots . .] \text { so making real tests with your students } \\
{[\ldots] \text { is very risky }[\ldots][\text { TW6-FG] }}\end{array}$ \\
\hline $\begin{array}{l}\text { Reasons for not using GLUE!-PS: lack of tech- } \\
\text { nical help/support }\end{array}$ & $\begin{array}{l}\text { (when outlining one focus group's conclusions about whether they } \\
\text { would use WebCollage/GLUE!-PS in real practice) yes, but [...] there } \\
\text { has to be more technical support [...] manuals, [online] help, help from } \\
\text { somebody [...] to have a guide [...] the provided worksheet is OK for } \\
\text { the task at hand, but in other cases you might be a bit lost [TW6-FG] }\end{array}$ \\
\hline $\begin{array}{l}\text { Tool reuse as an outstanding conceptual } \\
\text { problem }\end{array}$ & $\begin{array}{l}\text { (when particularizing the design of the example scenario with GLUE!- } \\
\text { PS) group } 12 \text { asks me to explain the concept of reuse and how to } \\
\text { configure it. After a brief explanation, they get it right away [TW6- } \\
\text { O] } \\
\text { (when particularizing the design of the example scenario with GLUE!- } \\
\text { PS) It seems that participants work without too much trouble. Sup- } \\
\text { port on our side is crucial at some points [...] I tell LP that it might } \\
\text { be nice to show an example of how the tools are reused in GLUE!-PS. } \\
\text { [...] Constant support is necessary [TW6-O] }\end{array}$ \\
\hline
\end{tabular}

The GLUE!-PS prototype, despite being experimental, was considered very usable by nonexpert teachers, even more so than the other, more established tools like Moodle or WebCollage. This positive opinion on the usability of GLUE!-PS was counter-balanced by some participants that highlighted the need of further usability enhancements that could be done (some of which have already been implemented, such as the editing of activity names and descriptions). The tool reuse features, especially, seemed to be still problematic to understand for some participants.

Finally, regarding GLUE!-PS's ability to provide useful run-time changes to common adaptation problems (T4), after briefly experimenting with the most common of them, they unanimously concluded that the problems presented are commonplace, and that solutions that GLUE!-PS provides are useful, intuitive and quick to implement (see Table 5.22). It is worth noting that the run-time change features that participants could test were limited by the possibilities of the respective LE adapters: part was tested in Moodle-based DLEs (post-deployment re-groupings before the student-generated data was added to the course), but most of the situations were applied to MediaWiki-based DLEs (run-time changes in the group formation and 
the activity resources), which are more adaptable due to them being an example of "dynamic deployment" (see Section 5.2.2).

Table 5.22: Findings and selected supporting evidence from evaluation happening TW6, around topic T4 (run-time changes)

\begin{tabular}{|c|c|}
\hline Finding & Selected supporting evidence \\
\hline $\begin{array}{l}\text { GLUE!-PS run-time change features cover } \\
\text { frequent problems }\end{array}$ & $\begin{array}{l}\text { (when particularizing the design of the example scenario in GLUE!- } \\
\text { PS) B and C (two participants) ask what happens if, in the middle } \\
\text { of the course, people do not come, etc. They have been playing with } \\
\text { GLUE!-PS, creating and deleting groups [TW6-O] } \\
\text { Teachers almost completely agreed with the presented "problematic } \\
\text { situations" being frequent (in a 1-8 Likert scale, avg }=7.19, \text { std=1.16) } \\
\text { [TW6-Q2] }\end{array}$ \\
\hline $\begin{array}{l}\text { Non-expert teachers considered run-time } \\
\text { solutions with GLUE!-PS useful }\end{array}$ & $\begin{array}{l}\text { (in the focus-groups, when discussing the presented run-time problems } \\
\text { and solutions) They think the presented problems are common, and } \\
\text { the solutions optimal. Also, they appreciate the fact that the problems } \\
\text { are highlighted (and that there is the possibility of a solution) [TW6- } \\
\text { O] } \\
\text { (when outlining one focus group's conclusions about the presented } \\
\text { run-time problems and GLUE!-PS solutions) all the problems looked } \\
\text { very common, and the solutions with GLUE!-PS intuitive, easy to do } \\
\text { [TW6-FG] } \\
\text { (when outlining one focus group's conclusions about the presented } \\
\text { run-time problems and GLUE!-PS solutions) we have commented that } \\
\text { the solutions were intuitive, easy, quick to implement [TW6-FG] } \\
\text { GLUE!-PS solutions to the run-time "problematic situations" were } \\
\text { considered useful (in a 1-8 Likert scale, avg=7.38, std=0.74) [TW6- } \\
\text { Q2] }\end{array}$ \\
\hline $\begin{array}{l}\text { Suggested run-time features: time } \\
\text { management }\end{array}$ & $\begin{array}{l}\text { (to the question: indicate other enactment problems that may emerge } \\
\text { when doing collaborative activities with ICT) Students that submit } \\
\text { their work beyond the deadlines - to allow (or not) flexibility in the } \\
\text { deadlines [TW6-Q2] } \\
\text { (when outlining one focus group's conclusions about other run-time } \\
\text { problems in blended CSCL) we discussed whether it could include } \\
\text { some way of managing deadlines, outdating of resources, links [TW6- } \\
\text { FG] }\end{array}$ \\
\hline
\end{tabular}

Moreover, teachers also suggested an aspect which currently is not being taken into consideration by the GLUE!-PS system: that of time management issues, such as setting deadlines for tasks or resources, and the run-time adaptations of those timings. This evidence comes to confirm similar assertions by the teachers in our authentic experiments, thus highlighting the importance of this aspect in the teachers' conception of orchestration. This also hints at a possible extension of the GLUE!-PS LF model to address these time management issues.

Overall, the workshop was considered a success both by participants and by the research team. The fact that this PD action was not hampered by the technical failures and other extraneous events of previous workshops and authentic experiments seems to correlate with a generally positive attitude towards the presented tools. This influence of the orchestration of the "situated action" itself into the evaluation results (which we already noticed in the evaluation of atomic patterns, see Section 4.5 can be used to temper the enthusiasm that we could extract from the gathered evidence. Also, it would be interesting to analyze how much of the success of this workshop and the positive opinions it garnered were caused by most of the teachers' participation in previous workshops which were aimed at conceptually supporting orchestration of blended CSCL activities (such as TW4). We hypothesize that the use of the conceptual and technological tools proposed in this thesis together might be more effective than their separate 
use, although this hypothesis should be tested empirically in the future.

\section{Partial conclusions and formative feedback}

The three evaluation happenings from this fourth evaluation iteration provided a wealth of new evidence about the usefulness of GLUE!-PS to support the orchestration of blended CSCL activities, delving into the nature of this support and its limitations. The partial conclusions from the different evaluation happenings, which used the improved GLUE!-PS prototype (especially the new GUI) at different stages of completion, have been represented graphically in Figure 5.44. These conclusions can also be grouped by topic in the following manner:

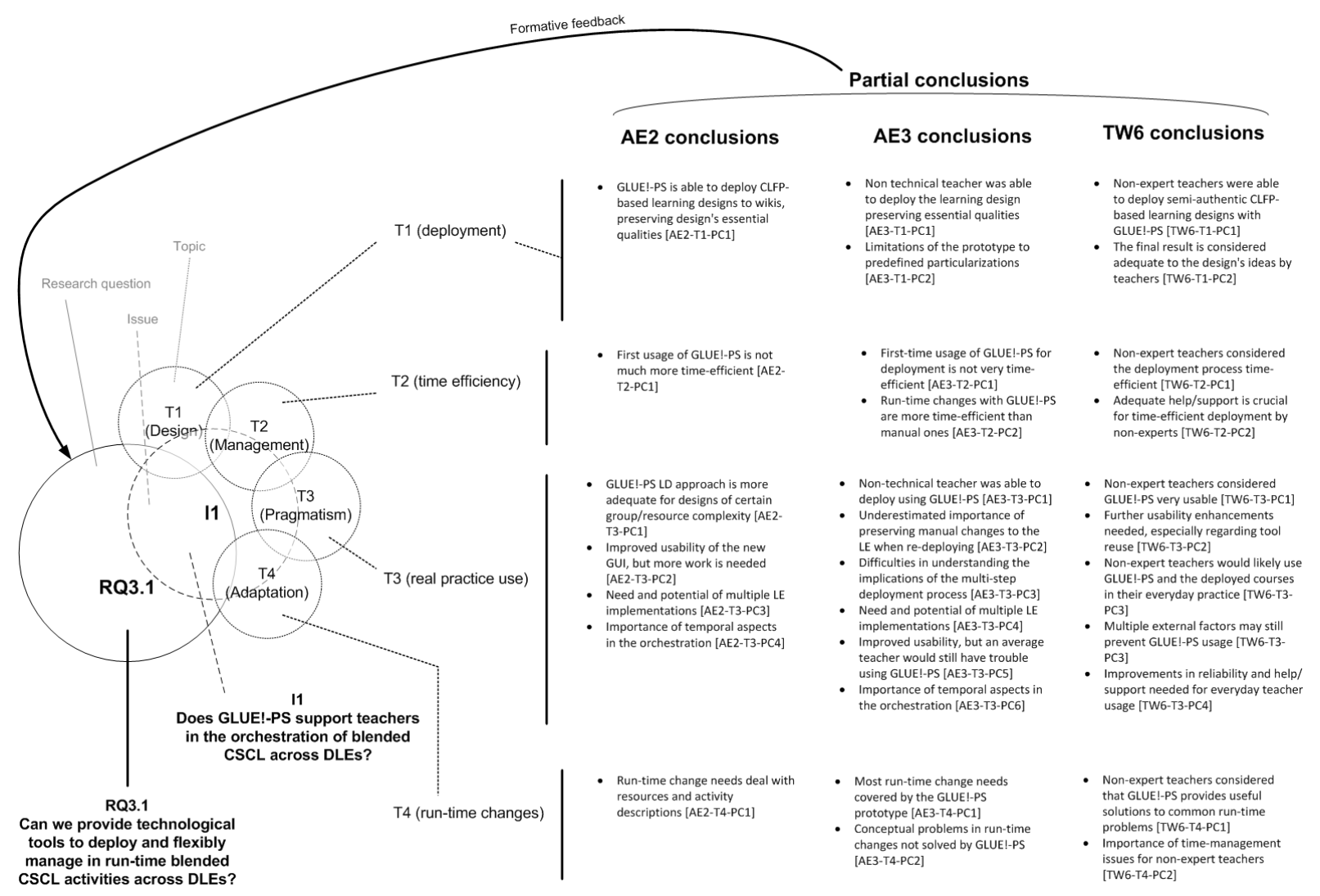

Figure 5.44: Graphical representation of the issues, topics and partial conclusions of the

Iteration \#4

T1 (deployment ability) From the authentic experiments and workshops performed we can generally conclude that GLUE!-PS has been able to deploy all the blended CSCL designs that expert and non-expert teachers have tried to implement, while preserving the design's essential qualities (AE2-T1-PC1, AE3-T1-PC1, TW6-T1-PC1, TW6-T1-PC2). However, these conclusions have been gathered only from one kind of blended CSCL scenario: those CSCL scenarios designed using the WebCollage authoring tool. Thus, they all share the common traits of being based on the idea of Collaborative Learning Flow Patterns 
(CLFPs), and being modeled using the IMS-LD specification (AE2-T1-PC1, AE3-T1-PC1, TW6-T1-PC1). Moreover, the use of GLUE!-PS in an authentic situation also revealed an important limitation of the current GLUE!-PS prototype: the fact that the teacher has to pre-define the particularization of the participants and tools before the enactment (AE3T1-PC2). Although this is not a limitation of the GLUE!-PS architecture or data model per se, the implementation of mechanisms for on-the-fly particularization might be more complex to implement in the GLUE!-PS LE adapters.

T2 (time-efficiency) Over the topic of time-efficiency of GLUE!-PS, especially in the deployment of learning designs across DLEs, we have somehow contradictory evidence. On the one hand, CSCL-expert teachers using it in authentic settings found that the (often iterative, as we saw in previous evaluations) design and deployment process can be time-consuming the first time a design is built from scratch (AE2-T2-PC1, AE3-T2-PC1). On the other hand, a much larger set of trans-disciplinar, non-expert teachers found the process timeefficient (TW6-T2-PC1). However, some of our other conclusions may shed some light into this contradiction: albeit the evidence gathered in the authentic experiments is more credible (since those designs were really enacted, as opposed to the workshop ones), it is also true that workshop teachers had a much greater scaffolding when designing (in the form of worksheets and example learning designs) (TW6-T2-PC2). On the other hand, all the teachers that experienced the run-time changes that were possible with the GLUE!-PS prototype, found them easy to make and generally time-efficient (AE3-T2-PC2).

T3 (use in real practice) Probably this topic is the most crucial of all for, and it is also the one for which more evidence has been gathered. Throughout the experiments and workshops we could see how the usability of the GLUE!-PS prototype was slowly improved, from being fit only for expert, technical teachers (AE2-T3-PC2) up to the point that a large set of non-technical teachers considered it usable (AE3-T3-PC1, AE3-T3-PC5, TW6T3-PC1). There is still, however, room for improvement, especially regarding the concept and management of student-generated resource reuse across different activities (TW6-T3PC2). Also, the GLUE!-PS authentic experiments also highlighted the need to provide different LE adapter implementations, in order to cater for the needs of different teachers in different situations (AE2-T3-PC3, AE3-T3-PC4), a need that the proposed GLUE!-PS architecture and data model facilitates.

In our workshop, non-expert teachers said that they would likely use GLUE!-PS and the deployed courses in their everyday practice (TW6-T3-PC3), although our more expert teachers using it in authentic situations were more careful in their statements, highlighting that the use of these technological tools might be advantageous only when there is a certain degree of group/resource complexity (AE2-T3-PC1). Also, it was found that the multi-step process imposed by the GLUE!-PS approach sometimes made it difficult to understand the implications of the teacher's actions in the final presentation of the activities across the DLE (AE3-T3-PC3). Other factors that may hamper the adoption of these tools can be the current unreliability and lack of adequate help support for the users of the prototype (TW6-T3-PC4), and others which are not even directly related to the proposal itself (e.g. institutional support, student and teacher attitudes, etc.) (TW6-T3-PC3).

Finally, an interesting aspect which emerged from the evaluations was the importance that teachers put in the specification (and eventual modification in run-time), of the time 
management aspects of the particularized learning design (i.e. submission deadlines, timed access to resources, etc.) (AE2-T3-PC4, AE3-T3-PC6, TW6-T4-PC2). This aspect may be transferred to the GLUE!-PS proposal in different ways, such as by extending the GLUE!PS LF data model with timing information, and/or providing a time-based view of the learning activities, in parallel with the current implementation of a more activity-based view of the learning design. This somehow relates the GLUE!-PS to the field of project/task management in computer-supported collaborative work (CSCW), which often take into account this duality between task and time.

T4 (run-time changes) Albeit the GLUE!-PS prototype currently only supports a few different kinds of run-time changes (basically the modification of group composition and resources in an activity), we have found that these features solve a number of very common run-time problematic situations in a intuitive and useful way (AE2-T4-PC1, AE3-T4-PC1, TW6-T4-PC1).

We have also found two interesting limitations to this run-time change support. One is the fact that GLUE!-PS does not solve the conceptual problems that these problematic situations often involve, especially in complex collaborative strategies (from the point of view of workflow), such as the Jigsaw (AE3-T4-PC2). This is a problem that increases with the scale of the particularized design (i.e. the number of groups, participants and resources), and is difficult to solve unless the restrictions of the strategy in use are taken into account - something that some LD authoring tools such as WebCollage do, but the current GLUE!-PS proposal cannot. Also, the current implementation of the GLUE!-PS prototype does not provide proper support to acknowledge and preserve manual changes done directly to the learning platform (AE3-T3-PC2). This is due to the current strategy of making the initial deployment and run-time changes a one-way process (from the LD tools to the DLE). There would be unquestionable benefits of making this process twoway, providing synchronism between the DLE and the GLUE!-PS; however, there is a clear tradeoff in this aspect, since implementing such functionality would require more advance programmatic APIs on the learning platform side, and would make the implementation of the GLUE!-PS LE adapters much more costly.

Once again, all these conclusions are limited by the methods and context in which our supporting evidence was gathered. Although we have tried to address the participant bias by gathering information from a trans-disciplinar set of teachers, these data still present a clear lack in the variety of learning designs that have been orchestrated using GLUE!-PS. Learning designs not based on the concept of CLFP and not expressed using the IMS-LD specification would be needed to better assess the scope of applicability of the GLUE!-PS proposal. Furthermore, the orchestration of a wider set of authentic situations should also be taken into consideration, using non-wiki-based DLEs (such as Moodle or even other learning platforms). Another issue to explore is the scalability of the approach to large numbers of groups and participants, since the results of happening AE3 hint at problems and inefficiencies when the number and complexity of groupings increase.

Thus, the following iterations in the research around GLUE!-PS should address one or more of these limitations in the gathered evidence, thus aiming at further authentic experiments, with less controlled subjects (some of the participants in the TW6 workshop are already good 
candidates for this), and using a wider variety of learning designs and learning platforms. At the same time, the usability, reliability and supporting materials for the GLUE!-PS prototype should be extended, in order to reach a stable release that practitioners and institutions can safely use in everyday practice. These efforts should be complemented with other peripheral efforts (such as contacts at the institutional level to make GLUE!-PS available at the University of Valladolid's official Moodle platform), in order to remove external obstacles to adoption which, no matter how well-known, would prevent the ultimate goal of GLUE!-PS, which is to support orchestration of blended learning in everyday CSCL practice.

\subsubsection{Wrapping up the evaluation: Global evaluation conclusions}

During these four iterations of evaluation, we have taken our proposal of the GLUE!-PS architecture and its common data model (GLUE!-PS LF), and we have used them to deploy and manage in run-time different learning designs (expressed in multiple different languages) across different kinds of Distributed Learning Environments. These evaluations have been analytical at first, and afterwards we have used them in learning scenarios and professional development actions of increasing authenticity and scope. As it was mentioned at the beginning of this Section, we were trying to find answers about one main research question:

RQ3.1 Can we provide technological tools to deploy and flexibly manage in run-time blended CSCL activities across DLEs?

Furthermore, following the tenets of the CSCL-EREM evaluation framework, we have tried to find answers to this complex question by exploring one evaluation Issue: "Does GLUE!-PS support teachers in the orchestration of blended CSCL across DLEs?" (I1). In turn, taking into account our definition of the different aspects of orchestration in TEL/CSCL (see Chapter 3), and our analysis of the GLUE!-PS proposal from that perspective (Section 5.3), we have chosen to explore that issue through four different dimensions of the orchestration support: the ability of GLUE!-PS in deploying learning designs preserving the design's essential qualities (T1), its time-efficiency in doing so (T2), the feasibility of using GLUE!-PS in everyday teaching practice (T3), and the usefulness of GLUE!-PS for flexibly managing the learning activities in run-time (T4). By gathering evidence around these topics, and taking into account the partial conclusions of each iteration in a process akin to a cross-case analysis [Sta06b], we can reach four global conclusions for our evaluation, which illuminate each of those topics, as represented in Figure 4.24

T1 Conclusion: GLUE!-PS allows non-expert teachers to deploy learning designs expressed in multiple LD languages into multiple DLEs, preserving the design's essential qualities as long as the learning platform and LE adapter does.... As we have seen several times during the analytical and empirical evaluations, the GLUE!-PS architecture is able to deploy learning designs expressed in a variety of LD languages into multiple DLEs built around different learning platforms, such as the Moodle VLE or the MediaWiki wiki engine. Although the usage of the GLUE!-PS LF as the central data model for our translations implies a certain loss of expressivity, we have found through analytical and empirical evaluations 
that the resulting deployed activities across the DLEs generally preserve the script's essential qualities, as long as the target learning environment (and the implementation of the corresponding LE adapter) complies with them. This fact, however, has been mostly tested with CLFP-based learning designs expressed using the IMS-LD specification. [Supported by partial conclusions AN1-T1-PC1, PT1-T1-PC1, TW3b-T1-PC1, TW5-T1-PC1, AE1-T1-PC1, AE2-T1-PC1, AE3-T1-PC1, TW6-T1-PC1, TW6-T1-PC2]

... with limitations regarding the up-front pre-definition, timing information and dependence on multiple external elements. However, apart from the aforementioned loss of expressivity, the current GLUE!-PS implementation assumes that the complete particularization of the learning design (e.g. group formation, group-specific resources, etc.) has to be done up-front, a practice that may clash with the orchestration customs of some teachers (as it happened in happening AE3). Teachers also found limiting the fact that there is no way of expressing time management aspects in GLUE!-PS, a fact that they circumvented by providing (manually) alternative time-based views of the learning activities. Also, it was noted that the loosely-coupled integration model proposed by GLUE!-PS implies a tradeoff between the ease of extending GLUE!-PS support to new platforms and LD languages, and the lack of control over the different services and elements involved in the deployment and run-time management of learning activities (which may lead to reliability problems). [Supported by partial conclusions AN1-T1-PC2, TW3b-T1-PC2, AE1-T1-PC2, AE3-T1-PC2]

T2 Conclusion: GLUE!-PS usage is arguably more time-efficient in the first deployment of a design, but it is clearly more time efficient when reusing the learning design, and for complex group/resource structures and run-time changes. The gathered evidence about the time-efficiency of the GLUE!-PS approach, which was gathered through authentic experiments and professional development workshops, is somehow inconsistent, maybe due to the variable stability of the GLUE!-PS prototypes throughout the different evaluation happenings. Although teacher use in professional development workshops showed that they appreciated the time-efficiency of the approach, teachers in authentic situations (provided with little tool usage scaffolding) experienced that the first-time design and deployment had to be built rather slowly and iteratively until the desired result was reached. However, teachers shared little doubts about the time-efficiency advantages of re-using learning designs through GLUE!-PS, in the case of deployments which showed complex group and resource structures and flows (e.g. peer reviews), or when doing run-time changes across the DLE. [Supported by partial conclusions PT1-T2-PC1, TW3b-T2-PC1, TW5-T2-PC1, AE1-T2-PC1, AE2-T2-PC1, AE3-T2-PC1, AE3-T2-PC2, TW6-T2-PC1, TW6-T2-PC2]

T3 Conclusion: A wide variety of teachers would use the GLUE!-PS system for CSCL designs of a certain complexity, since it adapts to the needs of the teacher, .... Throughout the different empirical evaluation happenings, a wide variety of the 55 teachers that informed our evaluation asserted that they would use the orchestration support provided by GLUE!PS for blended CSCL activities across DLEs. The usage of the GLUE!-PS prototype in authentic settings showed that one important advantage of this proposal is its modularity and extensibility, since it allows for different implementations of the LE adapters, which may produce different flavors of a deployed learning design, that cater for the contextual needs and preferences of the teacher. [Supported by partial conclusions TW5-T3-PC1, 
AE1-T3-PC3, АЕ2-T3-PC1, АЕ2-T3-PC3, АЕ3-T3-PC1, АЕ3-T3-PC4, АЕ3-T3-РC5, TW6-T3-PC1, TW6-T3-PC3]

... provided that the prototype is made more usable and reliable, and adequate support/training is provided. However, teachers also expressed a number of concerns or "buts" that could eventually prevent them from adopting the system in real practice. Although many of these obstacles are well-known and not directly related to GLUE!-PS (e.g. teacher and student attitudes towards ICTs or collaboration, or the lack of institutional support), others are more related to our efforts, such as the usability and reliability of the prototypes (which can certainly be improved), and the provision of more thorough support, either in the form of training actions and materials, or as online help within the application itself. [Supported by partial conclusions PT1-T3-PC2, TW3b-T3-PC1, TW5-T3-PC2, TW5-T3-PC3, AE1-T3-PC4, AE2-T3-PC2, TW6-T3-PC2, TW6-T3-PC4]

Also desirable would be to address the time management issues and the relationship between particularization and final learning platform presentation. The GLUE!-PS proposal, in its current incarnation, does not provide information and control about timing issues such as deadlines for activities and submissions, a feature that some participant teachers demanded. Also, it proved difficult for teachers to conceptualize the consequences of the design and particularization information across the different phases in the multi-step translations done by the GLUE!-PS approach. This forced teachers to use a "bricolage" approach to the design and deployment (trying and erring with the distribution of activities and resources), which was not greatly facilitated by the current GLUE!-PS prototype. Although these issues do not render the GLUE!-PS proposal worthless, they certainly should be explored more deeply in the future. [Supported by partial conclusions AE1-T3-PC1, AE1-T3-PC2, AE2-T3-PC4, AE3-T3-PC2, АE3-T3-PC3, АE3-T3-PC6]

T4 Conclusion: GLUE!-PS supports very commonplace resource and group run-time changes in an intuitive way for teachers, although it does not help with the conceptual problems of those changes. Although the implementation of run-time change features came rather late in the implementation of the GLUE!-PS prototype, they have been greatly appreciated by teachers. The group and resource changes that can be done in the GLUE!-PS user interface and applied across the DLE were considered commonplace and intuitive to apply. Albeit these changes are greatly dependent on the concrete learning platform features (especially the existence of programmatic APIs), the limited prototypes have shown how a minimal set of flexibility features can cover most of the problematic cases that teachers could think of. There are, however, other problems of a more conceptual nature when dealing with these run-time changes in complex collaborative learning (such as what Dillenbourg called "intrinsic constraints" of the script [Dil07d]), for which GLUE!-PS currently cannot offer much help. [Supported by partial conclusions AE1-T4-PC1, AE2-T4-PC1, AE3-T4-PC1, AE3-T4-PC2, TW6-T4-PC1]

\subsection{Conclusions, relevance and future work around GLUE!-PS}

The current emergence of software-as-a-service (SaaS) applications, and especially the socalled "Web 2.0" tools, together with the dominance of VLEs and other learning platforms to 


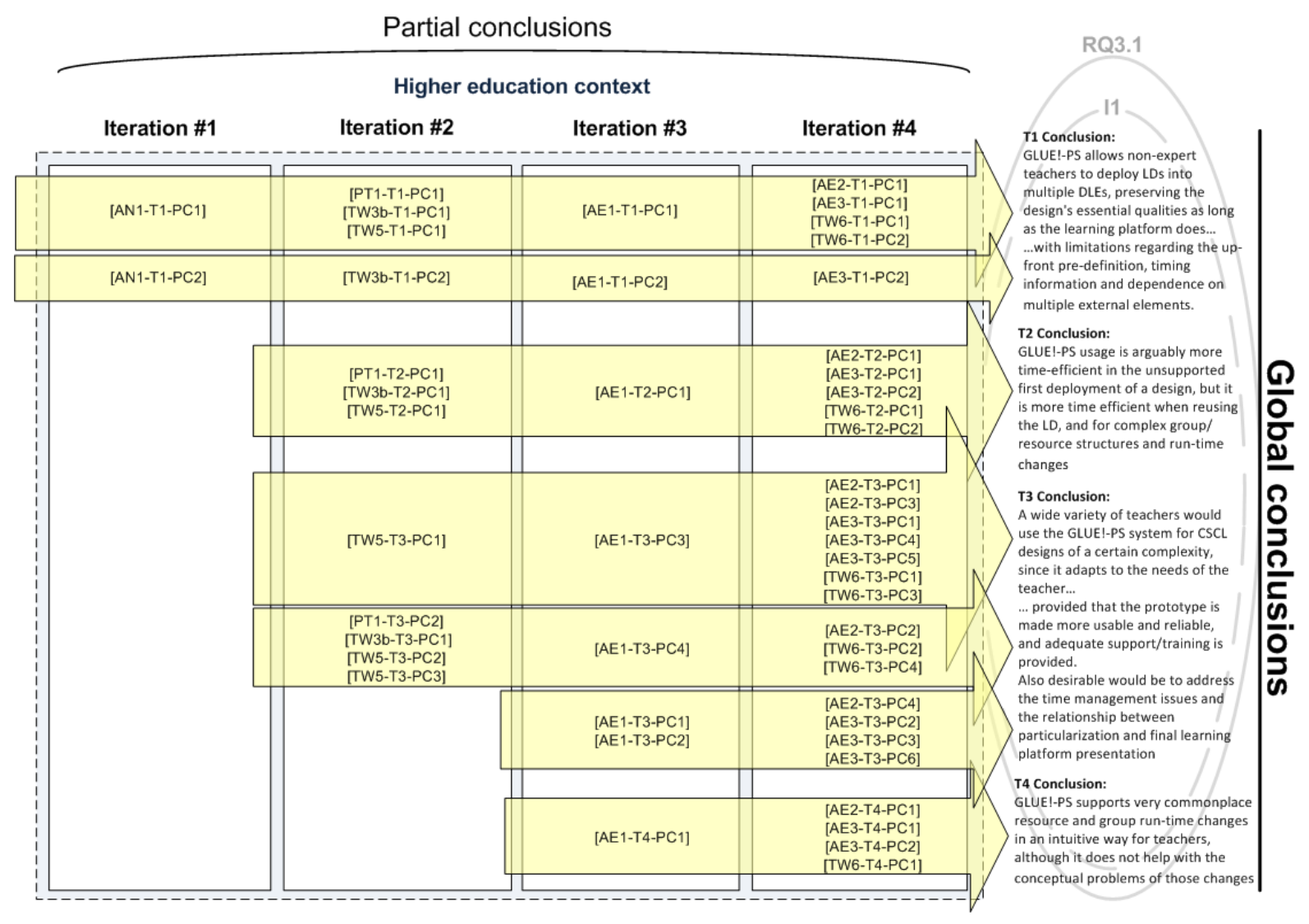

Figure 5.45: Graphical representation of the partial conclusions leading to the global evaluation conclusions on GLUE!-PS as a technological tool for orchestration 
support blended and distance learning, has recently given raise to several flavors of what we have called Distributed Learning Environments (DLEs, see Section 2.4.3). The labor of designing and enacting blended CSCL activities in this kind of technological context is a very complex and currently undersupported task, often to be performed by teachers who are not ICT experts. Thus, it can be considered a perfect example of what we have called "orchestration" throughout this dissertation. In this Chapter we have proposed, analyzed and evaluated the Group Learning Unified Environment - Pedagogical Scripting (GLUE!-PS), an architecture and underlying data model to support teachers in the orchestration of blended CSCL activities across DLEs.

Two main technological orchestration problems were detected in our review of CSCL mechanisms when working with DLEs (see Section 2.5): the lack of support for the deployment of learning designs expressed in different languages, to different DLEs based on widespread learning platforms (what we denominated the "deployment gap"); and the lack of run-time flexibility when enacting those activities through the DLE (which is known to be a major criticism to most LD approaches). The GLUE!-PS proposal consists in an architecture with two sets of adapters and a central element (the GLUE!-PS Manager service). This structure allows for the deployment and run-time management of learning designs, by performing two serial translations, from the original learning design format to a common data model (the GLUE!-PS lingua franca, or GLUE!-PS LF), and then from this data model to the target learning platform's own data model. This kind of architecture would theoretically allow for this kind of multi-LD-multi-LE translation, in an extensible way, at a reduced development cost... albeit with a certain loss of expressivity. In order to minimize unnecessary losses, the GLUE!-PS LF was modeled after the analysis of the most widespread learning platforms, in order to provide a model of the most commonly deployable characteristics of learning designs. By performing run-time changes to this common data model and re-deploying it again across the DLE in run-time, certain adaptation features would also be guaranteed.

By providing an open, extensible reference implementation of this GLUE!-PS architecture, including a user interface suitable for non-expert practitioners, we hypothesize that the adoption of learning design approaches (to CSCL and to TEL in general) by practitioners can be fostered. Moreover, the relative simplicity of the architecture and data model has been designed to maximize community acceptance also on the part of institutions and learning software developers. However, given our focus in supporting orchestration by teachers, we have been primarily concerned by the assessment of this proposal from the point of view of a (preferably non-CSCL-expert) practitioner. Thus, following the engineering method and guided by the CSCL-EREM evaluation framework, we have designed an iterative implementation and evaluation of the GLUE!-PS architecture, through a variety of analytical and empirical evaluation "happenings", including teacher professional development workshops and authentic experiments where the GLUE!-PS prototype has been used to orchestrate blended CSCL activities integrated in usual university curricula.

The conclusions of our evaluation, focused in the four main orchestration dimensions to which GLUE!-PS provides support (see Section 5.3), depict increasing evidences of usefulness as the GLUE!-PS prototypes iteratively improve in usability and reliability. By the end of this iterative process, non-expert teachers were able to design and deploy medium-complexity learning designs into two different DLEs using GLUE!-PS, and they were also able to emulate the flexible run-time solution to certain "problematic situations". The teachers considered the whole 
deployment process time-efficient, at least in certain cases, and usable in their everyday practice, with only a few natural caveats.

Our evaluation, however, also uncovered several obstacles to this time-efficiency and to teacher adoption in general. Some of the most interesting outstanding issues that emerged from our evaluations was the iterative, bricolage-like deployment process followed by some teachers when deploying, far from the ideal linear learning design life-cycle, due to the lack of visual information of the consequences that the teachers' actions throughout the process would have in the final DLE deployment. Also, the fact that the GLUE!-PS prototype now only supports an up-front particularization (also known as operationalization [Tch08] or instantiation [GS09]) of learning designs, which loads the teacher with such a task in deployment-time, was found limiting (especially since it is a task whose burden increases as the scale and complexity of the script increases). These outstanding issues make the first-time deployment of a design with GLUE!-PS still cumbersome. Although these problems have been tackled in a variety of ways by some LD approaches (see, e.g. the LAMS system Dal03]), they still remain unsolved for DLEs.

Overall, our evaluations hint towards two interesting directions. Regarding the problem of the "deployment gap", GLUE!-PS provides $a$ solution, but not the perfect solution. Indeed, GLUE!-PS makes apparent that, although deployment of learning designs across DLEs can be largely automated (in our case, thanks largely to the integration features of the GLUE! architecture), the intermediate instantiation phase (in which the design is particularized for the concrete educational setting) is still complex, burdensome and hard to grasp for non-expert teachers. Although support for this instantiation can be provided (see, for example, [VF09b], or (PR08), a general solution, valid for a wide variety of learning designs and usable by nonexperts, has not yet been provided. Other researchers, in a somewhat "lateral" leap, propose to distribute that kind of orchestration burden among participants (i.e. students) [Tch07] [Sha11].

Regarding the problem of run-time flexibility when enacting CSCL designs across DLEs, we have found that, surprisingly, in the GLUE!-PS architecture, very limited flexibility features can "go a long way", providing feasible solutions to many of the most common adaptation maneuvers that teachers find in everyday practice (see, e.g. the atomic pattern analysis of GLUE!-PS in Section 5.3). Our loose integration proposal, however, is very limited by the programmatic possibilities offered by the different target learning platforms (i.e. the capacity for an external entity such as GLUE!-PS to modify the internal structures of the platform from outside). As we saw in the case of Moodle-based DLEs, implementing such flexibility features can be difficult, although the modularity of the GLUE!-PS architecture allows each developer to implement as much (or as little) of this complexity as desired.

Nevertheless, the evaluations presented throughout this Chapter present a number of limitations that should be highlighted: the most glaring defect of the evaluations is the lack of variety in the learning designs that were deployed and managed in run-time (all of which were based on CLFPs and expressed in IMS-LD, although the current prototype also supports learning designs done with the Pedagogical Pattern Collector tool). Albeit CLFPs express common collaborative strategies and IMS-LD is the most widely used language in LD research, we certainly cannot assert that GLUE!-PS can deploy any learning design while preserving its essential qualities. Further evaluation with a range of widely-used learning design languages and strategies should be tried in the future. Indeed, these efforts have already started: one further authentic experiment using the Pedagogical Pattern Collector LD tool and a Moodle-based DLE with a large group of students (around 50) has recently been completed, and will be reported soon. 
Other weakness of the evaluations is the lack of generalizability of our conclusions, since the evidence was mostly gathered in "situated actions", thus veering towards the side of naturalistic/qualitative research. However, this issue was known by the research team right from the start, and we consciously chose this approach in order to better understand the multiplicity of the orchestration phenomenon, in absence of clear theories or frameworks to guide more experimental approaches.

There is also a limitation of perspective in the evaluations presented in this chapter. There exist other points of view which would be relevant to be explored in the future, such as assessing more formally whether the software development effort that GLUE!-PS requires to implement new LD-LE integrations, is really lower than, for example, 1-to-1 integrations such as the one alluded in [Ber05]. Also, further evidence could be gathered about the student experience in GLUE!-PS-orchestrated learning situations.

Finally, a number of divergent lines of future research work can already be signaled, starting where this dissertation stops. Apart from addressing the aforementioned methodological shortcomings in future evaluations of the GLUE!-PS prototypes, which should continue evolving iteratively, other interesting avenues for research include:

- With all its limitations, the GLUE!-PS system can prove to be the first stepping stone on which more advanced orchestration tools and systems can be based. These complementary systems could provide support in one or more of the orchestration aspects which GLUE!-PS does not address (see Section 5.3). Some of the clearest possibilities include: the potential use of the GLUE! and GLUE!-PS architectures for the development of Adaptive Collaborative Systems (ACS) is already discussed in Kar12]; we have already mentioned parallel research efforts into enhancing the design-based monitoring information provided to teachers, which already use the GLUE!-PS LF model to guide the gathering of monitoring data RT12]; the matching of tasks and tools, which is one of the difficulties of the instantiation process for non-experts (see, e.g. [RC12]) could also be integrated within the GLUE!-PS system in the moment of particularizing the learning design.

- Delving deeper in the problem of particularization of CSCL designs (or, more generally, TEL designs) might be another worthy research path, either following workflow-based approaches such as those proposed by [PR08 and Bor11]. Other alternative paths such as co-orchestration [Sha11] and student self-organization [Tch07] are even more attractive for the author, since they could be easily implemented over the general GLUE!-PS infrastructure. This kind of work could also lead to studies on the balance between teacher-led and self-organization of students, even going into the field of computer-supported collaborative work (CSCW).

- There are already efforts that try to extend the applicability of GLUE!-PS beyond the DLEs and the "web space", into other spaces where ubiquitous learning Bru08] may occur. For example, MC12a provides a first prototype of a GLUE!-PS-like system that allows for the deployment of CSCL activities that use Augmented Reality (AR) for learning.

- Another interesting path for research which has been barely scratched during this dissertation is the relationship between technological tools (such as GLUE!-PS) and conceptual tools (such as the atomic patterns presented in Chapter 4), and their joint effect in driving 
innovative change, e.g. in teacher everyday practice. In this dissertation's evaluations we have seen how a sequence of workshops that address both aspects garnered very positive feedback. This kind of "synergistic scaffolding" Tab04 of teacher professional development and innovation may also be worth considering for future work.

This way, we believe that the evidence of usefulness of the GLUE!-PS system provided in this Chapter, will be dwarfed by the future possibilities of its evolutions. This is only the beginning... 


\title{
Chapter 6
}

\section{Conclusions and future work}

\begin{abstract}
Summary: This chapter concludes the dissertation, by summarizing the overall research problem being tackled (the provision of tools that support orchestration of Technology-Enhanced Learning and especially blended CSCL activities), our proposed contributions, and how those contributions have been evaluated iteratively throughout the dissertation. The results of these evaluations lead us to conclude that the thesis objectives have been fulfilled, but also point us towards future lines of research work, which are also described in this chapter.

The number of publications related to the contents of this dissertation (including two papers in international peer-reviewed journals, a book chapter, and six international conference papers), and the influence of some of this thesis's contributions in recently-funded research projects at the author's research group (e.g. EEE-Web TIN2011-28308-C03-02), are first indicators of the success of our proposals, and the relevance of the aforementioned future work.
\end{abstract}

\subsection{Conclusions of the dissertation}

The progressive ubiquity of Information and Communication Technologies in our society at all levels is slowly but recklessly transforming educational settings, and it is changing the nature of practitioner's labor in the (physical or virtual) classroom. The emergence of new pedagogical approaches that use these new technologies has further transformed the lecture-oriented mission of the teacher. In the research fields of TEL and CSCL, the increasingly complex design and management of these multiple elements (activities, tools, groups, participants, and even contexts) has been termed, orchestration. This notion also emerges from a growing concern by researchers that do not see their advancements adopted in everyday learning and teaching practice.

This dissertation looked at this rather holistic notion of orchestration of TEL, and set out to propose, develop and evaluate tools (either technological and/or conceptual) that support such orchestration. We considered especially interesting the orchestration of blended CSCL activities (as a prototypical example of complex, technology-supported pedagogical approach), being supported through the use of what we have called Distributed Learning Environments (DLEs) which include not only a web learning platform to support the blended activities, but also other external learning tools, especially "Web 2.0" ones (an increasingly common trend in many educational institutions).

Following a typical engineering method along four phases of information, proposal, analysis and evaluation, we first deepened our knowledge into what was meant by "orchestration" in the 
fields of TEL and CSCL (Section 2.3), and what outstanding problems were considered most relevant (Section 2.5), especially in our technological and educational contexts of interest (DLEs and blended CSCL activities). The first problem that we encountered was a researcher problem: the multiplicity of meanings and connotations of the notion of orchestration in TEL, which was actually an obstacle in our endeavor (since such "fuzziness" could eventually prevent us from finding a clear focus for our evaluations). Other problems we found concerned the teacher's viewpoint more: TEL orchestration practices being so novel, together with the current low level of technical expertise of many teachers, led to a lack of clear conceptual advice for practitioners about how to orchestrate TEL and CSCL activities in their everyday teaching practice, and within the context and restrictions of their particular classrooms, from the design of activities to their flexible enactment. Finally, two technological problems were detected in current CSCL practice that used DLEs: the absence of technological support for the deployment of (blended) $C S C L$ designs across different flavors of DLEs (a "deployment gap" which in practice prevented the adoption of many computer-supported LD approaches by teachers), and the lack of support for flexibly managing and adapting those activities across DLEs (adaptation to emergent and contextual events is one of the main distinguishing aspects of orchestration). These technological problems were even more acute in the very common case of non-expert teachers who had to orchestrate blended CSCL scenarios using an institutionally-mandated learning platform as the center of their DLE.

Thus, with these four problems in mind, we proposed three partial goals, that addressed them:

To clarify the concept of orchestration in TEL/CSCL research. In order to help ourselves and other researchers in orchestration-related inquiries, we scoured the relevant TEL and CSCL literature, classifying and clustering orchestration-related research until a conceptual framework emerged (Chapter 3). This framework, " $5+3$ Aspects" detailed eight different dimensions or aspects to which researchers seemed to refer when talking about orchestration: Design, Management, Awareness, Adaptation, Role of actors, Theory, Pragmatism and Synergy. These eight aspects could be used to describe any orchestration effort (the first five aspects), and how it was done (the last three). We have subsequently used this framework as an lens to analyze and evaluate our other dissertation proposals, but we have also evaluated the framework itself. Through two panels of researchers, one with younger researchers in nearby research groups, and one with a set of internationally-recognized experts in TEL/CSCL orchestration, we have analyzed the completeness and usefulness of the conceptual framework. The results of the evaluations show that most of the participants in the studies saw the framework as a complete overview of the field of orchestration in TEL, that provides descriptive (rather than prescriptive) conceptual support for researchers. Albeit several expert researchers were reluctant to constrain their thinking to such frameworks, it was found that others considered it useful as a pedagogical tool to be used by younger researchers (e.g. their PhD students). This evaluation has not yet been published, although partial proposals leading to the framework have already been published in Pri11d and Pri11e. Thus, although this framework can be refined in further research iterations, e.g. by providing further practical support for researchers based on the framework, the "5+3 Aspects" framework has fulfilled the goal of clarifying the notion of orchestration for TEL researchers. Thus, for example, it can be used by researchers to frame and communicate about their different orchestration-related research efforts, as well as an analytical lens to analyze and 
evaluate the orchestration support of research proposals, as we did in our other contributions, and as it is also done by other researchers in GR12.

To provide non-expert teachers with conceptual support on the orchestration of blended CSCL activities in DLEs. From our 6-month observation of primary school (technology-enhanced) classrooms where teachers integrated and orchestrated a new CSCL tool in their everyday practice, we derived the notion of atomic patterns, described in Chapter 4 . Atomic patterns are recurrent, small-scale, contextualized elements of orchestration practice that teachers employ to design and manage in run-time their activities while complying with their classroom's contextual restrictions. The use of these patterns (elicited in a bottom-up fashion from the existing everyday practice of teachers) in two professional development workshops with primary school teachers (published in [Pri10a] [Pri11g] ) provided us with initial evidences of usefulness to support teachers' orchestration with ICTs, where other de-contextualized researcherdriven efforts had failed. The subsequent elicitation of a similar set of atomic patterns in the context of blended CSCL and DLEs, their combination with other pedagogical patterns (thus forming a multi-level pattern approach published in [Dim11b] Pri12a]) and their use in two more professional development workshops in the context of blended CSCL in higher education using DLEs served to further evaluate their usefulness and applicability. In our situated evaluations we have seen how these atomic patterns can be used in PD actions to "bridge the gap" between the de-contextualized advice that researchers often provide and the fully contextualized orchestration that teachers have to deliver in their everyday practice [Pri11f]. Although our evaluations showed that the usefulness for teachers was also related to other aspects such as the workshop design or the teachers' previous experience and beliefs, they were generally considered useful across different phases of the orchestration process. Thus, we can conclude that atomic patterns provide useful, concrete advice that can help non-expert teachers to "bootstrap" their orchestration of blended CSCL using DLEs, thus fulfilling our second partial goal. Not only that, but we have also explored other aspects of atomic patterns, as a tool to analyze, represent and support teacher innovation (some of which have been published already Dim11a [Pri11f] Pri11d Ros11 Pri11c).

To provide technological support for the orchestration of CSCL activities in DLEs (multi-LD, multi-LE). Following again the phases of the engineering approach, we analyzed the problem space of learning design (which encompasses a number of different languages and authoring tools), and the deployment possibilities over the landscape of currently widespread learning platforms, from VLEs to DLEs (see Appendix C). From this analysis, we proposed the Group Learning Unified Environment - Pedagogical Support (GLUE!-PS), which mainly defined an architecture but also a common data model (the GLUE!-PS lingua franca - GLUE!PS LF) representing the learning design characteristics that were most commonly deployable in widespread DLEs. This proposal was first evaluated analytically, and published in Pri11b. As it is described in Chapter 5 , a prototype implementation of the GLUE!-PS proposal was iteratively developed and evaluated in the following months, through pilot trials, authentic experiments with teacher-researchers and teacher workshops with a variety of trans-disciplinar teachers. The analysis of the GLUE!-PS from the point of view of the other two dissertation contributions, as well as the aforementioned evaluations themselves, highlighted the usefulness of the GLUE!-PS proposal in supporting several aspects of CSCL orchestration across DLEs 
(mostly the Design and Pragmatism aspects, but also the Management and Adaptation). The evaluations showed that a wide variety of non-expert teachers considered such support useful for everyday orchestration practice, not only in deploying learning designs, but also in providing intuitive and flexible run-time solutions to commonplace emergent problems. The evaluation happenings, especially those performed in authentic settings, also served us to explore the limits of this orchestration support: the use of "bricolage" in the deployment process, the difficulties in managing particularization of large/complex scenarios, or the lack of visual feedback about the final look of the deployment. Nevertheless, these limited GLUE!-PS prototypes have shown the potential of the proposal to support the deployment and run-time management of learning designs, for a wide variety of teachers, thus fulfilling our third partial objective. It is also interesting to note that peripheral aspects of this proposal have also been published, such as the features of the GLUE!-PS reference implementation [Pri12b], the loss of expressivity throughout the CSCL lifecycle [MC12b], or the use of GLUE!-PS along with the GLUE! architecture to deploy learning designs in Moodle-based DLEs AH12b.

This fulfilling of the three partial goals of the thesis leads us to safely assert that this dissertation has achieved its goal of proposing a variety of conceptual and technological tools to support researchers in conceptualizing orchestration, and non-expert teachers in orchestrating blended CSCL activities across Distributed Learning environments. However, we can also reflect that, in emergent fields such as orchestration, it is often the case that we do not learn so much from the answers that we provide to our research questions, but rather from the new questions that these (always incomplete) answers give birth to. Thus, from our contributions to the field of orchestration in TEL we can also extract a number of lessons that we have learnt throughout our dissertation.

From our research around GLUE!-PS and the limitations found to such a system in authentic settings we have learnt that some of the main limitations of current learning design approaches dwell, in fact, outside the reflective phase of design, in the treacherous path to implementation and enactment in "messy" authentic situations. Among the many pitfalls of this implementation process, the difficulties in visualizing, conceptualizing and organizing the particularization of an abstract learning design into the concrete participants, groups and tools is probably the most insidious. Finding novel ways of supporting this complex process is crucial if learning design is to be adopted on a large scale by TEL practitioners using a variety of learning platforms, and GLUE!-PS currently only provides a first stepping stone down that path. Multiple directions can be taken in this problem, either by supporting practitioners, or by off-loading part of that process to other actors, be them technological systems (e.g. using advanced workflow techniques) or human agents (e.g. by students co-orchestrating the scenario).

From our research on atomic patterns we have learnt about the uselessness of research innovations and tools that do not take into account the context where they should be used. This includes not only contextual restrictions such as time constraints or curricula, but also the attitudes, beliefs and experience of the different stakeholders affected by our innovations. Furthermore, we have learnt about the potential of small, carefully-orchestrated professional development actions to provoke conceptual change, and how that conceptual change may make the adoption of technological innovations more likely, provided that the other constraints make such innovation feasible and sustainable. In this dissertation we have just scratched the surface of this "synergistic interventions" issue. 
Finally, from our search for a unified conceptual framework for orchestration we have learned that the fuzziness of orchestration may be unavoidable in such a multi-disciplinary field. By participating in the growing research community interested in orchestration Pri11d] Dim11a, we have also come to appreciate that the common value of orchestration in TEL is not so much on whether we can agree on a common definition of it, but rather on the shared concern of trying to develop and integrate our research proposals into authentic learning and teaching settings, with all their contextual constraints and shortcomings, in a way that can really enhance learning. There is still a long way to go until we can agree what may be the best path to address that concern.

\subsection{Future lines of work}

As we have already outlined at the end of the different contribution chapters, our three contributions and the iterative research conducted on each of them, prompt us to propose possible paths for future research work around TEL/CSCL orchestration and the supporting tools proposed. Some of the contributions are evolutionary extensions of the research work presented so far, while others represent "lateral" (or, rather, "oblique") leaps into more divergent directions.

Among the "continuist" directions, we can mention:

- To complete a third iteration in the development of the "5+3 Aspects" conceptual framework for orchestration. At the end of Chapter 3 we already outline a proposal for this evolution, which should be complemented with further research instruments such as examples of application to different TEL research efforts, more concrete advice on how to structure its use, or concrete methods to evaluate an orchestration effort using the framework (indeed, the aspect-oriented evaluation of the GLUE!-PS system could represent a first step in this direction). Actually, it would be interesting to evaluate in a more extended timeframe the effects of using the framework throughout an orchestration-related research project (e.g. by case studies using it systematically to detect problems, propose new solutions and evaluate them).

Although this abstract framework is primarily intended for researchers, another interesting path to explore would be the application of this framework to actual teaching (orchestration) practice, which would require the development of a further set of tools and support to make it effective.

- During this dissertation we have used atomic patterns as a conceptual tool to support orchestration in two wildly different learning contexts (primary schools and university-level education), with a special focus on collaborative learning. Thus, it would be interesting to apply this atomic pattern approach to other pedagogical approaches which are equally complex to orchestrate (e.g. inquiry-based mobile learning). Eliciting atomic patterns from successful teachers' practice in these new contexts, combining them with other suitable patterns, and using them in PD actions and in authentic settings (possibly along with compatible technological innovations) represent other interesting avenues for 
research. Also, it would be interesting to evaluate the difference that these PD actions make over a solely technology-oriented intervention.

Moreover, our work with atomic patterns hinted that novel teachers tended to find them more useful than very experienced ones. Extrapolating this tendency, it would be interesting to test the usage of the multi-level pattern-based approach presented in this dissertation with pre-service teachers. We could hypothesize that carefully selected sets of atomic patterns could help to initiate them in the complex process of orchestrating complex TEL situations, a task that many younger teachers still find daunting, despite their supposed greater fluency with digital technologies.

- Although not strictly a research effort, given orchestration's emphasis on usage of innovations in authentic settings by "average teachers", we should continue working towards a wide adoption of the GLUE!-PS approach. This adoption implies, among other things, the improvement and release of the GLUE!-PS RI source code, and the development of further LD and LE adapters for the architecture. These actions can eventually convince institutions and learning software developers to adopt our approach, thus creating an ecosystem of adapter implementations that may enable teachers and institutions to enjoy de facto interoperability between LD authoring tools and widespread learning platforms.

Our research around the GLUE!-PS architecture and data model has a number of shortcomings that should be addressed in future efforts. One of the most glaring defects is the lack of concrete security mechanisms to ensure trust and identity among the different elements of the architecture, and the different actors involved (teachers, students, etc.). Although a solution in a similar architectural setting (the GLUE! architecture) has already been proposed in $\mathrm{AH} 12 \mathrm{a}]$, the particularities of the GLUE!-PS proposal, which acts as "master" to multiple learning platforms in potentially different domains, requires a more thorough analysis of the security issues across the system.

In our evaluation of atomic patterns we provided first hints of evidence that teachers would find useful the automated implementation of some of these patterns. Moreover, we have already analyzed the implications of such implementation in the case of several of the elicited atomic patterns. This research line of finding technological applications of atomic patterns as interesting features to design in TEL systems, is still largely unexplored. Indeed, we have first evidences of the usefulness of this kind of implementation, from a recent unreported authentic experiment with GLUE!-PS: in the orchestration of a learning design with a large number of students, the automation of just one single atomic pattern through GLUE!-PS was sufficient to transform the teacher opinion of the system from "largely unusable" to "magically convenient".

Finally, another immediate path for future research around GLUE!-PS is the extension of its applicability beyond web applications, into other technological contexts such as virtual 3D worlds or augmented reality resources. In fact, this last line of work has already been started [MC12a], and extensions of GLUE!-PS to use geotagged or marker-placed web and 3D resources. These advancements can extend the applicability of GLUE!-PS from web-based DLEs into enabling truly ubiquitous learning [Bru08].

There exist, however, more divergent paths for future research that our work in this dis- 
sertation has also made appealing:

- One interesting outstanding problem in the orchestration of blended CSCL with GLUE!PS, but which is generally applicable to any LD-oriented approach where large or complex sets of resources are used by different groups of changing granularity, is that of particularizing the abstract learning design to make concrete groups of concrete participants, and to let them use and exchange resources in a determined way. This particularization may be relatively easy for a small number of groups and participants, but the manual configuration of such particularization quickly becomes unwieldy, from a cognitive and a time-efficiency perspective. Thus, a more general exploration of the particularization of learning designs in different learning contexts, and especially the scaling up of such particularization, could be highly interesting and relevant for the TEL and CSCL communities. Currently emerging trends in online education such as Massive Online Open Courses (MOOCs, [McA10]) provide interesting testbeds for such exploration, where currently an LD-oriented CSCL approach is unthinkable (as it is a flexibly adaptive one). The suggestions of some authors of using a more distributed orchestration (i.e. co-orchestration [Sha11]) provides, in fact, an interesting path to start this exploration, as it is the gradual shift of the orchestration load from the teacher (or the system) to the students, in a sort of "fading" Wec07 of the orchestration.

- Another interesting path which has already been hinted at by the dissertation's findings is the exploration of the synergies among technological innovations (such as the GLUE!-PS system) and tools for conceptual change (such as atomic patterns), either applied to professional development interventions or any other innovation effort. This kind of synergy has already been pointed out by teacher educator frameworks such as TPCK Mis06 which proposes that successful integration of technology in teacher practice requires technological, pedagogical and content knowledge. The approach taken in this dissertation implied the implementation of a rather costly series of workshops in order to reap first benefits of such conceptual change. Finding variations of this approach that can be sustained and scaled up easily might prove a very relevant advancement towards a wider adoption of the technological and conceptual tools proposed in this thesis, but also could be applicable to many other TEL research advancements.

Overall, this thesis has tried to address the problem of orchestrating multiple ICT-enabled activities, providing answers to this complex question from three different perspectives. In this endeavor we have tried to keep in mind the growing concern of the TEL and CSCL research communities about the lack of adoption of many of our research-derived innovations, and thus we have proposed innovative yet pragmatic contributions. However, as we can see from the numerous list of outstanding questions and problems, we are still very far from a complete understanding of the problem, and it may very well be that it is these questions the ones that conform our larger contribution to the field. 



\section{Bibliography}

[Abd08] M. Abdulwahed, Z. K. Nagy, and R. Blanchard. Constructivist Project Based Learning Design, a Cybernetics approach. Journal of Education, Informatics and Cybernetics, $1(2): 1-8,2008$.

[Abd09] M. Abdulwahed and Z. K. Nagy. Applying Kolb's Experiential Learning Cycle for Laboratory Education. Journal of Engineering Education, 98(3):283-294, 2009.

[Adr93] W. R. Adrion. Research methodology in software enginering: summary of the Dagstuhl workshop on future directions on software engineering. SIGSoft Software Engineering Notes, 18(1):36-37, 1993.

[AH10] C. Alario-Hoyos and S. Wilson. Comparison of the main alternatives to the integration of external tools in different platforms. In Proceedings of the International Conference on Education, Research and Innovation (ICERI2010), 2010.

[AH12a] C. Alario-Hoyos. GLUE!: An architecture for the integration of external tools in Virtual Learning Environments. PhD Thesis, School of Telecommunications Engineering, University of Valladolid, Spain, 2012.

[AH12b] C. Alario-Hoyos, J.A. Muñoz Cristobal, L.P. Prieto, M.L. Bote-Lorenzo, J.I. AsensioPérez, and E. Gómez-Sánchez. GLUE! - GLUE!-PS: An approach to deploy non-trivial collaborative learning situations that require the integration of external tools in VLE. In Proceedings of the $1^{\text {st }}$ Moodle Research Conference, 2012.

[Ala04] M. Alavi. Distributed learning environments. Computer, 37(1):121-122, 2004.

[Ala09] H. S. Alavi, P. Dillenbourg, and F. Kaplan. Distributed awareness for class orchestration. In Learning in the Synergy of Multiple Disciplines - Proceedings of the $4^{\text {th }}$ European Conference on Technology Enhanced Learning, EC-TEL 2009, pages 211225. Springer, 2009.

[Ale77] C. Alexander, S. Ishikawa, and M. Silverstein. A Pattern Language: Towns, Buildings, Construction, volume 2 of Center for Environmental Structure Series. Oxford University Press, 1977.

[Amb02] S.W. Ambler. Agile Modeling: Effective Practices for EXtreme Programming and the Unified Process. J. Wiley, 2002. 
[Ang10] R. Anguita, S. García Sastre, S. Villagrá Sobrino, and I.M. Jorrín Abellán. Wikis y aprendizaje colaborativo: lecciones aprendidas (y por aprender) en la facultad de educación. Red U - Revista de Docencia Universitaria, Número Monográfico V. Número especial dedicado a WIKI y educación superior en España (II parte), 2010. Available from http://www.um.es/ead/Red_U/m5/ (Last visit: 25 Jun 2012).

[Aro92] E. Aronson and R. Thibodeau. The jigsaw classroom: A cooperative strategy for reducing prejudice. Cultural diversity in the schools, 2:231-256, 1992.

[Bal09] N. Balacheff, S. Ludvigsen, T. de Jong, A. Lazonder, and S. Barnes, editors. Technology-Enhanced Learning: Principles and Products. Springer, 2009.

[Bal10] N. Balacheff, R. Bottino, and F. Fischer. D1.1 The STELLAR vision and strategy statement. Deliverable, STELLAR Network of Excellence, 2010.

[Bar04] S. Barab and K. Squire. Design-based research: Putting a stake in the ground. Journal of the Learning Sciences, 13(1):1-14, 2004.

[Bar05] E. Bardram. Activity-based computing: support for mobility and collaboration in ubiquitous computing. Personal Ubiquitous Computing, 9:312-322, September 2005.

[Bea10] G. Beauchamp, S. Kennewell, H. Tanner, and S. Jones. Interactive whiteboards and all that jazz: the contribution of musical metaphors to the analysis of classroom activity with interactive technologies. Technology, Pedagogy and Education, 19(2):143-157, 2010 .

[Bec01] K. Beck, M. Beedle, A. Van Bennekum, A. Cockburn, W. Cunningham, M. Fowler, J. Grenning, J. Highsmith, A. Hunt, R. Jeffries, et al. Manifesto for agile software development. The Agile Alliance, 2001. Available online, at http://agilemanifesto. org/ (Last visit: 30 May 2012).

[Ben12] S. Bennett, A. Bishop, B. Dalgarnob, J. Waycott, and G. Kennedy. Implementing web 2.0 technologies in higher education: A collective case study. Computers $\mathscr{E}$ Education, 59(2):524-534, 2012.

[Ber05] A. Berggren, D. Burgos, J. M. Fontana, D. Hinkelman, V. Hung, A. Hursh, and G. Tielemans. Practical and pedagogical issues for teacher adoption of IMS Learning Design standards in Moodle LMS. Journal of Interactive Media in Education, 2005(2), 2005 .

[Bey10] Beyond Current Horizon Project. BCHP official site. Web site, http://www. beyondcurrenthorizons.org.uk/ (Last visit: 12 Nov 2010), 2010.

[Big99] J.B. Biggs and C. Tang. Teaching for quality learning at university. Open University Press Buckingham, 1999.

[Blu91] P.C. Blumenfeld, E. Soloway, R.W. Marx, J.S. Krajcik, M. Guzdial, and A. Palincsar. Motivating project-based learning: Sustaining the doing, supporting the learning. Educational Psychologist, 26(3-4):369-398, 1991. 
[Bor89] Hilda Borko and Carol Livingston. Cognition and improvisation: Differences in mathematics instruction by expert and novice teachers. American Educational Research Journal, 26(4):473-498, 1989.

[Bor11] O. Bordies, Y. Dimitriadis, C. Alario Hoyos, A. Ruiz Calleja, and A. Subert. Reuse of data flow designs in complex and adaptive scripts: A case study. In A. Daradoumis, editor, Intelligent Adaptation and Personalization Techniques in Computer-Supported Collaborative Learning. Springer Verlag, 2011.

[Bot07] L. Botturi and S.T. Stubbs. Handbook of visual languages for instructional design: Theories and practices. Information Science Publishing, 2007.

[Bow11] M. Bower and M. Wittmann. A comparison of LAMS and Moodle as learning design technologies - teacher education students' perspective. Teaching English with Technology, Special Issue on LAMS and Learning Design, 11(1):62-80, 2011.

[Bra08] A. Brasher, G. Conole, S. Cross, M. Weller, P. Clark, and J. White. CompendiumLD - a tool for effective, efficient and creative learning design. In Proceedings of the 2008 European LAMS Conference, 2008.

[Bro01] M. Brown and D. C. Edelson. Teaching by design: Curriculum design as a lens on instructional practice. In Paper presented at the Annual Meeting of the American Educational Research Association. 2001.

[Bru03] P. Brusilovsky and C. Peylo. Adaptive and intelligent web-based educational systems. International Journal of Artificial Intelligence in Education, 13(2-4):159-172, 2003.

[Bru08] B. C. Bruce. Ubiquitous learning, ubiquitous computing, and life experience. In Proceedings of the $6^{\text {th }}$ International Conference on Networked Learning (NLC2008), pages 583-590, 2008.

[Bur95] V. Burr. An introduction to social constructionism. Routledge, 1995.

[C. 09] C. R. A. Ana de Austria. "Ana de Austria" primary school official page. Web site, http://www.craanadeaustria.org, Last visit: 16 Feb 2009.

[Cae03] M. Caeiro, L. Anido, and M. Llamas. A critical analysis of IMS learning design. In Proceedings of the Computer Supported Collaborative Learning Conference, CSCL 2003, pages 363-367, 2003.

[Cae08] M. Caeiro. poEML: A separation of concerns proposal to instructional design. In L. Botturi and S. T. Stubbs, editors, Handbook of Visual Languages for Instructional Design: Theories and Practices, pages 183-207. IGI Global Publishing, 2008.

[Car09] A. Carell and I. Schaller. Scenario-based orchestration of Web 2.0 applications in university teaching and learning processes: a case study. International Journal of Web Based Communities, 5(4):501-514, 2009.

[Cha01] M. K. Chamberlain, C. B. Williams, F. S. Cowan, and F. Mistree. Orchestrating learning in a graduate engineering design course. In $13^{\text {th }}$ International Conference on Design Theory and Methodology, 2001. 
[Cha11] J. Chacón, D. Hernández-Leo, and J. Blat. From a pattern language to a pattern ontology. Approach for CSCL script design. In Proceedings of the International Conference on Computational Science and its Applications, Santander (Spain), 2011.

[Chi08] V.A. Chirikba. The problem of the Caucasian Sprachbund. In P. Muysken, editor, From Linguistic Areas to Areal Linguistics, pages 25-93. John Benjamins Publishing Company, 2008.

[Coc01] A. Cockburn. Writing effective use cases, volume 1. Addison-Wesley Boston, 2001.

[Coh04] M. Cohn. User stories applied: For agile software development. Addison-Wesley Professional, 2004.

[Col04] Allan Collins, Diana Joseph, and Katerine Bielaczyc. Design research: Theoretical and methodological issues. Journal of the Learning Sciences, 13(1), 2004.

[Con10a] M.A. Conde, F.J. García-Peñalvo, M.J. Casany, and M. Alier. Open Integrated Personal Learning Environment: Towards a New Conception of the ICT-Based Learning Processes. In Proceedings of the 3rd World Summit on the Knowledge Society, WSKS 2010, pages 115-124, 2010.

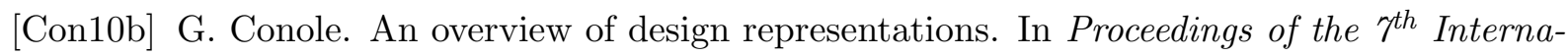
tional Conference of Networked Learning (NLC2010), pages 482-489, 2010.

[Con10c] G. Conole and P. Alevizou. A literature review of the use of web 2.0 tools in higher education. Report commissioned by the Higher Education Academy, The Open University, August 2010.

[Cre03] J.W. Creswell, V.L. Plano Clark, M.L. Gutmann, and W.E. Hanson. Advanced mixed methods research designs. In Handbook of mixed methods in social and behavioral research, pages 209-240. Sage Publications, 2003.

[Cub01] Larry Cuban. Oversold and Underused: Computers in the Classroom. Harvard University Press, 2001.

[Dal03] J. Dalziel. Implementing learning design: The learning activity management system. In Proceedings of the 20th Annual Conference of the Australasian Society for Computers in Learning in Tertiary Education (ASCILITE), 2003.

[Dal05] J. Dalziel. From re-usable e-learning content to re-usable learning designs: Lessons from LAMS. Retrieved from http://www.lamsfoundation.org/CD/html/ resources/whitepapers/Dalziel.LAMS.doc, 2005.

[Dal06] J. Dalziel. Lesson from LAMS for IMS Learning Design. In Proceedings of the $6^{\text {th }}$ International Conference on Advanced Learning Technologies (ICALT 2006), 2006.

[Dav06] B. David, R. Chalon, O. Delotte, G. Masserey, and M. Imbert. ORCHESTRA: Formalism to express mobile cooperative applications. In Y. A. Dimitriadis, I. Zigurs, and E. Gómez-Sánchez, editors, Groupware: Design, Implementation, and Use, $12^{\text {th }}$ International Workshop, CRIWG 2006. Lecture Notes in Computer Science, Volume 4154/2006, pages 163-178. Springer, 2006. 
[Dav09] B. David and R. Chalon. Orchestration modeling of interactive systems. In HumanComputer Interaction. New Trends. Proceedings of the $13^{\text {th }}$ International Conference HCI 2009. Part I, 2009.

[Dav10] H. Davis. The justification for a new learning environment at Southampton. LSL Seminar, University of Southampton, 2010. http://eprints.soton.ac.uk/271740/ (Last visit 11 May 2012).

[dC07] J. E. del Cid, L. de la Fuente-Valentín, S. Gutiérrez, A. Pardo, and C. D. Kloos. Implementation of a learning design run-time environment for the .LRN learning management system. Journal of Interactive Media in Education, Special Issue on Adaptation and IMS Learning Design, 2007.

[DeB11] A. H. DeBarger, W. Penuel, C. J. Harris, and P. Schank. Teaching routines to enhance collaboration using classroom network technology. In F. Pozzi and D. Persico, editors, Techniques for Fostering Collaboration in Online Learning Communities: Theoretical and Practical Perspectives. IGI Global Publishing, 2011.

[Dec99] Bologna Declaration. Joint declaration of the European Ministers of Education convened in Bologna on the 19th of june. available online at http://ec.europa.eu/ education/policies/educ/bologna/bologna.pdf (Last visit: 2 Jan 2011), 1999.

[Del95] S. Delamont. Teachers as artists. In L. W. Anderson, editor, International Encyclopaedia of Teaching and Teacher Education. Pergamon Press, 1995.

[Dem08] S. Demetriadis and A. Karakostas. Adaptive collaboration scripting: A conceptual framework and a design case study. In Proceedings of the International Conference on Complex, Intelligent, and Software Intensive Systems, pages 487-492, 2008.

[Der10] M. Derntl, S. Neumann, D. Griffiths, and P. Oberhuemer. Investigating teachers' understanding of IMS Learning Design: yes they can! In Proceedings of the European Conference on Technology-Enhanced Learning (EC-TEL 2010), 2010.

[Der11] M. Derntl, S. Neumann, D. Griffiths, and P. Oberhuemer. The conceptual structure of IMS Learning Design does not impede its use for authoring. IEEE Transactions on Learning Technologies, 5(1):74-86, 2011.

[DiG06] C. DiGiano, D. Tatar, and K. Kireyev. Learning from the post-it: Building collective intelligence through lightweight, flexible technology. In Conference on ComputerSupported Cooperative Work 2006, 2006.

[Dil99] P. Dillenbourg. What do you mean by "collaborative learning"? In P. Dillenbourg, editor, Collaborative Learning. Cognitive and Computational Approaches, pages 1-19. Elsevier Science, 1999.

[Dil02a] P. Dillenbourg. Over-scripting CSCL: The risks of blending collaborative learning with instructional design. In P. A. Kirschner, editor, Three Worlds of CSCL. Can We Support CSCL, pages 61-91. Open Universiteit Nederland, 2002.

[Dil02b] P. Dillenbourg, D. K. Schneider, and P. Synteta. Virtual learning environments. In Proceedings of the $3^{\text {rd }}$ Hellenic Conference "ICT in Education", pages 3-18, 2002. 
[Dil06] P. Dillenbourg and P. Jermann. SWISH: A model for designing CSCL scripts. In F. Fischer, H. Mandl, J. Haake, and I. Kollar, editors, Scripting ComputerSupported Collaborative Learning-Cognitive, Computational, and Educational Perspectives. Springer, 2006.

[Dil07a] P. Dillenbourg and F. Fischer. Basics of computer-supported collaborative learning. Zeitschrift für Berufs-und Wirtschaftspadagogik, 21:111-130, 2007.

[Dil07b] P. Dillenbourg and P. Jermann. Designing integrative scripts. In F. fischer, H. Mandl, J. Haake, and I. Kollar, editors, Scripting computer-supported collaborative learning: Cognitive, computational and educational perspectives. Springer Computer-supported Collaborative Learning Series, 2007.

[Dil07c] P. Dillenbourg and P. Jermann. Designing interactive scripts. In F. Fischer, I. Kollar, H. Mandl, and J. Haake, editors, Scripting computer-supported collaborative learning: Cognitive, computational and educational perspectives. Springer, 2007.

[Dil07d] P. Dillenbourg and P. Tchounikine. Flexibility in macro-scripts for computersupported collaborative learning. Journal of Computer Assisted Learning, 23(1):1-13, 2007.

[Dil08a] P. Dillenbourg and F. Hong. The mechanics of CSCL macro scripts. International Journal of Computer-Supported Collaborative Learning, 3(1):5-23, 2008.

[Dil08b] Pierre Dillenbourg. Integrating technologies into educational ecosystems. Distance Education, 29(2):127-140, 2008.

[Dil09a] P. Dillenbourg, Sanna Järvelä, and Frank Fischer. The evolution of research in computer-supported collaborative learning: from design to orchestration. In Nicolas Balacheff, Sten Ludvigsen, Ton de Jong, Ard Lazonder, and Sally Barnes, editors, Technology-Enhanced Learning: Principles and Products, pages 3-19. Springer, 2009.

[Dil09b] Pierre Dillenbourg. Exploring neglected planes: social signs and class orchestration. In Community Events Proceedings of the International Conference of ComputerSupported Collaborative Learning (CSCL2009), pages 6-7, 2009.

[Dil10] P. Dillenbourg and P. Jermann. Technology for classroom orchestration. In M. S. Khine and Saleh I. M., editors, New Science of Learning: Cognition, Computers and Collaboration in Education. Springer, 2010.

[Dil11a] P. Dillenbourg, M. Sharples, F. Fischer, I. Kollar, P. Tchounikine, Y. Dimitriadis, L. P. Prieto, J. A. Asensio-Pérez, J. Roschelle, C. K. Looi, M. Nussbaum, and A. Diaz. Trends in orchestration: Second research and technology scouting report. STELLAR NoE Deliverable, D1.5, Jul 2011. available at http://www.stellarnet.eu/kmi/deliverables/20110818_stellar___d1.5___ trends-in-orchestration.pdf the STELLAR website (Last visit: 18 Jan 2012).

[Dil11b] P. Dillenbourg, G. Zufferey, H. Alavi, P. Jermann, S. Do-Lenh, Q. Bonnard, S. Cuendet, and F. Kaplan. Classroom orchestration: The third circle of usability. In Proceedings of the International Conference of Computer-Supported Collaborative Learning (CSCL2011), 2011. 
[Dim07] Yannis Dimitriadis, Juan I. Asensio-Pérez, Davinia Hernández-Leo, J. Roschelle, J. Brecht, D. Tatar, S. Chaudhur, C. DiGiano, and C.M. Patton. From sociallymediated to technology-mediated coordination: A study of design tensions using Group Scribbles. In Proceedings of the Computer Supported Collaborative Learning 2007 Conference, CSCL 2007, pages 184-186, July 2007.

[Dim11a] Y. Dimitriadis, L. P. Prieto, and J.I. Asensio-Pérez. A response from Y. Dimitriadis, L. Prieto and J. Ignacio. In P. Dillenbourg, editor, D1.5 Trendscouting Report: "Classroom Orchestration", pages 23-27. STELLAR NoE, 2011. Available online at http://www.stellarnet.eu/kmi/deliverables/20110818_stellar___d1.5_-_ trends-in-orchestration.pdf (Last visit: 08 May 2012).

[Dim11b] Y. Dimitriadis, L. P. Prieto, and S. Villagrá-Sobrino. Designing for enactment: Multilevel patterns and routines in teacher practice. Paper presented at the "Art and Science of Learning Design" international workshop (ASLD11), Oct 2011.

[dlFV07] L. de la Fuente-Valentin, A.P. Sanchez, and C.D. Kloos. Using learning design to deploy and administer engineering courses. In Proceedings of the 37th Annual Frontiers In Education Conference (FIE 'O7), 2007.

[dlFV10] L. de la Fuente-Valentín, M. Pérez-Sanagustín, P. Santos, D. Hernández-Leo, A. Pardo, C. Delgado-Kloos, and J. Blat. System orchestration support for a flow of blended collaborative activities. In Proceedings of the 2010 International Conference on Intelligent Networking and Collaborative Systems, INCOS '10, pages 415-420, Washington, DC, USA, 2010. IEEE Computer Society.

[Dod09] J. M. Dodero, J. Torres, I. Aedo, and P. Díaz. Beyond descriptive EML: Taking control of the execution of complex learning processes. In II Simposio Pluridisciplinar sobre Diseño, Evaluación y Descripción de Contenidos Educativos Reutilizables (SPDECE), 2009.

[Doe09] M. Doebeli, B. Notari. Over-computing CSCL macro scripts? Gaining flexibility by using WikiPlus instead of specialized tools for authoring macro scripts. In Proceedings of the $9^{\text {th }}$ International Conference on Computer Supported Collaborative Learning, pages 482-486, 2009.

[Dom06] J. Domingue, S. Galizia, and L. Cabral. The choreography model for IRS-III. In Proceedings of the $39^{\text {th }}$ International Conference on System Sciences 2006, January 2006.

[Dou03] M. Dougiamas and P. C. Taylor. Moodle: Using learning communities to create an open source course management system. In ED-MEDIA 2003: World Conference on Educational Multimedia Hypermedia 8 Telecommunications, 2003. Available from http://dougiamas.com/writing/edmedia2003/ (Last visit: Feb 9, 2011).

[Dur09] G. Durand and S. Downes. Toward Simple Learning Design 2.0. In Computer Science Education, 2009. ICCSE '09. 4th International Conference on, pages 894 -897, July 2009 . 
[Eck95] W.W. Eckerson. Three tier client/server architecture: Achieving scalability, performance, and efficiency in client server applications. Open Information Systems, 10(3):120, 1995 .

[El191] C.A. Ellis, G.L. Gibbs, and G.L. Rein. Groupware: Some issues and experiences. Communications of the ACM, 43(1):39-58, 1991.

[Eng80] S. Engelmann. Direct instruction. Educational Technology Publications, 1980.

[Eng87] Y. Engeström. Learning by expanding: an activity-theoretical approach to developmental research. Orienta-Konsultit, Helsinki, Finland, 1987.

[Eng99] Y. Engeström, R. Miettinen, and R.-L. Punamäki-Gitai, editors. Perspectives on Activity Theory. Cambridge University Press, 1999.

[Eri82] F. E. Erickson. Classroom discourse as improvisation: Relationships between academic task structure and social participation structures in lessons. In L. C. Wilkinson, editor, Communicating in the classroom, pages 153-182. Academic Press, 1982.

[Ert99] P. A. Ertmer. Addressing first- and second-order barriers to change: Strategies for technology integration. Educational Technology Research and Development, 47(4):4761,1999 .

[Esc07] J.P. Escobedo, L. de la Fuente, S. Gutiérrez, A. Pardo, and C. Delgado-Kloos. Implementation of a learning design run-time environment for the .LRN learning management system. Journal of Interactive Media in Education (JIME), July 2007. http://jime.open.ac.uk/2007/07/escobedo-2007-07-paper.html.

[Faw89] J. Fawcett. Analysis and Evaluation of Conceptual Models of Nursing, chapter Conceptual models and theories, pages 1-40. F.A. Davis Company, 1989.

[Fer08] C. Ferraris, C. Martel, and L. Vignollet. Modelling the "Planet Game" case study with LDL and implementing it with LDI. Journal of Interactive Media in Education (JIME), Special issue on Comparing Educational Modelling Languages on the'Planet Game' Case Study, December 2008. http://jime.open.ac.uk/jime/article/view/ 2008-20.

[Fer10] V. M. Ferracutti. Integración de servicios telemáticos para el apoyo al proceso de enseñanza/aprendizaje en el MUI-TIC. Master's Thesis, School of Telecommunications Engineering, University of Valladolid, Spain, 2010.

[Fie99] R. Fielding, J. Gettys, J. Mogul, H. Frystyk, L. Masinter, P. Leach, and T. BernersLee. Hypertext transfer protocol-http/1.1, 1999.

[Fie02] R. Fielding and R. N. Taylor. Principled design of the modern Web architecture. ACM Transactions on Internet Technology, 2(2):115-150, 2002.

[Fin02] S. Fincher and I. Utting. Pedagogical patterns: Their place in the genre. In Proceedings of the rth Annual Conference on Innovation and Technology in Computer Science Education, New York, NY, 2002. ACM Press. 
[Fis04] B. Fishman, R. Marx, P. Blumenfeld, J. Krajcik, and E. Soloway. Creating a framework for research on systemic technology innovations. The Journal of the Learning Sciences, 13(1):43-76, 2004.

[Fis06] F. Fischer and P. Dillenbourg. Challenges of orchestrating computer-supported collaborative learning. In Paper presented at the $8^{\text {th }}$ Annual Meeting of the American Educational Research Association (AERA), 2006.

[Fis07] F. Fischer, I. Kollar, H. Mandl, and J. Haake, editors. Scripting computersupported collaborative learning: Cognitive, computational and educational perspectives. Springer, 2007.

[For02] E. A. Forman and E. Ansell. Orchestrating the multiple voices and inscriptions of a mathematics classroom. Journal of the Learning Sciences, 11(2/3):251-274, 2002.

[For07] A. Forte and A. Bruckman. Constructing text: Wiki as a toolkit for (collaborative?) learning. In Proceedings of the 2007 international symposium on Wikis, pages 31-42. ACM, 2007.

[Gag78] N. L. Gage. The Scientific Basis of the Art of Teaching. John Wiley, 1978.

[Gag05] R. M. Gagne, W. W. Wager, K. C. Golas, J. M. Keller, and J. D. Russell. Principles of instructional design. Performance Improvement, 44:44-46, 2005.

[Gam95] E. Gamma, R. Helm, R. Johnson, and J. Vlissides. Design Patterns: Elements of Reusable Object-Oriented Software. Addison-Wesley, 1995.

[Gam02] E. Gamma. Design patterns: Ten years later. In M. Broy and E. Denert, editors, Software Pioneers: Contributions to Software Engineering, pages 688-700. SpringerVerlag, 2002.

[Gar85] H. Gardner. Frames of mind: The theory of multiple intelligences. Basic books, 1985.

[Gar05] J.J. Garrett et al. Ajax: A new approach to web applications, 2005.

[Gla67] Barney G. Glaser and Anselm L. Strauss. The Discovery of Grounded Theory: Strategies for Qualitative Research. Aldine Publishing Company, 1967.

[Gla95] R. L. Glass. A structure-based critique of contemporary computing research. Journal of Systems and Software, 28(1):3-7, 1995.

[Goo92] C. Goodwin and A. Duranti. Rethinking context: An introduction. In Rethinking context: Language as an interactive phenomenon, pages 1-42. Cambridge University Press, New York, 1992.

[GR12] I. Gutiérrez-Rojas, Crespo-García R. M., and C. Delgado-Kloos. Enhancing orchestration of lab sessions by means of awareness mechanisms. In Proceedings of the rth European Conference on Technology Enhanced Learning (EC-TEL 2012), 2012.

[Gra00] S. Graham and K. R. Harris. The role of self-regulation and transcription skills in writing and writing development. Educational Psychologist, 35(1):3-12, 2000. 
[Gra05] C. R. Graham. Blended learning systems: Definition, current trends, and future directions. In Handbook of blended learning: global perspectives, local designs, pages 3-21. Pfeiffer, 2005.

[Gre96] J.G. Greeno, A.M. Collins, L.B. Resnick, et al. Cognition and learning. In R. Calfee and D. Berliner, editors, Handbook of Educational Psychology, pages 15-46. Prentice Hall International, 1996.

[Gre01] J.C. Greene, L. Benjamin, and L. Goodyear. The merits of mixing methods in evaluation. Evaluation, 7(1):25-44, 2001.

[Gru10] M. Gruber, Christian Glahn, Marcus Specht, and Rob Koper. Orchestrating Learning using Adaptive Educational Designs in IMS Learning Design. In Sustaining TEL: From Innovation to Learning and Practice, pages 123-138. Springer, 2010.

[GS09] E. Gómez-Sánchez, M.L. Bote-Lorenzo, I.M. Jorrín-Abellán, G. Vega-Gorgojo, J.I. Asensio-Pérez, and Y. Dimitriadis. Conceptual framework for design, technological support and evaluation of collaborative learning. International Journal of Engineering Education, 25(3):557-568, May 2009.

[Gub81] E.G. Guba. Criteria for assessing the trustworthiness of naturalistic inquiries. A Journal of Theory, Research, and Development on Educational Communication and Technology, 29(2):75-91, 1981.

[Haa07] J. Haake and H.R. Pfister. Flexible scripting in net-based learning groups. In F. Fischer, I. Kollar, H. Mandl, and J. M. Haake, editors, Scripting Computer-Supported Collaborative Learning, pages 155-175. Springer, 2007.

[Ham11] R. Hamalainen and K. Vahasantanen. Theoretical and pedagogical perspectives on orchestrating creativity and collaborative learning. Educational Research Review, $6(3): 16,2011$.

[Har09] A. Harrer, D. Kohen-Vacs, B. Roth, N. Malzahn, U. Hoppe, and M. Ronen. Design and enactment of collaboration scripts: an integrative approach with graphical notations and learning platforms. In Proceedings of the 9th international conference on Computer supported collaborative learning - Volume 2, CSCL'09, pages 198-200. International Society of the Learning Sciences, 2009.

[Hay80] J.R. Hayes and L.S. Flower. Identifying the organization of writing processes. Cognitive processes in writing, pages 3-30, 1980.

[Her08] R. Hermans, J. Tondeur, J. Van Braak, and M. Valcke. The impact of primary school teachers' educational beliefs on the classroom use of computers. Computers E Education, 51(4):1499-1509, 2008.

[HG08] J.A. Hernandez-Gonzalo, E.D. Villasclaras-Fernandez, D. Hernandez-Leo, J.I. Asensio-Perez, and Y. Dimitriadis. InstanceCollage: A graphical tool for the particularization of role/group structures in pattern-based IMS-LD collaborative scripts. In Proceedings of the Eighth IEEE International Conference on Advanced Learning Technologies, 2008. ICALT'08, 2008. 
[HL06a] D. Hernández-Leo, E. D. Villasclaras-Fernández, I. M. Jorrín-AbellánJ. I. AsensioPérez, Y. Dimitriadis, I. Ruiz-Requies, and B. Rubia-Avi. Collage, a collaborative learning design editor based on patterns. Educational Technology \& Society, 9(1):58$71,2006$.

[HL06b] D. Hernández-Leo, E.D. Villasclaras-Fernandez, J.I. Asensio-Pérez, Y. Dimitriadis, and S. Retalis. CSCL scripting patterns: Hierarchical relationships and applicability. In Proceedings of the Sixth International Conference on Advanced Learning Technologies (ICALT 2006), pages 388-392, 2006.

[HL07] D. Hernández-Leo. A pattern-based design process for the creation of CSCL macroscripts computationally represented with IMS-LD. PhD Thesis, School of Telecommunications Engineering, University of Valladolid, Spain, 2007.

[HL08] D. Hernández-Leo, E. D. Villasclaras-Fernández, J. I. Asensio-Pérez, Y. Dimitriadis, M. Bote-Lorenzo, and I. Jorrín-Abellán. The added value of implementing the planet game scenario with Collage and Gridcole. Journal of Interactive Media in Education (JIME), Special issue on Comparing Educational Modelling Languages on the'Planet Game' Case Study, December 2008. http://jime.open.ac.uk/jime/article/view/ 2008-22.

[HL09] D. Hernández-Leo, E. D. Villasclaras-Fernández, J. I. Asensio-Pérez, and Y. Dimitriadis. Generating CSCL scripts: From a conceptual model of pattern languages to the design of real scripts. In P. Goodyear and S. Retalis, editors, Technology-Enhanced Learning: Design Patterns and Pattern Languages, pages 49-64. Sense Publishers, 2009 .

[HL10a] D. Hernández-Leo, J. I. Asensio, Y. Dimitriadis, and E.D. Villasclaras. Pattern languages for generating CSCL scripts: From a conceptual model to the design of a real situation. In P. Goodyear and S. Retalis, editors, E-learning, design patterns and pattern languages, pages 46-64. Sense Publishers, Rotterdam, The Netherlands, 2010.

[HL10b] D. Hernández-Leo, I.M. Jorrín-Abellán, E.D. Villasclaras, J.I. Asensio-Pérez, and Y. Dimitriadis. A multicase study for the evaluation of a collaborative learning pattern-based visual design approach. Journal of Visual Languages and Computing, 21(6):313-331, 2010.

[Hop93] H. U. Hoppe, N. Baloian, and J. Zhao. Computer support for teacher-centered classroom interaction. In Proceedings of the International Conference on Computers in Education, ICCE'93, volume 4, pages 459-475, 1993.

[Hou00] American heritage® dictionary of the english language, 2000.

[Hub94] M. Huberman and M. B. Miles. Qualitative data analysis. Sage Publications, Newbury Park, CA, USA, 1994.

[Hum02] M. Humphreys and T. Hyland. Theory, practice and performance in teaching: professionalism, intuition and jazz. Educational Studies, 28(1):5-15, 2002. 
[IMS03a] IMS Global Learning Consortium. IMS Learning Design v1.0 final specification, 2003. Accesible at http://www.imsglobal.org/learningdesign/.

[IMS03b] IMS Global Learning Consortium. IMS simple sequencing v1.0 final specification, 2003. Accesible at http://www.imsglobal.org/specificationdownload.cfm.

[IMS03c] IMS Global Learning Consortium. IMS simple sequencing v1.0 final specification best practice and implementation guide, 2003. Accesible at http://www .imsglobal. org/specificationdownload.cfm.

[IMS07] IMS Global Learning Consortium. IMS content packaging v1.2 public draft 2 specification, 2007. Accesible at http://www.imsglobal.org/specificationdownload.cfm.

[IMS10] IMS Global Learning Consortium. IMS Basic Learning Tool Interoperability v1.0, 2010. Accesible at http://www.imsglobal.org/lti/.

[IMS11] IMS Global Learning Consortium. IMS Common Cartridge v1.1 specification, 2011. Accesible at http://www.imsglobal.org/cc/

[IMS12] IMS Global Learning Consortium. IMS GLC Learning Tools Interoperability Implementation Guide. Final Version 1.1, 2012. Accesible at http://www.imsglobal.org/ 1ti/ (Last visit 11 May 2012).

[Ip03] A. Ip and E. Canale. Supporting collaborative learning activities with SCORM. In Proceedings of the EDUCASE in Australasia 2003, pages 669-678, 2003.

[JA07] I.M. Jorrín-Abellán and B. Rubia-Avi. What the eye doesn’t see: An inquiry (cowiki) based learning case study. In 3rd International congress of Qualitative Inquiry. Technology, Identity and Qualitative Inquiry, 2007.

[JA09a] I. M. Jorrín-Abellán and R. E. Stake. Does ubiquitous learning call for ubiquitous forms of formal evaluation?: An evaluand oriented responsive evaluation model. Ubiquitous Learning: An International Journal, 1(3), 2009.

[JA09b] I. M. Jorrín-Abellán and R. E. Stake. The needlework in evaluating a CSCL system: The evaluand-oriented responsive evaluation model. In Proceedings of the $9^{\text {th }}$ International Conference on Computer Supported Collaborative Learning, pages 68-72, 2009.

[Jac92] I. Jacobson, M. Christerson, P. Jonsson, and G. Overgaard. Object-oriented software engineering: a use case driven approach. Addison-Wesley, 1992.

[Jan05] J. Janssen and H. Hermans. How to integrate learning design into existing practice. In R. Koper and C. Tattersall, editors, Learning Design - A Handbook on Modelling and Delivering Networked Education and Training. Springer, 2005.

[Jer09] P. Jermann, G. Zufferey, B. Schneider, A. Lucci, S. Lépine, and P. Dillenbourgh. Physical space and division of labor around a tabletop tangible simulation. In Proceedings of the $9^{\text {th }}$ International Conference on Computer Supported Collaborative Learning, pages 345-349, 2009. 
[JIS12] JISC infoNET. Virtual learning environments. Online, 2012. Available from http://www.jiscinfonet.ac.uk/InfoKits/effective-use-of-VLEs/ intro-to-VLEs/introtovle-intro (Last visit: 22 Jun 2012).

[Joh04a] P. John and R. Sutherland. Teaching and learning with ICT: new technology, new pedagogy? Education and Communication and Information, 4(1):101-109, 2004.

[Joh04b] R.B. Johnson and A.J. Onwuegbuzie. Mixed methods research: A research paradigm whose time has come. Educational Researcher, 33(7):14, 2004.

[Jon99] D. Jonassen. Designing constructivist learning environments. In C.M. Reigeluth, editor, Instructional-Design Theories and Models: A New Paradigm of Instructional Theory, volume 2 of Instructional design theories and models, pages 215-239. Lawrence Erlbaum Associates, 1999.

[Jon05] C. Jones, L. Dirckinck-Holmfeld, and B. Lindström. A relational, indirect, mesolevel approach to cscl design in the next decade. International Journal of ComputerSupported Collaborative Learning, 1(1):35-56, 2005.

[Jun05] I. Jung. ICT-pedagogy integration in teacher training: Application cases worldwide. Educational Technology \& Society, 8(2):94-101, 2005.

[Jur05] A. S. Jurow and L. Creighton. Improvisational science discourse: Teaching science in two K-1 classrooms. Linguistics and Education, 16:275-297, 2005.

[Kal08] Y. Kali. The design principles database as means for promoting design based research. In A. E. Kelly, R. A. Lesh, and J. Y. Baek, editors, Handbook of design research methods in education: Innovations in science, technology, engineering and mathematics learning and teaching, pages 423-438. Lawrence Erlbaum Associates, Mahurah, N.J., 2008 .

[Kar09] A. Karakostas and S. Demetriadis. Adaptation patterns in systems for scripted collaboration. In Proceedings of the $9^{\text {th }}$ International Conference on Computer Supported Collaborative Learning, pages 477-481, 2009.

[Kar11] A. Karakostas and S. Demetriadis. Adaptation patterns as a conceptual tool for designing the adaptive operation of CSCL systems. Educational Technology Research and Development, 59(3):327-349, 2011.

[Kar12] A. Karakostas, L.P. Prieto, and Y. Dimitriadis. Opportunities and challenges for adaptive collaborative support in distributed learning environments: Evaluating the GLUE! suite of tools. In Proceedings of the $12^{\text {th }}$ IEEE International Conference on Advanced Learning Technologies (ICALT 2012), 2012.

[Kat10] D. Katsifli. The impact of blackboard software on education globally over the past 10 years, with a focus on the measurable benefits from using blackboard learn software and related technologies. Technical report, Blackboard Inc., 2010. http://www.lms.unimelb.edu.au/elo/resources/The_impact_of_ Blackboard_software_on_education_globally_(20100204W).pdf (Last visit: 12 May 2012). 
[Ken07] S. Kennewell, H. Tanner, S. Jones, and G. Beauchamp. Analysing the use of interactive technology to implement interactive teaching. Journal of Computer Assisted Learning, 2007.

[Ker95] B. Kernfeld. What to Listen for in Jazz. Yale University Press, 1995.

[Kni06] H. Kniberg. Scrum and XP from the Trenches. How we do Scrum. Technical report, Crisp, 2006. Available at http://www.metaprog.com/csm/ ScrumAndXpFromTheTrenches.pdf (Last visit: 11 Jun 2012).

[Kob07] L. Kobbe, A. Weinberger, P. Dillenbourg, A. Harrer, R. Hämäläinen, P. Häkkinen, and F. Fischer. Specifying computer-supported collaboration scripts. International Journal of Computer-Supported Collaborative Learning, 2(2/3):211-224, 2007.

[Kol06] I. Kollar, F. Fischer, and F. Hesse. Collaboration scripts - a conceptual analysis. Educational Psychology Review, 18:159-185, 2006.

[Kop04] R. Koper and J. Manderveld. Educational modeling language: Modelling reusable, interoperable, rich and personalised units of learning. British Journal of Educational Technology, 35(5):537-551, 2004.

[Kop05] R. Koper and C. Tattersall. Learning Design: a Handbook on Modelling and Delivering Networked Education and Training. Springer, 2005.

[Kop06] R. Koper. Current research in learning design. Educational Technology 85 Society, $9(1): 13-22,2006$.

[Kos96] Timothy D. Koschmann. CSCL, Theory and Practice of an Emerging Paradigm. Routledge, 1996.

[Kov01] M. Kovalainen, K. Kumpulainen, and V. Satu. Orchestrating classroom interaction in a community of inquiry: Modes of teacher participation. Journal of Classroom Interaction, 36(2):17-28, 2001.

[Kra09] W. Kraan. Google Wave and teaching and learning. Blog post, 2009. http://blogs. cetis.ac.uk/wilbert/2009/05/29/google-wave-and-teaching-learning/.

[Lar02] C. Larman. Applying UML and patterns: An introduction to Object-Oriented Analysis and Design and the Unified Process. Prentice Hall Professional, Upper Saddle River, NJ, USA, 2002.

[Lau02] D. Laurillard. Design tools for e-learning. In Keynote presentation for ASCILITE 2002, 2002.

[Lau11] D. Laurillard, P. Charlton, B. Craft, D. Dimakopoulos, D. Ljubojevic, G. Magoulas, E. Masterman, R. Pujadas, E. Whitley, and K. Whittlestone. A constructionist learning environment for teachers to model learning designs. Journal of Computer Assisted Learning, 2011.

[Law08] N. Law, W.J. Pelgrum, and T. Plomp. Pedagogy and ICT use in schools around the world: Findings from the IEA SITES 2006 study, volume 23. Springer Verlag, 2008. 
[Law11] N. Law, D. Laurillard, and Y. Lee. Learning design as a medium for scaffolding teacher learning and collaboration. In Proceedings of the $9^{\text {th }}$ International Conference on Computer-Supported Collaborative Learning (CSCL 2011), volume 1, pages 526$533,2011$.

[Lej09a] A. Lejeune, M. Ney, A. Weinberger, M. Pedaste, L. Bollen, T. Hovardas, U. Hoppe, and T. de Jong. A graphical modeling language for computer-based learning scenarios. In Proceedings of the Ninth IEEE International Conference on Advanced Learning Technologies. ICALT 2009., pages 734-735, Los Alamitos, CA, USA, 2009. IEEE Computer Society.

[Lej09b] A. Lejeune, M. Ney, A. Weinberger, M. Pedaste, L. Bollen, T. Hovardas, U. Hoppe, and T. de Jong. Learning activity spaces: Towards flexibility in learning design? In Proceedings of the Ninth IEEE International Conference on Advanced Learning Technologies. ICALT 2009., 2009.

[Len07] A. Lenhart and M. Madden. Social networking websites and teens: An overview. Technical report, PEW Internet and American Life Project, 2007. http://pewinternet. org/PPF/r/198/report_display.asp (Last visit 11 May 2012).

[Leu01] B. Leuf and W. Cunningham. The Wiki Way: Quick Collaboration on the Web. Addison-Wesley, London, UK, 2001.

[Lég11] F. Légaré, D. Stacey, S. Gagnon, S. Dunn, P. Pluye, D. Frosch, J. Kryworuchko, G. Elwyn, Gagnon M. P., and Graham I. D. Validating a conceptual model for an inter-professional approach to shared decision making: a mixed methods study. Journal of Evaluation in Clinical Practice, 17(4):554-564, 2011.

[Lin75] H.A. Linstone and M. Turoff. The Delphi method: techniques and applications. Addison Wesley Publishing Company, 1975.

[Lou12] J. Louvel, T. Templier, and T Boileau. Restlet in Action. Manning Publications Co., Greenwhich, CT, USA, 2012.

[Luc08] R. Luckin. The learner centric ecology of resources: a framework for using technology to scaffold learning. Computers \& Education, 50:449-462, 2008.

[Mac10] S. MacNeill and W. Kraan. Distributed learning environments: A briefing paper. Technical report, JISC Center for Educational Technology and Interoperability Standards (CETIS), 2010. http://wiki.cetis.ac.uk/images/6/6c/Distributed_Learning. pdf.

[Mag09] C. Magdea, J. Meekb, J. Wellensc, and T. Hooleyd. Facebook, social integration and informal learning at university: It is more for socialising and talking to friends about work than for actually doing work? Learning, Media and Technology, Special Issue: Learning and social software, researching the realities, 34(2):141-155, 2009.

[Mar06a] C. Martel, L. Vignollet, C. Ferraris, and G. Durand. LDL: a language to model collaborative learning activities. In Elaine Pearson and Paul Bohman, editors, Proceedings of World Conference on Educational Multimedia, Hypermedia and Telecommunications 2006, pages 838-844, Chesapeake, VA, June 2006. AACE. 
[Mar06b] A. Martínez, Y. Dimitriadis, E. Gomez-Sanchez, B. Rubia-Avi, I. Jorrin-Abellan, and J.A. Marcos. Studying participation networks in collaboration using mixed methods. International Journal of Computer Supported Collaborative Learning, 1(3):383-408, 2006.

[MC12a] J. A. Muñoz-Cristóbal, J. I. Asensio-Pérez, L. P. Prieto, I. M. Jorrín-Abellán, Y. Dimitriadis, and A. Martínez-Monés. Helping educators to deploy CSCL scripts into mainstream VLEs that integrate third-party web and Augmented Reality tools. Paper accepted in the Workshop on Digital Ecosystems for Collaborative Learning, in conjunction with International Conference of the Learning Sciences, (ICLS 2012), 2012.

[MC12b] J.A. Muñoz-Cristóbal, L.P. Prieto, J.I. Asensio-Pérez, I.M. Jorrín-Abellán, and Y. Dimitriadis. Lost in translation from abstract learning design to ICT implementation: a study using Moodle for CSCL. In Submitted to the European Conference on Technology-Enhanced Learning (EC-TEL 2012), 2012.

[McA10] A. McAuley, B. Stewart, G. Siemens, and D. Cormier. The MOOC model for digital practice. Technical report, University of Prince Edward Island, Social Sciences and Humanities Research Council's Knowledge synthesis grants on the Digital Economy, 2010 .

[Mcc10] Deborah Mccutchen. of Writing Knowledge, Processing, and Working Memory : Implications for a Theory of Writing. Online, (907469330), 2010.

[Mer94] M. D. Merrill and D. Twitchell. Instructional Design Theory. Educational Technology, 1994.

[Mer07] N. Mercer and K. Littleton. Dialogue and development of teacher thinking: a sociocultural approach. Routledge, 2007.

[Mer10] Merriam-Webster Dictionary. Merriam-Webster Online. Retrieved December 2010.

[Mia05] Y. Miao, K. Hoeksema, H. U. Hoppe, and A. Harrer. CSCL scripts: modelling features and potential use. In Proceedings of the 2005 Conference on Computer Support for Collaborative Learning (CSCL2005), CSCL '05, pages 423-432. International Society of the Learning Sciences, 2005.

[Mil00] G.E. Mills. Action Research: A Guide for the Teacher Researcher. Prentice-Hall, Inc., 2000 .

[Mis06] P. Mishra and M. J. Koehler. Technological pedagogical content knowledge: A framework for integrating technology in teacher knowledge. Teachers College Record, 108(6):1017-1054, 2006.

[MM08] A. Martínez-Monés, S. Villagrá-Sobrino, R. Santos-Fernández, R. Anguita-Martínez, and I.M Jorrín-Abellán. Social network analysis support for an IBL wiki-based course. Proceedings of the Workshop Real-Time methods at International Conference of the Learning Sciences, ICLS 2008, 2008.

[Mon03] P. B. Monday. Web Service Patterns: Java Edition, chapter 4: Exploring the Architecture Adapter Pattern. Apress, 2003. 
[Moo01] J.A. Moon. Short courses $\&$ workshops: improving the impact of learning, training \&6 professional development, chapter Chapter 6: Instruction and the facilitation for learning: the facilitation of learning for impact, pages 104-123. Routledge, 2001.

[Moo05] D. L. Moody. Theoretical and practical issues in evaluating the quality of conceptual models: current state and future directions. Data 86 Knowledge Engineering, 55:243$276,2005$.

[Mor06] S. Moran, M. Kornhaber, and H. Gardner. Orchestrating multiple intelligences. Educational Leadership, 64(1):22-27, 2006.

[Mor08] Y. Mor and N. Winters. Participatory design in open education: a workshop model for developing a pattern language. Journal of Interactive Media in Education, 2008.

[MS10] K. Mäkitalo-Siegl, J. Zottmann, F. Kaplan, and F. Fischer. The classroom of the future - an introduction. In K. Mäkitalo-Siegl, J. Zottmann, F. Kaplan, and F. Fischer, editors, Clasroom of the Future: Orchestrating Collaborative Spaces, pages 1-12. Sense Publishers, 2010.

[Nat07] G. Natriello. Imagining, seeking, inventing: the future of learning and the emerging discovery networks. Learning Inquiry, 1(1):7-18, 2007.

[Neu10] S. Neumann, L. Klebl, D. Griffiths, D. Hernández-Leo, L. de la Fuente-Valentín, H. Hummel, F. Brouns, M. Derntl, and P. Oberhuemer. Report of the results of an IMS Learning Design expert workshop. International Journal of Emerging Technologies in Learning, 5(1):58-72, 2010. Available online at http://dspace.ou.nl/ bitstream/1820/2154/2/iJET_onlineaccess_version.pdf

[Nir07] Jitti Niramitranon, Mike Sharples, Chris Greenhalgha, and Chiu-Pin Lin. SceDer and COML : Toolsets for learning design and facilitation in one-to-one technology classroom. In Proceedings of the ICCE 2007, 2007.

[Nir10] Jitti Niramitranon, Mike Sharples, Chris Greenhalgh, and Chiu-Pin Lin. Orchestrating Learning in a One-to-One Technology Classroom. 2010 6th IEEE International Conference on Wireless Mobile and Ubiquitous Technologies in Education, pages 96$103,2010$.

[Nod08] T. Nodenot, X. Caron, P. A. Le Pallec, and P. Laforcade. Applying model driven engineering techniques and tools to the planets game learning scenario. Journal of Interactive Media in Education (JIME), Special issue on Comparing Educational Modelling Languages on the 'Planet Game' Case Study, December 2008. http://jime.open.ac.uk/jime/article/view/2008-18.

[Nus11] M. Nussbaum, P. Dillenbourg, F. Fischer, C. K. Looi, and J. Roschelle. How to integrate CSCL in classroom life: Orchestration. In Proceedings of the $9^{\text {th }}$ international conference on Computer-Supported Collaborative Learning (CSCL 2011), page 1199, 2011.

[O'C07] C. O'Connor and S. Michaels. When is dialogue "dialogic"? Human Development, 50:275-285, 2007. 
[O'D92] A. M. O'Donnell and D. F. Danserau. Scripted cooperation in student dyads: a method for analyzing and enhancing academic learning and performance. In R. HertzLazarowitz and N. Miller, editors, Interaction in cooperative groups: The theoretical anatomy of group learning, pages 120-141. Cambridge University Press, 1992.

[O'R05] T. O'Reilly. What is web 2.0? design patterns and business models for the next generation of software. Web site, Last visit: 1 April 2010, September 2005. http: //www.oreilly.de/artikel/web20.html.

[Osg03] R.T. Osguthorpe and C.R. Graham. Blended learning environments: Definitions and directions. Quarterly Review of Distance Education, 4(3):227-233, 2003.

[Pac10] N. Pachler, C. Daly, Y. Mor, and H. Mellar. Formative e-assessment: Practitioner cases. Computers \& Education, 54(3):715-721, 2010.

[Pap80] S. Papert. Mindstorms: Children, computers, and powerful ideas. Basic Books, 1980.

[Pap03] M.P. Papazoglou and D. Georgakopoulos. Service-oriented computing. Communications of the ACM, 46(10):24-28, 2003.

[Pel03] C. Peltz. Web services orchestration and choreography. IEEE Computer, 36(10):46-52, October 2003.

[Pel10] J.W. Pellegrino. Perspectives on the Integration of Technology and Assessment. Journal of Research on Technology in Education, 43(2):119-134, 2010.

[Pen07] W. Penuel, B. Fishman, R. Yamaguchi, and L. Gallagher. What makes professional development effective? strategies that foster curriculum implementation. American Educational Research Journal, 44(4):921-958, 2007.

[Pla06] Plan Avanza. Las TIC en la educacion. Available online at http://www.oei.es/tic/ TICCD.pdf (Last visit: 12 Nov 2010)., 2006.

[PR08] L. Palomino-Ramírez, M.L. Bote-Lorenzo, J.I. Asensio-Pérez, and Y. Dimitriadis. LeadFlow4LD: Learning and data flow composition-based solution for learning design in CSCL. In Proceedings of the $14^{\text {th }}$ International Workshop on Groupware, CRIWG 2008, pages 266-280, 2008.

[Pri09] L. P. Prieto. An exploration of teacher enactment of CSCL activities in computerintegrated classrooms. Master's Thesis, Telecommunications Engineering School, University of Valladolid, Spain, 2009.

[Pri10a] L. P. Prieto, S. Villagrá-Sobrino, Y. Dimitriadis, I. M. Jorrín-Abellán, A. MartínezMonés, and R. Anguita-Martínez. Recurrent routines in the classroom madness: pushing patterns past the design phase. In Proceedings of the rth International Conference on Networked Learning (NLC2010), pages 499-507, 2010.

[Pri10b] Luis Pablo Prieto. The many faces of orchestration: towards a (more) operative definition. Report. Available (privately) from http://gsic.tel.uva.es/wikis/gs2/ images/d/d4/20100402-Orchestration-enviado.pdf., April 2010. 
[Pri11a] L. P. Prieto. Going from learning design to its enactment in web learning environments (i): Requisites and learning design side. Technical report, University of Valladolid, January 2011.

[Pri11b] L. P. Prieto, J. I. Asensio-Pérez, Y. Dimitriadis, E. Gómez-Sánchez, and J. A. MunozCristóbal. GLUE!-PS: A multi-language architecture and data model to deploy TEL designs to multiple learning environments. In Proceedings of the European Conference on Technology-Enhanced Learning (EC-TEL 2011), 2011.

[Pri11c] L. P. Prieto, Y. Dimitriadis, and S. Villagrá-Sobrino. Representing learning design and classroom orchestration through atomic patterns. Paper presented at the "Art and Science of Learning Design" international workshop (ASLD11), Oct 2011.

[Pri11d] L. P. Prieto, Y. Dimitriadis, S. Villagrá-Sobrino, I. M. Jorrín-Abellán, and A. Martínez-Monés. Orchestrating CSCL in primary classrooms: One vision of orchestration and the role of routines. In Proceedings of the 9th international conference on Computer-SupportedCollaborative Learning (CSCL 2011), 2011. Paper presented at the Workshop "How to integrate CSCL in classroom life: Orchestration". Available online at http://www.gsic.uva.es/ lprisan/CSCL2011_WSOrchestration_Prieto_ submission.pdf.

[Pri11e] L. P. Prieto, M. Holenko-Dlab, M. Abdulwahed, I. Gutiérrez, and W. Balid. Orchestrating technology enhanced learning: a literature review and a conceptual framework. International Journal of Technology-Enhanced Learning (IJTEL), 3(6):583-598, 2011.

[Pri1f] L. P. Prieto, S. Villagrá-Sobrino, Y. Dimitriadis, P. Schank, W. Penuel, and DeBarger A. H. Mind the gaps: Using patterns to change everyday classroom practice towards contingent CSCL teaching. In Proceedings of the International Conference of Computer-Supported Collaborative Learning (CSCL2011), volume 1, pages 518-525, 2011.

[Pri11g] L. P. Prieto, S. Villagrá-Sobrino, I. M. Jorrín-Abellán, A. Martínez-Monés, and Y. Dimitriadis. Recurrent routines: analyzing and supporting orchestration in technology-enhanced primary classrooms. Computers \& Education, 57(1):1214-1227, 2011.

[Pri12a] L. P. Prieto, S. Villagrá-Sobrino, Y. Dimitriadis, and I. M. Jorrín-Abellán. Orchestrating classroom CSCL: a multi-level pattern approach for design and enactment. In R. Luckin, P. Goodyear, B. Grabowski, S. Puntambeker, J. Underwood, and N. Winters, editors, Handbook of Design in Educational Technology. Routledge, 2012.

[Pri12b] L.P. Prieto, J.A. Muñoz Cristóbal, J.I. Asensio-Pérez, and Y. Dimitriadis. Making learning designs happen in distributed learning environments with GLUE!-PS. In Submitted to the European Conference on Technology-Enhanced Learning (EC-TEL 2012), 2012.

[PS09a] M. Pérez-Sanagustín, J. Burgos, D. Hernández-Leo, and J. Blat. Considering the intrinsic constraints for groups management of TAPPS and Jigsaw CLFPs. In Proceedings of International Conference on Intelligent Networking and Collaborative Systems (INCoS 2009), 2009. 
[PS09b] M Pérez-Sanagustín, D. Hernández-Leo, and J. Blat. Conditioning factors for group management in blended learning scenarios. In Ninth International Conference on Advanced Learning Technologies, pages 233-235, 2009.

[PS09c] M. Pérez-Sanagustín, D. Hernández-Leo, and J. Blat. Towards supporting orchestrated computer supported collaborative learning scenarios. IEEE Multidisciplinary Engineering Education, 4(3):83-88, September 2009.

[PS11] M. Pérez-Sanagustín. Operationalization of collaborative blended learning scripts: a model, computational mechanisms and experiments. PhD Thesis, Department of Information and Communication Technologies. Universitat Pompeu Fabra. Barcelona, Spain, 2011.

[RC12] A. Ruiz-Calleja, G. Vega-Gorgojo, J. I. Asensio-Pérez, M. L. Bote-Lorenzo, E. GómezSánchez, and C. Alario-Hoyos. A linked data approach for the discovery of educational ICT tools in the web of data. Computers and Education, 2012.

[Ret06] S. Retalis, P. Georgiakakis, and Y. Dimitriadis. Eliciting design patterns for e-learning systems. Computer Science Education, 16(2):105-118, 2006.

[Ret10] S. Retalis, M. Katsamani, P. Georgiakakis, G. Lazakidou, O. Petropoulou, and T. Kargidis. Designing collaborative learning sessions that promote creative problem solving using design patterns. In Proceedings of the th International Conference on Networked Learning (NLC2010), pages 490-498, 2010.

[Ric99] L. Richards. Using NVivo in Qualitative Research. Sage, 1999.

[Ric03] V. Richardson. Constructivist pedagogy. The Teachers College Record, 105(9):16231640, 2003.

[Ric07] L. Richardson and S. Ruby. RESTful Web Services. O'Reilly Series. O'Reilly, 2007.

[Ris00] L. Rising and N.S. Janoff. The Scrum software development process for small teams. IEEE Software, 17(4):26-32, 2000.

[Ros02] J. Roschelle and R. Pea. A walk on the wild side: How wireless handhelds may change computer-supported collaborative learning. International Journal of Cognition and Technology, 1(1):145-168, 2002.

[Ros07] J. Roschelle, D. Tatar, S.R. Chaudbury, Y. Dimitriadis, C. Patton, and C. DiGiano. Ink, improvisation, and interactive engagement: Learning with tablets. Computer, 40(9):42-48, 2007.

[Ros11] J. Roschelle, C. Patton, P. Schank, W. Penuel, C. K. Looi, W. Chen, L. P. Prieto, S. Villagrá-Sobrino, and Y. Dimitriadis. CSCL and innovation: in classrooms, with teachers, among school leaders, in schools of education. In Proceedings of the $9^{\text {th }}$ International Conference on Computer-Supported Collaborative Learning (CSCL 2011), Jul 2011. 
[RT12] M.J. Rodríguez-Triana, A. Martínez-Monés, J.I. Asensio-Pérez, and Y. Dimitriadis. Towards a monitoring-aware design process for CSCL scripts. In 18th International Conference on Collaboration and Technology, CRIWG'12, Duisburg, Germany, 2012.

[Sal92] G. Salomon. What does the design of effective CSCL require and how do we studyits effects? SIGCUE Outlook, 21(3):62-68, 1992.

[Saw01] R. K. Sawyer. Creating Conversations: Improvisation in Everyday Discourse. Hampton Press, 2001.

[Saw04] R. Keith Sawyer. Creative teaching: Collaborative discourse as disciplined improvisation. Educational Researcher, 2004.

[Sch99] Alan H. Schoenfeld. Models of the teaching process. Journal of Mathematical Behavior, 18(3):243-261, 1999.

[Sch02] K. Schwaber and M. Beedle. Agile software development with Scrum. Prentice Hall, 2002 .

[Sev08] C. Severance, J. Hardin, and A. Whyte. The coming functionality mash-up personal learning environments. Interactive Learning Environments, 16(1):47-62, 2008.

[SF11] M.J. Sánchez-Franco, A.F. Villarejo-Ramos, and F.A. Martín-Velicia. Social integration and post-adoption usage of social network sites an analysis of effects on learning performance. Procedia - Social and Behavioral Sciences, 15(1):256-262, 2011.

[Sha09] L. Sha and J. van Aalst. A 2x3 model of student-directed formative assessment in collaborative knowledge creation. Computers in Education, pages 205-212, 2009.

[Sha11] M. Sharples. A response from M. Sharples. In P. Dillenbourg, editor, D1.5 Trendscouting Report: "Classroom Orchestration", pages 20-22. STELLAR NoE, 2011. Available online at http://www.stellarnet.eu/kmi/deliverables/20110818_stellar_ _-d1.5_-_trends-in-orchestration.pdf (Last visit: 08 May 2012).

[She09] N. Shechtman and J. Knudsen. Bringing out the playful side of mathematics: Using methods from improvisational theater in professional development for urban middle school math teachers. Play and Culture Series, 11, 2009.

[Sim82] H.A. Simon. Models of bounded rationality. The MIT Press, 1982.

[Sla01] R. E. Slavin and N. A. Madden (Eds.). Success for all: Research and reform in elementary education. Erlbaum, 2001.

[Sle82] D. Sleeman and J.S. Brown. Intelligent tutoring systems. In Intelligent tutoring systems, volume 5091. Academic Press, 1982.

[So08] H.J. So and T.A. Brush. Student perceptions of collaborative learning, social presence and satisfaction in a blended learning environment: Relationships and critical factors. Computers ES Education, 51(1):318-336, 2008.

[SRI08a] SRI. Group Scribbles official website. Web site, http://groupscribbles.sri.com (Last visit: 12 Nov 2010), 2008. 
[SRI08b] SRI International. Contingent pedagogies for conceptual teaching and learning project. Web site, http://ctl.sri.com/projects/displayProject.jsp?Nick= contingent (Last visit: 28 Jul 2009), 2008.

[Sta89] S.L. Star and J.R. Griesemer. Institutional ecology, translations and boundary objects: Amateurs and professionals in Berkeley's Museum of Vertebrate Zoology. Social Studies of Science, 19:387-420, 1989.

[Sta95] R. E. Stake. The art of case study research. Sage Publications, 1995.

[Sta05] Robert Stake. Qualitative case studies. In N. Denzin and Y. Lincoln, editors, The SAGE Handbook of Qualitative Research, pages 443-466. Sage, 2005.

[Sta06a] G. Stahl, T. Koschmann, and D. \& Suthers. Computer-supported collaborative learning: An historical perspective. In R. K. Sawyer, editor, Cambridge handbook of the learning sciences. Cambridge University Press, 2006.

[Sta06b] R. Stake. Multiple Case Study Analysis. The Guilford Press, 2006.

[Sta10] R. Stake. Qualitative Research: Studying How Things Work. The Guilford Press, 2010 .

[Sta11] G. Stahl, H. Spada, N. Miyake, and N. Law. Introduction to the proceedings of CSCL 2011. In G. Stahl, H. Spada, N. Miyake, and N. Law, editors, Proceedings of the 9th international conference on Computer-SupportedCollaborative Learning (CSCL 2011), pages vii-xi, 2011.

[Ste08] M. K. Stein, R. Engle, M. Smith, and E. Hughes. Orchestrating productive mathematical discussions: Five practices for helping teachers move beyond show and tell. Mathematical Thinking and Learning, 10(4):313-340, 2008.

[STE10a] STELLAR Network of Excellence. Orchestrating learning - STELLAR. Web site, Last visit: 1 April 2010, 2010. http://www.stellarnet.eu/d/1/1/Orchestrating_ learning.

[STE10b] STELLAR Network of Excellence. Stellar: The network for technology enhanced learning. Web site, Last visit: 1 April 2010, 2010. http://www.stellarnet.eu/.

[Sti00] M.J. Stiles. Effective learning and the virtual learning environment. In Proceedings of the 6th European University Information Systems Congress (EUNIS 2000), pages 171-180, 2000.

[Str07] J.W. Strijbos and F. Fischer. Methodological challenges for collaborative learning research. Learning and Instruction, 17(4):389-393, 2007.

[Sud93] D. Sudnow. Ways of the Hand: The Organization of Improvised Conduct. MIT Press, 1993.

[Sut06] D.D. Suthers. Technology affordances for intersubjective meaning making: A research agenda for CSCL. International Journal of Computer-Supported Collaborative Learning, 1(3):315-337, 2006. 
[Sut09] R. Sutherland and M. Joubert. D1.1: The STELLAR vision and strategy statement. Deliverable, STELLAR Consortium, 2009. Available from http://dspace.ou.nl/ bitstream/1820/2823/1/20090929_d1-1__vision-and-strategy.pdf.

[Tab04] I. Tabak. Synergy: A complement to emerging patterns of distributed scaffolding. Journal of the Learning Sciences, 13(3):305-335, 2004.

[Tat07] Deborah Tatar. The design tensions framework. Human-Computer Interaction, 22(4), 2007.

[Tch07] P. Tchounikine. Directions to acknowledge learners' self-organization in CSCL macroscripts. In J.M. Haake, S.F. Ochoa, and A. Cechich, editors, Groupware: Design, Implementation and Use, volume LNCS n 4715. Springer Berlin / Heidelberg, 2007.

[Tch08] P. Tchounikine. Operationalizing macro-scripts in CSCL technological settings. International Journal of Computer Supported Collaborative Learning, 3(2):193-233, 2008.

[Tch11] P. Tchounikine. A response from P. Tchounikine. In Trends in orchestration: Second research and technology scouting report, pages 38-43. STELLAR NoE, Deliverable, D1.5, 2011.

[Tho09] P. Thoeny. TWiki ( - the open source enterprise wiki and web 2.0 application platform. Web site, http://www.twiki.org/, Last visit: 20 August 2009.

[Tre03] L. Treleaven. A new taxonomy for evaluation studies of online collaborative learning. In T.S. Roberts, editor, Online Collaborative Learning: Theory and Practice. Idea Group Press, Hershey, Pennsylvania, 2003.

[Van03] L. Vandergrift. Orchestrating strategy use: Toward a model of the skilled second language listener. Language Learning, 53(3):463-496, 2003.

[Vas10] E. Vass and K. Littleton. Peer collaboration and learning in the classroom. In K. Littleton, C. Wood, and J. K. Staarman, editors, International Handbook of Psychology in Education, pages 105-135. Emerald, 2010.

[VF09a] E. D. Villasclaras-Fernández, D. Hernández-Leo, J. I. Asensio-Pérez, Y. Dimitriadis, and L. de la Fuente-Valentín. Interrelating assessment and flexibility in IMS-LD CSCL scripts. In Proceedings of the SFC-2009 Workshop: Scripted vs. Free Collaboration: Alternatives and Paths for Adaptable and Flexible CS Scripted Collaboration, pages 39-43, 2009.

[VF09b] E.D. Villasclaras-Fernández, J.A. Hernández-Gonzalo, D. Hernández-Leo, J.I. Asensio-Pérez, Y. Dimitriadis, and A. Martínez-Monés. InstanceCollage: A tool for the particularization of collaborative IMS-LD scripts. Educational Technology $\&$ Society, $12(4): 56-70,2009$.

[VF11] E. D. Villasclaras-Fernández, J. I. Asensio-Pérez, D. Hernández-Leo, Y. Dimitriadis, L. de la Fuente-Valentín, and A. Martínez-Monés. Implementing computerinterpretable CSCL scripts with embedded assessment: A pattern based design approach. In F. Pozzi and D. Persico, editors, Techniques for Fostering Collaboration in 
Online Learning Communities: Theoretical and Practical Perspectives, pages 261-277. IGI Global Publishing, 2011.

[vH06] M. van Harmelen. Personal learning environments. In Proceedings of the Sixth IEEE International Conference on Advanced Learning Technologies, ICALT '06, pages 815816, Washington, DC, USA, 2006. IEEE Computer Society.

[Vig08a] L. Vignollet, C. Ferraris, C. Martel, and D. Burgos. A transversal analysis of different learning design approaches. Journal of Interactive Media in Education, 2008(2), 2008.

[Vig08b] L. Vignollet, C. Martel, and C. Ferraris. Description of the 'Planet Game' case study and guidelines to the authors. Journal of Interactive Media in Education (JIME), Special issue on Comparing Educational Modelling Languages on the'Planet Game' Case Study, December 2008. http://jime.open.ac.uk/jime/article/view/2008-18.

[Vil09] Sara Villagrá and Luis P. Prieto. Informe de investigación: Cigales 2008-2009. Technical report, Grupo de Sistemas Inteligentes y Colaborativos (GSIC), University of Valladolid, Spain, 2009. Available online at http://gsic.uva.es/ lprisan/ VillagraSobrino2009_InformeFinalCigales2008-2009.pdf.

[VS11] S. Villagrá-Sobrino and L.P. Prieto. The making of a routine catalogue. Unpublished technical report, University of Valladolid, 2011. Available online at http://www.gsic . uva.es/ lprisan/Villagra2011_RoutineAnalysis.pdf (Last visit: 25 May 2012).

[VS12] S. Villagrá-Sobrino. El desarrollo profesional del profesorado centrado en el uso de rutinas de diseño y prácticas colaborativas con TIC en Educación Primaria. PhD Thesis, Faculty of Education and Social Work. University of Valladolid, Spain, 2012.

[W3C09] W3C Consortium. Widget packaging and configuration. W3C Candidate Recommendation, dec 2009. http://www.w3.org/TR/widgets/ (Last visit 11 May 2012).

[Wal05] L. Walker and K. Avant. Strategies for Theory Construction in Nursing. Pearson Prentice Hall, 2005.

[Wat03] M. Watts. The orchestration of learning and teaching methods in science education. Canadian Journal of Science, Mathematics and Technology Education, 2003.

[Wat06] D Watson. Understanding the relationship between ict and education means exploring innovation and change. Education and Information Technologies, 3-4(11):199-216, 2006 .

[Wec07] C. Wecker and F. Fischer. Fading scripts in computer-supported collaborative learning: the role of distributed monitoring. In Proceedings of the 8th iternational conference on Computer supported collaborative learning, CSCL'07, pages 764-772. International Society of the Learning Sciences, 2007.

[Wei91] M. Weiser. The computer for the $21^{\text {st }}$ century. Scientific American, 165(3):94-104, 1991. 
[Wei07] A. Weinberger, D. Clark, P. Dillenbourg, D. Diziol, V. Sampson, K. Stegmann, N. Rummel, F. Hong, H. Spada, B. Mclaren, T. Brahm, and F. Fischer. Orchestrating learning activities on the social and the cognitive level to foster CSCL. In Proceedings of the $8^{\text {th }}$ International Conference on Computer Supported Collaborative Learning, pages 38-47. International Society of the Learning Sciences, 2007.

[Wei09] A. Weinberger, I. Kollar, Y. Dimitriadis, K. Mäkitalo-Siegl, and F. Fischer. Computersupported collaboration scripts: Perspectives from educational psychology and computer science. In N. Balacheff, S. Ludvigsen, T. de Jong, A. Lazonder, and S. Barnes, editors, Technology-Enhanced Learning: Principles and Products. Amsterdam University Press, 2009.

[Wel06] G. Wells and R. Mejia-Arauz. Dialogue in the classroom. Journal of the Learning Sciences, 15(3):379-428, 2006.

[Wha02] J. Whalen, M. Whalen, and K. Henderson. Improvisational choreography in teleservice work. British Journal of Sociology, 53(2):239-258, 2002.

[Whi11] S. White and H.C. Davis. Making it rich and personal: crafting an institutional personal learning environment. International Journal of Virtual and Personal Learning Environments, 2011.

[Wil06] S. Wilson, O. Liber, M. Johnson, P. Beauvoir, P. Sharples, and C. Milligan. Personal Learning Environments: Challenging the dominant design of educational systems. In Proceedings of the Joint International Workshop on Professional Learning, Competence Development and Knowledge Management (LOKMOL and L3NCD), pages 67$76,2006$.

[Win09] N. Winters and Y Mor. Dealing with abstraction: Case study generalisation as a method for eliciting design patterns. Computers in Human Behavior, 25(5):10791088, 2009.

[Wor09] World Wide Web Consortium (W3C). XForms 1.1 W3C Recommendation, 2009.

[Yin87] R. J. Yinger. By the seat of your pants: An inquiry into improvisation and teaching. In Paper presented at the Annual Meeting of the American Educational Research Association, 1987.

[Zel98] M.V. Zelkowitz and D.R. Wallace. Experimental models for validating technology. IEEE Computer, 31(5):23-31, 1998.

[Zha03] Yong Zhao and Kenneth A. Frank. Factors affecting technology uses in schools: An ecological perspective. Technical report, Michigan State University, 2003. 


\title{
Appendix A
}

\section{The Orchestration Interview Guide (OIG): A research instrument derived from the ' $5+3$ Aspects' framework}

\begin{abstract}
Summary: In this appendix, we include the first researcher instrument derived from the $5+3$ Aspects' conceptual framework for orchestration in TEL/CSCL, as described in Section 3.4. This questionnaire has been used, both in its Spanish and English translations, during this dissertation. This appendix includes the English version of the instrument, as presented to the international expert researchers in study RP2 (see Section 3.3).
\end{abstract}

\author{
TEL/CSCL orchestration: \\ A reflection guide based on the ' $5+3$ ' framework \\ Luis P. Prieto (lprisan@gsic.uva.es) \\ GSIC/EMIC group, University of Valladolid
}

\begin{abstract}
In technology-enhanced learning (TEL), the concept of 'orchestrating learning' has been proposed as a metaphor of the classroom design and enactment, with an emphasis on the teacher's point of view, and the multiple constraints that teachers and students have to face when teaching and learning in authentic settings. Based on a recent literature review and conceptual framework, this document provides an interview guide for researchers, trying to characterize the orchestration of an authentic TEL setting, through its 8 main aspects. It can be used as a reflection guide or as an interview guide in order to know more (through an interviewee, e.g. a teacher) about how orchestration happens in an educational setting. This questionnaire makes special emphasis on recurrent patterns and best practices in orchestration, but it should be easy to customize it to analyze other aspects and phenomena under the 'orchestration' umbrella.
\end{abstract}

\section{Interviewee:}

Interviewer: 


\section{Date:}

Place:

\section{Educational context:}

\section{A.1. General guidelines}

- You can answer the questions by editing on the document itself, or annotating in paper the most interesting answers that come to mind.

- Probably the number of questions in this document is excessive for the typical interview (or mental exercise) timeframe. Thus, it is better to make emphasis in some aspects and go faster over others. The document highlights more prioritary questions (in bold face), medium-priority questions (in normal type) and lower-priority ones (in italics). However, this a priori categorization could be dependent on the educational context and the focus of the research.

- If using this guide to interview someone else, it is recommended to gather some previous information about the context (subject matter, general design of the course, presentiality, ICTs involved, gaining access to the LMS, if applicable, etc), in order to try and predict which orchestration aspects will be worthier of focus during the interview.

- In general, 8 aspects are too many to cover in a e.g., 60 minute interview. Thus, it is recommended to timebox each section (e.g. will not dedicate more than 7 minutes to each aspect), and to re-order them so that more prioritary (for our research focus) ones are touched upon at the beginning.

\section{A.2. Questioning guide}

\section{A.2.1. Design/Planning}

- Describe briefly the learning design and dynamics of the concerned course.

- How was the design/planning of the course activities done? Was it done by the same teacher that has to enact it, or by different people? Was the design done collaboratively by multiple people?

- Did the designers use specific tools or processes to do the design (e.g. LD tools like WebCollage, design through LD patterns, etc)? Did they further use any kind of heuristic or strategy to generate the design?

- Were the learning objectives explicitly defined? Were they content-related, or competencebased, or something else? 
- Is there a clear connection between these learning objectives and the designed learning activities? What is the relationship?

- (in case that more than one activity design is available) What is the design's granularity, time-wise (minutes, days, weeks...)? Are there recurrent elements in the available designs (e.g. kinds of activities, tool usage, groupings, etc)?

- Did the teacher do any modification/particularization to the original learning design, to make it a better fit for the concrete context (e.g. different classroom, students, etc) where it would be enacted? What modifications?

- What was the rationale behind the choice of technologies to be used during the learning activities? How were they chosen? Did teachers force the tools onto students, suggest technological tools, or left the choice entirely to the students?

- How did the teachers go from the learning design artifacts (e.g. plans), to the concrete technology instances that were used in the enactment (deployment)? Who did it? Was it manual or automatic? Was it done entirely before the enactment, or progressively as the course progressed? Which problems, tricks, heuristics etc were found in the process? Which parts were more tedious or simple? How long did this process take?

\section{A.2.2. Regulation/Management}

- Which parts of the course are more difficult to manage/regulate?

- (Here, direct the interviewee to the episodes/parts of the learning design which are more difficult to manage)

- Who managed how and when to go from one activity to the next one? Was this management done socially (using just oral communication, via email...) or through technology (e.g. automated)?

- How strong was the regulation of learning activities? Was it possible for students (and teachers) to step out of the script/design?

- Did any of the used technologies play a central role in the course (e.g. an LMS), over the others? How did the different technologies relate to one another?

- Were there recurrent elements in the coordination of activities (e.g. an email was always sent to mark the end of one activity and the beginning of the next one)? Was there any particular reason to do it so?

- Which problems arose when managing/regulating the learning activities? How were they solved? Did the teacher have any rules or tricks to solve these problems?

- Apart from the transitions between activities, was there any kind of regulation about how each activity had to be done? Was that regulation social or technological (was it spoken instructions, the use of a tool that only allowed for a particular type of operation, etc)? Did there exist recurrent element in how this regulation was done? What was the rationale behind this way of doing things? 
- How was the group formation done (if applicable)? Was it imposed by the teacher or decided by the students? Was it fixed throughout the course activities or was it changing? What was the rationale behind the group formation?

- How was the time management of activities done? Was it social or automatic?

\section{A.2.3. Adaptation/Flexibility/Intervention}

- Did the enactment of the learning activities occur exactly as it was designed? Which changes were necessary and why?

- (Here, direct the interviewee to the episodes/parts of flexibility mentioned)

- Did the learning design contemplate explicitly the possibility of changes during enactment? How was this expressed? Did the chance of change modify the choice or configuration of the technological support?

- Do the situations that provoked the changes occur frequently? How probable are they?

- Were the changes made using the technological support, or did the change consist on dismissing the technological support in favor of e.g. pen and paper? Were these changes facilitated in any way by the chosen technologies' functionalities (i.e. do these technologies allow for changes with ease)? How does the teacher access those changes in the technology? Are there recurrent elements in these technological changes?

- Were changes necessary due to the learning design being incomplete or erroneous? Did they imply changes in the script of activities, or were they just minor variations of the design?

- Does the application of changes have recurrent elements (even if the causes for the change are different)?

- Were changes in the temporal plan of the activity needed? And in the group formation? Were these aspects flexible by design, or did they have to be modified unexpectedly?

\section{A.2.4. Awareness/Assessment}

- Are there any mechanisms for the monitorization of the learning activities during their enactment? Are there any specific assessment activities in the design? Do these activities occur towards the end of the design, or also throughout the design?

- Does the technological support enable the monitorization of learning activities in a simple way? How does the teacher access to those features? Are there recurrent elements?

- Did the need for monitorization and assessment modify the learning design? And the technologies and tools' choice and deployment (i.e. is the deployment done in a way that makes it possible/easy to monitor)? What are the rules or rationales that teachers use to take this aspects into account? 


\section{A.2.5. Roles of the teacher and other actors}

- Did the teacher coordinate the unfolding of the learning activities, or was it done by students themselves, or a combination of both? What is the rationale behind doing it the way it was?

\section{A.2.6. Pragmatism/Practice}

- Were there contextual restrictions of the concrete educational setting that prompted for changes in the original activity design? And in its instantiation and deployment?

- Was the technology adapted to the learning activities to perform, or was it the other way around? Which form did those adaptations take? Are there any recurrent elements in that adaptation?

- Would the teacher have liked to enact the design or its technological implementation in a different way? What restrictions prompted him/her no to do it that way? Are there any recurrent restrictions?

- What are the rules and heuristics that the teacher uses to orchestrate activities in a pragmatic manner (as opposed to what she would like to do, or what seems to be a better option theoretically or pedagogically)?

\section{A.2.7. Alignment/Synergy}

- (Here, direct the interviewee's attention to the episodes or parts of the course where more than one kind of tool/scaffolding is used)

- Are there any recurrent combinations of technologies in the course's design? Is there any particular reason for these combinations?

- Are there any combined uses of technology that appear frequently during the enactment? In what kinds of activities? Is there any particular reason for that way of using available technologies?

- Does the teacher express any explicit relationship between the learning objectives and the used technologies? Which concrete uses of those technologies support more clearly those objectives? Are there any recurrent elements?

\section{A.2.8. Models/Theories}

- Which beliefs, theories or models did the teacher explicitly use to design the activities? Which rationales or heuristics were used to translate between the general theories and the concrete design? Are there any recurrent elements?

- Which theories or principles were used to coordinate the learning activities? Were there any rules or heuristics to make decisions on-the-fly during enactment? 
- (If there is a varied set of observations or interviews) Is there any correlation between the theory/model used and the concrete ways of orchestrating the activities? Are there any recurrent elements? 


\section{Appendix B}

\section{Orchestration Atomic Patterns Catalogue (OAPC)}

In this appendix we include a table with a classified list of all the atomic patterns uncovered during the dissertation (see Chapter 4), both in primary schools contexts and in higher education contexts. This catalogue is classified according to which orchestration aspect (Chapter 3 ) it represents the most, although we also list other aspects which the atomic pattern represents to a lesser extent.

Table B.1: Master catalogue of uncovered atomic patterns, categorized by their primary orchestration aspect. Bold face atomic patterns are those which are deemed more frequent or more useful (e.g. for use in professional development actions).

\begin{tabular}{|l|c|l|}
\hline Atomic pattern & Primary aspect & Secondary aspect(s) \\
\hline Adaptive prompting during collaboration & Adaptation & Awareness, Theory \\
\hline Advance the Advanced & Adaptation & Management \\
\hline $\begin{array}{l}\text { Ask students about the topics to cover in the } \\
\text { course }\end{array}$ & Adaptation & Roles, Theory \\
\hline Assign Moderator & Adaptation & Roles, Management, Design \\
\hline Challenging learning material & Adaptation & Management \\
\hline Change task from face-to-face to online & Adaptation & Management, Practice, Design \\
\hline Extend time limits and try again & Adaptation & Management, Practice \\
\hline Group Heterogeneity based on Prior Domain Knowledge & Adaptation & Design, Management \\
\hline Increased support for groups of novices & Adaptation & Management, Practice \\
\hline Lack of Confidence & Adaptation & Management \\
\hline Optional activities & Adaptation & Design, Practice \\
\hline Pen and paper "plan B" & Adaptation & Synergy, Practice \\
\hline Reform groups in face of the current attendants & Adaptation & Management, Design \\
\hline Spontaneous use of additional ICT & Adaptation & Practice, Synergy \\
\hline Successive deployment of activities & Adaptation & Design, Practice \\
\hline Use wiki to adapt the session script & Adaptation & Awareness, Practice, Management \\
\hline \hline "Freeze" intermediate artifact & Awareness & Management \\
\hline Attach initial evaluation & Awareness & Theory \\
\hline Contact information & Awareness & Theory, Roles \\
\hline Correct your own mistakes & Awareness & Roles, Management, Theory \\
\hline Disable anonymity & Awareness & Management, Adaptation \\
\hline Discuss selected results & Awareness & Adaptation, Management \\
\hline Establish work protocols and tools & Awareness & Theory, Management, Roles \\
\hline Explicit rationale & Awareness & Theory, Management \\
\hline Explicit rationale in a debate & Awareness & Theory, Management \\
\hline Formative assessment/Feedback & Awareness & Design, Theory \\
\hline
\end{tabular}


Table B.1 (continued from previous page)

\begin{tabular}{|c|c|c|}
\hline Atomic pattern & Primary aspect & Secondary aspect(s) \\
\hline Group space & Awareness & Theory, Design \\
\hline Individual test/Exam & Awareness & Design, Practice \\
\hline Make monitoring explicit & Awareness & Theory \\
\hline Meta-reflection & Awareness & Theory, Design \\
\hline Monitoring the task & Awareness & \\
\hline Monitoring web browser & Awareness & Practice, Synergy \\
\hline On -the- fly assessment & Awareness & Adaptation, Management \\
\hline On-the-fly peer assessment & Awareness & Design, Roles \\
\hline Oral presentation & Awareness & Design \\
\hline Peer review & Awareness & Roles, Design \\
\hline Prevent idea copying & Awareness & Management, Practice \\
\hline Probe with false information & Awareness & Management, Theory \\
\hline $\begin{array}{l}\text { Provide rubrics at the beginning of the } \\
\text { course/experience }\end{array}$ & Awareness & Theory, Design \\
\hline Provide students with rubrics & Awareness & Theory, Design \\
\hline Publish group decision & Awareness & Theory \\
\hline Publish partial products & Awareness & Theory, Management \\
\hline Questioning randomly during evaluation & Awareness & Management, Practice \\
\hline Questionnaire & Awareness & Design \\
\hline $\begin{array}{l}\text { Questionnaire to get structured/aggregated in- } \\
\text { formation }\end{array}$ & Awareness & Adaptation, Design \\
\hline Quick look at the results & Awareness & Adaptation \\
\hline Random evaluation of group work & Awareness & Practice, Management \\
\hline Solve doubts & Awareness & Practice \\
\hline Student revises the result in the whiteboard & Awareness & Management, Adaptation, Roles \\
\hline Students detect mistakes & Awareness & Management, Practice, Roles \\
\hline Submit generated artifact & Awareness & Practice \\
\hline Synthesis & Awareness & Design, Theory \\
\hline Take and publish notes & Awareness & Roles, Theory, Practice \\
\hline Task evaluation & Awareness & Design, Management \\
\hline Teacher as participant in student groups & Awareness & Practice, Roles, Design \\
\hline $\begin{array}{l}\text { Use different colors of post-it notes to distin- } \\
\text { guish teams }\end{array}$ & Awareness & Practice, Management \\
\hline Use ICTs for real-time monitoring & Awareness & Synergy, Design \\
\hline $\begin{array}{l}\text { Use shared whiteboard to make student con- } \\
\text { cepts explicit }\end{array}$ & Awareness & Synergy, Adaptation \\
\hline Use student opinions as base for debate & Awareness & Adaptation \\
\hline Use whiteboard to exemplify & Awareness & Management, Synergy \\
\hline Use wiki to give feedback & Awareness & Synergy, Design, Practice \\
\hline Use wiki to track group progress & Awareness & Design, Practice \\
\hline Wander and solve doubts & Awareness & Practice, Management \\
\hline Written feedback on the same artifact & Awareness & Synergy \\
\hline Bibliographical search & Design & \\
\hline Brainstorming & Design & Management, Awareness \\
\hline Calibrate task difficulty according to student level & Design & Adaptation, Management, Practice \\
\hline Choose research topic & Design & Adaptation, Roles \\
\hline Classification & Design & Management, Awareness \\
\hline Clues & Design & Management \\
\hline Collaborative enigma & Design & Management, Awareness \\
\hline Conceptual mapping & Design & Management, Awareness \\
\hline Conceptual mapping & Design & Awareness \\
\hline Conflict* & Design & Management, Awareness \\
\hline Debate/Discussion & Design & Awareness, Theory \\
\hline Demonstration & Design & Practice, Management \\
\hline Discussion synthesis & Design & Roles, Adaptation, Theory \\
\hline Distributed problem solving & Design & Management, Awareness \\
\hline $\begin{array}{l}\text { Explicit connection between activity and learn- } \\
\text { ing objectives }\end{array}$ & Design & Practice, Management, Theory \\
\hline $\begin{array}{l}\text { Explicit relationship between discussion and } \\
\text { course concepts }\end{array}$ & Design & Practice, Management, Theory \\
\hline Jigsaw & Design & Management, Theory \\
\hline
\end{tabular}


Table B.1 (continued from previous page)

\begin{tabular}{|c|c|c|}
\hline Atomic pattern & Primary aspect & Secondary aspect(s) \\
\hline Jigsaw* & Design & Management, Awareness \\
\hline Lab group work & Design & Practice \\
\hline Large group deliverable timeline & Design & Management, Adaptation, Practice \\
\hline Make a report & Design & Awareness, Practice \\
\hline Make references to the students" "real life" & Design & Practice \\
\hline Meta-evaluation & Design & Theory, Awareness \\
\hline Meta-reflection & Design & Theory, Roles \\
\hline Peer review using Moodle Workshop & Design & Management, Synergy \\
\hline Prepare a presentation & Design & Awareness \\
\hline Prepare backup materials & Design & Management, Practice \\
\hline Propose research problems/challenges & Design & Adaptation, Roles \\
\hline Pyramid & Design & Management \\
\hline Question/representation of information & Design & Management, Awareness \\
\hline Quiz show in teams & Design & Management \\
\hline Reciprocity* & Design & Management, Awareness \\
\hline Reuse artifact as template & Design & Management, Synergy \\
\hline Reuse generated artifacts & Design & Management, Synergy \\
\hline Running gag & Design & Management, Roles \\
\hline Strategies to group formation & Design & Adaptation, Management \\
\hline Support the task with non-ICT resources & Design & Practice, Synergy \\
\hline Synthesis of existing resources & Design & Practice \\
\hline Synthesis/comparison among presentations & Design & Theory, Awareness \\
\hline Teacher chooses tools & Design & Management, Synergy, Roles \\
\hline Teacher determines group formation & Design & Roles, Management, Adaptation \\
\hline Unregulated activity & Design & Roles, Management, Practice \\
\hline Use .LRN to structure activities & Design & Synergy, Management \\
\hline Use graphics to support a task & Design & Practice, Awareness \\
\hline Use pre-existing artifacts & Design & Management \\
\hline Use results of a task in a different task & Design & Management, Synergy \\
\hline Use wiki to structure activities & Design & Management, Practice \\
\hline Use/Read peer artifacts & Design & Roles, Theory \\
\hline Where is on the image? & Design & Management, Awareness \\
\hline 1:1 computer use & Management & Design \\
\hline Aggregate several artifacts into one & Management & Synergy \\
\hline Control the order of the group tasks & Management & \\
\hline Coordination through email & Management & Synergy, Practice \\
\hline Emphasize collaboration rules & Management & Practice, Awareness \\
\hline Establish rules and routines & Management & Practice \\
\hline Explanation of activity objectives & Management & Design, Practice \\
\hline Form groups & Management & Theory, Practice \\
\hline Free activity flow & Management & Roles, Theory, Practice, Design \\
\hline Group computer use & Management & Design \\
\hline Include an activity in the final score & Management & Awareness, Design \\
\hline Insert mini-lecture in a debate & Management & Roles, Practice \\
\hline $\begin{array}{l}\text { Make an activity face-to-face to ensure interac- } \\
\text { tion }\end{array}$ & Management & Design, Practice \\
\hline Mark a temporal limit & Management & Practice \\
\hline Mediate in collaborative tasks & Management & Practice, Adaptation \\
\hline Prevent tools usage & Management & Practice \\
\hline Punishment & Management & Adaptation, Practice \\
\hline Remind about debate rules & Management & Practice \\
\hline Step by step & Management & Practice \\
\hline Submission via email & Management & Synergy, Awareness \\
\hline Summarize & Management & Design, Practice \\
\hline Time management alerts & Management & Design, Adaptation, Practice \\
\hline Use a group as example of a general problem & Management & Awareness \\
\hline Use pre-existing groups & Management & Design \\
\hline Use projector and script to support explanation & Management & Awareness, Practice, Synergy \\
\hline Use tablets in couples & Management & Design \\
\hline Use wiki to structure a presentation/session & Management & Synergy, Design, Practice \\
\hline
\end{tabular}


Table B.1 (continued from previous page)

\begin{tabular}{|c|c|c|}
\hline Atomic pattern & Primary aspect & Secondary aspect(s) \\
\hline Voting/poll & Management & Design, Awareness, Adaptation \\
\hline Anticipate script & Practice & Management \\
\hline Backup ICT infrastructure & Practice & Management, Design, Adaptation \\
\hline Centralized file repository & Practice & Management, Design \\
\hline $\begin{array}{l}\text { Distribute participants physically to facilitate } \\
\text { interaction }\end{array}$ & Practice & Management, Synergy \\
\hline Divide and conquer & Practice & Adaptation, Design, Management \\
\hline Introduction to the subject/experience & Practice & Design \\
\hline Lesson/Practice about specific tool usage & Practice & Management, Design \\
\hline $\begin{array}{l}\text { Link different learning environments in the } \\
\text { course }\end{array}$ & Practice & Awareness, Management \\
\hline Mention course routines & Practice & Management \\
\hline Provide tool usage guide/manual & Practice & Management, Design \\
\hline Revise related concepts & Practice & Management, Design \\
\hline Solve technological infrastructure problems & Practice & Adaptation \\
\hline Summarize learned concepts & Practice & Management \\
\hline Take a break in the session & Practice & \\
\hline Test task to exemplify tool use & Practice & Design \\
\hline Tips and tricks in tool usage & Practice & Design \\
\hline Assign management roles to students & Roles & Management \\
\hline Guest speaker & Roles & Theory, Design \\
\hline Peer helping & Roles & Theory \\
\hline Students choose their roles in a group & Roles & Management \\
\hline Students choose their tools & Roles & Management, Synergy \\
\hline Students choose their work topic & Roles & Management, Adaptation \\
\hline Students determine group formation & Roles & Management \\
\hline Teacher as moderator & Roles & Management \\
\hline Display artifact during the discussion & Synergy & Practice, Management \\
\hline Use ICT for persistence of intermediate artifacts & Synergy & Design, Awareness \\
\hline Use IWB to trace conversation/debate & Synergy & Awareness, Practice \\
\hline Use IWB/projector to support explanation & Synergy & Awareness, Practice \\
\hline Use Moodle to automate submissions & Synergy & Management, Practice, Design \\
\hline Use open-access ICT to do peer review & Synergy & Awareness, Design \\
\hline Use projector/IWB to exemplify tool usage & Synergy & Awareness, Practice \\
\hline Use spreadsheet to create rubrics & Synergy & Awareness, Practice \\
\hline $\begin{array}{l}\text { Use traditional blackboard to show static infor- } \\
\text { mation }\end{array}$ & Synergy & Practice \\
\hline Use wiki to do collaborative writing & Synergy & Design \\
\hline Use wireless mouse as a remote & Synergy & Practice, Management \\
\hline Democratic reward assignment & Theory & Management \\
\hline Equal rewards to all teams & Theory & Management \\
\hline
\end{tabular}




\title{
Appendix $\mathrm{C}$
}

\section{An analysis of existing Learning Design languages and learning platforms}

\begin{abstract}
Summary: This appendix contains a more detailed depiction of the analysis that was performed during the dissertation, in order to ascertain the most common features of both widespread learning design languages, and widespread learning platforms such as Virtual Learning Environments (VLEs). This analysis concludes with several remarks that originated the structure of the GLUE!PS architecture and data model, as they have been presented in Chapter 5.
\end{abstract}

\section{C.1. Analysis of Learning Design languages}

The design of learning situations (understood widely as the preparation of instructional materials, activities, information resources and/or evaluation), not only is a crucial step in the orchestration of such situations, but it has been present in education, in one form or another, for ages. In modern times, educational science has proposed slightly different flavors and methodologies of design (e.g. instructional design [Mer94], learning design [Lau02] ...), with varying degrees of acceptance by the practitioners. With the advent of ICT, distance learning and e-Learning, the dream of being able to model computationally, automatically execute and reuse learning situations has been driving many research and standardization efforts [Kop05.

However, despite all these efforts, learning design, in the sense of computationallyexpressed, reusable designs, as promoted by the IMS Consortium, is not widely used outside large institutions specialized in distance learning. For many practitioners the design of learning situations is restricted to aide memoires scribbled in a notebook, or high-level lesson plans required by educative administrations, while in other cases it is not even in written form, and it lives on the teacher's head. One of the main reasons behind the lack of learning design adoption in the teacher community is the scarcity of usable learning design tools [Neu10]. Even if there are learning design tools that try to overcome this limitation (such as Collage [HL06a]), an even bigger obstacle lies in the setup and execution of learning designs, which currently is restricted to the few learning environments that provide scripting support (either through IMSLD or proprietary mechanisms), or to IMS-LD players that are not integrated in the learning 
environment, thus posing the same problems as the usage of external tools for learning, that the GLUE! architecture tries to solve. These problems not only are hampering the adoption of learning design in everyday teaching practice, but also of technology-enhanced learning in general (because of time and complexity constraints of most real educational scenarios preclude experimental or time-consuming practices).

Thus, the current situation is that, a) even if there is a de facto standard for learning design (IMS-LD IMS03a]), it is not widely used and it has often been criticized, specially because of its complexity and the difficulties of its setup and execution (see, e.g. Cae03 (Mia05); b) there exist a good number of learning design tools, with different degrees of usability by non-experts, but they use different underlying languages to express learning designs; and c) there is a majority of practitioners that use informal and/or non-computational learning design languages, either for their own use or for administrative purposes. The proposed GLUE!-PS architecture attempts to overcome these limitations: its adapter architecture would allow for the usage of already existing IMS-LD designs (labeled Units of Learning, or UoL), plus it would allow users of other learning design languages and tools to setup their activities in a variety of learning environments. It would even enable the apparition of new learning design tools based in the current widely used but rarely exploited informal learning design languages, increasing their expected benefits from the start, by allowing for the deployment of those designs in a variety of learning environments.

In this section we will review some of the main learning design languages and specifications, in search of trends and common concepts that can help us in defining the GLUE!-PS data model. According to the requirements outlined in the previous sections, we have selected design languages considering acceptance in the community, also prioritizing those that veer towards teacher-centrism (as opposed to those requiring deep technical knowledge). This includes mainly the IMS-LD specification, but also other general-purpose learning design languages (LAMS, LDL, LAS, SCY-SE, CompendiumLD), as well as a few examples of informal design languages, so as to remain as close as possible to actual learning design practice in a variety of situations. Several learning design languages, especially graphical languages, have been proposed in literature, such as Collage HL06a or MoCoLADe Har09]. However, since they are able to export their data into IMS-LD format, for now we will not analyze them, considering them as "under the IMS-LD umbrella".

\section{C.1.1. IMS Learning Design}

The IMS-LD specification IMS03a is, with all probability, the most widely used computerinterpretable learning design language. The specification was created by the IMS Global Learning Consortium with the aim of "supporting pedagogical diversity and innovation, while promoting the exchange and interoperability of e-learning materials". Currently, the specification is supported by a few VLEs such as .LRN (with the GRAIL engine), as well as a number of IMS-LD "player" tools that support the execution of IMS-LD sequences (including the GUI-less reference engine implementation, CopperCore, and other players that incorporate a user interface, such as CopperCore Player, OpeNET LD, SLeD or the Reload LD Player). There also exist a number of LD authoring tools, including CopperAuthor, Reload LD, ASK LDT, Collage, ReCourse, CoSMoS, MOT+ and Prolix GLM. 


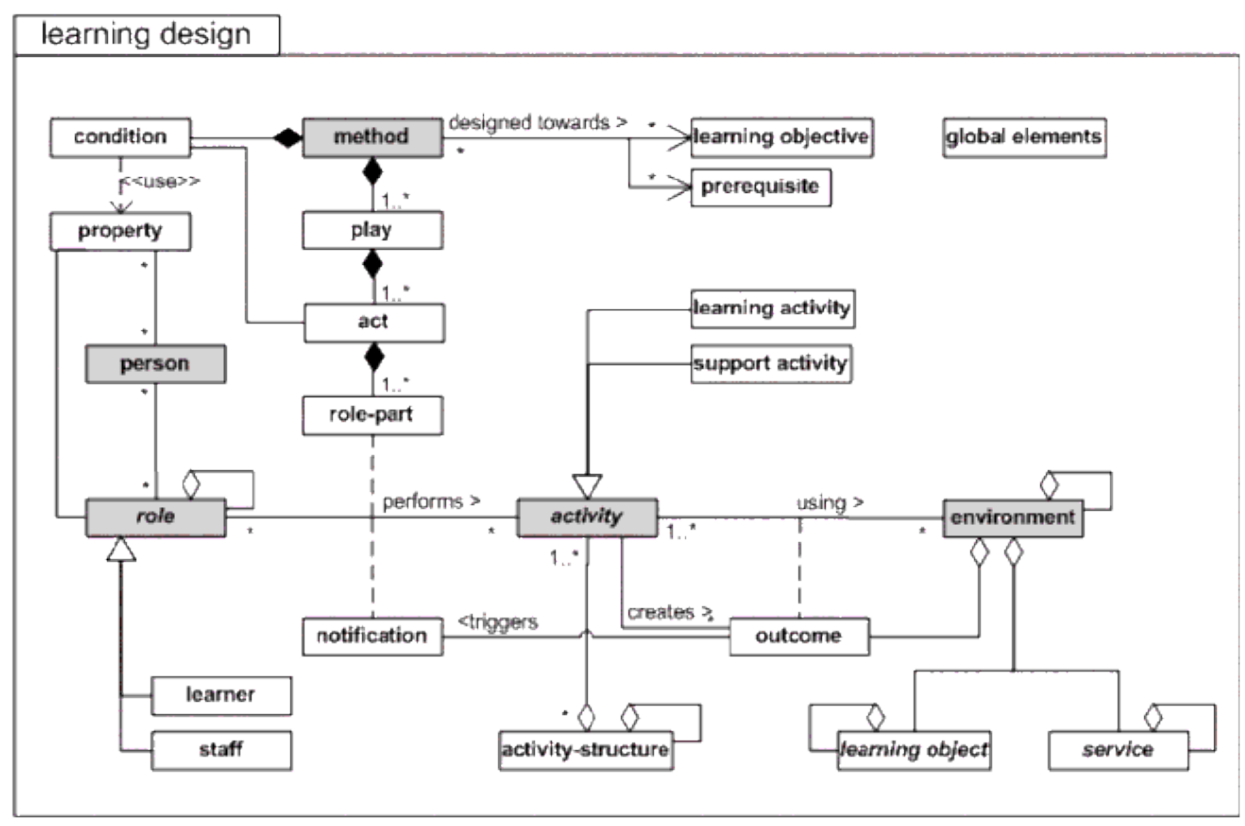

Figure C.1: IMS-LD metamodel, taken from IMS03a

IMS-LD is based around the idea of an Educational Modeling Language, a 'meta-language' trying to capture learning design practice, and which, in the end boils down to: "a Method prescribing various Activities for learner and staff Roles in a certain order. Each activity refers to a collection of specific objects and services (called the 'Environment') needed to perform the activity. In order to support the description of individualized learning designs, learner Properties, Conditions, and Notifications are needed". The IMS-LD specification structures activities around a theatrical metaphor, with plays, acts and role-parts to give structure to the learning activities. This general meta-model (see figure C.1) is made concrete throughout the specification, and it is finally expressed in what is called a "Unit of Learning" (UoL), which basically consists on a XML file and a number of resources referenced in it.

It is also important to note that IMS-LD defines 3 levels of compliance, of increasing complexity: Level A provides the basic concepts (method, activities, roles, environments), while level B provides properties and conditions (for more elaborate sequences and interactions), and level $\mathrm{C}$ adds notifications on top of that.

As it has already been pointed out, IMS-LD has been criticized for its complexity of implementation, lacking features in managing complex (sp. highly parallel) activity flows and a long etcetera [Cae03] Mia05 dlFV07] [Dod09 [Neu10. These factors, as well as its original conception as a way for modeling mainly online learning courses, explain its scarce adoption outside of large institutions specialized in online learning ([Jan05] provides an interesting depiction of the complexity of implementing IMS-LD in a real institution). 


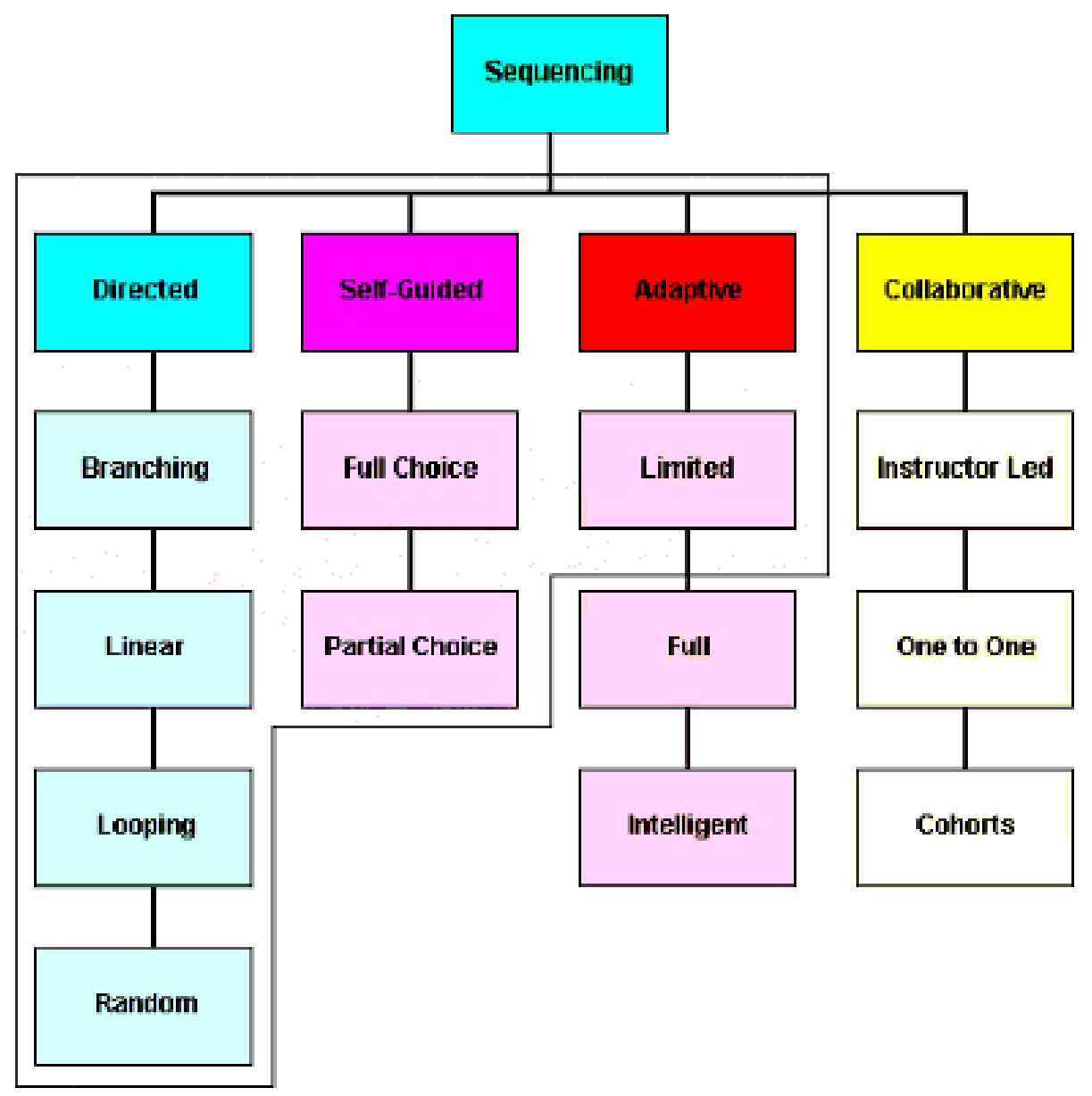

Figure C.2: IMS-SS sequencing modes, taken from [IMS03b]

\section{Similar, but not quite the same: IMS Simple Sequencing}

As an aside note, there is another IMS specification which speaks about the sequencing of learning activities: the Simple Sequencing specification (IMS-SS [IMS03b]). This specification is used to declare the relative order in which electronic learning activities are to be presented to a learner, and to decide which contents are delivered during presentation. The specification relies on a rather limited concept of learning activity as "a pedagogically neutral unit of instruction, knowledge, assessment, etc. It can have sub-activities and may be nested to an arbitrarily deep level. Each activity may have a tracking status associated for each learner that is assigned to experience the activity. Activities can be attempted any number of times [...] can be suspended, abandoned, exited normally, etc." IMS03c]. Figure C.2 shows the available sequencing modes in IMS-SS.

In IMS-SS, each activity has a number of objectives (typically used to record test scores). Also activities may include additional resources (e.g. glossaries, context sensitive services...). The sequencing is defined through a (rather complex) set of sequencing rules and limit conditions. At run-time, the sequencing is done by combining the definition information model (basically, 
an IMS-CP XML file defining an activity tree plus the sequencing instructions) and the tracking information (which is collected as the learner works with and completes the activities).

The main limitation of IMS-SS with respect to IMS-LD is that it does not consider multiuser activities (as needed e.g. in collaborative activities). In fact, IMS-SS has a underlying pedagogical model reminiscent of the classic behaviorist instructional design Gag05, which is often dismissed by modern pedagogical approaches as too simplistic.

IMS-SS is an extension to the IMS Content Packaging specification [IMS07]. There is no clear list of learning environments that support this specification. Since it is a IMS Content Packaging extension, the compliance with IMS-CP does not automatically mean that IMS-SS is supported. Seemingly, the ADL SCORM 2004 implementations are based on IMS-SS with further refinement:1. Also, the Icodeon platform ${ }^{2}$ has released an engine implementing IMS-SS.

\section{C.1.2. LAMS}

The Learning Activity Management system (LAMS ${ }^{3}$ ) is a learning environment "inspired $[\ldots]$ in EML and the IMS-LD specification", which provides functionality "for user administration, student run-time delivery of sequences, teacher run-time monitoring of student sequences and, most importantly, teacher authoring/adaptation of sequences" Dal03. It is in this aspect as a learning design/authoring tool that we will talk about LAMS here. Its capabilities as a VLE will be discussed alongside other VLEs in the next report.

In a similar vein to IMS-LD, LAMS designs are pedagogically neutral, and revolve around what they call a Graphic Workflow Model, a sequence of activities chosen from a limited palette of tools/activity types: questions, polls, discussion forum, chat, noticeboard, shared resources, journal, assessment, and even offline activities (not run on the computer). Learning designs (called sequences in LAMS speech) are created by dragging and dropping activities onto the learning space, and then creating transitions, optional elements or gates/branches to specify the path that learners take through the sequences. The activities also support grouping, and each activity can be used in either whole class (the default mode for the tools) or small group modes.

\section{C.1.3. LDL}

The Learning Design Language (LDL $[\mathrm{Mar06a}]$ ) is a meta-model proposal for learning design, competing with IMS-LD. Contrary to IMS-LD (which claims to be pedagogically neutral), LDL is proposed to address the peculiar challenges of collaborative learning activities. Moreover, it is claimed that LDL is teacher-centered, allowing teachers to design and describe activities (as opposed to specialized instructional designers to which IMS-LD seems to be directed given its complexity).

LDL does not conceive learning activities as a sequence of steps or a hierarchical tree, but rather as a set of exchanges between participants, occurring in a pedagogical context (contents,

\footnotetext{
${ }^{1}$ According to a JISC Briefing Paper on IMS-SS, http://www.icodeon.com/pdf/ss2brief.pdf. At the time of this writing, the ADL website was down.

2 http://www.icodeon.com

3 http://www . lamsinternational .com/
} 


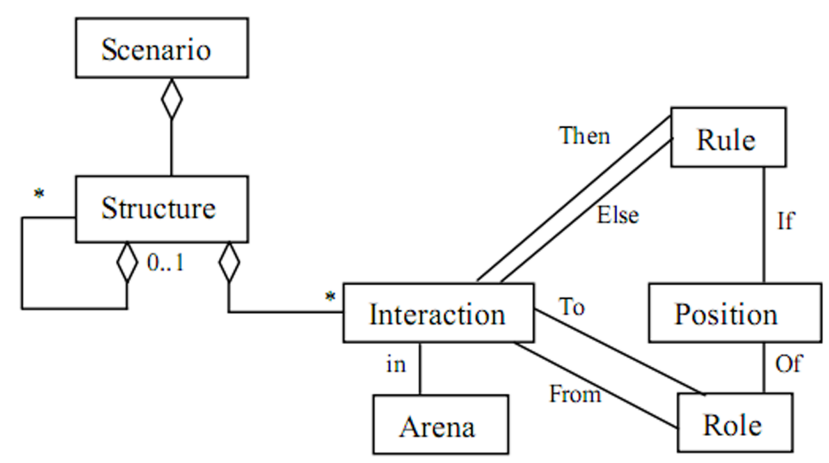

Figure C.3: LDL simplified meta-model, taken from Mar06a

objectives, rules). Activities may also have dependencies among them. In the LDL philosophy, the nature and unfolding of the activity is largely unexpected, and teachers should have the means to modify this context dynamically. The (collaborative) learning situations are also classified around a few canonical situations (frontal or independent, open or free, and collective).

LDL considers that scenarios (similar to what we have called "abstract designs") have to be operationalized in order to be deployed in a learning environment. This operationalization includes choosing the participants, attributing roles to them, and selecting the services and contents required by the scenario.

The LDL meta-model is organized around these scenarios, in which the main element is the concept of interactions and their structure (how the exchanges are to be organized, e.g. in a sequence). Once the interactions are clear, the roles involved are defined, as well as the arenas (i.e. the pedagogical contexts mentioned above, e.g. specific contents or services) where they will take place. The rules that will govern the interactions are then defined and, finally, each role's position (point of view) is described. This schema is depicted in Figure C.3.

LDL also considers other more esoteric notions such as positions (rather ill-defined, including participants' perceptions, reactions, availability, value... of an activity) and observables (things that can be observed, such as interaction state, activity progress, etc). These notions try to address the challenge of observing and subsequently modifying the scenario as it unfolds (which is also one of the main tenets of what we have called "orchestrating learning").

In general, this learning design approach, albeit interesting, has not been widely implemented (although Mar06a mentions a Learning Design Infrastructure for its deployment and execution). Also the learning design tools that would be used to create such designs were not clearly specified.

\section{C.1.4. LAS (SCY-SE)}

In a similar conceptual strand as LDL is the SCY (Science Created by You) project 4 which aims to "build a flexible, open-ended [science] learning environment that truly engages and empowers adolescent learners". In order to facilitate the design of these constructivist science

${ }^{4}$ http://www.scy-net.eu/ 


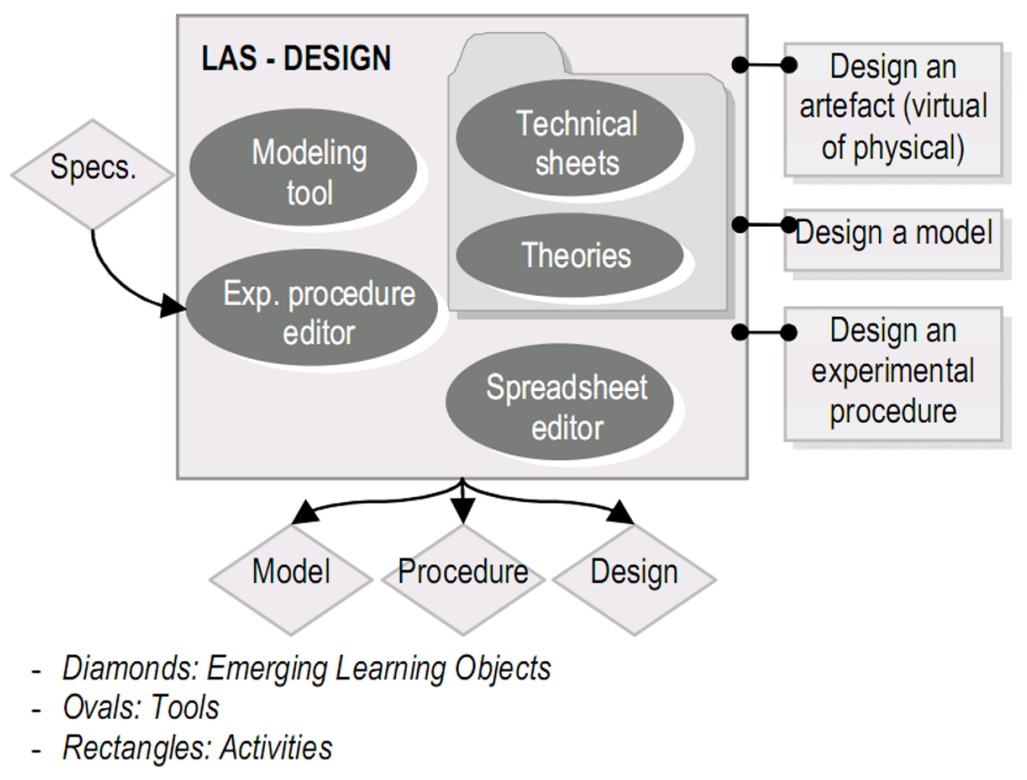

Figure C.4: "Design" LAS example, taken from Lej09b

learning scenarios, the project proposes a SCY Scenario Editor (SCY-SE), based on what they call Learning Activity Spaces (LAS Lej09b]), and its graphical representation [Lej09a, as an alternative to IMS-LD that is flexible, interoperable with existing platforms and contents, while maintaining pedagogical neutrality and usability for practitioners.

LAS is defined as "a coherent and intuitive set of activities supported with specific tools and scaffolds". These LASs are described in terms of what they call "Emerging Learning Objects" (ELOs), artifacts created by students. Thus, a learning scenario can be defined as a collection of LASs and the various learning paths between them. The main concepts involved in LASs are activities, ELOs, tools and scaffolds. Each activity requires an input in the form of a learning object or ELO from a previous LAS. Also, each activity results in at least one ELO. Activities are supported by tools, which may or may not have scaffolding characteristics. Figure C.4 shows an example LAS, showing its main components.

The (graphical) design language models scenarios at three levels of abstraction: the $L A S$ (composed by activities, ELOs and tools). LASs are related to one another to form a scenario, with the addition of other components that define the learning setting: individual vs. collaborative nature, location, technological support etc. Once this scenario has been contextualized, it becomes a mission that learners may undertake (i.e. execute). It is important to note that this approach does not prescribe a linear sequence of activities, but rather provide a LAS with a number of tools and scaffolds that are available to students at any time while they are in the LAS. Also, further flexibility is achieved by activities coming with alternative assignments.

As far as we know, the SCY project only provides functionality for design of scenarios, although support for configuration, adaptation and monitorization of scenarios is expected in the future. 


\section{C.1.5. CompendiumLD}

CompendiumLD ${ }^{5}$ is a design tool developed by the Learning Design project at the Open University, in order to support course design process. Basically, CompendiumLD intends to be a visualization tool for learning design, which is based around a previous mindmapping application (Compendium), thus providing a "flexible visual interface managing the connections between information and ideas" Bra08].

CompendiumLD was developed in a large institution such as OU, specialized in online learning, upon the realization that developing a formal LD specification was a difficult endeavor, and that different individuals saw the design process in very different ways. CompendiumLD intends to be a simple and flexible mind tool that helps in the "messy process" of learning design.

From a purely technical perspective, CompendiumLD is a mindmapping tool that basically provides three kinds of entities: nodes, links between nodes and additional attributes/properties for a node. However, CompendiumLD proposes a number of learning-related entities (basically, node types with different attributes), which can be flexibly combined and linked with each other, to represent activities and its components: assignments, outputs/outcomes, resources, roles, tasks and tools. Other components are also available, such as conditional forks, activity templates, approaches to design, etc. All these LD-specific components have a name, and a number of special attributes (e.g. for a tool, the kind of tool such as a wiki) in order to further detail the learning designs. It is worth noting that activities (and their components) can be nested within other activities (in a sort of an activity tree), thus allowing to define activities at various levels of granularity. Figure C.5 shows an example of an activity modeled with CompendiumLD.

\section{C.1.6. Ad-hoc, non-computational languages}

As a way to show current learning design examples in everyday teaching practice, we will provide here the schema of the lesson planification for a real course at the University of Valladolid (Spain). This is just an isolated example, and by itself it does not provide evidence of what the practitioners do in terms of learning design. In fact, probably there is no such thing as "what practitioners do". However, I think that this example illustrates current learning design practices that could be utilized to promote the adoption of learning design and TEL, just by providing semi-automated means of deployment and management of the execution of these very basic designs (thus making useful an effort only done before for administrative purposes, which may not even be followed in the real lessons).

\section{The MUITIC case: a graduate program for research in ICT}

The "ICT Research Master" 6 (MUITIC being the Spanish acronym) at the University of Valladolid is a graduate level course within the new European Space for Higher Education (ESHE), which aims towards the acquisition of a solid methodological, scientific and technological base, in the field of ICT. This master follows a blended learning approach, involving

5 http://compendiumld.open.ac.uk/

6 http://muitic.tel.uva.es/ 


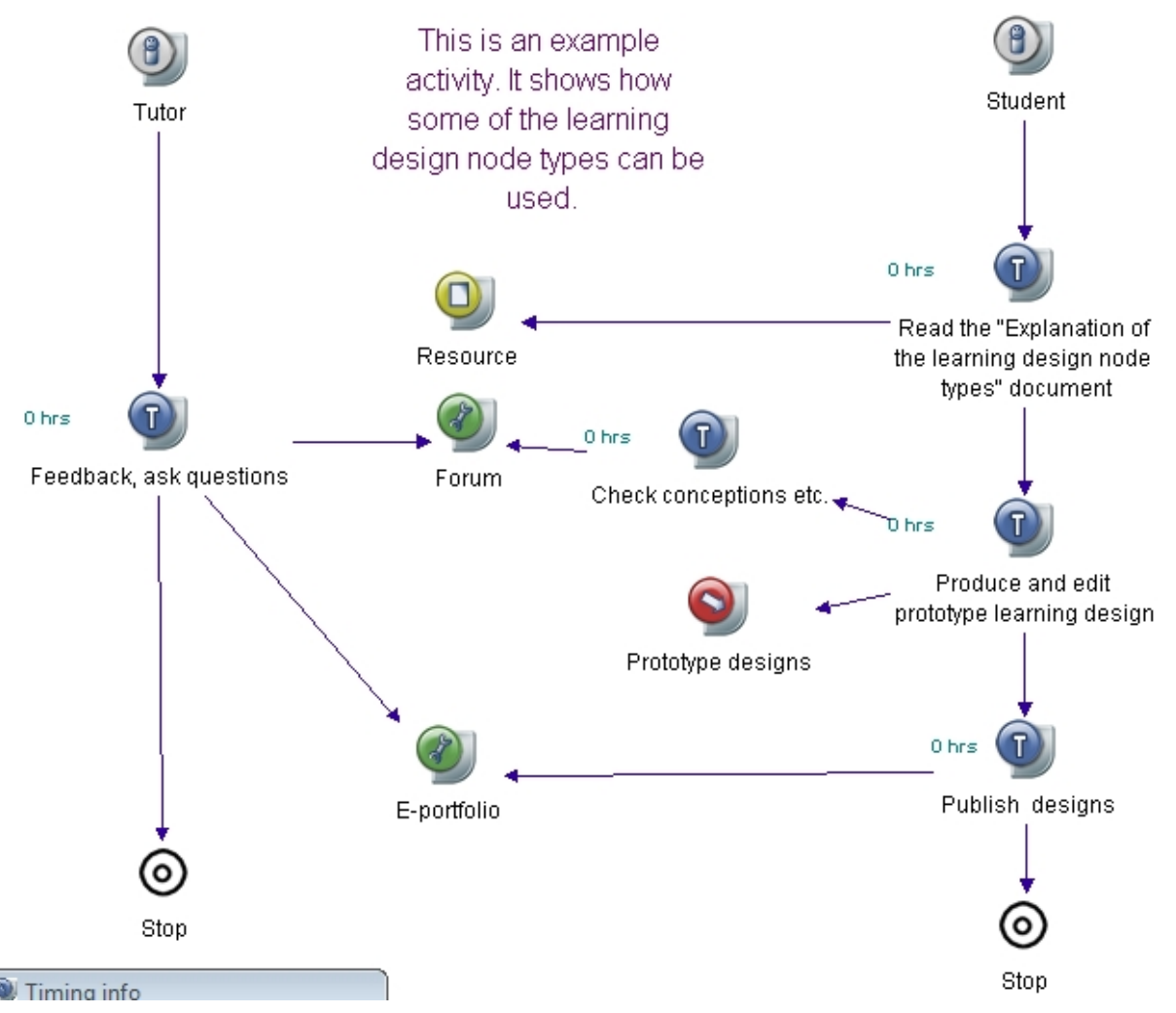

Figure C.5: Example activity in CompendiumLD 
\begin{tabular}{|l|l|}
\hline Denominación de la asignatura & TECNOLOGIAS EMERGENTES EN SISTEMAS TELEMÁTICOS \\
\hline
\end{tabular}

Atención: El plan de trabajo que aquí se presenta es una planificación orientativa de la asignatura. Si bien el objetivo es seguir lo más fielmente posible dicha planificación, no debe entenderse como algo totalmente cerrado e inflexible, sino que puede modificarse y adaptarse si las circunstancias asi lo requieren.

\begin{tabular}{|c|c|l|l|}
\hline $\begin{array}{c}\text { Sesión } \\
\text { presencial }\end{array}$ & Fecha/Horas & \multicolumn{1}{|c|}{ Actividad en la sesión } & \multicolumn{1}{c|}{$\begin{array}{c}\text { Otra información } \\
\text { y fechas límite }\end{array}$} \\
\hline 1 & $\begin{array}{c}19 \text { Oct. } \\
(19: 00-21: 00)\end{array}$ & $\begin{array}{l}\text { Presentación Asignatura } \\
\text { Presentación participantes } \\
\text { Debate inicial: ¿Qué es la Web 2.0 y la } \\
\text { Web semántica? }\end{array}$ & $\begin{array}{l}\text { Cuestionario de experiencia } \\
\text { previa y expectativas } \\
\text { Lecturas iniciales y } \\
\text { formulario de contenido }\end{array}$ \\
\hline 2 & $\begin{array}{l}21 \text { Oct. } \\
(16: 00-19: 00)\end{array}$ & $\begin{array}{l}\text { Debate sobre lecturas iniciales. } \\
\text { ldentificación y justificación de temas a } \\
\text { tratar en la asignatura. }\end{array}$ & Lecturas sobre Web 2.0 \\
\hline 3 & $\begin{array}{l}26 \text { Oct. } \\
\text { Debate sobre lecturas Web 2.0. } \\
\text { Presentación de formatos de } \\
\text { agregación de contenidos (RSS/Atom) }\end{array}$ & Lecturas sobre "Mash-up" \\
\hline 4 & $\begin{array}{l}28 \text { Oct. } \\
(16: 00-19: 00)\end{array}$ & $\begin{array}{l}\text { Debate sobre lecturas "Mash-up" } \\
\text { Reparto de tareas: herramientas para } \\
\text { la creación de "Mash-up" }\end{array}$ & $\begin{array}{l}\text { Documentación y pruebas } \\
\text { de herramientas de "mash- } \\
\text { up" especifica } \\
\text { Elaboración de presentación }\end{array}$ \\
\hline- & 2 Nov. & . . . . . & $\begin{array}{l}\text { Documentación y pruebas } \\
\text { de herramientas de "mash- }\end{array}$ \\
\hline
\end{tabular}

Figure C.6: Example of MUITIC integrated working plan

both face-to-face and online activities. The master's steering committee establishes that, for each subject within the course, a "teaching guide" and a "integrated working plan" have to be provided by the corresponding teacher(s). As the reader may have guessed, the elaboration of these guides an plans requires an effort in learning design, even if at a very high level. In this case, these designs have to be expressed in the following non-computational language:

Teaching guides are formatted as table-like forms, detailing first a number of general information parameters about the subject (name, workload, language, teachers etc). Then, the educational context of the subject is established (including pre-requisites, relationships with other subjects, workload and presentiality), and especially the general and specific competences to be acquired (i.e. the expected learning outcomes) and its general goals. Furthermore, the different content units of the subject are detailed (context, learning goals, summary of the contents, bibliography, learning methodology and assessment), as well as their (high-level) temporization. The reader is asked to refer to the working plan for a detail of the learning activities.

Integrated working plans are also formatted as tables, which detail the face-to-face sessions to be held, their timing (date/time), and an overview of the activities to be held during the session (just the title). Furthermore, there is a field for additional information, which seems to be used to account for the online activities to be performed between face-to-face sessions (the face-to-face session being a sort of deadline for those activities). Figure C.6 shows an excerpt of one of these working plans. 


\section{C.2. Discussion: common trends in the analyzed Learning De- sign languages}

As we can see from the sampler in the previous section, there is a wide variety of learning design languages, from very expressive and detailed languages to flexible and loosely defined ones. However, we can also detect a number of common trends among many, if not all the analyzed languages:

- All the design languages sport, in one way or another, the notion of activity, often as the central concept in the language. Although it varies from language to language, some common characteristics of these learning design activities is that they are performed by one or more participants (persons or groups), using a number of tools, resources or services. This pre-eminence of activity over e.g. content is a hallmark of modern pedagogical approaches and theories, such as Activity Theory [Eng99] and other constructivist pedagogies.

- This concept of activity has a number of common properties, such as learning goals (or objectives), rules or instructions for its completion, as well as the production of outputs, intermediate artifacts that may be reused in later activities.

- Another common feature is the activity structure, normally in the sense that activities can be nested into one another, in arbitrarily deep tree-like structures.

- Many learning design languages define sequences of activities (again, this may also include nested sequences inside sequences), or other timing criteria for the execution of the activities. The mechanisms and level of enforcement of these sequences varies greatly from language to language (due to differences in the pedagogical approach).

- Another important aspect of the activities is its social structure (i.e. whether they are performed individually, in groups, and which roles each participant plays). At least, the concepts of person and group seem unavoidable. The usage of the concept of roles is more contested, since it appears almost exclusively in collaborative learning activities (as opposed to other pedagogical approaches).

- Other common features of many of the analyzed languages is the presence of a higher-level container for the activities (be it in the form of IMS-LD's Unit of Learning, or SCY-SE and LDL's Scenarios). However, it is not clear if this kind of container is not just a higher-level activity. A similar concept is that of environment, sometimes used as a wrapping concept around the tools, services and resources that are used in an activity.

- Some of the languages reviewed also provide some sort of activity templates (such as SCYSE's LASs, or LAMS's activity types), in order to provide some basic scaffolding structure (e.g. usual combinations of tools) over the extremely malleable concept of activity. A different kind of "templating" can be found in Collage [HL06a], which provides templates of activity structures (in IMS-LD speech, Methods).

It is worth noting that the purpose of many of these learning design languages is to create abstract designs that can be reused across different educational scenarios. However, in our case 
we aim to contextualize those designs and reify them in a concrete web learning environment including external tools. Thus, we should expect our "lingua franca" to include concepts that are not present in any of the learning design languages. Specially, the concept of instances (either of persons, groups or tools that they use) appears to be one of these additional concepts to take into account.

Also, this centrality of the concept of activity (defined as a structured set of data representing a human activity, including its goal, the people that participate in it, the services and resources to be used for the activity and other data about the activity and its state) connects (at least conceptually) with the "activity-centered computing" approach Bar05 in the field of computer-supported cooperative work (CSCW). It is too soon to assert that the technological solutions found in that area will be reusable or adaptable to our domain, but we may certainly look there for inspiration.

\section{C.3. Learning environments and Learning Design scripting}

In this section we will analyze some of the main learning environments for blended learning (specially the so-called VLEs). This includes, for example, the Moodle, LAMS and Dokeos VLEs, but also other pieces of software that can be used as the center of a blended learning environment, such as the MediaWiki software for collaborative writing or the Elgg social networking platform. We understand that a learning environment is in this context any piece of software that can serve to centralize learning activities, even if not all of them happen inside that piece of software (e.g. using Moodle as the access point for activities involving both a Moodle forum and a Google Docs document). Given the problem that we are trying to solve (going from learning designs to its enactment on a concrete ICT infrastructure) and our goal of maximizing community acceptance, we will pay special attention to learning environments that are popular among teacher: 7 .

\section{C.3.1. Moodle}

As of this writing, Moodle 8 is one of the most successful VLEs, with almost 50,000 registered installations and 40 millon users worldwide9. This VLE was originally developed as an effort to apply social constructionism Bur95 theories to online learning (e.g. engaging in reflective dialogue within a community of learners, sp. by reading and writing collaboratively) Dou03. Other aspects of its philosophy are the modularity of its implementation (Moodle is composed by a small core and a large number of modules for different functionalities, which can be combined in different customized distributions of the software), role flexibility (e.g. students can act as teachers in a certain activity, if desired) and adaptability (it is possible to adjust the courses' schedule or to add new activities on the fly).

\footnotetext{
${ }^{7}$ I have not found trustworthy usage statistics for the main VLEs, but going through their own websites and the registered sites they know about, the most widely used are Moodle (almost 50,000 sites and 40 million users), Dokeos (more than 10,000 sites and 3 million users), .LRN ("more than half a million users"), Claroline (more than 1700 organizations using it), Sakai (more than 350 sites), OLAT (more than 150 sites) or LAMS (around 100 sites listed). Blackboard (the main proprietary LMS), Atutor and Desire2Learn do not list user base figures.

http://moodle.org

${ }^{\mathrm{s}}$ As listed in Moodle's statistics site, http://moodle.org/stats/(Last visit: 5 Feb 2011)
} 
Design philosophy: bricolage As described in Ber05], one important aspect of the Moodle philosophy of operation is its approach to learning design, which is said to be one of the reasons for its wide acceptance among teachers. Rather than the "normative design" advocated by IMS$\mathrm{LD}$ and most of its implementing tools, in which the design is completed before the deployment and execution of the activities, Moodle encourages a more open, unstructured, "bricolage-like" design of the courses. In the educational concept of bricolage Pap80, the teacher refines and iterates the learning design, often as the course is being taught, to handle unexpected occurrences and opportunities. In this kind of pedagogy, the ability to deploy complex activity structures is less important than the ability to tweak them as the needs arise during the course. This concept of bricolage is very much in line with the idea of orchestration as the modification of the activities to react to occurrences in the classroom, and our system should support or, at least, allow that kind of operation when used in conjunction with a learning environment such as Moodle (see requirements FR7 and OR10, in Pri11a).

Moodle architecture and concepts 10 In a nutshell, Moodle is structured around courses, which contain activities and resources. Courses can be arranged in different formats, such as by topic (the course is composed of a number of topics, which are flat structures of activities and resources, e.g. if the course is objective-based, with topics taking different amounts of time), weekly (the course is divided time-wise in weeks where activities and resources are placed, e.g. if the teacher wants all students to do the same activities at the same time) or socially (arranged around a social forum and its discussion topics, e.g. in the case of free-form courses or non-course educational efforts). Other course formats are also possible, such as LAMS courses (if you want to use LAMS designs from inside Moodle) or SCORM courses (creates a course from a SCORM package, but apparently does not allow usual Moodle tools to be used with it), and a number of user-contributed course formats (project course format, timeline course format, shared activities course format...).

As we have mentioned, the courses are basically a set of activities and resources. The concept of activity here is closely related to that of a tool: in a typical Moodle distribution there are 20 different kinds of activities, each one mapping to a kind of tool available in the VLE (e.g. forum, polls, wikis, assignments, SCORM players). The basic idea in Moodle is to structure activities into groups, to guide learners through learning paths (e.g. activities building on the results of the previous ones). The tools (in Moodle speech) are other functions like blogs, messaging, participant lists etc, intended for building the community of learners, as well as other general functions such as reports or gradebooks. In Moodle there is also the distinction between activities/resources and blocks, but it is largely a matter of presentation (activities/resources appear on the central area of the GUI, blocks appear on the sidebars, often as views of data available elsewhere).

Other fundamental Moodle concepts include users (with their profiles, roles, capabilities and permissions), authentication (done by Moodle or by and external database, e.g. LDAP), enrollment (which users are enrolled in which courses, maybe connecting with other institutional services), groups and grouping: 11

\footnotetext{
${ }^{10}$ Taken mostly from http://docs.moodle.org/

${ }^{11}$ The difference between these two concepts is tricky to understand and to manage. Generally, groups are used to work in parallel in the same activity, such as several classrooms doing the same course (there are several
} 
Going deeper into the technical side, Moodle is developed in PHP language, using the typical 3-tier architecture, with a database holding all relevant data, a presentation layer that dictates how information is presented to the users, and a controlling logic that handles the interactions between the other two. The Moodle interface is made up of sections (normally in the center of the interface, managed by the teacher or course developer, and made up of text and/or activities) and blocks (normally on the sidebars, pieces of installed system-wide functionality that teachers can select to customize the course).

Compliance with educational standards As it has been mentioned, Moodle can handle ADL SCORM packages, creating isolated SCORM resources or complete SCORM courses (this is important since many institutions, especially in the UK and US, require SCORM compatibility for their contents). Regarding IMS standards, Moodle can import IMS Content Packages (IMS$\mathrm{CP}$, intended for simple, rather static contents). There were some efforts in integrating Moodle and IMS-LD (see Ber05]), but they seem to be discontinued now ${ }^{12}$, and at most will only be achieved through LAMS's own IMS-LD compatibility (see below in the LAMS section) and its integration with Moodle. The work is much more active regarding IMS Common Cartridge (IMS-CC): importing CC packages is available in Moodle since version 1.9.7 (although it is experimental and disabled by default ${ }^{13}$, with IMS-CC export still unimplemented. Moodle 2.0 is listed in the IMS page as Basic LTI compliant ${ }^{14}$. Compliance with other IMS standards such as QTI will be limited to the aspects covered by IMS-CQ15.

\section{C.3.2. LAMS (Learning Activity Management System)}

LAMS ${ }^{16}$ is an open source virtual learning environment that was developed with the purpose of making learning design accessible for teachers, especially in the field of online learning, beyond the mere piling and sequencing of educational contents Dal03. LAMS positions itself as a more activity-based (rather than absorption-based), multi-learner (rather than single-learner) environment. Albeit it recognizes great influence from EML and IMS-LD, LAMS deliberately avoided being a reference implementation of those specifications, its main mission being to "provide a complete solution to the needs of teachers and learners using a Learning Design approach" Dal05, since there are aspects of the IMS-LD spec which have been said to be difficult to implement or understand [Ber05], specially regarding collaborative work, one of the main focuses of LAMS. Nevertheless, LAMS shares with IMS-LD its goal of reusability of learning designs, and it includes from the very beginning the idea of repositories of learning designs that teachers can share and reuse.

modes of visibility, so that students can/cannot see the other groups' work). You must belong to a group before joining a grouping. If you want to have different groups doing entirely different activities, you put the group (or set of groups) into a grouping. However, groupings is an experimental feature in Moodle, which is disabled by default (probably, due to its management being difficult to understand and error-prone). More on this at http://docs.moodle.org/en/What_is_the_difference_between_groups_and_groupings\% $3 F$

${ }^{12}$ http://moodle.org/mod/forum/discuss.php?d=128149.

13 http://docs.moodle.org/en/IMS_Common_Cartridge_import

14 http://www.imsglobal.org/cc/statuschart.html.

15 http://moodle.org/mod/forum/discuss .php?d=154888.

16 http://lamsinternational.com 
LAMS architecture and concepts The main LAMS concepts regarding its understanding of learning design are described in the previous report [Pri11a $]$, and so here we will concentrate more on the aspects of LAMS as a LMS and its "controller" capabilities. Conceptually, LAMS, as an implementation of a Learning Design system, has several components: an authoring component (similar to what we have called a learning design tool), a monitoring component, a controller component (sometimes also called the "learner" component) and the various activity tools Dal05. In the LAMS 2.0 architecture there are also other services, and it considers the concept of external tools (which comply with the LAMS tool contract through a tool-specific wrapper called a tool adapter ${ }^{17}$.

One of the most important concepts of LAMS regarding its relationship with external tools and its support to the enactment of learning designs, is that of LAMS's "tool contract"18. This contract basically specifies an API, a set of requirements that all tools desiring to be integrated with the LAMS "controller" have to follow. This interface covers authoring, monitoring, learner and administration capabilities of LAMS, including technical details for the deployment of the tool Dal06]. Basically, it dictates how tools have to share information among themselves and with the LAMS controller. In practice, this means that the tools have to be implemented using the Spring framework, following the LAMS skins for user interface, be packaged in jar files, be deployed using LAMS's tool deployment utility and provide a number of URLs for the different tool functions like preview, export, monitor, administration, etc. Thus, LAMS advocates a model of tight integration with external tools, with equally tight requirements and a high barrier of entry for tool providers or integration parties.

Regarding its support for learning designs, as we saw in the previous report [Pri11a, LAMS supports the concept of sequence, which is edited using the Author (graphical) user interface. These sequences are basically series of tools (from LAMS's toolset that includes fora, questionnaires, notebooks etc), representing activities to be done with those tools, which can also be branched, synchronized, or done in groups.

Compliance with educational standards LAMS's compliance with the most related educational standard (IMS-LD) has been mentioned by its creator from the very beginning of the system Dal03] [Dal05], but always set in a future, when the shortcomings of the specification are fixed. Since the evolution in the IMS-LD specification has stalled, it is doubtful that this compliance will ever be achieved 19 . LAMS is currently listed in IMS's list of IMS-CC and IMS-BLTI compliant VLEs as "under development" 20 . However, I have not found further documentation on the status of these efforts.

Requirements for LAMS, requirements for GLUE! In many aspects, LAMS's functionality is very similar to that of GLUE!-PS (or, at least, a part of LAMS, since LAMS aims to

\footnotetext{
${ }^{17}$ Apparently, only a few example tool adapters have been developed, such as a Moodle forum adapter (http: //wiki.lamsfoundation.org/display/lams/Moodle+Tool+Adapter). Moreover, this adapter is not transparent since it requires changes to the Moodle code.

${ }^{18}$ LAMS 2.0 and up. See http://wiki.lamsfoundation.org/display/lams/Tool+Contract.

${ }^{19}$ According to http://edutechwiki.unige.ch/en/LAMS\#Standardization_of_LDs_and_services, LAMS technically can export to IMS-LD level A. However, the way the XML is formatted means that the interpreter has to know about LAMS tools to be able to understand it. Thus, in practice, LAMS-generated IMS-LDs can only be run by LAMS.

20 http://www . imsglobal.org/cc/statuschart.html
} 
be a "complete solution" while the GLUE!-PS is part of one). LAMS authoring and sequencing capabilities are quite advanced (including branching, optional activities, etc), and this makes it difficult to imagine both LAMS and the GLUE!-PS working side by side. Feasible scenarios could include deploying learning designs developed with LAMS in other learning environments and external tools, or deploying external designs into LAMS with external tools 21 . Nevertheless, due to these similarities, some of the requirements of the LAMS system [Dal05] could also be used for our GLUE!-PS, adding to the list that was presented in the previous report Pri11a ${ }^{22}$;

- Instantiate designated tools at specific points in time with specific students

- Run multiple versions of the same tool with different groups

- Synchronize learners across collaborative activities where required

- Centrally manage tool instantiation and shut-down processes (to ensure quality of service from tools during the running of a sequence),

- Know how to provide instructions/content into a tool as a result of an authoring process

- Provide a way of monitoring learner progress within each tool (preferably in real-time), together with recording student contributions and activities for tracking purposes.

- Manage the transition of students from one tool to another (including tracking of current location, and providing the ability to view outcomes from past tools, but not allow access to future tools which require completion of current activities before the future tools become available)

\section{C.3.3. Dokeos}

Dokeo $^{23}$ is another big player in the open source VLE arena, with thousands of installations not only in universities, but also in medical, business and administration institutions. Its basic pedagogical approach is close to instructional design and traditional teaching, although it also includes tools that encourage constructivism (like wikis, forums, blogs, etc). In fact, Dokeos is built around the "rapid learning" paradigm, which intends to make the development of elearning (in the sense of course materials and contents, rather than activities or other elements of learning design) as fast and cost-effective as possible. This is much in line with the SCORM philosophy, and Dokeos is very focused on producing and using SCORM courses.

\footnotetext{
${ }^{21}$ However, both scenarios seem of limited usefulness to me (due to the ease of use and rich coupling among components in LAMS, it might be easier to just rebuild the learning design by hand using LAMS, for example).

${ }^{22}$ Due to the composition of the GLUE! architecture, some of these requirements do not map to GLUE!-PS but to other elements of the architecture, or require the collaboration of the GLUE!-PS and other elements. However, we have included them in the report since they illustrate the functionality that the final system should have, if at all possible

$\sqrt[23]{\text { http: //www . dokeos.com }}$
} 
Dokeos architecture and concepts In Dokeos, as in many other VLEs, everything is contained in courses. Within each course, the student can see a number of available tools, from forums to chats or surveys. From a teacher perspective, Dokeos authoring capabilities are structured around the creation of contents (e.g. pages, documents, multimedia presentations, SCORM courses, etc), although it is also possible to create sequences of activities (called learning paths) using the different tools provided in Dokeos (documents, tests, links, assignments, forums or SCORM imports) through a simple sequence authoring interface. Dokeos tracks the progress of the student along this path, as a way for teachers to assess the progress of the students' learning. As far as I could see, no concept of group work is present in the platform, apart from the fact that students can interact through some of the tools, like a forum or a chat.

Compliance with educational standards Dokeos is compliant with SCORM 1.2, both for importing courses, as well as to export contents (like presentations) as SCORM courses.

\section{C.3.4. Blackboard}

Despite being the most widely deployed proprietary LMS for years, the lack of publicly accessible documentation has made impossible an analysis of this platform (at least within the timeframe of this report). However, Blackboard is listed as compliant with IMS Common Cartridge and IMS Basic LTI in its version 92.

\section{C.3.5. MediaWiki}

Albeit their original purpose was to accumulate knowledge and ease communication among people ${ }^{25}$ (or maybe precisely because of that), a number of teachers and researchers have explored the use of wikis for educational purposes Con10c], very often playing a central role in the learning environment, similar to that of the VLEs described so far. In order to provide a wider view of what we mean by "learning environment", MediaWiki 26 (MW), one of the most popular open source wiki engines, will be described and analyzed here as another VLE. Since there is no official or standardized way of using a wiki as a VLE, we will depict here just one possible way of doing it, taken from a real world blended learning case involving an heterogeneous learning environment, which has been in action for several years now: the "ICT in Education" course in University of Valladolid's Faculty of Education and Social Work27. Thus, the contents of this section should not be taken as normative, but rather as illustrative of another possible kind of heterogeneous learning environment.

MediaWiki architecture and concepts One of the most salient features of most wiki engines is their almost complete lack of structure. In MediaWiki, as in most wikis, the central

\footnotetext{
${ }^{24}$ http://www.imsglobal.org/cc/statuschart.html

${ }^{25}$ Originally, knowledge about design patterns, among programmers, see http://c2.com/cgi/wiki? WikiHistory

${ }^{26}$ http://mediawiki.org

${ }^{27}$ http://www.gsic.uva.es/TIC/
} 
concept is the page, an HTML document that is easily editable by users using a simplified syntax that mimics the most often used features of HTML. A thin layer of structure is added in the form of categories (groups of related pages) and namespaces. MediaWiki also includes other functionalities like WYSIWYG editors for even simpler editing, history of page changes, or the concept of users. Given its original purpose, MediaWiki and most wikis lack features that are common in VLEs, such as restrictive or fine-grained access control, groupings of users or the concept of activity or tool altogether.

From a technological point of view, MediaWiki is a web application implemented in PHP, with a database layer for persistence of data. MediaWiki provides a core wiki engine, and additional functionality can be added in the form of community-developed extensions, small pieces of software (including PHP code and database entities) that can be installed alongside the core. In MediaWiki there are a large number of extensions in different states of development, implementing all sorts of functionalities, from discussion forums to bibliographic reference management or the embedding of external web tools.

Compliance with educational standards Since MediaWiki was not developed with an educational purpose in mind, it does not comply, nor interoperate with any of the educational standards mentioned so far (IMS-LD, SCORM, IMS-CC, etc).

Using MediaWiki as a VLE As we have mentioned, wikis such as MediaWiki have been used with educational purposes for some time now, especially in their role as collaborative writing tools. There exist, however, ways of using a wiki as the central element of the learning environment (equivalent to a VLE), using not only its collaborative writing features but also other tools either internal (in the form of extensions) or external (by linking them or embedding them in the wiki pages). The following description corresponds to an authentic scenario at the University of Valladolid, but there could be other ways of developing this kind of wiki-centric learning environment.

This course has a complex learning design involving learning activities such as collaborative writing, readings, discussions, presentations, video production, etc. To complete those activities, students have to use a variety of tools, including the wiki itself, a forum and other extensions, as well as external services (e.g. WIX, Youtube or Prezi). The design is replicated for 9 different groups of students, with slightly modified contents for each group depending on their specialty. Each group course has a main page, where the sequence of activities (organized by the different lesson dates) is depicted as a hierarchical list of bullets, containing instructions and links to the resources needed (e.g. as files uploaded to the wiki) or particular activity pages. Also, the course calendar (a Moodle calendar, in fact) is embedded in the course's main page. An activity page has instructions for its completion, plus one section for each group or individual that has to enact it (in small-group or individual activities), where students write their assignments, or external tools are linked/embedded.

Having a course organized like this around a wiki has a considerable advantage of having the learning design clearly described for students and teachers, and being flexible in the face of unexpected occurrences such as absent students, latecomers, modification of the quantity or nature of activities, etc, just by editing the wiki page containing the design. However, this kind of operation is not without its problems: even for 9 identical learning designs, each of them has 
to be copied manually and reviewed, changing all the links and page names to avoid naming problems (which can lead to data loss if not done carefully). Also, this approach requires that teachers create all the external tool instances (e.g. GoogleDocs documents, Youtube channels, etc) manually, updating the corresponding links in the main and in the different activity wiki pages. In this way, making a copy of an existing learning design has been estimated by one of the teachers as taking about 15 hours, while making the original design would take even more time. All these shortcomings could be mitigated or solved by using a system such as the GLUE!-PS to deploy different instances of the same learning design.

Regarding the integration of MediaWiki as a VLE with the GLUE! architecture, a first analysis has been already done, and can be briefly summarized as:

Gained affordances the GLUElet Manager would allow for the integration of external tools in wiki pages without the need of developing an extension (provided that the tool adapter has been developed for other VLEs); the GLUE!-PS would be used to create a page structure (similar to the one described above) translated from the learning design.

Problems Most of the main problems with using MW as a VLE derive from the fact that, even if both systems have registered, identifiable users, they have very different philosophies with regard to user access rights: in MW access rights are generic, not per-page, and there is no notion of groups of users. Even if there exist extensions to add groups and more fine-grained access rights, their usage is discouraged.

Implementation There are several alternatives to the integration of MW and GLUE!, depending on how much of the typical VLE access rights we want to enforce. The most feasible implementation route would involve Gluelets (in GLUE! speech, an instance of an external tool) as a special kind of wiki page, containing a template with the instance information (either by referring to the instance identifier in GLUE!, or to the activity identifier). When the page is accessed, the GLUElet Manager provides the embedded instance corresponding to the information on the wiki page.

\section{C.3.6. Elgg}

Although it is advertised as a "networking engine", Elgg 28 has been often associated with the personal learning environment (PLE) movement vH06, as a way to implement such learning environments in which students take control and responsibility for their own learning. We analyze it briefly here in order to give a wider sense of a learning environment where GLUE! (and especially GLUE!-PS) could be helpful.

Elgg architecture and concepts As a software for building social environments, Elgg includes the concept and management of users, their relationships (through the FOAF technology) and groups. It also allows to manage contents (e.g. files), $R S S$ feeds and other snippets of functionality in the form of widgets.

Technically speaking, Elgg is developed in PHP with a MySQL database for the backend. Elgg is arranged around a relatively small core functionality, which can be extended with a variety of plugins like blogs, microblogs, wiki-like functions, bookmarks, messaging, etc.

${ }^{28}$ http://elgg.org 
Compliance with educational standards As in the case of MediaWiki, due to its noneducational nature, Elgg is not compatible with any of the common educational standards, as far as I know.

\section{C.4. A slightly different view: Using standards to deploy learn- ing designs}

This and the previous report Pri11a seem to operate under the rationale that the GLUE!PS has to adapt learning design concepts to learning environment concepts, in order to deploy and support the enactment of teachers' learning designs. However, there is an alternative (or maybe complimentary) approach: to use the learning environments' compliance with educational standards as a way of deploying the learning designs, by providing a GLUE!-PS adapter for those standards. Here we will briefly consider the main educational standards that could fit in this role, analyzing how feasible it is their usage for the purpose of deploying learning designs.

SCORM 29 Although SCORM is one of the most widely supported standards for interoperability of educational content in LMSs (it is supported, e.g. by Blackboard, Moodle, Dokeos...), it is a well known weakness of the specification that "two different learners cannot share any [...] data elements to pass information between themselves" Ip03. This limitation alone rules out SCORM for the deployment of learning designs, since collaborative learning and group work are among the main activities that the GLUE!-PS should support.

IMS Learning Design (IMS-LD) ${ }^{30}$ Even if this specification is (quite obviously) a learning design language, since there exist VLEs (such as .LRN) and standalone tools (such as the Reload Player) that support the enactment of designs using this specification, we could think of scenarios where learning designs (developed with non-IMS-LD authoring tools) are deployed using GLUE!-PS into IMS-LD compliant learning environments. However, the limited adoption of the specification makes this kind of scenario relatively unimportant when compared with e.g. providing good Moodle support.

IMS Content Packaging (IMS-CP) ${ }^{31}$ IMS-CP defines the way learning contents are to be packaged for distribution across systems. Thus, this specification only relates to the content, and not to the activities and their sequencing, as IMS-LD does 32 . As such, IMS-CP only would serve our deployment purposes partially (just for the contents). The adoption of IMS-CP is wider than IMS-LD's, but nevertheless using it as an output format would only solve part of our problems (there is still important design information about users, groups and activities that is missing from the specification).

\footnotetext{
${ }^{2 \mathrm{~g}}$ http://www .adlnet.gov/Technologies/scorm/default.aspx

30 http://www .imsglobal.org/learningdesign/

31 http://www .imsglobal.org/content/packaging/

${ }^{32}$ In fact, IMS-LD and IMS-CP are designed to be complimentary, one specifying the contents and the other specifying the pedagogical method.
} 
IMS Common Cartridge (IMS-CC) + IMS Basic Learning Tools Interoperability (IMS-BLTI) ${ }^{33}$ In a similar way to IMS-CP, the Common Cartridge specification is focused primarily in achieving error-free import of content into any compliant LMS. However, IMS$\mathrm{CC}$ is explicitly said to support blended learning and collaborative learning (although its main emphasis is in instructor-led learning) and the idea of "launching and exchanging data with external applications". In fact, the content part of the cartridge is done with IMS-CP version 1.2 (or a subset of it), and the external applications part is done with the Basic LTI specification.

The IMS-CC data model has four main parts: the content resources (or "learner experience data"), optional (or "supplemental") resources, operational data for controlling the LMS's behavior (e.g. authorization), and descriptive metadata about the cartridge. The supported content types include: web content, associated content, QTI assessments and question banks, authorization data, discussion topics (to initiate/populate discussions e.g. in a forum) and web links (URLs).

Regarding the interaction with external tools, Basic LTI just dictates that an URL has to be provided inside the cartridge for launching the external tool, and that an OAuth authentication scheme should be used for securing the message interactions between the LMS and the external tools. However, Basic LTI does not cover the management of the external tool's lifecycle (e.g. the creation of instances, or their configuration).

Thus, we could think of deploying a (contextualized) learning design (expressed in the GLUE!-PS lingua franca), by converting it to a IMS-CC cartridge and importing it from an IMS-CC compliant VLE ${ }^{34}$. However, doing this would have several shortcomings: since IMS-CC does not have any notion of users or groups (beyond roles such as instructor or student, which are used for access rights to the content), much of the information in collaborative activities might be lost, and would have to be completed in a non-standard manner, e.g. by creating a copy of the resource/tool for each group and modifying the access rights in the VLE after the import. Also, the concept of activity is not supported "per se" in the specification, although activities could be mimicked as IMS-CC resources that contain the activity's objects or services. Finally, external tools in the form of GLUElets could be included with relative ease as Basic LTI links 35 .

\section{C.5. Summing up: towards first proposal for GLUE!-PS}

From the analyses of the learning design languages and the learning environments done in this and the previous report, we can already see a number of trends and common concepts, which will serve us to further clarify the GLUE!-PS data model and the main implementation strategies. The main conclusions that we can derive from the analyses include...

... regarding the GLUE!-PS data model (or lingua franca):

\footnotetext{
$\sqrt[3]{\text { http://www.imsglobal.org/cc/alliance.html }}$

${ }^{34}$ See http://www.imsglobal.org/cc/statuschart.html

${ }^{35}$ We should look closely at this, since I'm not sure that the GLUElet Manager complies with Basic LTI's expected behaviors for tool providers.
} 
- As we saw when analyzing the learning design languages, most of the design specifications and tools are centered around the concept of activity (or a close equivalent). These activities relate one or more tools and resources with the goals of their usage, as well as the participants that take place in the activity and rules or instructions for its completion. At the abstract design level, both the tools and the participants of an activity may remain undefined or incomplete, but in the deployment and enactment process both have to be defined, with concrete activities referencing concrete tool instances and specific participants and groups.

- Learning design languages have either role-based (e.g. IMS-LD) or group-based (e.g. LAMS) definitions of the participants in a group or an activity. However, most learning environments are group-based (if groupings exist at all). This makes me think that group-based participant definitions would be more convenient, although this can make the translation from role-based design languages a complex task. Also, the concrete way of integrating GLUE!-PS in learning environments that do not have a strong grouping functionality (like LAMS does) remains an open question, and the solution will likely be context- and teacher-dependent.

- Learning design languages and learning environments organize activities in a variety of activity structures, sometimes hierarchical, sometimes sequential, with varying levels of hierarchy depth and breadth. The lingua franca should support as many of these organizational schemes as possible, without imposing arbitrary limitations. In some cases, an implicit sequence is derived from the order in which activities are displayed. Also, many learning environments have tools or resources (e.g. a community forum) which is not associated with any activity (although parts of it (e.g. a discussion thread) might be thought of as resources in a concrete activity.

- Most learning environments have some kind of top-level activity/resource/tool container (e.g. a course). Although most learning designs do not require this kind of concept, our lingua franca should take it into account so that learning environment entities (e.g. activities, resources or participants) can be referenced from GLUE!-PS.

... regarding the design and implementation of the GLUE!-PS:

- As we anticipated, the functionalities and concepts offered by the analyzed learning environments are much more heterogeneous than in the learning design side: some environments provide the concept of activities, others do not; some have the concept of groupings, others do not; some provide the concept of activity sequences, with varying levels of regulation, others do not provide it at all. By default, the strategy in the implementation of the GLUE!-PS should be to provide in each case the functionality that the teacher is missing from the learning environment. This, however, would mean providing practically all the functionalities of a scripting solution in the vein of LAMS (minus the user management, which is present in all the environments), in a modular way, so as to use only the parts that are needed. Making such a system easily configurable and usable by a common teacher (who probably is used to the "plain old VLE interface") will undoubtedly prove a great challenge. 
- Using educational standards (more concretely IMS-CC and Basic LTI) to deploy learning designs to the learning environment can be a cost-effective solution to reach a large user base with relatively low development effort (since many of the main VLEs already support them). However, many central learning design concepts are missing from these specifications (like groups and activities), and workarounds should be found to overcome these limitations, which would probably be VLE-dependent. There are also proposals for the inclusion of IMS-LD-like functions in Common Cartridge [Dur09, but this inclusion in the standard and its implementation by the majority of VLEs is more than uncertain. 



\title{
Appendix D
}

\section{GLUE!-PS Lingua Franca (GLUE!-PS LF)}

\begin{abstract}
In this appendix we include a more detailed description of the GLUE!-PS LF data model, used as the main data vehicle in across the GLUE!-PS architecture proposed in Chapter 5. Thus, here we specify not only the main entities used in the model, but also their relationships and attributes.
\end{abstract}

As described in Section 5.2.3, we have analyzed the most relevant learning design (LD) languages, as well as the most widespread learning environments (e.g. VLEs and others), aiming to determine which are the main learning design characteristics which are deployable in distributed learning environments Pri11b]. The results of this analysis, classified by aspects extracted from different LD and CSCL script conceptual frameworks, are summarized in Figure D.1.

From this analysis's results we have defined a data model to serve as lingua franca (i.e. a common intermediate language) for the translation between learning design concepts (generally abstract, de-contextualized) and learning platform concepts (normally tied to a specific run-time context). The basic entities of this data model can be observed in Figure D.2, while its basic properties can be observed in the central row of the table in Figure D.1.

\section{D.1. Overview of the data model}

As it can be seen in Figure D.2, each design is composed of a number of activities, which can be structured as a tree/sequence (even if only for presentation purposes, given that many currently widespread learning environments do not really support complex activity sequencing). Each activity can be performed by participants, playing different roles, and can be mediated by one or more resources. These resources can, in turn, be either static documents whose location is known, or tools which have to be instantiated (e.g. Google Documents is a tool which is instantiated to produce multiple individual shared documents), so that each group performing the activity can access their (instances of) resources independently.

Moreover, for each design we can choose to perform one or more deployments, each of which is a particularization of such design for a concrete learning environment installation. In 


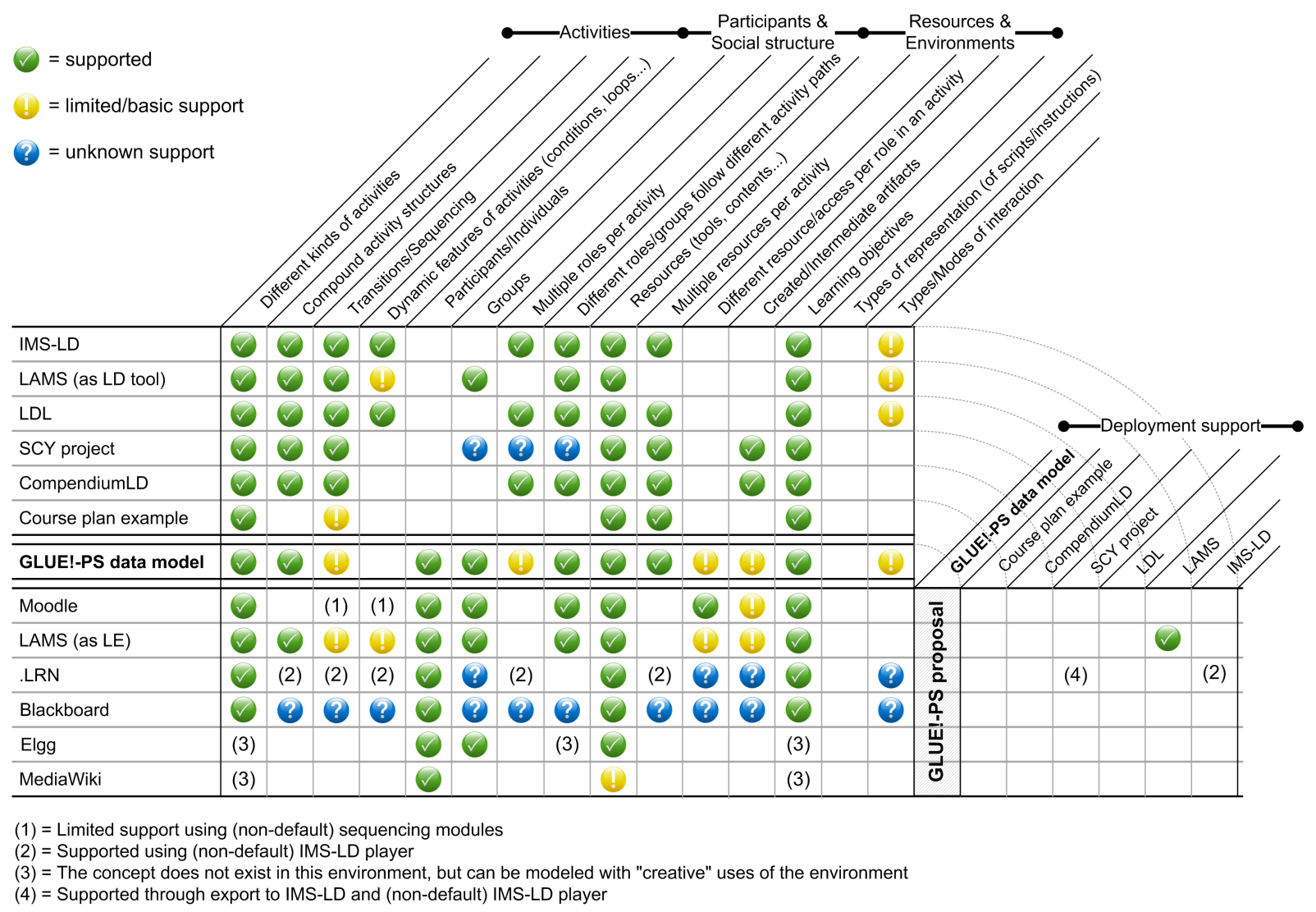

Figure D.1: Summary of the main scripting characteristics supported by learning design languages and learning environments, extracted from [Kol06] Kob07] Wei09

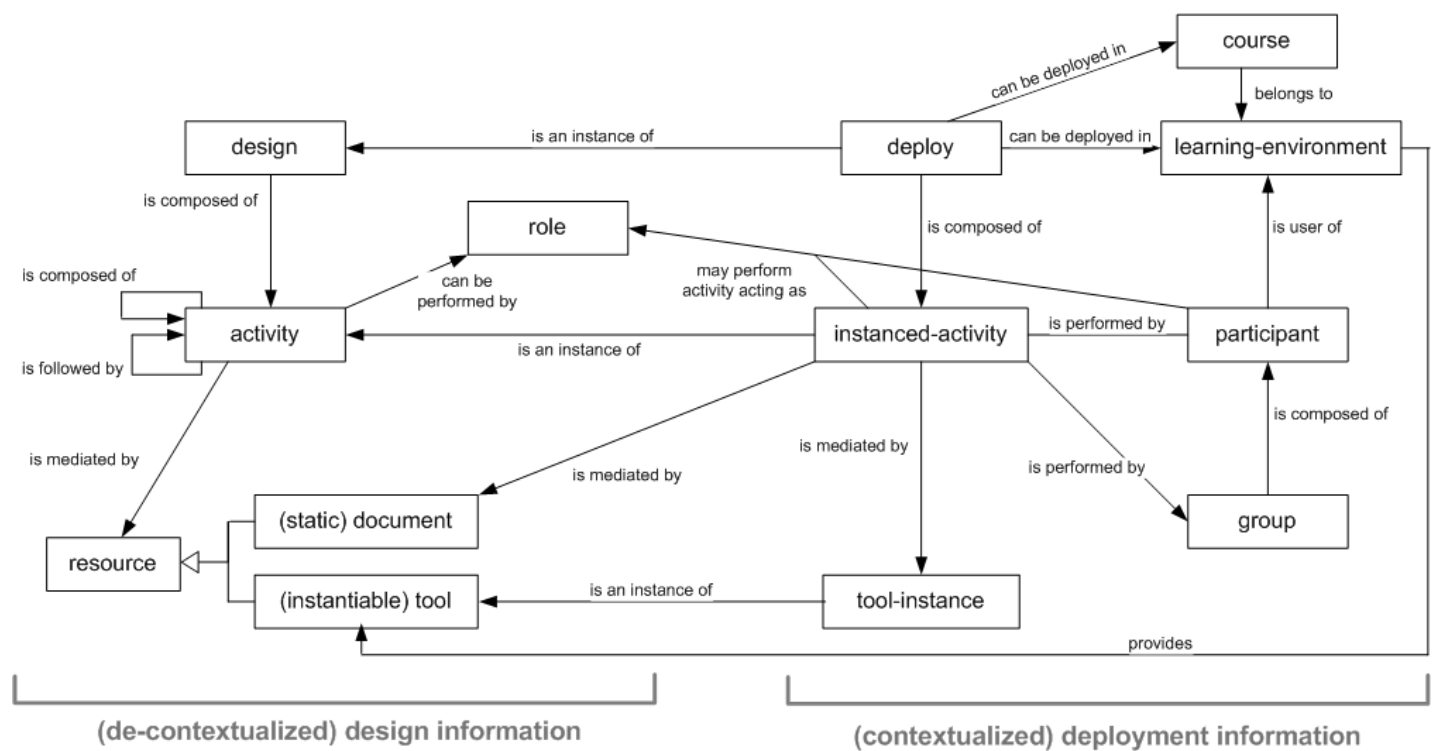

Figure D.2: GLUE!-PS data model 
a deployment, the concrete participants (i.e. the users of the learning platform) will take part in instanced activities, each one particularized from one of the design activities. We define one of these instanced activities per group performing the activity. Moreover, each of the instanced activities will be mediated by a series of resources, be them documents whose location we know, or tool instances derived from the design's tools for the activity.

\section{D.2. Detailed description of the data model}

Below we describe more fully each of the aforementioned entities and their attributes:

\begin{tabular}{|r|l|}
\hline \multicolumn{1}{|l|}{ design } & \\
\hline \hline Attribute & Description \\
id & $\begin{array}{l}\text { Unique identifier of the design (unique, at least, within } \\
\text { a GLUE!-PS installation) } \\
\text { Design's (short) name } \\
\text { description } \\
\text { Description of the design as a whole, rationale, guiding } \\
\text { principles, etc. } \\
\text { LD language or authoring tool in which the design was } \\
\text { originally expressed (e.g. "IMS LD") } \\
\text { Identifier of the user who created/uploaded the design } \\
\text { author } \\
\text { timestamp } \\
\text { Dabjectives and time in which the design was created (or last } \\
\text { modified) } \\
\text { Learning objectives of the design (array) } \\
\text { Reference to the first activity of the design's activity } \\
\text { sequence/tree } \\
\text { Design in its original format/language, in case it can } \\
\text { be useful for the instantiation/deployment (binary) } \\
\text { Possible roles used within the design (array) } \\
\text { Resources (either documents or tools) which are used } \\
\text { within the design (array) }\end{array}$ \\
resources
\end{tabular}




\begin{tabular}{|r|l|}
\hline activity & \\
\hline \hline Attribute & Description \\
name & $\begin{array}{l}\text { Unique identifier of the design (unique, at least, within } \\
\text { a GLUE!-PS design) } \\
\text { Title or short name for the activity } \\
\text { Detailed description of the activity, including partici- } \\
\text { pant instructions, etc. } \\
\text { description } \\
\text { Identifiers of the following activity/ies (array) } \\
\text { moctivity } \\
\text { Social level in which the activity occurs (class - in- } \\
\text { dividual - group - groupopen) } \\
\text { Identifier of this activity's children activity/ies (array) } \\
\text { childrenSequenceMode } \\
\text { Sequencing mode of the children activities (0 - se- } \\
\text { quence - 1 - parallel - 2 - choose one) } \\
\text { Identifier of this activity's parent activity (if appropri- } \\
\text { ate) } \\
\text { Identifier/s of the resource/s that will mediate in this } \\
\text { activity (array) } \\
\text { Identifier/s of the role/s that perform this activity (if } \\
\text { any) } \\
\text { Reference to an URL within the learning platform } \\
\text { where the activity has been deployed (if applicable) } \\
\text { Indicates whether this activity should be deployed to } \\
\text { the DLE or not (true - false) }\end{array}$ \\
location \\
toDeploy
\end{tabular}

\begin{tabular}{|r|l|}
\hline \multicolumn{2}{|l|}{ role } \\
\hline \hline Attribute & Description \\
\hline id & Unique identifier (at least, within a GLUE!-PS design) \\
name & Short name for the role \\
description & Description or specific instructions for this role \\
isTeacher & Indicates whether this role corresponds to a teacher or \\
& similar actor (true - false) \\
\hline
\end{tabular}




\begin{tabular}{|c|c|}
\hline \multicolumn{2}{|l|}{ resource } \\
\hline Attribute & Description \\
\hline & Unique identifier (at least, within a GLUE!-PS design) \\
\hline name & Resource's short name \\
\hline instantiable & $\begin{array}{l}\text { Indicates whether a resource is a document whose lo- } \\
\text { cation is known at design-time (false) or a tool that } \\
\text { has to be instantiated before use by a concrete group } \\
\text { of participants (true) }\end{array}$ \\
\hline location & $\begin{array}{l}\text { Resource's URL. In the case of tools, it corresponds } \\
\text { with the URL of the Gluelet Manager or the LE } \\
\text { adapter that manages them. In the case of documents } \\
\text { which were embedded in a learning design (e.g. a IMS- } \\
\text { LD UoL), the files can be stored in a local file repos- } \\
\text { itory and be referenced here relatively to the server's } \\
\text { root, e.g. "/path/to/file" }\end{array}$ \\
\hline toolKind & $\begin{array}{l}\text { (applies only to tools) Indicates whether a tool is built } \\
\text { into the learning platform or it is external to the learn- } \\
\text { ing platform (internal - external) }\end{array}$ \\
\hline toolType & $\begin{array}{l}\text { (applies only to tools) Installation that serves this tool } \\
\text { (same as in Gluelet Manager) }\end{array}$ \\
\hline toolData & $\begin{array}{l}\text { (applies only to tools) Other parameters or relevant } \\
\text { data that may be of use to the service that creates } \\
\text { tool instances }\end{array}$ \\
\hline
\end{tabular}




\begin{tabular}{|c|c|}
\hline deploy & \\
\hline Attribute & Description \\
\hline & Unique identifier (at least, within a GLUE!-PS instal- \\
\hline & \\
\hline designId & $\begin{array}{l}\text { Identifier of the design in GLUE!-PS from which this } \\
\text { deployment is derived }\end{array}$ \\
\hline name & Short name for the deployment \\
\hline learningEnvironment & $\begin{array}{l}\text { Reference to the learning environment installation } \\
\text { that will serve as center of the DLE }\end{array}$ \\
\hline course & $\begin{array}{l}\text { Identifier of the LE course wherein this deployment } \\
\text { should be performed }\end{array}$ \\
\hline author & GLUE!-PS user that performs the deployment \\
\hline timestamp & $\begin{array}{l}\text { Date/time when the deployment was created/last } \\
\text { modified }\end{array}$ \\
\hline deployData & $\begin{array}{l}\text { Additional parameters for the deployment, to be used } \\
\text { by the specific LE adapter (e.g., modality, course in- } \\
\text { formation, etc.). Generally expressed in the following } \\
\text { format: "iparam } 1_{i}=\text { ivalue } 1_{i} ; \text { iparam } 2 i=\text { ivalue } 2 ; ; \ldots . "\end{array}$ \\
\hline instancedActivities & $\begin{array}{l}\text { List of instanced activities that are part of this deploy- } \\
\text { ment, deriving from the deployment's original design's } \\
\text { activities, one for each group that performs such ac- } \\
\text { tivity (array) }\end{array}$ \\
\hline toolInstances & $\begin{array}{l}\text { List of tool instances to which reference is made } \\
\text { throughout this deployment, one for each tool and } \\
\text { group performing an activity (array) }\end{array}$ \\
\hline participants & $\begin{array}{l}\text { List of participants (e.g. LE users) which are involved } \\
\text { in this design's particularization (array) }\end{array}$ \\
\hline groups & $\begin{array}{l}\text { List of participant groups that take part in the activ- } \\
\text { ities of this particularized design (array) }\end{array}$ \\
\hline staticDeployURL & $\begin{array}{l}\text { URL where we can find a LE-specific static form of } \\
\text { this deployment (e.g. a Moodle course backup), if the } \\
\text { selected LE supports this kind of deployment }\end{array}$ \\
\hline liveDeployURL & $\begin{array}{l}\text { URL where we can find this deployment within the } \\
\text { learning platform where it has been deployed using } \\
\text { programmatic APIs, if the selected LE supports this } \\
\text { kind of deployment }\end{array}$ \\
\hline inProcess & $\begin{array}{l}\text { Specifies whether a deployment is being performed } \\
\text { programmatically at the moment in the selected LE } \\
\text { installation, if the LE supports this kind of deploy- } \\
\text { ment }\end{array}$ \\
\hline
\end{tabular}




\begin{tabular}{|c|c|}
\hline \multicolumn{2}{|c|}{ learning-environment } \\
\hline Attribute & Description \\
\hline id & $\begin{array}{l}\text { Unique identifier (at least, within a GLUE!-PS instal- } \\
\text { lation) }\end{array}$ \\
\hline name & Short name for the learning platform installation \\
\hline type & $\begin{array}{l}\text { Identifier for the kind of learning platform of this in- } \\
\text { stallation (Moodle - MediaWiki - SharePointLMS), } \\
\text { which also indicates the LE adapter to be used }\end{array}$ \\
\hline credentials & Credentials for accessing the LE (if any) \\
\hline author & $\begin{array}{l}\text { Identifier of the user that has defined this learning } \\
\text { platform installation (and who provides the creden- } \\
\text { tials) }\end{array}$ \\
\hline location & $\begin{array}{l}\text { Learning platform's access URL (e.g. welcome page, } \\
\text { login page) }\end{array}$ \\
\hline internalTools & Built-in tools available within the LE (array) \\
\hline externalTools & $\begin{array}{l}\text { External tools available in the DLE (through Gluelet } \\
\text { Manager) (array) }\end{array}$ \\
\hline courses & $\begin{array}{l}\text { LE's available courses in which the author can deploy } \\
\text { a particularized design (array) }\end{array}$ \\
\hline
\end{tabular}

\begin{tabular}{|r|l|}
\hline \multicolumn{2}{|l|}{ course } \\
\hline \hline Attribute & Description \\
id & Unique identifier (at least, within a particular learning \\
environment installation) \\
name & Short name for the course \\
relativeUrl & Course's access point, relative to the LE's main access \\
& URL \\
vleParameters & Course-specific parameters, LE-dependent (if any) \\
participants & List of participants in the course, including their re- \\
& spective roles (array) \\
\hline
\end{tabular}

\begin{tabular}{|r|l|}
\hline \multicolumn{2}{|c|}{ instanced-activity } \\
\hline \hline Attribute & Description \\
id & $\begin{array}{l}\text { Unique identifier (at least, within a deployment) } \\
\text { Identifier of the deployment to which this instanced- } \\
\text { activity belongs }\end{array}$ \\
activityId & $\begin{array}{l}\text { Identifier for the activity from which this instanced- } \\
\text { activity is a particularization } \\
\text { Identifier for the group which performs this instanced- } \\
\text { activity } \\
\text { resourceIds } \\
\text { toolinstanceIds } \\
\text { instanced-activity (array) } \\
\text { location } \\
\text { List of identifiers of the tool instances associated to } \\
\text { this instanced-activity (array) } \\
\text { URL where the deployed equivalent of this instanced- } \\
\text { activity can be accessed in the learning platform }\end{array}$ \\
\hline
\end{tabular}




\begin{tabular}{|r|l|}
\hline \multicolumn{1}{|l|}{ tool-instance } \\
\hline \hline Attribute & Description \\
id & $\begin{array}{l}\text { Unique identifier (at least, within a deployment) } \\
\text { Short name for the tool instance } \\
\text { deployId }\end{array}$ \\
Identifier of the deployment to which this too-instance \\
belongs \\
resourceId \\
Identifier of the tool (resource) from which this entity \\
is an instantiation \\
URL where this tool-instance can be accessed, be it \\
either within the LE (if it is a built-in tool, and it has \\
a distinct URL), or in the Gluelet Manager service (if \\
it is an external tool) \\
In the case of a tool reuse, this field contains the iden- \\
tifier of the tool which is being reused
\end{tabular}

\begin{tabular}{|r|l|}
\hline \multicolumn{1}{|l|}{ participant } \\
\hline \hline Attribute & Description \\
id & Unique identifier (at least, within a deployment) \\
deployId & $\begin{array}{l}\text { Participant's username } \\
\text { Identifier of the deployment to which this participant } \\
\text { belongs }\end{array}$ \\
learningEnvironmentData & $\begin{array}{l}\text { LE-specific participant data (e.g. his local identifier at } \\
\text { the LE), as well as other data about the participant } \\
\text { ishich might be useful for the LE adapter } \\
\text { Indicates whether this participant is a teacher or plays } \\
\text { a similar staff role (true - false) }\end{array}$ \\
\hline
\end{tabular}

\begin{tabular}{|r|l|}
\hline \multicolumn{2}{|l|}{ group } \\
\hline \hline Attribute & Description \\
id & Unique identifier (at least, within a deployment) \\
deployId & $\begin{array}{l}\text { Group's short name } \\
\text { Identifier of the deployment to which this group be- } \\
\text { longs } \\
\text { participantIds } \\
\end{array} \begin{array}{l}\text { Identifiers of the participants that belong to the group } \\
\text { (array) }\end{array}$ \\
\hline
\end{tabular}




\title{
Summary in Spanish
}

\author{
Resumen en español
}




\section{Preámbulo}

Las siguientes páginas hasta completar el documento contienen el resumen en lengua española de la tesis "Supporting orchestration of blended CSCL scenarios in Distributed Learning Environments" (Soporte a la orquestación de escenarios CSCL mixtos en Entornos de Aprendizaje Distribuidos). Este resumen contiene el índice de la tesis doctoral traducido al español (para facilitar la búsqueda y referencia de las diferentes secciones de la versión original inglesa), así como resúmenes de los contenidos de cada capítulo. En estos resúmenes se hace especial énfasis en las contribuciones aportadas por la presente tesis, así como de las conclusiones que se derivan de cada una de ellas (especialmente, de su evaluación). Nótese que las referencias bibliográficas que aparecen en estos resúmenes deben buscarse en la lista de referencias de la versión original (página 335). Los apéndices de la tesis, por su contenido más específico o técnico, no han sido resumidos.

En el primer capítulo se describe el contexto general de la tesis, así como sus objetivos y la metodología seguida para conseguirlos. La tesis se centra en el concepto emergente de "orquestación" en el Aprendizaje Mejorado por Tecnología (TEL, de sus siglas en inglés), que es la coordinación de múltiples actividades de aprendizaje, que se desarrollan a distintos niveles sociales y usando una variedad de herramientas. En concreto, la tesis propone herramientas tecnológicas y conceptuales para soportar este tipo de coordinación en el Aprendizaje Colaborativo Soportado por Ordenador (CSCL, en inglés), en el contexto tecnológico de los Entornos de Aprendizaje Distribuidos (DLEs, en inglés). En este capítulo también se describe cómo se proponen tres contribuciones hacia dicho objetivo, siguiendo el método de ingeniería, y cómo estas contribuciones se evaluarán usando técnicas de método mixto, enmarcadas en un modelo receptivo y orientado al evaluando para evaluar CSCL (CSCL-EREM, en inglés). Además, en este primer capítulo también se dibuja la estructura general del resto del documento.

El segundo capítulo profundiza en el contexto de investigación de la tesis, proporcionando una revisión del trabajo relacionado más relevante relacionado con el tema de la tesis. Así, se describe el CSCL, y cómo se puede promover a través del denominado aprendizaje mixto (blended), que combina actividades presenciales y no presenciales. A partir de los campos de investigación de TEL y CSCL se describe en profundidad la noción de "orquestación", como concepto que enfatiza la complejidad del trabajo del profesor en entornos TEL y CSCL auténticos. Esta revisión nos conduce a una primera síntesis de la literatura TEL/CSCL sobre orquestación. En este capítulo también se revisan las nociones de entornos de aprendizaje que suelen usarse para dar soporte al aprendizaje mixto (VLEs y PLEs). También se describe cómo estas plataformas de aprendizaje se están extendiendo con herramientas externas, dando lugar así a Entornos de Aprendizaje Distribuidos (DLEs). La orquestación del aprendizaje en este tipo de entornos distribuidos implica múltiples problemas, algunos de los cuales también se describen en este capítulo: la falta de un concepto de "orquestación" claro, la dificultad de desplegar diseños de aprendizaje y adaptarlos en tiempo de ejecución, así como la falta de guías o buenas prácticas para realizar esta orquestación. Estos problemas son los que se explorarán durante el resto de la tesis.

El Capítulo 3 propone una de las contribuciones de la tesis: una nueva definición de "orquestación” y el marco conceptual ' $5+3$ Aspectos', como herramienta conceptual para ayudar al investigador TEL a trabajar sobre este tema. Este marco puede ser usado por los investigadores como "lente analítica" para caracterizar la orquestación en entornos educativos concretos, 
así como para enmarcar y comunicarse acerca de su trabajo con otros investigadores. El capítulo también describe cómo este marco ha sido evaluado por dos paneles de investigadores TEL/CSCL (uno con expertos internacionales, otro con un grupo más joven de investigadores). Esta evaluación nos muestra cómo los investigadores expertos consideran este marco como completo y útil, mientras que tiene un cierto valor pedagógico para los investigadores más jóvenes. Este capítulo también presenta un primer instrumento de investigación (un cuestionario y guía de reflexión), y se aportan primeras evidencias de su utilidad.

El cuarto capítulo presenta los patrones de diseño como una manera común de comunicar, crear y reflexionar sobre el conocimiento de practicantes en una variedad de prácticas complejas. Un concepto similar se ha aplicado a la práctica de la orquestación durante esta tesis, primero en escuelas de educación primaria y luego en prácticas de CSCL mixto con DLEs en educación superior. El capítulo propone estos "patrones atómicos" como otra de las contribuciones de la tesis, una herramienta conceptual para orquestar estos complejos ecosistemas de herramientas y actores. El capítulo describe cómo se extrajo un catálogo de patrones atómicos de la observación de prácticas CSCL auténticas (primero en educación primaria y luego en educación superior), parra luego evaluar un subconjunto de ellos a través de su aplicación iterativa en una serie de talleres de desarrollo profesional docente. Describimos también cómo durante la evaluación usando métodos mixtos, los docentes reaccionaron favorablemente a este tipo de soporte, aunque se apuntó también a interacciones con varios factores (como las creencias y experiencia previa de los profesores, o el formato de los propios talleres).

El Capítulo 5 señala dos de los principales problemas tecnológicos de la orquestación de CSCL mixto en DLEs: la falta de soporte tecnológico para que un docente despliegue sus diseños de aprendizaje a lo largo de un DLE, así como para la gestión flexible de dichas actividades desplegadas. Este capítulo también propone la principal contribución tecnológica de la tesis: el Entorno Unificado de Aprendizaje en Grupo - Guiado Pedagógico (GLUE!-PS por las siglas en inglés). Esta arquitectura y modelo de datos fueron extraídos de una revisión de la variedad de lenguajes y herramientas de Diseño de Aprendizaje (en inglés, LD), así como de las principales plataformas de aprendizaje populares en la actualidad. También se presenta el desarrollo y evaluación iterativas de la propuesta GLUE!-PS a través de experiencias analíticas y auténticas, así como de talleres con profesores. De esta evaluación se deriva que el GLUE!-PS no sólo representa un soporte potente para la orquestación de aprendizaje en DLEs, sino que también permite el desarrollo de soportes a la orquestación más avanzados.

Finalmente, el sexto capítulo concluye la tesis propiamente dicha, resumiendo el problema general de investigación que la concierne (la provisión de herramientas que den soporte a la orquestación de actividades TEL y CSCL), las contribuciones propuestas, y cómo esas contribuciones se han evaluado iterativamente a lo largo de la tesis. Describe también cómo de dichas evaluaciones podemos concluir que los objetivos de la tesis han sido alcanzados, indicando también posibles líneas de trabajo futuro. También describe cómo el número de publicaciones relacionadas con los contenidos de la tesis y su influencia sobre algunos proyectos de investigación financiados recientemente, pueden servir de indicadores del éxito de las propuestas y de la relevancia de trabajo futuro. 


\section{Índice General}

Abstract

Resumen

\begin{tabular}{lll}
\hline & Introducción & 1
\end{tabular}

1.1 Objetivos de la tesis y contribuciones . . . . . . . . . . . . . . . . . . . . 4

1.2 Metodología de investigación . . . . . . . . . . . . . . . . 8

1.3 Estructura del documento . . . . . . . . . . . . . . . . . . . . . 15

2 Contexto de investigación: Orquestación en TEL y Entornos de Aprendizaje $\begin{array}{ll}\text { Distribuídos para soportar CSCL mixto } & 17\end{array}$

$2.1 \quad$ Introducción . . . . . . . . . . . . . . . . . . . . 17

$2.2 \quad$ Desde TEL hasta el Aprendizaje Colaborativo Soportado por Ordenador mixto 19

2.2.1 Aprendizaje Colaborativo Soportado por Ordenador (CSCL) . . . . . . . . 19

2.2 .2 Diseño de Aprendizaje, guiado CSCL y el ciclo de vida del CSCL . . . . . 20

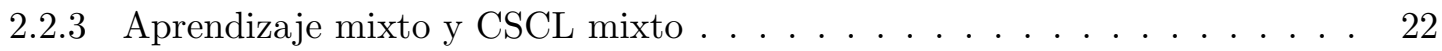

2.3 Orquestación en TEL y CSCL . . . . . . . . . . . . . . . . . . . . 23

2.3 .1 Una revisión de literatura sobre orquestación . . . . . . . . . . . . . . 24

2.3 .2 Ejemplos de investigación bajo el "paraguas" de la orquestación . . . . . . 26

$2.3 .3 \quad$ Primer intento de síntesis sobre la orquestación $\ldots \ldots \ldots \ldots$. . . . . 28

2.4 Entornos de Aprendizaje Virtuales, Personales y Distribuidos (VLEs, PLEs y

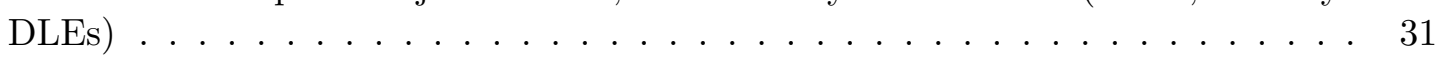

$2.4 .1 \quad$ Entornos Virtuales de Aprendizaje (VLEs) $\ldots \ldots \ldots$. . . . . . . . . . . 31

2.4.2 Entornos Personales de Aprendizaje (PLEs) y otras plataformas de apren-

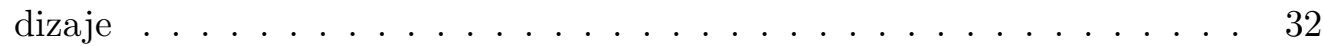

2.4.3 Entornos de Aprendizaje Distribuidos (DLEs): Más allá del VLE/PLE . . 34

2.5 Orquestando CSCL mixto en DLEs: Práctica actual y problemas de orquestación 37

2.5.1 Hacia una visión sintetizada de la orquestación en TEL y CSCL . . . . . . 37

2.5 .2 Prácticas docentes en la orquestación de DLEs: la necesidad de guía con-

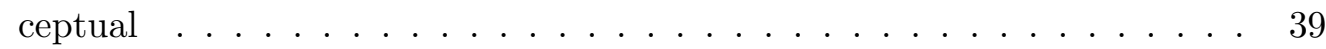

2.5.3 Diseño de Aprendizaje y el "hueco de despliegue" . . . . . . . . . . . . . . 40

2.5.4 Flexibilidad: Desde la improvisación al problema de la gestión y adaptación en tiempo real de las actividades de aprendizaje en DLEs . . . . . . . . . 43

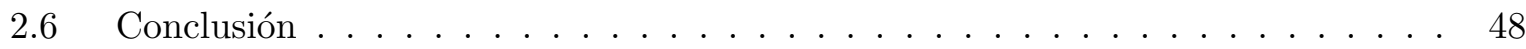

3 '5+3 Aspectos': Un marco conceptual para la orquestación en TEL 51 
$3.1 \quad$ Introducción: Relevancia de la orquestación en TEL . . . . . . . . . . . . . . . . 51

$3.1 .1 \quad$ Una nota sobre metodología $\ldots \ldots \ldots \ldots \ldots$. . . . . . . . . . 54

$3.2 \quad 5+3$ Aspects': Una definición y marco conceptual para la orquestación de Aprendizaje Mejorado con Tecnología . . . . . . . . . . . . . . . . . 54

$3.2 .1 \quad$ Aspectos de la orquestación $\ldots \ldots \ldots \ldots \ldots$. . . . . . . . . . . . . . . . . . . . . . . .

3.2 .2 ¿Qué es orquestación? Una definición . . . . . . . . . . . . . . . . . . 61

$3.3 \quad$ Evaluación de '5+3 Aspectos': Dos paneles de investigadores . . . . . . . . . . . 61

$3.3 .1 \quad$ Contexto y método de la evaluación . . . . . . . . . . . . . . . 62

3.3.2 Un panel de investigadores TEL/CSCL relacionados (RP1) . . . . . . . . 66

3.3.3 Un panel más amplio de investigadores TEL/CSCL sobre orquestación reconocidos internacionalmente (RP2) $\ldots \ldots \ldots . \ldots . \ldots . \ldots 77$

3.3 .5 Cerrando la evaluación de $5+3$ Aspectos' $\ldots \ldots \ldots$. . . . . . . . . . 94

3.4 Un instrumento de investigación derivado de ' $5+3$ ': Una guía de entrevis-

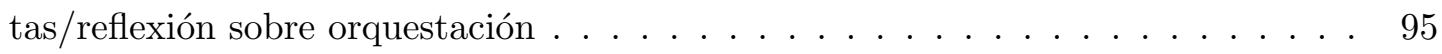

$3.4 .1 \quad$ Estructura de la guía $\ldots \ldots \ldots \ldots$. . . . . . . . . . . . . . . . . . . . . . . . . . . . . . . . . . .

3.4 .2 Evidencias preliminares de utilidad . . . . . . . . . . . . . . . . . . 98

$3.5 \quad$ Conclusiones: ¿Hacia un nuevo marco? . . . . . . . . . . . . . . . . . . . . . 101

4 Patrones atómicos como herramientas conceptuales para la orquestación 105

$4.1 \quad$ Introducción: El papel de los patrones en la orquestación . . . . . . . . . . . 105

4.1 .1 Una nota sobre metodología . . . . . . . . . . . . . . . . . . . . 108

$4.2 \quad$ Patrones atómicos para orquestación . . . . . . . . . . . . . . . . . . . 110

$4.2 .1 \quad$ El origen de los patrones atómicos . . . . . . . . . . . . . . . . . . 111

4.2 .2 ¿Qué es un "patrón atómico?" . . . . . . . . . . . . . . . . . . . . 116

4.2.3 Los patrones atómicos frente a otras aproximaciones basadas en patrones. 120

4.2.4 Analizando los patrones atómicos desde una perspectiva de la teoría de la

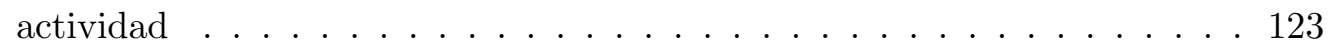

4.3 Una aproximación basada en patrones multi-nivel para la orquestación de acti-

vidades CSCL en DLES . . . . . . . . . . . . . . . . . . 125

4.3 .1 Visión general de la aproximación . . . . . . . . . . . . . . . 126

$4.3 .2 \quad$ Uso de la aproximación . . . . . . . . . . . . . . . . . . . . . . . . 127

4.3 .3 Ventajas, desventajas y desafíos de la aproximación . . . . . . . . . . . . . . . 132

$4.4 \quad$ Otros usos de los patrones atómicos . . . . . . . . . . . . . . . . . . 133

$4.4 .1 \quad$ Representando la orquestación a través de patrones atómicos . . . . . . . 133

$4.4 .2 \quad$ Usando los patrones atómicos para desarrollar tecnología . . . . . . . . . . . 137

4.5 Evaluando los patrones atómicos: Cuatro talleres de desarrollo profesional del

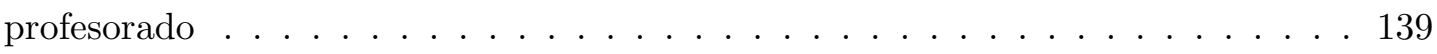

$4.5 .1 \quad$ Contexto y método de la evaluación $\ldots \ldots \ldots$. . . . . . . . . . . . 140

4.5.2 Iteración \#1: Primer intento de taller de profesorado basado en patrones atómicos en educación primaria (TW1) . . . . . . . . . . . . . 145

4.5.3 Iteración \#2: Un segundo taller de profesorado basado en patrones atómicos en educación primaria (TW2) . . . . . . . . . . . . . . . . . 154

4.5.4 Iteración \#3: Generación de un nuevo catálogo de patrones atómicos, y un primer taller de profesorado en educación superior (TW3) . . . . . . . . . 160

4.5.5 Iteración \#4: Un segundo taller de profesorado en educación superior (TW4) 175

4.5 .6 Cerrando la evaluación de los patrones atómicos . . . . . . . . . . . . . 192 
4.6 Conclusiones, relevancia y trabajo futuro sobre los patrones atómicos . . . . . . 197

5 GLUE!-PS: Una arquitectura y modelo de datos para la orquestación de diseños de aprendizaje en Entornos Distribuidos de Aprendizaje $\quad 201$

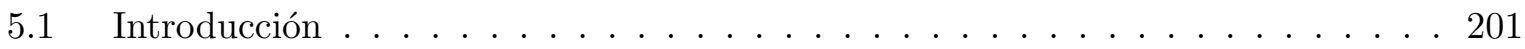

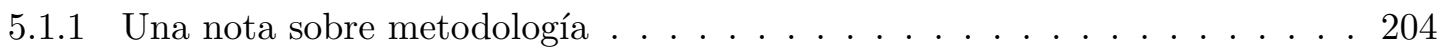

5.2 GLUE! Guiado Pedagógico (GLUE!-PS) . . . . . . . . . . . . . . . . . . . . 206

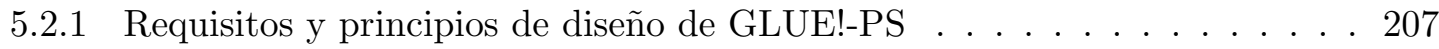

5.2 .2 Arquitectura de GLUE!-PS $\ldots \ldots \ldots \ldots$. . . . . . . . . . . . . 212

5.2.3 Lingua Franca de GLUE!-PS (GLUE!-PS LF): Un modelo de datos para desplegar y gestionar diseños de aprendizaje en DLEs . . . . . . . . . . . 220

5.2 .4 Funcionalidad de GLUE!-PS: Principales casos de uso . . . . . . . . . . 223

$5.3 \quad$ Analizando GLUE!-PS como herramienta de orquestación . . . . . . . . . . . . 236

5.3 .1 GLUE!-PS desde el marco conceptual de orquestación '5+3 Aspectos'. . . 236

5.3 .2 Analizando el soporte a la orquestación de GLUE!-PS a través de patrones

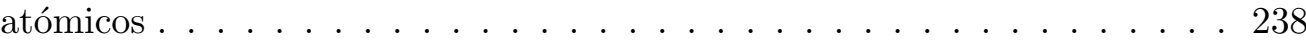

5.4 Implementación de referencia de GLUE!-PS (GLUE!-PS RI) . . . . . . . . . . . 244

5.4 .1 Tecnologías implicadas . . . . . . . . . . . . . . . . 245

5.4 .2 Evolución de GLUE!-PS RI . . . . . . . . . . . . . . . . . . . . 246

5.4 .3 Estado actual de GLUE!-PS RI . . . . . . . . . . . . . . . . . . . 246

5.5 Evaluando GLUE!-PS ． . . . . . . . . . . . . . . . . . 255

5.5 .1 Contexto y método de la evaluación . . . . . . . . . . . . . . . . . 255

5.5 .2 Iteración \#1: Una primera evaluación analítica de GLUE!-PS . . . . . . . 260

5.5 .3 Iteración \#2: Evaluando el primer prototipo de GLUE!-PS en talleres . . 266

5.5.4 Iteración \#3: Extendiendo el soporte a VLEs de GLUE!-PS - una experiencia basada en wikis. . . . . . . . . . . . . . . . . . . 282

5.5.5 Iteración \#4: Añadiendo soporte a cambios en tiempo de ejecución y evaluación en talleres y experiencias basadas en wikis . . . . . . . . . . . . 293

5.5 .6 Cerrando la evaluación: Conclusiones globales de la evaluación . . . . . . . 319

$5.6 \quad$ Conclusiones, relevancia y trabajo futuro sobre GLUE!-PS . . . . . . . . . . . . 321

$\begin{array}{lll}6 & \text { Conclusiones y trabajo futuro } & 327\end{array}$

6.1 Conclusiones de la tesis . . . . . . . . . . . . . . . . . . . . 327

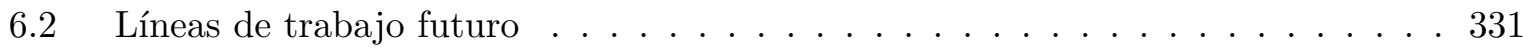

$\begin{array}{ll}\text { Referencias } & 335\end{array}$

A La Guía de Entrevistas de Orquestación (OIG): Un instrumento de investigación derivado del marco '5+3 Aspectos'] 361

A.1 Guías generales . . . . . . . . . . . . . . . . . . . . 362

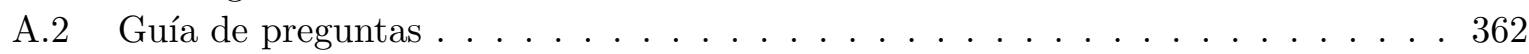

A.2.1 Diseño/Planificación . . . . . . . . . . . . . . . . . . . 362

A.2.2 Regulación/Gestión . . . . . . . . . . . . . . . . . . . . 363

A.2.3 Adaptación/Flexibilidad/Intervención . . . . . . . . . . . . . . . . . 364

A.2.4 Percepción/Evaluación . . . . . . . . . . . . . . . . . . . . . 364

A.2.5 Roles del profesor y otros actores . . . . . . . . . . . . . . . . 365 
A.2.6 Pragmatismo/Práctica . . . . . . . . . . . . . . . . . 365

A.2.7 Alineamiento/Sinergia . . . . . . . . . . . . . . . . . . . . 365

A.2.8 Modelos/Teorías . . . . . . . . . . . . . . . . . 365

B Catálogo de Patrones Atómicos de Orquestación (OAPC) 367

C Un análisis de lenguajes de Diseño de Aprendizaje (LD) y plataformas de aprendizaje $\quad \mathbf{3 7 1}$

C.1 Análisis de lenguajes de Diseño de Aprendizaje (LD) . . . . . . . . . . . . . . . 371

C.1.1 IMS Learning Design . . . . . . . . . . . . . . . . . . . . . . . . 372

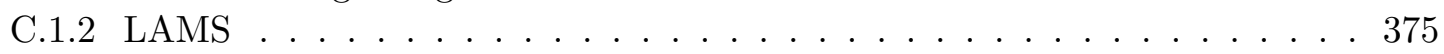

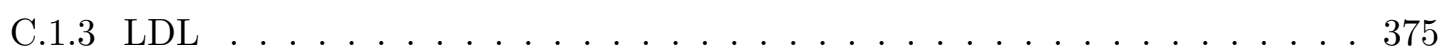

C.1.4 LAS (SCY-SE) . . . . . . . . . . . . . . . . . . 376

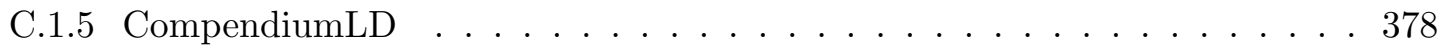

C.1.6 Lenguajes ad-hoc, no computacionales . . . . . . . . . . . . . . . 378

C.2 Discusión: tendencias comunes en los lenguajes de Diseño de Aprendizaje anali-

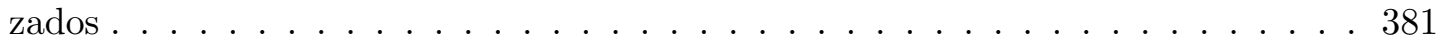

C.3 Entornos de aprendizaje y guiado de Diseño de Aprendizaje . . . . . . . . . . . 382

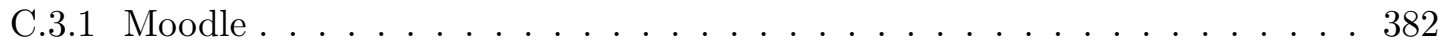

C.3.2 LAMS (Learning Activity Management System) . . . . . . . . . . . . . . . . 384

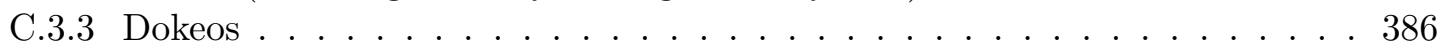

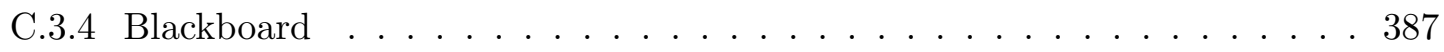

C.3.5 MediaWiki . . . . . . . . . . . . . . . . . . . . . . 387

C.3.6 Elgg . . . . . . . . . . . . . . . . . . . . . . . . . . . . 389

C.4 Una visión ligeramente diferente: Usando estándares para desplegar diseños de

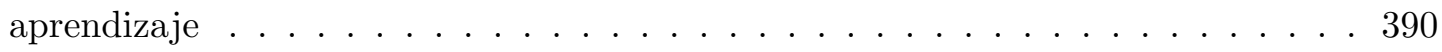

C.5 $\quad$ Resumiendo: hacia un primera propuesta de GLUE!-PS . . . . . . . . . . . . . . 391

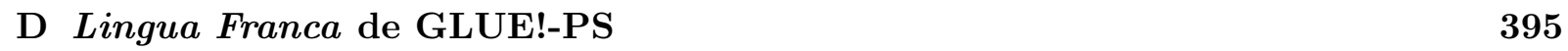

D.1 Descripción general del modelo de datos . . . . . . . . . . . . . . . . . . . . 395

D.2 $\quad$ Descripción detallada del modelo de datos . . . . . . . . . . . . . . . . . . 397 


\section{Introducción}

El uso creciente de las Tecnologías de la Información y las Comunicaciones (TICs) en el campo de la educación abre múltiples nuevas oportunidades y aproximaciones tanto en docencia como en el aprendizaje, que estudia el campo de investigación del Aprendizaje Mejorado con Tecnología (en inglés, TEL [Bal09]). Sin embargo con estas nuevas oportunidades también aparecen nuevos retos en el uso de estas tecnologías. Algunas tendencias en la investigación y la práctica del TEL (como por ejemplo el uso de aprendizaje mixto Gra05 y una variedad de aproximaciones pedagógicas no tradicionales, y su soporte por parte de diversas plataformas de aprendizaje [Dil02b] y herramientas "Web 2.0" [Con10b]) han llevado a los investigadores a señalar la complejidad de coordinar el "ecosistema de la clase" [Luc08. Esta coordinación se ha denominado "orquestar el aprendizaje" [Bal10], y es considerado uno de los mayores retos en el área de TEL en el futuro inmediato. Orquestar implica la coordinación de las "intervenciones de apoyo a través de múltiples actividades de aprendizaje que ocurren a diferentes niveles sociales" Fis06], y enfatiza especialmente la labor de los docentes en entornos de aprendizaje auténticos (por oposición a experimentos más controlados, [Dil09b]). Este tipo de coordinación es especialmente compleja y crítica en el caso del Aprendizaje Colaborativo Soportado por Ordenador (en inglés, CSCL).

Uno de los escenarios más frecuentes por todo el mundo, especialmente en instituciones de educación superior, es el de un profesor que diseña actividades de aprendizaje (para lo cual existen múltiples herramientas y lenguajes de diseño), y las pone en práctica usando un Entorno Virtual de Aprendizaje (en inglés VLE) u otra plataforma de aprendizaje similar impuesta por su institución. A veces, el docente desea usar también herramientas externas a dicho VLE, usando así lo que algunos han denominado Entorno de Aprendizaje Distribuido (DLE, [Mac10]). Sin embargo, en general la transición entre el diseño de aprendizaje y la infraestructura TIC usada para dicho aprendizaje (dentro del antes mencionado DLE) actualmente se realiza de manera manual, en un proceso tedioso y sujeto a errores. Otro tanto ocurre en el caso de que, debido a eventos emergentes, el docente quiera modificar dichas actividades "desplegadas" en el DLE de manera flexible durante el tiempo de ejecución (el docente debe hacer las modificaciones manualmente en cada una de las herramientas implicadas).

Aparte de estos problemas derivados del soporte tecnológico, existen otros problemas de naturaleza conceptual que los practicantes de TEL/CSCL deben afrontar para promover dicho "aprendizaje orquestado": actualmente no existen guías ni consejos claros sobre cómo usar y combinar la variedad de herramientas (tanto TIC como no-TIC) que el profesor tiene a su disposición en el contexto concreto de su clase. Probablemente por ello, y a pesar de los programas de desarrollo profesional docente sobre tecnología, las TICs no han sido adoptadas de manera 
general para la docencia, excepto de las maneras más básicas [Hop93] [Cub01] [Wat06] [Her08] (lejos, por ejemplo, de prácticas pedagógicas complejas como el CSCL).

En este contexto, la presente tesis tiene como objetivo proporcionar herramientas tecnológicas y conceptuales para dar soporte a la orquestación de Aprendizaje Mejorado con Tecnología mixto, centrándose sobre todo en las actividades con un componente colaborativo (en las que la orquestación es aún más crucial), en el contexto tecnológico de los Entornos de Aprendizaje Distribuidos (DLEs). Dada la relativa novedad e indefinición de este problema, también se busca una clarificación del concepto de orquestación en sí mismo, que pueda servir al autor y a otros investigadores a proponer y evaluar innovaciones relacionadas con esta orquestación. Sin embargo, dado que resolver todos los problemas que implica la orquestación supera el alcance de una tesis doctoral, aquí nos hemos concentrado en tres problemas concretos:

- La orquestación trata sobre el diseño y gestión en tiempo real de una situación de aprendizaje para conseguir de manera sinérgica una serie de objetivos de aprendizaje [Fis06 Bal10. El campo del Diseño de Aprendizaje (LD, en inglés) ha estudiado este diseño durante décadas y ha propuesto una serie de herramientas y lenguajes para realizarlo. Sin embargo, actualmente pocos diseños de aprendizaje se pueden desplegar y ejecutar en las plataformas de aprendizaje ampliamente disponibles, y menos en DLEs. Además, tras este despliegue es necesario poder modificar la infraestructura resultante de manera sencilla y flexible, durante la ejecución de las actividades. Así, estos dos aspectos (el despliegue de un diseño y su adaptación en tiempo de ejecución) actualmente no cuentan con un soporte claro, e implican que el docente realice una serie de operaciones manuales en cada una de las herramientas TIC del DLE. [Problema tecnológico para los docentes]

- Teniendo en cuenta que la mayoría de los docentes no son expertos en TICs o en TEL, encontrar buenos usos pedagógicos de las TIC cuando se orquestan escenarios TEL mixtos es tan problemático como las propias dificultades tecnológicas ya mencionadas [Law08. Así, la orquestación de TEL es una práctica compleja difícil de expresar, enseñar y ejecutar. [Problema conceptual para los docentes]

- Además, la "orquestación del aprendizaje" es una metáfora de nuevo cuño, con límites y definiciones poco definidos. Por ello, es difícil para el investigador comunicarse claramente y acumular conocimiento sobre el tema, al menos hasta que se encuentre una definición más clara del proceso y sus principales aspectos. [Problema conceptual para investigadores]

\section{Objetivos y contribuciones de la tesis}

Dado el contexto de investigación mencionado arriba, podríamos formular el objetivo global de la tesis como: "Diseñar, implementar y evaluar herramientas tecnológicas y conceptuales para soportar la orquestación de CSCL mixto que usa DLEs". Para alcanzar este objetivo, se propone la aproximación a los tres problemas de orquestación específicos que acabamos de mencionar, dando así lugar a tres objetivos parciales de la tesis (véase también la Figura 1.1, página 7):

1. Clarificar el concepto de orquestación en la investigación TEL/CSCL. Debido a su reciente aparición, la investigación sobre el problema de la orquestación es heterogénea 
y carece de un marco teórico o conceptual común. Así, una de las principales contribuciones que propone esta tesis es una definición y marco conceptual del proceso de orquestación, que ayude a la comunidad científica (y a otros actores) a encontrar soluciones de soporte a la orquestación. De hecho, también se pueden proponer instrumentos de investigación concretos derivados de dicho marco, para analizar y caracterizar dicha orquestación.

2. Proporcionar a los docentes soporte conceptual sobre la orquestación de actividades CSCL mixtas en DLEs. Para orquestar una experiencia de aprendizaje, no sólo hay que diseñar unas actividades con un cierto objetivo pedagógico, sino que también hay que tomar acciones durante su ejecución que estén alineadas con dichos objetivos. En este sentido, buenas prácticas formalizadas como patrones [Ale77] son una forma común de destilar, comunicar y reflexionar sobre prácticas complejas a distintos niveles de granularidad. Otra de las contribuciones de la presente tesis es la propuesta de patrones atómicos contextualizados como herramientas conceptuales para dar soporte a la orquestación de actividades de aprendizaje mixto que implican múltiples TICs. También se propone una aproximación para la extracción, desarrollo y evaluación de este tipo de buenas prácticas (p.ej. para su uso en desarrollo profesional docente).

3. Proporcionar soporte tecnológico a la orquestación de actividades CSCL en DLEs. La actual separación entre los diseños de aprendizaje, expresados en una variedad de lenguajes, y sus respectivos despliegues a lo largo de diferentes DLEs, puede cubrirse mediante una infraestructura tecnológica que traduzca entre dichos diseños y su implementación en plataformas de aprendizaje. Así, esta tesis propone el diseño, desarrollo y evaluación de tal infraestructura tecnológica, que apoye la orquestación de CSCL mixto en DLEs, sin atar a los usuarios a una implementación concreta de DLE o de diseño del aprendizaje. Más concretamente, se propone un modelo de datos que sirva de lingua franca para traducir entre los conceptos de los diferentes lenguajes de Diseño del Aprendizaje, y los distintos entornos de aprendizaje. También se propone la arquitectura e implementación de referencia de una infraestructura tecnológica basada en este modelo de datos. Estas contribuciones no sólo supondrían una solución al problema del despliegue, sino que también podría usarse para la gestión en tiempo de ejecución de las actividades de aprendizaje mixto en DLEs, haciendo así factible para un docente no experto la orquestación de tales actividades.

Aunque las contribuciones propuestas atacan cada uno de los problemas de orquestación mencionados de manera independiente, y pueden usarse de manera separada, no son completamente independientes. De hecho, estas contribuciones se han informado mutuamente a lo largo del proceso de investigación, y pueden ser usadas de manera combinada (por ejemplo, clasificando los patrones atómicos en función de los aspectos del marco conceptual, o usando los patrones atómicos y luego la infraestructura tecnológica en acciones de desarrollo profesional docente sobre la orquestación de CSCL en DLEs). 


\section{Metodología de investigación}

Durante la tesis doctoral se han usado diferentes metodologías de investigación, a diversos niveles y con diferentes objetivos. Los tres pilares metodológicos han sido:

Método de ingeniería. Dado que el problema de la orquestación se enmarca en el campo multidisciplinar del TEL (y del CSCL), hemos elegido un método híbrido e iterativo de investigación como marco general de la tesis: el método de ingeniería [Adr93. Este método propone cuatro fases que se siguen de manera iterativa, informando, proponiendo, analizando y evaluando las propuestas. Esta aproximación orientada a la ingeniería pero a la vez multidisciplinar señala la gran importancia de la fase de evaluación para demostrar la validez de las propuestas. Así, debemos señalar que aunque los resultados de evaluación se presentan al final de cada contribución, esta evaluación se ha realizado a lo largo de todo el proceso, alimentando las demás fases de siguientes iteraciones.

Dentro de este marco general, hemos seguido un método observacional [Zel98], recogiendo datos relevantes durante el desarrollo de experiencias reales. Este tipo de métodos tiene sus desventajas (p.ej. no siempre es posible generalizar sus resultados), pero creemos que es el más adecuado dado el énfasis de la orquestación en el uso de TEL en entornos auténticos, y nuestro interés en evaluar lo apropiado de nuestras propuestas para entornos auténticos (más que en medir los efectos de dichas propuestas). Las actividades realizadas de manera iterativa durante la tesis para cada una de las contribuciones puede verse, de manera simplificada, en la Figura 1.2 de la página 9. Sin embargo, hemos de hacer hincapié en la iteratividad del proceso seguido (que no se aprecia claramente en dicha figura).

Modelo de evaluación: CSCL-EREM. Las fases evaluativas de nuestro método de ingeniería se han desarrollado usando una aproximación naturalista, realizándose dentro de lo posible en entornos educativos auténticos. Para ello, hemos seguido las indicaciones del Modelo de Evaluación Receptivo orientado al Evaluando para CSCL (CSCL-EREM, véase [JA09b]), que intenta superar algunas de las dificultades de la evaluación de innovaciones en el campo del CSCL. Así, el modelo propone guías claras y accionables para los practicantes del CSCL a la hora de evaluar un sistema CSCL, dando de esa manera una organización particular a la complejidad del campo del CSCL.

Este modelo propone "Issues" como organizadores conceptuales del estudio de evaluación, tensiones entre dos maneras de tratar al evaluando. También propone tres facetas (perspectiva, contexto y método) que resumen las principales características que deben tenerse en cuenta mientras se realiza la evaluación. También define cuatro itinerarios prácticos en función del objeto de evaluación. Asimismo, se propone una representación gráfica de estas características, y recomendaciones a la hora de escribir el informe de evaluación. En general, el modelo aconseja el uso de técnicas de recogida de datos mixtas, a partir de una variedad de informantes, en una serie de Happenings (eventos de recogida de datos) donde se concentra la mayoría del trabajo de evaluación.

De esta manera, para cada una de las evaluaciones descritas en los capítulos de contribución (capítulos 3, 4 y 5), se describen las tres facetas del modelo CSCL-EREM (perspectiva, contexto y método de la evaluación), y los datos recogidos en cada uno de los happenings de evaluación. 
Aproximación de método mixto. El marco CSCL-EREM propone el uso de recogidas de datos profusas basadas en el método mixto Gre01] Cre03], que se considera generalmente apropiado para explorar los múltiples factores que afectan las situaciones TEL y CSCL Sut06] [Str07]. Durante la tesis hemos usado un método de evaluación mixto tomado de [Mar06b], que combina técnicas de recogida de datos cuantitativas (para mostrar tendencias) y cualitativas (para confirmar o rechazar las tendencias, entenderlas e identificar rasgos emergentes), aunque se han priorizado las cualitativas.

Este método de evaluación mixto define una serie de issues para centrar la atención del evaluador, así como una serie de categorías de análisis. Este esquema de issues y categorías se refina progresivamente (e.g. a través de las diferentes iteraciones del método de ingeniería). Se recogen datos cuantitativos y cualitativos sobre el perfil de los participantes, el uso de las contribuciones y las reflexiones de los participantes tras los eventos. Para ello se usan una variedad de técnicas como cuestionarios tanto abiertos como cerrados, observaciones, entrevistas, grupos de debate, etc., que pueden variar en cada una de las evaluaciones, debido a las circunstancias concretas de tiempo o accesibilidad de las fuentes. Del análisis cuantitativo (e.g. estadística descriptiva) y cualitativo se extraen una serie de conclusiones parciales a confirmar o rechazar por triangulación Gub81, y produciendo realimentación formativa para la siguiente iteración, hasta cristalizar eventualmente en una serie de conclusiones globales de la evaluación. 
416 


\section{Contexto de investigación: Orquestación en TEL y Entornos de Aprendizaje Distribuidos para soportar CSCL mixto}

A medida que las Tecnologías de la Información y las Comunicaciones (TICs) se usan más frecuentemente en el campo de la educación, la complejidad tecnológica y pedagógica de las situaciones de aprendizaje auténticas ha llevado a los investigadores a hablar de la "orquestación" del Aprendizaje Mejorado por Tecnología (en inglés, TEL). Esta orquestación generalmente implica la coordinación de los procesos de aprendizaje de los estudiantes a lo largo de las diferentes herramientas, tareas y niveles sociales del escenario Fis06. Bajo este nuevo significado, la orquestación se ha planteado como uno de los retos de investigación más importantes en el campo del TEL [Sut09], y resulta especialmente crítico en el caso del Aprendizaje Colaborativo Soportado por Ordenador mixto (blended CSCL). Esta tesis gira sobre esta noción emergente de la orquestación de TEL, e intenta proporcionar herramientas tecnológicas y conceptuales para dar soporte a diferentes actores (mayormente profesores, pero también investigadores) en esta compleja coordinación. En este capítulo se revisa la literatura TEL y CSCL para enmarcar el problema de investigación de la tesis, tanto desde el punto de vista pedagógico como tecnológico. Asimismo, se motiva a través de esta revisión de literatura la existencia y relevancia de tres problemas de orquestación concretos.

\section{Desde TEL hasta el Aprendizaje Colaborativo Soportado por Or- denador mixto}

El Aprendizaje Mejorado por Tecnología (en inglés, TEL) es un campo de estudio cuyo objetivo es ayudar y mejorar la docencia y el aprendizaje a través de la tecnología [Joh04a] Bal09]. La orquestación ha sido denominada uno de los "Grandes Desafíos" del TEL en general Sut09, aunque en esta tesis exploraremos este problema sobre todo a través de aquellos procesos TEL que intentan promover el aprendizaje a través de la colaboración (o sea, el Aprendizaje Colaborativo Soportado por Ordenador o CSCL [Sta06a $]$ ), integrando tanto actividades presenciales como no presenciales (aprendizaje mixto, o blended learning, Gra05). 
Koschmann Kos96 define el CSCL como un "campo de investigación multidisciplinar inspirado por el poder del aprendizaje colaborativo y por la promesa de las tecnologías digitales para soportar dicho aprendizaje colaborativo" Sta11]. La principal característica del CSCL es que se aproxima al soporte de las interacciones entre estudiantes, con el profesor jugando un papel de mediador o facilitador [Sta06a], usando una variedad de métodos cualitativos y cuantitativos, a menudo combinando ambos para desarrollar entendimientos más ricos de fenómenos complejos.

Una manera común de dar soporte al CSCL es a través de lo que se denomina guiado (scripting), que implica el diseño de secuencias de actividades dirigidas a hacer el proceso de colaboración más productivo Dil07c. Así, los scripts colaborativos son diseños de aprendizaje para asistir a los practicantes en organizar y estructurar (o sea, orquestar) la colaboración, y que sirven para guiar a los estudiantes a través de flujos de datos y procesos de aprendizaje complejos, para mejorar los beneficios educativos [Fis07]. Dentro de estos scripts, los scripts CSCL generalmente implican que el diseño tiene algún tipo de representación computacional Mia05.

Este guiado forma parte del campo más general del Diseño de Aprendizaje (LD, en inglés) Kop05. Este diseño incluye la preparación de los materiales, actividades, información, recursos y evaluación para la instrucción. Esta preparación ha sido parte de las labores docentes desde siempre, aunque existen diversas variantes de este diseño, que han tenido diferentes grados de aceptación por los docentes. Con la llegada de las TICs, el aprendizaje a distancia y el e-learning, se ha intentado modelar computacionalmente este diseño para ejecutarlo de manera automática y poder reutilizar las situaciones de aprendizaje. La práctica del LD en general (y del guiado CSCL en particular) asume un cierto "ciclo de vida" de las actividades de aprendizaje. Este ciclo ha sido definido de diferentes maneras, por ejemplo siguiendo los procesos de diseño, instanciación, ejecución y evaluación de las actividades GS09.

El TEL y el CSCL cubren tanto el aprendizaje presencial en el que la tecnología interviene, como el aprendizaje a distancia, así como las combinaciones de ambas modalidades. Esta última combinación normalmente se denomina aprendizaje mixto [Osg03] [Gra05] (blended learning, en inglés). De hecho, el uso de estas aproximaciones mixtas al aprendizaje (p.ej. para facilitar la transición entre actividades en clase y actividades en otros contextos) es un rasgo típico de la educación moderna, especialmente en educación superior (véase el Espacio Europeo de Educación Superior, Dec99]). El hecho de que estas nuevas legislaciones favorecen el uso de pedagogías "activas" como el CSCL hacen que el CSCL mixto sea un escenario muy relevante en el futuro próximo, especialmente en educación superior.

De hecho, muchas instituciones de educación superior utilizan plataformas software denominadas Sistemas de Gestión de Aprendizaje (LMSs) o Entornos de Aprendizaje Virtuales (VLEs). Otros utilizan aproximaciones y plataformas ligeramente diferentes (p.ej. Entornos de Aprendizaje Personales, o PLEs), o el uso de herramientas de la "Web 2.0" para dar soporte al aprendizaje mixto, por lo que estas plataformas y herramientas constituyen el principal contexto tecnológico de la tesis.

\section{Orquestación en TEL y CSCL}

El diccionario define la "orquestación" como el "organizar cosas para alcanzar un efecto deseado" Hou00 o "colocar o combinar para alcanzar un efecto máximo o simplemente deseado" 
[Mer10]. Para cosechar los beneficios de situaciones de aprendizaje mejoradas con tecnología o colaborativas, los docentes deben coordinar diferentes actividades, en las que estudiantes agrupadas de distintas formas usan diversas herramientas [Fis06 [Dil09a, a veces incluso a través de contextos diferentes (p.ej. en el antes mencionado aprendizaje mixto). Se ha realizado una revisión de la literatura TEL/CSCL buscando referencias a esta "orquestación", así como de ejemplos de investigaciones dicen promoverla, para así poder aportar una primera síntesis de su definición y principales componentes.

En el campo del CSCL los promotores más importantes de la metáfora de la orquestación en los últimos años han sido Fischer y Dillenbourg, que definieron la orquestación como el "proceso de coordinar productivamente las intervenciones de apoyo a través de múltiples actividades de aprendizaje que ocurren a diversos niveles sociales" [Fis06]. Este concepto a su vez tiene otras implicaciones y dimensiones, tales como la gestión de la dimensión cognitiva de dicho proceso, la adaptación de las actividades diseñadas a los eventos de la clase o la dimensión más tecnológica de coordinar las transacciones entre los diferentes componentes software del escenario. Así, podemos observar que la coordinación del flujo de actividades que propugna el guiado CSCL (scripting) es una parte de dicha orquestación, como también lo es la representación computacional de dicho guiado (p.ej. usando la especificación IMS-LD [IMS03a]) para automatizar el flujo de trabajo en un entorno de aprendizaje.

Con la información de la antes mencionada revisión de literatura se ha destilado una posible definición operativa de qué es la orquestación de actividades TEL y CSCL, tanto de una manera gráfica (Figura 2.4 en la página 30), como textual:

La orquestación es el proceso complejo de coordinar una situación de enseñanza/aprendizaje, desde el punto de vista del profesor. La orquestación intenta gestionar (o guiar sutilmente) las diferentes actividades que ocurren en diferentes contextos educativos y niveles sociales, usando diferentes recursos y herramientas de una manera sinérgica. Esta orquestación es especialmente crítica en las transiciones y concurrencias entre estos elementos, y a menudo es guiada por un diseño (en forma de script o no) que puede ser modificado de manera flexible durante la ejecución (automatizada o no) de las actividades, en respuesta a eventos emergentes.

\section{Entornos de Aprendizaje Virtuales, Personales y Distribuidos (VLEs, PLEs y DLEs)}

Los Entornos Virtuales de Aprendizaje (en inglés, VLEs) son plataformas software para el aprendizaje, que se han definido como "los componentes en los que aprendices y tutores participan, en interacciones online de diversos tipos, incluyendo aprendizaje a distancia" [JIS12] . Diversos estudios han reportado que los VLEs son beneficiosos para educadores y estudiantes Kat10. Puesto que la mayoría de los VLEs permiten la definición de actividades y estructuras sociales (p.ej. grupos) y el uso de una variedad de herramientas (p.ej. chats o foros), se puede considerar a los VLEs como ejemplos de sistemas CSCL ampliamente usados en la actualidad en entornos auténticos [Jon05]. Dadas las definiciones de orquestación aportadas (ver más arriba), también podemos decir que los VLEs pueden ser una pieza clave en la orquestación del CSCL mixto que use dichas plataformas. 
Aunque los VLEs son las plataformas más aceptadas por el amplio apoyo institucional con que cuentan, los VLEs no son las únicas plataformas de aprendizaje de amplia difusión. Los Entornos de Aprendizaje Personales (PLEs [vH06]) son sistemas que permiten a los estudiantes tomar el control y gestionar su propio conocimiento (por oposición a los VLEs en los que el educador u otro miembro de la plantilla selecciona y gestiona las herramientas y recursos que los estudiantes deberían usar) [Sev08]. También existen otras plataformas que se han usado en los últimos años para soportar el TEL, como pueden ser las wikis [Leu01] o las redes sociales. De hecho, en los últimos años tanto los docentes como los investigadores han empezado a explorar nuevas herramientas tecnológicas disponibles en la Web (p.ej. las llamadas herramientas "Web 2.0" O'R05, de las que wikis y redes sociales son parte). Todas estas plataformas son muy similares desde el punto de vista arquitectónico, ya que funcionan como "integradores" o puntos de acceso hacia otros servicios ofrecidos por la misma plataforma o por terceros. Por ello, las denominaremos "plataformas de aprendizaje" de manera más general.

Existen estudios que han revelado la preocupación de los docentes sobre el uso de los VLEs, especialmente en cuanto a la carga que implica el preparar en ellos las situaciones de aprendizaje Bow11, o el número reducido de herramientas de que disponen Con10a Bow11. Sobre todo debido a este último, que limita la cantidad y naturaleza de las situaciones de aprendizaje que los docentes pueden proponer, varias iniciativas han emergido en los últimos años para expandir los entornos de aprendizaje más allá de las definiciones de VLE y PLE típicas [Mac10]. [AH10] y Mac10] describen varias de estas aproximaciones para integrar nuevas herramientas en entornos existentes, que llamaremos de manera general "Entornos de Aprendizaje Distribuidos (DLEs).

Una posible variante de DLE es el Entorno Unificado de Aprendizaje en Grupo (en inglés, GLUE!), una arquitectura orientada a servicios para la integración de herramientas externas, desarrolladas en múltiples tecnologías, en diferentes VLEs y plataformas de aprendizaje. Durante esta tesis, nuestras propuestas tecnológicas se enfocarán principalmente en DLEs basados en la arquitectura GLUE, por tres razones: su capacidad para gestionar el ciclo de vida de las herramientas externas (y así, poder orquestarlas de manera semi-automática); su diseño pedagógicamente agnóstico aunque orientado al educador; y el hecho de que da soporte a las dos primeras plataformas que se evaluarían durante la tesis. Sin embargo, debe señalarse que ni GLUE! ni nuestra propia propuesta tecnológica (ver Capítulo 5) se restringe a esas dos plataformas.

\section{Orquestando CSCL mixto en DLEs: Práctica actual y problemas de orquestación}

Después de revisar las fuentes de literatura relevantes para la orquestación de TEL y CSCL, de aprendizaje mixto y de los Entornos de Aprendizaje Distribuidos, se observa cómo la orquestación puede ser un desafío complejo. A continuación se señalan algunos de los retos implicados, como introducción a las contribuciones de la tesis propiamente dichas concernientes a estos problemas, y que se presentan en los siguientes capítulos:

Hacia una visión sintetizada de la orquestación en TEL y CSCL. Como se ha visto en la revisión de literatura, el uso de la palabra "orquestación" en investigación sobre TEL y 
CSCL no es excesivamente coherente, ya que distintos autores remarcan múltiples aspectos de esa orquestación. Aunque hemos intentado aportar una nueva definición de orquestación, ésta no basta para operacionalizar un esfuerzo de investigación solamente sobre ella. Los investigadores necesitan otros apoyos para comunicar las diferencias y similitudes entre sus distintos trabajos concernientes a la orquestación, y es necesario contar con una visión holística que nos permita analizar y eventualmente evaluar propuestas de investigación desde el punto de vista de la orquestación. Nuestra hipótesis es que el tener un marco conceptual más claro sobre qué es y qué implica la orquestación, no sólo ayudará a la hora de proponer herramientas para soportarla, tanto en aspectos particulares como desde un punto de vista global. Un marco conceptual de este tipo se propone en el Capítulo 3, como una de las principales contribuciones de la tesis.

Prácticas docentes en la orquestación de DLEs: la necesidad de guía conceptual. A medida que se introducen las TICs en la educación, el aula (física o virtual) se está convirtiendo en un complejo ecosistema de herramientas y tecnologías [Zha03]. Por otro lado, la investigación pedagógica viene proponiendo métodos y prácticas más complejas que el flujo unidireccional de información típico de las lecciones magistrales Bru08. Sin embargo, la introducción de nuevas tecnologías por sí misma no asegura una mejor experiencia de aprendizaje, pues a menudo se observa cómo las TICs son aplicadas a la educación de una manera que sólo promueve la infrautilización de la tecnología y la imitación de tecnologías anteriores Hop93 Cub01 Wat06 Her08. Aunque la metáfora de la orquestación tiene una larga historia en la literatura educativa, hay una gran escasez de estudios sobre cómo los docentes deben orquestar múltiples TICs. En el caso concreto de los DLEs, dado su reciente nacimiento, no hemos encontrado ninguna descripción detallada de cómo los profesores deben acercarse a la práctica en dichos entornos. Así pues, no tendremos más remedio que emplear métodos inductivos de investigación (bottomup) Gla67 Bar04 para proponer herramientas conceptuales dirigidas a los profesores, p.ej. en forma de buenas prácticas. Puesto que la orquestación de actividades colaborativas en una clase mejorada con tecnología es un proceso complejo que debe tener en cuenta multitud de factores contextuales, técnicos y pedagógicos, podemos intentar aplicar el uso de patrones. Los patrones de diseño Ale77] son una manera de representar soluciones exitosas a problemas recurrentes en un campo de práctica (originalmente, en arquitectura, pero ahora aplicado a múltiples campos), describiendo el núcleo de dicha solución de manera que pueda ser reutilizado en diferentes contextos.

Diseño de Aprendizaje y el "hueco de despliegue". Como ya se ha mencionado, los lenguajes de Diseño de Aprendizaje computacionales no han conseguido tener una amplia aceptación. Una de las posibles razones podría ser la escasez de herramientas de autoría usables por los docentes Neu10. Sin embargo, aun en presencia de este tipo de herramientas (p.ej. Collage HL06a para diseños CSCL), un mayor obstáculo es la particularización y despliegue (automáticos) de los diseños de aprendizaje. Actualmente, existen pocas alternativas para este despliegue, que no aten al practicante a una implementación específica de herramienta de autoría o entorno de ejecución. Aunque estándares como IMS-LD pretendían la interoperabilidad entre herramientas de autoría y entornos de ejecución, casi ningún gran proveedor de plataformas de aprendizaje se ha adherido a la especificación. Otros esfuerzos de investigación que tratan de ir desde el diseño de las actividades hasta su ejecución Fer08 Nod08 tampoco son apropiados para profesores no expertos, ya que requieren un profundo conocimiento de sus respectivas 
tecnologías y lenguajes. Así, actualmente un docente que trabaja sobre un VLE (a menudo provisto/ordenado por su institución) tiene muy pocas probabilidades de poder desplegar sus diseños de una manera sencilla. A menudo, este despliegue debe hacerse manualmente, lo cual es un proceso tedioso y sujeto a errores - especialmente en el caso del CSCL donde muchas veces hay configuraciones de grupos y recursos que varían en el tiempo. El caso es aún más delicado en el caso de los DLEs, ya que son entornos descentralizados, en los que el docente debe acceder a diferentes dominios para gestionar los recursos que usarán los estudiantes. Además, en caso de desear reutilizar/repetir el mismo diseño, dicho proceso debe realizarse de nuevo íntegramente.

Flexibilidad: Desde la improvisación al problema de la gestión y adaptación en tiempo real de las actividades de aprendizaje en DLEs. Como hemos visto, la orquestación no sólo se refiere a la preparación de las actividades de aprendizaje, sino también a la adaptación de las mismas ante eventos inesperados. Éste es un problema conocido de las aproximaciones de guiado, en las que los planes y expectativas se materializan en el soporte tecnológico: la flexibilidad de los diseños y de los entornos de ejecución. Una de las principales tensiones durante la ejecución de actividades de aprendizaje es la que existe entre el guiado y la improvisación Dim07] Tat07 Dil02a], ya que la forma concreta que toman las clases nunca está especificada hasta la última palabra Saw01. El guiado CSCL ha sido criticado a menudo por ofrecer un soporte demasiado rígido Dil02a Dil07d, que puede hacerlo inútil ante ciertos eventos inesperados que ocurran en el aula. En la literatura se han propuesto diversos mecanismos y herramientas para añadir flexibilidad a mecanismos de guiado (p.ej. [Dem08, |VF09a, Doe09]). Sin embargo, estos esfuerzos proponen invariablemente entornos ad-hoc auto-contenidos, no compatibles con los sistemas ampliamente difundidos y usados por los docentes, y mucho menos en un entorno tipo DLE.

Así, podemos concluir que no sólo desplegar diseños de aprendizaje (especialmente guiado CSCL) esta poco soportado en los entornos DLE basados en plataformas de aprendizaje extendidas. Tampoco existen soluciones flexibles para la orquestación de esos diseños desplegados en DLEs. Así, en esta tesis se propone una infraestructura tecnológica que permita este despliegue y adaptación en tiempo de ejecución de diseños de aprendizaje en Entornos de Aprendizaje Distribuidos (DLEs), descrita en el Capítulo 5. 


\section{3. '5+3 Aspectos': Un marco conceptual para la orquestación en TEL}

Una de las primeras preguntas que surge cuando la palabra "orquestación" aparece en una conversación entre investigadores de TEL es "¿Qué quieres decir con orquestación?". En la revisión de literatura sobre orquestación (Capítulo 2) se encontraron varias definiciones y esfuerzos de investigación sobre orquestación con relativamente pocos rasgos comunes. Creemos que una mayor claridad de conceptos sobre qué es la orquestación puede ayudar a los investigadores a estructurar y analizar las innovaciones en entornos TEL auténticos, además de ayudar a la comunidad científica TEL a comunicarse (dando una referencia común de términos) y acumular conocimiento al respecto de la orquestación. En este capítulo se presenta un marco conceptual para la orquestación en TEL, y una definición de orquestación derivada de él. Este marco conceptual, denominado ' $5+3$ Aspectos', será evaluado a lo largo de este capítulo con respecto a la siguiente pregunta de investigación: "¿Podemos proporcionar herramientas conceptuales a los investigadores para clarificar y dar soporte a la investigación relacionada con la orquestación?" (RQ1.1).

\section{'5+3 Aspects': Una definición y marco conceptual para la orques- tación de Aprendizaje Mejorado con Tecnología}

Se ha agrupado la revisión de literatura realizada en ocho temas principales, que se mencionan a menudo al hablar de orquestación en TEL/CSCL:

Diseño/Planificación de las actividades de aprendizaje, relacionado con los campos del Diseño de Aprendizaje [Kop05], y el concepto de "scripting", que se vió en el capítulo anterior (Sección 2.2.2). Sin embargo, este diseño y su posterior despliegue no siempre siguen un ciclo lineal Ber05].

Regulación/Gestión es otro aspecto importante, que engloba problemas como la gestión de clase, de flujo de datos, del tiempo o de los grupos [Dil07d] [Dil08b] [Nir10] [Dil10]. Esta regulación puede hacerse de manera manual/social, o automatizada/mediada por tecnología Dim07. 
Adaptación/Flexibilidad/Intervención Otro aspecto importante que se deriva de la revisión de literatura es el de orquestación como intervención [Dil07d] [Dil09a] [Dil10], cambiando o adaptando los planes establecidos en función del contexto local y las ocurrencias emergentes en el aula. De nuevo, esta adaptación puede hacerse mediante mecanismos sociales que son naturalmente flexibles, o bien a través de sistemas tecnológicos que sean lo bastante flexibles Dil07d] [Kar09].

Percepción/Evaluación Dado que el concepto de orquestación requiere hacer intervenciones en función del contexto y de los eventos emergentes, la percepción de lo que está ocurriendo (en el aula y en las mentes de los estudiantes) tiene una importancia crucial al orquestar un escenario Dil09a] [Bal10]. Esto también incluye mecanismos de evaluación (especialmente, evaluación formativa).

Roles del profesor y otros actores La mayor parte del corpus de literatura sobre orquestación se enfoca principalmente en la perspectiva del profesor [Dil10] [Bal10], donde la presencia de éste es esencial para conseguir la orquestación. Sin embargo no hay razones para desechar la idea de que la orquestación la realicen (total y parcialmente) los alumnos.

Estos cinco aspectos representan la parte sobre qué implica la orquestación en TEL. Sin embargo, hay en la literatura fuentes que enfatizan otros aspectos más bien relacionados con el cómo se realiza la orquestación:

Pragmatismo/Práctica Dillenbourg y otros han notado que las ideas sobre la orquestación tienen mucho que ver con hacer los resultados de la investigación en TEL accesibles para el docente medio, en entornos educativos auténticos (por oposición a experimentos más controlados) Dil09b Dil10 Ham11. Este énfasis en esfuerzos de investigación que cumplan con las restricciones de los entornos auténticos, y sean escalables y sostenibles parece ser una preocupación creciente en la literatura sobre orquestación.

Alineamiento/Sinergia Una de las características más citadas sobre experiencias de aprendizaje bien orquestadas Dil09a [Dil10 [Pri11f] es la coordinación de los elementos a orquestar hacia un "andamiaje sinérgico" [Tab04]. Este alineamiento con respecto a los objetivos de aprendizaje es quizás el principal reto de un profesor al orquestar.

Modelos/Teorías La complejidad de los escenarios de aprendizaje presentes y futuros urge el desarrollo de teorías más robustas sobre la orquestación [Nat07]. En la literatura se han propuesto diversos modelos sobre cómo orquestar, y los profesores lo hacen en las aulas en buena medida guiados por sus teorías, modelos e ideas (a veces implícitas) sobre el aprendizaje.

Así, teniendo en cuenta estos ocho aspectos, podríamos proponer una nueva definición sintética de la orquestación, que incluya la literatura revisada al respecto:

Orquestación es el proceso por el que los docentes y otros actores diseñan, gestionan, adaptan y evalúan las actividades de aprendizaje, alineando los recursos a su disposición para alcanzar el máximo efecto de aprendizaje, informados por la teoría y ciñéndose pragmáticamente a las restricciones contextuales del entorno. 
Este modelo conceptual puede representarse gráficamente (Figura 3.4 en la página 59). Sin embargo, en Pri11e los autores reconocen que hacen falta más datos empíricos para asegurar la utilidad de este marco para el investigador TEL. Una primera evaluación del marco se presenta también en este capítulo.

\section{Evaluación de '5+3 Aspectos': Dos paneles de investigadores}

Aparte de la evaluación subjetiva del marco que se pueda hacer a través de su uso en esta disertación, y de la realimentación (mayormente positiva) recibida de algunos de los miembros de la sub-comunidad científica dedicada a la orquestación en TEL, el marco necesita ser evaluado de manera más formal, respecto a su utilidad para una amplia gama de investigadores TEL/CSCL.

Hemos usado el marco de evaluación CSCL-EREM [JA09b] para diseñar la evaluación de esta segunda generación de herramientas conceptuales para investigadores de la orquestación. Para ello, se han propuesto dos paneles de evaluación (validación por consenso) sobre el marco conceptual, uno con investigadores más jóvenes, y otro con expertos internacionalmente reconocidos en el campo de la orquestación TEL/CSCL. Así, en estos estudios se ha explorado el issue "¿Clarifica '5+3 Aspectos' la noción de orquestación, dando soporte a la investigación relacionada con la orquestación?" (I1). A su vez, esta tensión evaluativa se explora a través de tres declaraciones temáticas (topics), concernientes al perfil y conocimientos previos de los participantes sobre la orquestación (T1), la completitud y coherencia del marco (T2) y la utilidad del marco para la práctica de la investigación (T3). Para la evaluación se ha utilizado una aproximación de método mixto (basada en Mar06b), recogiendo datos cualitativos y cuantitativos a través de cuestionarios en línea y analizándolos para conseguir unas primeras conclusiones parciales de cada estudio, que puedan usarse para destilar conclusiones más globales sobre la evaluación de ' $5+3$ Aspectos' como herramienta conceptual para investigadores.

\section{Un panel de investigadores TEL/CSCL relacionados (RP1)}

El primer panel de investigadores siguió un proceso en el que los participantes primero respondieron a un cuestionario de perfilado (incluyendo conocimientos y definiciones previas sobre orquestación), para luego ser expuestos a un breve material multimedia sobre el marco conceptual. Luego, responderían otro cuestionario evaluando la completitud y utilidad percibidas.

Los participantes fueron voluntarios pertenecientes a cuatro grupos de investigación españoles cercanos a las actividades del autor (los grupos GSIC-EMIC, CETIE, GTI y GAST, con base en Valladolid, Barcelona y Madrid), para asegurar un número mínimo de participantes con cierto interés en el tema de la orquestación TEL/CSCL. De estos grupos, finalmente 22 investigadores finalizaron el estudio. Así, el estudio se puede considerar un éxito, ya que un número elevado de participantes aportaron realimentación generalmente positiva sobre el marco. De las evidencias obtenidas, se pueden extraer ciertas conclusiones en torno a los temas de interés definidos.

El perfil previo de los participantes (T1) nos indicó que los conceptos previos de los participantes eran coherentes con la revisión de literatura realizada, aunque algunos investigadores 
mostraron cierto recelo ante el hecho de que la orquestación se estaba convirtiendo en una "palabra de moda" (buzzword) en TEL. El marco se consideró, de manera general, como lógico, claro y completo (T2), como también lo fueron las definiciones y representaciones aportadas (aunque con menor consenso). Hubo, sin embargo, algunas críticas sobre la estructura o la terminología elegidas al presentar el marco. Finalmente, el marco fue considerado moderadamente útil por los participantes, especialmente como visión global o lista de problemas a considerar en la práctica TEL/CSCL en entornos auténticos. Incluso si los conceptos del marco no se consideraron excesivamente novedosos, hubo indicios de que el marco podría tener un cierto valor pedagógico (para investigadores más noveles).

Sin embargo, dadas las limitaciones del estudio, todavía no era posible concluir la utilidad o completitud del marco, por lo que se realizó un estudio más amplio a continuación.

\section{Un panel más amplio de investigadores TEL/CSCL sobre orquestación reco- nocidos internacionalmente (RP2)}

Este estudio más amplio siguió la misma estructura y materiales usados en el estudio RP1 (con mínimas variaciones). Para este segundo estudio se confeccionó una lista de expertos reconocidos internacionalmente sobre la orquestación en TEL/CSCL. De todos los expertos contactados, finalmente 24 expertos completaron los cuestionarios evaluando el marco.

Los resultados de este panel de investigadores expertos fueron positivos, aunque más heterogéneos y menos entusiastas que en el estudio RP1. Muchos expertos consideraron el marco como relevante a los campos del TEL y del CSCL, señalando su capacidad para dar un marco más amplio dentro del que muchos investigadores podían localizar sus trabajos más focalizados. Sin embargo, también señalaron que el marco proporcionaba una herramienta descriptiva para el análisis, más que una guía normativa sobre cómo resolver los problemas de orquestación. Las principales conclusiones del estudio se pueden resumir de la siguiente manera, atendiendo a los diferentes temas de interés:

En este panel se observó que las concepciones previas de los investigadores expertos (T1) también eran coherentes con la revisión de literatura realizada, aunque sus definiciones tenían una mayor profundidad y riqueza conceptual. Algunos participantes también compartían el recelo hacia la indefinición y el uso liberal que se estaba haciendo del término orquestación, que ocultaba niveles de complejidad subyacentes en la práctica TEL/CSCL. En cualquier caso, el marco fue considerado lógico, claro y completo de manera general (T2), aunque de manera más atemperada. De hecho, se señaló que podía resultar "demasiado completo", de manera que toda práctica TEL/CSCL pudiera caber dentro de dicho paraguas. También se mencionó la notable ausencia de la tecnología de entre los aspectos del marco. De nuevo, el marco fue considerado útil por un número moderado de expertos (T3), con una amplia variedad de opiniones. Aunque los conceptos del marco no se consideraron revolucionarios, sí se confirmó su potencial interés pedagógico para su uso con investigadores noveles que se acercaban por primera vez al campo. Finalmente, hubo ciertas ideas generales emergentes de los datos recabados, especialmente respecto a la falta de acuerdo sobre el papel de los diferentes actores en la orquestación (p.ej., de los profesores, pero no sólo de ellos), o la conveniencia de un marco como el presentado. 


\section{Cerrando la evaluación de ' $5+3$ Aspectos'}

Como se puede observar, las conclusiones de ambos estudios fueron en gran medida paralelas, con los expertos internacionales jugando un papel más crítico dentro de su valoración positiva. Las concepciones previas de los investigadores (T1) confirmaron buena parte de nuestra revisión de literatura, e indicaron la relevancia del concepto y los peligros de dicha relevancia en forma de "modas científicas". El marco fue considerado en general lógico, claro y completo (T2), aunque debe trabajarse en definir mejor sus fronteras. También se puede concluir que una buena parte de los investigadores consultados encontraron el marco útil (T3) como visión global de la práctica TEL/CSCL, y como material pedagógico de iniciación al campo. También emergieron (T4) interesantes tensiones respecto al rol de los distintos actores y la conveniencia de este tipo de marcos conceptuales, y de la noción misma de orquestación.

Naturalmente, estas conclusiones deben ser vistas en el contexto de los estudios que las generaron, y las limitaciones de éstos en cuanto a recogida y análisis de los datos. Así, aunque las conclusiones no son generalizables al campo de TEL en su conjunto, si representan una cierta "sabiduría colectiva" al respecto de qué es la orquestación, y cómo el marco " $5+3$ Aspectos" ayuda en la práctica investigadora.

\section{Un instrumento de investigación derivado de ' $5+3$ ': Una guía de entrevistas/reflexión sobre orquestación}

Este capítulo también presenta un primer instrumento derivado del marco conceptual, que se ha usado principalmente para extraer prácticas recurrentes de orquestación en entornos educativos concretos (véase el siguiente capítulo). Este instrumento tiene una estructura simple, adecuada para su uso en una entrevista semi-estructurada de unos 60 minutos, incluyendo indicaciones para la entrevista (o la reflexión), y hasta 42 preguntas cubriendo los 8 aspectos de la orquestación que define el marco ' $5+3$ Aspectos'. El instrumento completo puede consultarse en el Apéndice A.

Este instrumento fue evaluado de manera preliminar durante los estudios sobre el marco de orquestación (como una parte opcional del estudio). Aunque los datos recogidos son escasos y heterogéneos (en total, sólo 11 investigadores lo usaron durante los estudios RP1 y RP2), se pueden extraer algunas conclusiones preliminares, tales como su aparente mayor utilidad para investigadores menos expertos, su utilidad como lista de elementos a considerar al implementar TEL/CSCL, o su capacidad para generar reflexiones y preguntas de investigación sobre la práctica del TEL. En cualquier caso, la guía debe ser depurada, especialmente si se desea que ofrezca más apoyo a la generación de soluciones de orquestación TEL/CSCL.

\section{Conclusiones: ¿Hacia un nuevo marco?}

Los resultados de las evaluaciones indican que los investigadores participantes, tanto a nivel local como internacional, vieron el marco como una visión general útil del campo de la orquestación, al menos a efectos descriptivos/analíticos (más que normativos sobre cómo orquestar). Especialmente notable fue el uso propuesto por varios participantes como material 
pedagógico para investigadores noveles, y como lista de elementos a tener en cuenta al diseñar innovaciones TEL/CSCL. Estas evaluaciones también constituyeron un acicate para revisar el marco propuesto en este capítulo, hacia una tercera encarnación de esta síntesis de la noción de orquestación. Estas revisiones, que amplían la noción de actores más allá del profesor, y que tienen más en cuenta el papel potencialmente ubicuo de la tecnología en la orquestación, darían como resultado nuevas definiciones de orquestación, así como nuevas representaciones del marco (Figura 3.19, en la página 104):

"La orquestación es el proceso de diseñar y gestionar en tiempo real los procesos de aprendizaje en un escenario TEL auténtico (incluyendo mecanismos de percepción y adaptación). Las responsabilidades en este proceso se comparten entre una serie de actores dependiendo del contexto (a menudo por parte de los docentes, pero posiblemente también de los estudiantes, investigadores o tecnologías), intentando alinear los recursos pragmáticamente hacia un efecto máximo, considerando sus modelos/teorías/creencias."

Aunque es poco probable que lleguemos a una "teoría unificada de la orquestación" en la que todos los investigadores coincidan, en este capítulo hemos propuesto una manera (al parecer, razonable) de organizar una noción actualmente difusa y confusa que, sin embargo, es considerada altamente relevante para las comunidades científicas de TEL y CSCL. Independientemente de la exactitud de nuestras propuestas, al menos servirán para comunicar, entender y discutir la creciente complejidad de la práctica educativa en entornos TEL reales, y a proponer nuevas soluciones fácilmente integrables en nuestro sistema educativo. 


\section{Patrones atómicos como herramientas conceptuales para la orquestación}

A pesar de su floreciente comunidad científica, el CSCL hasta ahora no ha conseguido influenciar la práctica educativa diaria a gran escala, en parte debido a la inherente complejidad de la adopción de esta pedagogía. La noción de orquestación se ha propuesto como tema que engloba esta complejidad. En el caso de la orquestación del CSCL mixto soportado por DLEs, podemos ver cómo la compleja tarea de orquestar puede ser problemática para los docentes, especialmente en aquellos contextos como la educación universitaria donde un profesor muchas veces pone en marcha cursos diseñados por él mismo.

En este capítulo describimos la segunda herramienta de apoyo a la orquestación, que intenta superar este reto: proporcionar a los docentes (especialmente aquellos no expertos) guías conceptuales sobre cómo manejar la orquestación, a lo largo del diseño y puesta en práctica de CSCL mixto en DLEs. Para ello, proponemos una variante de la aproximación de los patrones de diseño [Ale77], como descripciones de problemas recurrentes y núcleos de la solución a dichos problemas. A estos patrones específicamente pensados para apoyar la práctica de la orquestación los hemos denominado "patrones atómicos".

\section{Patrones atómicos para orquestación}

Los patrones atómicos fueron extraídos por primera vez del análisis de actividades diseñadas y puestas en marcha por docentes de educación primaria mientras trataban de integrar una nueva herramienta TIC de trabajo colaborativo en su práctica diaria en clases con amplia presencia de TICs. Este análisis mostró cómo los docentes diseñaban y ponían en práctica sus actividades con la nueva herramienta (incluyendo improvisaciones) usando un número limitado de elementos recurrentes o "rutinas". Nuestra hipótesis es que estos elementos recurrentes podrían ayudar a los docentes en la toma de decisiones recurrentes con respecto a problemas pedagógicos y tecnológicos de bajo nivel. Así, en esta tesis proponemos estos elementos, llamados "patrones atómicos" como herramientas conceptuales útiles para dar soporte a profesores no expertos en la labor de la orquestación de actividades TEL complejas.

En este caso, la variante de los patrones de diseño propuesta, se denominan "atómicos" debido a su pequeña granularidad y simplicidad, que los hace los elementos recurrentes más 
pequeños de práctica de la orquestación. La forma que suelen tomar estos patrones es muy sencilla, incluyendo un nombre identificativo, una breve descripción, así como una serie de breves ejemplos de uso del patrón en la práctica real. Además, estos patrones pueden ser clasificados en función de diversas dimensiones, ya sean la fase en la que se dan (p.ej. en el diseño vs. en la puesta en marcha), las herramientas que usa, el tipo de tarea donde suele aparecer, o el aspecto de orquestación que representan (véase el Capítulo 3). Las principales características de un patrón atómico son:

Heterogéneo Los patrones atómicos cubren muy diferentes tipos de prácticas recurrentes, desde tipos de actividad, uso de recursos, hasta maniobras de gestión de la clase.

Pequeña escala Aunque los patrones atómicos varían en su granularidad, por lo general tienden a ser más pequeños, más simples que otros patrones, que pueden llevar sesiones enteras en su puesta en práctica. Esta naturaleza más de bajo nivel les hace especialmente indicados para "rellenar los huecos" dentro de estos otros patrones más grandes, bien para resolver problemas pedagógicos de más bajo nivel, o para alinear los elementos contextuales a nuestra disposición, dentro del marco del patrón más grande.

Numerosos Debido a su pequeña escala (p.ej. en una sesión de 50 minutos hay tiempo para usar decenas de ellos), su número muchas veces es mayor que el de otros catálogos de patrones. A pesar de que estos elementos son recurrentes, las circunstancias del aula son tan variadas que no existen patrones atómicos que "valgan para todo", de manera que el docente debe manejar un buen número de ellos.

Informal Al contrario que los patrones de diseño originales, los patrones atómicos se definen usando muchos menos campos, de una manera más flexible y sencilla que permita usar gran número de ellos, y usarlos dentro de las restricciones temporales que impone la puesta en marcha en tiempo real.

Concreto/Contextual Finalmente, y en oposición a otros patrones de diseño, que intentan permanecer lo más abstractos que sea posible para facilitar su aplicabilidad en múltiples contextos, los patrones atómicos son muy contextuales. De hecho, a menudo hacen referencia explícita (incluso en su título) a características concretas de la clase, las herramientas, los grupos, etc. Esto les hace fácilmente localizables y reconocibles desde el punto de vista del docente.

\section{Una aproximación basada en patrones multi-nivel para la orques- tación de actividades CSCL en DLEs}

Aparte de la noción misma de "patrón atómico", y los diferentes catálogos de patrones extraídos de las prácticas observadas (véase el Apéndice B), esta tesis propone también una aproximación para la extracción, desarrollo, combinación (con otros patrones) y uso de los patrones atómicos (p.ej. en acciones de desarrollo profesional docente). Especialmente importante es el hecho de que distintos tipos de patrones, con distintos orígenes y niveles de contextualización, se pueden combinar para dar una mayor riqueza contextual a los diseños y las puestas en marcha de actividades de aprendizaje. Así, proponemos que los patrones atómicos pueden 
"cubrir el abismo" entre el consejo abstracto que dan muchos otros patrones de diseño, y la actuación contextualizada que el docente debe realizar en el aula [Pri11f].

La aproximación tiene cuatro fases: 1) la extracción de patrones atómicos a partir de práctica docente auténtica dentro de nuestro foco de interés (p.ej. CSCL mixto usando DLEs); 2) el catálogo de patrones es refinado y categorizado, para seleccionar los patrones con más potencial y facilitar su manejo posterior; 3) los patrones son combinados con otros patrones de diseño de más alto nivel, especialmente aquellos apropiados para nuestro foco de interés (p.ej. para CSCL, los Patrones de Flujo de Aprendizaje Colaborativo [HL09]); y 4) este catálogo combinado es usado en el campo de aplicación deseado (p.ej. en talleres de formación docente para la promoción de CSCL mixto). Eventualmente, el ciclo puede volver a comenzar, con extracciones o refinamientos adicionales del catálogo.

\section{Otros usos de los patrones atómicos}

Representando la orquestación a través de patones atómicos Aparte del uso como soporte para orquestación (es decir, el diseño y la puesta en marcha) por parte de los docentes, los patrones atómicos también pueden ser usados por investigadores como herramienta de análisis para entender cómo transcurre la orquestación de un episodio de práctica educativa concreto. Estos análisis pueden tomar una forma textual, o bien puede representarse gráficamente, incluyendo la secuencia de actividades, roles, herramientas usadas y los patrones atómicos usados en cada momento Pri11d.

Usando los patrones atómicos para desarrollar tecnología Otro posible uso de estos patrones atómicos es como herramienta de extracción de requisitos y funcionalidades a la hora de diseñar software educativo, especialmente aquel que tiene algún papel en la orquestación de las actividades de aprendizaje. De hecho, un análisis de este tipo se ha realizado durante la tesis doctoral, para determinar la hoja de ruta y funcionalidades de GLUE!-PS, un soporte tecnológico para la orquestación de CSCL mixto en DLEs (véase Capítulo 5. Sin embargo, esta utilidad no ha sido evaluada formalmente, algo que dejamos para trabajos futuros.

\section{Evaluando los patrones atómicos: Cuatro talleres de desarrollo profesional del profesorado}

Durante el desarrollo de la tesis se ha seguido el método de ingeniería Adr93] a la hora de investigar sobre los patrones atómicos como herramienta conceptual de soporte a la orquestación para docentes. Concretamente, las cuatro iteraciones de la investigación se han evaluado usando cuatro talleres de desarrollo profesional docente, uno por cada iteración. Estas experiencias de evaluación se han desarrollado en dos contextos educativos diferentes: las dos primeras en un colegio de educación primaria (centradas en la integración de una nueva herramienta colaborativa en clases presenciales auténticas), y las dos siguientes en la Universidad de Valladolid (más centrados en la orquestación de CSCL mixto usando DLEs). La elección de este tipo de "experiencias situadas" se debió al énfasis de la investigación en orquestación de abordar entornos 
educativos reales y de dirigirse a docentes medios, no expertos. Así, la evaluación ha transcurrido a lo largo de los siguientes eventos de recogida:

- Iteración \#1: Primer intento de taller de profesorado basado en patrones atómicos en educación primaria (TW1)

- Iteración \#2: Un segundo taller de profesorado basado en patrones atómicos en educación primaria (TW2)

- Iteración \#3: Generación de un nuevo catálogo de patrones atómicos y primer taller con profesores de educación superior (TW3)

- Iteración \#4: Un segundo taller de profesorado en educación superior (TW4)

La evaluación se ha centrado sobre todo en una tensión de evaluación predefinida (¿son los patrones atómicos útiles para los docentes a la hora de orquestar?), pero otra tensión también ha emergido en las últimas iteraciones de evaluación (¿es útil tener patrones atómicos embebidos en las tecnologías educativas, y de qué manera?). Estas tensiones se han explorado a través de diferentes dimensiones o temas, incluyendo la utilidad de los patrones a lo largo del ciclo de vida de las actividades, la influencia de la propia orquestación de los talleres en la utilidad percibida, o la influencia de la experiencia y creencias previas de los participantes en dicha utilidad.

Las conclusiones generales que se han extraído de la evaluación a través de las cuatro iteraciones, incluyen la idea de que los patrones atómicos fueron considerados útiles y cercanos a la práctica docente diaria por la mayor parte de los participantes. También se pudo concluir que los talleres, en su encarnación final tras varias depuraciones, eran adecuados para mostrar el potencial de los patrones atómicos (aunque, por tanto, es difícil separar el efecto de los patrones del de los talleres que los usaban). Se observó también cierta tendencia de percepción de mayor utilidad de los patrones por parte de los profesores más noveles, si bien la correlación al respecto no era perfecta. También pudimos concluir que los talleres y los patrones atómicos tenían un poder limitado para cambiar creencias y actitudes hacia el CSCL, aunque al menos ofrecían una visión más concreta y realista de cómo llevarlo a cabo. Respecto a la forma de incorporar patrones atómicos a la tecnología, los participantes parecían preferir la automatización de ciertos patrones sobre otras formas como los sistemas de recomendación.

\section{Conclusiones, relevancia y trabajo futuro sobre los patrones atómicos}

A lo largo de este capítulo se ha planteado la idea de utilizar "patrones atómicos" para dar solución al problema de la falta de guías concretas sobre cómo orquestar que sufren los docentes, especialmente los no expertos. Más aún, se ha propuesto una aproximación para la extracción, combinación y uso de estos patrones atómicos en acciones de formación de docentes hacia la práctica de la orquestación. Esta aproximación se ha probado en dos contextos concretos muy distintos (educación primaria y universitaria), a través de experiencias situadas en talleres de desarrollo profesional docente, con resultados prometedores. En general, los profesores participantes apreciaron la capacidad de los patrones atómicos para hacer más concretas las ideas de 
práctica CSCL que hasta entonces habían sido difusas. Aunque la evaluación realizada tiene diversas limitaciones (especialmente en cuanto a su generalizabilidad), se ha cumplido con creces el objetivo de proporcionar herramientas conceptuales para la orquestación. También se han señalado algunas direcciones futuras de investigación, como el dirigirse a profesores aún más noveles (incluso estudiantes de universidad), aplicar la aproximación propuesta a otras pedagogías o contextos, o la combinación de este tipo de patrones con otras herramientas tecnológicas para dar soporte a la orquestación, como la que se describe en el siguiente capítulo. 
434 


\section{GLUE!-PS: Una arquitectura y modelo de datos para la orquestación de diseños de aprendizaje en Entornos Distribuidos de Aprendizaje}

Tal y como se vió en el Capítulo2, la orquestación de actividades CSCL en el contexto de los Entornos de Aprendizaje Distribuidos (DLEs) conlleva varios problemas para los docentes: en primer lugar, no existe un soporte adecuado para el despliegue de sus diseños de aprendizaje a lo largo del DLE; además, la modificación en tiempo de ejecución de un diseño CSCL desplegado en un DLE obliga a realizar múltiples operaciones manuales en distintos dominios. De esta manera, podemos afirmar que los DLEs actuales son difícilmente "orquestables", al menos para un profesor bajo las restricciones típicas de un entorno educativo auténtico.

Este capítulo propone las principales contribuciones tecnológicas de la tesis: el Entorno Unificado de Aprendizaje en Grupo - Guiado Pedagógico (en inglés, GLUE!-PS). GLUE!-PS es una arquitectura orientada a servicios (y un modelo de datos subyacente) que permite desplegar diseños de aprendizaje (expresados en una variedad de lenguajes) sobre DLEs basados en plataformas de aprendizaje ampliamente difundidas. Es interesante notar que durante este capítulo se propone, analiza y evalúa el sistema GLUE!-PS desde el punto de vista del actor que normalmente realiza la orquestación: el docente (no experto en CSCL).

\section{Entorno Unificado de Aprendizaje en Grupo - Guiado Pedagógi- co (GLUE!-PS)}

El problema tecnológico antes mencionado (el despliegue y gestión en tiempo de ejecución de diseños de aprendizaje) tiene dos vertientes principales: la de procurar la interoperabilidad entre múltiples lenguajes de diseño de aprendizaje (LD) y múltiples plataformas de aprendizaje; y la de coordinar dichos diseños a lo largo del DLE (tanto al desplegar como al modificar en tiempo de ejecución). La arquitectura GLUE! AH12a integra plataformas de aprendizaje con herramientas externas, dando así varias implementaciones posibles de DLE - y por tanto, 
facilitando la coordinación de recursos a lo largo del DLE. Por tanto, en el desarrollo de GLUE!PS se usará la arquitectura GLUE! para solventar la segunda vertiente, concentrándonos en el caso de GLUE!-PS en el problema de la interoperabilidad entre múltiples lenguajes de diseño y múltiples plataformas de aprendizaje.

A pesar de los esfuerzos hacia la estandarización del diseño de aprendizaje (ejemplificados principalmente por la especificación IMS-LD [IMS03a]), dichos estándares no son utilizados ampliamente, y en la actualidad podemos encontrar múltiples lenguajes y herramientas de autoría distintos para describir diseños de aprendizaje. Otro tanto ocurre con las plataformas de aprendizaje, ya que existen múltiples variantes e implementaciones que generalmente no son interoperables entre sí (ni con las antes mencionadas herramientas de autoría). En esta situación, en vez de proponer una nueva herramienta o lenguaje de autoría, o un nuevo entorno de ejecución, proponemos una arquitectura destinada a hacer que los diseños de aprendizaje expresados en lenguajes existentes, puedan desplegarse en plataformas de aprendizaje ampliamente difundidas en la actualidad. Así, nuestro principal principio de diseño es buscar la máxima aceptación en la comunidad (educativa y de desarrolladores de los distintos sistemas), tratando de cubrir las necesidades de los docentes no expertos, en escenarios educativos auténticos.

Arquitectura de GLUE!-PS Siguiendo este principio de máxima aceptación, proponemos GLUE!-PS como un modelo de integración de bajo acoplamiento, en forma de arquitectura orientada a servicios (SOA, Pap03], de tres capas (ver Figura 5.4 en la página 213). En esta arquitectura, $\mathrm{m}$ herramientas/lenguajes de LD y n plataformas de aprendizaje se adaptan entre sí, a través de una capa software intermedia y dos juegos de adaptadores. Este uso del patrón Adaptador Gam95 Mon03 pretende reducir el esfuerzo de desarrollo, ya que el código de integración necesario es asumido en buena parte por dicha capa intermedia. La arquitectura también define unos contratos REST Fie02 sencillos, y usa los mecanismos y APIs nativos de las plataformas para desplegar los diseños y gestionarlos en tiempo de ejecución.

Estos dos juegos de adaptadores se usan para "envolver" los contratos y modelos de datos variables de las distintas herramientas de autoría y plataformas de aprendizaje, reduciéndolos a dos contratos genéricos y homogéneos. Así, se facilita la interoperabilidad de herramientas de LD y plataformas de aprendizaje sin necesidad de modificar sus respectivas implementaciones (favoreciendo así la aceptación por parte de los implementadores y de las instituciones). Esto también permite el desarrollo independiente de los distintos adaptadores (incluso por parte de terceros) y la extensibilidad del sistema a un coste bajo (ya que cada nuevo adaptador de LD permite automáticamente el despliegue en todas las plataformas ya soportadas, y viceversa). De esta manera, GLUE!-PS representa una solución de compromiso entre las diferentes expresividades de los lenguajes de LD y las funcionalidades variables que las plataformas de aprendizaje tienen actualmente. Además, desde el punto de vista del docente o de su institución, permite conservar las infraestructuras existentes (de LD o de entorno de aprendizaje) a la vez que da una solución eficiente en tiempo para el docente (comparada con las alternativas manuales actuales), al problema del despliegue y modificación de sus diseños.

Lingua Franca de GLUE!-PS (GLUE!-PS LF): Un modelo de datos para desplegar y gestionar diseños de aprendizaje en DLEs Resulta claro que el modelo de datos que se emplee en el elemento central de GLUE!-PS (denominado GLUE!-PS Manager) como lenguaje 
intermedio para traducir de lenguajes de LD a plataformas es uno de los factores decisivos para el éxito de la propuesta, ya que influye en el coste de desarrollo de los distintos elementos de la arquitectura, así como en la expresividad del diseño que se conserva durante el despliegue (que puede llegar a hacer que el diseño desplegado ya no cumpla los requisitos pedagógicos del diseño original). Así, hemos analizado y comparado los principales lenguajes de LD y plataformas de aprendizaje, para determinar las principales características de dichos lenguajes que son desplegables en tales plataformas (véase Apéndice C).

El resultado de este análisis ha servido para construir un modelo de datos que representa las capacidades de guiado de los lenguajes de LD existentes, en tanto en cuanto las plataformas de aprendizaje actuales las soportan. El modelo propuesto (que hemos llamado "lingua franca de GLUE!-PS", o GLUE!-PS LF) aparece en la Figura 5.7 de la página 223 cada diseño se compone de una serie de actividades, que pueden ser estructuradas en estructura de árbol y pueden ser secuenciadas. Cada actividad puede ser realizada por una serie de roles funcionales, y es mediada por uno o más recursos (que pueden ser a su vez herramientas o documentos). Un diseño de aprendizaje puede ser particularizado para su despliegue, y cada uno de estos despliegues es una contextualización de dicho diseño para un entorno de aprendizaje (o sea, un DLE) determinado. En dicho despliegue, los participantes concretos que toman parte en las actividades de aprendizaje deben ser especificados. Para cada actividad del diseño, existen una o más actividades instanciadas, una por cada grupo de participantes que la realiza. Cada grupo que realiza una actividad requerirá el uso de los antes mencionados recursos, o de instancias de las herramientas definidas en el diseño.

Analizando GLUE!-PS como herramienta de orquestación Aunque la presente tesis presenta tres contribuciones principales (el marco ' $5+3$ Aspectos', los patrones atómicos y el sistema GLUE!-PS) que deben entenderse como aproximaciones independientes al soporte de distintos aspectos de la orquestación, estas contribuciones se han informado mutuamente en su desarrollo. De hecho, el soporte a la orquestación ofrecido por GLUE!-PS se puede analizar bajo el punto de vista de los "aspectos de orquestación" propuestos en el Capítulo3, observándose que dicho soporte se produce principalmente en las dimensiones de Diseño, Gestión, Adaptación y Pragmatismo. Además, también se han utilizado los patrones atómicos propuestos en el Capítulo 4 para analizar el potencial soporte que este sistema tecnológico puede dar a dichos patrones, y para guiar el desarrollo de GLUE!-PS en función de la implementación de una serie de estos patrones atómicos. Así, este capítulo ofrece algunos ejemplos de cómo las contribuciones de la tesis descritas en los anteriores capítulos pueden ser usadas como "lente analítica" para conceptualizar trabajos de investigación relacionados con la orquestación.

Implementación de referencia de GLUE!-PS (GLUE!-PS RI) A lo largo del trabajo de la tesis, y con el principal propósito de evaluar la propuesta del GLUE!-PS, se ha desarrollado iterativamente un prototipo de la arquitectura y modelo de datos propuestos (que denominamos GLUE!-PS RI). Esta implementación de referencia incluye un prototipo del servicio GLUE!PS Manager, así como dos juegos de adaptadores de LD y LE mínimos (con dos adaptadores cada uno). Los adaptadores implementados cubren el principal lenguaje de diseño de aprendizaje (IMS-LD) así como los diseños realizados con otra herramienta de autoría, el Pedagogical Pattern Collector. Asimismo, se ha implementado un adaptador para la plataforma de aprendizaje más 
extendida (Moodle), y para otra plataforma más ligera y flexible, basada en un motor wiki (MediaWiki). Es importante señalar que esta implementación nos permite evaluar las ideas propuestas a través de una solución usable, pero de complejidad moderada, y facilita también el trabajo de potenciales implementadores de la arquitectura.

\section{Evaluando GLUE!-PS}

Tal y como se describe en el Capítulo 1, se ha seguido el método de ingeniería Adr93 para estructurar el trabajo de investigación sobre el GLUE!-PS como herramienta tecnológica de soporte a la orquestación de CSCL mixto en DLEs. Tal y como define este método, las propuestas se han evaluado de manera iterativa a medida que las propuestas y su implementación evolucionaban. Para realizar esta evaluación, al igual que se hizo para las contribuciones anteriores, hemos utilizado tanto el marco de evaluación CSCL-EREM [JA09b] como la aproximación de método mixto derivada de Mar06b.

La evaluación se ha centrado en analizar el soporte a la orquestación a través de temas que representaban las cuatro principales dimensiones de orquestación en las que GLUE!-PS supuestamente proporcionaba soporte: la capacidad para desplegar diseños, la eficiencia en tiempo, su potencial de uso en práctica real, y la capacidad para realizar cambios en tiempo de ejecución. Así, la evaluación se ha realizado a través de una variedad de técnicas, en las distintas iteraciones:

- En la primera iteración, la propuesta del GLUE!-PS se ha evaluado analíticamente, mediante la traducción de un diseño de aprendizaje, usado como benchmark por la comunidad de LD, desde dos lenguajes de diseño (IMS-LD y LDL) hasta su despliegue en dos plataformas de aprendizaje muy distintas (Moodle y MediaWiki).

- En la segunda iteración, un primer prototipo funcional de GLUE!-PS se ha evaluado a través de una serie de experiencias piloto de despliegue, así como a través de un taller de desarrollo profesional docente (ante profesores no expertos aunque mayoritariamente técnicos) y otro taller de profesores y profesores-investigadores expertos.

- En la tercera iteración se ha realizado una primera experiencia real de uso de GLUE!-PS, en una asignatura a nivel de Máster en la Universidad de Valladolid, en el que una profesora experta en CSCL desplegó un diseño de aprendizaje colaborativo hecho en IMS-LD, hasta un DLE basado en wikis.

- En la cuarta iteración se implementó la capacidad de modificación de actividades en tiempo de ejecución, y se realizaron dos experiencias reales más en cursos de la Universidad de Valladolid (con una profesora experta y otra más novel). Finalmente, también se evaluó el GLUE!-PS en otro taller de profesorado con docentes no expertos en CSCL, provenientes de múltiples disciplinas.

De la evaluación incremental a través de las cuatro iteraciones se ha concluido que GLUE!PS permite a los docentes no expertos desplegar diseños de aprendizaje expresados en múltiples lenguajes de diseño, a lo largo de múltiples DLEs, preservando las cualidades esenciales del 
diseño hasta el punto que las propias plataformas las soportan, aunque actualmente presenta limitaciones en su uso, debido a que los diseños deben particularizarse de antemano, a la ausencia de información y control sobre información temporal, y a la dependencia del sistema en la fiabilidad de diversos servicios externos.

También se ha observado que el uso del prototipo de GLUE!-PS tan sólo es ligeramente más eficiente en tiempo que las alternativas, en la primera definición y utilización del diseño. Sin embargo, GLUE!-PS es claramente más eficiente cuando el diseño se reutiliza, o cuando hay estructuras complejas de recursos y grupos.

Una amplia variedad de profesores indicaron que usarían el sistema GLUE!-PS en su práctica real diaria, al menos para diseños CSCL de cierta complejidad, ya que se adapta a las necesidades del docente, y siempre que se mejore la usabilidad y fiabilidad del prototipo, y se proporcione soporte y entrenamiento adecuados. También se señaló como deseable el tener funcionalidades de gestión de tiempos, y para la visualización de las consecuencias que las acciones sobre GLUE!-PS pueden tener sobre la presentación final de las actividades desplegadas.

Finalmente, se han recogido evidencias de que las capacidades de GLUE!-PS para modificar las actividades en tiempo de ejecución cubren la mayor parte de los escenarios problemáticos frecuentes, especialmente en cuanto a gestión de grupos y de recursos. Los participantes notaron que solucionar dichas situaciones problemáticas con GLUE!-PS era sencillo e intuitivo, aunque GLUE!-PS no aporta apoyo conceptual a dicho proceso.

\section{Conclusiones, relevancia y trabajo futuro sobre GLUE!-PS}

Al proporcionar una arquitectura abierta y extensible que incluye interfaces de usuario razonablemente usables, creemos que GLUE!-PS puede favorecer la adopción del Diseño de Aprendizaje por parte de los docentes. Asimismo, la relativa simplicidad de la arquitectura y del modelo de datos favorecen la adopción de este sistema por parte de la comunidad (de desarrolladores software así como de instituciones educativas).

La evaluación de los prototipos de GLUE!-PS, centrada en las cuatro principales dimensiones de orquestación que soporta, han extraído crecientes evidencias de utilidad (a medida que los prototipos del sistema estaban más completos y depurados). De esta manera, en el punto final de esta tesis, docentes no expertos han sido capaces de diseñar y desplegar actividades CSCL de complejidad media, a lo largo de dos DLEs distintos, usando GLUE!-PS. Los docentes consideraron este proceso eficiente y usable en su práctica diaria (si bien con algunos "peros"). Las evaluaciones también han descubierto algunos obstáculos para esta eficiencia y para la adopción por docentes en general, como es la naturaleza iterativa del despliegue que hacían los profesores en las experiencias reales, o el esfuerzo que representa la particularización previa de los diseños.

Las evaluaciones realizadas hasta el momento sobre GLUE!-PS presentan una serie de limitaciones, que deberían ser resueltas en el futuro, como por ejemplo la escasa variedad de los diseños realizados por los docentes durante las experiencias. Otras líneas de trabajo futuro relevantes incluyen la extensión o el uso de GLUE!-PS por parte de otros sistemas que proporcionen soporte más avanzado a la orquestación (p.ej. sistemas de monitorización), investigar más a fondo el problema de la particularización de los diseños CSCL/TEL (incluyendo la noción de co-orquestación con los alumnos), la extensión de la aplicabilidad de GLUE!-PS más allá del 
espacio web (p.ej. en escenarios educativos ubicuos o que usen la realidad aumentada), o la investigación de los efectos de combinar las herramientas tecnológicas y conceptuales de esta tesis, para promover innovaciones en la práctica educativa. 


\section{Conclusiones y trabajo futuro}

En los campos de investigación del TEL y del CSCL, el cada vez más complejo diseño y gestión de los múltiples elementos de un escenario educativo (actividades, herramientas, grupos, contextos, etc.) se ha denominado "orquestación" en los últimos años. Este término también emerge de una preocupación creciente por la falta de adopción de muchos de los avances realizados en estos campos, en la práctica educativa diaria. La presente tesis ha estudiado esta noción holística de orquestación en TEL, y se ha marcado como objetivo el proponer, desarrollar y evaluar herramientas (tecnológicas y conceptuales) que den soporte a esta orquestación. Especialmente, nos hemos centrado en la orquestación de actividades de CSCL mixto (como ejemplo de aproximación pedagógica compleja de orquestar), soportadas por Entornos de Aprendizaje Distribuidos (DLEs) que incluyan una plataforma de aprendizaje y herramientas de aprendizaje externas.

Durante la tesis se ha seguido un método de ingeniería en ciclos iterativos de información, proposición, análisis y evaluación. Tras una revisión de la literatura sobre el tema, detectamos varios problemas tecnológicos y conceptuales relevantes en el campo: a) la multiplicidad de significados de la palabra "orquestación" en el campo del TEL y del CSCL (punto de vista del investigador); b) la falta de guías conceptuales claras sobre cómo realizar la orquestación (punto de vista del profesor); y c) la ausencia de un soporte tecnológico adecuado para el despliegue y modificación en tiempo de ejecución de actividades CSCL mixtas a realizarse sobre DLEs.

Así, durante la tesis se han realizado una serie de acciones concernientes a los tres objetivos parciales derivados de estos problemas:

1. Clarificar el concepto de orquestación en el campo de TEL y CSCL. Tras revisar y clasificar una amplia selección de la literatura concerniente a la orquestación TEL/CSCL, hemos propuesto un marco conceptual (denominado '5+3 Aspectos', ver Capítulo 3), que organiza la orquestación en ocho aspectos: Diseño, Gestión, Evaluación, Adaptación, Rol de los actores, Teoría, Pragmatismo y Sinergia. La utilidad de este marco ha sido evaluada a través de dos paneles de investigadores (uno con investigadores más jóvenes y cercanos, y otro con expertos reconocidos internacionalmente). Los resultados de la evaluación mostraron que los participantes veían el marco como una completa visión general del campo que proporcionaba soporte descriptivo a los investigadores, y que tenía un cierto valor pedagógico para su uso con investigadores noveles. Así, podemos afirmar que este marco ha completado el objetivo marcado de clarificar las nociones del campo, y ya ha sido utilizado en práctica investigadora real tanto a lo largo de esta tesis, como en el trabajo de otros colegas GR12. 
2. Proporcionar soporte conceptual a los docentes no expertos, sobre la orquestación de CSCL mixto en DLEs. En este caso, la tesis propone la noción de "patrones atómicos" (descrita en el Capítulo 4) como herramienta conceptual útil en este sentido. Los patrones atómicos son elementos recurrentes en la práctica de la orquestación, de grano fino y contextualizados, que los docentes pueden utilizar para diseñar y gestionar en tiempo real sus actividades, ciñéndose a las restricciones de su contexto concreto. El uso de estos patrones se ha evaluado a través de cuatro talleres de formación con docentes, dos de ellos en educación primaria y otros dos en educación superior. La evaluación de los talleres nos proporcionó evidencias de su utilidad, especialmente al usarse en combinación con otros patrones pedagógicos de más alto nivel. Aunque esta utilidad se veía influenciada por otros factores como las creencias o la experiencia docente de los participantes, se ha podido concluir que los patrones atómicos proporcionan consejo concreto y útil que puede ayudar a los docentes no expertos en la orquestación de CSCL mixto usando DLEs.

3. Proporcionar soporte tecnológico a la orquestación de CSCL mixto en DLEs. Tras analizar el contexto de la práctica real del CSCL mixto y las distintas plataformas de aprendizaje ampliamente difundidas, hemos propuesto el Entorno Unificado de Aprendizaje en Grupo - Guiado Pedagógico (en inglés, GLUE!-PS), una arquitectura y modelo de datos subyacente que permite el despliegue de diseños de aprendizaje (expresados en múltiples lenguajes) sobre DLEs (basados en una variedad de plataformas de aprendizaje). Estas propuestas tecnológicas se evaluaron primero de manera analítica, y luego a través de pilotos, talleres con docentes y experiencias de uso en cursos reales. Las evaluaciones mostraron que una amplia variedad de profesores consideraron el soporte que GLUE!-PS proporciona como útil, tanto en el despliegue como en la modificación flexible de las actividades. Las evaluaciones en entornos reales también nos permitieron explorar los límites de este soporte a la orquestación: la implementación iterativa de diseños seguida por muchos docentes, las dificultades de gestionar la particularización de los diseños más complejos, o la falta de realimentación visual sobre el aspecto final del despliegue. En cualquier caso, los prototipos de GLUE!-PS han mostrado el potencial de la propuesta GLUE!-PS para soportar el despliegue y gestión en tiempo de ejecución, para docentes provenientes de múltiples disciplinas, cumpliendo así con nuestro tercer objetivo.

Este cumplimiento de estos tres objetivos parciales nos conduce a afirmar que la tesis ha alcanzado su objetivo de proponer herramientas conceptuales y tecnológicas que den soporte a los investigadores en entender la orquestación, y a los docentes en ponerla en práctica (en entornos de CSCL mixto con DLEs). El número de publicaciones asociadas a la tesis (incluyendo dos artículos en revistas internacionales, un capítulo de libro, y seis artículos en conferencias internacionales, entre otras) y la influencia de algunas de las contribuciones en proyectos de investigación financiados recientemente, dan indicadores adicionales de la relevancia del trabajo realizado.

En cuanto a las líneas de trabajo futuro que esta tesis ha abierto, podemos destacar algunas de corte más continuista/evolutivo con respecto al trabajo de la tesis: refinar y evaluar más ampliamente el marco ' $5+3$ Aspectos', la aplicación de los patrones atómicos al desarrollo inicial docente, la aplicación de GLUE!-PS a otros contextos y su extensión hacia soportes más avanzados para la orquestación. Sin embargo, también hay algunas líneas más rupturistas que puede ser interesante explorar: un estudio más extenso y profundo sobre los problemas y 
soluciones sobre la particularización de diseños de aprendizaje (incluyendo la posibilidad de una co-orquestación con los estudiantes), o la exploración de las sinergias entre las herramientas conceptuales y tecnológicas de la tesis, a la hora de conseguir innovaciones en la práctica docente en entornos reales. 
444 